

\section{Isabell Stamm}

Unternehmerfamilien 


\section{Isabell Stamm}

\section{Unternehmerfamilien}

Über den Einfluss des Unternehmens auf Lebenslauf, Generationenbeziehungen und soziale Identität

Verlag Barbara Budrich Opladen • Berlin • Toronto 2013 
Bibliografische Information der Deutschen Nationalbibliothek

Die Deutsche Nationalbibliothek verzeichnet diese Publikation in der Deutschen Nationalbibliografie; detaillierte bibliografische Daten sind im Internet über

http://dnb.d-nb.de abrufbar.

Alle Rechte vorbehalten.

(C) 2013 Verlag Barbara Budrich, Opladen, Berlin \& Toronto

www.budrich-verlag.de

$$
\begin{array}{ll}
\text { ISBN } & 978-3-8474-0050-9 \\
\text { eISBN } & 978-3-8474-0303-6 \\
\text { (eBook) }
\end{array}
$$

Das Werk einschließlich aller seiner Teile ist urheberrechtlich geschützt. Jede Verwertung außerhalb der engen Grenzen des Urheberrechtsgesetzes ist ohne Zustimmung des Verlages unzulässig und strafbar. Das gilt insbesondere für Vervielfältigungen, Übersetzungen, Mikroverfilmungen und die Einspeicherung und Verarbeitung in elektronischen Systemen.

Umschlaggestaltung: bettina lehfeldt graphic design, Kleinmachnow

Lektorat: Magdalena Kossatz, Berlin 


\section{Danksagung}

Die Ergebnisse dieser Arbeit sind zu einem großen Teil im Rahmen des interdisziplinären Forschungsprojektes „Generationen in der Erbengesellschaft", genauer gesagt in dem soziologischen Teilprojekt „Von Generation zu Generation - Der Nachfolgeprozess in Familienunternehmen" entstanden. Gefördert wurde dieses von der Volkswagenstiftung, die uns auch den Austausch mit anderen Nachwuchswissenschaftlern ermöglichte. Mein größter Dank gilt meinem Betreuer und dem Projektleiter Martin Kohli, der die Anregung zu diesem Thema gab, die Erarbeitung der Thesen kritisch begleitet hat und für Fragen immer zur Verfügung stand. Mit Nicole Schmiade habe ich kollegial zusammengearbeitet, intensive fachliche Diskussionen geführt und viele heitere und nachdenkliche Stunden der Interviewführung verbracht. Mit den Kollegen am Zentrum für Literatur- und Kulturwissenschaften in Berlin sowie an der Universität Zürich konnten wir einen offenen, interdisziplinären Dialog pflegen. Diese Arbeit wurde durch die Einbindung in das Institut für Soziologie an der Freien Universität Berlin sehr bereichert. Bedanken möchte ich mich bei Jochen Roose für die zügige Erstellung des Zweitgutachtens sowie für seine stete Gesprächsbereitschaft. Das Engagement der Kollegen in Auswertungsrunden oder dem Doktorandenkolloquium hat mich motiviert und die Arbeit inhaltlich geschärft. Für ihre konstruktiven Anregungen $\mathrm{zu}$ den einzelnen Kapiteln und dem methodischen Vorgehen danke ich Bettina Völter, Christina Lubinski, David Glowsky, Sabine Pokorny, Sylvia Kämpfer und Tanja Müller. Jana Windwehr und Maria Jessen haben mit sorgfältiger Korrekturarbeit den Text rund gemacht. Bei meinen Eltern bedanke ich mich für ihre Befürwortung meiner Ausbildung, meiner Mutter für ihren „Anonymitätscheck“ der Falldarstellungen, meinem Vater für stetige Literaturhinweise. Die besondere Allianz mit meiner Freundin Becky hat uns trotz Kleinkindern Zeit geschaffen, an unseren Dissertationen zu schreiben. Gewidmet ist die Arbeit meinem Mann Jens, dessen Vertrauen und liebevolle Unterstützung mir einen wichtigen Rückhalt gibt. 



\section{Kurzfassung}

In der vorliegenden Arbeit wird der Einfluss des Unternehmens auf das Leben und das Beziehungsgefüge in Unternehmerfamilien zum Gegenstand einer empirischen Untersuchung. Damit wird eine soziale Einheit soziologisch hinterfragt, die trotz ihrer Bedeutung für unsere moderne Ökonomie noch wenig Beachtung gefunden hat. Mit dem Begriff Unternehmerfamilie sind solche Familien gemeint, die Betriebsvermögen besitzen und/oder über ihre Tätigkeit im Unternehmen Einkommen generieren, deren familiärer Alltag also nicht an Erwerbsarbeit ausgerichtet ist, sondern von einem (oder mehreren) Unternehmen beeinflusst wird. Der transgenerationale Transfer von Eigentum und Leitung in solchen Unternehmen (kurz: der Nachfolgeprozess) wird als Reproduktionsmechanismus des Unternehmenseinflusses betrachtet. Das Ziel der Untersuchung besteht darin, analytisch zu fassen, auf welche Weise das Unternehmen auf das Leben von Unternehmerfamilien einwirkt. Die Bedingungen ihrer spezifischen Konstellation, die sich daraus ergebenden Herausforderungen, ebenso wie ihre Bewältigung werden offen gelegt und münden in eine empirisch fundierte Theorie über den Einfluss des Unternehmens auf Unternehmerfamilien als Familien eigener Art. Ein besonderer Fokus liegt auf den Auswirkungen für Solidarität, Identität und Struktur der Familie.

Die Arbeit gliedert sich in einen theoretisch-methodologischen sowie einen empirischen Teil. Zunächst werden die idealisierten Vorstellungen des Ganzen Hauses, der modernen Kernfamilie sowie der multilokalen Mehrgenerationenfamilie als mögliche Vergleichsgruppen für die Untersuchung von Unternehmerfamilien gedankenexperimentell herangezogen. Aus der Perspektive soziologischer Modernisierungstheorien erscheint die Verbindung von Unternehmen und Familie eine Familie eigener Art zu konstituieren, die mal anachronistisch, mal zukunftsweisend wirkt, aber die nie ganz in den gesellschaftlichen Idealvorstellungen familialen Handelns aufgeht. Ein anschließender Durchgang durch die bestehende Forschung schärft das Profil von Unternehmerfamilien als Untersuchungsgegenstand. Die sich wandelnde gesellschaftliche Bedeutung dieser sozialen Einheit wird dabei ebenso thematisiert wie ihre zentralen Merkmale. Darauf aufbauend wird ein konzeptionelles Modell über die Dimensionen des wechselseitigen Einflusses von Unternehmen und Familien entwickelt, das die spezifischen Strukturen und Bedingungen von Unternehmerfamilien theoretisch zu fassen sucht. Das vorgeschlagene UFFU-Modell wird schließlich auf den Generationswechsel angewandt. Im Zuge des Transferprozesses erneuern und verändern sich Familienunternehmen und Unternehmerfamilie gleichermaßen. Damit wird das 
Verständnis von Nachfolge als Übergang zwischen Akteuren um jenes einer simultanen Reproduktion von zwei Systemen ergänzt.

Zwei wesentlichen Erkenntnisse gehen aus diesem theoretischen Teil hervor: Zum einen legt die bestehende Forschung zu Unternehmerfamilien immer denselben Vergleichsmaßstab zu Grunde, nämlich eine Entwicklung vom Ganzen Haus zu einer bürgerlichen Kernfamilie. Doch gerade systemtheoretischen Erwägungen werfen die Frage auf, ob der Vergleichsmaßstab einer bürgerlichen Familie für die Untersuchung von Unternehmerfamilien nicht um neuere Ansätze ergänzt werden müsste. Zum anderen weisen Unternehmerfamilien eine strukturelle Eigenlogik auf, die sich aus der Verbindung von Unternehmen und Familie ableitet und über die Zeit hinweg konstant zu bleiben scheint. Das UFFU-Modell kann als Versuch gewertet werden, diese strukturelle Eigenlogik analytisch zu fassen. Hier wird die Annahme vertreten, dass sich der wechselseitige Einfluss in drei Dimensionen niederschlägt: Erstens, während die Familie über Leitung, Kontrolle und Eigentum die Geschicke des Unternehmens bestimmt, wirkt dieses in der „Macht"-Dimension auf die Einkommensstruktur, das Vermögen, die Lebenslaufgestaltung sowie Privilegien und Pflichten der Familie ein. Die Familien-Unternehmens-Konstellation zeichnet sich ferner durch ein spezifisches temporales Verständnis aus, dass sich als „Denken in Generationen“ und einen ausgeprägten Kontinuitätsanspruch äußert. Schließlich prägt der wechselseitige Einfluss die praktizierte Kultur sowohl im Unternehmen als auch in der Familie.

Dieser theoretische Teil liefert eine systematische Analyse des Untersuchungsobjektes Unternehmerfamilien und trägt damit dazu bei, eine Lücke in der bisherigen Forschung zu schließen. Es zeigt sich darüber hinaus, dass der gegenwärtig sehr prominente systemische Ansatz einige Schwächen aufweist, allen voran ein sehr ausgeprägter Fokus auf widerstreitende Logiken, Rollenkonflikte und Integrationsprobleme. Zu kurz kommt das Wechselspiel aus institutionalisierten Strukturen und den Konstruktionsleistungen der aktuell Handelnden. Es muss gelingen, die strukturelle Eigenlogik und ihre jeweilige Interpretation empirisch offen zu legen, um die effizienten Wege, wie Unternehmerfamilien ihre Lebensform nicht nur erträglich, sondern sogar attraktiv machen, zu verstehen. Es wird deutlich, dass ein Zugang zum Untersuchungsobjekt gewählt werden sollte, der das „Ganze Leben“ der Familien eigener Art erfasst. Einen solchen Zugang versprechen das Programm des Institutionalisierten Lebenslaufes (Kohli ergänzt um Elder) sowie der Ansatz der Alltäglichen Lebensführung (Voß, Kudera), die als theoretische Perspektiven eingeführt und ihre Kombination diskutiert wird. Auf dieser Grundlage gelingt es, zentrale Begriffe zu definieren und die Forschungsfrage dieser Arbeit näher einzugrenzen: Welche konstanten Routinen, Handlungsregeln und Beziehungskonstellationen bringt der Besitz und die Leitung eines „Familienunternehmens" für die Lebensführung von Unternehmerfamilien mit 
sich? Wie bewältigen und interpretieren die Familienmitglieder den Einfluss des Unternehmens auf ihren Lebenslauf und ihre Beziehungsmuster?

Damit reiht sich diese Arbeit in die deutschsprachige soziologische Forschung zu Unternehmerfamilien ein, die auf ihr Untersuchungsobjekt aus einer modernisierungstheoretischen Perspektive blickt. Im Unterschied zu einer rein systemtheoretischen Perspektive wird mit den Leitbegriffen Lebenslauf, Biografie und Alltägliche Lebensführung das ,ganze Leben“ der Unternehmerfamilien eingefangen. Auf diese Weise entsteht ein Ansatz, der versucht, die alltäglichen Tätigkeiten der Familienmitglieder zur Aufrechterhaltung der Unternehmerfamilie in ihrem zeitlichen Verlauf zu verstehen.

Zur Untersuchung der Fragestellung wird eine interpretative, biografietheoretische Vorgehensweise gewählt, die die Bedingungen, Merkmale und Auswirkungen des Unternehmenseinflusses auf die Unternehmerfamilie rekonstruiert. Insgesamt werden 32 narrativ-biografische Interviews mit Mitgliedern aus zwölf Unternehmerfamilien geführt. Die transkribierten Interviews werden einzeln fallrekonstruktiv nach Rosenthal ausgewertet. Auf diese Weise entsteht ein Verständnis für die Lebenslaufentscheidungen sowie die biografische Selbstdarstellung der Befragten. Abschließend werden die Interviews der einzelnen Familienmitglieder mittels Genogrammen in Verbindung gesetzt. Die Interpretationsarbeit wird exemplarisch an einem Fall dargestellt und auf diese Weise dem Kriterium der Nachvollziehbarkeit qualitativer Forschung entsprochen.

Die Ergebnisdarstellung beschreitet zunächst einmal hypothetisch den Lebensweg eines Familienunternehmers von der Kindheit bis ins Alter. Auf diese Weise gelingt es, den Einfluss des Unternehmens in den unterschiedlichen Lebensphasen zu beschreiben und Pfadabhängigkeiten zu identifizieren. Die Ergebnisse sind vielfältig und detailreich: So wird etwa in der Phase der Kindheit die Wucht der „Erlebniswelt" Unternehmen beschrieben, die voraussetzungsreiche Partnerwahl thematisiert oder das professionelle Wertesystem eines Familienunternehmers näher bestimmt. Aus dem empirischen Material werden vier typische Wege in das Familienunternehmen abgeleitet sowie jene strukturellen Faktoren benannt, welche darüber bestimmen welcher berufliche Weg eingeschlagen wird. Unabhängig davon zeichnet sich ein einheitliches narratives Muster der ,freien Wahl“ ab, das zur Rechtfertigung für den eingeschlagenen Lebensweg angewandt wird und den Code zur Individualisierung mit der Kontinuität des Familienunternehmens in Einklang zu bringen sucht. Mit Hinblick auf die alltägliche Lebensführung einer Unternehmerfamilie werden in Anlehnung an die Typenbildung von Sue Birley drei idealtypische Gruppen der praktizierten Grenzarbeit herausgearbeitet. Familienunternehmer müssen immer wieder aufs Neue bestimmen, wie stark das Unternehmen den Familienalltag beeinflusst, müssen aktive Grenzarbeit leisten, die als Ergebnis eines stetigen Verhandlungs- und Verarbeitungsprozesses von persönlicher Lebenserfahrung, den Wünschen des Part- 
ners und Gelegenheitsstrukturen verstanden werden kann. Ein weiteres wesentliches Ergebnis der empirischen Analyse ist die Erkenntnis, dass die Art und Weise, wie sich der Rückzug aus dem Unternehmen gestaltet, vielfältig ist und sich zum Teil drastisch von den Normalerwartungen an einen Lebenslauf unterscheidet. Familienunternehmer sehen sich anderen institutionellen und strukturellen Rahmenbedingungen ausgesetzt als abhängige Erwerbstätige und deshalb eignet sich der Begriff „Ruhestand“ als Bezeichnung für diese Lebensphase nur bedingt. Der Rückzug aus dem Unternehmen gestaltet sich als Aushandlungsprozess mit einer Vielfalt an potentiellen Orientierungspunkten, in dem individuelle Bedürfnisse mit den Erwartungen anderer Familienmitglieder und der Belegschaft ebenso wie mit gesellschaftlichen Altersnormen koordiniert werden müssen. Aus dem Interviewmaterial wird die These entwickelt, dass der Rückzug aus dem Familienunternehmen entlang zweier Orientierungspunkte des „Normallebenslaufes“ in der Arbeitsgesellschaft („Ruhestand-Modell“) einerseits und eines voll flexiblen Überganges in der Leistungsgesellschaft („Leistungs-Modell“) andererseits erfolge. Aus dieser Perspektive stellen sich die „Schwierigkeiten des Loslassens“ weniger als Unfähigkeit oder als pathologisch dar denn als eine spezifische Strategie, mit dem Ende der Leistungskraft und der Tatsache der eigenen Sterblichkeit emotional zu verfahren.

Es kann festgehalten werden, dass das Unternehmen den Lebenslauf der Familienunternehmer maßgeblich beeinflusst. Es ist die Verbindung von Familie und Unternehmen (nicht unbedingt das Unternehmen an sich), die einen Orientierungsrahmen für das Leben, eine bestimmte Sequenzialität im Lebenslauf sowie einen Fluchtpunkt in der Ausbildung von Identität schafft, auch wenn die Grenzen der einzelnen Lebensphasen ebenso wie ihr „Timing" im Lebenslauf weniger stark institutionalisiert erscheinen. Das Programm eines „Normallebenslaufes“ lässt sich nur bedingt auf die Familien eigener Art übertragen. Grund dafür scheint vor allem die besondere Qualität der Tätigkeit im Unternehmen zu sein, die sich nur schwer einfangen lässt mit den üblichen Begriffen der „Berufswahl“, der „Profession“ oder des „Ruhestandes“. Der Einfluss des Unternehmens scheint die Flexibilität in der Ausgestaltung des Lebenslaufes und der Lebensführung zu erhöhen und die Einflusskraft der institutionalisierten Vorgaben des Normallebenslaufes zumindest teilweise zu reduzieren. Familienunternehmer stehen vor der Aufgabe, die entstehenden Freiräume aktiv zu gestalten und zwar innerhalb des unternehmerfamilialen Kontextes - insofern wird die potentielle Eigenbestimmtheit begrenzt. Ein möglicher Einstieg in das Familienunternehmen, der Zeitpunkt des Einstieges, die Übertragungen von Unternehmensanteilen, die Rollen einzelner Personen innerhalb der Unternehmerfamilie uvm. sind das Ergebnis einer Koordination der Lebensphasen und direkter Aushandlungen unter den Familienmitgliedern. 
Die Besonderheiten dieses generationalen Settings in Unternehmerfamilien sind Gegenstand der weiteren Ergebnisdarstellung, die einem Dreischritt folgt: Erstens, das Unternehmen durchdringt die verschiedenen Beziehungskonstellationen zwischen den Mitgliedern einer Unternehmerfamilie. Das Unternehmen als Thema der familialen Kommunikation, die Verbundenheit der Lebensläufe sowie finanzielle Abhängigkeiten schaffen sehr spezifische Voraussetzungen für die Begegnungen der Generationen und für die Entfaltung von Solidarität und Konflikt. Die Unternehmerfamilie bildet einen spezifischen Verweisungszusammenhang, eine Figuration, die die Form eines unmittelbaren Aushandlungsprozesses annimmt. Zweitens, Unternehmerfamilien haben in diesem Prozess eine Reihe von Koordinierungsaufgaben zu bewältigen, zu denen vor allem die Positionsbestimmung in Familie und Gesellschaft, die Synchronisation der Lebensläufe sowie der materielle Transfer gehören. Dieser aktive und direkte Aushandlungsprozess läuft quasi lebenslänglich und zumeist eher subtil. Drittens, Unternehmerfamilien wenden spezifische Verhandlungsweisen in diesen Aushandlungsprozessen an. Die wesentlichen Elemente dieser wurden als Pädagogik der Nachfolge, Indizienanalyse der Lebensläufe und schließlich als Gesprächs- und Erzählkultur beschrieben.

Im Lichte dieser Ergebnisse tritt die Unternehmerfamilie sehr deutlich als Erinnerungs- und Erzählgemeinschaft hervor. Der langfristige soziale Konstruktionsprozess zwischen den Generationen durchdringt die Überzeugungen und Lebensskripte der Familienmitglieder und trägt zu einer andauernden narrativen Fortschreibung der Familiengeschichte bei. Es wäre jedoch zu kurz gegriffen, das Kollektiv der Unternehmerfamilie ausschließlich im Sinne eines konstruktivistischen Ansatzes analytisch zu fassen. Die Ergebnisse heben deutlich die phänomenale und kontraktuelle Seite der Generationenverbundenheit hervor. Die Unternehmerfamilie formt eine Erfahrungsgemeinschaft, die eine besondere Lebenswelt bewohnt, ebenso wie eine Zweckgemeinschaft, die durch ,harte Faktoren' wie Eigentum, Beruf, finanzielle Vorsorge für die Eltern etc. aneinander gebunden ist. Sie bildet ein Netzwerk an sich überlagernden Lebensläufen, das den Austausch zwischen den Generationen ermöglicht und auch erforderlich macht. Als Familien eigener Art bilden sie eine kollektive Identität, spezifische Mitgliedschaftsregeln und eine Außengrenze aus, die Anknüpfungspunkte für die individuelle Identität bilden, ein Zusammengehörigkeitsgefühl ebenso wie solidarische Handlungen zwischen den Generationen und Familienmitgliedern befördern können. Gleichzeitig verschärfen sich in einer solchen Gemeinschaft die Risiken der Exklusion, Rivalität, Entfremdung und Ungleichheit. Es lässt sich festhalten, dass sich durch den direkten Charakter der Generationenbeziehungen in Unternehmerfamilien die Generationenambivalenzen potenzieren.

Zum Abschluss wird der theoretische Teil der Arbeit mit den empirischen Ergebnissen konfrontiert, theoretische Bezüge hergestellt und offene 
Forschungsfragen formuliert. Das erarbeitete UFFU-Modell wird präzisiert und noch einmal die Spannung zwischen einer strukturellen Eigenlogik und Handlungsoptionen thematisiert. Schließlich dient die Habermas'sche Kolonialisierungsthese erweitert um seine Skizze einer Öffnungs-SchließungDynamik dazu, den gewählten Zugang zum „Ganzen Leben“ in Beziehung $\mathrm{zu}$ der bestehenden systemtheoretischen Forschung zu setzen. Die Unternehmerfamilie wird als dreifach erweiterte Lebenswelt gefasst. Das Unternehmen findet sich damit eben nicht mehr nur als systemischer Einfluss auf die Familie wieder, sondern wird ein fester Bestandteil der Alltagsarrangements, die erinnert, erzählt und erlebt werden. Entsprechend zeichnet sich als ein wesentlicher theoretische Anknüpfungspunkte dieser Arbeit eine lebensweltliche Fassung des Unternehmensbegriffes ab. 


\section{Abstract}

This empirical study focuses on how a business might influence the relational structure and way of life for an entrepreneurial family, thus it entails sociological scrutiny of a social unit that has received little research attention, despite its impact on our modern economy. The term 'entrepreneurial family' refers to a family that owns business assets and generates its income via occupational engagement in the business. Hence everyday life is not structured around gainful employment, but rather influenced by one or more businesses. The transgenerational transfer of ownership and leadership in such businesses (known as 'the succession process') is understood as the reproduction mechanism of this business influence. It is the aim of this study to conceptualize the ways in which businesses impact on the lives of entrepreneurial families. I unveil the conditions of their specific constellation and the resulting challenges, as well as the strategies developed to cope with these challenges; then I develop my analysis into an empirically grounded theory on the influence of a business on an entrepreneurial family as a unique family group. In particular, I focus on the issues of solidarity, identity and family structure.

This work consists of two parts: theoretical-methodological considerations and the presentation of empirical findings. I begin by discussing the idealised notions of the 'ganzes Haus' (family economy), the 'nuclear family', and the 'multi-local extended family structure', thus creating a frame of reference for the analysis of entrepreneurial families. From the perspective of sociological modernization theory, the relationship between a business and a family seems to result in a unique family group: seeming sometimes anachronistic, sometimes pioneering, they nonetheless never conform entirely to the socially idealised types of familial acting. My rigorous literature review establishes the basis of entrepreneurial families as my research objective. The evolving social meanings of this social unit are addressed along with its central characteristics. On this basis I develop a conceptual model for the reciprocal influence of the business and the family, which theorizes the specific structures and conditions of entrepreneurial families. Finally, I apply my interpreted UFFU-Model to the succession process. During the generational transfer both the family business and the entrepreneurial family are regenerated and changed. Hence by focusing on the simultaneous reproduction of these two systems, an understanding of succession as transition between actors emerges.

The theoretical part of my work promotes two central insights. On the one hand, the existing theoretical and empirical research on entrepreneurial families continuously applies the unchanging standard of comparison: the 
development from the family economy to a bourgeoisie family. Yet current contributors - particularly those writing from a systems theory perspective raise the question as to whether this standard of comparison should be enriched by newer approaches. On the other hand, entrepreneurial families betray a structural character derived from the intertwinement of a family and a business, and this appears to be historically consistent. The UFFU-Model can be interpreted as an attempt to analytically grasp this structural character. It advocates the idea that the reciprocal influence can be manifested threefold. Firstly, while the family controls the fate of the business via management, ownership and control (e.g. board activities), the business impinges on the entrepreneurial family in this 'power' dimension via assets, life course structures, privileges and duties. The family-business-constellation is further characterized by a specific temporal understanding manifesting itself as 'thinking in generations' and demonstrating distinctive aspirations for continuity. Finally, the reciprocal influence shapes both the business and family culture.

This theoretical and systematic analysis of the research objectives closes a gap in existing research. Furthermore, it becomes obvious that the currently en vogue systems theory approaches have their weaknesses, with an overly strong focus on antagonistic logics, role conflicts and paradoxes leading the way. The interplay between institutionalized structures and the constructed performances of the actors are hardly touched upon. It is necessary to focus on the structural character and its interpretations in order to understand the efficient ways in which entrepreneurial families make their form of living not only tolerable, but attractive as well. An approach that embraces the 'total life' of these unique families in this endeavour is imperative. Such an approach offers the program of an institutionalized life course (Kohli complemented by Elder) as well as the theory of an everyday conduct of life (Alltägliche Lebensführung, Voß, Kudera). Both perspectives are introduced and their combination is discussed. On this basis, central terms can be defined, and the research question for the empirical investigation can be specified as follows: What constant routines, rules and relational constellations do the ownership and management of a family business hold for an entrepreneurial family's conduct of life? How do members of such families cope with and interpret the influence of the business on their life course and their relational patterns?

On this basis, my arguments coincide with German sociological research on entrepreneurial families, which views the research object from the perspective of modernization theories. In contrast to a pure systems approach, the central concepts of life course, biography and conduct of life offer analytical tools to grasp the 'total life' of entrepreneurial families. This study presents an approach that attempts to understand the everyday activities of family members who work to sustain the entrepreneurial family over time. 
The methodological design applies an interpretative, biographical approach that reconstructs the conditions, characteristics and consequences of the business influence on the entrepreneurial family. I conducted a total of 32 narrative biographical interviews with twelve entrepreneurial families and have analysed the interview material via the biographical case reconstruction method (Rosenthal). This enables an understanding for life course decisions while also examining the biographical self-representation of the interviewees. Finally, genograms put the individual interviews into a family context. The interpretative process is comprehensively explained on a sample case, which meets the criteria of intersubjective replicability required for qualitative research.

The presentation of results follows a hypothetical life path of a family entrepreneur from childhood into old age. Thus I am able to examine the influence of the business on the life of entrepreneurial family members at different stages in their lives and to identify dependencies along the way. The results are multifaceted and rich in detail. For instance, a child is shown to be exposed to the vehemence of the business as world of experience; the process of mate selection is revealed to be complicated by additional expectations which support the life style of an entrepreneurial family; the composition and application of a family entrepreneur's professional value system is exposed. Four typical ways into the family business can be identified from the interview material, demonstrating how deliberate structural features can determine the path taken. Independent of the actual route, a uniform narrative pattern of 'free choice' can be identified; this is used as a biographical justification strategy, facilitating compromise between the social code of individualization and the continuity of the family project. With regard to the entrepreneurial family's everyday conduct of life and with reference to the work of Sue Birley, three ideal groups of active boundary construction can be identified. Family entrepreneurs have to constantly redefine the intensity in which the business influences their everyday life; they have to perform active boundary construction, which can be understood as the ongoing process of negotiating and coping with personal life experience, along with a partner's desires and opportunity structures. Another essential finding from the empirical analysis concerns the way family entrepreneurs enact their withdrawal from the business, which may differ drastically from the norms of common living. Family entrepreneurs are confronted with very different institutional and structural circumstances than are dependent employees, thus the term 'retirement' has limited suitability. Withdrawal from the business can be understood as a negotiation process, in which individual needs have to be coordinated with the expectations of family members and employees, as well as with social age norms. The interview material indicates that withdrawal from the business may follow one of two opposed directions: either the idea of a 'threefold life course' ('retirement-model') or that of a fully flexible transi- 
tion ('performance model'). Viewed from this perspective the difficulties of 'letting go', frequently referred to in the research literature, no longer come across as indicative of pathological behaviour, but rather as a specific strategy to emotionally deal with the end of one's economical potential and the fact of human mortality.

It can be stated that the business significantly influences the life course of family entrepreneurs. It is the intertwinement of family and business (und not necessarily the business itself) that creates an orientation framework for life, a sequence of life stages, and a vanishing point in the development of identity, even though the timing and execution of life stage transitions is less strongly institutionalized. The model of a threefold life course can only be applied to these unique families to a limited extent. The major reason for this has to be seen in the quality of the occupational engagement in the business, which can hardly be described by the common terms 'career choice', 'profession' or 'retirement'; the influence of the business seems to increase the flexibility of life choices and conduct, while in turn at least partially reducing the impact of the institutionalized standard threefold life course. Family entrepreneurs have to actively configure the emerging flexibility within the entrepreneurial family context - in this respect the potential for self-determination is not limitless. A possible entry into the family business, the timing of that entry, the transfer of company stock, and the roles played by individuals within the entrepreneurial family (as well as much more) all result from the combined influence of established life stages and immediate negotiations between family members.

Moving on to focus on the particularities of this generational setting in entrepreneurial families, I present my results in three parts. Firstly, I note how the business pervades the various relational constellations in entrepreneurial families. The business as topic of familial communication, as well as the related life courses and financial dependencies are all contributing factors in the creation of very specific premises for generational encounters and for the emergence of solidarity and conflict. The entrepreneurial family forms a particular referential context, a figuration (Elias), taking the shape of an immediate negotiation process. Secondly, entrepreneurial families have to solve a number of coordination tasks in this process, most vitally the determination of ones position within the family and society, the synchronisation of life courses, and the transfer of assets. This active and straightforward negotiation process takes place over the course of a lifetime and is invariably subtle. Thirdly, entrepreneurial families apply specific negotiation strategies in this process, described as the pedagogy of succession, the permanent analysis of life course evidence, and finally a conversational and narrative culture.

In the light of these findings, the entrepreneurial family stands out as a community of shared memories and narratives. The long-term social construction process from generation to generation pervades the individual be- 
liefs and life plans of family members and contributes to an ongoing narrative: the perpetuation of family history. It would however be short sighted to conceive of entrepreneurial families exclusively in such a constructivist manner. The findings clearly emphasize the phenomenal and contractual side of generational bonds. Hence the entrepreneurial family also forms a community of experience occupying a very specific life-world and a community of convenience bound together by 'hard factors' such as assets, occupation, financial provision for one's parents, etc. They create a network of overlapping life courses that both enable and require interchange between generations. As unique families they establish a collective identity, specific membership rules and group borders, thereby promoting vital links for individual identity, along with a sense of togetherness and models of joint behaviour and interaction between family members and generations. At the same time, the risks of exclusion, rivalry, alienation, and inequality amplify in such communities. It can be stated that the immediate character of the generational relations in entrepreneurial families increases generational ambivalence (Lüscher).

At the close of this work, I confront the theoretical considerations and empirical findings, discuss theoretical connections, and formulate open research questions. The discussed UFFU-Model is presented and, once again, the tension between the structural character and courses of action within entrepreneurial families is discussed. Finally, the thesis of colonialization as formulated by Habermas - which he later complemented with the idea of an opening-closing-dynamic - serves as a vehicle with which to relate the selected approach to the 'total life' of entrepreneurial families to prominent systems theory approaches. The entrepreneurial family can be understood as a triply enlarged life-world. Consequently, the business can be analytically viewed as a firm component of the everyday arrangement of entrepreneurial families; rather than imposing only systemic influence on the family it resides at the centre of all that is remembered, narrated, and experienced. Accordingly, this work offers a number of key links for the development of a life-world approach to businesses. 



\section{Inhaltsverzeichnis}

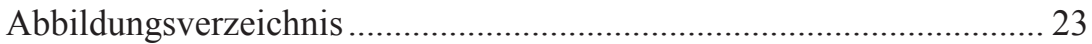

I. Tatort Unternehmerfamilie.............................................................. 25

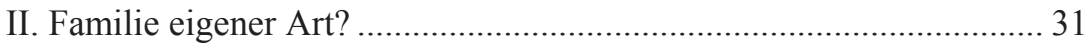

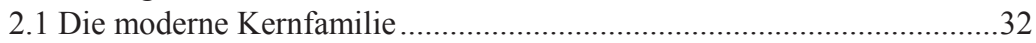

2.2 Die Auflösung des „Ganzen Hauses“ „......................................................35

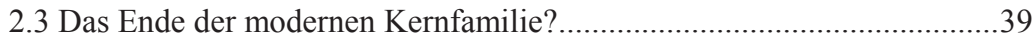

2.4 Zwei Hypothesen zum Status von Unternehmerfamilien .........................44

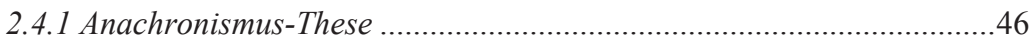

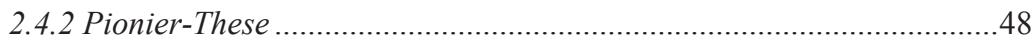

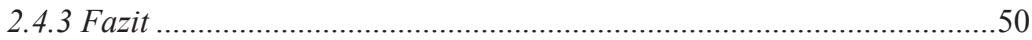

III. Die Besonderheiten von Unternehmerfamilien .............................. 51

3.1 Überblick über die Forschungslandschaft ..............................................

3.2 Gesellschaftliche Bedeutung von Unternehmerfamilien ..........................56

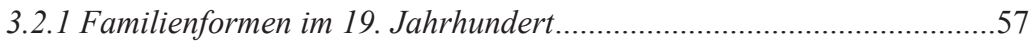

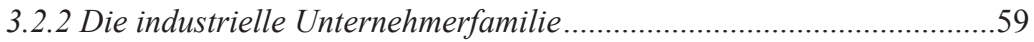

3.2.3 Unternehmerfamilien in der ersten Hälfte des 20.

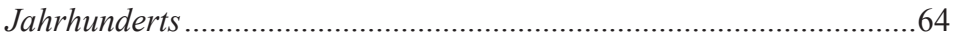

3.2.4 Familienunternehmen als Motor der deutschen Wirtschaft..................65

3.2.5 Unternehmerfamilien in der zweiten Hälfte des 20.

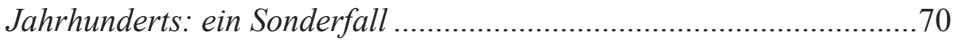

3.2.6 Enge und weite Fassung von Unternehmerfamilien ..............................74

3.3 Systemtheoretischer Zugang: Koevolution und Rollenkonflikt................75

3.4 Dimensionen des Unternehmenseinflusses auf die Familie.......................82

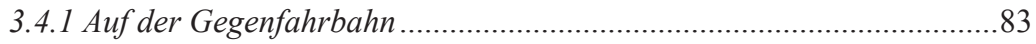

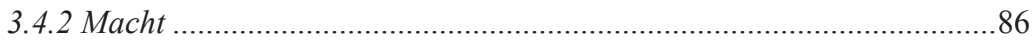

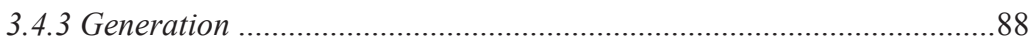

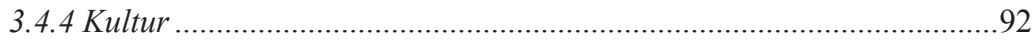

3.5 Generationswechsel aus Familienperspektive ………………..................94

IV. Theoretischer Ansatz: Ein Zugang zum „Ganzen Leben“..........................101

4.1 Anachronismus, Paradox oder doch Pionier? .........................................101

4.2 Die strukturelle Eigenlogik von Unternehmerfamilien...........................106

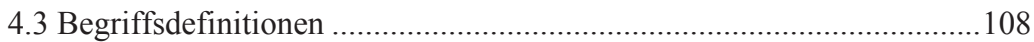

4.4 Forschungslücke: Ein Zugang zum „Ganzen Leben“.............................111

4.4.1 Lebenslauf- und Biografieforschung (Kohli, Elder) ...........................112

4.4.2 Alltägliche Lebensführung (Voß, Kudera).........................................117 
4.4.3 Die Analyseebenen Lebenslauf, Biografie und Alltägliche

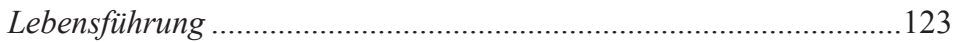

4.4.4 Eingrenzung und Präzisierung der Forschungsfrage .........................126

V. Grundzüge der empirischen Vorgehensweise ................................. 129

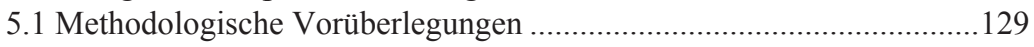

5.2 Lebensgeschichten als Zugang zur Alltagswelt.....................................132

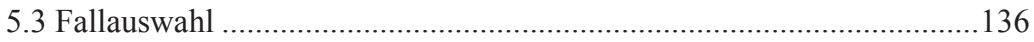

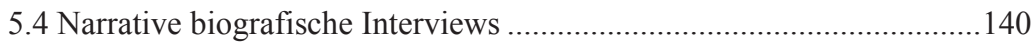

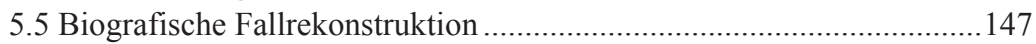

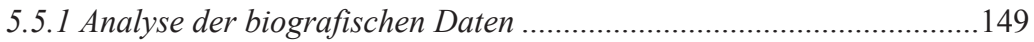

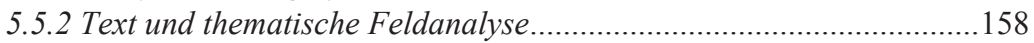

5.5.3 Rekonstruktion der erlebten Lebensgeschichte..................................163

5.5.4 Kontrastierung von erzählter und erlebter Lebensgeschichte .............171

5.5.5 Rekonstruktion der Familiengeschichte ...........................................173

5.6 Theoriebildung und Generalisierbarkeit der Ergebnisse ........................178

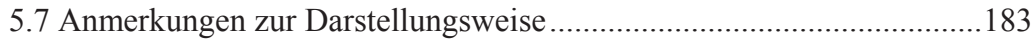

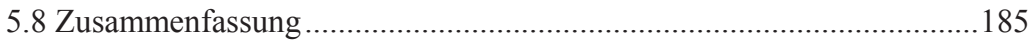

VI. Lebenslauf und Lebensführung des Familienunternehmers............ 187

6.1 Das Aufwachsen in Unternehmerfamilien...........................................187

6.1.1 Das Unternehmen als Erlebniswelt .................................................188

6.1.2 Die Mitarbeit im Familienunternehmen ............................................192

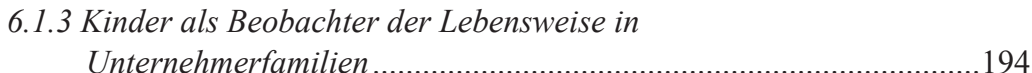

6.1.4 Berufliche Orientierung und Identitätsfindung .................................197

6.1.5 Fazit: Unternehmen als familialer Raum ........................................200

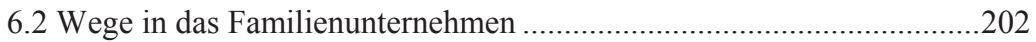

6.2.1 Berufswahl, Unternehmensgründung und Nachfolge .........................204

6.2.2 Typische Wege ins Familienunternehmen ........................................208

6.2.3 Strukturelle Einflussfaktoren ............................................................218

6.2.4 Der Eintritt ins Unternehmen als biografische
Herausforderung

6.2.5 Warum ,entscheiden “ sich Familienunternehmer für das

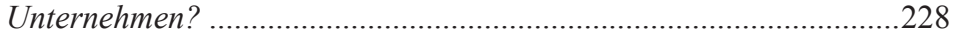

6.3 Familiales Handeln in Unternehmerfamilien........................................229 


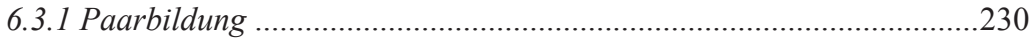

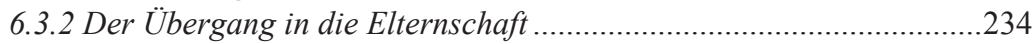

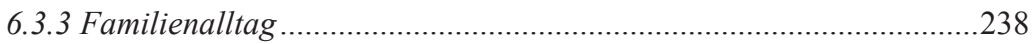

6.3.4 Fazit: Die lebensprägende Bedeutung des Familienalltags ................246

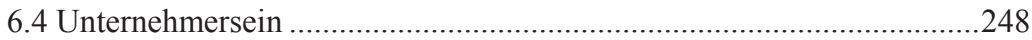

6.4.1 Typische Elemente in unternehmerischen

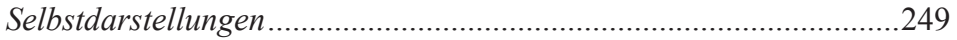

6.4.2 Das professionelle Wertesystem des

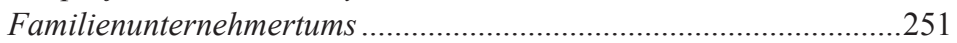

6.4.3 Fazit: Die Identitätsschaffende und Sozialdifferenzierende

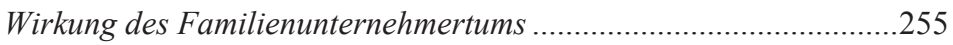

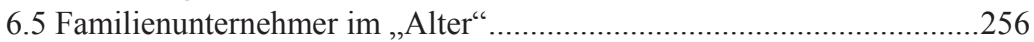

6.5.1 Der Ruhestand von Familienunternehmern in der

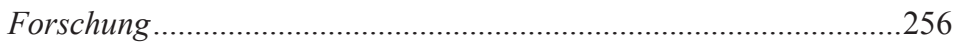

6.5.2 Eine Kritische Diskussion des Begriffes Ruhestand ...........................261

6.5.3 Inkorporation einer Leistungskultur ...................................................263

6.5.4 Fazit: Das Ruhestandsmodell und das Leistungsmodell ....................267

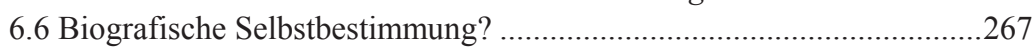

VII. Familiale (Generationen-)Beziehungen in Unternehmerfamilien ......................................................................... 275

7.1 Dimensionen der Generationenbeziehungen ........................................275

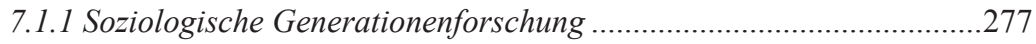

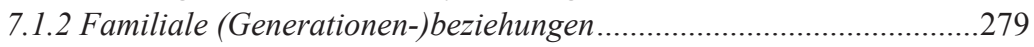

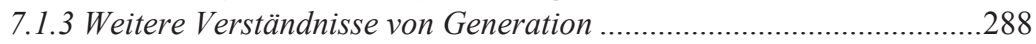

7.1.4 Unternehmerfamilien als Figuration ...............................................2295

7.2 Koordinierungsaufgaben in Unternehmerfamilien ...............................298

7.2.1 Positionsbestimmungen in Familie und Gesellschaft .........................298

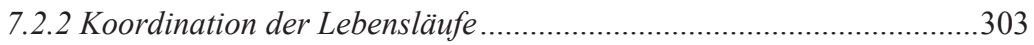

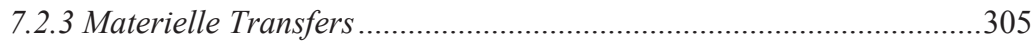

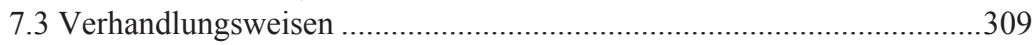

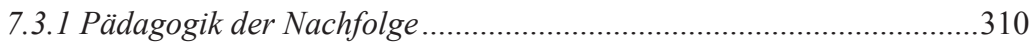

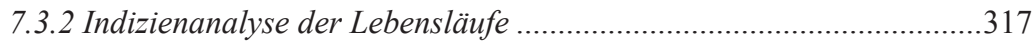

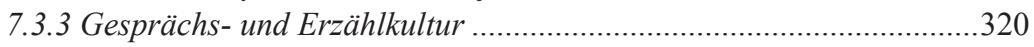

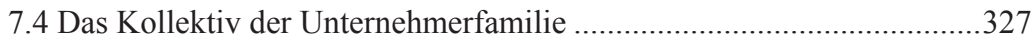




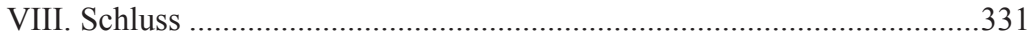

8.1 Strukturen und Handlungen in Unternehmerfamilien .............................331

8.1.1 Noch einmal: Die strukturelle Eigenlogik von

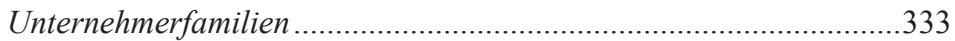

8.1.2 Handlungsspielräume und Strategien in

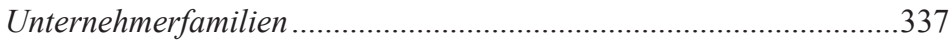

8.2 Unternehmerfamilien als kolonialisierte Lebenswelt?...........................339

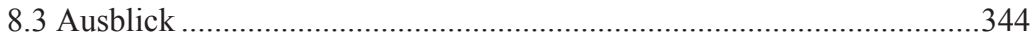

Literaturverzeichnis..................................................................... 347

Anhang: Falldarstellungen ............................................................ 371 


\section{Abbildungsverzeichnis}

Abbildung 1: Übersicht idealtypischer Familienformen............................. 45

Abbildung 2: Das UFFU-Modell des wechselseitigen Einflusses ................. 86

Abbildung 3: Übersicht der Entwicklungskonzepte ................................... 90

Abbildung 4: Modell zum Generationswechsel........................................... 98

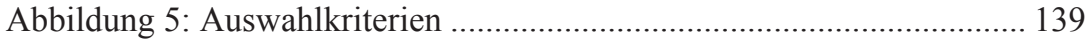

Abbildung 6: Transkriptionsregeln ......................................................... 146

Abbildung 7: Ausschnitt aus der Sequenzübersicht Michael Treuninger... 160

Abbildung 8: Genogramm der Familie Treuninger .................................. 174

Abbildung 9: Vertikale und Horizontale Generationenbeziehungen.......... 294

Abbildung 10: Indizienanalyse der Lebensläufe......................................... 319

Abbildung 11: Überarbeitetes UFFU-Modell ............................................ 336

Abbildung A 1: Erläuterung der im Genogramm verwendeten Symbole... 371

Abbildung A 2: Genogramm der Familie Carstens.................................... 373

Abbildung A 3: Genogramm der Familie Claas ......................................... 375

Abbildung A 4: Genogramm der Familie Clemens ................................... 377

Abbildung A 5: Genogramm der Familie Hagemann ................................. 380

Abbildung A 6: Genogramm der Familie Treuninger ................................ 382

Abbildung A 7: Genogramm der Familie Lösch ....................................... 385

Abbildung A 8: Genogramm der Familie Naumann.................................... 387

Abbildung A 9: Genogramm der Familie Niest........................................ 390

Abbildung A 10: Genogramm der Familie Vester/Wagner ......................... 392

Abbildung A 11: Genogramm der Familie Singermayer............................ 394

Abbildung A 12: Genogramm der Familie Übermann .............................. 397

Abbildung A 13: Genogramm der Familie von Moline.............................. 399 



\section{Tatort Unternehmerfamilie}

Während eines Beachvolleyballspieles bekommt der 15-jährige Allergiker Florian plötzliche Krämpfe und leidet unter Atemnot, nachdem er zuvor ein Fitnessgetränk zu sich genommen hat - wenig später ist der Schüler tot. Erste Untersuchungen ergeben, dass dem Getränk eine wesentlich höhere als die gesetzlich zulässige Menge an Lebensmittelfarbstoff zugesetzt war. So beginnt eine der mehr als 800 Folgen von Deutschlands ältester und beliebtester Krimiserie. ${ }^{1}$ Über acht Millionen Zuschauer (Das Erste 2010) verfolgen, wie die Ermittlungen des Kieler Kommissars Borowski direkt zu dem Familienunternehmen Kallberg führen. Die aus einem landwirtschaftlichen Betrieb erwachsene Molkerei vermarktet ihr Spitzenprodukt „Vitanale“ aggressiv. Als Borowski das Unternehmen zum ersten Mal betritt, findet er sich in einem düsteren Kuhstall wieder. Die Tiere sind eng aneinandergepfercht und durchlaufen wie auf einem Fließband den Melkprozess. Er hört, wie sich etwas weiter hinten zwei Personen unterhalten: „Wenn Du verkaufen willst, dann kannst Du mich gleich mit entsorgen“" sagt Großvater Kallberg wutentbrannt zu seiner Tochter Liane. Die ehrgeizige Firmenchefin im weißen Kittel reagiert entsetzt auf die Todesnachricht des Jungen. Bei einer Begehung des Betriebes fällt ein Einbruch auf. Sabotage scheint damit sicher. Die Ermittlungen führen über den Vater von Florian und einen Umweltaktivisten zurück zu den Kallbergs. Schritt für Schritt tauchen die Zuschauer tiefer in die Geschichte dieser Familie ein:

Der Großvater übernahm den Hof schon im jugendlichen Alter von 15 Jahren, als sein Vater plötzlich verstarb. Das Unternehmen war der Mittelpunkt seines Lebens. Es fällt ihm schwer, sich zu lösen und von der jüngeren Generation nicht mehr gebraucht zu werden. Eigentlich sollte sein älterer Sohn Paul die Nachfolge antreten, doch seine Idee eines Biobauernhofes widersprach den Vorstellungen des Vaters. Paul verzichtet auf sein Erbe und bewirtschaftet wenig gewinnbringend sein eigenes Land. Stattdessen übernimmt die jüngere Schwester Liane, die zuvor bei einem Lebensmittelhersteller in der Schweiz arbeitete, die Führung im Familienunternehmen. Die allein erziehende Mutter einer Tochter baut den Hof zu einem industriellen Betrieb um. Die Verwendung von gesundheitsschädlichen Farbstoffen bringt die Familienmitglieder gegen sie auf. Liane will verkaufen und neu anfangen,

1 Konkret handelt es sich um die Episode „Borowski und eine Frage des reinen Geschmacks", Erstausstrahlung am 24. Oktober 2010 in der ARD. Siehe auch: http://www.daserste.de/tatort/sendung.asp?datum=24.10.2010, zuletzt geprüft am 29.01.2011. 
die Tochter soll eine gute Ausbildung auf einem Internat erhalten. Als der Großvater von diesen Plänen erfährt, manipuliert er eine Charge des Vitamingetränkes. Die Überdosis ruft bei gesunden Menschen - mit der Ausnahme des Allergikers Florian - nur Übelkeit hervor und soll dem Ruf der Firma und damit ihrem Verkaufswert schaden. Alles soll so bleiben, wie es ist.

Die Tatortepisode spielt stereotypisch mit den sich überlagernden Motivebenen in Unternehmerfamilien. Der Großvater steht für die Tradition, Pflichtbewusstsein und Loyalität. In seinem ganzen Auftreten verkörpert er den bodenständigen Landwirt, der ein enges Vertrauensverhältnis zu seinen Mitarbeitern pflegt. Die Konflikte mit seinen Kindern haben ihn zu einem gebrochenen Mann werden lassen, der sogar vor der Schädigung seines eigenen Betriebes nicht zurückschreckt. Liane, die nur als zweite Wahl die Nachfolgerrolle übernimmt, spielt die starke, aber einsame Macherin. Sie schwankt zwischen offensichtlichen Schuldgefühlen und kühler Berechnung des Marktwertes ihres Unternehmens. Paul dagegen verkörpert den von Prinzipien geleiteten Rebell. Seine innovativen Ideen lassen sich nicht mit der Tradition, seine moralischen Vorstellungen nicht mit dem Gewinnstreben vereinbaren. Die Begegnungen der Familienmitglieder sind stark inszeniert und leben von den vielschichtigen Spannungen zwischen ihnen. Das Unternehmen scheint die zwischenmenschlichen Beziehungen ausgekühlt und die Familienstrukturen zerrüttet $\mathrm{zu}$ haben. Individuelle Lebensentwürfe müssen hinter dem Fortbestand der Organisation zurücktreten.

Ein solches Setting eines Generationen überspannenden Familienstreites, der sich mit unternehmerischen Interessen paart, scheint dramaturgisch interessanter als eine Welt kaltblütiger Krimineller. Es gibt kaum eine abwechslungsreichere Krimiserie als den von föderalen Strukturen geprägten Tatort, der immer wieder aktuelle gesellschaftliche Konflikte aufgreift und diese in unterschiedlichen Milieus platziert. ${ }^{2}$ Dennoch spielen in über 40 Jahren Tatort mehr Fälle in solchen Unternehmerfamilien als etwa im Rotlichtmilieu oder der Drogenszene. ${ }^{3}$ Die Tatortmacher bedienen sich eines Gegenstands mit literarischer Tradition. In der fiktiven Bearbeitung von Unternehmerfamilien scheinen ökonomische Macht und vertraute Familienbeziehungen auf sonderbar konzentrierte Weise aufeinander zu treffen. Es ist diese eigenartige Verbindung, die über Generationen aufrechterhalten wird und schließlich in

2 Der Literaturwissenschaftler Dennis Gräf 2010 untersucht aus diesem Grund den Tatort als kulturellen Speicher. Anhand seiner Analysen der Kommissarfiguren, der behandelten Themen sowie der dargestellten Milieus in ausgewählten Episoden versucht er den gesellschaftlichen Wandel der letzten Jahrzehnte nachzuzeichnen.

3 Eine Schlagwortsuche in einem der Tatortarchive, in diesem Fall auf der Fanseite: www.tatort-fans.de, ergab etwa für „Unternehmer“ 45 Folgen, für „Drogen“ 38 Folgen, für „Prostituierte“ 16 Folgen. Diese kurze Suche vermittelt lediglich einen oberflächlichen Eindruck und stellt keine wissenschaftlich gehaltvolle Analyse dar. 
buddenbrookscher Manier zu zerbrechen droht, von der die Faszination für diese Dynastien ausgeht. Immer wieder stehen sich Alt und Jung, Übergeber und Übernehmer, Eltern und Kinder, Erblasser und Erben gegenüber. Damit gewinnen Unternehmerfamilien ihre Dynamik nicht zuletzt aus der Brisanz der Beziehungen zwischen den Generationen. Denn diese führt - zumindest in der künstlerischen Darstellung - unweigerlich zu Intrigen und Versöhnungen, zu Erbstreitigkeiten und Familienzwistigkeiten.

Die mediale Beschäftigung mit realen Unternehmerfamilien hat darüber hinaus Konjunktur: Erst jüngst zeigte das ZDF einen aufwändigen Dreiteiler über die Familie Krupp ${ }^{4}$ und der NDR die Dokumentationsreihe „Norddeutsche Dynastien", in der unter anderen die Deichmanns, Underbergs und Wempes vorgestellt wurden. ${ }^{5}$ In Tageszeitungen oder den einschlägigen Wirtschaftsmagazinen findet man kurze Unternehmerdarstellungen, und die Zahl von Auftragsbiografien über Unternehmerclans steigt stetig an. ${ }^{6}$ Mit Blick auf die Berichterstattung in den letzten Jahren - so zumindest mein persönlicher Eindruck - zeichnet sich ein sehr unterschiedliches Bild von Familienunternehmen und ihren Familien in der öffentlichen Wahrnehmung ab: Während Familienunternehmen als „Hidden Champions“, gute Arbeitgeber, als Verkörperung des nachhaltigen Kapitalismus und überhaupt als Rückgrat der deutschen Wirtschaft verstanden werden, haftet den dazugehörigen Familien etwas Kauziges an. Nicht selten werden sie mit ,patriarchalischen Familienclans" in Verbindung gebracht, in denen Leben und Arbeit eng verbunden sind und sich Autonomie und Individualität nur gegen Widerstand entfalten können. Missgunst, Neid, Profitgier, Geschwisterrivalität, Ehebruch oder sonstige familiäre Probleme sind deshalb Bestandteil der öffentlichen Normalerwartung gegenüber mehrgenerationalen Unternehmerfamilien. So passt es ins Bild, wenn ein Nachfolger nicht in die Fußstapfen der Vorfahren treten will oder die dritte Generation das Unternehmen in den Ruin treibt. Kurzum, die Normalerwartung an solche Unternehmerfamilien ist, dass sich in ihnen Probleme, Dramen und unterdrückte Wünsche zuspitzen.

Auch aus soziologischer Perspektive fällt die Unternehmerfamilie aus dem Rahmen. Mit den üblichen modernisierungstheoretischen Ansätzen lässt sich diese soziale Einheit nur schwer fassen. In eine Gesellschaft, in der sich Arbeit und Leben in zwei Bereiche differenziert haben (funktionale Differenzierung) und in der die Norm eines eigenständigen Lebensentwurfs gilt (Individualisierung), wollen Unternehmerfamilien und ihre Mitglieder, deren Lebensplanung in dieser Verbindung aufgeht, nicht so recht passen. Stattdes-

4 "Krupp - Eine deutsche Familie" Erstausstrahlung 22., 23. und 25. März 2009 (drei Teile) im ZDF.

5 Die Reihe „Norddeutsche Dynastien“ läuft seit 2009 im NDR.

6 Als Beispiele seien hier jene der Familien Neckermann Veszelits 2005, Reemtsma Lindner 2008, Wertheim Fischer und Ladwig-Winters 2008 und Oetker Jungbluth 2006 genannt. 
sen erinnern sie an die traditionelle Haushaltsökonomie und an das Leben im Ganzen Haus ${ }^{7}$. Unternehmerfamilien erscheinen - zumindest auf den ersten Blick - als anachronistisch. Gleichsam entsteht durch diese Konstellation ein sehr modernes Problem: Die Unternehmerfamilie befindet sich am Schnittpunkt zweier Systeme mit unterschiedlichen Handlungslogiken. Sie muss paradoxe Handlungserwartungen koordinieren und ist gefordert, ihre Grenze zwischen Arbeit und Leben selbst herzustellen. Das Unternehmen lässt die Grenzen zwischen Privatheit und Firma verschwimmen, durchdringt die Lebensführung der Familienmitglieder und stellt die Generationenbeziehungen vor die Aufgabe konfligierende systembedingte Erwartungen zu koordinieren. Es entsteht ein spannungsreiches Verhältnis aus festen Strukturen und Traditionsbeständen sowie flexiblen Gestaltungsräumen jenseits gesellschaftlicher Institutionalisierungen.

In der vorliegenden Arbeit soll dieser Einfluss des Unternehmens auf das Leben und das Beziehungsgefüge in Unternehmerfamilien zum Gegenstand einer empirischen Untersuchung werden. Damit wird eine soziale Einheit soziologisch hinterfragt, die trotz ihrer Bedeutung für unsere moderne Ökonomie noch wenig Beachtung gefunden hat. Mit dem Begriff Unternehmerfamilie sind solche Familien gemeint, die Betriebsvermögen besitzen und/oder über ihre Tätigkeit im Unternehmen Einkommen generieren, deren familiärer Alltag also nicht an Erwerbsarbeit ausgerichtet ist, sondern von einem (oder mehreren) Unternehmen beeinflusst wird. Der transgenerationale Transfer von Eigentum und Leitung in solchen Unternehmen (kurz: der Nachfolgeprozess) wird als Reproduktionsmechanismus des Unternehmenseinflusses betrachtet. $^{8}$

Entstanden ist die vorliegende Studie im Rahmen des Forschungsprojektes „Von Generation zu Generation“, die von 2007-2010 an der Freien Universität Berlin von Martin Kohli geleitet wurde. ${ }^{9}$ Während das Forschungsprojekt seinen Fokus auf den Nachfolgeprozess in Familienunternehmen legte, besteht das Ziel dieser Untersuchung darin, analytisch zu fassen, auf welche Weise das Unternehmen auf das Leben von Unternehmerfamilien ein-

7 Mit dem Begriff des „Ganzen Hauses“ wird in der Familiensoziologie die familiale Lebensweise in vormodernen Gesellschaften beschrieben, näheres siehe Kapitel 2.

8 In Kapitel 3 werden diese Begriffe im Lichte der aktuellen Forschung ausführlich diskutiert und in Kapitel 4 eine Definition angeboten.

9 Gefördert wurde dieses als ein Teilprojekt im Rahmen des Forschungsverbundes „Generationen der Erbengesellschaft“ im Programm „Schlüsselthemen der Geisteswissenschaften“ der VolkswagenStiftung. In diesem Kontext wurden von der Verfasserin die narrativen Interviews erhoben und analysiert, wie in Kapitel 5 näher beschrieben. Das Forschungsdesign umfasste darüber hinaus eine parallele qualitative Untersuchung von italienischen Unternehmerfamilien und eine quantitative Untersuchung von Haushaltsdatensätzen. Letztere wurde von Nicole Schmiade durchgeführt. Ein Teil der Vorarbeiten und erste Gedanken sind im Rahmen des Projektes publiziert und auf Kongressen und Tagungen zur Diskussion gestellt worden. 
wirkt. Das Bezugsobjekt ist deshalb zunächst die alltägliche Lebenswelt der Unternehmerfamilien. Die Bedingungen ihrer spezifischen Konstellation, die sich daraus ergebenden Herausforderungen, ebenso wie ihre Bewältigung sollen offen gelegt werden und in eine empirisch fundierte Theorie über den Einfluss des Unternehmens auf Unternehmerfamilien als Familien eigener Art münden. Besonderer Fokus liegt auf den Auswirkungen des Unternehmens auf die Solidarität, Identität und Struktur der Familie.

Die Arbeit gliedert sich in einen theoretischen-methodologischen sowie einen empirischen Teil. In Kapitel II wird zunächst die „Normalfamilie“ im Lichte soziologischer Modernisierungstheorie als theoretische Vergleichsgruppe für Unternehmerfamilien skizziert. Dieses Vorhaben ist angesichts der unüberblickbaren Fülle an Beiträgen sowie der vielen, teils konträren Ansätze ein riskantes Unterfangen. Der Literaturüberblick muss zwangsläufig selektiv und unvollständig bleiben, besonders im Bereich der Individualisierungstheorie und Familiensoziologie. Mit Hilfe von Lehrbüchern, klassischen Aufsätzen und einführender Literatur sollen deshalb diejenigen Merkmale von modernen Familien, Arbeitsweisen und Lebenslaufgestaltungen vorgestellt werden, die als Konsens oder zumindest als zentrale Ansätze gelten können. Ziel ist nicht, den Facettenreichtum der soziologischen Diskussion zu diesen Themen wiederzugeben, sondern zu veranschaulichen, dass Unternehmerfamilien im Lichte dieser theoretischen Ansätze und empirischen Forschungsergebnisse in zweierlei Hinsicht - eben als Anachronismus oder Paradox - auffällig sind.

Nach dieser theoretischen Einführung werden in Kapitel III zentrale Ergebnisse und Kontroversen der lückenhaften Forschung zu Unternehmerfamilien zusammengefasst. Der anfängliche Überblick über die Forschungslandschaft zeichnet die Auseinandersetzung mit dem Untersuchungsobjekt in den verschiedenen Wissensgebieten nach, bevor jenseits disziplinärer Grenzen die Ergebnisse der verschiedenen Studien und Forschungsvorhaben näher erläutert werden. Im Einzelnen wird anhand historischer Überlegungen die sich wandelnde gesellschaftliche Bedeutung von Unternehmerfamilien diskutiert, zeigt ein systemtheoretischer Zugang Rollenkonflikte und Paradoxien auf, wird ein theoretisches Modell über die Dimensionen des Unternehmenseinflusses auf die Familie entwickelt und am Beispiel des Generationenwechsels aus Familienperspektive angewandt.

Die Zwischenbetrachtung (Kapitel IV) schlägt eine Brücke zwischen diesen theoretischen Vorarbeiten sowie der empirischen Anlage der Studie. Die bestehenden Erkenntnisse zum Untersuchungsobjekt Unternehmerfamilie werden in Beziehung gesetzt zu den im Vorfeld entwickelten Thesen aus modernisierungstheoretischer Sicht. Dadurch wird deutlich, dass ein Zugang zum Untersuchungsobjekt gewählt werden sollte, der das „Ganze Leben“ der Familien eigener Art erfasst. Einen solchen Zugang versprechen das Programm des Institutionalisierten Lebenslaufes (Kohli ergänzt um Elder) sowie 
der Ansatz der Alltäglichen Lebensführung (Voß, Kudera), die als theoretische Perspektiven eingeführt und diskutiert werden. Auf dieser Grundlage gelingt es, zentrale Begriffe zu definieren und die Forschungsfrage dieser Arbeit näher einzugrenzen.

Das Kapitel V beschreibt die Vorgehensweise der empirischen Untersuchung. Die detaillierte Darstellung des Forschungsprozesses und der Interpretationsarbeit soll dem Kriterium der Nachvollziehbarkeit der analytischen Ergebnisse entsprechen.

Die folgenden Kapitel VI und VII stellen die empirischen Befunde vor. Zunächst wird der Einfluss des Unternehmens auf das Leben der Mitglieder einer Unternehmerfamilie in den unterschiedlichen Lebensphasen beschrieben. Die aus dem empirischen Material generierten theoretischen Verallgemeinerungen zeichnen ein Portrait moderner Unternehmerfamilien. Die dabei hervortretenden Besonderheiten von unmittelbaren und spannungsreichen Generationenbeziehungen sowie einem ausgeprägtem „Wir-Gefühl“ werden danach einer intensiven Analyse unterzogen.

Kapitel VIII bildet den Abschluss dieser Arbeit. Wichtigen Befunde werden kurz zusammengefasst, theoretische Bezüge hergestellt und offene Forschungsfragen formuliert. Im Mittelpunkt steht dabei die Spannung zwischen der strukturellen Eigenlogik und den Handlungsoptionen von Unternehmerfamilien. Zudem wird der Versuch unternommen den gewählten $\mathrm{Zu}-$ gang zum „Ganzen Leben“ in Beziehung zu der bestehenden systemtheoretischen Forschung zu Unternehmerfamilien zu setzen. 


\section{Familie eigener Art?}

Es war Bruno Hildenbrand (2002) der erstmals Unternehmerfamilien als „Familien eigener Art“ bezeichnete. Gemeint ist damit, dass sich diese familiale Lebensweise strukturell von anderen so stark unterscheidet, dass sie einen eigenen Typ formt. Um diese These zu untermauern, setzt Hildenbrand das Familienunternehmen in Relation zu der traditionellen Familienform des oikos - des Ganzen Hauses - ebenso wie zu der modernen Kernfamilie. Er kommt zu dem Fazit, dass Familienbetriebe eine Einheit sind, ,in der aus der Logik der Sache heraus Strukturmerkmale des ,ganzen Hauses' notwendig konflikthaft gepaart sind mit solchen der ausdifferenzierten Kernfamilie“ (Hildenbrand 2002:118). Hildenbrand löst mit dieser Vorgehensweise ein wichtiges analytisches und methodisches Problem, das ganz bewusst an den Anfang dieser Arbeit gestellt wird.

Denn die nahe liegende Frage nach einer geeigneten Vergleichsgruppe war für die vorliegende Studie zunächst schwierig zu beantworten. Es schien sehr willkürlich, eine spezifische Gruppe wie etwa die „Manager“ oder „Arbeiterfamilien“"im Vorfeld zu bestimmen, wenn die konstitutiven Merkmale des Untersuchungsobjektes noch gar nicht feststehen. Ebenso problematisch ist es allerdings, die Besonderheiten der Unternehmerfamilie anhand einer impliziten alltagsweltlichen Vorstellung der „Normalfamilie“ zu beurteilen. Aus diesem Grund soll im Folgenden der bestechende Gedanke Hildenbrands aufgegriffen werden und eine theoretische Vergleichsgruppe für Unternehmerfamilien auf Basis von theoretischen Idealtypen für „normale“ Familien entworfen werden.

Aus der Perspektive soziologischer Modernisierungstheorien haben sich unterschiedliche Typen familialer Lebensformen als über einige Jahrzehnte hinweg historisch stabil erwiesen und üben eine starke Orientierungsfunktion für das familiale Handeln einer Gesellschaft aus. Innerhalb der soziologischen Theoriebildung kommt dabei der modernen Kernfamilie, die lange Zeit als die „Normalfamilie“ bezeichnet wurde, eine besondere Stellung zu: Sie dient einerseits als Fluchtpunkt dieses Modernisierungsprozesses und wird anderseits als seine Grundlage verstanden. Diesem Umstand trage ich insofern Rechnung, als der folgende Abschnitt nicht chronologisch sortiert ist. Stattdessen nutze ich zwei klassische soziologische Aufsätze, um die moderne Kernfamilie als theoretischen Ausgangspunkt zu fassen. In zeitlicher Perspektive nach hinten gerichtet flankiere ich diese Familienform mit dem Idealtypus des „Ganzen Hauses“, als Modell aktueller und zukünftiger Familienformen führe ich das Konzept der „Multilokalen Mehrgenerationenfamilie“ 
ein. In meiner Darstellung verbinde ich die drei idealtypischen Familienformen mit theoretischen Vorstellungen zum Modernisierungsprozess und konfrontiere diese kritisch mit empirischen Befunden.

In einem zweiten Schritt wird die Unternehmerfamilie gedankenexperimentell in Relation zu diesen Familienformen gesetzt und damit der Möglichkeitsraum für die Merkmale und Besonderheiten von Unternehmerfamilien geöffnet. Der theoretische Vergleich geht insofern über Hildenbrand hinaus, als explizit auch Pluralisierungs- und Destandardisierungstendenzen der familialen Lebensformen in die Analyse einbezogen werden. Ganz im Sinne eines hermeneutischen Gedankenexperimentes werden jedoch zunächst alle Erkenntnisse der bisherigen Forschung zu Unternehmerfamilien ausgeblendet und deren zentrale Ergebnisse und Kernargumente erst im nächsten Kapitel aufgegriffen.

Diese theoretische Hinführung hat nicht das Ziel, die familiensoziologische und modernisierungstheoretische Literatur mit ihren oft konträren Beiträgen auch nur annähernd vollständig abzubilden. Zugegebenermaßen wird ein vereinfachtes und unterkomplexes Bild gezeichnet, das nicht der Vielfalt der empirischen Wirklichkeit Rechnung trägt. Es soll kurz zentrale Begrifflichkeiten vorstellen, die das ,,analytische Handwerkszeug“ für die Ergebnispräsentation bilden. Dieses Kapitel dient dazu, die Eckpfeiler jener Familienformen zu skizzieren, denen in unterschiedlichen historischen Perioden eine starke Orientierungsfunktion für familiales Handeln zukam.

\subsection{Die moderne Kernfamilie}

Was ist eine „normale“ Familie? Auf diese Frage werden in den klassischen Beiträgen zur Familiensoziologie klare Antworten gegeben. Mit Bezug auf zwei einschlägige Aufsätze - zum einen Emile Durkheims „La famille conjugale“ (2011 c1921), zum anderen Talcott Parsons „The Kinship System of the Contemporary United States“ (1954) - lassen sich die wesentlichen Kriterien beispielhaft ableiten: Die moderne Familie tritt als Kernfamilie auf, sie besteht also aus einem Ehepaar mit unverheirateten (und noch nicht volljährigen) Kindern. Innerhalb dieses Zusammenhanges von zwei Generationen und zwei Geschlechtern entfaltet sich eine typische Rollenstruktur, die Handlungserwartungen an die eheliche Paarbeziehung ebenso wie an die ElternKind-Beziehung stellt. Der Vater erwirtschaftet auf dem Arbeitsmarkt Güter und Geldeinkommen, während sich die Mutter um Haushalt und Erziehung kümmert. Zu den Kindern herrscht ein emotionales, aber nicht gleichberechtigtes Verhältnis. Parsons weist deshalb dem Vater eine ,instrumentelle“, der Mutter eine „expressive“ Führungsrolle zu. Die Verbindung der Eheleute 
markiert das beständige Element moderner Kleinfamilien, weshalb Durkheim den Begriff der Gattenfamilie vorschlägt. Beide verweisen darauf, dass die Partnerwahl frei, also ohne den Einfluss der Verwandtschaft, und von Emotionen geleitet erfolgt. Abgesehen vom Endogamieverbot gilt als einzige Orientierung dafür, wer wen heiraten und eine Familie gründen darf, die Vorstellung romantischer Liebe.

Die typische Kernfamilie lebt in einem eigenen Haushalt, getrennt von den Eltern beider Ehegatten, und ist ökonomisch unabhängig. Für ihre Kinder wiederum besteht die Verpflichtung, sich von den Eltern zu emanzipieren, wodurch das Stadium der Adoleszenz zu einem Übergang im Lebenslauf wird. Es entstehen Ketten von verbundenen, neolokalen Familien, die eine Unterscheidung in Herkunfts- und Reproduktionsfamilie ermöglichen. Mit der Heirat und beruflichen Unabhängigkeit der Kinder schwächt sich die Bindung zu den Alten ab, was eine Vereinsamung des alten Paares zur Konsequenz haben kann (Parsons 1954:195). Familiale Solidarität ist in der Kernfamilie stark ausgeprägt, innerhalb des Verwandtschaftssystems (schon z.B. gegenüber Großeltern) wesentlich geringer.

Parsons sieht in der „Isolation“ der amerikanischen Kernfamilie einen Unterschied zur europäischen Familie. Jenseits des Atlantiks (aus Parsons Perspektive) erhalten wesentlich mehr Kernfamilien finanzielle Unterstützung von der Herkunftsfamilie oder erben etwa ein Haus oder einen beruflichen Status (insbesondere einen Hof oder ein Familienunternehmen) (Parsons 1954:184). In den Vereinigten Staaten sei eine solche Solidarität entlang der Verwandtschaftslinien ein Phänomen der höheren Klassen. In elitären Familien spiegelt sich das historische Muster europäischer Aristokratien wider, nur spürbar weniger verpflichtend. Parsons hebt hervor, dass diese Art der generationalen Solidarität eng mit Eigentum (insbesondere einem Familiensitz) und dem Erhalt eines bestimmten Status in der lokalen Gemeinschaft verbunden ist. Auch Durkheim geht auf das Eigentum ein und prognostiziert, dass Veränderungen in den bestehenden Erbschaftsregelungen notwendig sein werden. Indem innerhalb der Familie einige Vorteile übermittelt werden, die sich Personen nicht aus eigener Leistung geschaffen haben, sondern die aus einer privilegierten Stellung gegenüber anderen resultieren, wird sie zum Mittler sozialer Ungleichheit.

Durkheim und Parsons sind sich darüber einig, dass die Familie einen Bereich der Privatheit formt, der als Komplementär zur Arbeitswelt wichtige Funktionen erfüllt. Erstens ist Familie der Ort der Sozialisation. In diesem Prozess bilden sich die wesentlichen Züge der Persönlichkeit eines Individuums aus, das auf dem Arbeitsmarkt seine Fähigkeiten feilbieten kann. Wertorientierungen, Normen und anderes lebensweltliches Wissen wird in Familien übermittelt und von ihren Mitgliedern internalisiert. Zweitens trägt die Familie über ihre Anerkennungs- und Sanktionsmechanismen zur Aufrechterhaltung und Stabilisierung dieser Persönlichkeit bei. In modernen Familien 
zählt die ganze Person. Beziehungen zueinander sind unkündbar. Obwohl die Familienmitglieder ihre Berufsrollen zum Erwerb von Geldeinkommen als Individuen und nicht aufgrund ihres familialen Status ausüben, sehen sowohl Parsons als auch Durkheim, dass sich der berufliche Status einer oder zweier Person/en (meist des Vaters) auf die ganze Familie auswirkt. Für Durkheim macht zudem die Arbeit nur dann Sinn, wenn etwas geschaffen wird (materiell oder immateriell), das über das eigene Leben hinaus erhalten bleibt und den Personen nützlich ist, die wir lieben. Der Orientierungspunkt Familie wirkt entsprechend sinnstiftend und als eine wichtige Motivationsquelle im Hinblick auf Erwerbsarbeit.

Schließlich muss die Familie auch als ein Ort von öffentlichem Interesse verstanden werden, wie Durkheim ausführt. Der Staat greift in die Familie ein, indem er beispielsweise den Übergang von Vermögen regelt oder die Ehe legitimiert. Nach Durkheim trägt der Staat auf diese Weise zum Machtausgleich in den vom Vater dominierten Familien bei. Das institutionalisierte System der Familie ist insofern eine soziale Einheit in einer funktional differenzierten Gesellschaft.

Die moderne Kernfamilie, wie sie Parsons und Durkheim beschrieben, wird in vielerlei Hinsicht als „Normalfamilie“ bezeichnet. Zum einem gilt diese insbesondere über weite Teile des 19. und 20. Jahrhunderts hinweg als gesellschaftliches Ideal, an dem sich familiales Handeln ausrichtet. In den 1950er und 1960er Jahren erlebt die bürgerliche Kernfamilie ihren Höhepunkt (Schneider 2002:513) und ist auch empirisch die häufigste Familienform. Aus heutiger Perspektive wird ein Festhalten an dem Modell ,Normalfamilie“ jedoch vielfach kritisiert, weil damit immer auch ein normativer Anspruch verbunden sei, der ,abweichende“ Formen abwertet (Burkart 2008:140), und die jüngere empirische (und historische) Familienforschung dieses Bild als unterkomplex entlarvt hat. Dennoch kommt diesem Bild in der soziologischen Theoriebildung insofern ein zentraler Stellenwert zu, als die moderne Kernfamilie (von dem Begriff der „Normalfamilie“ hat man sich distanziert) als ein wichtiger Referenzpunkt dient, und zwar sowohl in der Interpretation der historischen Entwicklungen als auch bei der Deutung jüngerer Veränderungen und Zukunftsprognosen. Während die beiden modernisierungstheoretischen Leitideen der funktionalen Differenzierung und der Individualisierung eine Entwicklung hin zur modernen Kernfamilie beschreiben, verweisen Pluralisierungs- und Destandardisierungsthesen auf eine Auflösung dieser Familienform in der Spätmoderne.

In diesem Sinne wird im Folgenden am Modell der modernen Kernfamilie festgehalten und zwischen dem historisch vorgelagerten Idealtypus (im Weberschen Sinne) der Hausgemeinschaft bzw. des Ganzen Hauses und der Zukunftsvision der multilokalen Mehrgenerationenfamilie eingereiht. Beide Formen des familialen Handelns werden auch im Hinblick auf ihr Verhältnis zu Arbeit und Eigentum beschrieben. 


\subsection{Die Auflösung des „Ganzen Hauses“}

Durkheim und Parsons gehen von einer bipolaren Entwicklung der Familienformen aus. Im Laufe der Modernisierung habe sich die Familie von einer großen häuslichen Gemeinschaft in eine kleine, emotionale, soziale Einheit gewandelt. Wie muss man sich diese frühere Familienform vorstellen? Gemeinhin wird die traditionelle Familie mit einer romantischen Vorstellung der Großfamilie assoziiert. Mehrere Generationen leben harmonisch in einem Haushalt zusammen. Großeltern und alleinlebende Verwandte sind integriert, und es herrscht ein hohes Maß an Verantwortungsgefühl für hilfsbedürftige Angehörige. Diese Vorstellung hält sich zwar hartnäckig als Stereotyp, hat sich aber als falsch erwiesen - und damit ist auch das Durkheimsche Kontraktionsgesetz widerlegt. Der Familienhistoriker Michael Mitterauer spricht deshalb vom „Mythos der vorindustriellen Großfamilie“ (1980). Durch seine Untersuchungen, in der er unter anderem das durchschnittliche Heiratsalter (25-30 Jahre) zu dem mittleren Sterbealter der Verheirateten (55-60 Jahre) in Beziehung setzt, kann er zeigen, dass es nur einen Korridor von fast dreißig Jahren für die gemeinsame Lebenszeit von Großeltern und Enkeln gibt. Stattdessen wird die Hausgemeinschaft als vorherrschende Lebensform im vorindustriellen Europa identifiziert (16.-18. Jahrhundert), das so genannte „Ganze Haus“.

In dieser Familienform ${ }^{10}$ ist die Ehe ebenfalls ein wichtiges Element der sozialen Integration, aber sie ist eingebettet in einen weiteren sozialen Kontext: die Hausgemeinschaft und Ständegesellschaft. Kaum jemand lebt als Ehepaar nur mit Kindern zusammen, sondern die Haushalte umfassen neben der eigentlichen Kernfamilie meist auch ledige Verwandte und nichtverwandte Arbeitskräfte. Die Bezeichnung „Ganzes Haus“ spielt auf den Umstand an, dass sich hier unter einem Dach Wohnen, Arbeiten und Leben vereinen. In der Hausgemeinschaft sind persönliche Bedürfnisse zweitrangig, es geht um die soziale und wirtschaftliche Absicherung der Mitglieder. Innerhalb des Haushaltes gibt es wenig Raum für Individualität, Privatheit oder Intimität. Sexuelle oder emotionale Beziehungen stehen nicht im Mittelpunkt, die Hausgemeinschaft ist in erster Linie eine ökonomische Arbeitsgemeinschaft.

Die Gemeinschaft ist patriarchal strukturiert. Der Hausherr vertritt das Haus nach außen und trägt die Verantwortung für die Abwicklung von Geschäften. Er sorgt dafür, dass der Lebensunterhalt der Mitglieder gesichert ist, dass sie innerhalb der Gemeinschaft von äußeren Gefahren geschützt und im Krankheitsfall versorgt werden. Die Solidarität zwischen den Familien-

10 Die Beschreibung des „Ganzen Hauses“ in den nächsten beiden Absätzen bezieht sich im Wesentlichen auf die Zusammenfassung von Burkart 2008:116ff. 
mitgliedern bezieht sich auf das Ganze Haus und nicht nur auf die Kernfamilie. Auch der sittlich-moralische Lebenswandel der Hausgemeinschaft wird vom Hausherrn verantwortet. Die familiale Organisation zielt darauf ab, das häusliche Vermögen in der Familie zu erhalten - die Familienmitglieder leben und besitzen gemeinsam. Persönliche Bindungen und individuelle Identität scheinen dem Überleben des Kollektivs nachgelagert zu sein.

An dieser Stelle sei noch einmal betont, dass das „Ganze Haus“ ebenso als theoretisch überspitzte soziale Einheit zu gelten hat wie die „moderne Kernfamilie“ im Abschnitt zuvor. In der empirischen Realität finden sich diese Idealtypen höchst selten in ihrer Reinform. Die historische Sozialforschung hat darüber hianus sehr treffend und detailreich beschreiben, dass dieser Typus nicht allen Bevölkerungsschichten anzutreffen war insbesondere nicht in besitzlosen Klassen (Dienstleute, Tagelöhner etc.). In Kapitel 3.2 wird noch ausführlicher auf die historische Forschung zu Unternehmerfamilien eingegangen.

Die dahinter stehende Frage ist - und damit haben sich die Sozialwissenschaften seit ihrem Bestehen auseinandergesetzt -: Wie vollzieht sich ein so grundlegender Wandel der familialen Lebensformen? Die Diskussion ist geprägt von einer Vielfalt von Erklärungsansätzen, die mit plakativen Schlagworten versehen sind: Industrialisierung, Rationalisierung, Domestizierung, Urbanisierung, Enttraditionalisierung, Demokratisierung oder Säkularisierung. Zwei zentrale Leitlinien dieser Diskussion sollen im Folgenden kurz skizziert werden, zum einen die These einer funktionalen Differenzierung, zum anderen die These einer fortschreitenden Individualisierung.

Die strukturfunktionalistischen und systemischen Ansätze (etwa Durkheim, Parsons, Habermas und Luhmann) verstehen den Prozess der Modernisierung als einen, in dem sich Institutionen und Organisationen herausbilden, die sich auf bestimmte, für die Erhaltung der Gesellschaft wichtige Funktionen konzentrieren (2009:10). Beispiele solcher gesellschaftlicher Subsysteme sind etwa das Bildungssystem, die Gerichtsbarkeit, das Wirtschaftssystem, organisierte Religion und eben auch Familie. Ein zentraler Argumentationsstrang, der sich unmittelbar an die These einer funktionalen Differenzierung der Gesellschaft anschließt, ist jener vom Funktionsverlust der Kernfamilie. Im Zuge dieses Prozesses wird die Kernfamilie von der Einheit der Hausgemeinschaft funktional isoliert und auf eine „Gefühlsgemeinschaft“ reduziert (Beck-Gernsheim 1986:212). Ständische Bindungen werden obsolet, und die Einheit von Familie und Wirtschaftsgemeinschaft zerbricht. Der Kernfamilie verbleiben wenige gesellschaftliche Funktionen, zu denen die Reproduktion, Sozialisation, Stabilisierung der Persönlichkeit und Statuszuweisung zählen (Burkart 2008:144f.). Eine besondere Erwähnung verdient in diesem Zusammenhang die Transformation des Geschlechterverhältnisses. Für Frauen und Männer werden nun unterschiedliche Wesenszüge angenommen, die sich aus der Biologie der Menschen natürlich legitimieren ließen: Während dem 
Mann eine „Versorgerrolle“ zugesprochen wird, gilt die Frau als „Gefühlsspezialistin“.

Die Individualisierungsthese ist als ein Konglomerat aus Beschreibungen der Lebensbedingungen moderner Gesellschaften zu verstehen, eher als eine „vage Entwicklungshypothese“ (Hill und Kopp 2006:313) denn eine geschlossene Theorie. ${ }^{11}$ In einem allgemeinen Sinn beschreibt Individualisierung bestimmte subjektiv-biografische Aspekte des Zivilisationsprozesses. Modernisierung führe zur Herauslösung der Einzelnen aus historisch vorgegebenen Sozialformen und -bindungen, zum „Verlust von traditionalen Sicherheiten im Hinblick auf Handlungswissen, Glauben und leitende Normen (Entzauberungsdimension)“ (Beck 1986:206) und zu neuen Formen sozialer Einbindung. Individuelle Beziehungen erscheinen nun nicht mehr eingebettet in klassen- oder schichtspezifische Konfigurationen, sondern werden nach individuellen Präferenzen gewählt und genutzt (Hill und Kopp 2006:313). Die Modernisierung der Gesellschaft führt zum Verlust kollektiver Bindung und zur Individualisierung sowohl im Sinne von Autonomie als auch von Vereinzelung (Hill und Kopp 2006:316). Die Familie ist eines von vielen Feldern, auf denen sich der breite Trend zur Individualisierung bemerkbar macht. Die Leistung der Kernfamilie sieht auch die Individualisierungstheorie in der Sozialisation von rationalen und individuellen Persönlichkeiten, deutet dies jedoch weniger pessimistisch als strukturfunktionalistische Ansätze. Die Kernfamilie trägt zur sozialen und emotionalen Absicherung in riskant gewordenen Gesellschaften bei. Sie fungiert als geschützter Raum vor gesellschaftlichen Umbrüchen, hier herrscht eine Balance zwischen Individualismus und sozialer Verantwortlichkeit (Hill und Kopp 2006:310). Insofern ist die Reduktion der Bedeutung der Familie keineswegs gleichzusetzen mit ihrer funktionalen Irrelevanz (Hill und Kopp 2006:315). Im Gegenteil, die ,Sinnentleerung marktmäßig organisierter (Arbeits-)Beziehungen wird in der Familie kompensiert, sie wird zu der identitätsstiftenden Institution schlechthin“" (Hill und Kopp 2006:315). Sie schafft den Ersatz für die Deutungsmuster und Sozialbeziehungen, die mit der Modernisierung aufgelöst werden (Beck-Gernsheim 1986:213), sie verleiht dem Leben Sinn und Verankerung.

Die Ausdifferenzierung von Individuallagen geht gleichzeitig mit einer hochgradigen Standardisierung einher. An die Stelle traditioneller Bindungen und Sozialformen (soziale Klasse, Kleinfamilie) treten die sekundären Instanzen und Institutionen der ausdifferenzierten Erwerbsarbeit sowie die um sie herum entstandenen wohlfahrtsstaatlichen Sicherungssysteme, die den Lebenslauf des Einzelnen prägen. Der von Martin Kohli beschriebene Pro-

11 Die Individualisierungstheorie wurde von einer Reihe von Arbeiten Mitte der 1980er Jahre angestoßen und entscheidend geprägt. Dazu gehören etwa Beck 1983; 1986, BeckGernsheim 1983; 1986 und Kohli 1985. 
zess der Institutionalisierung des Lebenslaufs (siehe hierzu auch Kapitel 4) beschreibt, wie sich ein Wandel ,,von einem Muster der Zufälligkeit der Lebensereignisse zu einem des vorhersehbaren Lebenslaufes“" (Kohli 1985:5) vollzogen hat. Der Lebenslauf selbst wird zu einer gesellschaftlich integrierenden und strukturierenden Instanz. Er umfasst neben einer Sequenzialität im Sinne eines geordneten (und chronologisch festgelegten) Ablaufs der wesentlichen Lebensereignisse und der Kontinuität im Sinne einer verlässlichen, auch materiell gesicherten Lebensspanne eine Biografizität im Sinne eines Codes von personaler Entwicklung und Emergenz, also einen Übergang zu einer biografischen, d. h. vom Ich aus strukturierten und verzeitlichten Selbst- und Weltauffassung (Kohli 1985, 1994, 2007). Die ,institutionellen“ Lebenslaufmuster sind geprägt vom Eintritt und Austritt in bzw. aus Bildungssystem und Erwerbsarbeit, sozialpolitischer Fixierung des Rentenalters etc. Es tritt eine Vereinheitlichung bezüglich der Abfolge individueller Lebensläufe und des Familienzyklus ein (Huinink und Wagner 1998:94ff.). Der institutionalisierte Lebenslauf konstituiert ein Vergesellschaftungsprogramm, das an den Individuen als den neuen sozialen Einheiten ansetzt (Kohli 1985:3). Soziale Ordnung bzw. Kontrolle entsteht nicht mehr aus einer stabilen Lebenslage, sondern vielmehr aus einem regelhaften, verlässlich erwartbaren Lebenslauf (Kohli 1994). Es etabliert sich ein regelhafter Familienzyklus, der in vormodernen Gesellschaften weniger an bestimmte Altersmarken gebunden war (Kohli 1985:6). Heiraten, Kinder haben, bis zum Alter von mindestens 55 Jahren zusammen mit dem Ehepartner überleben (Empty Nest Phase) wird zur selbstverständlichen Normalität. Mit Blick auf die unterschiedlichen Lebensläufe von Männern und Frauen muss an dieser Stelle darauf verwiesen werden, dass sich geschlechtstypische Biografien herausbilden, die zwischen Eheleuten abgestimmt und in ihrem Zusammenhang begriffen werden sollten.

Obwohl in modernen Gesellschaften Haushalt und Betrieb getrennt existieren, können Kernfamilien also keineswegs unabhängig von den wirtschaftlichen und staatlichen Systemen betrachtet werden. Die Kernfamilie begibt sich in eine starke Abhängigkeit von Erwerbseinkommen und Transferleistungen ihrer Mitglieder (Hill und Kopp 2006:309). Familienökonomische Ansätze (etwa Becker und Pies 1996:101ff.), die den Rational-Choice- und Austauschtheorien zugeordnet werden können, bezeichnen Familien weiterhin als „Produktionsgemeinschaften“, die über die Bündelung von Ressourcen und eine innerhalb der Familie arbeitsteilige Produktion versuchen, eine vorteilhafte Ausgestaltung des Alltags zu erreichen (Hill und Kopp 2006:114).

Auch die vorherrschenden normativen Vorstellungen hinsichtlich Eigentum und Vererbung unterliegen einem Wandel und korrespondieren mit der neuen familialen Lebensweise in Kernfamilien (Beckert 2008:84): Eheleute werden als Zugewinngemeinschaft verstanden, dass heißt, sie besitzen ge- 
meinsam und generieren arbeitsteilig weiteres Vermögen. Nach ihrem Ableben soll dieses zu gleichen Teilen auf die leiblichen Kinder verteilt werden. ${ }^{12}$ Diese Idealvorstellung findet ihren Ausdruck unter anderem in der Vorschrift eines Pflichtteiles für die Kinder.

Diese kurze Heranführung an zentrale Ideen soziologischer Modernisierungstheorie verdeutlicht, dass familiale Strukturen selten konstant sind, sondern das Ergebnis familialen Handelns, das den Einflüssen sich ändernder gesellschaftlicher Rahmenbedingungen und Persönlichkeitsmuster unterliegt (Hill und Kopp 2006:305). Immer wieder bilden sich jedoch „Leitbilder“ familialen Handelns heraus, die über einen gewissen Zeitraum Orientierung bieten. Im Zuge von funktionaler Differenzierung und Individualisierung entstehen Wahlfreiheiten und Autonomie ebenso wie ein erhöhtes Bedürfnis nach Bindung und Nähe. Beides findet aus modernisierungstheoretischer Perspektive seine Entsprechung in der modernen Kernfamilie, die einhergeht mit einer spezifischen Gestaltung der Lebensführung und einem Normallebenslauf.

\subsection{Das Ende der modernen Kernfamilie?}

Die gesellschaftliche Entwicklung endet nicht mit der modernen Kernfamilie. Aus der Perspektive der historischen Familienforschung ist diese lediglich ein Übergangsstadium im permanenten Wandel familialer Strukturen (siehe etwa von Trotha 1990). Globalisierung, Ökonomisierung, Entstofflichung, Beschleunigung, Entfremdung, Tertiarisierung oder Flexibilisierung sind die zentralen Schlagworte, die den Vorgang einer weiteren Veränderung moderner Gesellschaften fassen sollen. Einigkeit herrscht darüber, dass der Prozess der Modernisierung mit all seinen Facetten auf einem neuen Niveau fortschreitet. Degele und Dries (2005) schlagen deshalb eine Einteilung der „Moderne“ in drei Epochen vor: die Frühmoderne (16. bis Ende 18. Jahrhundert), die Hoch- oder Industriemoderne (19.-20. Jahrhundert) und die Spätmoderne (etwa ab den 1970er Jahren). ${ }^{13}$ Mit dem Übergang in die Spätmoderne werden die Grenzen des Fortschritts ersichtlich (wie etwa der Bericht des Club of Rome anschaulich darstellt Meadows 1972), und die Modernisierungseuphorie lässt nach. Gleichzeitig scheint sich das Tempo der gesell-

12 Diesen normativen Grundsatz konnte eine Reihe von empirischen Arbeiten im Bereich der intergenerationellen Transferforschung belegen (etwa Kohli 2004, 1999; Kohli und Künemund 2001; Albertini et al. 2006.

13 Die Epochengrenzen verstehen Degele und Dries als flexibel. Die Bezeichnung „Spätmoderne“ soll eine Alternative zu dem ideologisch aufgeladenen Begriff der „Postmoderne“ bieten, so Degele/Dries 2005:88. 
schaftlichen Entwicklung (und insbesondere der funktionalen Differenzierung und Individualisierung) zu erhöhen.

Aus einer historischen Perspektive verändern sich ab den 1960er/70er Jahren die Lebensbedingungen in Deutschland grundlegend. Ein relativ hoher allgemeiner Wohlstand eröffnet materielle Gestaltungsmöglichkeiten. Die Bildungsexpansion, die rechtliche Gleichstellung der Frau sowie die steigende Frauenerwerbstätigkeit bringen die traditionellen Geschlechterrollen ins Wanken. Die selbstverständliche Lebensweise der modernen Kernfamilie wird zunehmend in Frage gestellt (Hill und Kopp 2006:313). Beck und BeckGernsheim führen aus, wie in einem zweiten „Individualisierungsschub“ die Individuen gänzlich aus den diversen gesellschaftlichen Einbettungen und Abhängigkeiten freigesetzt werden. In der Spätmoderne mutieren Individuen zum „Sinnzentrum“ und „Planungsbüro“ ihrer eigenen Biografie (Beck 1986:217). Anonyme und selbst geknüpfte soziale Netze treten an die Stelle von nahen verwandtschaftlichen Bindungen. Als unmittelbare Folge von Individualisierungsprozessen lasse sich empirisch eine zunehmende Pluralisierung von Lebensstilen und Lebensformen beobachten: etwa Patchworkfamilien, Mehrgenerationenfamilien, Wohngemeinschaften, Alleinerziehende mit we-chselnden „Lebensabschnittspartnern“ oder gleichgeschlechtliche Lebensgemeinschaften (siehe etwa Peukert 2007 oder Nave-Herz 2007). Die Pluralisierung der Lebens- und Familienformen ist eng mit der kulturellen Liberalisierung von Ehe und Familie (Kaufmann 2005) verbunden und wird häufig als der charakteristische Trend in (spät)modernen Gesellschaften gehandelt (Hill und Kopp 2006:306). Das so genannte „living apart together“ (Hoffmann-Nowotny und Hans-Joachim 1995:340) erscheint als ein typisches Element neuer familialer Lebensweisen. Erwachsene Familienmitglieder leben demnach zunehmend ,getrennt" zusammen, verringern also den Teil des Alltags, den sie gemeinsam verbringen, und pflegen ihren eigenen Lebensstil. Auf diese Weise können sie der dominanten Ideologie von Gleichheit und Individualität entsprechen (Hill und Kopp 2006:309).

An die Stelle homogener „Normalbiografien“ und Gruppenidentitäten treten vielfältige Milieus und Lebensstilvarianten - die Individuen sind gefordert, ihren eigenen, unverwechselbaren Stil zu entwickeln, um der herrschenden Individualisierungsnorm zu entsprechen.

„In der vollmobilen, flexibilisierten Bildungs- und Arbeitsgesellschaft wird die eigene Biografie letztlich zum regelrechten Risikounternehmen, das die Individuen zu permanenter Selbstanpassung, Selbstgestaltung und -vermarktung zwingt" (Degele und Dries 2005:88).

Kohli (1985) und Wohlrab-Sahr (1997) sehen in dieser Steigerung der Individualisierung Ambivalenzen zwischen einer prinzipiellen Wahlmöglichkeit 
auf struktureller Ebene (die mit neuen strukturellen Zwängen einhergeht) und einem Bedeutungsgewinn eines individuellen Zurechnungsmusters auf kultureller Ebene (Scherger 2007:35). Die fortschreitende Destandardisierung des Lebenslaufes wird besonders an weiblichen Biografien offenkundig, die sich gleichzeitig am Arbeitsmarkt und am Familienzyklus orientieren. ${ }^{14}$ Damit wird zunehmend die Vereinbarkeit von Beruf und Familie stärker in den Mittelpunkt gerückt (Hill und Kopp 2006: 326). Gleichzeitig vollzieht sich ein Funktions- und Bedeutungswandel von Kindern, und die qualitativen Ansprüche an die Eltern-Rolle steigen (Nave-Herz 2007:127). Für beide Geschlechter nehmen die Relevanz von Daseinsfragen und die Suche nach Identität in der Partnerschaft zu (Hill und Kopp 2006:316).

Beck und Beck-Gernsheim (1990) veranschaulichen, wie sich die (hoch)modernen Kernfamilien schließlich in (spät)modernes Liebes- und Beziehungschaos entwickeln. Die liebenden Individuen sind gefordert, ihre steigenden Selbstansprüche zu koordinieren. Während in der Kernfamilie die „Bündelung von Lebensplänen, Lebenslagen und Biografien weitgehend Verbindlichkeit" (Beck 1986:163) besaß, scheint es nun keine allgemein akzeptierten Formen des Zusammenlebens zu geben. Besonders durchlässig müssen die neuen Lebensweisen hinsichtlich zunehmend flexibler und mobiler Arbeitsweisen sein. „Der doppelte ,Lebensentwurf“, der Arbeit und Familie integriert und flexible Wechsel zwischen diesen Feldern ermöglicht und fördert, wird zum Normalfall“ (Hill und Kopp 2006:326). Was „Familie“ ist, muss verhandelt, abgesprochen und begründet werden (Beck und BeckGernsheim 1990:13). Auf diese Weise entsteht eine Dauerreflexivität hinsichtlich möglicher Identitäten, Lebenspläne und Lebensstile (Hettlage 1992:76). Familiale Wirklichkeit unterliegt einer steten Neu- und Redefinition, die statt konsistenter biografischer Konstrukte von Fall zu Fall mobilisierbare Selbst- und Fremdbilder provoziert (Schmidt und Moritz 2009:46; Hahn 1988). Dabei geben die institutionellen und lebensgeschichtlichen Rahmungen Bausätze vor, die sich in Form von Bastelbiografien kombinieren lassen. Auf der einen Seite entstehen mehr Wahlmöglichkeiten, wie man leben und familial handeln möchte. Auf der anderen Seite besteht nun auch eine Notwendigkeit, die ergriffene Form zu rechtfertigen (Degele und Dries 2005:86).

Mit Blick auf die Empirie (auch und gerade in historisch vergleichender Perspektive) wird diese idealisierende Beschreibung der Spätmoderne und besonders die Pluralisierungsthese immer wieder angezweifelt, eingeschränkt und relativiert. So ist die Vielfalt der Lebensformen keinesfalls ein neues Phänomen, und die meisten heute bekannten Lebensformen haben bereits

14 Jüngste Forschung weist darauf hin, dass eine Tendenz dahin besteht, dass auch männliche Biografien nicht mehr länger nur Arbeitsmarkt orientiert sein werden. Zum Wandel der Vater-Rolle siehe etwa Nave-Herz 2007:54f. oder Jurczyk und Lange 2009. 
einmal existiert (Schmidt und Moritz 2009:44) - sie stehen aber in einem Gegensatz zu dem zuvor dominanten Modell der Kernfamilie. Burkart und Kohli geben zu bedenken, dass sich familiale Strukturen heute mehr denn je milieuspezifisch ändern (Burkart 2008:46f.; Burkart und Kohli 1992:235ff.). Zudem sind insbesondere die nichtehelichen Lebensformen oft nur Übergangsphasen im Lebenslauf (Burkart 2008:34). Insgesamt scheint die aktuelle Diskussion eher um eine Zustandsbeschreibung zu kreisen, sie ist gekennzeichnet von empirischen Analysen der Scheidungsraten, Geburtenzahlen, der Häufigkeit von Ein-Personen-Haushalten oder der steigenden durchschnittlichen Lebenserwartung. Es gibt kaum ein anderes Feld sozialwissenschaftlicher Analyse, das auf Basis statistischer Daten zu so kontroversen Einschätzungen kommt (Burkart 2008:15). Ebenso uneindeutig sind die empirischen Ergebnisse hinsichtlich einer Destandardisierung des Lebenslaufes (Burkart 2008:250). Die arbeitsmarktseitigen Veränderungen (zentrale Schlagworte sind etwa Ausbildungsphase als dauerhaftes Provisorium, Generation Praktikum, Anstieg von ungesicherten und Teilzeitarbeitsverhältnissen usw.) scheinen sich bislang weit weniger dramatisch auf den institutionalisierten Lebenslauf auszuwirken als erwartet (Kohli 2007:263; Scherger 2007). Einigkeit scheint darüber zu bestehen, dass von der Kernfamilie der 1950er und 1960er Jahre mit ihren Rollenstrukturen heute keine große Orientierungsfunktion mehr ausgeht.

Eine neue Definition von „Familie“ wird zwar viel diskutiert, steht aber noch aus (Burkart 2008:299ff.), wobei sich eine Tendenz dahin gehend feststellen lässt, dass nun eher von privaten Beziehungen als von Familie gesprochen wird. Die Zukunftsvisionen von Familie schließen eine Entkoppelung der Reproduktion vom persönlichen Zusammenleben (durch Reproduktionsmedizin) oder die vollständige Auslagerung der Kindeserziehung aus der Familie nicht aus. Einige malen überspitzt sogar das Ende der Familie schlechthin an die Wand ${ }^{15}$. Ob es ein neues Leitbild familialen Zusammenlebens geben wird und wie sich dieses gestaltet, ist noch relativ unklar. Ein viel versprechendes Konzept ist die multilokale Mehrgenerationenfamilie (Lauterbach 2004; Bertram 2000).

Der Begriff verweist unter anderem auf zwei „neue“ Merkmale familialer Lebensformen, die sich aus den veränderten ökonomischen Randbedingungen, aus dem demografischen Wandel (insbesondere der nachhaltig gestiegenen Lebenserwartung und dem Rückgang der Geburtenzahlen) und der Erosion kultureller Werte ergeben. Erstens, Familien lassen sich nicht

15 Eine Diskussion über das Ende der Familie wird vor allem im englischsprachigen Raum geführt. Prominente Vertreter dieser skeptischen Zukunftsdeutung der Familie erkennen eine Erziehungs-, Bindungs- und Wertekrise aktueller familialer Lebensformen. Jedoch hat sich die These bislang noch nicht als empirisch tragfähig erwiesen Hill und Kopp 2006:323. Ehe und Familie seien immer noch zentrale Lebenswerte, die weiterhin als wichtiges Orientierungsmodell im Lebenslauf junger Erwachsener fungieren (siehe auch Burkart 2008:13). 
zwangsläufig mit Haushalten gleichsetzen, sie werden ,,multilokal“. Die Ergebnisse der Generationenforschung zeigen, dass familiale Solidarität wesentlich belastbarer ist als bisher angenommen. Eine netzwerktheoretische Perspektive lenkt den Blick auf die praktizierten Beziehungen von Familienmitgliedern über den Lebenslauf hinweg und zeigt, dass Familien nicht zerfallen, nur weil sie nicht im selben Haushalt leben (Burkart 2008:212). Die „neu“ entdeckte Multilokalität von Familie stellt für Bertram (2000:117) keine neue Praxis familialen Handelns dar, sondern entlarvt vielmehr die These einer isolierten Kernfamilie als Mythos. Dennoch kommt dem neolokalen Prinzip in der Spätmoderne mit ihren wachsenden Mobilitätsanforderungen des Arbeitsmarktes ebenso wie dem Bedürfnis, einen eigenen Lebensstil zu pflegen, eine erhöhte Bedeutung zu. Empirische Untersuchungen zeigen, dass die Wohnortentfernung in den allermeisten Fällen sehr gering ist (etwa getrennte Haushalte im gleichen Haus oder nur wenige Kilometer zwischen den Wohnungen), aber mit steigendem Bildungsgrad der erwachsenen Kinder zunimmt (Burkart 2008:212; Kohli et al. 2005b).

Zweitens, die vertikalen Beziehungen (zwischen Kindern, Eltern, Großeltern und Urgroßeltern) werden immer wichtiger, wohingegen die horizontalen Bande zu Geschwistern, Cousinen und Vettern an Bedeutung verlieren (Bertram 2000:118). Familien umfassen immer häufiger mindestens drei, häufig sogar vier Generationen (Lauterbach 2004:224). Die lange gemeinsame Lebenszeit von Großeltern und Enkeln, immer öfter auch Urenkeln, ist historisch gesehen eine Besonderheit. Die persönliche Verbundenheit zwischen den Familienmitgliedern erstreckt sich über den Lebenslauf hinweg und nimmt in den verschiedenen Lebensphasen unterschiedliche Beziehungsqualitäten an. Damit verbunden ist eine Veränderung der Eltern-KindBeziehung, die nun zunehmend zu gleichberechtigten Lebenspartnern werden. Kinder stellen emotional und materiell die wichtigste Investition der Eltern dar. Ihr Verhältnis ist mehr denn je von Zuwendung und Unterstützung, weniger von Autorität und Abhängigkeit geprägt. Auch nach dem Verlassen des Elternhauses bleiben sie nachhaltig verbunden. Rosenmayr prägt in diesem Zusammenhang den Begriff einer „Intimität auf Distanz“ (Bertram 2000:109). Den Großeltern und anderen (Wahl-)Verwandten kommt in diesen Familien eine wachsende Bedeutung in der Erfüllung familialer Funktionen zu. Sie sind Vorbild in der Sozialisation der Enkel, unterstützen die Eltern durch finanzielle und zeitliche Ressourcen, sie tragen zur Generationen übergreifenden Solidarität und Kontinuität bei (Bengston 2001:7).

Die Beziehungen in multilokalen Mehrgenerationenfamilien sind gekennzeichnet durch eine gemeinsam erlebte Familiengeschichte, ein Gefühl der Verantwortlichkeit für andere Generationen, eine emotionale Bindung und die Bereitschaft zu Unterstützungsleistungen (Bertram 2000:103). Empirisch gesehen kommt diesem Familientypus schon heute eine erhebliche Bedeutung zu. Es ist jedoch noch nicht absehbar, inwiefern sich dieser auch als 
(politische) Ordnungsvorstellung durchsetzt und welche Folgen diese neue Art der familialen Lebensweise für den Umgang mit Eigentum haben wird. ${ }^{16}$

Insgesamt lässt sich also ein allmähliches Aufweichen der Kernfamilie, wohl aber nicht ein Ende der Familie insgesamt beobachten. Welche Lebensweise(n) letztlich eine Orientierungsfunktion für familiales Handeln bzw. private Beziehungen übernimmt(nehmen), bleibt allerdings noch offen. Diese kurzen Erläuterungen verdeutlichen, dass das Verständnis einer „normalen" Familie in einem historischen und gesellschaftlichen Kontext zu sehen ist und einem andauernden Wandel unterliegt.

\subsection{Zwei Hypothesen zum Status von Unternehmerfamilien}

Familien, die Eigentum an einem Unternehmen besitzen und darin eine leitende Funktion übernehmen, werden gemeinhin als Unternehmerfamilien bezeichnet. Sie beeinflussen die strategische Ausrichtung eines Unternehmens meist über mehrere Generationen hinweg. Nimmt man nun die oben skizzierten idealtypischen Familienformen aus modernisierungstheoretischer Perspektive als Normalerwartungen an, lassen sich gedankenexperimentell einige Thesen über die Eigenschaften und Merkmale von Unternehmerfamilien formulieren, die über eine Minimaldefinition hinausgehen. Es lässt sich darüber spekulieren, wie sich das Innenverhältnis solcher Familien gestaltet, was die Grundlagen der Identitätsbildung sind und mit welcher Außenwirkung sich Unternehmerfamilien konfrontiert sehen. Es lassen sich Aussagen zu möglichen Konfliktlinien und Potentialen dieser Familien treffen. Je nachdem welche der drei Familienformen man als Vergleichsgruppe wählt, scheinen unterschiedliche Aspekte differenzierend oder verbindend $\mathrm{zu}$ wirken. Sehr überspitzt lassen sich zwei konträre Hypothesen formulieren. Erstens, während der Entwicklungsphase vom Ganzen Haus hin zur Kernfamilie wirken Unternehmerfamilien als Anachronismen. Zweitens, in der Entwicklungsphase von der Kernfamilie zur multilokalen Mehrgenerationenfamilie wirken Unternehmerfamilien als Pioniere.

16 Denkbar ist, dass Eigentum vermehrt als persönliches, individuelles und temporäres Eigentum angesehen wird. Beckert weist darauf hin, dass sich erbrechtlich gesehen der Familienbegriff auf nicht biologisch verwandte Personen wie z.B. Adoptivkinder ausdehnt Beckert 2008:85f.. Zudem könnte das Mittel der Testierfreiheit künftig genutzt werden, um Vermögen über die Generationenfolge hinweg zu transferieren (etwa von Großeltern auf Enkel). 
Abbildung 1: Übersicht idealtypischer Familienformen

\begin{tabular}{|c|c|c|c|}
\hline & Ganzes Haus & Kernfamilie & $\begin{array}{c}\text { Multilokale } \\
\text { Mehrgernationen- } \\
\text { familie }\end{array}$ \\
\hline $\begin{array}{l}\text { Grund- } \\
\text { einheit }\end{array}$ & Hausgemeinschaft & $\begin{array}{c}\text { Ehepaar mit ledigen, } \\
\text { nichtvolljährigen Kindern }\end{array}$ & $\begin{array}{l}\text { Netzwerk aus persön- } \\
\text { lichen Beziehungen }\end{array}$ \\
\hline Solidarität & $\begin{array}{c}\text { Bezieht sich } \\
\text { auf das ganze Haus }\end{array}$ & $\begin{array}{c}\text { In der Kernfamilie stark } \\
\text { ausgeprägt, im Verwandt- } \\
\text { schaftsnetz eher schwach }\end{array}$ & $\begin{array}{c}\text { Erstreckt sich über } \\
\text { mehrere Generationen } \\
\text { und schließt Wahlver- } \\
\text { wandtschaft ein }\end{array}$ \\
\hline $\begin{array}{c}\text { Eltern- } \\
\text { Kind- } \\
\text { Beziehung }\end{array}$ & $\begin{array}{c}\text { Kinder als kleine } \\
\text { Erwachsene; } \\
\text { Arbeitskraft }\end{array}$ & $\begin{array}{c}\text { Autoritär } \\
\text { Kindheit als geschützter } \\
\text { Raum }\end{array}$ & $\begin{array}{l}\text { Partnerschaftlich; } \\
\text { Gestiegene Anforde- } \\
\text { rungen an die Eltern }\end{array}$ \\
\hline $\begin{array}{l}\text { Rolle von } \\
\text { Großeltern }\end{array}$ & $\begin{array}{l}\text { Geringe gemeinsa- } \\
\text { me Lebenszeit von } \\
\text { Großeltern und En- } \\
\text { keln }\end{array}$ & $\begin{array}{c}\text { Verbindung zu den Eltern } \\
\text { schwächt nach dem Aus- } \\
\text { zug aus dem Elternhaus } \\
\text { ab. }\end{array}$ & $\begin{array}{c}\text { Kontinuität, Unterstüt- } \\
\text { zen in der Sozialisati- } \\
\text { on der Enkel; Materiel- } \\
\text { le und immaterielle } \\
\text { Transferleistungen }\end{array}$ \\
\hline $\begin{array}{l}\text { Rollen- } \\
\text { struktur }\end{array}$ & $\begin{array}{l}\text { Vater als Haushalts- } \\
\text { vorstand vertritt das } \\
\text { Haus nach außen } \\
\text { und sorgt für Le- } \\
\text { bensunterhalt, Absi- } \\
\text { cherung und Sitten } \\
\text { der Gemeinschaft. }\end{array}$ & $\begin{array}{c}\text { Mann = Versorger } \\
\text { (Einkommen auf dem Ar- } \\
\text { beitsmarkt) } \\
\text { Frau = Gefühlsspezialistin } \\
\text { (Haushalt, Erziehung, } \\
\text { Wohlbefinden) }\end{array}$ & $\begin{array}{l}\text { Ideologie von Indivi- } \\
\text { dualität und Gleichheit; } \\
\text { Gleichstellung der } \\
\text { Frau }\end{array}$ \\
\hline $\begin{array}{l}\text { Partner- } \\
\text { wahl }\end{array}$ & $\begin{array}{l}\text { Nach Standesord- } \\
\text { nung; Verwandt- } \\
\text { schaft hat Mitspra- } \\
\text { che }\end{array}$ & $\begin{array}{c}\text { Ideal der romantischen } \\
\text { Liebe; Ehe auf Lebenszeit }\end{array}$ & $\begin{array}{l}\text { Verhandlung über Art } \\
\text { und Weise des Zu- } \\
\text { sammenlebens; „Le- } \\
\text { bensabschnittspart- } \\
\text { nerschaften“ }\end{array}$ \\
\hline Wohnform & $\begin{array}{c}\text { Ehepaar mit Kin- } \\
\text { dern, ledigen Ver- } \\
\text { wandten und Ange- } \\
\text { stellten leben in ei- } \\
\text { nem Haushalt }\end{array}$ & $\begin{array}{c}\text { Ehepaar mit Kindern lebt in } \\
\text { einem Haushalt }\end{array}$ & $\begin{array}{c}\text { Großeltern, Paar, (er- } \\
\text { wachsene) Kinder le- } \\
\text { ben in getrennten } \\
\text { Haushalten }\end{array}$ \\
\hline $\begin{array}{l}\text { Arbeits- } \\
\text { stätte }\end{array}$ & Unter einem Dach & Getrennt von Haushalt & $\begin{array}{l}\text { Verschwimmen der } \\
\text { Grenzen zwischen } \\
\text { Freizeit und Arbeit; } \\
\text { Vereinbarkeit von Fa- } \\
\text { milie und Beruf }\end{array}$ \\
\hline $\begin{array}{c}\text { Ein- } \\
\text { kommen }\end{array}$ & $\begin{array}{c}\text { Aus gemeinschaftli- } \\
\text { cher Produktion }\end{array}$ & Aus Erwerbsarbeit & $\begin{array}{c}\text { Arbeitskraftunterneh- } \\
\text { mer }\end{array}$ \\
\hline Eigentum & $\begin{array}{l}\text { Kollektives Einkom- } \\
\text { men des Hauses }\end{array}$ & $\begin{array}{c}\text { Zugewinngemeinschaft der } \\
\text { Eheleute }\end{array}$ & Individualvermögen \\
\hline Vererbung & $\begin{array}{l}\text { Vermögen soll in der } \\
\text { Familie erhalten } \\
\text { werden; strenge }\end{array}$ & $\begin{array}{l}\text { Vermögen soll gleich unter } \\
\text { den leiblichen Kindern ver- } \\
\text { teilt werden }\end{array}$ & $\begin{array}{l}\text { Denkbar: Individuelle } \\
\text { Gestaltung des Nach- } \\
\text { lasses; zunehmender }\end{array}$ \\
\hline
\end{tabular}




\begin{tabular}{|cccc|}
\hline \hline & $\begin{array}{c}\text { Erbregelung (z.B. } \\
\text { Fideikommiss) }\end{array}$ & & $\begin{array}{c}\text { Einbezug von nicht- } \\
\text { verwandten Personen } \\
\text { und Überspringen der } \\
\text { Generationenfolge }\end{array}$ \\
\hline \hline Identität & Aus Gemeinschaft & $\begin{array}{c}\text { Biographisierung des insti- } \\
\text { tutionalisierten Lebenslaufs }\end{array}$ & $\begin{array}{c}\text { Individuen als „Sinn- } \\
\text { zentren“ der eigenen } \\
\text { Biographie }\end{array}$ \\
\hline \hline $\begin{array}{c}\text { Familie } \\
\text { als... }\end{array}$ & $\ldots$...Gemeinschaft & ...Ort der Privatheit & $\ldots$ Sinnstifter \\
\hline \hline
\end{tabular}

Quelle: eigene Darstellung

\subsubsection{Anachronismus-These}

Die charakteristische Verbundenheit von Unternehmen und Familie ähnelt der grundlegenden Struktur des Ganzen Hauses sehr. Arbeiten und Leben sind eng verschränkt, werden gar nicht als zwei unterschiedliche Bereiche wahrgenommen. Die Familie zeichnet sich durch ihre Funktionsvielfalt aus: Sie bietet eine Ausbildung, einen Arbeitsplatz, Kapital und Sicherung im Krankheitsfall und schafft eine kollektive Identität. Das Ziel, das Unternehmen in der Familie zu erhalten, zählt mehr als das Wohlbefinden einzelner Individuen. Auch das Verständnis von Eigentum als Familienvermögen, das möglichst im Ganzen von der nächsten Generation bewahrt werden soll, scheint mit einer Unternehmerfamilie kompatibel. Die Familienmitglieder bilden eine Solidargemeinschaft, die sich insbesondere durch gemeinsames Arbeiten und Eigentum auszeichnet.

Im Zuge der Entwicklung hin zur Kernfamilie als wesentlicher Orientierungspunkt familialen Handelns wird die Unternehmerfamilie als legitime Lebensweise jedoch zunehmend in Frage gestellt. In eine Gesellschaft, in der sich Arbeiten und Leben in zwei Bereiche differenziert haben und in der die Norm eines eigenständigen Lebensentwurfs gilt, wollen Unternehmerfamilien und ihre Mitglieder, deren Lebensplanung in dieser Verbindung aufgeht, nicht so recht passen (Stamm 2009:23). Ihre spezifische Struktur steht in einem inneren Konflikt zu den Merkmalen moderner Kernfamilien. Unternehmerfamilien könnten sich einerseits den Modernisierungstendenzen verschließen und sowohl der funktionalen Differenzierung als auch der Individualisierung trotzen. Sie wäre dann traditionelle soziale Einheiten, die immer weiter an den Rand der Gesellschaft und unter Rechtfertigungsdruck geraten. Anderseits könnten sich Unternehmerfamilien ganz den Idealen der modernen Kernfamilie verschreiben und in konsequenter Weise das Unternehmen von der Familie trennen. Dann jedoch würden sie nicht mehr als Unternehmerfamilien fortbestehen. Diese beiden bedrohlichen Lesarten stellen zwei 
extreme, aber auch unwahrscheinliche Pole dar, zwischen denen sich Unternehmerfamilien positionieren lassen.

Wie könnten Unternehmerfamilien den Normen der Kernfamilie als Ideal familialen Handelns entsprechen? Eine Trennung von Leben und Arbeiten, aber auch der Generationen könnte sich in räumlich getrennten Haushalten bzw. Betriebsstätten niederschlagen. Darüber hinaus könnte sich die familiale Kommunikation dahin gehend verändern, dass unternehmensbezogene Themen nicht im Raum der Familie diskutiert werden. Die Familienmitglieder üben sich darin, unterschiedliche Rollen zu spielen, was jedoch aufgrund der sich überlappenden Handlungsfelder nicht durchgängig gelingen kann. Geschäftsbeziehungen könnten quer stehen zur emotionalen Beziehung der Familienmitglieder zueinander und auf diese Weise zu Konflikten führen. Notwendig wird ein erhöhtes Maß an Abgrenzungsarbeit. Ein zunehmendes Verständnis des Unternehmens als Arbeitsstätte könnte wiederum die Verbundenheit mit dem und das Engagement für den Familienbetrieb reduzieren.

Ebenfalls ist denkbar, dass sich das Programm eines institutionalisierten Lebenslaufes in Unternehmerfamilien zunehmend entfaltet. Die Dreiteilung des Lebenslaufes, der Familienzyklus ebenso wie Freiheiten in der Berufsund Partnerwahl verschlechtern jedoch die Chancen auf eine Reproduktion der Unternehmerfamilie. Es steigt das Risiko, dass die Lebensläufe der Familienmitglieder nicht synchronisierbar und ihre Lebensvorstellungen unvereinbar sind. In Unternehmerfamilien sind die Wahlmöglichkeiten der Familienmitglieder nicht nur durch marktförmige (Arbeits-, Heiratsmarkt, etc.) oder wohlfahrtsstaatliche Strukturen beschränkt, sondern hängen auch in hohem Maße von anderen Familienmitgliedern ab. Eine ökonomische Unabhängigkeit der Familienmitglieder voneinander scheint kaum realisierbar, insbesondere für jene Kinder, die den Weg einer Nachfolge einschlagen. Selbst wenn sich für die Alterssicherung der Eltern und Großeltern private und wohlfahrtsstaatliche Alternativen ausbilden, sind sie über den materiellen Transfer des Unternehmenseigentums ebenso wie über das Unternehmen als Einkommensquelle aneinander gebunden.

Vor diesem Hintergrund wird offensichtlich, dass die Generationen in Unternehmerfamilien unmittelbarer und vielfältiger miteinander verbunden sind als dies in modernen Kernfamilien der Fall ist. Sie bilden eine wirtschaftliche Solidargemeinschaft, ihre Lebensläufe sind eng verschachtelt, und sie sind Bestandteil einer gemeinsamen Unternehmens-FamilienGeschichte, die Identität und Motivation verleiht. Entsprechend reichhaltig sind auch die Konfliktpotentiale zwischen den Generationen. Gerade in der Außendarstellung werden diese Besonderheiten von Unternehmerfamilien ihren Mitgliedern ein erhöhtes Maß an Rechtfertigung und Biografizität abverlangen. Die wichtige Entwicklungsaufgabe einer Lösung der Kinder von den Eltern ebenso wie die Konstitution einer individualisierten Persönlichkeit muss im Rahmen der spezifischen Struktur von Unternehmerfamilien erfol- 
gen. Versuchen Familienunternehmer in der Rolle des Haushaltsvorstandes zu verharren, besteht die Gefahr, sich mit den Kindern, die sich eher an den modernen Normen orientieren könnten, zu überwerfen und damit den Fortbestand der Unternehmerfamilie zu bedrohen. Familienunternehmer müssen Modernisierungsrisiken wagen. Unternehmerfamilien geraten im Laufe des Modernisierungsprozesses unter Druck: Sie sind gefordert, die strukturelle Koppelung von Arbeiten und Leben, die ihnen zueigen ist und per se traditionell erscheint, in ihren Biografien und ihrem Selbstverständnis, also auf einer kommunikativ-kulturellen Ebene, zu kompensieren. Sie müssen sich dem ersten Eindruck, nämlich dem, ein anachronistisches Lebensmodell zu verfolgen, entgegen stellen und mit Ambivalenzen umgehen.

\subsubsection{Pionier-These}

Das Verhältnis von Arbeit und Leben ändert sich mit dem zweiten Modernisierungsschub erneut. Die Grenzen der funktionalen Systeme werden durchlässig, die institutionelle Entlastung für die Inhalte von Lebenslaufentscheidungen schwindet. (Kohli 1994). In diesem Licht erscheint die Koppelung von Arbeit und Leben, wie sie in Unternehmerfamilien praktiziert wird, in hohem Maße mit den Vorstellungen spätmoderner Lebensweisen zu korrespondieren. Während sich für die Arbeitnehmer die Eckpfeiler ihrer alltäglichen Lebensführung und biografischen Planungen wandeln, haben Unternehmerfamilien Erfahrung im Umgang mit paradoxen Systemanforderungen, Risiken, Ambivalenzen und sich überschneidenden Rollenerwartungen. Familienunternehmer stehen auf der anderen Seite der abhängigen Beschäftigten, entsprechend wird ihr Leben weniger von Erwerbsarbeit und den Wohlfahrtssystemen, sondern vielmehr unmittelbar durch Absatz-, Zuliefer- oder Finanzmärkte und andere externe Einflüsse bestimmt (Stamm et al. 2009a). Ihre Lebensentwürfe gründen sich schon länger auf einer ,potenziellen“ Eigenbestimmtheit. Die Absicherung ihrer Erwerbsrisiken und des Ruhestandes obliegt ihrer individuellen Wahl, ${ }^{17}$ es gibt keine formalen Ausbildungen für ihren Beruf, keine festgelegten Altersgrenzen für den Rückzug aus dem Unternehmen, keine klaren Grenzen zwischen Betrieb und Familie. Familienunternehmer sind gefordert, berufliche und private Anforderungen in zeitlicher, räumlicher und organisatorischer Hinsicht eigenständig $\mathrm{zu}$ koordinieren. Damit erfüllen Familienunternehmer einige der Voraussetzungen, die Günter Voß (Voß und Pongratz 1998; Voß 2001) mit seinem „Arbeitskraftunter-

17 Familienunternehmer als Eigentümer von Unternehmensanteilen mit maßgeblichem Einfluss auf die Geschicke des Unternehmens sind nicht generell sozialversicherungspflichtig oder können sich von dieser Pflicht befreien lassen. Hierfür greifen bei der Krankenversicherung oder bei der Rente unterschiedliche Kriterien (z.B. Anteilshaber, Tätigkeitsfeld, Beitragsbemessungsgrenze, Anzahl der Mitarbeiter, etc.). 
nehmer" beschreibt. In Unternehmerfamilien besteht einerseits das positive Potenzial einer hohen Zeitsouveränität und einer optimierten Vereinbarkeit von Arbeit und Leben. Anderseits sind dafür eine hohe gestalterische Leistung und personale Stabilität sowie eine situative Offenheit und Flexibilität erforderlich. Insofern kann behauptet werden, dass Familienunternehmer zu Pionieren der biografischen Selbstbestimmung werden. ${ }^{18}$

Sehr einleuchtend scheinen darüber hinaus die Parallelen zwischen Unternehmerfamilien und multilokalen Mehrgenerationenfamilien. Unternehmerfamilien sind insofern multilokal, als die verschiedenen Generationen in unterschiedlichen, vom Betrieb getrennten Haushalten leben, die eventuell über eine Region bzw. Stadt verteilt sind. Jene Familienmitglieder, die nicht aktiv im Unternehmen arbeiten, können in einem weiteren Umkreis verteilt leben, stehen jedoch als Solidaritäts- und Netzwerkressource zur Verfügung. Die unternehmerische Tätigkeit verlangt von den Familienmitgliedern erhöhte räumliche und geistige Flexibilität, wenn es gilt, sich auf neue Technologien, Produkte, Standorte und Vertriebswege einzulassen. Zudem sind Unternehmerfamilien als Mehrgenerationenfamilien angelegt. Großeltern, Eltern und Kinder sind über ein familiales Projekt verbunden. Gemeinsam verfolgen sie die Kontinuität des Familienunternehmens, was materielle (Eigentum) und immaterielle (Wissen, Identität) Transferleistungen erfordert. Die prominente Rolle der Großeltern in Mehrgenerationenfamilien entspricht dem unmittelbaren Generationenverhältnis, wie es in Unternehmerfamilien praktiziert wird. Insofern können Unternehmerfamilien als Vorreiter einer familialen Solidarität, die sich über die Kernfamilie hinaus erstreckt, verstanden werden, wie sie sich in breiten Teilen der familialen Lebensformen, unterstützt und getrieben vom Wandel der sozialen Wohlfahrtssysteme und einer Politik des Förderns und Forderns, immer mehr durchsetzt. ${ }^{19}$

Es scheint, überspitzt formuliert, als ob ein einst als anachronistisch betrachtetes Lebensmodell, das in der Verbindung von Unternehmen und Familie aufgeht, gar zum Pionier spätmoderner Lebensformen wird. Diese Entwicklung dürfte zu einer Entspannung von generationalen Konflikten in Unternehmerfamilien beitragen und auch den Rechtfertigungsdruck der eigenen Biografie reduzieren. Neue Konfliktlinien lassen sich hingegen im Geschlechterverhältnis vermuten. Es ist fraglich, inwiefern in einer Struktur, die seit Generationen mit einer autoritären Führungsrolle des Firmen- und Familienpatriarchen verbunden ist, Mütter und Töchter nun zu gleichberechtigten Eigentümerinnen und Geschäftsführerinnen aufsteigen können. Die Ideologie

18 Diese Gedanken sind weiter in Stamm et al. 2009a ausgeführt und werden in Kapitel 6 wieder aufgegriffen.

19 An dieser Stelle soll nicht behauptet werden, dass Unternehmerfamilien schon länger anderen demografischen Strukturen unterliegen (weniger Kinder, ältere Familienmitglieder). Die Parallelen werden vielmehr auf einer Einstellungs- und Beziehungsebene vermutet. 
der Gleichheit als wesentlicher Bestandteil spätmodernen familialen Handelns könnte zu erheblichen Konflikten und Veränderungen innerhalb von Unternehmerfamilien führen.

\subsubsection{Fazit}

Die beiden entwickelten Thesen legen nahe, dass Unternehmerfamilien in der Tat Familien eigener Art bilden. Sie haben einen konstanten strukturellen Kern, der fallweise als antiquiert oder zukunftsweisend erscheint. Die Orientierung an den gesellschaftlichen Idealvorstellungen hinsichtlich familialen Handelns wirkt auf das Innen- und Außenverhältnis von Unternehmerfamilien ein und könnte der Motor für Veränderungen, aber auch Konflikte sein. Diese typisierenden Erwägungen eröffnen einen Möglichkeitsraum, wie Unternehmerfamilien sein könnten, und sensibilisieren dadurch für deren Besonderheiten. 


\section{Die Besonderheiten von Unternehmerfamilien}

Im Anschluss an die gedankenexperimentellen Erwägungen zu den Besonderheiten von Unternehmerfamilien soll im Folgenden die bestehende Forschung zu diesen Familien eigener Art dargestellt werden. Ziel dieses Kapitels ist es, das Profil von Unternehmerfamilien als Untersuchungsgegenstand zu schärfen und mögliche Forschungsaufgaben zu benennen. Jenseits disziplinärer Grenzen werden die zentralen Diskussionsaspekte und Forschungsbeiträge zu Unternehmerfamilien aufgearbeitet, zusammengeführt und weiterentwickelt. Dabei zeigt sich, dass einige der vorgestellten Überlegungen bereits Gegenstand von empirischer Forschung und Theoriebildung waren, andere hingegen bislang keine Beachtung gefunden haben. Ein Überblick über die Forschungslandschaft verdeutlicht, dass die Familie des Familienunternehmens bisher häufig im Schatten des Unternehmens stand (Abschnitt 1). Die folgenden Abschnitte schlagen hingegen eine Familienperspektive vor. Die Leitfrage der historischen und gesellschaftlichen Bedeutung von Unternehmerfamilien legt einige wesentliche strukturelle Merkmale dieser Familien eigener Art offen (Abschnitt 2). Im nächsten Abschnitt wird der in der Forschungspraxis gängige systemtheoretische Zugang zu Unternehmerfamilien diskutiert und es werden weitere spezifische Merkmale im Lichte von modernisierungstheoretischen Leitideen herausgearbeitet (Abschnitt 3). Darauf aufbauend wird ein konzeptionelles Modell über die Dimensionen des wechselseitigen Einflusses von Unternehmen und Familien entwickelt, das die spezifischen Strukturen und Bedingungen von Unternehmerfamilien theoretisch zu fassen sucht (Abschnitt 4). Das vorgeschlagene Modell wird abschließend auf den Generationswechsel - in seiner Funktion als Reproduktionsprozess der Familie eigener Art - angewandt (Abschnitt 5). Die begriffliche Bestimmung der Unternehmerfamilie und die Erweiterung der bestehenden Theoriebasis stehen damit im Mittelpunkt dieses Kapitels.

\section{1 Überblick über die Forschungslandschaft}

Im Gegensatz zur Popularität von Unternehmerfamilien in Literatur und Film hält sich das wissenschaftliche Interesse an diesen spezifischen Familien in Grenzen. Die Forschungslandschaft gestaltet sich zusammenhanglos, wenig theoriegeleitet und lückenhaft. Die Unschärfe des Untersuchungsgegenstan- 
des Unternehmerfamilie zeigt sich schon darin, dass die Begriffe Familienunternehmen und Unternehmerfamilie oft synonym verwandt werden.

Innerhalb der Soziologie ist die Beschäftigung mit Unternehmerfamilien nicht weit verbreitet. Lediglich in der Landsoziologie kann die Analyse von Bauernfamilien als soziale Gebilde im Spannungsverhältnis zwischen Tradition und Moderne als etabliertes Forschungsthema angesehen werden. Unter dem Aspekt erschwerter Individualisierungsbedingungen für ihre Mitglieder gilt das Interesse vor allem der Hofnachfolge (Abel 1953; Bohler und Hildenbrand 1997; Timmermann und Vonderach 1993; Lauterbach und Shanahan 1998; Breuer 2009). Diesen meist qualitativen Studien ist gemein, dass die Verbindung von Familie und Unternehmen zu einer Strukturbedingung im Prozess der Individualisierung wird.

Ausgehend von dieser Beobachtung gerät auch die Struktur und Übergabepraxis von Familien mit kleinen und mittleren Unternehmen zunehmend in den Blick. Schon früh entstanden einige Arbeiten im Bereich des Handwerkes (Künemund und Beate Mücke 1990; Bertaux und Bertaux-Wiame 1981). Franz Breuer führte eine Reihe von empirisch-explorativen Projekten zur Nachfolge-Problematik in landwirtschaftlichen, gastronomischen und handwerklichen Familienunternehmen durch. Auf dieser Basis entwickelte er eine theoretische Vorstellung von Weitergabe-/Übernahme-Prozessen, die er nicht nur in Unternehmerfamilien wirksam sieht, sondern auf ,,persönliche Objekte" allgemein anwendet (2009). Auch Bruno Hildenbrand erweiterte seine anfänglich landsoziologischen Untersuchungen von Bauernfamilien auf kleine und mittelständische Betriebe (etwa Apotheken, Hotels, etc.) und stellt den Begriff der „Familien eigener Art“ vor, der Unternehmerfamilien als spezifische Gattung markiert und ihre inneren Konflikte als zentrales Merkmal hervorhebt. Daniela Jäkel-Wurzer (2010) greift diesen Begriff auf und buchstabiert ihn im Lichte einer Sozialisationstheorie und immer im Vergleich zur modernen Kernfamilie aus (siehe 3.3). Ihre Präsentation wesentlicher Strukturmerkmale von Unternehmerfamilien leistet einen Beitrag zur Theoriebildung in diesem Bereich. Ihre empirische Untersuchung der Dynamik von Vater-Mutter-Tochter-Beziehungen unterstreicht die Besonderheiten der Familien eigener Art aus familiensoziologischer Perspektive.

Einen zentralen Impuls für die Beschäftigung mit der „Familie des Familienunternehmens" liefert die Sammelmonographie von Fritz B. Simon aus dem Jahr 2002. Darin stellt Simon die Unternehmerfamilie als „Kopplung von Familie und Unternehmen als Systeme, die nicht nur unterschiedlichen, sondern teilweise kontradiktorischen Regeln folgen" (Simon 2002:13), als System zwischen Gefühl und Geschäft vor. Simon prägt mit diesem und späteren Beiträgen (etwa Simon et al. 2005) eine systemtheoretische Auseinandersetzung mit der Thematik. In jüngster Zeit finden sich in dieser Tradition vereinzelte Arbeiten zu Identitäts- (Bäcker 2008) und Individualisierungskonflikten (Wenk 2007; Büttner 2007), zu paradoxen Anforderungen in Un- 
ternehmerfamilien (Klett 2005) und zu den Besonderheiten weiblicher Nachfolge (Jäkel-Wurzer 2010). Den weiterhin meist qualitativen empirischen Studien ist gemein, dass die entwickelten Strategien zum Umgang mit bzw. zur Bewältigung der Strukturbedingungen in Unternehmerfamilien im Mittelpunkt stehen.

Das geringe Interesse wirtschaftssoziologischer Forschung an Unternehmerfamilien überrascht. Denn Untersuchungen zu Eigentumsstrukturen von europäischen Unternehmen (wie etwa von LaPorta et al. 1998 oder Faccio und Lang 2002 zu concentrated ownership) heben die zentrale Bedeutung von Familien als Anteilseignern hervor. Insofern scheinen sie eine gestaltende Größe moderner Märkte und wirtschaftlichen Handelns zu sein. Innerhalb der Diskussion zu den unterschiedlichen Ausprägungen des Kapitalismus („Varieties of Capitalism“) spielt die Verbindung von Familie und Unternehmen ebenfalls eine untergeordnete Rolle (siehe etwa Hall und Soskice 2001; Amable 2003; Whitley 2002). Dabei ließe sich gerade Granovetters Ansatz (1985) einer Einbettung ökonomischer Handlungen in soziale Strukturen für eine Analyse von Unternehmerfamilien ausbauen, die als ein Teil des engen Netzwerkes sozialer Beziehungen verstanden werden können, auf denen sich Unternehmensbeziehungen gründen. Dieser Netzwerkgedanke ist in der Forschung zu Wirtschaftseliten (siehe etwa Hartmann 1996, 2008) prominent, in deren Fokus hauptsächlich angestellte Manager großer Firmen und weniger Unternehmerfamilien stehen. Untersuchungen verweisen auf ein relativ geschlossenes Milieu mit spezifischem Habitus und Rekrutierungsstrategien. Auch die Forschung zu industriellen Clustern rückt einen Netzwerkgedanken in den Mittelpunkt (etwa Porter 1990).

Die Entstehung von Unternehmertum bildet den Mittelpunkt des Entrepreneurship Research, einer Richtung, die sich in der Tradition des Schumpeterschen Prozesses der schöpferischen Zerstörung überwiegend mit Innovation, unternehmerischem Entdecken, Risiko und Unsicherheit beschäftigt. Seitdem Gartner (1989) postulierte, dass die Frage: „Who is an Entrepreneur?" der falsche Ansatz sei, lässt sich das interdisziplinäre Feld in zwei Schulen unterteilen. Der ,traits'-Ansatz beschäftigt sich mit der Person, den Charaktereigenschaften und den Möglichkeitsstrukturen von Gründern (z.B. Kellermanns et al. 2008). Diese soziologisch geprägten Beiträge heben vor allem den Einfluss der familialen Sozialisation auf die Gründungsneigung einiger Individuen hervor (etwa Matthews und Human 2004). Die Familie gilt als wichtige Kontextvariable, über die Wissen, Kontakte, Finanzressourcen und auch Risikoeinstellungen transportiert werden können. Die ,emerging organizations'-Perspektive hingegen versteht Entrepreneurship als einen Prozess, in dem neue unternehmerische Handlungsfelder entdeckt, bewertet und ausgeschöpft werden (Fallgatter 2004:25). Vertreter dieser Richtung sehen in einer angemessenen Strategie die entscheidende Variable für eine gelungene Gründung. Die Familie tritt hier insofern als „missing variable“ (Dyer 2003) 
auf, als durch neue Lebensformen und Familienverständnisse sich auch neue Felder für unternehmerische Aktivitäten öffnen (Aldrich und Cliff 2003). Ganz im Sinne Granovetters, der eine Mittelposition zwischen einer überund einer untersozialisierten Herangehensweise einnimmt, stellt Martin Ruef (2010) einen dritten Weg vor: Entrepreneurship sei eine kollektive Handlung zur Gründung einer spezifischen sozialen Einheit - der „entrepreneurial group“ (Ruef 2010:7). Entgegen der weitläufigen Vorstellung des „Soloentrepreneurs" (Ruef 2010:13) argumentiert Ruef Gründungswillige hätten ein aktives Interesse daran, andere als Miteigentümer, Angestellte, Investoren, Berater oder Helfer zu rekrutieren. Entrepreneurship werde damit zu einer Verbindung von Individuen gemäß ihres materiellen und zeitlichen Engagements, ihrer Zugehörigkeit zu sozialen Netzwerken sowie ihrer Identität und Zielvorstellung. In seiner Beschreibung solcher entrepreneurial groups weist Ruef darauf hin, dass diese mehrheitlich auf ehelichen und verwandtschaftlichen Beziehungen (strong ties) gründen, die für eine solide Vertrauensbasis bürgten. Die Familie erhält damit eine wichtige Funktion im Gründungsgeschehen.

Wesentlicher Bezugspunkt der wirtschaftshistorischen Auseinandersetzung mit Unternehmerfamilien ist die unter anderem von Alfred Chandler geprägte These einer gradlinigen Entwicklung von kleinen, familiengeführten Eigentümerunternehmen hin $\mathrm{zu}$ großen Konzernen mit einer strikten Trennung von Eigentum und Management. Die Verbindung von Familie und Unternehmen wird in dieser Perspektive zum Wegbereiter des modernen Kapitalismus. Auf lange Sicht jedoch würden die Familienunternehmen - relativ schnell, in zwei oder drei Generationen - schließen oder, falls erfolgreich, sich in Manager-Unternehmen verwandeln. Dieses lineare Verständnis des Lebenszyklus von Familienunternehmen wurde oft mit der Komplexität großer Konzerne erklärt, die sich schlecht mit familiären Strukturen leiten ließen (Colli 2003:13). Neben einer Reihe von Monographien, die die Verstrickungen von Unternehmens- und Familiengeschichte an namhaften, meist wohlhabenden Beispielen aufarbeiten (etwa Priemel 2007; Landes 2006), finden sich auch vergleichende (James 2005) und theoriebildende Arbeiten (Kocka 1979; Schäfer 2007; Lubinski 2010). Im Sinne Chandlers kommen sie zu dem Schluss, dass Familienunternehmen eine vorteilhafte Konstellation in Zeiten von unreifen Märkten bieten. Die Beschäftigung mit der Rolle von Unternehmerfamilien nach 1945 ist hingegen sehr gering ausgeprägt. Die Arbeiten von Colli (2003) und Lubinski (2010) ebenso wie ein Aufsatz von Kocka (2002) betonen jedoch, dass der Einfluss von Familien auf die Wirtschaft nicht nachgelassen, sich aber verändert habe. Auf Basis ihrer empirischen Arbeit zeigt Lubinski, wie sich der familiale Einfluss auf die Corporate Governance-Struktur auswirkt, Teil der Unternehmenskultur wird und zur Institutionalisierung sehr spezifischer Wirtschaftspraktiken führt. Umgekehrt 
kann die Historikerin zeigen, dass das Unternehmen zu einem Familienprojekt mit Gemeinschaft stiftender Kraft wird.

Eine ganz ähnliche Entwicklung der Hauptargumentationslinien findet sich im interdisziplinären Feld des Family Business Research wieder. Typisch für die Beschäftigung mit Familienunternehmen in den 1960-70er Jahren ist die Konzentration auf die Stärken und Schwächen von Familienunternehmen als erfolgreiche wirtschaftliche Organisationen, wobei das große Manager-Unternehmen als Maßstab angelegt wird (Colli und Rose 2003:341342). Seit den 1980er Jahren hat sich das exponentiell wachsende Feld stärker auf Familienunternehmen an sich konzentriert. Mittels empirischer Studien konnte gezeigt werden, dass Familienunternehmen in den meisten Industriestaaten die Mehrheit aller Unternehmen stellen und damit kein randständiges oder auslaufendes Modell sind. Family Business Research beschäftigt sich immer systematischer und empirisch fundierter (Bird et al. 2002:346) mit einer Vielzahl von Themen rund um Familienunternehmen, wobei sich ein deutlich positiver Unterton der Beiträge erkennen lässt. In einem Überblicksartikel gliedert Sharma (2004) die inhaltlichen Schwerpunkte in vier Ebenen: Beschäftigung mit Schlüsselpersonen (Individualebene); interpersonelle Beziehungen wie etwa Verträge, Zusammenarbeit oder Nachfolge (Gruppenebene); Corporate Governance, Ressourcenmanagement und Strategie (Organisationsebene); sowie volkswirtschaftliche Bedeutung und kulturelle Einbettung von Familienunternehmen (gesellschaftliche Ebene). Insgesamt ist die Forschung auf den ersten beiden Ebenen wesentlich stärker ausgeprägt. Zwar hat noch immer keine der zahlreichen Definition (für einen Überblick siehe etwa Flören 2002 und Pfannenschwarz 2006) von Familienunternehmen allgemeine Akzeptanz gefunden, dennoch besteht große Einigkeit darüber, dass der wechselseitige Einfluss von Familie und Unternehmen das zentrale Charakteristikum und damit konstitutiver Gegenstand des Feldes ist (Sharma 2004:9). Die Idee der Reziprozität hat zwei Folgen: Zum einen kann eine Entwicklung hin zu einem graduellen Verständnis von Familienunternehmen statt einer Dichotomisierung gegenüber Nicht-Familienunternehmen beobachtet werden (so argumentiert etwa Björnberg und Nicholson 2007). Einschlägig erscheint in diesem Zusammenhang vor allem die von Joe Astrachan, Sabine Klein und Kosmas Smyrnios entwickelte Skala zur Erfassung des Familieneinflusses in Unternehmen über die drei Kanäle Macht, Erfahrung und (Unternehmens-) Kultur (Astrachan et al. 2002; Klein et al. 2005). Zum anderen findet eine stärkere Konzeptualisierung der Familienseite statt. Im Jahr 2008 titelte beispielsweise die jährliche Konferenz der „International Family Enterprise Research Academy (IFERA), The Entrepreneuring Family“ und weckte Erwartungen für eine stärkere Beachtung dieses Aspektes. Darüber hinaus existiert seit 2005 das Global STEP Project, das ganz explizit die Familie als Analyse-Ebene einbezieht. Im Rahmen dieser Initiative stehen weltweit Besitzer und Leiter von Familienunternehmen mit 
Forschern in engem Austausch und diskutieren etwa, wie sich der Unternehmergeist über mehrere Generationen aufrechterhalten kann (siehe dazu etwa Nordqvist und Zellweger 2010). Trotz allem fokussieren die Beiträge mehrheitlich den Erfolg des Unternehmens, der durch das Engagement der Familie befördert oder durch ihre internen Konflikte in Gefahr geraten könne. Diese organisationale Perspektive mag sich aus der Dominanz wirtschaftswissenschaftlicher Beiträge ebenso wie aus der engen Verknüpfung des Forschungsfeldes mit der Beratungs- und Unternehmenspraxis erklären. Die umgekehrte Richtung des ,wechselseitigen“ Einflusses findet keine systematische Beachtung. Trotzdem gibt es eine Reihe von Anknüpfungspunkten etwa in Beiträgen zur Zusammenarbeit der Familienmitglieder, Sozialisation oder Nachfolge.

Es lässt sich zusammenfassend festhalten, dass in diesen heterogenen Beiträgen die Familie in den Schatten des Unternehmens und seiner Entwicklung fällt. Der oft genannte Einbettungsbegriff drängt die Familie an den Rand der Strukturbedingungen. Die Familie versorgt den wirtschaftlichen und biografischen Akteur mit ökonomischem, sozialem und kulturellem Kapital. Einerseits wirkt sie dabei als Ressource für den wirtschaftlichen Erfolg des Unternehmens. Andererseits wird ihre spezifische Konstellation zu einem Fallstrick für Individualisierungsbestrebungen ebenso wie für Unternehmensinnovation und -wachstum. Das zunehmende Interesse an der Familienseite, das sich in den letzten zehn Jahren beobachten lässt, hat zu einer beginnenden Konzeptionalisierung von Unternehmerfamilien als Forschungsgegenstand beigetragen, die dem Tenor einer Anachronismus-These (siehe hierzu Kapitel II) folgt. Es mangelt weitgehend an einer systematischen Aufarbeitung der inneren Dynamik dieser sozialen Einheit ebenso wie der Auswirkungen des Unternehmenseinflusses auf das Familiengeschehen. Im Folgenden sollen jenseits disziplinärer Grenzen die zentralen Diskussionslinien und Forschungsbeiträge $\mathrm{zu}$ Unternehmerfamilien aufgearbeitet und integriert werden. Ziel ist es, das Profil von Unternehmerfamilien als Untersuchungsgegenstand zu schärfen und mögliche Forschungsaufgaben zu benennen.

\subsection{Gesellschaftliche Bedeutung von Unternehmerfamilien}

In der wirtschaftshistorischen Entwicklung Deutschlands spielen Unternehmerfamilien eine herausragende Rolle. Wer kennt sie nicht, die Flicks, die Quandts oder die Siemens, die über Generationen hinweg mit ihren wirtschaftlichen Imperien die Industrialisierung vorangetrieben haben? Die folgenden Überlegungen konzentrieren sich auf die gesellschaftliche Bedeutung von Unternehmerfamilien vom 19. bis ins frühe 21. Jahrhundert. Um es 
gleich vorwegzunehmen: Weder die historische noch die gegenwärtige Rolle von Unternehmerfamilien in Wirtschaft und Gesellschaft können - nicht zuletzt aufgrund des lückenhaften Forschungsstandes - nachhaltig eingeschätzt und dargestellt werden. Dennoch scheint eine Auseinandersetzung mit dieser Frage lohnenswert, da sie zu einem besseren Verständnis dieser Familien eigener Art ebenso wie zur begrifflichen Klärung wesentlich beiträgt.

\subsubsection{Familienformen im 19. Jahrhundert}

In den 1830er und 1840er Jahren setzt in Deutschland um einige Jahrzehnte später als etwa in Großbritannien die Industrialisierung und damit ein tief greifender Wandel der Agrargesellschaft ein (Henning 1995). Das 19. Jahrhundert ist entsprechend geprägt von einer großen Vielfalt an Familienformen, einem Nebeneinander von traditionellen und modernen Lebensweisen. Mit Blick auf die materiellen Grundlagen und die wirtschaftliche Organisation gruppiert Andreas Gestrich (2010) - wie in der historischen Familienforschung üblich - die Familienformen schichtspezifisch in vier Gruppen:

Erstens, die traditionellen Bauernfamilien, die sich als Hausgemeinschaft verstehen und nach Sicherung der Nahrung sowie des sozialen Status streben. Alle Familienmitglieder sind in den Produktionsprozess eingebunden und bilden eine Konsumeinheit. Der Hof ist Grundlage der Versorgung aktueller und künftiger Generationen. Gestrich weist darauf hin, dass sich in diesen Familien am ehesten das Ideal des „Ganzen Hauses“ finden lässt. Die Proletarisierung und Verarmung der Bauern schreitet im Laufe des 19. Jahrhunderts schnell voran. Die landlosen Mittel- und Unterschichten arbeiten zusätzlich im Handwerk oder der Fabrik und entfernen sich von der Lebensweise der Familienwirtschaft. In der jungen Bundesrepublik leben nur noch 9\% der Bevölkerung von der Landwirtschaft (Gestrich 2010:11). Diese Bauernfamilien verfügen als Eigentümer ihres Betriebes über hohe Vermögenswerte, aber erzielen ein relativ geringes Einkommen aus der Bewirtschaftung (Gestrich 2010:13). Trotz allem sieht Gestrich die Familienwirtschaft im ländlichen Bereich als einen Traditionsbestandteil, der sich bis in die Nachkriegszeit hält.

Zweitens, im handwerklichen Familienbetrieb lebt die Kernfamilie des Meisters mit den Gesellen und Gesinden als Wohn- und Arbeitsgemeinschaft. Der Alltag wird von der gemeinsamen Tätigkeit strukturiert, und es herrscht eine patriarchalische Ordnung. Im Laufe des 19. Jahrhunderts gründen die Gesellen vermehrt ihren eigenen Haushalt, sind allerdings häufig auf ein zusätzliches Einkommen der Frau angewiesen. Die Einkommensstreuung ist nicht nur zwischen Meister und Gesellen, sondern auch innerhalb der verschiedenen Zünfte sehr ausgeprägt (Gestrich 2010:14). Im Laufe des 20. Jahrhunderts lassen sich starke Verdrängungs- und Konzentrationsprozesse 
im Handwerk beobachten, die einerseits die Zahl der Handwerksbetriebe drastisch reduzieren, aber andererseits die Einkommen selbständiger Handwerker ansteigen lassen.

Drittens, Arbeiterfamilien sind für ihren Lebensunterhalt ausschließlich auf ein Einkommen aus abhängiger Beschäftigung angewiesen. Die stark schwankenden Individuallöhne der Männer reichen im Kaiserreich bei der Mehrheit der Arbeiter nicht aus, um eine Familie zu versorgen. Die Erwerbstätigkeit der Frau, meist in Heimarbeit, ist deshalb weit verbreitet, ebenso wie Kinderarbeit. Zusätzliche Einnahmequellen bieten Untervermietungen und Kleingärten. Der kontinuierliche Reallohnanstieg nach dem Ersten Weltkrieg verbessert die Situation dieser land- und besitzlosen Bevölkerungsschicht (Gestrich 2010:15). Eine ähnliche Situation lässt sich für untere Angestellte beobachten.

Viertens, die bürgerlichen Familien zeichnen sich durch wirtschaftliche Selbständigkeit aus, die ihnen eine Privatisierung und Emotionalisierung der Familie ermöglicht. In den Haushalten der Beamten, höheren Angestellten, freien Berufe oder der Fabrikanten herrscht eine starke Betonung unterschiedlicher Geschlechterrollen. Kindheit wird als geschützter Raum definiert, und in die Ausbildung der meist wenigen Kinder wird viel investiert. ${ }^{20}$ Ein hohes Einkommen ermöglicht es dieser Schicht, das Familienvermögen $\mathrm{zu}$ erhalten und künftigen Generationen materiell sichere Startbedingungen mitzugeben. Gestrich weist etwa darauf hin, dass über die „Hälfte der Personen, die zwischen 1851 und 1873 in Berlin ein Unternehmen führten, [...] dieses bereits von ihren Eltern geerbt [hatten I.S.]“" (Gestrich 2010:20).

Diese Aufzählung lässt sich noch um die Adelsfamilien ergänzen, die eine Minderheitenposition einnehmen, aber in hohem Maße mit politischer Macht und Vermögen ausgestattet sind. Sie zeichnen sich durch ihr spezifisches Heirats- und Erbverhalten aus, das je nach Konfession und Landschaft erheblich variiert, aber im Großen und Ganzen dynastischen Motiven folgt. Besonders auffallend im 19. Jahrhundert ist die Bereitschaft von nachgeborenen Söhnen zum Verzicht auf das Erbe sowie die hohe Quote unverheirateter Töchter. Voraussetzung für eine solche Strategie zum Erhalt von Vermögen und Status in der Stammlinie sind gut dotierte Alternativen für die nichterbenden Kinder, die eine standesgemäße Lebensführung ermöglichen, wie etwa reichskirchliche Ämter oder eine Militärlaufbahn (Reif 1999:30f.). Im Laufe des 19. und 20. Jahrhunderts erlebt der deutsche Adel einen dramatischen Wandel und Fall. Es wird geschätzt, dass der Anteil der Adeligen (Hochadel, Niederadel und Noblierte) an der Gesamtbevölkerung von etwa $0,5 \%$ (ca. 140.000 Personen) im Jahr 1815/30 auf weniger als 0,1\% (ca. 6070.000 Personen) im Jahr 1925 sank (Reif 1999:8f.). 
Ein zentraler Punkt wird schon bei einer solch oberflächlichen Betrachtung deutlich: Die Verquickung von Betrieb und Familie war im 19. Jahrhundert eher der Normalfall und ein Schicht übergreifendes Phänomen. Gerade deshalb stechen die Arbeiterfamilien ebenso wie das Bildungsbürgertum (Beamte, Angestellte) als „neue“ soziale Erscheinung so hervor. Scheinbar macht es jedoch einen großen Unterschied für die Lebensweise einer Familie, ob diese einen landwirtschaftlichen, handwerklichen oder eben industriellen Betrieb besitzt und führt. Je nach Sektor gestaltet sich das Familienleben entlang unterschiedlicher räumlicher und sozialer Bedingungen. In Bauernfamilien gelten andere Regeln für das gemeinschaftliche Arbeiten und Leben als etwa in den handwerklichen Zünften. Von beiden unterscheidet sich grundlegend das eher urban geprägte Leben der bürgerlichen Fabrikanten.

Mit Max Weber könnte man an dieser Stelle einwenden, dass zusätzlich die verschiedenen „Betriebslogiken“ in Betracht gezogen werden sollten. So könnte man die bäuerlichen und handwerklichen Betriebe des frühen 19. Jahrhunderts einer traditionellen, auf Bedarfsdeckung ausgerichteten Wirtschaftsweise und die industriellen Betriebe eher der aufkeimenden kapitalistischen Wirtschaftsweise mit ihrer rationalen Gewinnabsicht zurechnen. Bekanntlich vertritt Weber die These, dass sich der Wandel hin zu einer kapitalistischen Ökonomie okzidentaler Prägung über den Prozess der „Rationalisierung" vollzieht. Dieser beinhaltet neben einer grundlegende Neuordnung des ökonomischen Systems (dazu zählen etwa privates Eigentum, freie Lohnarbeit, Kapitalrechnung oder Kapitalmärkte) (Weber 1958:239f.) auch einen kulturellen Wandel. Die Wahlverwandtschaft zwischen der protestantischen Ethik und dem Geist des Kapitalismus öffnet die Tür, so die These, zu einer „Entzauberung“ der Lebensführung (Weber 1988:7).

Dem modernen, kapitalistischen Unternehmer (und seiner Familie) kommt in diesem Spiel eine Schlüsselrolle zu. Er verkörpert wie kein anderer das rastlose Erwerbsstreben, die strenge Loyalität, die sittliche Redlichkeit, das rational berechenbare Handeln, die Abwertung des Konsums gegenüber dem Erwerb und die Unterordnung der eigenen Person unter ihre Aufgaben all jene Merkmale, die Weber unter dem „Ethos“ der innerweltlichen Askese zu fassen sucht. Dem modernen Unternehmer ist ein spezifischer Habitus zueigen, der auf ein Leben für die kapitalistische Unternehmung ausgerichtet ist. Wesentlicher Bestandteil seiner Lebensweise, so Weber, ist auch eine ökonomische Trennung von Haushalt und Betrieb.

\subsubsection{Die industrielle Unternehmerfamilie}

In der Tradition Webers untersucht Jürgen Kocka die Entstehung und Entwicklung der Industrialisierung in Deutschland und zeichnet dabei ein detailliertes Bild der industriellen Unternehmerfamilie im 19. Jahrhundert. Die 
jungen Fabrikanten sind mit einer überwältigenden Mehrheit Nachfahren von Handwerkern, Verlegern oder Händlern (Kocka 1979:104; ähnliches stellt auch Schäfer fest 2007:61f.). Insofern sieht Kocka eine große familiäre und soziale Kontinuität, die er mit zwei wesentlichen Faktoren zu begründen sucht: Erstens, die Familie nimmt eine dominierende Rolle im Generationen übergreifenden Transfer von Wissen und damit in der Qualifikation der ersten Generation industrieller Unternehmer ein (Kocka 1979:106). Sie genießen eine private Erziehung, absolvieren eine praktische Ausbildung in der Firma des Vaters oder eines anderen Verwandten und sammeln erste Berufserfahrungen im Ausland. Zweitens, die Söhne von Kaufleuten und Handwerkern haben in der Regel zumindest einen kleinen Betrag geerbt und können auf eigene Ersparnisse und Vermögensressourcen als Startkapital zurückgreifen. Kredite werden ebenfalls oft innerhalb der Familien auf Basis von Vertrauen gewährt. Kocka betont darüber hinaus die Bedeutung von Endogamie als familiärem Mechanismus zur Kapitalbeschaffung (Kocka 1979:110f.) und die Rolle der weiblichen Verwandten in der Pflege sozialer Kontakte (Kocka 1979:114). Das Wohlergehen der Familie dient - nicht selten im Kampf gegen potentielle Armut - als eine treibende Kraft für unternehmerisches Engagement und Bereitschaft zum Risiko (Kocka 1994:259). Viele Unternehmer legitimieren ihr Streben nach Leistung, Erfolg und Expansion mit einer starken Familienorientierung oder richten ihre Arbeit an ethischen und religiösen Grundsätzen aus (Kocka 1994:260). Insofern ist die Unternehmerfamilie der industriellen Revolution eine empathische und wirtschaftliche Solidargemeinschaft mit einem Generationen überspannenden Familienbewusstsein.

In seiner historischen Studie zu sächsischen Unternehmern von 1850 bis 1940 beschreibt Michael Schäfer (2007) die Übergabe der Unternehmen in die nächste Generation als Regelfall. Er stellt fest, dass im Vergleich zu den Lebensläufen der Unternehmensgründer der industriellen Revolution die schulischen und beruflichen Karrierewege der Unternehmensnachfolger deutlich mehr Ähnlichkeit miteinander aufweisen. Die Ausbildung ist stark auf die Bedürfnisse des jeweiligen Betriebes abgestimmt und dabei berufsund fachbezogen, international und auf frühe Führungsverantwortung ausgerichtet. Im Fall der von Schäfer untersuchten Nachfolger tritt fast die Hälfte im Alter von 25 bis 29 Jahren in eine leitende Stellung ein (Schäfer 2007:121). Zwischen dem Eintritt der Nachfolger und dem Rückzug der Seniorchefs beobachtet Schäfer hingegen - und das entspricht auch den Ergebnissen jüngerer Forschung - eine relativ lange Periode des gemeinsamen Arbeitens beider Generationen, die nicht selten mit Führungskonflikten einhergeht. Der Fortbestand des Unternehmens über mehrere Generationen verschafft den Unternehmerfamilien soziales Ansehen, das sie als symbolisches Kapital gegenüber Gläubigern, staatlichen Instanzen und auch Kunden in die Waagschale werfen können. Schäfer führt weiter aus, dass Unternehmerfami- 
lien als Zeit übergreifende Gemeinschaften, die in einer chronologischen Linie vom Gründer bis zu seinen Nachfahren reichen, als Ressource für familiale Loyalität und Sinngebung dienen (Schäfer 2007:221). Innerhalb der Familie bildet sich ein Pflichtgefühl gegenüber dem Vater und den Vorfahren aus, die Kontinuität des „Mannesstammes“ (Schäfer 2007:137) nicht abreißen zu lassen. An dieser Terminologie wird deutlich, dass Schäfer vor allem die männliche Linie und die Führungsnachfolge im Blick hat, wohingegen das imaginäre Konstrukt einer individuellen Verortung innerhalb der Generationen übergreifenden Gemeinschaft unabhängig von Geschlecht und späterer Führungsbeteiligung ist (Lubinski 2010:118). Auch Boch weist in seinem historischem Rückblick auf die Unternehmensnachfolge in Deutschland darauf hin, dass auf diese Weise nicht nur die Arbeit einen zusätzlichen Sinn erhielt, sondern das unternehmerische Handeln auch einen erweiterten Zeithorizont - beide machen es leichter, auf kurzfristige Vorteile zu verzichten (Boch 1999:176).

Im Laufe der Zeit differenzieren sich Unternehmerfamilien von Handwerkern und Bauern ebenso wie von Aristokraten und werden Teil einer dichten und geschlossenen wirtschaftsbürgerlichen Klasse (zu der beispielsweise noch die angestellten Manager zählen) mit hoher geographischer Mobilität, geschäftlichen und technischen Kenntnissen, sozialen Kontakten und finanziellen Ressourcen (Kocka 1994:252). Kocka folgt Fritz Redlich in der Annahme, dass das junge kapitalistische Wirtschaftssystem in Deutschland wie in anderen Industrieländern das Produkt von ein paar hundert Familien sei (Kocka 1975:33). Jedoch tragen die Vorbehalte der Oberschicht (Adelige, Beamte, Akademiker) gegenüber der unbürokratischen Art der Geschäftsmänner, die Risiken eingehen und nach Gewinn streben, dazu bei, dass der soziale Status der durchschnittlichen Unternehmerfamilie in Deutschland Anfang des 19. Jahrhunderts relativ niedrig ist (Kocka 1994:255). Daher entspricht die gesellschaftliche Anerkennung und politische Macht der Unternehmer nicht unbedingt ihrem wirtschaftlichen Wohlstand. Erst im späten 19. Jahrhundert entsteht eine soziale, kulturelle und rechtliche Konstellation, die den Aufstieg einer unternehmerischen Elite begünstigt (Kocka 1975:35).

Jürgen Kocka sieht in der industriellen Unternehmerfamilie eine bestimmte Mischung aus Rückwärtigkeit und Modernität, die sich als sehr förderlich für das Wirtschaftswachstum erweist (Kocka 1994:272). Unternehmerfamilien bewahren ihren erwerbswirtschaftlichen Funktionalismus: Kinderreichtum, patriarchalische Ordnung, Tendenz zur Berufsvererbung und zur sozialen Endogamie und Sicherung einer eindrücklich erfahrbaren intergenerationellen Familienidentität (Kocka 1979:176). Trotzdem spricht Kocka davon, dass sich „etwas Neues in neuer Umgebung“ (ebd.) beobachten lässt. Die industriellen Unternehmer verstehen ihre Familien als einen geschützten, privaten Innenraum. Die romantische Verinnerlichung und Emotionalisierung der Familie, die zunächst im Bildungsbürgertum die Vorstellung von 
Familie veränderte, macht auch ,,vor den Türen der Unternehmerhäuser nicht halt" (Kocka 1979:122). Als bürgerliche Familien entwickeln auch Unternehmerfamilien Ansätze zur Selbstbestimmung in Distanz zur ökonomischen Rationalität (Kocka 1979:126). Im Bereich der Eheschließung erfolgt beispielsweise die Wahl der Heiratspartner immer seltener entlang des familienstrategischen Kalküls, stattdessen steht die gegenseitige Zuneigung der angehenden Eheleute im Vordergrund. Gleichwohl erfolgt die Partnerwahl meist innerhalb schon bestehender sozialer Kontakte (Verkehrskreise) und orientiert sich an einem spezifischen Einstellungsset, so halten Brautleute ,standesgemäßes“" Heiraten für wichtig und die Zustimmung der Eltern für unabdingbar (Kocka 1979:127). Insofern kann auch die Liebesehe kompatibel mit der Geschäftspolitik sein und die gleiche Wirkung erzielen wie eine Zweckehe - muss es aber nicht. Die hier drohenden Konflikte zwischen den Generationen sind sicherlich nicht $\mathrm{zu}$ unterschätzen. $\mathrm{Zu}$ einem ganz ähnlichen Schluss, nämlich dem, dass auch Unternehmerfamilien den gewandelten Vorstellungen über emotionale Bindung als Fundament folgen, kommt auch Harold James (2005), der in vergleichender wirtschaftshistorischer Perspektive die Unternehmerfamilien Haniel, Wendel und Falck untersucht. Die Anpassung erfolge jedoch nicht ohne Hindernisse. Die vermeintliche Unvereinbarkeit zwischen Liebe und ökonomischer Rationalität könne für chronische psychische Konflikte sorgen (James 2005:21).

Die Konsequenz einer solchen Annäherung an bürgerliche Ideale für die Verbindung von Unternehmen und Familie liegt auf der Hand: Die Aufgabe, familiäre, individuelle und unternehmerische Interessen $\mathrm{zu}$ koordinieren, wird auf die einzelnen Familienmitglieder übertragen, die sich nun an abstrakten Wertvorstellungen statt an der (haus-)väterlichen Autorität orientieren. Dass dies auch zu dramatischen Problemen führen kann, wird am Beispiel des materiellen Transfers im Erbgang deutlich. Kocka weist darauf hin, dass es häufig zu Nachfolgekrisen in Familienunternehmen kommt, weil sich der Anspruch, das Erbteil an mehrere Kinder auszuzahlen, nicht mit der Liquidität des Unternehmens vereinen lässt (Kocka 1979:131). Auch Boch stellt, unter Rückgriff auf eine Studie von Dirk Schumann zu Bayerns Unternehmern in Gesellschaft und Staat von 1834 bis 1914, fest, dass die Gleichbehandlung der Familienmitglieder vor der besonderen Förderung des Nachfolgers steht, d.h. in der Regel das Betriebsvermögen zu gleichen Teilen vererbt wird - und das auch zwischen Töchtern und Söhnen (Boch 1999:167). Ungeachtet dessen wird nicht selten versucht, durch festgelegte Auszahlungsmodalitäten wie etwa gestaffelte Ausbezahlung der Erbteile zu verhindern, dass der Bestand des Betriebes bedroht wird.

Kocka beobachtet, wie einige Unternehmen, die ein enormes Wachstum erlebten, ihre Corporate Governance-Struktur hin zu Gesellschaften mit beschränkter Haftung ( $\mathrm{GmbH}$ und $\mathrm{AG})$ anpassen. Die zunehmend unüberschaubarer werdenden Organisationen führen nicht selten zu einem veränder- 
ten, oft distanzierten Verhältnis der einzelnen Familienmitglieder zum Unternehmen. Zudem bringt es die formale Trennung von Führung und Eigentum mit sich, dass externe Kapitalgeber oder Manager integriert werden müssen und sich die Rollen der verschiedenen Familienmitglieder differenzieren. Kocka weist zudem auf eine Reihe von Veränderungen im Laufe des 19. Jahrhunderts hin, die zu einer Schwächung der Verbindung von Familie und Unternehmen beitragen. Die technischen und kaufmännischen Kenntnisse eines Unternehmers werden zunehmend über das öffentliche Bildungssystem vermittelt, die Finanzmärkte entwickeln sich, neue Unternehmensgesetze werden verabschiedet, Kartelle und professionelle Netzwerke machen die familialen Verbindungen überflüssig. Trotzdem können der unternehmerische Habitus und die entsprechenden informellen Kontakte nur im Raum der Familie angeeignet bzw. entwickelt werden. Darüber hinaus diene die Familie als wesentliches Element der Unternehmenskultur. Alles in allem hält Kocka fest, dass um 1914 der industrielle Kapitalismus der familiären Ressourcen nicht mehr so sehr bedarf wie im Jahr 1850, weil sich Alternativen entwickelt haben (Kocka 1979:135).

Es kann als historischer Fakt gelten, dass die bürgerliche Unternehmerfamilie zur Lösung vorindustrieller Probleme und zur Entwicklung des Kapitalismus wesentlich beigetragen hat (Kocka 1979:117). Angesichts der fortschreitenden Trennung von Betrieb und Familie in ökonomischer wie auch emotionaler Hinsicht ebenso wie der Reifung und Etablierung institutioneller Strukturen im kapitalistischen Wirtschaftssystem und den modernen Familienformen sieht Kocka jedoch die Vorteile der Verbindung von Familie und Unternehmen schwinden. Er vertritt damit die allgemeine Forschungsmeinung in der Wirtschafts- und Sozialgeschichte, dass Funktionen, die vormals die Familie erfüllte, nun von marktförmigen Strukturen übernommen werden (Lubinski 2010:11-12). Kaelble und Spode (1990) sprechen in diesem Zusammenhang sogar von einem fünffachen Funktionsverlust der Unternehmerfamilie. Kocka schlussfolgert daraus, dass die Unternehmerfamilie lediglich ein Übergangsstadium von der traditionalen Hausgemeinschaft hin zur bürgerlichen Familie ohne Unternehmensanschluss sei (Kocka 1979:117). Die Parallelität dieser These zu Chandlers Vorstellung einer deterministischen Entwicklung vom Familienunternehmen hin zu modernen Großunternehmen ist erstaunlich. Eben dies kritisiert Schäfer, der die Verbindung von Unternehmen und Familie mit ihren spezifischen Strukturen, Beziehungen und Bedeutungszuschreibungen auch im Übergang zum 20. Jahrhundert als stabil ansieht (Schäfer 2007:100). 


\subsubsection{Unternehmerfamilien in der ersten Hälfte des 20. Jahrhunderts}

Die deutsche Wirtschaft und mit ihr das Unternehmertum erlebt in der wechselhaften ersten Hälfte des 20. Jahrhunderts eine Berg- und Talfahrt zwischen politischer Einflusskraft und blühenden Geschäften sowie staatlicher Kontrollwirtschaft und Weltwirtschaftskrise. Unter anderem hat sich Adam Tooze (2007) ausführlich mit der Geschichte der Wirtschaft im Nationalsozialismus auseinandergesetzt. ${ }^{21}$ Für die Weimarer Zeit bis 1929 stellt er fest, dass das Großunternehmertum den wirtschaftlichen Kurs in noch nie dagewesener Weise beeinflusste (Tooze 2007:142), sich dann aber einer Diskussion um staatliche Regulation ausgesetzt sah. Hitler und seine Helfer mobilisierten die deutschen Industriellen als Finanzgeber im Wahlkampf 1933 mit der Aussicht auf ein Ende der Linken in Deutschland und wirtschaftliches Wachstum (Tooze 2007:129). Für Unternehmer war ein Anschluss an die NSDAP von ökonomischem Vorteil, jedoch wäre es zu vereinfachend, alle Unternehmer als linientreue Parteimitglieder zu verstehen. Mit dem 1934 verabschiedeten „Gesetz zur Ordnung der nationalen Arbeit“ verschiebt sich das Machtgleichgewicht in der deutschen Wirtschaft zugunsten der Unternehmer und Firmenchefs (und zu Ungunsten der Gewerkschaften), die ,in dieser autoritären Atmosphäre richtiggehend aufblühten“ (Tooze 2007:130). Im Laufe der Zeit installiert der Nationalsozialismus eine staatliche Wirtschaftsverwaltung, die zu immer mehr Interventionismus und Bürokratie tendiert (z.B. Regulierung von Kapitalflüssen oder Infrastruktur). Das Reichswirtschaftsministerium konnte über die sogenannten Reichsgruppen, denen beizutreten jedes Unternehmen verpflichtet war (Tooze 2007:136), unternehmerisches Handeln engmaschig überwachen. Insbesondere die nationalsozialistischen Rüstungsprogramme erwiesen sich für die deutsche Industrie zunächst als förderlich. Als anschauliches Beispiel führt Tooze den Flugzeugbau an, der die Produktion in kurzer Zeit von ein paar hundert Flugzeugen pro Jahr auf mehrere tausend steigern konnte (Tooze 2007:156). Die staatlich gelenkte Expansion ging in einigen Sektoren mit erheblichen Gewinnsteigerungen einher. Nach 1938 und mit dem Beginn des Zweiten Weltkrieges nahm die Rüstungspolitik irrsinnige Dimensionen an, was zu Lasten anderer Ressourcen und Produkte ebenso wie der Preisstabilität ging (Tooze 2007:410). Der Status eines „kriegswichtigen Betriebes“ bedeutete für Unternehmerfamilien ein Einkommen über die Kriegsjahre hinweg. Zudem konnten wichtige Familienmitglieder im Unternehmen verbleiben - falls sie sich nicht freiwillig an die Front melden. Gleichzeitig wurden ihre Betriebe so zu Zielen der Bombenangriffe durch die Alliierten.

21 Die Literatur zu Unternehmen im Nationalsozialismus ist sehr umfangreich. Ebenfalls einschlägig sind die Arbeiten von Herve Joly. 
Mit dem Ende des Zweiten Weltkrieges begann für Unternehmerfamilien, wie für die deutsche Gesellschaft insgesamt, die Zeit der Besatzung und des Wiederaufbaus. Maschinen wurden demontiert, Rohstoffe und Betriebsmittel standen kaum zur Verfügung, ehemals kriegswichtige Betriebe wurden entnazifiziert, was oft bedeutete, dass ein externer Interims-Geschäftsführer das Unternehmen verwaltete. Es kann mit einiger Plausibilität behauptet werden, dass Familien in diesen Krisenzeiten erneut als wichtiger Motor für den ökonomischen Wiederaufbau fungieren. Familiale Loyalität, Willenskraft und Ressourcen tragen dazu bei, dass viele Unternehmen wieder zurück in Familienbesitz und -führung gelangen oder neue Unternehmen gegründet werden. Kocka konstatiert eine hohe familiale und soziale Kontinuität zwischen der Zeit des Nationalsozialismus und den Anfängen der Bundesrepublik (Kocka 2002:10). Er betont, dass sich „,die allermeisten Unternehmer auf den Boden des parlamentarisch-demokratischen Systems der entstehenden Bundesrepublik" (Kocka 2002:10) stellen, sei es aus Überzeugung oder einer strategischen Anpassung an die neuen Macht- und Werteverhältnisse. Es kann vermutet werden, dass in mehrgenerationellen Unternehmerfamilien mit ihrer Kontinuität in Vermögen und beruflicher Tätigkeit ein Generationenkonflikt über die Frage der Täterschaft während der NS-Zeit noch drastischer angelegt ist als in anderen Familien: ${ }^{22}$ Es müssen Wege gefunden werden, wie die Kollektividentität der Solidargemeinschaft an die Familientradition anschließen kann. Die NS-Zeit als Tabuthema in der Familie - also eine Strategie des Schweigens - scheint aufgrund der täglichen Begegnung der Familienmitglieder im operativen Geschäft und des Vermögens als Existenzgrundlage besonders wahrscheinlich.

\subsubsection{Familienunternehmen als Motor der deutschen Wirtschaft}

Beginnend mit der zweiten Hälfte des 20. Jahrhunderts verändert sich die Markt- und Wettbewerbssituation grundlegend. Mit dem Wandel vom Verkäufer- zum Käufermarkt werden Werbung und Marketing zu zentralen unternehmerischen Tätigkeitsfeldern. Neue Technologien, eine zunehmende Internationalisierung und später Globalisierung, wirtschaftliche Krisen ebenso wie veränderte Finanzmärkte machen es erforderlich, in hohem Maße mit unvorhersehbaren Wechselwirkungen, Risiken, durchlässigen Grenzen, Mehrdeutigkeit und Kontingenz umzugehen.

In den Wirtschaftswunderjahren gelingt es der deutschen Wirtschaft, wieder zu den bedeutendsten Industrienationen der Welt aufzuschließen und diese Position seither zu behalten. Dabei unterscheidet sich der kontinental-

22 Die Thematisierung der NS-Zeit in Familien ist disziplinenübergreifend erforscht worden. Wesentlich sind etwa Welzer et al. 2005, 2002 oder Rosenthal 1999. 
europäische Kapitalismus vom angelsächsischen Modell durch die engen Verbindungen von Staat, Markt und Familie, die ihn zu einer sozialen Variante des Kapitalismus werden lassen. ${ }^{23}$ Auffällig ist die Bedeutung mittelständischer Unternehmen für das ökonomische Wachstum und die Wertvorstellungen dieser spezifischen Ausprägung des Kapitalismus. Familienunternehmen bleiben weiterhin ein wesentlicher Bestandteil der Unternehmenslandschaft. Im Zuge dieser Veränderungsprozesse lassen sich zwei zentrale Konsequenzen für Familienunternehmen beobachten: Erstens, wie schon im 19. Jahrhundert ziehen sich Familien immer mehr aus der Leitung und auch dem Eigentum an großen Unternehmen zurück. Zweitens, die Managementphilosophien unterliegen einem Wandel, der eine erneute Veränderung der Unternehmens-Familien-Beziehung verlangt. Auf beide Punkte soll im Folgenden näher eingegangen werden.

\subsubsection{Familienunternehmen gleich KMU?}

Die historische Studie zu Familienunternehmen in Westdeutschland von Christina Lubinski (2010) verdeutlicht, dass der Familieneinfluss bei großen Unternehmen immer mehr zur Disposition gestellt wird und sich ein Trend zu „professionellen“ Corporate Governance-Strukturen beobachten lässt. Für eine quantitative Vorstudie nutzt Lubinski das Hoppenstedt-Adress-Register. Sie zieht im Jahr 1960 ein Sample von insgesamt 310 Unternehmen, die mehr als 250 Beschäftigte haben und entweder in Düsseldorf oder in München ansässig sind, und recherchiert zusätzliche biografische und betriebliche Daten. 165 dieser Unternehmen sind familienbeeinflusst, die große Mehrheit davon (103 Fälle) wird vollständig von einer Familie oder einer Person besessen (meist 2-3 Familienmitglieder) und befindet sich in zweiter oder einer späteren Generation (Lubinski 2010:55f.). 58\% der familienbeeinflussten Unternehmen firmieren in Rechtsformen mit persönlicher Haftung (Einzelkaufleute, OHG, KG) (Lubinski 2010:60). Die absolute Mehrheit der Unternehmen besitzt mindestens einen Vertreter der Familie in den Führungsgremien. Lubinski verfolgt diese Unternehmen bis in das Jahr 2008 weiter und kann damit Aussagen über die Aufrechterhaltung und Institutionalisierung des Familieneinflusses treffen. Viele dieser Unternehmen (88) nehmen eine Umfirmierung in eine Rechtsform mit beschränkter Haftung vor, was die Aufnahme externer Kapitalgeber erleichtert, aber auch zu einer zunehmenden Trennung von Familien und Unternehmen führt. Mehr als die Hälfte der Unternehmen (85) geben in diesem Zeitraum von fünfzig Jahren den Familieneinfluss definitiv auf (Lubinski 2010:66).

23 Einschlägige Arbeiten in diesem Bereich sind etwa: Amable 2003; Hall und Soskice 2001 oder Whitley 2002. 
Studien zur volkswirtschaftlichen Bedeutung von Familienunternehmen zu Beginn des 21. Jahrhunderts zeigen, dass diese dennoch in den meisten europäischen Ländern die große Mehrheit aller Unternehmen bilden. Laut einer Analyse von Werner Freund am Institut für Mittelstandsforschung (IfM) in Bonn (2004) sind 95,1 Prozent aller deutschen Unternehmen mit einem jährlichen Umsatz über 50.000 Euro Familienunternehmen. Sabine Klein (2004) findet in ihrer Erhebung aus dem Jahr 2000, dass 71 Prozent der von ihr befragten Unternehmen mit mehr als einer Million Euro Jahresumsatz Familienunternehmen darstellen. Der Anteil an Familienunternehmen sinkt drastisch auf immerhin noch 34\% in der Gruppe der Unternehmen mit einem Jahresumsatz von 50 Millionen Euro und mehr, so eine Studie der Stiftung Familienunternehmen aus dem Jahr 2007. ${ }^{24}$ Familienunternehmen sind also eher kleine und mittlere Unternehmen, und deshalb hat die Gleichsetzung mit dem Begriff Mittelstand (in seiner quantitativen Variante) eine gewisse Berechtigung. Damit erklärt sich auch, warum Familienunternehmen nur unterproportional zum Bruttoinlandsprodukt ebenso wie zur Zahl der Arbeitsplätze beitragen: Im Auftrag der Stiftung Familienunternehmen (2007) ermittelte das IfM Bonn, dass die Familienunternehmen 41,5 Prozent der Umsätze aller deutschen Unternehmen erwirtschaften und ca. 57,3 Prozent aller sozialversicherungspflichtigen Beschäftigungsverhältnisse stellen. Trotzdem lautet das Fazit der aktualisierten Studie zur volkswirtschaftlichen Bedeutung von Familienunternehmen der Stiftung Familienunternehmen (2009), dass die 500 größten Familienunternehmen krisenresistenter als nicht-familiengeführte DAX-Unternehmen seien, denn ihnen gelinge es, auch in wirtschaftlich unsicheren Zeiten Arbeitsplätze zu schaffen und den Umsatz stabil zu halten. Die Entwicklung börsennotierter Familienunternehmen kann seit 2010 auch anhand eines eigenen Index des DAX plus family Index, abgelesen werden.

Seit Beginn der 1990er Jahre veröffentlicht das IfM Bonn auch statistische Hochrechnungen von zukünftigen Unternehmensübergaben. Die Berechnungen basieren auf der Umsatzsteuerstatistik, dem Mikrozensus, Angaben zu den Sozialversicherungen sowie einer Reihe von Umfragen zur Nachfolge in Deutschland. Das IfM geht davon aus, dass in der Zeit von 2010 bis 2014 in 110.000 Familienunternehmen, also in 3\% aller Familienunternehmen, eine Nachfolgelösung gefunden werden muss (2010:20). In den allermeisten Fällen (86\%) erfolgt der Transfer aufgrund des Alters des Übergebers. Im Vergleich zu den bisherigen Schätzungen ist diese Zahl der Nachfolgen deutlich, nämlich auf ein Drittel, gesunken (Freund 2004). Ein wesentlicher Grund dafür liegt in einem neuen Schätzverfahren, das die über-

24 Die Gegenüberstellung dieser Zahlen ist insofern problematisch, als in den verschiedenen Studien die Definition und Operationalisierung von Familienunternehmen uneinheitlich ist. Aus diesem Grund können die präsentierten statistischen Werte lediglich als Richtgrößen verstanden werden. 
nahmewürdige Substanz eines Unternehmens nicht mehr anhand von Umsatz, sondern von Gewinn bestimmt. Eine Reihe von Studien beschäftigt sich darüber hinaus mit der Übergabe in Familienunternehmen auf der Ebene einzelner Bundesländer oder Branchen (z.B. Künemund und Beate Mücke 1990; Schüßler et al. 2004; Engelmann et al. 2005). Deutlich wird hierbei immer wieder der hohe Wille der Kinder, das Unternehmen zu erhalten, der aber nicht immer der tatsächlichen Übergabepraxis entspricht.

Die hier angeführten Statistiken vermitteln jedoch kein repräsentatives Bild. Den meisten großen Datensätzen fehlt die notwendige Verbindung von betrieblichen Informationen, Individual- und Haushaltsdaten. In der Forschung zu Familienunternehmen wird deshalb oft die Nachnamensgleichheit von Eigentümern und Geschäftsführern eines Unternehmens als Indiz für verwandtschaftliche Verhältnisse genutzt (z.B. Freund 2004). Diese Operationalisierung scheint vor dem Hintergrund plausibel, dass Familienunternehmen in der Vergangenheit in männlicher Linie geführt und weitergegeben wurden. Je stärker sich jedoch moderne Lebensweisen (z.B. nichteheliche Lebensgemeinschaften) und emanzipierte Übergabepraktiken in Unternehmerfamilien verbreiten, desto unzuverlässiger wird dieser Indikator für familiäre Beziehungen. Eine aktuelle Umfrage der Intes Akademie für Familienunternehmen weist etwa darauf hin, dass inzwischen jedes vierte Familienunternehmen von einer Frau geführt wird (o.A. 2011) - viele von ihnen tragen andere Nachnamen als ihre väterlichen Vorgänger. Des Weiteren sei darauf hingewiesen, dass für die Schätzung zukünftiger Nachfolgen das gesetzliche Rentenalter als Orientierungswert angesetzt wird (Hauser et al. 2010:18), jedoch keine Statistiken über das tatsächliche Rentenalter von Unternehmern vorliegen.

\subsubsection{Ein neuer Geist des Kapitalismus?}

Neben der Anzahl an Familienunternehmen in der zweiten Hälfte des 20. Jahrhunderts lässt sich auch nach der Qualität der Familien-UnternehmensVerbindung fragen. In diesem Zusammenhang scheint die „Revolutionierung des Managements“ (Kocka 2002:12), die unter anderem Jürgen Kocka beobachtet, eine besondere Rolle zu spielen:

Während in den 1950er und 1960er Jahren eine zunehmende Bedeutung systematisch produzierten Wissens in der Planung, Koordination und Kontrolle wirtschaftlicher Betriebe im Sinne eines Scientific Managements zu verzeichnen ist, setzt das postfordistische Management auf Netzwerke, Koordination mit oft informellen Mitteln, die auf sozialer Kohäsion beruhen. Die „Entstofflichung und Entgrenzung der Ökonomie“ (Albert 1999) hat zur Folge, dass Hierarchie- und Marktbeziehungen durch quasi politische Aushandlungsprozesse ergänzt werden (Kocka 2002:12). In der Unternehmens- 
führung bezeichnet man Unternehmungen nun als ,,proaktive, strukturpolitische Akteure" (Schneidewind 1998), die in hohem Maße mit Instabilität und Unsicherheit umgehen müssen. Kocka betont, dass vor diesem Hintergrund Unternehmenskultur, Loyalität und Identifikation der Einzelnen mit dem Unternehmen umso wichtiger geworden sind (Kocka 2002:13-14). Des Weiteren vermutet er, dass die Unternehmer an der Schwelle zum 21. Jahrhundert andere Orientierungen, Fähigkeiten und Verhaltensweisen aufweisen dürften als noch 50 Jahre zuvor (Kocka 2002:14).

In einem ausführlichen qualitativen Vergleich zwischen der Management-Literatur der 1960er Jahre und der 1990er Jahre zeigen Luc Boltanski und Eve Chiapello die darunter liegenden normativen Verschiebungen. Sie vertreten die These, dass sich der "Geist des Kapitalismus" im Laufe der Zeit gewandelt habe. Das 19. Jahrhundert war geprägt von dem familienweltlichen Geist des Kapitalismus (Boltanski und Chiapello 2003:55f.), dessen zentrale Figur der Unternehmer bildete, der die „neue“ kapitalistische Wirtschaftshaltung mit Familienorientierung verband. Im Gegensatz zu diesem Familienkapitalismus steht der „Konzernkapitalismus“ (Boltanski und Chiapello 2003:57), der die Organisation in den Mittelpunkt rückte und den Firmendirektor als heroische Figur betrachtete. Zentrale Merkmale sind Massenproduktion, strenge Hierarchien, Technikgläubigkeit, Kollaboration mit dem Staat und Rationalität. Dieser Geist des Kapitalismus erlebte von 1930 bis 1960 seinen Zenit. Die Veränderungen des kapitalistischen Geistes gehen mit tief greifenden Wandlungen der Lebens- und Arbeitsbedingungen einher. Der jeweilige Geist legitimiert den Akkumulationsprozess und schafft soziale Identität, setzt aber auch normative Grenzen. Ein neuer Geist kann sich nur etablieren, so Boltanski und Chiapello, wenn es gelingt, die Kritik an dem vorhergehenden normativen System konstruktiv aufzunehmen und zu integrieren. In ihrer Analyse versuchen sie den ,,neuen“ (dritten) Geist des Kapitalismus näher zu bestimmen. In dessen Mittelpunkt stehen vernetzte Unternehmen und die Selbstentfaltung durch Projektvielfalt. Die Wertigkeit der Netzwerkmenschen bemisst sich an ihrer Aktivität, also der Fähigkeit „Projekte ins Leben zu rufen oder sich den von anderen initiierten Projekten anzuschließen" (Boltanski und Chiapello 2003:155). Sie können andere begeistern, Vertrauen erwecken, scheuen kein Risiko, handeln autonom und vertrauen ihrer Intuition und ihrem Talent. In einer solchen vernetzten Welt verschwindet die Unterscheidung zwischen Privat- und Berufsleben. Boltanski und Chiapello sprechen davon, dass die bis dahin bestehende Arbeitswelt dekonstruiert wird (ebd.:261ff.): Die interne Arbeitsorganisation sowie das Produktionssystem verändern sich grundlegend (z.B. durch Change-Management), die Gewerkschaften verlieren an Bedeutung, die sozialen Klassen werden in Frage gestellt und die Beschäftigungsverhältnisse werden bei wachsender Arbeitsbelastung zunehmend unsicher. In ihrer Zentrierung von Persönlichkeit sowie direkter Kontakte ähneln sich der familienweltliche und 
der postfordistische, vernetzte Kapitalismus stark. In der idealtypischen Beschreibung der beiden Franzosen kommt es zu einem Wiedererstarken der kleinen, oft in Händen einer Familie befindlichen Unternehmen (Boltanski und Chiapello 2003:182). Jedoch verliere die Unternehmerfamilie als Kollektiv sowohl in ihrer identitätsstiftenden als auch in ihrer kontrollierenden Funktion an Bedeutung. Die Unternehmerfamilie darf die individuelle Wahl nicht begrenzen, Beziehungen nicht vorbestimmen (Boltanski und Chiapello 2003:183).

\subsubsection{Unternehmerfamilien in der zweiten Hälfte des 20. Jahrhunderts: ein Sonderfall}

Der Wandel des ökonomischen Systems geht einher mit grundlegenden Veränderungen der Lebensführung. Die Stichworte der Individualisierung, Pluralisierung und Netzwerke sind bereits in Kapitel II behandelt worden. Die abhängige Erwerbsarbeit hat sich als materielle Lebensgrundlage für breite Teile der Bevölkerung durchgesetzt, und der allgemeine Wohlstand ist gestiegen. Der Abbau von ständischen und regionalen Unterschieden, die allgemeine Ausdehnung von öffentlichen Aktivitäten im Sozialisationsbereich und die Normierung der Erfahrungsbereiche durch die Medien haben, so Gestrich, die demografische Struktur und die ökonomische Bedeutung der Familie verschiedener Gesellschaftsschichten einander angeglichen (Gestrich 2010:2). In der Forschung werden Familien nun anhand der personellen Konstellation, der Haushaltsgröße oder der Kinderzahl differenziert und nicht mehr anhand ihrer materiellen Basis (z.B. Nave-Herz 2007). Das bedeutet allerdings auch, dass die Unternehmerfamilien (seien sie nun handwerkliche, landwirtschaftliche oder industrielle Familien) in der zweiten Hälfte des 20. Jahrhunderts zum Sonderfall werden.

Statistische Angaben zu Unternehmerfamilien in Deutschland sucht man vergebens. Wir wissen nichts über die Struktur und Lebensweise von Unternehmerfamilien, ihre durchschnittliche Kinderzahl, das durchschnittliche Haushaltseinkommen oder typische Bildungswege. Fest steht lediglich, dass es sich um eine relativ kleine Gruppe handelt: $\mathrm{Zu}$ Beginn des 21. Jahrhunderts ist nur ein Bruchteil der Bevölkerung selbständig tätig. Die Daten des Sozio OEkonomischen Panels aus dem Jahr $2007^{25}$ zeigen, dass lediglich 6\% aller Erwerbstätigen in Deutschland selbständig sind bzw. in $10 \%$ aller Haushalte mindestens eine Person selbständig ist, wenn auch mit zunehmender Tendenz (jeweils gemessen an allen SOEP-Teilnehmern). Auch der Anteil solcher Personen, die Betriebsvermögen besitzen, ist mit knapp 5\% bzw. 
knapp 8\% aller Haushalte gering. Während also der Anteil aller Familienunternehmen an allen Unternehmen nach wie vor sehr hoch ist, ist die mengenmäßige Bedeutung von Unternehmerfamilien gemessen an allen Familien relativ niedrig.

Aufgrund des sehr lückenhaften Forschungsstandes lassen sich nur sehr wenige Aussagen über die Lebensweise dieser spezifischen Gruppe treffen. Insbesondere im Vergleich zu dem facettenreichen Bild der industriellen Unternehmerfamilie wirken die Familien eigener Art in der zweiten Hälfte des 20. Jahrhunderts blass. Auf den ersten Blick scheint zumindest die Annahme plausibel, dass Unternehmerfamilien tendenziell reicher sind als andere Familien. Indizien dafür liefern nicht nur die alljährlichen „Reichenlisten“, die vom Forbes Magazin oder Manager Magazin veröffentlicht werden. Sie illustrieren die außergewöhnliche Rolle von unternehmerischer Aktivität und des Eigentums an Betriebsvermögen für die Vermögensbildung ebenso wie den anhaltenden Einfluss von Familien in der Bildung und oder dem Erhalt von Vermögen. Auf den Zusammenhang zwischen Unternehmertum und Reichtum deutet darüber hinaus auch die soziologische Forschung zu Vermögenden in Deutschland hin. Dem Armuts- und Reichtumsbericht kann man etwa entnehmen, dass sich in der Gruppe der vermögensstarken Personen weit überdurchschnittlich viele Selbständige bzw. Rentner finden (Grabka et al. 2007:144). Der „Vermögensvorsprung“ zu anderen Berufsgruppen sinkt zwar, wenn ein Teil des Betriebs- und Privatvermögens für den Aufbau einer privaten Altersvorsorge berücksichtigt wird, bleibt aber in den meisten Einkommensgruppen erheblich (Grabka et al. 2007:252). Im Zeitraum von 1998 bis 2006 ist das Armutsrisiko von Selbständigen zwar leicht angestiegen, ist aber immer noch am geringsten im Vergleich zu allen anderen Erwerbsgruppen (Grabka et al. 2007:121). In der Studie „Vermögen in Deutschland" von Wolfgang Lauterbach, Thomas Druyen und Matthias Grundmann, die auf eine Selbstklassifizierung als Unternehmer zurückgreift, findet man unter der Gruppe der Reichen vor allem Freiberufler, selbständige Akademiker und Unternehmer (Lauterbach und Shanahan 1998:71). Weiter können sie beobachten, dass gerade Unternehmer mit mittleren und größeren Betrieben zu den Reichsten in der Bevölkerung zählen.

„Jeder fünfte ist in der kleinen Gruppe der Reichen ein Unternehmer mit mehr als 10 Mitarbeitern, unter den HNWIs [High Net Worth Individuals; min. $€ 1$ Mio freiverfügbares Kapitel, I.S.] ist es sogar mehr als jeder Dritte, in der Mittelschicht hingegen sind es nur 0,6\%" (Lauterbach und Shanahan 1998:72).

Angesichts der starken Konzentration von Unternehmen in der Hand weniger, oft reicher Familien besteht für Kocka kein Zweifel, dass in Deutschland weiterhin eine Wirtschaftselite aus Unternehmern, Kapitalisten, Managern und ihren Angehörigen besteht (Kocka 2002:15). Weiter geht er davon aus, 
dass diese exklusive Gruppe hohe Selbstrekrutierungsraten aufweist - was die soziale Herkunft betrifft, sogar exklusiver als noch vor einigen Jahrzehnten (Kocka 2002:14). Die soziologische Elitenforschung in Deutschland, die von Michael Hartmann maßgeblich geprägt wird, betont ebenfalls, dass die Rolle sozialer Herkunft immer wichtiger werde. Denn der Kapitalismus des 21. Jahrhunderts setzt auf persönliche Eigenschaften wie Kommunikationsund Motivationsfähigkeiten, die Kunst der Selbstdarstellung, Common-Sense oder Führungs- und Moderationstechniken. Unter Rückgriff auf Pierre Bourdieu argumentiert Hartmann, dass sich im Laufe der familiären Sozialisation ein Unternehmerhabitus herausbilde. Und gerade diese „feinen Unterschiede" des inkorporierten kulturellen Kapitals sorgen für die Abgeschlossenheit des Unternehmermilieus (Lubinski 2010:175).

Andererseits greift jüngste Forschung den Aspekt einer drohenden Prekarisierung des Unternehmertums auf. Unter sozialpolitischen Gesichtspunkten bedenklich ist zu beobachten, dass nicht alle Selbständigen ausreichend Kapital für das Alter ansparen. Die Zahl der Betroffenen beläuft sich auf $15 \%$ der Selbständigen bzw. 12,2\% der Population der Selbständigenhaushalte (Grabka et al. 2007:257). Zudem haben Zeitarbeit, befristete Verträge und Ich-AGs zu einem sprunghaften Anstieg der Zahl von Mikro- bzw. Soloselbständigen geführt. Bögenhold und Fachinger stellen fest, dass nach der subjektiven Wahrnehmung der Höhe des Haushaltsnettoeinkommens etwa $30 \%$ der Solo- bzw. Mikro-Selbständigen unterhalb der Armutsschwelle liegen (Bögenhold und Fachinger 2010:77). Aus diesem Grund grenzen Bührmann und Pongratz (2010) die neuen, flexiblen Formen selbständiger Arbeit vom „Normalunternehmer“ ebenso wie vom Normalarbeitsverhältnis ab. Auch in der Schichtungssoziologie unterscheidet man die sehr heterogene Gruppe der Selbständigen weiter. Etwa zwei Drittel bis drei Viertel der Selbständigen liegen in Bezug auf ihre materielle Situation und ihre Lebenschancen in der oberen und mittleren Mitte der Gesellschaft, und nur eine Minderheit rangiert eher im unteren Bereich (2008:140). Die Gruppe der Unternehmer, Handwerker, Händler und Bauern wird deshalb oft unter dem Stichwort ,alter Mittelstand“26 gefasst (von dem sich der neue Mittelstand aus Angestellten und Beamten abgrenzt).

Für eine Konzeptionalisierung von Unternehmerfamilien scheint eine Abgrenzung zu der sehr heterogenen Gruppe der Selbständigen notwendig. Dabei sollten die bestehenden Unterschiede zwischen den Besitzerfamilien von kleinen Handwerksbetrieben und großen Pharmakonzernen, von Werbe-

26 Innerhalb der Sozialstrukturanalyse wird der Begriff „Mittelstand“ verwandt, um eine mittlere Position innerhalb der gesellschaftlichen Strukturierung zu bezeichnen. Dieser Begriff ist von mittelständischen Unternehmen zu differenzieren, die sich aufgrund ihrer Größe (Umsatz, Mitarbeiter) und Normvorstellungen von kleinen und großen Unternehmen unterscheiden. 
agenturen und Handelsketten nicht nivelliert werden. Die Größe des Unternehmens, gemessen an der Mitarbeiterzahl, sticht als Differenzierungsmerkmal hervor und scheint Auswirkungen auf den jeweiligen Lebensstil der Unternehmerfamilie zu haben.

Im Vergleich zur industriellen Unternehmerfamilie gilt auch heute noch, dass die Verbindung von Familie und Unternehmen gleichzeitig ein Armutsrisiko und eine relativ hohe Chance auf Wohlstand birgt. Hinsichtlich des sozialen Status von Unternehmerfamilien lassen sich hingegen keine fundierten Aussagen treffen.

Und wie steht es um die Orientierung an bürgerlichen Idealen? Kocka merkt an, dass sich Unternehmerfamilien von der bürgerlichen Art der Familie losgelöst und sich von ihrer strikten Orientierung an Bildungs- und moralischen Idealen getrennt hätten. Gleichzeitig will er am Begriff der Bürgerlichkeit und der damit verbundenen Kultur, die Selbständigkeit und Leistung hochschätzt, im Kontext von Unternehmerfamilien festhalten (Kocka 2002:15). Lubinski stellt in ihrer qualitativen Arbeit eine „erzählte Bürgerlichkeit" als markantes Element der Selbstdarstellung von Unternehmerfamilien fest. In ihrer Auswertung des reichhaltigen Archiv- und Interviewmaterials der industriellen Familien Bagel, Rodenstock und Deckel arbeitet sie zentrale Thesen zu typischen Praktiken des Eigentums, der Nachfolge und Sozialisation, der kollektiven Identität und der Unternehmenskultur heraus. Die Unternehmerfamilie wird nach Lubinski zu einem sozialen Konstrukt, das insbesondere über Erzählungen aufgebaut und perpetuiert wird. In „Generationenerzählungen“" werden das Verhältnis von Familie und Unternehmen sowie seine Kontinuität zum Thema eines intergenerationellen Deutungsprozesses (Lubinski 2010:130) und die Nachfolge zu einem symbolischen Akt. Diese historischen Erzählungen folgen meist der Form von Schöpfungsgeschichten. In der Regel gehen sie von der Person eines Gründers und den besonderen Umständen der Gründung aus, schreiben dann den einzelnen Generationen je spezifische Perioden zu und behandeln Firmensowie Familiengeschichte gleichermaßen (Lubinski 2010:139). Solche Generationenerzählungen sind Bezugspunkt für Identität, Individualität, ein spezifisches Werteset und Zugehörigkeit sowohl im Raum des Unternehmens (Unternehmenskultur, Marketing) als auch in der Gemeinschaft der Unternehmerfamilie (Selbstverständnis, Wir-Gefühl).

Lubinski beobachtet, dass das bürgerliche Arbeitsethos dabei tief in der Selbstdarstellung der Unternehmerfamilien verankert ist, und spricht deshalb von „erzählter Bürgerlichkeit“ (Lubinski 2010:140). Zentrale Werte, die immer wieder auftreten, sind unter anderem: Innovationsfähigkeit, Sparsamkeit, Arbeitseifer, Bildung, Familiensinn, öffentliches Engagement sowie komplexe Werte der Selbständigkeit (Lubinski 2010:140). Des Weiteren sind typische wirtschaftsbürgerliche Ideale wie ein patriarchalisches Senioritätsprinzip, der Erhalt des Erbes für künftige Generationen sowie eine klassische 
Rollenverteilung zwischen den Geschlechtern zentraler Bestandteil dieser Generationenerzählungen. Insofern kann Lubinski nur einen langsamen Wertewandel in diesen Familien feststellen (Lubinski 2010:286).

Im Lichte dieser historisch-kulturwissenschaftlichen Darstellung von Unternehmerfamilien des späten 20. Jahrhunderts scheint sich die Unternehmerfamilie der industriellen Revolution in eine narrative Fiktion zu verwandeln - von einer empathischen und wirtschaftlichen Solidargemeinschaft hin zu einer Erinnerungs- und Erzählgemeinschaft. Eine solche konstruktivistische Lesart steht allerdings in einem Spannungsverhältnis zu anhaltenden strukturellen Verschränkungen von Familie und Unternehmen, beispielsweise in Form der Tatsache, dass das Unternehmen nach wie vor wesentliche Grundlage der Vermögensbildung oder berufliche Wirkungsstätte ist.

\subsubsection{Enge und weite Fassung von Unternehmerfamilien}

Um die gesellschaftliche Bedeutung von Unternehmerfamilien noch einmal auf den Punkt zu bringen: Bis ins 19. Jahrhundert war die Verbindung eines bäuerlichen oder handwerklichen Betriebes und der Hausgemeinschaft die dominierende Lebensweise. Im Zuge der Industrialisierung entstehen neben einer Reihe von Familienformen, die nun nicht länger an einen Betrieb gebunden sind (die Arbeiter-, Angestellten- und Beamtenhaushalte) auch die industriellen Unternehmerfamilien, die in hohem Maße mit ökonomischer Macht ausgestattet sind und zu einem festen Bestandteil der wirtschaftsbürgerlichen Klasse werden. Im Laufe der zweiten Hälfte des 20. Jahrhunderts verändert sich die gesellschaftliche Bedeutung der Verbindung von Familie und Unternehmen erneut: Während Familienunternehmen immer noch die Mehrheit der Firmenpopulation stellen, werden Unternehmerfamilien zum Sonderfall. Diese Überlegungen geben Anstoß zu einer engeren und einer weiteren Konzeptionalisierung von Unternehmerfamilien. Einerseits könnte man behaupten, dass Unternehmerfamilien erst im Zuge der Industrialisierung und der Herausbildung einer kapitalistischen Gesellschaft entstanden sind - und diesen Prozess sogar gestaltend prägten. In dieser engen Lesart verbindet sich das gewinnorientierte Wirtschaftshandeln mit der Idealvorstellung einer bürgerlichen Familie. Insofern ist aber auch das Verständnis der „Unternehmerfamilie“ an spezifische historische Umstände gebunden, und es stellt sich die Frage, ob solche Familien in Zukunft noch eine Rolle spielen werden. Andererseits könnte man die Unternehmerfamilie als ein dynamisches Gebilde verstehen, das sich an die jeweiligen Wirtschaftsweisen und familialen Lebensformen anpassen kann. Als historisch stabil haben sich dann die Hausgemeinschaft (Bedarfsdeckung und Ganzes Haus) ebenso wie die industrielle Unternehmerfamilie (Erwerbswirtschaft und bürgerliche Familie) erwiesen. Angesichts der gesellschaftlichen Umwälzungen der letzten 
Jahrzehnte lässt sich aus dieser Perspektive überspitzt formulieren: Können wir eine neue Form der Verbindung von flexiblen Organisationen und postmodernen Familien beobachten? Welche Bedingungen gelten für diese Verbindung heute und was sind die neuen Koordinationsmechanismen und Konfliktfelder?

\subsection{Systemtheoretischer Zugang: Koevolution und Rollenkonflikt}

Ein wesentlich differenzierteres, aber abstraktes Bild von Unternehmerfamilien der Gegenwart zeichnen systemtheoretische Analysen. Sie liefern eine konzeptionelle Vorstellung von typischen Merkmalen, Handlungsregeln und Konfliktfeldern. Dieser theoretische Zugang ist insbesondere innerhalb der deutschsprachigen Familienunternehmensforschung weit verbreitet. Dabei schmücken Venn-Diagramme häufig die theoretischen Überlegungen zu Familienunternehmen. Ihre komplexe Struktur wird mittels zweier oder mehrerer sich überlappender Kreise verbildlicht, wobei die Kreise soziale Systeme symbolisieren, denen eigene Organisationsstrukturen, Mitgliedschaftsregeln und Reproduktionslogiken zugesprochen werden, die aber in dieser spezifischen Konstellation miteinander verbunden sind. In der schlichtesten Variante dieser Kreismodelle verschränken sich die Systeme „Familie“ und „Unternehmen“. Vielfache Verwendung findet auch das Modell der Wirtschaftswissenschaftler Renato Tagiuri und John Davis, die zusätzlich einen Kreis „Eigentum“ einführen. Darüber hinaus gibt es Diskussionen, ob der Unternehmenskreis weiter ergänzt oder differenziert werden sollte, beispielsweise um einen Kreis „Kontrollorgane“ wie etwa Beiräte (für einen Überblick siehe Klett 2005:40f. oder Wiechers 2006:17ff.). Kritische Stimmen geben jedoch $\mathrm{zu}$ bedenken, dass die beiden letztgenannten eher Spielfelder mit spezifischen Regeln darstellen als eigenständige soziale Systeme. Im Laufe der letzten Jahrzehnte hat sich das 2-Kreise-Modell als das robustere erwiesen, das in der aktuellen Forschung vermehrt Anwendung findet (Wiechers 2006:24).

Den Kern dieser Modelle bildet die Vorstellung eines wechselseitigen Einflusses von mindestens zwei Systemen, wobei das Aufeinandertreffen der unterschiedlichen Systemlogiken interne Widersprüchlichkeiten verursacht. Während in der wirtschaftswissenschaftlichen Forschung zu Familienunternehmen eher von offenen Systemen die Rede ist, ${ }^{27}$ wird in der sozialwissenschaftlichen Forschung eher im Luhmannschen Sinne von sich gegenseitig

27 Ein jüngeres Modell, das der Idee offener Systeme folgt, ist etwa „The Bulleye“ von Torsten Pieper und Sabine Klein 2007. 
beobachtenden, geschlossenen Systemen gesprochen (Wiechers 2006; Klett 2005; Simon 2002b). Typischerweise wird unter Familie ein soziales System verstanden, das sich aus ungehemmter Interaktion herstellt. In den seltensten Fällen können Familien ihre Mitglieder auswählen und ebenso wieder ausschließen. Das System, dem ein hoher Grad an Emotionalität unterstellt wird, muss keine spezifische Leistung erbringen und stellt insofern eine „Gegenwelt zur übrigen Gesellschaft" (Klett 2005:71) dar. Gegenstand der familiären Kommunikation sind zugeschriebene Eigenschaften der Personen, die sich im Laufe der Biografie und einer gemeinsamen Geschichte herausbilden (Klett 2005:72). Im Familiengedächtnis formt sich eine Erwartungskollage gegenüber jeder Person und bildet die Grundlage für Identität.

Das Unternehmen realisiert als Organisation innerhalb des Funktionssystems Wirtschaft komplexe Entscheidungszusammenhänge (Klett 2005:65). Diese sozialen Systeme, denen ein hoher Grad an Rationalität unterstellt wird, thematisieren Kompetenz und Qualifikationen. Die Mitgliedschaft erfolgt anhand spezifischer Kriterien (Selektion), kann jederzeit gekündigt werden, ist vertraglich festgehalten und wird kurzfristig materiell entlohnt. Simon fasst, wie schon Luhmann selbst, die beiden beziehungsstiftenden Medien der antagonistischen Systeme formelhaft in Liebe vs. Geld zusammen (Simon 2002b:13).

Die Vertreter eines systemtheoretischen Ansatzes argumentieren, dass in Familienunternehmen bzw. Unternehmerfamilien beide Systeme strukturell gekoppelt sind und sich co-evolutionär entwickeln (Simon et al. 2005:17). Das Verhältnis von Familie und Unternehmen ist weder vorab definiert noch beliebig (Wiechers 2006:164f.). Sie nehmen höchst selektiv und individuell aufeinander Bezug. Trotzdem scheint sich, folgt man Ralph Wiechers, eine gegenseitige Dienstbarkeit und Wahrnehmung als Ressource zu entwickeln (Wiechers 2006:168). Der Begriff der strukturellen Kopplung, den Fritz Simon, Rudolf Wimmer und Torsten Groth verwenden (2005:21), betont hingegen stärker die gegenseitige Abhängigkeit, die sich im Laufe der ungleichzeitigen Entwicklung herausbildet. Beide Systeme finden einander in ihrer Umwelt und nehmen in kommunikativer Weise Bezug aufeinander. Sie bezeichnen sich mit Verweis auf das jeweils andere System als Familienunternehmen (= das Unternehmen) und als Unternehmerfamilie (= die Familie) (Wiechers 2006:26). ${ }^{28}$ Erst das Unternehmen als Fremdreferenz für die Familie lässt diese zur Unternehmerfamilie werden (Wiechers 2006:158) - wir

28 Das Verständnis von Unternehmerfamilien in dieser Arbeit folgt dieser begrifflichen Differenzierung, die unter anderem von Ralph Wiechers 2006 vorgeschlagen wurde. Die Begriffe Unternehmerfamilie und Familienunternehmen werden also nicht synonym verwandt, sondern beziehen sich auf je unterschiedliche Sphären. Jedoch wird im Rahmen dieser Arbeit der Begriff Unternehmerfamilie statt dem von Wiechers genutzten Begriff der Unternehmensfamilie verwandt. Nähere Erläuterungen dazu finden sich in der Zwischenbetrachtung (Kapitel 4). 
haben es also mit einer kontextabhängigen Bezeichnung zu tun. Familienunternehmen und Unternehmerfamilien haben sich zu Einheiten ausdifferenziert, die nicht einfach gleichgesetzt werden können (Klett 2005:97).

Folgt man dieser Systemperspektive konsequent, so besteht die Besonderheit der Unternehmerfamilie darin, dass sie in ihrer familialen Kommunikation Bezug auf das Unternehmen nimmt und dieses damit Teil der Familienidentität ebenso wie der Persönlichkeit der einzelnen Mitglieder werden kann. Das Unternehmen wird innerhalb der Unternehmerfamilie zu einer sinn- und identitätsstiftenden Instanz. Jäkel-Wurzer (2010) folgt Hildenbrand (2002) in der Annahme, dass in den privaten Raum der Familie ein rationales System hineinragt. Die Unternehmerfamilie wird ,dauerhaft mit Impulsen einer spezifischen Handlungslogik konfrontiert“ (Jäkel-Wurzer 2010:27), die ein Mischungsverhältnis aus Privatheit und Öffentlichkeit entstehen lässt. Diesen Umstand bezeichnet Jäkel-Wurzer als, Öffnung'. Diese Öffnung der Unternehmerfamilie verursache nun veränderte Bedingungen für die Entfaltung von (unbegrenzter) Solidarität. Gleichzeitig besteht in Unternehmerfamilien ein Kontinuitätsanspruch, also ein Anspruch der Fortführung durch spätere Generationen, der eine Bindung der Familienmitglieder an das Unternehmen erfordert und der Kohärenz bedarf. Der entstehende Widerspruch macht es erforderlich, dass Unternehmer-familien ,,der Öffnung ,Kräfte“ entgegensetzen, welche die Nachkommen auch angesichts des Einwirkens einer rational motivierten Handlungslogik dauerhaft an die Gemeinschaft binden." (Jäkel-Wurzer 2010:27). Die Handlungs- und Orientierungsmuster der jeweiligen Systeme verlieren im Zuge ihrer wechselseitigen Durchdringung ihre verbindliche Funktion. Die Unternehmerfamilie ist gefordert, diese durch individuelle Referenzen zu ersetzen. Aus dieser Konstellation heraus ist die strukturelle Gestalt der Unternehmerfamilie anders als jene der bürgerlichen Familie.

Durch die Systemperspektive tritt der paradoxe und spannungsgeladene Charakter von Unternehmerfamilien zu Tage, denn die beiden Systemlogiken stehen sich nicht widerspruchsfrei gegenüber. Es gilt gleichzeitig Gewinne zu erwirtschaften, Leistung zu belohnen und flexibel auf dem Markt zu agieren und andererseits Einkommen zu sichern, das Gleichheitsprinzip zu wahren und Identität zu schaffen. David Klett (2005) führt aus, wie sich im Raum des Familienunternehmens Kompetenz und Herkunft gegenüber stehen. Bei Entscheidungen über Gehalt, Positionen oder Nachfolge gilt nicht mehr eine „reine“ personenindifferente Kompetenzorientierung, sondern Mitglieder der Unternehmerfamilien werden ,wie feste Adressen“ (Klett 2005:99) ins Kalkül einbezogen, und ein Herkunftsargument kommt zum Tragen (Klett 2005:106). Im Raum der Unternehmerfamilie können die unterschiedlichen Einflussmöglichkeiten der Mitglieder im Unternehmen (Selektion) Gegenstand des familiären Gleichheitsprinzips sein und die Familie unter Selektionsdruck geraten (Klett 2005:114). Simon, Wimmer und Groth definieren 
insgesamt sechs typische Paradoxien (2005:150ff.): (1) Die Familie ist gleichzeitig Ressource und Gefahr für das Unternehmen; (2) die Qualität der Entscheidungen bestimmen einerseits Emotionen, aber andererseits ökonomische Rationalität; (3) als Grundlage von Gerechtigkeit (z.B. im Transfer von Eigentum) zählt in der Familie das Gleichheitsprinzip, während im Unternehmen ungleich anhand von Kompetenzen und Hierarchien selektiert wird; (4) Familien als Eigentümer wollen Shareholder sein, ohne wie Investoren zu handeln; (5) die faktische Abhängigkeit des Unternehmens von seiner Umwelt widerspricht dem Ideal der Autonomie der Familie; (6) und schließlich steht der Identitätserhalt der Familie der Wandlungsfähigkeit des Unternehmens entgegen. Simon stellt fest, dass die Kommunikation unter den Familienmitgliedern konflikt- und paradoxieanfälliger ist als in anderen Familien, ,da keine allgemein akzeptierten Zeichen, Schemata oder Prozeduren verfügbar sind, die zweifelsfrei festlegen, welcher Kontext wann als relevant zu erachten ist." (Simon 2002a:42).

Aus der Akteursperspektive ergeben sich aus den jeweiligen Systemen bestimmte Rollenerwartungen und aus den identifizierten Paradoxien spezifische Rollenkonflikte. Die leichte Übersetzbarkeit auf eine Handlungsebene stellt eine weitere Stärke eines systemischen Zugangs zu Unternehmerfamilien dar. Anfang der 1980er Jahre leiten Tagiuri und Davis aus ihrem 3Kreise-Modell (Familie, Unternehmen, Eigentum) sieben unterschiedliche Rollentypen ab (siehe Wiechers 2006:19f.): (1) Ein Familienmitglied, das weder Anteile am Unternehmen hält noch im Unternehmen mitarbeitet; (2) ein Anteilseigner, der weder zur Familie gehört noch im Unternehmen arbeitet; (3) ein Mitarbeiter, der weder Teil des Unternehmens ist noch Anteile hält; (4) ein Familienmitglied, das am Unternehmen beteiligt ist, nicht jedoch in diesem arbeitet; (5) ein Mitarbeiter des Unternehmens, der am Unternehmen beteiligt ist, nicht jedoch zur Familie zählt; (6) ein Familienmitglied, das im Unternehmen arbeitet, jedoch nicht über Anteile verfügt; (7) und ein Familienmitglied, das sowohl Anteile des Unternehmens hält als auch im Unternehmen arbeitet.

Diese Rollenvielfalt hat gleichzeitig positive und negative Effekte auf das Unternehmen wie auf die Familie. Aus diesem Grund sprechen Tagiuri und Davis von „bivalent attributes“. Hohe familiale Identität, Loyalität und kurze Entscheidungswege werden in der Regel auf der Habenseite verbucht, während mangelnde Objektivität, Rivalitäten und Entscheidungsverzögerungen der Sollseite zugeschrieben werden (Tagiuri und Davis 1996:207). Ein rollentheoretischer Zugang wird in der Regel für die Analyse von Konflikten, Dilemmata und Zielkongruenzen herangezogen (Müller 2008; Wiechers 2006:20) und hat sich zu einem stabilen Erklärungsmuster für intrapsychische Probleme einzelner Akteure entwickelt (Wiechers 2006:18).

In der Management-Literatur dienen die Systemperspektive und ihr Blick auf Zielkonflikte und Paradoxien dazu, Risiken-Chancen-Analysen durchzu- 
führen und konkrete Handlungsempfehlungen darüber abzuleiten, wie die Überlebensfähigkeit der Familienunternehmen gesichert werden kann (z.B. Aronoff und Ward 1994; Simon 2002b; Ward 2004; Fleming 2000). Während in den Anfängen der Forschung zu Familienunternehmen sowie in der populären Ratgeberliteratur eine strikte Trennung beider Sphären als Patentrezept gehandelt wurde, dominiert heute die Einsicht, dass der gegenseitige Einfluss gerade das konstituierende Merkmal darstellt und nicht ausgeklammert werden kann (Tagiuri und Davis 1996:206; Wiechers 2006:160). Eine Unternehmerfamilie, die eine defensive Position bezieht und ihren Einfluss auf das Unternehmen minimiert, wäre nicht länger unternehmerisch. Stattdessen sollen mittels eines gezielten Managements die organisationale, familiale und juristische Eigendynamik zeitlich und sachlich gesteuert und aufeinander abgestimmt (Simon et al. 2005:19), also die Koevolution der Systeme gefördert werden (Simon et al. 2005:38). Dabei rückt die Fähigkeit, mit Paradoxien umzugehen, in den Mittelpunkt - eine Eigenschaft, die insbesondere mehrgenerationellen Großfamilien zugeschrieben wird. Die Familie dient als sinnstiftende Bezugsgröße mit Langfristcharakter, als Quelle einer von festen Werten getragenen Unternehmenskultur, als mögliches Reservoir begabter Unternehmer für das Topmanagement, als Reputationsquelle für die Pflege einer starken Marke, etc. Simon, Wimmer und Groth gehen davon aus, dass sich im Laufe der Zeit Mechanismen zur Beobachtung und Balancierung der Paradoxien institutionalisieren. Auch Kirsten Baus (2007) sieht die dynastischen Familien als Vorbild, in denen sich Strukturen institutionalisieren, die Stabilität schaffen und Handlungsregeln vorgeben und auf diese Weise die Steuerbarkeit des Unternehmens erhöhen. Die Empfehlung lautet, dass ein aktives „Grenzmanagement“ (Simon et al. 2005:157) bzw. „Familienmanagement" (Wiechers 2006) betrieben werden sollte, das eine Familienstrategie expliziert. Doch welche Konsequenzen hat diese Empfehlung auf die familiale Kommunikation, Loyalität, das Heiratsverhalten oder die Kinderzahl? Denkt man diese Vorgehensweise im Raum der Familie weiter, widerspricht sie den normativen Vorstellungen einer modernen Gesellschaft. Und genau darin besteht eine große Schwäche des gegenwärtigen systemischen Ansatzes, denn gezielt wird auf das „Überleben“ des Unternehmens statt auf die Reproduktion des wechselseitigen Einflusses. Es ist eine Argumentation eben aus der Perspektive des Familienunternehmens heraus und weit davon entfernt, ,beide“ Seiten in gleicher Weise in Betracht zu ziehen. Eine systematische Analyse der Familienseite, der Bedeutung des Einflusses eines Unternehmens auf die Unternehmerfamilie sowie hier entstehender Rollenkonflikte stellt daher eine relevante Forschungslücke dar.

Unter Berücksichtigung einer gesellschaftlichen Dimension müssen die beschriebenen inneren Konflikte einer Familienunternehmen-Unternehmerfamilie-Konstellation noch um Spannungen nach außen ergänzt werden. Hier binden sich zwei Systemtypen aneinander, welche sich im historischen Ver- 
lauf gesellschaftlich auseinander entwickelt haben. Die Unternehmerfamilie ist damit gefordert, sich neben einer Balance der inneren Paradoxien gegenüber einer differenten Außenwelt zu positionieren (Jäkel-Wurzer 2010:29). Aktuelle Forschung kommt darin überein, dass sich in Unternehmerfamilien weder die Vorstellung eines eigenständigen Lebensprogrammes voll entfalten (diesem steht der Kontinuitätsanspruch entgegen) (Jäkel-Wurzer 2010:24; Büttner 2007:9; Stamm et al. 2009a) noch eine funktionale Differenzierung voll zum Tragen kommen kann (Jäkel-Wurzer 2010:26; Stamm et al. 2009a). Ebenso steht die Unternehmerfamilie der These einer Isolierung der Kernfamilie entgegen: „Verbunden über die Verantwortung für einen gemeinsamen Besitz, muss diese der entstehenden Entfremdung innerhalb erweiterter verwandtschaftlicher Netzwerke entgegenwirken" (Jäkel-Wurzer 2010:28). Trotzdem kann sich auch die Wirkung des Ganzen Hauses nicht entfalten, da die Unternehmerfamilie mit den Ansprüchen an eine moderne Kernfamilie, wie etwa Affektivität, Intimität und Individualität, ebenso wie an eine moderne Unternehmensführung konfrontiert wird. Hildenbrand weist darauf hin, dass Unternehmerfamilien, die dennoch an der Tradition kompromisslos festhalten, besonders von psychosozialen Krisen bedroht sind (Hildenbrand 2002:121). Die Unternehmerfamilie sieht sich unter dem Druck, einerseits ihre Struktur zu erhalten, andererseits sich den Entwicklungen der Moderne zu stellen. Im Vergleich zum Modell der bürgerlichen Kernfamilie ist deshalb Unternehmerfamilien eine ,zwangsläufig konflikthafte Dynamik“ (Jäkel-Wurzer 2010:26) zueigen. Wie diese Ausführungen wiederum verdeutlichen, rangieren Unternehmerfamilien im Lichte soziologischer Modernisierungstheorien uneindeutig zwischen Tradition und Moderne, wobei die bestehende Forschung zu Unternehmerfamilien eher eine anachronistische Lesart impliziert. ${ }^{29}$

Aus dieser systemtheoretischen Perspektive lassen sich folgende Charakteristika von Unternehmerfamilien zusammenfassend festhalten:

29 Im Übrigen kann auch für den Typus einer modernen Kernfamilie, der sich im Laufe der Modernisierung von dem Modell des „male breadwinner“ löst, eine solche konflikthafte Dynamik in Anspruch genommen werden. 
- Wechselseitiger Einfluss von mindestens zwei Systemen

- Systeme sind strukturell gekoppelt und entwickeln sich koevolutionär

- Familie bezeichnet sich mit Bezug auf das Unternehmen als Unternehmerfamilie (kontextabhängige Bezeichnung)

- Aufeinandertreffen der Systemlogiken erzeugt interne Widersprüchlichkeiten

- Paradoxer und spannungsgeladener Charakter

- Fähigkeit, mit Paradoxien umzugehen, hat sich insbesondere in Großfamilien entwickelt

- Aus Akteursperspektive: Rollenkonflikte, intrapsychische Probleme

- Unternehmerfamilie muss sich gegenüber gesellschaftlichen Normvorstellungen positionieren $\rightarrow$ äußere Spannungen

- Langfristige Besonderheit zeigt sich in der Generationenfolge und Anteilsvererbung

Eine wesentliche Schwäche des Dreiklangs aus Kreismodellen, Systemgedanken und Rollenzuschreibungen ist darin zu sehen, dass sie eine implizite Bewertung vornehmen: Unternehmen und Familie sind sich gegenseitig eine Last: „mehr Anspruchsgruppen, mehr konkurrierende und sich widersprechende Anforderungen, mehr Dilemmata für die beteiligten Akteure" (Klett 2005:41). Der Fokus auf Konflikte und Probleme versperrt den Blick auf den Gesamtzusammenhang (Wiechers 2006:24) und mag dazu verleiten, die Akteure als Opfer einer bestimmten Struktur zu begreifen. Eva Maria Bäcker zeigt hingegen in ihrer Untersuchung zu Identitätsverständnissen von Familienunternehmern, dass der Grad an „Überlappung“ beider Systeme bzw. des wechselseitigen Einflusses auch eine Konstruktionsleistung der Beteiligten ist. Ob Unternehmen und Familie als getrennte Sphären oder als Einheit praktiziert werden, ist das Ergebnis aus einem Wechselspiel zwischen aktuellen Handlungen und institutionalisierten Strukturen. $\mathrm{Zu}$ einem ähnlichen Ergebnis kommen auch Sue Birley, Dennis NG und Andrew Godfrey (1999) mit ihrer Klassifikation von Einstellungen von Familienunternehmern als „Family Rules“, „Family Out“ oder „Family Business Jugglers“. denbrand weist darauf hin, dass Unternehmerfamilien zweifellos über effiziente Wege verfügen, diese Lebensform nicht nur erträglich, sondern sogar attraktiv machen (Hildenbrand 2002:144). Schließlich kann die systemische Perspektive auch nicht erklären, warum unter diesen Bedingungen eine Fortsetzung des Paradoxes überhaupt möglich ist. In Sachen Reproduktion und Kontinuität erreicht dieser theoretische Ansatz die Grenzen seiner Reichwei-

30 Auf beide Untersuchungen wird im Rahmen der Ergebnisdarstellung noch einmal näher eingegangen. 
te, was sich insbesondere in der Betrachtung des Nachfolgedilemmas aus einer Akteursperspektive zeigt (siehe hierzu auch Abschnitt 5). Es fehlt an einer systematischen Analyse der Selbstverständlichkeiten, der gelingenden Kommunikation und des Alltags in Unternehmerfamilien, um die Schwächen dieses theoretischen Zugangs auszubalancieren.

\subsection{Dimensionen des Unternehmenseinflusses auf die Familie}

Auf der Grundlage dieser systemtheoretischen Erwägungen soll im Folgenden ein Modell entwickelt werden, das Unternehmerfamilien als soziale Gebilde näher zu fassen sucht. Die bereits herausgearbeiteten Eigenschaften werden dabei um die bestehenden, aber fragmentarischen empirischen Forschungsergebnisse zu Unternehmerfamilien ergänzt, wobei ein besonderer Fokus auf das Alltags- und Beziehungsgeschehen gelegt wird. Den Schlüssel zu einer solchen Systematik bildet der schon oft beschworene wechselseitige Einfluss von Unternehmen und Familie, der das Feld der Familienunternehmensforschung begründet, sich wie dargestellt historisch gewandelt hat und den Ansatzpunkt theoretischer Erwägungen darstellt. Dabei wird dieser Einfluss so gefasst, dass er unterschiedlich stark und in verschiedenen Dimensionen anzutreffen ist. Ferner greift die folgende Systematik die systemtheoretische Trennung in Familienunternehmen und Unternehmerfamilien in zwei soziale Gebilde auf, die über den wechselseitigen Einfluss verbunden sind, aber nicht ineinander aufgehen. Eine solche konzeptuelle Differenzierung unterstreicht die Abhängigkeit beider als strukturelle Komplemente. Der Fokus auf Unternehmerfamilien soll perspektivisch als ein Gegengewicht zu der bestehenden Familienunternehmensforschung wirken und eine ganzheitliche Perspektive ermöglichen. ${ }^{31}$

Damit folgt dieses Vorhaben den Spuren der Forschergruppe um Kathryn Stafford, Ramona Heck und Mary Winter (1999; Heck et al. 2000, 2000; Heck et al. 2006) mit ihrem „Sustainable Family Business Model“, die mit ihrem systemtheoretischen Ansatz ebenfalls für eine gleichgewichtige Perspektive auf Familie und Unternehmen plädieren. ${ }^{32}$ Allerdings wird in den näheren Ausführungen zu diesem Modell lediglich die Unternehmensseite mit der Familie als sozialem Kontext behandelt. Der umgekehrte Fall, der im Rahmen dieser Arbeit im Mittelpunkt steht, wird mit einem kurzen ,vice ver-

31 Bis dahin kann man jedoch zu Recht kritisieren, dass der Fokus auf Unternehmerfamilien etwas einseitig ist und nur innerhalb eines Systems (nämlich der Familie) argumentiert.

32 Dieses Modell wurde von Olson et al. 2003 empirisch getestet. Eine ausführliche Erläuterung des Modells findet sich auch bei Heck et al. 2006. 
sa" abgehandelt - das ist einer der Gründe, warum nicht dieses Modell als theoretischer Ausgangspunkt gewählt wurde.

Ganz konkret wird im Folgenden in Anlehnung an die F-PEC Skala ein theoretisches Modell über die Dimensionen des Unternehmenseinflusses auf die Familie entwickelt. Insofern wird ein empirisches Messinstrument als theoretisches Gerüst zweckentfremdet. Diese Systematik ist rein konzeptioneller Natur und hat einen vorläufigen Charakter. Sie soll dazu dienen, bereits erforschte Aspekte neu zu ordnen, Forschungslücken aufzuzeigen und kann sich darüber hinaus als eine Art Kompass in der empirischen Arbeit als hilfreich erweisen. Gleichsam kann erst die Empirie diese Systematik validieren, insofern muss sie offen für Revision und Überarbeitung bleiben.

\subsubsection{Aufder Gegenfahrbahn}

Joe Astrachan, Sabine Klein und Kosmas Smyrnios stellen im Jahr 2002 eine Skala vor, mit der sich zukünftig Familienunternehmen im Rahmen quantitativer Forschung besser identifizieren und klassifizieren lassen sollen. Auf Basis einer ausführlichen Inhaltsanalyse zahlreicher Definitionen und konzeptueller Modelle zu Familienunternehmen sucht das Forscherteam die häufigen und relevanten Themen in drei Dimensionen des Einflusses einer Familien auf das Familienunternehmen zu fassen und legt empirische Messgrößen dafür fest. Mittels Macht, Erfahrung und Kultur kann ein Unternehmen nun mehr oder weniger stark von einer Familie beeinflusst werden. Mit ihrem Ansatz, familialen Einfluss zu messen, verabschiedet sich das Forscherteam von der alten Dichotomie zwischen Familien- und Nicht-Familienunternehmen und legt stattdessen ein kontinuierliches Verständnis zu Grunde (Astrachan et al. 2002:46; Chrisman et al. 2005:244; Colli 2003:26).

Auf diese Weise entsprechen sie dem Konzept der Familiarität (familiness), das zuerst von Habbershon und Williams (1999; 2003) ${ }^{33}$ eingeführt wurde. Seither findet die F-PEC-Skala als Messinstrument breite Anerkennung (z.B. Bird et al. 2002; Chrisman et al. 2005; Holt et al. 2007), wird empirisch getestet (beispielsweise Klein et al. 2005; Holt et al. 2007) und in zahlreichen Studien angewandt (etwa Craig und Moores 2005; Jaskiewicz et al. 2005; Jaskiewicz und Klein 2007). Sie gilt als ein zuverlässiges Instrument zur Bewertung des Ausmaßes und der Qualität von familiärem Einfluss in drei Dimensionen eines Unternehmens (Cliff und Jennings 2005; Holt et al. 2007; Klein et al. 2005:321 und 333).

Die Analyse von Astrachan, Klein und Smyrnios zeigt, dass sich die meisten Beschreibungen von Familienunternehmen auf ein spezifisches Mus-

33 Für weiterführende Literatur siehe etwa das Sonderheft des ET\&P im Mai 2005 mit einer Einleitung von Chrisman, Chua und Steier 2005: 237-247 oder Pearson et al. 2008. 
ter an Eigentum, Governance und Management beziehen (2002:48). Die Familie mag einen signifikanten Teil der Anteile am Unternehmen halten, Mitglied in den Kontrollgremien oder der Unternehmensführung sein. Das Forscherteam fasst dies als drei Kanäle zusammen, über welche die Familie „Macht“ auf das Unternehmen ausüben kann. Diese Klassifizierung kann auf eine lange Tradition innerhalb der Forschung zu Familienunternehmen zurückblicken und lässt Raum dafür, sowohl direkte als auch indirekte Formen des Machtanspruchs in Familienunternehmen einzubeziehen (Klein et al. 2005:34; Faccio und Lang 2002; Lester und Cannella, JR 2006; Barca und Becht 2001).

In der zweiten Dimension „Erfahrung“ rekurrieren Astrachan et al. auf das Wissen, das in Familienunternehmen über die Generationenfolge und durch aktive oder interessierte Familienmitglieder akkumuliert wird. In der Ausarbeitung dieser Kategorie verweist das Forscherteam auf eine Diskussion innerhalb der Familienunternehmensforschung (2002:49), die bis heute noch nicht abgeschlossen ist, nämlich die Frage, ab wann ein Familienunternehmen beginnt, ein solches zu sein: Bereits mit der Intention, ein Unternehmen an die nächste Generation zu übergeben (z.B. Chua et al. 1999; Heck et al. 2000; Birley 1986; Barach und Ganitsky 1995; Ward 1987) mit dem festen Plan der Übergabe (Gallo 1998; Flören 2002; Pfannenschwarz 2006:349) oder aber erst mit mindestens einer vollzogenen Übergabe (Rosenblatt et al. 1985; Ward und Aronoff 1996). Den drei Gruppen ist gemeinsam, dass sie sich auf einen spezifischen Zeitrahmen von Familienunternehmen beziehen (Zellweger 2007). Sie können als unterschiedliche Manifestationen eines Denkens entlang eines generationalen Kontinuums, das von einer volatilen zu einer faktischen Dimension reicht, verstanden werden. Die Autoren bemühen sich, keine klare Position zu beziehen und konzentrieren sich stattdessen darauf, was als allgemein akzeptiert gilt: Jede Nachfolge und jedes weitere involvierte Familienmitglied mehrt die Erfahrung eines Familienunternehmens (Astrachan et al. 2002:49; Klein et al. 2005:325). ${ }^{34}$

Die dritte Kategorie der F-PEC-Skala bezieht sich auf die Besonderheiten der Unternehmenskultur in Familienunternehmen, die in der Literatur meist als diffuse und heterogene Beschreibungen der so genannten "Soft Factors“ auftauchen. Die „Finnish Family Entrepreneurship Working Group” beschreibt etwa den sozialen Charakter von Familienunternehmen wie folgt: Die Eigentümerfamilie verleiht dem Unternehmen und seinen Produkten eine persönliche Note (Finish Ministry of Industry and Trade 2006:31); auf der Management-Ebene äußert sich dies in informellen Entscheidungs- und Organisationsstrukturen, Flexibilität und langfristiger Planung; auf einer per-

34 An dieser Stelle seien zwei Punkte kritisch angemerkt: Zum einem implizieren die Autoren mit ihrer Operationalisierung, dass ein Unternehmen über die Generationenfolge "familialer" wird, zum anderen wirkt das unterliegende Verständnis von Wissen akkumulativ. 
sönlichen Ebene in einem höheren Verpflichtungsgefühl, bestimmten unternehmerischen und familialen Werten und einem ausgeprägten Gemeinschaftssinn (Finish Ministry of Industry and Trade 2006:31f.). Häufig avanciert der Familienunternehmer als moderne, verantwortungsbewusste Führungspersönlichkeit zu einem gatekeeper dieser Kultur. Astrachan et al. nehmen diese Elemente einer Unternehmenskultur, verstanden als eine spezifische Sammlung von Werten und Normen, die innerhalb der Organisation geteilt werden, als weitere Dimension des familialen Einflusses auf (Astrachan et al. 2002:50; Klein et al. 2005:325). Sie messen dabei die Überlappung von familialen und unternehmerischen Werten (Astrachan et al. 2002:50) ebenso wie das Engagement der Familienmitglieder. ${ }^{35}$

Nimmt man nun die Vorstellung eines wechselseitigen Einflusses ernst (wie etwa von Stafford et al. 1999; Heck et al. 2006 gefordert), wirkt das Unternehmen in den gleichen Dimensionen, nur mit umgekehrten Vorzeichen, auf die Familie ein und konstituiert diese als Unternehmerfamilie. Grob gesagt: Das Unternehmen wirkt in der Machtdimension als zentrale Einkommensquelle (aus Arbeit oder Vermögen) ebenso wie als Option im Lebenslauf der Familienmitglieder. Die verschiedenen Ämter, die Familienmitglieder im Unternehmen innehaben, verschaffen ihnen eine Reihe von Privilegien, aber auch Verpflichtungen. In der zweiten Dimension, die ich als „Generation" bezeichnen möchte, wirkt das Unternehmen als eine Generationen überspannende Klammer. Es ist ein gemeinsames Projekt, das in der Familie weitergegeben wird und $\mathrm{zu}$ institutionalisierten Handlungsregeln führen kann. Ebenso formt das Unternehmen in der Unternehmerfamilie eine spezifische, dynastische Kultur mit einem ausgeprägten Gemeinschaftssinn.

Anhand dieser Grundidee sollen nun empirische Forschungsergebnisse zu Unternehmerfamilien sortiert werden. Dabei wird ein wesentlicher Vorteil der F-PEC-Skala auf die Analyse von Unternehmerfamilien übertragen: diese können nun mehr oder weniger stark von einem Unternehmen beeinflusst sein.

35 Cliff und Jennings 2005 kritisieren die empirische Validität der Operationalisierung der kulturellen Dimension. 
Abbildung 2: Das UFFU-Modell des wechselseitigen Einflusses

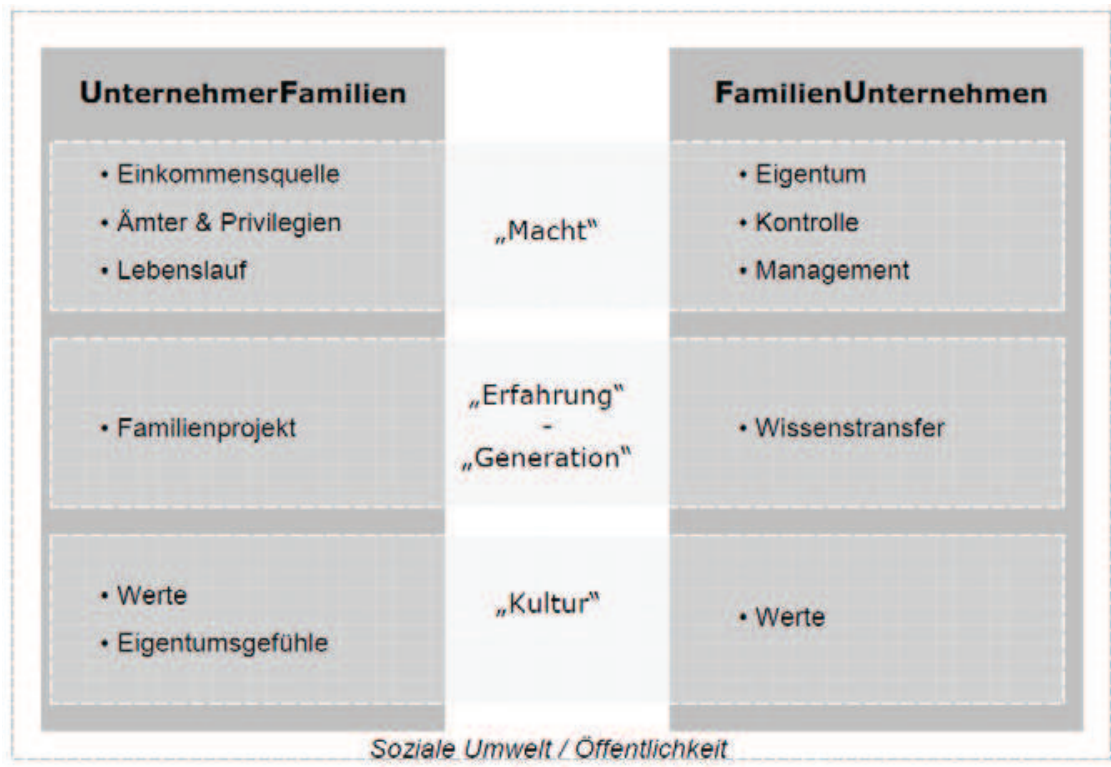

Quelle: eigene Darstellung

\subsubsection{Macht}

Die drei von Astrachan et al. identifizierten Wege, wie die Familie das Familienunternehmen in der Macht-Dimension beeinflusst, schlagen sich ebenfalls auf der Familienseite nieder. Management, Kontrolle und Eigentum stellen unterschiedliche Einkommensquellen für die Unternehmerfamilie dar und legen ihren Mitgliedern Verpflichtungen und Verantwortung auf. Das Unternehmen beeinflusst den Lebenslauf einzelner Familienmitglieder ebenso wie ihre Beziehung zueinander.

Der Besitz von Unternehmensanteilen, ausgeschüttete Dividenden, Gewinne, Privatentnahmen oder die Gehälter von angestellten Familienmitgliedern tragen in unterschiedlichem Ausmaß zum Haushaltseinkommen bei und binden damit die Unternehmerfamilie finanziell an das Unternehmen (Gantzel 1962; Pieper 2007; Kormann 2011). Martin Erhardt (2001) zeigt in seiner steuerlichen Analyse der Nachfolge in Familienunternehmen ausführlich, dass das Unternehmen die materielle Basis für den Wohlstand und das Einkommen der Unternehmerfamilie und für die private Vorsorge für Lebens- 
laufrisiken von abhängigen Familienmitgliedern bildet. Familienunternehmer nehmen als Selbständige im deutschen Sozialversicherungssystem einen Sonderstatus ${ }^{36}$ ein: Die Absicherung der „Erwerbsrisiken“ und des Ruhestandes obliegt ihrer individuellen Wahl, und der Gestaltungsraum ist breit: freiwillige Beiträge zu den gesetzlichen Sozialversicherungen, private Vorsorge, Aufbrauchen von stillen Reserven, Verkauf von Unternehmensanteilen etc. (Stamm et al. 2009b). Damit „dürfen“ oder „müssen“ sie ihre privaten Risiken hinsichtlich Krankheit, Berufsunfähigkeit und Rente zunächst privat absichern oder freiwillig in die gesetzlichen Systeme einzahlen. Insbesondere bei kleineren Unternehmen wird das Unternehmen selbst zum Bestandteil dieser Risikovorsorge in Form von Verkaufserlösen, Abfindungen, Raten, Renten, dauernden Lasten, sonstigen wiederkehrenden Zahlungen oder Bezügen aus einer Stiftung. Mit der Vorsorge für den Ruhestand von Familienunternehmern haben sich Ueli Kieser (2011) für die Schweiz und Andreas Wiedemann sowie Bertram Layer (2011) für Deutschland näher befasst. Insgesamt haben die finanziellen Grundlagen von Unternehmerfamilien bislang jedoch nur sehr wenig Beachtung gefunden.

Die unterschiedlichen Positionen, die Familienmitglieder im Unternehmen innehaben, erlegen ihnen Verpflichtungen und Verantwortung auf. Als Anteilseigner, Mitglied des Familienbeirates oder des Aufsichtsrates müssen Unternehmerfamilien rechtliche und normative Erwartungen der Fürsorge für das Unternehmen und seine Angestellten erfüllen. Gesetze, Verhaltenscodes und Unternehmensethik fordern von der Unternehmerfamilie Solidarität gegenüber dem Unternehmen ein.

Schließlich durchdringt und formt das Familienunternehmen die Lebensläufe von einigen oder allen Familienmitgliedern sowie ihre Beziehung zueinander. Es sind bereits einige Versuche unternommen worden, Erkenntnisse aus der Entwicklungspsychologie ebenso wie einer Soziologie des Lebenslaufs auf Unternehmerfamilien und ihre Mitglieder zu übertragen. Die Forschungsergebnisse illustrieren, welchen Effekt das Unternehmen auf die Unternehmerfamilie in unterschiedlichen Lebensphasen hat. Dabei lassen sich drei markante Punkte ausmachen, denen besonders viel Interesse entgegengebracht wurde. Hinsichtlich Kindheit und Erziehung in Unternehmerfamilien wird betont, wie Kindern bewusst wird, dass sie aus einer Familie stammen, deren Vorfahren ein Unternehmen aufgebaut haben, wie sie beobachten, auf welche Weise ihre Eltern mit dem Unternehmen umgehen, und schließlich, wie dieses Verhalten der Eltern die Kinder prägt (Davis und Tagiuri 1989; Erdmann 1999; Lansberg 1999; Siefer 1996; Churchill und Hatten 1987). Als zweites großes Forschungsthema findet sich die Frage, warum sich die jüngere Generation für einen Eintritt in das Familienunternehmen entscheidet. Stavrou und Swiercz (1998) finden heraus, dass insbeson-

Siehe hierzu auch Fußnote 16. 
dere die aktuelle Marktsituation des Unternehmens und die Möglichkeiten zur Emanzipation von der Familie eine zentrale Rolle spielen. Hinsichtlich der Suche nach einem potentiellen Nachfolger stellt die Forschung einen starken Gender-Effekt fest und beschreibt, wie Töchter als mögliche Kandidaten übersehen oder anders behandelt werden (Jäkel-Wurzer 2010; Haubl und Daser 2006; Rosenblatt et al. 1985; Hamilton 2006; Keese 2002; Vera und Dean 2005). Dunn (1999) und Wenk (2007) betonen den Eintritt des Nachfolgers in das Familienunternehmen als eine Statuspassage im Lebenslauf, die biografische Arbeit erfordert. Schließlich haben sich einige Studien mit dem Abschied des Seniors (a ,Hero's Farewell') beschäftigt und verschiedene Arten des Rücktritts identifiziert (Lansberg 1999; Sonnenfeld 1988). In diesem Zusammenhang wird immer wieder die Schwierigkeit des sich Lösens vom Familienunternehmen betont (z.B. Ibrahim et al. 2001; Bernhard und Jaskiewicz 2011; Levinson und Wofford 2000; Baumgartner 2009). ${ }^{37}$ All diese Studien sehen eine Notwendigkeit zur Synchronisation der Lebensläufe der Familienmitglieder - besonders derer von Nachfolger und Übergeber -, damit ein Generationswechsel gelingen kann. Andere Lebensphasen, wie etwa die Partnerwahl oder Familienplanung, das Geschwisterverhältnis oder die Arbeit im Familienunternehmen jenseits eines anstehenden Generationenwechsels sind weit weniger gut erforscht (Büttner 2007). Ebenso fehlt es an einem ganzheitlichen Bild. Phyllis Moen (1998) hat einen lebenslauftheoretischen Zugang zu Unternehmerfamilien vorgestellt, der dafür als Richtschnur dienen könnte. Sie schlägt vor, einen Fokus auf das Timing, den Prozess und den Kontext der Leben von Unternehmerfamilien zu legen (siehe Kapitel IV). ${ }^{38}$

\subsubsection{Generation}

In Analogie zum „Denken in Generationen“ in Familienunternehmen könnte man sich fragen, inwiefern das Unternehmen die generationale Zeit in Unternehmerfamilien beeinflusst. Daniela Jäkel-Wurzer (2010) hat einen Kontinuitätsanspruch in Unternehmerfamilien herausgearbeitet (siehe hierzu Abschnitt 3), der die Verbundenheit der Generationen nicht nur über die Abstammungslinie und Heirat, sondern auch über ein gemeinsames Projekt betont. In der Terminologie von Ivan Lansberg (1999) sind es diese „Shared

37 Kim und DeVaney 2003 stellen in ihrer Untersuchung eine relativ hohe Zahl an Familienunternehmern fest, die sich ein Altersteilzeitmodell vorstellten können. Vor dem Hintergrund einer hohen emotionalen Verbundenheit mit dem Unternehmen scheint der Wunsch nach einem graduellen Ausstieg plausibel.

38 In dem Konzept der Führungsnachfolge, das von Dyck et al. 2002 vorgelegt wurde, wird ebenfalls in ähnlicher Weise eine Differenzierung in Timing, Technik und Kommunikation vorgeschlagen. 
Dreams", die in Unternehmerfamilien den Takt angeben. Miller und LeBreton Miller (2005) suchen diesen zeitlichen Aspekt ebenfalls unter dem Begriff „Kontinuität“ zu fassen und unterscheiden dabei in eine Vergangenheits- und eine Zukunftskomponente (Miller und Le Breton-Miller 2005:37f.). Ihre Ergebnisse lassen sich auf Unternehmerfamilien übertragen:

Das Wissen über die Familiengeschichte, die um das Unternehmen kreist, ist Teil des Familienlebens. Von Generation zu Generation wird das Familienunternehmen zum Thema von Erzählungen und Familienmythen, die zu einem Traditionsbewusstsein beitragen und die junge Generation mit den Leistungen der Vorfahren und Eltern konfrontieren. Mit der Zeit institutionalisieren sich spezifische Arten und Weisen, wie über das Unternehmen gesprochen und mit diesem umgegangen wird. In Unternehmerfamilien mag es deshalb ein großes Geschichtsbewusstsein ebenso wie eine ausgeprägte dynastische Perspektive auf die Familientradition geben (Klein 2004:68). Durch die Firma mögen die vergangenen Generationen zu einem Bestandteil persönlicher Identität werden und so den individuellen Lebenslauf transzendieren (Schäfer 2007:226). Dies enthält Anklänge an die traditionellen Bauernfamilien, in denen der Eigentümer weniger als Person denn als Repräsentant seiner Abstammungslinie aufgefasst wird.

Die Zukunftskomponente spiegelt sich in dem ausgesprochenen Wunsch wider (siehe hierzu auch Abschnitt 2.4), dass die Kinder oder andere Verwandte das Familienprojekt fortführen mögen. Zentrales Element einer solchen dynastischen Denkweise in Unternehmerfamilien (Lambrecht 2005; Schäfer 2007:225) ist die Vorstellung vom Unternehmen als Garant für Wohlstand oder Arbeitsplatzsicherheit für kommende Generationen.

Innerhalb der Forschungsliteratur scheint Einigkeit darüber zu bestehen, dass sich beide Komponenten (also die Retrospektive ebenso wie die Prospektive) im Laufe der Zeit intensivieren und sich in Form von Handlungsregeln institutionalisieren. Die verschiedenen Entwicklungsstadien von Unternehmerfamilien werden häufig in einem Drei-Stufen-Modell beschrieben. Dabei findet insbesondere das Modell, das von Gersick et al. (1997) entwickelt und von Lansberg (1999) aufgegriffen wurde, breite Verwendung. In der Stufe des „Controlling Owner“ liegen Eigentum und Leitung in den Händen des Unternehmers. Dieser gestaltet nicht nur maßgeblich die Geschicke des Unternehmens, sondern ist auch innerhalb seiner Kernfamilie sehr präsent und dominiert den Nachfolgeprozess (Lansberg 1999:31). In der zweiten Stufe, dem „Sibling Partnership“ hält ein Geschwisterteam mehr oder weniger gleichmäßig Anteile am Unternehmen und wirkt auf die Unternehmensführung ein. Ist das Eigentum hingegen über ein weit verzweigtes Verwandtschaftsnetz gestreut, so handelt es sich um die dritte Stufe, das „Cousin Consortium". Hier hat sich eine komplexe Unternehmensstruktur herausgebildet, die sich über verschiedene Stämme erstreckt und der Familie einen dynastischen Charakter verleiht. Jaffe und Lane (2004) schlagen eine ähnliche Klas- 
sifizierung vor, nur unter anderem Namen. Sie betonen, dass in jeder dieser Familienformen eine unterschiedliche Strategie im Umgang mit dem Unternehmen praktiziert wird. Auch das Modell der Forschergruppe um Fritz B. Simon ebenso wie die Typologie von Ralph Wiechers sehen die gleichen Abstufungen vor, sie versuchen jedoch, stärker an übliche Bezeichnungen in der Familienforschung anzuschließen.

Abbildung 3: Übersicht der Entwicklungskonzepte

\begin{tabular}{|c|c||c|c|}
\hline & $\begin{array}{c}\text { Gersick et al. 1997 } \\
\text { Lansberg 1999 }\end{array}$ & Jaffe, Lane 2004 & $\begin{array}{c}\text { Simon et al 2005 } \\
\text { Wiechers 2006 }\end{array}$ \\
\hline \hline $\begin{array}{c}\text { Eigentum und Kontrolle } \\
\text { liegen in der Hand ei- } \\
\text { ner Person, oft des } \\
\text { Gründers selbst. }\end{array}$ & $\begin{array}{c}\text { Controlling } \\
\text { Owner }\end{array}$ & Entrepreneurship & Kleinfamilie \\
\hline $\begin{array}{c}\text { Eigentum und Leitung } \\
\text { teilt sich zwischen Ge- } \\
\text { schwistern. }\end{array}$ & $\begin{array}{c}\text { Sibling } \\
\text { Partnership }\end{array}$ & Musiness & Großfamilie \\
\hline $\begin{array}{c}\text { Eigentum (und Leitung) } \\
\text { liegt in einem weit ver- } \\
\text { zweigten Verwandt- } \\
\text { schaftsnetz. }\end{array}$ & Consortium & Business & Dynasty \\
& & & Mehrstämmige - dy- \\
nastische & \\
\hline
\end{tabular}

Quelle: eigene Darstellung

Die einzelnen Stufen beschreiben zunächst einen statischen Zustand. Die Unternehmerfamilie wird anhand von zwei Kriterien, die eigentlich unabhängig von der Verwandtschaftsstruktur sind - nämlich Eigentum und Leitung -, charakterisiert. Im Vordergrund steht dabei, welcher Teil der Familie faktisch in das Unternehmen involviert ist (Größe des Personenkreises). Vernachlässigt werden hingegen Verbindungen jenseits eines Arbeits- oder Besitzverhältnisses wie etwa emotionale Verstrickungen mit dem Unternehmen.

Gleichsam impliziert die Reihenfolge der Stufen eine genealogische Entwicklung. Ausgehend von einer Ursprungsfamilie (Kernfamilie) verändert sich über die Generationenfolge die Struktur der Unternehmerfamilie 
und damit die Art und Weise, wie das Unternehmen auf die Familie einwirkt. Mit der Zeit institutionalisieren sich Regeln, wie dieser Einfluss zugelassen und gestaltet wird. So zeigt beispielsweise eine von Sonfield und Lussier (2004) durchgeführte Befragung von knapp 150 Familienunternehmen im Raum New York und Massachusetts, dass beim Übergang von der ersten auf die zweite Generation weniger Nachfolgeplanung vorgenommen wird als bei späteren Generationswechseln.

Entsprechend fordert Lansberg (1999), dass nicht nur in unterschiedliche Familientypen, sondern auch in Formen der Nachfolge differenziert werden sollte. Er schlägt vor, immer dann von „recycled transitions“ zu sprechen, wenn sich eine bestehende Leitungs- und Eigentumsstruktur wiederholt, also beispielsweise ein Controlling-Owner das Unternehmen an ein Kind übergibt und damit lediglich ein Protagonistenwechsel erfolgt. Eine Nachfolge im Sinne eines ,evolutionary process“ bringt eine fundamentale Veränderung in der Autoritäts- und Kontrollstruktur des Systems in Form zunehmender Komplexität mit sich (etwa von einem Geschwisterteam hin zu einer Führung durch Cousins), die gegenteilige Variante, also eine ,,devolutionary succession", besteht in der Reduktion von Komplexität. Ein Generationswechsel dieser beiden letzten Typen trägt zu Unsicherheit und Ambiguität bei und bedarf neuer Aushandlungsprozesse und Handlungsregeln. Bei einer RecyclingVariante können die Nachfolger hingegen auf das Erfahrungswissen der Vorgänger zurückgreifen. Diese Stufenmodelle vermitteln den Eindruck, dass es insbesondere von den Strategien der Anteilsvererbung und Führungsteilhabe am Unternehmen abhängt, welche Form der Unternehmerfamilie sich langfristig herausbildet.

Diese Vorstellung einer evolutionären Entwicklung der Unternehmerfamilie wird nicht selten deterministisch gefasst und verbündet sich mit einem Narrativ des Scheiterns. Der Buddenbrooks-Effekt, das Gesetz der 3. Generation oder die survival rate legen es nahe, dass die Unternehmerfamilie die drei Stadien durchläuft, um dann spätestens in der dritten Generation zu zerbrechen. Während der wissenschaftliche Beweis dieser Entwicklungslogik auf mehr als wackeligen Beinen steht (siehe hierzu Stamm und Lubinski 2011), scheint es angebracht, für eine Erklärung der jeweiligen Form einer Unternehmerfamilie auch den gesellschaftlichen Kontext einzubeziehen. Das vorherrschende Wirtschafts- und Rechtssystem haben wie das Wohlfahrtsund Familienregime ebenfalls Auswirkungen auf die Struktur einer Unternehmerfamilie sowie ihre Generationenfolge (Stamm et al. 2011).

Zusammenfassend lässt sich behaupten, dass in Unternehmerfamilien sowohl auf horizontaler als auch auf vertikaler Ebene die Generationen zusätzlich über ein gemeinsames Projekt verbunden werden. Das Unternehmen wird zu einem identitätsstiftenden Element dieser Gemeinschaft und beeinflusst, was als „Grenze“ der Familie wahrgenommen wird. Über die Generationenfolge kann sich die jeweilige Form der Unternehmerfamilie verändern, 
in jedem Fall scheinen sich jedoch Geschichtsbewusstsein und Erwartungen an künftige Generationen zu intensivieren. Insofern wird das Unternehmen ein konstitutives Element in der Wahrnehmung von Zeit in Unternehmerfamilien, das Lebenslaufentscheidungen und -orientierungen beeinflusst. Empirische und theoretische Arbeiten zu diesem temporalen Aspekt liegen jedoch nur vereinzelt vor.

\subsubsection{Kultur}

Die dritte Dimension des Einflusses eines Unternehmens auf die Unternehmerfamilie fasst die kulturellen Aspekte. Anknüpfend an die Idee, dass Unternehmern ein spezifisches Werteset und damit verbunden ein eigener Lebensstil zu eigen ist, könnte man in der wachsenden Literatur zu Unternehmensethik und der sozialen Verantwortung von Unternehmen Beiträge zu einer Kultur der Unternehmerfamilie vermuten. Doch diese Verbindung scheint eine Leerstelle in der aktuellen Forschung zu bilden. Stafford et al. (1999) integrieren zwar in das Sustainable Family Business Model (SFBM) den Hinweis, dass jedes Familienunternehmen eine soziale Verantwortung innerhalb seiner jeweiligen Gemeinde trage (2006:93), aber Aussagen über die Wahrnehmung einer solchen Verantwortung von Seiten der Familie sowie zu anderen spezifischen Normen von Unternehmerfamilien bleiben aus.

Innerhalb der Forschung zu Familienunternehmen steht die These im Raum, dass nur ein positives Familienklima zuträglich für den Unternehmenserfolg sei. Wie sich dieses Klima allerdings gestaltet, bleibt offen. Åsa Björnberg und Nigel Nicholson haben im Jahr 2007 diese These zum Gegenstand einer quantitativen Untersuchung gemacht. Mittels der „Familienklimaskala", die in die Dimensionen Familienkommunikation, Zusammenhalt und intergenerationale Beziehungen unterteilt ist, untersuchen sie Unternehmerfamilien und Nicht-Unternehmerfamilien. Sie kommen zu dem Schluss, dass der Einfluss eines Unternehmens keinen signifikanten Effekt auf das Familienklima hat (Björnberg und Nicholson 2007:239). Die einzige Besonderheit ist darin zu erkennen, dass die Autorität älterer Generationen in Unternehmerfamilien ausgeprägter erscheint (2007:240). Auch Barbara Dunn (1999) betont in ihrer qualitativen Untersuchung von Unternehmerfamilien in Schottland die Besonderheit der unterschiedlichen Familien, die ihre je eigene Geschichte - sowohl in einem historischen als auch in einem narrativen Sinne - von der komplexen Verbindung von Familie und Unternehmen aufweisen, weshalb keine einheitliche Kultur, Wertesysteme oder Regeln in solchen Familien ausfindig gemacht werden können (Dunn 1999:46).

Gleichzeitig erkennt Dunn spezifische Muster einer emotionalen Dynamik in Unternehmerfamilien. Sie seien gefordert, die Entwicklung von familialen und unternehmerischen Projekten in Einklang zu bringen (Dunn 
1999:49). Dies mache auf der Ebene des Individuums wie auch im Umgang der Generationen miteinander eine emotionale Auseinandersetzung erforderlich. Vorhandene Ängste etwa vor dem Übergang in den Ruhestand können zu spezifischen Handlungsweisen führen, die sich über die Generationenfolge zu Regeln verdichten und auf diese Weise konservieren. Dunn schlussfolgert zudem, dass sich die Verbindung von Familie und Unternehmen ganz besonders während Statuspassagen im Familienzyklus bekräftigt (Dunn 1999:51). In diesem Zusammenhang scheinen zwei Konzepte erwähnenswert, zum einen die Theorie des psychologischen Eigentums (a), zum anderen eine Klassifizierung praktizierter Grenzarbeit (b):

$\mathrm{Zu}$ (a): Familienmitglieder empfinden, dass sie Mühe, Zeit und psychologische Energie in das Unternehmen investiert haben und deshalb ein intimes Wissen über dieses besitzen. Dieser Umstand führt zu einem gefühlten Eigentumsverständnis, das jenseits von rechtlichen und wirtschaftlichen Grenzen operiert (Lubinski 2009; Pierce et al. 2001, 2003; Zellweger und Astrachan 2008; Bernhard und Jaskiewicz 2011). Ein solches ,psychologisches Eigentum“ mag zu einem höheren Engagement von Familienmitgliedern im Unternehmen führen, zu persönlicher Aufopferungsbereitschaft, der Neigung, Risiko und Verantwortung auf sich zu nehmen, ebenso wie dem Widerwillen, das Unternehmen zu teilen oder zu Ambivalenzen im Umgang mit Veränderung. Dieses Konzept zielt darauf, die Grundlage einer spezifischen Kultur in Unternehmerfamilien zu erklären.

$\mathrm{Zu}$ (b): Sue Birley und ihre Kollegen (Birley et al. 1999; Birley 2001) haben in ihren Studien zu Einstellungen in Unternehmerfamilien gegenüber dem Unternehmen drei markante, oben bereits kurz angedeutete Muster identifiziert: Die Gruppe „Family-Rules“ unterstützt eine frühe Einbindung der Kinder in das Unternehmen, erkennt die Verantwortung der Familie gegenüber dem Unternehmen an und befürwortet lange Amtszeiten von Familienmitgliedern in zentralen Unternehmensfunktionen. Im Gegensatz dazu versucht die Gruppe „Family-Out“, die beiden Sphären von Familie und Unternehmen getrennt zu halten. Kinder beispielsweise sollten keine Anteile in jungen Jahren erhalten, und Familienmitglieder sollten keine finanziellen Privilegien aus dem Unternehmen beziehen. Die letzte Gruppe der „FamilyBusiness-Jugglers" versucht, eine Balance zwischen beiden Bereichen zu finden - eine spezifische Variante des allgemein bekannten Problems der Work-Life-Balance. Diese Differenzierung lässt vermuten, dass sich mehr als nur eine typische Kultur in Unternehmerfamilien finden lässt.

Eine intensive kulturwissenschaftliche Bearbeitung dieses Themenkomplexes oder ein ethnographischer Zugang zu Unternehmerfamilien würden das Wissen um diese Familien eigener Art fundieren. Ebenso scheint eine Rückkopplung zu den verschiedenen Stadien von Unternehmerfamilien gewinnbringend. Zentrale Fragen bleiben derzeit unbeantwortet: Was sind typische Werte und Normen in solchen Familien? Besteht eine Unternehmerkul- 
tur, was macht diese aus und wie gelingt es, den Unternehmergeist über Generationen hinweg zu pflegen?

\subsection{Generationswechsel aus Familienperspektive}

Das vorgestellte UFFU-Modell, das den wechselseitigen Einfluss von Familie und Unternehmen in den Mittelpunkt rückt, erlaubt es auch, die Literatur zur Nachfolge, dem seit gut zwanzig Jahren meist beforschten Themenkomplex innerhalb der Literatur zu Familienunternehmen, ${ }^{39}$ neu zu ordnen und um eine Familienperspektive zu ergänzen.

Die Familienunternehmensforschung stellt die Regelung der Nachfolge vor allem als ein komplexes Managementproblem in einer sich stetig wandelnden Umwelt dar (siehe etwa Aronoff 1998; Chittoor und Das 2007; Dunemann und Barrett 2004; Le Breton-Miller et al. 2004). Es herrscht große Einigkeit darüber, dass die Nachfolge in Familienunternehmen ein Geflecht von simultanen und zum Teil konträr ablaufenden Prozessen sei. ${ }^{40}$ Lambrecht spricht sogar von Nachfolge als einem „lebenslangen“ Prozess (Lambrecht 2005:267). Der Übergang erfolge zwischen zwei Gruppen - den Übergebern und den Übernehmern - wobei die beiden Gruppen spezifische Aufgaben und Rollen, die im Laufe der Nachfolge wechselseitig aufeinander Bezug nehmen, erfüllen. Wendy Handler führt dafür den Begriff des „mutual role adjustment" ein (Handler 1990:43). Diese Gruppen stehen meist in einem verwandtschaftlichen Verhältnis zueinander und gehören unterschiedlichen Generationen an - denn sonst endet der familiale Einfluss auf das Unternehmen rasch. Deshalb scheint Aronoffs (1998) Vorschlag - zumindest in den meisten Fällen - von „Generationswechsel“ statt von „Unternehmensnachfolge" zu sprechen, attraktiv. Übertragen wird die "Macht" über das Familienunternehmen, es erfolgen also ein Übergang in der Unternehmensleitung bzw. Unternehmenskontrolle sowie ein Transfer von Unternehmensanteilen. Eingebettet sei dieser Prozess in ein komplexes Wechselspiel mit den anderen Dimensionen der Erfahrung und Kultur.

Im Vordergrund der meisten theoretischen und empirischen Untersuchungen der Nachfolge steht der kontinuierliche Erfolg des Familienunter-

39 Dies stellen unter anderem die Review-Artikel von Bird et al. 2002:341 und Zahra und Sharma 2004:334 fest. Für einen Überblick der Literatur siehe beispielsweise Wortman 1994; Klett 2005:34f.; Casillas und Acedo 2007; Dyer, JR und Sànchez 1998 oder Sharma 2004.

40 Siehe etwa die Definitionen von Dumas et al. 1995; Rogers et al. 1996; Seeghitz 2000; Erhardt 2001; Ibrahim et al. 2001; Sharma et al. 2001; Flören 2002; Sharma et al. 2003; Dunemann und Barrett 2004 oder Chittoor und Das 2007. 
nehmens ganz im Sinne einer gelungenen Übergabe des Staffelstabes, der maßgeblich zum Sieg in einem Rennen beiträgt (etwa Dyck et al. 2002). Die „Familie“ taucht lediglich als eine Positionsbezeichnung auf. Sie wird zu einem Rekrutierungspool für die Unternehmensleiter oder Eigentümer. Die Beziehungen zwischen den einzelnen Akteuren werden dann bedeutsam, wenn sie als Gefährdungspotenziale in dem strategischen Spiel der Organisation auftreten. Nicht so recht zu diesem Bild wollen Beiträge zur Versorgung von Familienmitgliedern oder $\mathrm{zu}$ den schwierigen Autonomisierungsbestrebungen von Unternehmerkindern passen.

Diesen Vorgängen innerhalb der Unternehmerfamilie ebenso wie ihrem Einfluss auf den Generationswechsel widmet sich Franz Breuer (2009) mit seiner Analyse von Objekt-Transfers, die im Zusammenhang dieser Arbeit besonders relevant erscheint. Breuer stellt eine Theorie für jegliche Vorgänger-Nachfolger-Übergänge auf, die sich als soziale Schemata beschreiben lassen (etwa Kaufen, Schenken, Transplantieren, Spenden, Wählen), jedoch mit einer wesentlichen Einschränkung: es geht nur um solche Objekte, „die sich durch ein bedeutsames Ausmaß an transaktionaler Verbundenheit mit einer Person bzw. einer identifikatorischen Aufgeladenheit und Wertigkeit des Objektes für die Person auszeichnen" (Breuer 2009:12) oder in anderen Worten um „persönliche Objekte“ (ebd.). Dabei unterscheidet Breuer in zwei Transfer-Konstellationen: Entweder erfolgt ein Wechsel der Protagonisten oder des Objektes, in jedem Fall liegt eine dreistellige Relation vor (Triade). Breuer arbeitet als die zentralen Variablen der Vorgänger-NachfolgerÜbergänge den Kontext, die Zeitperspektiven und Deutungsmuster heraus. Unter Fokussierung eines individuellen Akteurs, so Breuer, kann ein Objekt-/ Protagonistenwechsel als kritisches Lebensereignis oder als Statuspassage aufgefasst werden (Breuer 2009:82), was die persönliche Verarbeitung in der Lebensgeschichte in den Mittelpunkt rückt. $\mathrm{Zu}$ verschiedenen Zeitpunkten (Phasen, Altersstufen) des Lebenslaufs werden die Objekte unterschiedlich wahrgenommen, konnotiert und wertgeschätzt (Breuer 2009:153). Der Transfer wird insofern zu einem Aushandlungs- und Passungsprozess, als sich innerhalb von fixierten Merkmalen die Charakteristika und Qualitäten der interpersonalen Beziehungen verhandeln lassen (Breuer 2009:112).

In der Entwicklung seiner Theorie diente Franz Breuer seine empirische Arbeit zu Familienunternehmen als wichtige Grundlage. In Kapitel 9 geht er ausführlich auf den Transferprozess von Familienunternehmen als persönlichen Objekten ein. Er behandelt als Transfer-Variante die altersbedingte, familieninterne Übergabe eines Klein- und Kleinstunternehmens (Personalunion von Eigentümer und Leiter) an nur einen Nachkommen. Der von Breuer skizzierte idealtypische Verlauf erfolgt in vier Schritten: erstens, die implizite Auswahl und Sozialisation des Nachfolgers; zweitens, der „ExplizitheitsSwitch“, also eine nun offizielle Verhandlung und Verkündung der Nachfolgemodalitäten; drittens, eine meist sehr lange Phase des gemeinsamen Arbei- 
tens im Betrieb (Kohabitation); viertens, die Unternehmensrepräsentanz durch den Nachfolger. Charakteristischerweise sind die Lebensläufe beider Parteien eng miteinander verwoben. Für diese Art von Beziehung sind emotionale Ambivalenzen und Oszillationen im Verlauf einer langjährigen gemeinsamen Familiengeschichte typisch (Breuer 2009:163). „Aus der widersprüchlichen Gemengelage im familiären und objektbesitzbezogenen Rollengefüge ergeben sich häufig Schwierigkeiten und Friktionen hinsichtlich des „Loslassens“ aufseiten der Eltern (Vater, Mutter) und der „Ablösung“, des Autonomie- und Autoritätsgewinns des Sohns bzw. der Tochter als Nachfolger/in" (Breuer 2009:165). Mit jeder weiteren Übergabe verändern sich die Bedingungskonstellationen und Unternehmensstrukturen. Es vergrößert sich vor allem das Maß individueller und kollektiver Bewusstheit der Akteure für das Nachfolge-Thema. Im Laufe der Generationenkette steigt der Verpflichtungsgrad gegenüber der Familientradition..$^{41}$ Trotzdem hat der zeithistorische Wandel der Kontexte, des Milieus, des Zeitgeistes etc. einen Effekt auf den Vorgänger-Nachfolger-Übergang. So wird ein Familienbetrieb „heutzutage eher verkauft oder stillgelegt, als dass ein Nachkomme gegen seinen Willen zur Übernahme bzw. Weiterführung verpflichtet oder gezwungen wird“" (Breuer 2009:276f.).

Die Erläuterungen von Franz Breuer zu den komplexen VorgängerNachfolger-Übergängen sind sehr aufschlussreich. Dennoch stellt sich die Frage, ob es ausreicht, ein Familienunternehmen als ein persönliches Objekt zu fassen - besonders vor dem Hintergrund der Multidimensionalität, die in der Familienunternehmensforschung immer wieder hervorgehoben wird. Mit Hilfe des UFFU-Modells gelingt es, den Blick von einem Übergang zwischen Akteuren auf die simultane Reproduktion von zwei Systemen zu lenken. Im Zuge des Transferprozesses erneuern und verändern sich Familienunternehmen und Unternehmerfamilie gleichermaßen. Übertragen wird also nicht das „Familienunternehmen“ als persönliches Objekt, sondern vielmehr (wie oben beschrieben) die Macht über dieses. Aus der entwickelten Systematik zum wechselseitigen Einfluss lässt sich analog ableiten, wie sich der Generationswechsel aus einer Familienperspektive darstellt. Der Übergang von Eigentum und Management des Familienunternehmens bedeutet für die Unternehmerfamilie dreierlei: Erstens, im Zuge des Generationswechsels wird in erheblichem Maße die Einkommensstruktur und die materielle Versorgung der einzelnen Familienmitglieder umstrukturiert. Das Vermögen wird zu Lebzeiten oder als Erbe an die nächste Generation transferiert (Erhardt 2001:21). Der Bezug von Gehältern aus der Tätigkeit als Geschäftsführer, Aufsichtsratsmitglied, etc. endet oder verändert sich. Betroffen davon sind nicht nur Übergeber und Übernehmer, sondern auch alle anderen abhän-

41 Diese Ausführungen unterstreichen noch einmal die Aussagen, die unter dem Abschnitt zu Generationen herausgearbeitet wurden. 
gigen Familienmitglieder. Die Nachfolge stellt zweitens einen Übergang in den Berufs- und Familienbiografien der Unternehmerfamilien dar. Dieser Umstand ist besonders offensichtlich im Fall des ausscheidenden oder eintretenden Geschäftsführers, Vorstands, etc., gilt allerdings auch für Familienmitglieder, die nur unterstützend tätig sind. Drittens verändert sich im Generationswechsel die Geltung und Wirkungskraft von Rechten und Pflichten der Unternehmerfamilie - in faktischer wie in affektiver Weise. ${ }^{42}$

Die Übertragungsprozesse werden von den Generationenstrukturen und der Familienkultur getragen und wirken auf sie zurück. Eine Studie, die sich etwa mit der Übertragung von Werten im Zuge der Nachfolge (verstanden als Sozialisationsprozess) beschäftigt, ist die von Garcia-Alvarez und LopezSintas (2006 und 2002). Sie zeigen, wie Gründer ihren Unternehmergeist anstelle reiner Managementqualitäten betonen und je nach Typ ein spezifisches Set an psychosozialen Werten weitergeben möchten, zu denen Selbstverwirklichung, Teamgeist oder Familientradition gehören können. Auch Lambrecht (2005) hebt in seinem Modell des Generationswechsels den Übergang der weichen Faktoren, bestehend aus Unternehmergeist, Freiheit, Werten, Erfahrung, Erziehung und Bildung (Lambrecht 2005:279) hervor. Lloyd Steier (2001) fasst diese als soziales Kapital zusammen. Gerade bei dynastischen Unternehmerfamilien haben sich häufig bestimmte Verhaltensmuster und -normen institutionalisiert.

Mit dieser auf dem UFFU-Modell basierenden Systematik zum Generationswechsel in Familienunternehmen und Unternehmerfamilien gelingt es, die Reproduktion beider Systeme zu fassen, unabhängig von der Größe des jeweiligen Unternehmens bzw. der Familie. Darüber hinaus lassen sich nun auch die in der Literatur viel beschriebenen Nachfolgedilemmata oder Faktoren, die Nachfolge verhindern (DeMassis et al. 2008:183), plausibel integrieren.

Allen voran seien die entstehenden normativen Konflikte beim Transfer des Eigentums bzw. des Vermögens erwähnt: Der materielle Transfer stellt die beteiligten Akteure vor die Aufgabe, die finanzielle Stabilität des Unternehmens mit der Versorgung des Ausscheidenden (und gegebenenfalls weiterer von diesem versorgter Familienmitglieder) zu koordinieren. Darüber hinaus gilt es, die verbriefte Mitsprache im Unternehmen im Sinne einer effektiven Entscheidungskompetenz zu regeln und dabei gleichzeitig eine im Sinne familiärer Gerechtigkeitsvorstellungen anerkannte Lösung der intergenerationellen Transfers zu finden. Die Forschung hierzu hat für weite Teile Europas und Nordamerika ergeben, dass in der Familie inter vivos-Transfers von den Eltern im allgemeinen (altruistisch) nach den unterschiedlichen Bedürfnissen der Kinder gesteuert werden, während für Vererbung heute normativ die Gleichbehandlung aller Kinder oberste Maxime ist und auch fak-

42 Diese drei Punkte wurden bereits in Schmiade, Stamm, Kohli 2009: 5-6 vorgestellt. 
tisch weit überwiegt (Light und McGarry 2004 oder Kohli 2004). Einerseits also gilt es, den Nachfolger mit ausreichenden Anteilen auszustatten, die es ihm ermöglichen, das Unternehmen angemessen zu beeinflussen. Andererseits sollen alle Kinder einer Unternehmerfamilie im Erbgang finanziell gleich behandelt werden (Stamm 2009). Das ist nicht leicht, wenn überproportional viel Vermögen in der Firma liegt und das Privatvermögen klein ist. Hildenbrand (Hildenbrand 2002) weist darauf hin, dass die Entscheidung einer Familie, ein Kind als Nachfolger im Erbgang zu bevorzugen, als Versuch gewertet werden kann, das kulturelle und erbrechtliche Gleichbehandlungsgebot zu unterlaufen. Das Ziel einer klaren Regelung von Entscheidungsgewalt und Unternehmensführung kann dem Ziel einer Gleichbehandlung der Kinder entgegenstehen. Regina Wenk lenkt den Blick darüber hinaus auf den Umstand, dass der Transfer des Eigentums mit dem Transfer von kulturellem und sozialem Kapital gekoppelt ist und entsprechend die Ungleichheit zwischen Geschwistern in Unternehmerfamilien noch verstärken dürfte (Wenk 2007:209ff.).

Abbildung 4: Modell zum Generationswechsel

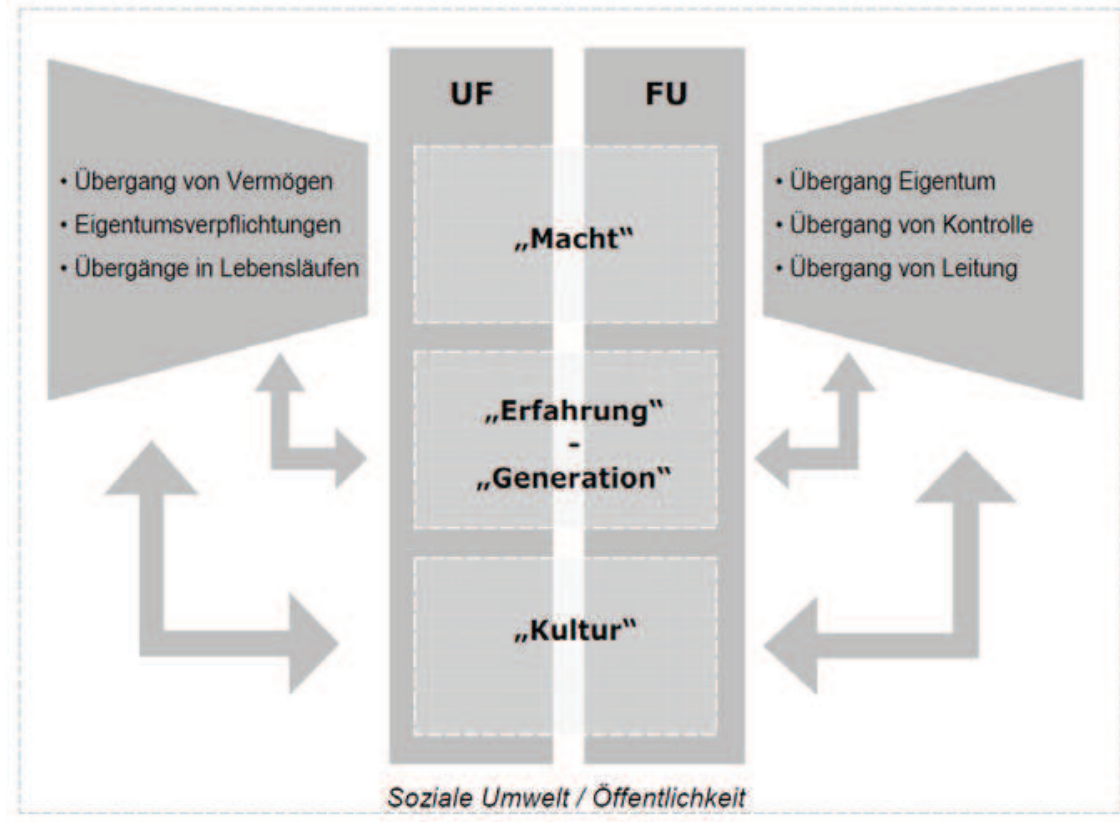

Quelle: leicht modifizierte Darstellung von Stamm, Schmiade, Kohli 2009:182 
Nicht weniger kompliziert hinsichtlich der zu erfüllenden und teils konträren normativen Erwartungen erscheint die Neubesetzung von Schlüsselpositionen im Unternehmen mit Familienmitgliedern, die sich als ein Übergang im Lebenslauf dieser Personen gestaltet. Klett weist (wie in Abschnitt 4.3. bereits ausgeführt) darauf hin, dass innerhalb einer Organisation die Selektion eines Kandidaten anhand von Kompetenzkriterien erfolgen sollte, während innerhalb der Familie die bloße Herkunft qualifiziert. Die empirische Forschung mahnt darüber hinaus, dass die Adaption des elterlichen Lebensstils nicht länger als Selbstverständlichkeit angenommen werden darf (Breuer 2000:475). Vielmehr handle es sich um eine berufliche Entscheidung von Individuen (Timmermann und Vonderach 1993:144). Stellte die Übernahme des väterlichen Betriebes für den Sohn einst eine attraktive Möglichkeit zur Sicherung von Bildung und Lebensunterhalt der Familie dar, treten durch den Geltungsverlust traditioneller Orientierungen individuelle Bedürfnisse in den Vordergrund (Jäkel-Wurzer 2010:31). In den Lebensgeschichten ist dabei weniger die Kluft zwischen Familienbetrieb und eigenständigem Lebenslauf bedeutsam als die Koordinierungsaufgabe, sich innerhalb und mit dem Familienbetrieb zu individualisieren (Wenk 2007:219). Die Verbindung von Unternehmen und Familie muss, wenn sie gelingen soll, trotz aller Unwegsamkeiten einen individuellen Lebensentwurf erlauben (Timmermann und Vonderach 1993:217). Der Familienunternehmer steht vor dem Balanceakt, eine stabile Identität im Spannungsfeld zwischen Familie und Unternehmen in einer ausdifferenzierten Gesellschaft zu wahren (Bäcker 2008:37). Es lässt sich festhalten, dass jedes Mitglied einer Unternehmerfamilie eine Karriere im Familienunternehmen als eine Option unter vielen biografisieren muss, um der normativen Individualisierungserwartung gerecht zu werden. Die materielle und emotionale Verstrickung der Familienmitglieder miteinander und mit dem Unternehmen erfordert ein höheres Maß an „biografischer“(Wenk) bzw. „Individualisierungsarbeit“ (Bäcker). Um noch einmal auf Breuer zurückzugreifen: Die Mitglieder der Unternehmerfamilie bewegen sich zwischen der Gebundenheit an die Strukturvorgaben aus der Familien-Unternehmens-Konstellation und der Freiheit von Handlungsspielräumen, die sich in modernen Gesellschaften ergeben (Breuer 2009:123).

Im Laufe des Generationswechsels reproduziert und transformiert sich damit der wechselseitige Einfluss von Familie und Unternehmen. Nachfolge in Familienunternehmen und Unternehmerfamilien bedeutet, dass sich von Generation zu Generation Fragen der Unternehmensstrategie, Organisation und Innovation, ebenso wie des familiären Miteinanders, der Vererbung und Entwürfen des Lebenslaufes neu stellen. Es ist davon auszugehen, dass sich die spezifischen Muster und Antworten, wie Nachfolge gelöst wird, im historischen Zeitverlauf wandeln. $\mathrm{Zu}$ vermuten sind darüber hinaus regional unterschiedliche Strategien. Immer wieder gilt es, die jeweils vorherrschenden 
Vorstellungen und gesellschaftlichen Orientierungsmuster in diesen Prozess zu integrieren. 


\section{Theoretischer Ansatz: Ein Zugang zum „Ganzen Leben“}

Die Zwischenbetrachtung dient als vorläufiges Resümee über den Charakter und die Eigenarten von Unternehmerfamilien und legt die Stoßrichtung der empirischen Arbeit fest. Die Thesen zu Unternehmerfamilien, die sich aus den theoretischen Vorüberlegungen und dem Stand der Forschung ergeben, werden zusammengefasst und zentrale Begriffe definiert. Ein alltagsweltlicher Zugang zu dem Einfluss des Unternehmens auf die Familie wird als Desiderat erkannt, die soziologischen Ansätze eines Institutionalisierten Lebenslaufs und der Alltäglichen Lebensführung werden vorgestellt und eine Kombination der Perspektiven diskutiert, um auf dieser Grundlage die Forschungsfrage zu präzisieren.

\subsection{Anachronismus, Paradox oder doch Pionier?}

In Kapitel II wurden die idealisierten Vorstellungen des Ganzen Hauses, der modernen Kernfamilie sowie der multilokalen Mehrgenerationenfamilie als mögliche Vergleichsgruppen für die Untersuchung von Unternehmerfamilien gedankenexperimentell herangezogen. Aus der Perspektive soziologischer Modernisierungstheorien erscheint die Verbindung von Unternehmen und Familie eine Familie eigener Art zu konstituieren, die nie ganz in den gesellschaftlichen Idealvorstellungen familialen Handelns aufgeht. Während Unternehmerfamilien eine Reihe von strukturellen Ähnlichkeiten mit der Familienform des Ganzen Hauses aufweisen, sind die Parallelen zu der modernen Kernfamilie, die sich von ihren ökonomischen Funktionen sowie der Ursprungsfamilie weitgehend emanzipiert hat, weniger offensichtlich. Unternehmerfamilien erscheinen in diesem Lichte als Anachronismen. Mit der zunehmenden Bedeutung von multilokalen Mehrgenerationenfamilien im Zuge fortschreitender Modernisierung ändert sich die Bewertung von Unternehmerfamilien: Sie wirken geradezu als Pioniere dieser Form familialen Handels.

Bezieht man die beiden plakativen Thesen zur Bewertung von Unternehmerfamilien auf den in Kapitel III dargestellten Forschungsstand, so zeigt sich, dass die Anachronismusthese insbesondere in der Soziologie weit verbreitet ist, die Idee der Unternehmerfamilien als Pioniere hingegen keinen 
Eingang in die Forschung gefunden hat. Es lässt sich jedoch eine zunehmende Beschreibung von Unternehmerfamilien als Paradoxe beobachten und damit eine neue Qualität der Unternehmens-Familien-Verbindung. Die reduktionistische Bewertung von Unternehmerfamilien als zukunftsweisend oder antiquiert soll an dieser Stelle nicht fortgeführt werden. Als fruchtbar hat sich allerdings der dahinter liegende Gedanke von unterschiedlichen Idealen familialen Handelns als Vergleichsgruppen für Unternehmerfamilien erwiesen.

Die sozial- und wirtschaftshistorische Forschung, die sich im Wesentlichen mit der Entstehung und Entwicklung großer industrieller Familienunternehmen und ihrer Familien auseinandergesetzt hat, arbeitet sich in besonderem Maße an der Unternehmerfamilie als spezifische Mischung zwischen Rückwärtigkeit (Ganzes Haus) und Modernität (Kernfamilie) ab. Zusammenfassend werden die Besonderheiten der industriellen Unternehmerfamilien wie folgt charakterisiert: Hier verbinden sich eine ökonomische Abhängigkeit von den Eltern und ihrem Wirtschaftsbetrieb im Hinblick auf einen Arbeitsplatz (Tendenz zur Berufsvererbung) und der Absicherung von Lebensrisiken - also erwerbswirtschaftliche und sozialstaatliche Funktionen - mit dem Ideal der Familie eines privaten, geschützten Innenraumes, in dem die Generationen ein emotionalen Verhältnis pflegen. Als Beleg dafür, dass die Ideale der bürgerlichen Familie, allen voran die romantische Verinnerlichung und Emotionalisierung der Familie, innerhalb von industriellen Unternehmerfamilien wirkmächtig sind, wird auf die sich durchsetzende Praxis der freien Wahl des Heiratspartners verwiesen. Industrielle Unternehmerfamilien sind geprägt von einem geteilten Familienbewusstsein, dass als Quelle von Solidarität, Loyalität und Sinngebung dient. Als eigenständige Gruppierung innerhalb der sich formenden wirtschaftsbürgerlichen Klasse genießen sie ein steigendes soziales Ansehen. Sie sind oft in hohem Maße mit finanziellem und symbolischem Kapital ausgestattet.

Die Anpassung an die gewandelten Vorstellungen emotionaler Bindung erfolgt jedoch nicht ohne Hindernisse: Die historischen und soziologischen Untersuchungen von Unternehmerfamilien zeigen Konflikte zwischen den Generationen hinsichtlich der Verständnisse von Privatheit, Berufsvererbung und Partnerwahl auf und legen die pathologische Seite (bspw. Minderwertigkeitsgefühle) offen. Die Konsequenz einer solchen Annäherung an bürgerliche Ideale für die Verbindung von Unternehmen und Familie ist darin zu sehen, dass die Aufgabe, familiäre, individuelle und unternehmerische Interessen zu koordinieren, nun auf die einzelnen Familienmitglieder übertragen wird, die sich an abstrakten Wertvorstellungen statt an der (haus-) väterlichen Autorität orientieren. Die einzelnen Familienmitglieder sind gefordert, einen individuellen Lebensentwurf innerhalb der strukturellen Logik der Unternehmerfamilie zu biografisieren. Auf der Ebene der Familie und des Un- 
ternehmens gilt es, Autonomie gegenüber den Eltern und Kompetenz gegenüber externen Kandidaten zu behaupten.

Auch aus ökonomischer Perspektive erscheinen Familienunternehmen als spezifische Mischung aus Rückwärtigkeit und Modernität. Es kann als historischer Fakt gelten, dass Unternehmerfamilien zur Lösung vorindustrieller Probleme und zur Entwicklung des industriellen Kapitalismus wesentlich beigetragen haben. Mit der Entfaltung des industriellen Kapitalismus entstehen jedoch zunehmend marktförmige Alternativen zur Familie als Ressource. Die technischen und kaufmännischen Kenntnisse eines Unternehmers werden zunehmend über das öffentliche Bildungssystem vermittelt, die Finanzmärkte entwickeln sich, neue Unternehmensgesetze werden verabschiedet, Kartelle und professionelle Netzwerke machen die familialen Verbindungen abkömmlich. Boltanski und Chiapello beschreiben eine Veränderung der Managementphilosophie hin zum Konzernkapitalismus mit dem Fabrikdirektor als gatekeeper. Die Verbindung von Unternehmen und Familie gerät also auch aus dieser Richtung zunehmend unter Legitimierungsdruck.

Wie im weiteren Verlauf des dritten Kapitels deutlich wird, weisen Untersuchungen $\mathrm{zu}$ Unternehmerfamilien der Gegenwart hinsichtlich der typischen konfliktreichen Merkmale dieser Familien eigener Art bemerkenswerte Parallelen zu der historischen Forschung auf, die industrielle Unternehmerfamilien als soziale Einheiten deklariert, die nur innerhalb eines spezifischen historischen Settings denkbar sind. Büttner (2007) betont etwa die Vielzahl ökonomischer Funktionen (Arbeitsstelle, Ausbildungsplatz, Kapitalanalyse, Sozialversicherung), die innerhalb der Unternehmerfamilie geleistet werden können und müssen. Hildenbrand, als weiteres Beispiel, ordnet die fehlende Trennung von Arbeiten und Wohnen, die erweiterte Haushaltsfamilie, die auch nicht verwandte Personen einbezieht, sowie die Orientierung an der Familientradition als typische Merkmale eines Ganzen Hauses insbesondere landwirtschaftlichen Betrieben und Hoteliersfamilien $\mathrm{zu}$ (Hildenbrand 2002:129). Bäcker (2008) erklärt sogar, dass die Auswirkungen der funktionalen Differenzierung vor dem traditionellen Familienunternehmen „Halt" gemacht hätten und Unternehmerfamilien in „solider Gegensätzlichkeit“ zu modernen Familien stehen (Bäcker 2008:239).

Die Tradition der Familie und die strukturellen Ähnlichkeiten zum Ganzen Haus stellen eine Herausforderung für die Erfüllung moderner Individualisierungsnormen dar, dienen aber gleichzeitig auch als Quelle von Individualität und Solidarität. Tradition ist in Unternehmerfamilien mehr als eine Residualkategorie, stattdessen ist die alltägliche Lebensführung solcher Familien durchsetzt von traditionalen Elementen. Unternehmerfamilien verdeutlichen einmal mehr, dass sich die Vorstellung einer Unvereinbarkeit von Tradition und Moderne als unhaltbar erweist (Degele und Dries 2005:19). Die von Hildenbrand auf Unternehmerfamilien angewandte Denkfigur der „Traditionserhaltung durch Traditionsbruch“ erscheint attraktiv (Hildenbrand 
2002:121). Durch die Integration moderner, rational geprägter und affektiv getragener Handlungsorientierungen in traditionelle Muster gelingt es Unternehmerfamilien, Kontinuität herzustellen. Im Kontext einer modernen Gesellschaft müssen Unternehmerfamilien in der Lage sein, sich als widersprüchliche Einheit zu organisieren (ebd.). Dazu gehört ein erhöhtes Maß an Aktivität. So ist beispielsweise die Trennung von Privatheit und Öffentlichkeit in Unternehmerfamilien nicht das Ergebnis einer externen, räumlichen Gegebenheit, sondern eines aktiven Hervorbringungsprozesses (Hildenbrand 2002:135). Auch Jäkel-Wurzer beschreibt dieses Spannungsverhältnis zwischen Traditionserhalt und den gesellschaftlichen Entwicklungen der Moderne, zu welchem sich Unternehmerfamilien positionieren müssen (JäkelWurzer 2010:24).

Um es noch einmal auf den Punkt zu bringen: Die hohe Übereinstimmung in der Beschreibung von industriellen und gegenwärtigen Unternehmerfamilien lässt den Schluss zu, dass die jeweiligen Analysen immer denselben Vergleichsmaßstab zu Grunde legen, nämlich eine Entwicklung vom Ganzen Haus zu einer bürgerlichen Kernfamilie. Es sollte jedoch bedacht werden, dass zwar eine Reihe von Merkmalen der modernen Kernfamilie (wie etwa die Vorstellung einer freien Partnerwahl oder der Kindheit als geschützter Raum) ihre Fortsetzung in spätmodernen Familienformen finden, sich dennoch familiales Handeln und insbesondere die herrschenden Rollenvorstellungen grundlegend gewandelt haben. Insofern scheint die Frage berechtigt, ob der Vergleichsmaßstab einer bürgerlichen Familie für die Untersuchung von Unternehmerfamilien nicht um neuere Ansätze ergänzt werden müsste.

Die hohe Übereinstimmung in der Beschreibung von Unternehmerfamilien lässt darüber hinausgehend noch einen zweiten Schluss zu: Unternehmerfamilien weisen einen konstanten strukturellen Kern auf, der Raum dafür lässt, aktuell geltende Vorstellungen zu familialem und unternehmerischem Handeln zu integrieren. Weitere Indizien für diese Thesen finden sich vor allem in der systemtheoretischen Forschung zu Unternehmerfamilien.

Kapitel III geht ausführlich auf den lückenhaften Forschungsstand und die fehlenden empirischen Informationen zu Unternehmerfamilien der Gegenwart ein. Ganz im Gegensatz zu Familienunternehmen, die immer noch die Mehrheit der Firmenpopulation stellen, haben sich Unternehmerfamilien zum Sonderfall entwickelt. Ihren spezifischen Charakter fasst eine systemtheoretische Perspektive als ein Produkt eines Widerstreits von zwei gleich begründeten Sinngehalten (Unternehmen und Familie). Hier binden sich zwei Systemtypen aneinander, welche sich im historischen Verlauf gesellschaftlich auseinander entwickelt haben. Der private Raum der Familie wird dauerhaft mit Impulsen des rationalen Systems des Unternehmens konfrontiert. Die Handlungs- und Orientierungsmuster der jeweiligen Systeme verlieren im Zuge ihrer wechselseitigen Durchdringung ihre verbindliche Funktion 
und verlangen von der Unternehmerfamilie aktive Grenzarbeit. Bei eingehender Betrachtung erweist sich die Unternehmerfamilie als Paradox mit internem Zusammenhang. Die Mitglieder solcher Familien bilden in besonderem Maße ein Geschick im Umgang mit Ambivalenzen aus. Folgt man dieser Systemperspektive konsequent, so besteht die Besonderheit der Unternehmerfamilie darin, dass sie in ihrer familialen Kommunikation Bezug auf das Unternehmen nimmt und dieses damit Teil der Familienidentität ebenso wie der Persönlichkeit der einzelnen Mitglieder werden kann. Das Unternehmen wird innerhalb der Unternehmerfamilie zu einer sinn- und identitätsstiftenden Instanz.

Diese systemtheoretische Perspektive wirkt insbesondere dann erhellend, wenn sie in Bezug zu einem sich verändernden Charakter des Kapitalismus und der damit einhergehenden Wirtschafts- und Lebensweise gestellt wird. Der Netzwerkkapitalismus sieht eine zunehmende Durchlässigkeit der Grenzen zwischen Arbeit und Freizeit, zwischen Unternehmen und persönlichen Beziehungen vor. In Kapitel III wurde die Entstehung neuer, flexibler Formen der Selbständigkeit ebenso wie eine notwendige Abgrenzung der „Normalunternehmer" von diesen diskutiert. Boltanski und Chiapello heben das projektförmige Arbeiten als Kern der neuen Wirtschaftsweise hervor. Die Verbindung von Unternehmen und Familie erfährt insofern einen Bedeutungszuwachs, als in Unternehmerfamilien Netzwerkmenschen sozialisiert werden, die im flexiblen Umgang mit Ambivalenzen geübt sind. Die Fähigkeiten zur Beobachtung und Balance von Paradoxien und zur Integration von ökonomischen Erfordernissen in die eigene Lebensplanung sowie eine entsprechende Positionierung gegenüber der Außenwelt sind im erstarkenden Netzwerkkapitalismus zunehmend nützlich.

Die aktuelle Forschung hebt zudem deutlich hervor, dass sich Unternehmerfamilien der Gegenwart von der bürgerlichen Art der Familie losgelöst haben. Trotzdem bleibt eine „Bürgerlichkeit“ als markantes Element in der Selbstdarstellung von Unternehmerfamilien erhalten. In „Generationenerzählungen“ (Lubinski) werden das Verhältnis von Familie und Unternehmen, seine Kontinuität sowie eine Kultur der Selbständigkeit und Leistung zum Thema eines intergenerationellen Deutungsprozesses. Verbunden über die Verantwortung für einen gemeinsamen Besitz sowie über eine geteilte Familiengeschichte, stehen die Generationen in einem engen Solidaritätsverhältnis wie dies für multilokale Mehrgenerationenfamilien beschrieben wird. Als Erinnerungs- und Erzählgemeinschaft beziehen sie ihre personale und kollektive Identität aus einem gemeinsamen Projekt. Jedoch verliert die kontrollierende Funktion der Familie an Bedeutung, die Unternehmerfamilie darf die individuelle Wahl nicht begrenzen, Beziehungen nicht vorbestimmen (Boltanski und Chiapello 2003:183). Insofern erweist sich das Konzept der multilokalen Mehrgenerationenfamilie als möglicher Vergleichsmaßstab für 
Unternehmerfamilien als tragfähig, wie bereits in den gedankenexperimentellen Ausführungen in Kapitel II vermutet wurde.

Die hier zusammengefassten Erwägungen zur Unternehmerfamilie der Gegenwart deuten an, dass sich Unternehmerfamilien in einer postmodernen Gesellschaft mit einer projektförmigen, flexiblen Wirtschaftsweise und einem solidarischen Mehrgenerationenverbund als stabile soziale Einheit erweisen können. Trotz oder gerade wegen ihrer traditionalen Elemente erscheinen Unternehmerfamilien nun als Grenzgänger, die geübt sind im Umgang mit Ambivalenzen. Ebenso wie industrielle Unternehmerfamilien, sind diese postmodernen Unternehmerfamilien an einen spezifischen historischen Kontext gebunden, sie weisen jedoch wie oben gezeigt eine Vielzahl an Parallelen auf.

Insofern bekräftigt sich hier noch einmal die These, die im Übrigen auch von Franz Breuer (2009:123) vertreten wird, dass sich die Mitglieder einer Unternehmerfamilie zwischen der Gebundenheit an einen strukturellen Kern, der sich direkt aus der Verbindung von Familie und Unternehmen ableitet, sowie der Freiheit von Handlungsspielräumen bewegen, die es erlauben, die jeweils herrschenden Vorstellungen von unternehmerischem und familialem Handeln zu integrieren. Unternehmerfamilien unterscheiden sich strukturell von den Familienformen des Ganzen Hauses, der modernen Kernfamilie und der multilokalen Mehrgenerationenfamilie, beinhalten allerdings einige Merkmale der jeweiligen Formen. Welchen Merkmalen nun in der Innenund Außenbeziehung von Unternehmerfamilien mehr oder weniger Bedeutung beigemessen wird, hängt vom jeweils vorherrschenden gesellschaftlichen Kontext ab.

\subsection{Die strukturelle Eigenlogik von Unternehmerfamilien}

Es schließt sich also nahtlos die Frage an, wie sich die strukturelle Eigenlogik von Unternehmerfamilien gestaltet. Mit Blick auf die Gegenwart wird mit dem vorgestellten Modell zum wechselseitigen Einfluss von Unternehmerfamilien und Familienunternehmen (kurz UFFU-Modell) eine Ordnungsmatrix vorgestellt, die empirische und theoretische Ergebnisse einordnen und Forschungslücken benennen kann. In Analogie zur F-PEC Skala, einem in der Familienunternehmensforschung etablierten Instrument, wird dargelegt, auf welche Weise das Unternehmen auf die Familie einwirkt. Reflektiert man nun über diesen analytischen Vorstoß im Lichte der vorangegangenen soziohistorischen und systemtheoretischen Erwägungen, so könnte dieser als erster, vorläufiger Versuch betrachtet werden, die strukturelle Eigenlogik von 
Unternehmerfamilien auf einer theoretischen Ebene, aber auf Basis bisher geleisteter empirischer Forschung, zu fassen.

In dem Modell wird die Annahme vertreten, dass sich der wechselseitige Einfluss in drei Dimensionen niederschlägt: Erstens, während die Familie über Leitung, Kontrolle und Eigentum die Geschicke des Unternehmens bestimmt, wirkt dieses in der „Macht“-Dimension auf die Einkommensstruktur, das Vermögen, die Lebenslaufgestaltung sowie Privilegien und Pflichten der Familie ein. Die Familien-Unternehmens-Konstellation zeichnet sich ferner durch ein spezifisches temporales Verständnis aus, dass sich als „Denken in Generationen“ und einen ausgeprägten Kontinuitätsanspruch äußert. Schließlich prägt der wechselseitige Einfluss die praktizierte Kultur sowohl im Unternehmen als auch in der Familie.

Das analytische Potenzial dieses Modells, das über eine reine Ordnungsmatrix der Literatur hinausgeht, zeigt sich in dem Anwendungsbeispiel der Nachfolge. Im Zuge des Transferprozesses erneuern und verändern sich Familienunternehmen und Unternehmerfamilie gleichermaßen. Übertragen wird nicht „nur“ das „Familienunternehmen“ als persönliches Objekt, sondern vielmehr die Macht an diesem. Damit wird das Verständnis von Nachfolge als Übergang zwischen Akteuren um jenes einer simultanen Reproduktion von zwei Systemen ergänzt. Mit jeder weiteren Übergabe verändern sich die Bedingungskonstellationen und Unternehmensstrukturen. Es vergrößert sich vor allem das Maß individueller und kollektiver Bewusstheit der Akteure für das Nachfolge-Thema. Im Laufe der Generationenkette steigt der Verpflichtungsgrad gegenüber der Familientradition. Trotzdem hat der zeithistorische Wandel der Kontexte, des Milieus, des Zeitgeistes etc. einen Effekt auf den „Vorgänger-Nachfolger-Übergang“ (Breuer). Es gelingt, die wesentlichen Prozesse, die sowohl unternehmens- als auch familienseitig ablaufen, zu fassen, ohne dabei den Gestaltungs- und Interpretationsraum der Familienmitglieder einzugrenzen. Insofern hält das Modell die Balance zwischen strukturellen Vorgaben und Handlungsoptionen.

Die Feststellung eines strukturellen Kerns darf nicht dazu führen, dass (gerade alte) Unternehmerfamilien als unveränderlich begriffen werden. Sie durchlaufen, wie familiales Handeln im Allgemeinen, einen Wandel und orientieren sich an den jeweils geltenden gesellschaftlichen Idealvorstellungen, doch immer unter der Auflage, gleichzeitig Unternehmen und Familie zu balancieren. In der Betrachtung von Unternehmerfamilien ist deshalb eine gewisse historische Tiefe wünschenswert, die auch Generationeneffekte bedenkt. Je nach zeithistorischen Umständen lassen sich unterschiedliche Herausforderungen und Problemlagen für die Familienmitglieder vermuten, die ihre je spezifischen Strategien und Bewältigungsformen erfordern. In anderen Worten, die Lebensführung von Unternehmerfamilien variiert trotz dieser strukturellen Eigenlogik im Laufe der Zeit. 


\subsection{Begriffsdefinitionen}

Die Argumentation der systemtheoretischen Diskussion zu Familienunternehmen und Unternehmerfamilien legt es nahe, den wechselseitigen Einfluss von Familie und Unternehmen als konstitutives Element festzulegen. Dabei gehen die organisationale und familiale Einheit nicht vollständig ineinander auf. Die Unternehmerfamilie wird deshalb als eine vom Unternehmen maßgeblich beeinflusste Familie verstanden, die sich trotz ihrer engen Bande von dem Familienunternehmen differenzieren lässt.

In ihrem Lehrbuch zu Familienunternehmen definiert Sabine Klein Unternehmerfamilien als ,,institutionalisierte Familien“ (2004:11). Zwei wesentliche Merkmale sieht sie als essentiell: Es muss ein familiales Kollektiv vorhanden sein, beispielsweise alle direkten Abkömmlinge und Ehepartner einer definierten Ursprungsehe. Dieses Kollektiv nimmt sich der Aufgabe an, die Familie und ihren Einfluss mittels unternehmerischer Tätigkeit zu erhalten. Ein solches familiales Projekt erfordert Handlungsregeln, die den angehörenden Familienmitgliedern eng definierte Grenzen für ihre persönlichen Entscheidungen setzen. Diese Definition scheint anschlussfähig an das weitere Verständnis der „entrepreneurial groups“ von Martin Ruef. Demnach bilden Unternehmerfamilien über einen längeren Zeitraum hinweg eine stabile Gruppe, nicht nur zur Gründung, sondern auch zum Erhalt eines Unternehmens. Wer Mitglied dieser Gruppe ist, bestimmt sich nicht nur über Verwandtschaft allein, sondern über das mehr oder weniger hohe materielle und zeitliche Investment, soziale Netzwerke, Identität und gemeinsame Ziele (Ruef 2010:7). Sowohl Klein als auch Ruef sehen den Aspekt einer kollektiven Handlung als wesentlich an.

Mit Blick auf die theoretischen Überlegungen der vorangegangenen Kapitel besteht die Gefahr einer solchen akteurszentrierten Definition jedoch in einer Überbetonung des zielgerichteten, strategischen Handelns in Unternehmerfamilien, die sich ergebende strukturelle Zwänge und ihre Bewältigung vernachlässigt. Eine wesentliche Quintessenz der bisherigen Betrachtung von Unternehmerfamilien ist darin zu sehen, dass sich aus der Verbindung von Familie und Unternehmen strukturelle Komponenten ergeben, die einerseits die Handlungsmöglichkeiten der Familienmitglieder beschränken, sich andererseits aber durchaus von ihnen gestalten und interpretieren lassen.

Im Sinne einer weiten Fassung von Unternehmerfamilien (siehe hierzu Kapitel 3.2) ist die soziale Einheit Unternehmerfamilie offen für unterschiedliche Formen familialen und unternehmerischen Handelns. Verschiedene Vorstellungen, Orientierungsmuster und Normen hinsichtlich Leben und Arbeiten, die mit den jeweiligen zeithistorischen Umständen, der Lebenszeit der Familienmitglieder und auch der Bestandsdauer der Unternehmerfamilie variieren, erweisen sich als tragfähig für diese soziale Einheit. 
Insofern soll im Folgenden der Definition von Unternehmerfamilien kein spezifisches Konzept von „Familie“ (etwa die bürgerliche Kleinfamilie) oder von „Unternehmen“ (etwa der mittelständische Industriebetrieb) zu Grunde gelegt werden. Klein weist zu Recht darauf hin, dass sich durch den Verzicht auf eine solche Definition die Grenzen einer Mitgliedschaft in Unternehmerfamilien nur schwer vergleichen lassen. Andererseits wird es auf diese Weise möglich, auch die historischen Wandlungen der Familien über die Zeit und ihre eigene Grenzdefinition zu fassen, was insbesondere bei sehr alten Unternehmerfamilien eine Rolle spielen dürfte. Zentraler Ansatzpunkt für die hier vertretene Definition von Unternehmerfamilien ist entsprechend die strukturelle Eigenlogik von Unternehmerfamilien und ihre Bearbeitung durch die Familienmitglieder. Aus diesem Grund dient das vorläufige UFFUModell als Grundlage für die folgende Definition von Unternehmerfamilien:

Eine Unternehmerfamilie wird als eine Familie definiert, die maßgeblich von einem Unternehmen beeinflusst wird. Dieser Einfluss findet über die drei Dimensionen Macht, Generation und Kultur statt. Unternehmerfamilien beziehen einen wesentlichen Teil ihres Einkommens aus der Arbeit im und dem Besitz am Unternehmen. Die beruflichen und familialen Lebensläufe der Mitglieder einer Unternehmerfamilie werden vom Unternehmen durchdrungen und geformt. Aus dem Besitz von Unternehmensanteilen ergeben sich Eigentumsverpflichtungen und Privilegien (Macht). Das Unternehmen wird in diesen Familien eigener Art zu einem Generationen überspannenden Projekt mit Kontinuitätsanspruch und beeinflusst die Zukunftsvorstellungen und Vergangenheitsdeutungen ihrer Mitglieder. Über die Zeit institutionalisieren sich spezifische Handlungsregeln und ergeben sich Pfadabhängigkeiten (Generation). Schließlich findet sich in Unternehmerfamilien eine spezifische Kultur, die ein unternehmerisches Element über Generationen aufrechterhält. In ihrem Außenverhältnis wird Unternehmerfamilien ein je spezifischer sozialer Status zugewiesen (Kultur).

Es wird offensichtlich, dass in Unternehmerfamilien das Unternehmen sowohl die Innen- als auch die Außenbeziehungen beeinflusst. Im Laufe der vorangegangenen Diskussion fand dabei vor allem das Generationenverhältnis in Unternehmerfamilien immer wieder Beachtung, das als direkt, unmittelbar und konfliktreich dargestellt wird. Deutlich wurde auch, dass für eine tragfähige Analyse der Beziehungsverhältnisse der jeweilige gesellschaftliche Kontext, geltende Normen und Lebensvorstellungen einzubeziehen sind. Jäkel-Wurzer (2010:3) betont mit ihrem strukturlogischen Modell, dass sich das jeweilige Beziehungsgefüge auf mehreren Ebenen auswirkt: Individuum, Familie und Unternehmen, Gesellschaft.

Die hier vorgenommene Betonung der strukturellen Einflüsse und des Zusammenhanges mit einer Organisation würde es nahe legen, die von Ralph Wiecher genutzte Begrifflichkeit der Unternehmensfamilien (business family) aufzugreifen und auf diese Weise die personelle Engführung des Ter- 
minus Unternehmerfamilie (entrepreneurial family), der eher auf die unternehmerische Tätigkeit, verbunden mit einem spezifischen Einstellungsset und Habitus, verweist, zu vermeiden. Dennoch wird im Folgenden der in der Forschungspraxis und Alltagssprache geläufigere Begriff der Unternehmerfamilie beibehalten. Dieser soll als kollektive Bezeichnung für alle Formen des Unternehmens-Familien-Nexus aus Familienperspektive genutzt werden, also auch ganz explizit solche Familien einbeziehen, die zwar ein oder mehrere Unternehmen besitzen, darin aber nicht operational tätig sind. Der Begriff macht ferner weder größen- noch branchenbezogene Einschränkungen und umfasst alle Familienmitglieder, die materiell oder emotional mit dem Unternehmen verbunden sind. ${ }^{43}$ Zugegebenermaßen wird der Begriff Unternehmerfamilien damit zu einer breiten Kategorie unterschiedlicher Typen dieser Familien eigener Art. Er bezieht sowohl die mehrstämmige Familiendynastie, die mit einem weltweit tätigen, an der Börse notierten Pharmakonzern verbunden ist, als auch die Familie mit Migrationshintergrund, die gemeinsam eine kleine Reinigung betreibt, ein. Es gibt nicht die Familie des Familienunternehmens, wie Simon betont (2002b:9), aber sie zeichnen sich alle durch eine spezifische strukturelle Eigenlogik aus, die sich aus der Verbindung von Unternehmen und Familie ergibt. Eine weitere Klassifizierung von Unternehmerfamilien ist sicherlich sinnvoll. Insbesondere die sozialgeschichtlichen Erwägungen lassen vermuten, dass sich die Lebensweise von Unternehmerfamilien erheblich unterscheidet, je nachdem, ob die Familie einen kleinen oder großen, einen landwirtschaftlichen oder industriellen, einen regionalen oder internationalen Betrieb besitzt und leitet. Bereits bestehende Typologien von Familienunternehmen (für eine Übersicht siehe etwa Schäfer 2007:35f.) könnten als Ausgangspunkt genutzt und auf ihre Übertragbarkeit auf Unternehmerfamilien geprüft werden. So wäre es beispielsweise denkbar, den Typ der „Family Offices“, die Eigentum an Unternehmen als PortfolioBestandteil ihres Vermögens verstehen, auszugliedern. Eine derartige Klassifizierung sollte jedoch vor allem Ergebnis empirischer Arbeit sein. Deshalb wird an dieser Stelle von einer Typologie der Unternehmerfamilien Abstand genommen und dieser Aspekt in der Schlussbetrachtung noch einmal aufgegriffen.

Im Rahmen dieser Arbeit kommt darüber hinaus dem Nachfolgeprozess eine wesentliche Bedeutung zu. Wiederum wird weniger eine akteurszentrierte Perspektive eingenommen, die Nachfolge in erster Linie als eine Entscheidung versteht. Stattdessen sollen Nachfolge und Generationswechsel synonym für die Reproduktion der strukturellen Eigenlogik von Unternehmerfamilien (und parallel des Familienunternehmens) verstanden werden. Es

43 Damit geht die hier vorgestellte Definition von Unternehmerfamilien über die von Uhlaner vorgeschlagene Definition hinaus, die nur solche Familienmitglieder einschließt, die Anteile am Unternehmen halten oder darin arbeiten Uhlaner 2006:126. 
handelt sich um einen kontinuierlichen Prozess, der den Zusammenhang von Unternehmen und Familie bestätigt, verfestigt und erneuert.

\subsection{Forschungslücke: Ein Zugang zum „Ganzen Leben“}

Die bisherigen Ausführungen haben eine Vielzahl von Forschungslücken im Bereich Unternehmerfamilien deutlich werden lassen, die nicht alle im Zuge dieser Arbeit aufgegriffen werden können. Das eklatanteste Defizit besteht jedoch darin, dass sich die Forschung zu Unternehmerfamilien bislang nur sehr punktuell gestaltet und eine systematische Analyse fehlt. Die intensive Aufarbeitung des Forschungsstandes über disziplinäre Grenzen hinweg sowie die Entwicklung des UFFU-Modells leisten einen analytischen Beitrag zu einer solchen Systematik, benötigen jedoch eine empirische Fundierung. Es zeigt sich darüber hinaus, dass der gegenwärtig sehr prominente systemische Ansatz einige Schwächen aufweist. Dazu gehören neben einer überproportionalen Betonung der Unternehmensseite ein sehr ausgeprägter Fokus auf widerstreitende Logiken, Rollenkonflikte und Integrationsprobleme, die den Blick auf den Gesamtzusammenhang versperren. Schließlich kann die systemische Perspektive auch nicht erklären, warum unter diesen Bedingungen eine Fortsetzung des Paradoxes Unternehmerfamilie überhaupt möglich ist. $\mathrm{Zu}$ kurz kommt das Wechselspiel aus institutionalisierten Strukturen und den Konstruktionsleistungen der aktuell Handelnden. Es fehlt an einer systematischen Analyse der Selbstverständlichkeiten, der gelingenden Kommunikation und des Alltags in Unternehmerfamilien, um die Schwächen dieses theoretischen Zugangs auszubalancieren. Die sich damit verknüpfende Forderung besteht nicht darin, die Probleme und Konflikte klein zu reden oder gar zu negieren, sondern darin, die gefundenen Strategien zum Umgang mit diesen und zu ihrer Lösung in die Analyse einzubeziehen. Es muss gelingen, die strukturelle Eigenlogik und ihre jeweilige Interpretation offen zu legen, um die effizienten Wege, wie Unternehmerfamilien ihre Lebensform nicht nur erträglich, sondern sogar attraktiv machen, zu verstehen. Erforderlich ist dafür ein Ansatz, der das Leben als Ganzes erfasst und nicht von vornherein den Lebenszusammenhang in „Arbeit“ hier und „der Rest des Lebens“ dort aufteilt. Zwei soziologische Forschungsansätze haben sich einen solchen $\mathrm{Zu}-$ gang explizit auf die Fahnen geschrieben: zum einen die Lebenslauf- und Biografieforschung, zum anderen die subjektorientierte Soziologie mit dem Konzept der Alltäglichen Lebensführung. Beide sollen im Folgenden kurz vorgestellt werden. 


\subsubsection{Lebenslauf- und Biografieforschung (Kohli, Elder)}

In seinem klassischen Ansatz der „Institutionalisierung des Lebenslaufes“ beschreibt Martin Kohli (1985, oder auch 1994 oder 2007) den historischen Prozess der Entstehung und Erosion eines standardisierten Normallebenslaufes in zwei Individualisierungsschüben, wie diese bereits kurz in Kapitel II skizziert wurden. Mit Blick auf die langfristige Modernisierungsgeschichte westlicher Gesellschaften stellt Kohli fest, dass sich der Ablauf von Lebenszeit und Lebensalter zu zentralen Strukturprinzipien herausgebildet hat. Eine Grundbedingung für diese Entwicklung ist eine zunehmende zeitliche Stabilität des Lebens, dessen Verlauf zu einer vorhersehbaren Größe wird, mit der das Individuum „rechnen“ und die nicht jederzeit durch einen überraschend eintreffenden Tod beendet werden könnte (Scherger 2007:20). Die Verzeitlichung und Chronologisierung des Lebenslaufes werden durch die neu entstehenden altersgeschichteten Systeme öffentlicher Rechte und Pflichten (etwa Schulsystem, Wahlrecht, Sozialversicherungssystem) vorangetrieben (Kohli 1985:8ff.). Kohli schreibt dabei dem Arbeitsmarkt mit seinen Altersnormen eine besondere Bedeutung zu und identifiziert das Bildungs- und Rentensystem als organisatorische Träger einer Sequenzierung der Lebensphasen. Evident ist die Organisation des Lebenslaufes um die Erwerbsarbeit, durch die jeweils eine Phase der Vorbereitung, der Aktivität und des Ruhestandes entsteht. Insbesondere das „Alter“ als eigenständige, zeitlich abgegrenzte Lebensphase stelle ein Novum dar. Kohli spricht davon, dass die soziale „Pragmatik der Altersgrenzen“ (Kohli 1985:10) auch die lebensweltlichen (Zeit-)Horizonte der Individuen strukturiert. Im Selbstverständnis der Individuen dient nun weniger der historische bzw. der jahreszeitliche Turnus als Verlaufsachse des Lebens, sondern sie konstituieren ein eigenständiges Ablauf- bzw. Entwicklungsprogramm ihres individuellen Lebens (Kohli 1985:12). Das Leben wird zu einem Projekt, das es selbst zu gestalten gilt (Kohli 1992:289). Es entstehen Standardantworten, auf die in der subjektiven Deutung des Lebenslaufes zurückgegriffen werden kann und die eine langfristige, reflexive Lebensplanung erleichtern. Die Vertreter der Individualisierungsthese (allen voran Beck, aber auch Kohli) gehen davon aus, dass das Ablaufprogramm des Lebenslaufes mit seinen flankierenden sozialstaatlichen Institutionen erst die Sicherheit und Kontinuität schafft, die individualisiertes biografisches Handeln ermöglicht und gleichsam eine biografische Kompetenz bzw. eine Fähigkeit zum "doing biography" erforderlich macht. Kohli prognostiziert Mitte der 1980er Jahre eine „Sprengung des chronologischen Korsetts" durch einen zweiten Individualisierungsschub, die zu einer Destandardisierung und Differenzierung der Lebenslaufmuster führen könnte. In den folgenden Jahrzehnten stellt Kohli (etwa 1993 oder 2007) eine überraschende Hartnäckigkeit des institutionalisierten Lebenslaufprogramms fest. 
Sowohl die Veränderungen in der Arbeitswelt als auch in den privaten Lebensformen hätten weit weniger drastische Auswirkungen auf die Sequenzialität des Lebens als zunächst erwartet. Altersgrenzen haben sich als resistent erwiesen und entfalten weiterhin eine starke Steuerungswirkung, selbst wenn diese in einem konfliktreichen Verhältnis zu einigen wesentlichen Aspekten des Wertekanons, allen voran der Flexibilität, und den systemischen Anforderungen zeitgenössischer Gesellschaften stehen (Kohli 2000:21 oder Kohli 2007:17). Zusammenfassend lassen sich weiterhin Tendenzen einer Destandardisierung und Differenzierung beobachten, von einem neuen Lebenslaufregime kann hingegen noch nicht gesprochen werden.

Den Kern dieses klassischen Ansatzes bildet die starke These, dass sich der Lebenslauf in modernen Gesellschaften als Institution, also ein verfestigtes strukturelles und normatives Muster, durchsetzt und deshalb als eigenständige gesellschaftliche Strukturdimension aufzufassen ist (Kohli 1985:1). Mit „Institutionalisierung“ meint Kohli also nicht nur die sozialstaatliche Rahmung des Lebenslaufes, sondern ein ganzheitliches Muster an komplex zusammenhängenden gesellschaftlichen Regelungen, die der Forderung, den Lebenslauf teleologisch $\mathrm{zu}$ ordnen, unterliegen und auf diese Weise eine Form gesellschaftlicher Integration bilden. Der Lebenslauf fasst, wie Scherger resümiert, als „Institution höheren Grades die Anforderungen und Handlungsangebote verschiedener anderer Institutionen ans Individuum zusammen und bündelt sie" (Scherger 2007:56). Es entsteht ein Muster, das einer einheitlichen, nicht notwendigerweise widerspruchsfreien, aber auf das Individuum zugeschnittenen Logik folgt.

Kohli bezeichnet den Lebenslauf in Anlehnung an Schütz und Luckmann als eine „Kontinuitätsidealisierung“ (Kohli 1986:191). Gemeint ist damit, dass das Leben in seiner Länge und in seinem Ablauf bestimmte idealisierte Formen annehmen kann, von denen eine strukturbildende Kraft ausgeht. Diese Ideale z.B. des dreigeteilten Normallebenslaufes werden nicht von allen geteilt und in Handeln umgesetzt. Das institutionalisierte Lebenslaufprogramm ist insgesamt als Anspruch oder als Leitnorm zu verstehen, eine Art „Normalitätsunterstellung“, die gleichzeitig auch die Existenz von ,sinnvollen“ Abweichungen ermöglicht (Scherger 2007:62). Die faktische Verbreitung eines Normal-Lebenslaufs, wie er sich statistisch erfassen lässt, muss sowohl von seiner normativen Verankerung als auch von sozialpolitischen Modellen differenziert, aber nicht isoliert betrachtet werden.

Die Institution des Lebenslaufes ist in den Worten von Monika WohlrabSahr eine „Ordnung, richtiger Zeit' und ,richtigen Zusammenhangs"“ (Wohlrab-Sahr 1992:12). Sie beinhaltet Altersnormen, Alterstypisierungen oder auch normativ geprägte Vorstellungen über die persönliche Entwicklung im Lebenslauf, die für bestimmte Lebensereignisse bestimmte Zeiten festschreiben. Auf einer objektiven Ebene stellt sich der Lebenslauf als Abfolge von Lebensereignissen dar, die in einem Zusammenhang stehen und nicht isoliert 
und situativ betrachtet werden können. Die Ablaufstruktur der Lebensereignisse liefert sozusagen das Material für die subjektive Interpretation des Lebens als Phasen und Übergänge. Die Individuen konstruieren einen bestimmten inhaltlichen Zusammenhang der Ereignisse, eine übergreifende Geschichte ihrer Biografie. Insofern sind spätere Ereignisse, Zukunftsvorstellungen und Erwartungen nur auf der Grundlage vorausgegangener Lebensgeschichte zu verstehen und zu erklären.

An dieser Stelle lässt sich der Ansatz Kohlis um einige von Glen H. Elder Jr, einem prominenten Vertreter der amerikanischen Lebenslaufsoziologie, eingeführte Grundbegriffe ergänzen. In seiner bekannten Studie der „Children of the Great Depression“ (Elder 1974) stößt Elder auf die Bedeutsamkeit gemeinsam erlebter historischer Ereignisse für die Lebenslaufgestaltung von Alterskohorten bzw. von historischen Generationen im Sinne Mannheims. Diese frühe Arbeit prägt seine Theoriebildung sowie weitere empirische Forschung. Elder differenziert zwischen drei temporalen Dimensionen des Lebenslaufes, die jeweils unterschiedliche analytische Perspektiven eröffnen (Elder 1975:167): a.) der individuelle Lebenslauf von der Geburt bis zum Tod umfasst den Prozess des Alterns und lenkt den Blick auf Sozialisation und Adaption als lebenslangen Prozess, b.) die soziale Zeit des Lebenslaufes drückt sich in einem sequentiellen Muster und Konfigurationen sozialer Rollen (Ehe, Ruhestand, etc.) aus, die im Wesentlichen durch Alter(n)snormen strukturiert werden. Sie geben ein angemessenes Alter für den Auszug aus dem Elternhaus, die Hochzeit oder den Ruhestand vor und sind mit einem informellen System an Belohnungen und negativen Sanktionen für die Einhaltung dieser Altersgrenzen verbunden, c.) die historische Zeit, in der sich Personen anhand ihres Geburtsjahres lokalisieren lassen und dann gemeinsam mit ihrer Kohorte die Altersschichten durchlaufen.

Zunächst betont Elder die Aussagekraft der historischen Dimension und fordert, gerade in Analysen mehrerer Generationen diese dritte Zeitebene einzubeziehen (Elder 1975). Später jedoch weist er dem „Alter“ und dem „generationalen Status“ ein unterschiedliches analytisches Potenzial zu (im Folgenden Elder 1985b:41; ähnlich Elder 1995:127): Die Präzision des chronologischen Alters entspricht nicht der zeitlosen Natur eines generationalen Status. Die Abfolge von Kind, Eltern und Großeltern ist universal, sie gilt in allen Zeiten und an allen Orten. Zudem können Personen einer familialen Generation eine Altersvarianz von mehr als 40 Jahren aufweisen, wenn etwa eine Frau bereits mit 30 Jahren oder erst mit 70 Jahren zum ersten Mal Großmutter wird. Das Alter trägt den historischen Kontext und Erkenntnisse über Altersdifferenzierung in die Analyse des Lebenslaufes herein, während die intergenerationale Analyse die gegebene Natur der Familienbeziehungen betont, den wechselseitigen Einfluss ihrer Lebensläufe und -pläne sowie die Generationen übergreifenden Prozesse, durch die historische Erfahrung und Einfluss übertragen werden. 
Die Dynamik des Lebenslaufes spiegelt sich langfristig in Form von Verlaufsmustern (trajectories) wieder, zu denen etwa Arbeit, Ehe, die Entwicklung von Verdienst oder Selbstbewusstsein zählen; kurzfristig zeigt sie sich in Form von Übergängen (transitions), einzelnen Lebensereignisse wie etwa Hochzeit, Scheidung, Einstellung oder Kündigung (Elder 1985b:31). Übergänge sind immer in Verlaufsmuster eingebettet, die ihnen eine spezifische Form und Bedeutung verleihen. Verlaufsmuster des Lebens zeichnen sich ab, wenn man die verschiedenen Stadien aufeinander folgender Jahre verbindet, etwa die unterschiedlichen Stadien der Beschäftigung, des Verdienstes oder der Gesundheit. Jedes Verlaufsmuster besteht aus einer Sequenz an Lebensereignissen und Übergängen, Veränderungen der Stadien, die mehr oder weniger abrupt erfolgen (Elder 1985b:31-32). Ein Verlaufsmuster kann also als ein Pfad verstanden werden, der durch den Prozess des Alterns bzw. den Lauf durch die Altersstruktur definiert wird.

Bei der Analyse einzelner Lebensläufe darf dabei nicht der breitere Kontext, in den sie eingebettet sind, vergessen werden. Das gilt sowohl für die historischen Umstände als auch die konkrete familiale Situation. Scheidung, Wiederheirat oder berufliche Verfehlungen sind nicht nur innerhalb des individuellen Lebenslaufes bedeutsam, sondern beeinflussen auch die Leben der Familienmitglieder und umgekehrt. Das Verwandtschaftsnetz bildet eine Matrix an sozialen Einflüssen und Unterstützung, Hindernissen und Optionen (Elder 1995:120). Diesen Umstand, dass die Leben verschiedener, meist verwandter Personen eng miteinander verbunden sind, bezeichnet Elder als „linked lives“, ein weiterer Begriff, der heute zum Standardvokabular in der Lebenslaufforschung zählt.

Stärker als Kohli betont Elder die Unterschiedlichkeit von Lebensläufen und Zeitplänen, von Optionen und Ressourcen und von Rollensequenzen und Aktivitäten (Elder 1975:174). Er interessiert sich für die Synchronisation der unterschiedlichen, miteinander verbundenen Lebenspfade (Elder 1995:105), die ineinander greifenden Verlaufsmuster und Übergänge, sowohl innerhalb eines Lebensabschnittes als auch über das Leben hinweg oder zwischen verschiedenen (verwandten) Personen. Elder ruft dazu auf, ein Lebensereignis in seinem historischen und familialen Kontext, seinem prozesshaften Gewordensein sowie seiner zeitlichen Platzierung im Lebenslauf zu analysieren. In den letzten Jahren widmet er zudem der Ebene von „human agency“ und „self-regulation“ eine erhöhte Aufmerksamkeit. Er weist darauf hin, dass Lebensentscheidungen nicht nur aus der Situation heraus erklärt werden können, sondern dafür auch die Situationsinterpretation, die Erfahrungen und die Lebensgeschichte einer Person herangezogen werden müssen. Elder schreibt den subjektiven Deutungen der Individuen, als einem kontinuierlichen Prozess der Konstruktion und Rekonstruktion, eine einflussreiche und handlungswirksame Rolle zu (Elder 1985b:43 oder Elder 1995:110). Insofern zeigt Elder ein zunehmendes Interesse an jener subjektiven Ebene des Le- 
benslaufes, die durch Kohli als Biografie gefasst und in seinen Abhandlungen zur Biografieforschung (etwa Kohli 1981 oder Fischer und Kohli 1987) genauer definiert wurde.

Der Lebenslauf lässt sich entsprechend aus zwei Perspektiven betrachten: einerseits den objektiven Regelungen, die ihn als Ablauf bestimmter Sequenzen und Karrieren konstituieren; anderseits als subjektive Deutungen dieser objektiven Regelungen, als biografische Handlungen, die sich von einem vorstrukturierten normativen Orientierungsrahmen anleiten lassen (Scherger 2007:20-21). Diese beiden Analyseebenen lassen sich der Lebenslaufforschung einerseits, verstanden als quantitative Untersuchung der objektiven Strukturen des Lebens(ver)laufes (etwa Elder, Mayer, etc.), und der Biografieforschung andererseits (etwa Rosenthal, Bude etc.), als qualitative Analyse der Orientierungsmuster, kohärenten Geschichten und individuellen Deutungen der Lebensereignisse, zuordnen. Beide Forschungsstränge haben sich in den letzten dreißig Jahren etabliert und sind als Sammelbecken unterschiedlicher Ansätze zu verstehen. Gemeinsam formen sie, was Elder (1995) als Lebenslauf-Paradigma bezeichnet. Die Besonderheit des klassischen Ansatzes von Kohli ist darin zu sehen, dass hier explizit und nicht nur ansatzweise beide Seiten einbezogen werden. Strukturen, Handlungen und subjektive Deutungen stehen in einem interdependenten, dynamischen und oft spannungsreichen Verhältnis. Das feste Gerüst an Handlungsorientierungen im Bereich der Lebensführung entlastet von Handlungsdruck, schränkt aber auch freies Handeln ein (Kohli 1985:19f.). Versteht man den Lebenslauf im Sinne Kohlis als Institution, lässt sich gerade die Verbindung der verschiedenen Teilbereiche des Handelns in den Blick bekommen, was sich in den letzten Jahren als einer der Schwerpunkte der Lebenslauf- und Biografieforschung entwickelt hat (Scherger 2007:22). Kohli formuliert, wie auch Bude, immer wieder den Anspruch, das „ganze Leben“ (Kohli 1981:291) oder die „Totalität des Lebens“ (Kohli 1981:290) erfassen zu wollen. Es ist sein Ziel, die Lebenslage von Menschen nicht nur aus den aktuellen sozialen Bedingungen, sondern auch aus ihrer Geschichte und damit auch aus subjektiver Erfahrung zu erklären (Voß 1991:100). Der Ansatz des Institutionalisierten Lebenslaufes fordert damit eine integrative Position nicht nur hinsichtlich einer ganzheitlichen Erfassung der funktional ausdifferenzierten Lebensbereiche, sondern auch hinsichtlich einer Dialektik aus Struktur und Deutungen.

Die Kritik am Ansatz Kohlis reibt sich im Wesentlichen an der starken Akzentuierung des Erwerbsbereiches. Kohli wird vor allem vorgeworfen, sich zu stark auf den traditionellen männlichen Normallebenslauf zu konzentrieren und damit den Bereich der Familie und der häuslichen Arbeit zu vernachlässigen. Scherger fasst die Kritik und die Entgegnungen Kohlis treffend zusammen (Scherger 2007:23): Kohli argumentiert, dass der überwiegende Bezug auf den männlichen Normallebenslauf implizit darauf verweise, dass Frauen im traditionellen Modell des männlichen Ernährers eben über ihre 
Ehemänner vergesellschaftet sind und auch in ihrer Lebenslaufgestaltung nicht nur ökonomisch von diesen Männern abhängen. Mit der zunehmenden Frauenerwerbstätigkeit löse sich diese Abhängigkeit aber auf und Frauen hätten selbst am Programm des Normallebenslaufs teil. Kohli habe es aber zumindest versäumt, dies in der klassischen Formulierung seiner Thesen explizit auszuführen.

Der Ansatz des Institutionalisierten Lebenslaufes, ergänzt um einige zentrale Begriffe von Glen Elder, bietet ein analytisches Gerüst, das dafür geeignet scheint, das Leben von Unternehmerfamilien in seiner Verflochtenheit $\mathrm{zu}$ untersuchen. Der ganzheitliche Blick einer solchen Lebenslaufperspektive lenkt den Fokus auf die Zusammenhänge und Wechselwirkungen zwischen einzelnen Lebenspfaden, Institutionen und Personen. Entsprechend kann der Einfluss des Unternehmens auf die Unternehmerfamilie in seiner Multidimensionalität erfasst werden. Inhaltlich lässt der starke Fokus auf den Erwerbsbereich die Unternehmerfamilie jedoch als ,sinnvolle Abweichung“ erscheinen. Sowohl Kohli (etwa in Kohli 1992) als auch Elder (1994) ziehen eine familieninterne Nachfolge als Beispiel des „Ganzen Hauses“ heran und damit einer Gesellschaft, die stärker durch Altersgruppen denn durch chronologisches Alter organisiert war und in der generationaler Verbundenheit noch eine existenzsichernde Bedeutung zukam. An Unternehmerfamilien lässt sich untersuchen, inwiefern auch eine „Randgruppe“ oder ein „Extremfall“ innerhalb des Erwerbssystems vom Programm des Institutionalisierten Lebenslaufes erfasst wird. Entfalten die üblichen Altersnormen auch im Bereich der selbständigen Erwerbstätigkeit ihren lebenslaufstrukturierenden Einfluss? In welchem Verhältnis stehen die Lebensläufe der Familienmitglieder in Unternehmerfamilien? Antworten auf diese Fragen lassen sich aus der Warte des Institutionalisierten Lebenslaufes als eine inhaltliche Erweiterung lesen.

\subsubsection{Alltägliche Lebensführung (Voß, Kudera)}

Der Forschungsansatz zur Alltäglichen Lebensführung, der im Rahmen der Münchener „Subjektorientierten Soziologie“ im Laufe der 1990er Jahre entstanden ${ }^{44}$ und seither ausgebaut und verfeinert worden ist, nimmt ebenfalls das gesamte Leben von Individuen in seiner Komplexität in den Blick und konzipiert das Verhältnis von Arbeit und Leben weniger als eine fixe Struktur denn als eine kontinuierliche, anspruchsvolle Aufgabe (Gottschall und Voß 2003:22). Das „Ganze“ wird als ein faktisches Resultat der komplexen

44 Entstanden ist dieses Konzept im Kontext des Forschungsprojektes „Alltägliche Lebensführung“ unter der Leitung von Karl Martin Bolte, unter Mitarbeit von Luise Behringer, Sylvia Dietmaier, Wolfgang Dunkel, Karin Jurczyk, Wener Kudera, Maria S. Rerrich und Günter Voß. Siehe etwa Behringer 1998; Jurczyk und Maria S. Rerrich 1993; Kudera und Voß Gerd 2000; Projektgruppe "Alltägliche Lebensführung" 1995 oder Voß 1991. 
Alltagshandlungen verstanden, als eine Praxisform und weniger als ein Sinnzusammenhang (Voß 2000:322). Es geht um das relativ stabile ,individuelle Arrangement" der Tätigkeiten von Personen in ihren verschiedenen Lebensbereichen oder um die Art und Weise, wie das Alltagsleben von Menschen in den für sie relevanten Lebenssphären zusammengehalten wird. Im Wesentlichen ist Lebensführung deshalb als ein stabiles Konglomerat aus Routinen, Gewohnheiten und gegenseitigen Absprachen in Familie, Betrieb und Freizeit zu verstehen, das für die Person im Alltag entlastend wirkt. Bei der Untersuchung von Lebensführung interessiert nun nicht so sehr die Beschaffenheit der einzelnen gesellschaftlichen Bereiche, die eine Person in ihrer Lebensführung berührt, sondern ,wie diese im Alltag miteinander ,verwoben“ sind“ (Voß 1991:131). Dieses System der Alltäglichen Lebensführung besitzt eine an das Subjekt gebundene, aber von ihr nur teilweise steuerbare strukturelle Eigenlogik. Und genau diese stellt einen zentralen Erkenntnisgegenstand der Lebensführungsforschung dar. Thematisiert wird, wie sich unter gegebenen, konkreten Bedingungen das Handeln der Person formt, so dass daraus eine „beschreibbare Struktur der Lebensführung wird“ (Voß 1991:131).

Der Ansatz der Alltäglichen Lebensführung nimmt wichtige Anregungen aus der Lebenslauf- und Biografieforschung auf. Beide Konzepte fordern explizit, sowohl die gesellschaftlichen Bedingungen bzw. Konstitutionen der Lebensgestaltung als auch die subjektiven Leistungen der individuellen „Verarbeitung“ bzw. die personale Konstruktion dieser Momente in der Gestaltung der eigenen Lebensform zu beachten. Sowohl in der „Biografie“ als auch in der „Lebensführung“ stehen Individuum und Gesellschaft in einem dialektischen Zusammenhang. Der Ansatz der Alltäglichen Lebensführung kritisiert den starken Fokus auf die diachrone Struktur des Lebenslaufes in der Biografieforschung. Die Komplexität des „ganzen Lebens“ werde durch den Blick auf die Biografie als durchgeformter Sinnzusammenhang, der Lebensanfang und Lebensende verbindet, selektiv reduziert (Voß 1991:101).

Konkret benennt Voß (1991) in seinen grundlegenden Überlegungen zur „Alltäglichen Lebensführung als Arbeit“ drei zentrale Differenzen der beiden Ansätze $^{45}$ : Erstens, das Konzept der Alltäglichen Lebensführung räumt dem Alltag eine praktische Vordringlichkeit vor der Biografie ein (Voß 1991:107). Ein Verständnis des alltäglichen Lebens wird als Basis für dessen

45 Günter Voß versteht das Konzept der Alltäglichen Lebensführung als einen allgemeinen heuristischen Bezugsrahmen Voß 1991:12, der sich klar zu bestehenden theoretischen Konzepten zu Alltag, Lebenswelt und Lebensführung positioniert. Intensiv diskutiert er die Arbeiten von Karl Marx sowie neuere marxistische Konzepten, die soziologischen Freizeitforschung, die Frauenforschung und die Gesellschaftsphilosophie von Jürgen Habermas, in denen er eine Dichotomisierung des „Lebens“ erkennt und zu überwinden sucht. Darüber hinaus grenzt er die praktische Lebensführung von ihrer aktiven Stilisierung mit der Funktion der expressiven Distinktion ab, wie diese in der Lebensstilforschung analysiert wird. 
langzeitlichen Verlauf verstanden. Erst in der alltäglichen Praxis entstehen die Anschlüsse für eine Lebensgeschichte mit Vergangenheit und Zukunft. Damit liegt der Fokus auf einer Logik des „Hier und Jetzt“, und das „Woher“ und „Wohin“ findet erst in zweiter Linie Beachtung. Zweitens, das Vermittlungsglied in der Subjekt-Gesellschaft-Dialektik ist von jeweils unterschiedlicher Qualität. Während Kohli vorschlägt, den Lebenslauf als Institution zu fassen, plädiert Voß aus einer subjektorientierten Sicht dafür, das System der alltäglichen Lebensführung als subjektive Konstruktion zu verstehen ohne dabei gesellschaftliche Prägungen in Abrede stellen $\mathrm{zu}$ wollen(Voß 1991:109). Drittens, wie die Biografie weist auch die Lebensführung eine „innere Form bzw. eine „funktionale Gestalt“ auf, die es zu rekonstruieren gilt. Dabei unterscheidet Voß die Analyseebenen der expliziten Selbststilisierung einer Person (was in etwa einer biografischen Perspektive entspricht), der latenten Regeln der Lebenspraxis und des sozialen Charakters der personalen Formen von Lebensführung.

Das Lebensführungskonzept fußt also auf den gleichen Grundannahmen und Zielen wie die Lebenslauf- und Biografieforschung, ist jedoch, in den Worten von Günter Voß, ,systematisch quer“ (Voß 1991:112) dazu angelegt: Während mit dem Blick auf die Lebensführung der synchrone, alltägliche Zusammenhang des Lebens in Erscheinung tritt, offenbart der Blick auf den Lebenslauf und die Biografie die Diachronie des „Lebens“. Während sich also die einen auf die Breite der alltäglichen Erfahrung konzentrieren, steht für die anderen das Leben in seiner Länge im Vordergrund.

Innerhalb des Ansatzes der Alltäglichen Lebensführung spielt der Begriff der Arbeit eine tragende Rolle. Dieser wird in einer sehr weiten Fassung als Tätigkeit verstanden. Die grundlegende Struktur der Lebensführung ergibt sich aus der Verteilung der alltäglichen Tätigkeiten auf die Lebensbereiche einer Person, die einen Zusammenhang formen. Die Alltägliche Lebensführung wird durch die „Arrangements“ in den einzelnen Lebensbereichen selbst zu einem temporalen, räumlichen, sachlichen, sozialen und medialen „Arrangement“ (Voß 1991:263). Es wird unterstellt, dass Lebensführung eine relativ autonome, konstruktive Leistung einer Person darstellt (Voß 2000:322). Diese Konstruktionsleistung ist in der Regel kein Produkt bewusster Planung und Organisation, sondern bedient sich praktischer Mechanismen, wobei Routinisierung eine herausgehobene Bedeutung zukommt. Dennoch kann die Koordination der unterschiedlichen Tätigkeiten Gegenstand von Reflektion (werden) und so potentiell rationalisiert werden. Voß geht davon aus, dass die Gesamtkomposition der Lebensführung über eine operative Tiefenstruktur verfügt und sich gleichzeitig Tendenzen zu einer harmonischen gestalthaften Schließung sowie einer Stilisierung beobachten lassen, die Lebensführung als eine ganzheitliche Form erscheinen lassen und in dieser erfassbar machen. Über den Lebenslauf hinweg weist die Lebensführung eher stabile Phasen der Repetition und eher instabile Phasen der 
Neuordnung auf. Innerhalb des Ansatzes der Alltäglichen Lebensführung wird der Person ein hohes Maß an Gestaltungsmöglichkeiten und -freiheiten im flexiblen Umgang mit Lebensressourcen und Lebensbedingungen zugeschrieben (Voß 1991:258). Trotzdem darf Lebensführung nicht mit der Kontingenz des Alltags verwechselt werden, sondern ist als aktive Begrenzung der potentiellen Vielfalt zu begreifen.

Die aktive Konstruktionsleistung der Person ist immer schon in gesellschaftliche Prozesse und Strukturen eingebunden. Im Laufe des Lebens verinnerlichen und erfahren die Personen kulturell stabilisierte Orientierungsmuster für die Gestaltung ihres Lebens, Verteilungsmuster der Tätigkeiten in bestimmten sozialen Lagen oder biografischen Situationen. Der Gestaltungsspielraum wird auf diese Weise von Entscheidungen entlastet, aber auch deutlich eingeengt (Voß 1991:313). Solche „Normalitätsfolien“, wie Voß sie nennt, signalisieren den Personen, welche Formen von Lebensführung sich in der Praxis bewährt haben. Sie erleichtern beispielsweise die Vermittlung zwischen unterschiedlichen Rollen. Aus der Perspektive der Alltäglichen Lebensführung wird der Person eine sozial integrierende Funktion in einer ausdifferenzierten Gesellschaft zugeschrieben.

„Es sind die Personen, die mittels ihrer Lebensführung die sozial getrennten und eigensinnigen Bereiche zu sich hin und damit faktisch miteinander vermitteln. Die Personen sind es, die so unterschiedliche Sphären wie die Familie und den Beruf, unter einen Hut ${ }^{6}$ bekommen müssen“ (Voß 1991:327).

Das Konzept der Alltäglichen Lebensführung wurde seit den 1990er Jahren theoretisch weiterentwickelt (etwa hinsichtlich der Identitätskonstruktion von Personen Behringer 1998) und in empirischen Studien zu Ein-PersonenSelbständigen oder Telearbeitern angewandt (siehe etwa die diversen Beiträge in dem Sammelband von Gottschall und Voß 2003). Günter Voß hat gemeinsam mit Hans Pongratz dieses Konzept als theoretischen Bezugsrahmen für ihre Analyse der sich wandelnden Arbeitsstrukturen im Posttaylorismus angewandt und daraus die These des „Arbeitskraftunternehmers“ entwickelt, auf die schon einige Male im Laufe dieser Arbeit verwiesen wurde. Aus den arbeits- und industriesoziologischen Debatten der 1990er Jahre, die vor allem um eine zunehmende Durchsetzung entstandardisierter Arbeitszeiten, neue Arbeitsformen wie etwa die Telearbeit oder die Forderung nach mehr Unternehmerkultur und Unternehmensethik kreisen, diagnostizieren sie (Voß 2000:310ff.) eine neue Qualität des betrieblichen Zugriffes auf Arbeitskraft, nämlich eine, die zu einer ,ganzheitlichen“ Erfassung der Person tendiert und das ganze alltägliche Leben tangiert. Auf diese Weise wird die Trennung von Arbeit und Leben, die maßgeblich durch das Lohnarbeitsverhältnis bestimmte wurde, aufgeweicht und von einer ,zunehmenden und neuartigen 
,Interessiertheit ' durchdrungen. Das gesamte Leben der Arbeitenden wird verstärkt zum expliziten Objekt betrieblicher Strategie“" (Voß 2000:319). Voß/Pongratz vertreten die These, dass sich die Ware Arbeitskraft strukturell verändere und die vorherrschende Form verberuflichter Arbeiternehmer durch einen neuen Typus ergänzt und langfristig sogar ersetzt werde (etwa Voß und Pongratz 1998:132), wobei Pongratz (2003) später die These dahingehend erweitert, dass eine dauerhafte Pluralität unterschiedlicher Arbeitskrafttypen für wahrscheinlicher gehalten wird.

Dieser neue Arbeitskrafttyp muss zunehmend als strategisch handelnder Unternehmer der eigenen Arbeitskraft auftreten (Voß 2000:327; Voß und Pongratz 1998:145), er muss seine Arbeitskraft weitgehend selbst organisieren, kontrollieren und anbieten. Die Begrifflichkeit des „Unternehmers“ verweist also auf eine spezifische Funktion im kapitalistischen Produktionsprozess ganz im Sinne eines marxistischen Verständnisses: auf der einen Seite der Unternehmer, der im Streben nach einer möglichst profitreichen Kapitalverwertung in effizienter Weise Ressourcen einsetzt, auf der anderen Seite der Arbeiter, der seine Arbeitskraft gegen eine Lohnzahlung ausbeuten lässt. Sowohl die Betriebsführung des Unternehmers als auch die Lebensführung des Arbeiters stellen systematische Tätigkeitsformen dar, unterscheiden sich aber in ihrer praktischen Zielsetzung. Aus diesem Grund warnt Voß davor, die Analogie der strategischen Betriebsführung und der alltäglichen Lebensführung zu überziehen. Stattdessen fragt er nun nach den veränderten alltagspraktischen Strategien, die „Arbeitskräfte“ der neuen Qualität des ganzheitlichen, betrieblichen Zugriffes entgegenstellen (Voß 2000:324). Er stellt fest, dass die konkrete Ausgestaltung des Alltags und insbesondere des Verhältnisses zwischen Arbeit und Privatheit in einem immer stärkeren Maße aktiv und individuell an die jeweilige Situation anzupassen sind:

„Individuelle Zeitökonomie wird zunehmend bedeutsam, Alltagstechnik muss selbstverständlich und kompetent zur Unterstützung eingesetzt werden, die individuellen Fähigkeiten müssen sorgsam gepflegt und systematisch weiterentwickelt werden, soziale Beziehungen müssen geschickt gestaltet werden, Konsum ist aktive Reaktion wie auch bewusste Investition, Motivierung, Ablenkung und Belohnung, räumliche Mobilität ist unausweichlich und muss geschickt arrangiert werden uvm.“" (Voß 2000:326).

Die Sphären von Arbeit und Leben treten so in ein wesentlich komplexeres und wechselseitig durchdrungenes Verhältnis, das verstärkt aktiv und individuell gestaltet wird. Die Lebensführungen der Personen unterscheiden sich damit stärker voneinander. Ihnen ist jedoch gemein, dass die Intensität des Erwerbsbereiches nun einen stärker prägenden Einfluss ausübt. 
Das Konzept der Alltäglichen Lebensführung in Kombination mit der These des Arbeitskraftunternehmers liegt damit ganz auf einer Linie mit Max Webers Vorstellungen einer aktiven Lebensführung und seiner These eines Rationalisierungsprozesses im sich entwickelnden Kapitalismus. Voß et al. nehmen für sich in Anspruch, die These, die Max Weber nur für eine Elite, das calvinistische Unternehmer- und Großbürgertum, formuliert hätte, zu verallgemeinern und (neben anderen Faktoren) als eine Folge sich erweiternder Formen des betrieblichen Zugriffs auf die Subjektivität von Arbeitskräften und deren Leben zu fassen. Allerdings wird hier die Rationalisierungsthese Webers ebenso wie sein Verständnis einer Wahlverwandtschaft zwischen der asketischen Lebensführung der calvinistischen Unternehmer und der rationalen Lebensweise sehr eng ausgelegt. Auffällig ist darüber hinaus, dass im Kontext des „Arbeitskraftunternehmers“ die Begrifflichkeit des „Unternehmers" nur im Sinne seiner Funktion als strategischer Betriebsführer angeführt und ansonsten systematisch vermieden wird. Geht es um die konkrete Beschreibung der Lebensführung, werden „Selbständige“, ,freie Berufe" und ,autonom eingesetzte Fach- und Führungskräfte“ als diejenigen Berufstätigen benannt (Voß 2000:333; Voß und Pongratz 1998:145), an denen sich bisher diese neue Qualität der Lebensführung beobachten ließ, die sich nun auf eine breitere gesellschaftliche Gruppe ausweite. Auch in den empirischen Untersuchungen wird der klassische Unternehmer, dessen Lebensführung Weber so reichhaltig beschreibt, vernachlässigt und nur solche Formen der Arbeitskraft analysiert, die in einem mediären Verhältnis zwischen Weisungsabhängigkeit und Selbständigkeit stehen, beispielsweise AlleinSelbständige, freie Mitarbeiter oder weibliche Telearbeiter.

Das blasse Verständnis des „Unternehmers“ im Konzept der Alltäglichen Lebensführung verwundert. In einem subjektorientierten Ansatz tauchen „die“ Unternehmer nur in ihren funktionalen Rollen auf. Und obwohl Unternehmer und Selbständige als zentrale Referenzpunkte und Vergleichsgruppen für eine „Entgrenzung von Arbeit und Leben“ (Gottschall und Voß 2003:23) gehandelt werden, fehlt es an ihrer genaueren begrifflichen Bestimmung und Abgrenzung ebenso wie an einer empirisch fundierten Analyse ihrer gegenwärtigen Alltagspraxis. Stattdessen wird m.E. mit allgemeinen Annahmen und klassischen Idealvorstellungen verfahren. Hinsichtlich einer Analyse von Unternehmerfamilien eröffnet das Konzept der Alltäglichen Lebensführung dennoch eine Chance, die gelebte Verbindung von Unternehmen und Familie ganzheitlich zu fassen. Zugleich kann die hier angestrebte empirische Untersuchung dazu beitragen, eine wesentliche Lücke dieses Konzeptes zu schließen. 


\subsubsection{Die Analyseebenen Lebenslauf, Biografie und Allägliche Lebensführung}

Sowohl der Ansatz des Institutionalisierten Lebenslaufes als auch jener der Alltäglichen Lebensführung stellen Herangehensweisen an das ,ganze Leben" dar. Wie systemtheoretische Ansätze operieren sie unter der Prämisse einer funktional differenzierten Gesellschaft. Sie interessieren sich allerdings weniger für die einzelnen Handlungsbereiche und ihre Logiken, sondern stellen ihren Zusammenhang in den Vordergrund. Beide Ansätze nehmen für sich in Anspruch, einen spezifischen Modus gesellschaftlicher Integration zu beschreiben: Die unterschiedlichen Lebenssphären und Rollen verbinden sich in synchroner Weise in der alltäglichen Lebensführung und in diachroner Weise im Lebenslauf, wobei beide Theorieangebote von einem dialektischen Verhältnis aus Handlungen und Strukturen ausgehen. Sie erfassen damit exakt jenes Wechselverhältnis zwischen einer strukturellen Eigenlogik, die sich aus der Verbindung von Unternehmen und Familie ergibt, sowie den aktiven Konstruktionsleistungen der Familienmitglieder.

Darüber hinaus stehen beide Ansätze in einem konstitutiven Verhältnis zueinander. Der Lebenslauf kann als eine Abfolge der alltäglichen Lebensführungen zu verschiedenen Zeitpunkten verstanden werden. Die getroffenen Tätigkeitarrangements müssen sich den Lebensumständen und Lebensereignissen anpassen, sie bilden aktuelle Möglichkeiten und Präferenzen ab. Im Prozess des Alterns entsteht eine Pfadabhängigkeit, die sich in der jeweiligen Gestaltung der alltäglichen Lebensführung ausdrückt. Gemeinsam bilden Lebenslauf und alltägliche Lebensführung die Vergangenheit der Person, den Erfahrungsraum, aus dem sich die biografische Selbstsicht und Lebensplanung speist. Alltägliche Lebensführung wiederum gewinnt durch den biografischen Horizont Sinn und lebenszeitliche Perspektive. Insofern ist „die alltägliche Lebensführung die individuelle alltagspraktische Ausgestaltung der Biografie" (Hildebrandt et al. 2009:26).

Trotz dieses konstitutiven Verhältnisses ist jedoch festzuhalten, dass beide Perspektiven unterschiedliche Logiken und Zeitstrategien verfolgen, wie Hildenbrandt ausführt (2009:26-27). Alltagshandeln ist einer Reproduktionslogik und synchronen Zeitorganisation verpflichtet, der Bewältigung von vielfältigen, gleichzeitig aufeinander treffenden Interessen und Anforderungen. Sowohl der Lebenslauf als auch die Biografie hingegen folgen einer Entwicklungslogik des Lebens und einer diachronen Zeitorganisation, die sich stärker an dem Nacheinander aufeinander aufbauender Lebensphasen orientieren. Alltägliche Lebensführung einerseits und der biografisch reflektierte Lebenslauf andererseits finden immer gleichzeitig und ergänzend statt, da einzelne Lebensbereiche und Lebensphasen nicht „stillgelegt" werden können. (Hildebrandt et al. 2009:26-27). Gemeinsam ergeben sie die kreative 
Dynamik des Lebens, die sich als spannungsreiche Kombination aus Stabilität und Innovation darstellt.

Im Zuge dieser Arbeit soll der Versuch unternommen werden, beiden Ansätzen gerecht zu werden. Jeder für sich gibt Hinweise darauf, wie mit dem Untersuchungsobjekt Unternehmerfamilie verfahren werden kann, und stellt ein spezifisches Erkenntnisinteresse in den Vordergrund. Aus der Perspektive eines Institutionalisierten Lebenslaufes kann die Unternehmerfamilie als ein zeitlich stabiles Kollektiv verstanden werden, das über das Leben mehrerer Generationen hinweg gemeinschaftlich ein Projekt verfolgt. Eine lebenslauf- und biografietheoretisch fundierte Analyse lässt Antworten erwarten auf Fragen nach dem Grad des Einflusses des Unternehmens auf die Lebenslaufgestaltung einzelner Familienmitglieder, dem Zusammenhang der Leben der verschiedenen Familienmitglieder oder der Tradierung spezifischer Handlungsmuster. Aus der Perspektive der Alltäglichen Lebensführung kann die Unternehmerfamilie als ein praktisches Arrangement unterschiedlicher Lebensbereiche gefasst werden, das Fragen danach ermöglicht, wie diese Lebensbereiche koordiniert werden und welche Lebensführungsmuster sich herausbilden. Aber erst durch die Kombination beider Ansätze kann es gelingen, nicht nur die Mechanismen und sozialen Formen der Unternehmerfamilien offen zu legen, die sich in der alltäglichen Lebensführung herausbilden und als stabil erweisen, sondern auch ihren Pfad im Leben einzelner Familienmitglieder sowie über die Generationenfolge hinweg nachzuzeichnen und zu verstehen. Der Einfluss des Unternehmens kann auf diese Weise in der Breite des täglichen Lebens ebenso wie in der Tiefe der aufeinander folgenden Lebensereignisse untersucht werden. Umgekehrt trägt die empirische Untersuchung von Unternehmerfamilien zur inhaltlichen Ergänzung beider Ansätze bei.

Phyllyis Moen (1998) stellt in ihrem Aufsatz "A life course approach to the entrepreneurial family" eine interessante Forschungsagenda vor, die der Komplexität dieser sozialen Einheit Rechnung trägt. Zwar knüpft Moen überwiegend an die Lebenslaufperspektive Glen Elders sowie Erkenntnisse aus der amerikanischen Forschung zu Altern und Lebenslauf an, zudem haftet dem etwas unscharf verwendeten Begriff der ,entrepreneurial family“ der Unterton einer „Gründungsfamilie“ an. Dennoch liefern ihre zahlreich formulierten Forschungsfragen, die bislang einer empirischen Umsetzung entbehren, fruchtbare Ansatz- und Aufmerksamkeitspunkte, die sich leicht auf die in dieser Arbeit angestrebte Kombination der Ansätze zum Institutionalisierten Lebenslauf und der Alltäglichen Lebensführung sowie der Unternehmerfamilie als Familie eigener Art erweitern und übertragen lassen.

Eine Lebenslaufperspektive, so Moen, fordere die traditionellen Arten, wie das Wechselspiel aus Unternehmen und Familie untersucht wird, heraus. Es gelingt, die eingeschlagenen Lebenswege, Rollenveränderungen und Statuspassagen, also den dynamischen Entwicklungsprozess, nachzuzeichnen 
und auf diese Weise die Verwobenheit von Arbeit, Familie und Gemeinschaft, die sich mit dem Altern der Familienmitglieder sowie dem Voranschreiten der Geschichte wandeln, herauszuarbeiten. Mittels der Kategorien Timing, Prozess und Kontext stellt Moen drei Lebenslaufthemen vor, die das Verständnis für die Eigenarten von Unternehmerfamilien erhöhen.

(1) Timing (zeitliche Abstimmung) bezieht sich auf die Auftretenshäufigkeit, Dauer und Abfolge von Rollen. Informationen über die Dauer und soziale Form der unternehmerischen Aktivität erhalten, setzt man sie zum Alter und den Lebensphasen der Familienmitglieder ins Verhältnis, eine zusätzliche Qualität. Moen betont, dass etwa das Timing einer Unternehmensgründung bzw. einer Nachfolge im Leben einer Person nicht unabhängig von anderen Arbeits- und Familienentscheidungen ebenso wenig wie von kulturellen Altersnormen über den ,richtigen Zeitpunkt“ einer beruflichen Veränderung gesehen werden kann.

(2) Die Kategorie Prozess legt den Fokus darauf, wie unterschiedliche Rollen ausgespielt werden. Moen fordert, dass es die Art und Weise zu analysieren gilt, in der einzelne Lebensbereiche und die einzelnen Lebensläufe der Familienmitglieder zusammenwirken, in welchem Verhältnis beispielsweise Arbeits- und Familienverpflichtungen oder die beruflichen Entscheidungen von Geschwistern stehen. Hinter dieser Forderung steht auch die Vermutung Moens, dass die Lebensläufe der Individuen in Unternehmerfamilien in einem hohen Maß über Zeit und Generationen verbunden sind (linked lives).

(3) Kontext beschreibt eine Sammelkategorie sozialer Einflüsse auf die Form von Unternehmerfamilien. Moen fasst darunter in erster Linie persönliche (Familienstand, Bildung, Familiengröße) und historisch gebundene (Krisenereignisse, Wertvorstellungen, Normen) Faktoren. Besondere Aufmerksamkeit widmet sie zudem den Aspekten Generation, Geschlecht, situative Imperative und später auch Gesundheit, die für ein Verständnis der Rollenaufteilungen, gelebten Verbindungen zwischen den Lebensbereichen und Lebenslaufentscheidungen ausschlaggebend sein könnten. Beispielsweise könne eine plötzliche Erkrankung zu einer vorgezogenen Nachfolgesituation führen oder die Pflege von Familienmitgliedern (Kinder, Kranken oder Alten) Kapazitäten aus dem Unternehmen abziehen.

Im Sinne der oben ausgeführten Ansätze lässt sich jede dieser Kategorien einmal in der Entwicklungslogik und diachronen Zeitstruktur des Lebenslaufes und der Biografie und einmal in der Reproduktionslogik und synchronen Zeitstruktur der alltäglichen Lebensführung analysieren. Auf der Analyseebene der Alltäglichen Lebensführung können die zeitliche Abstimmung und Koordination verschiedener Lebensbereiche (Timing) sowie die stabilen Strategien zur Koordination verschiedener Lebensbereiche (Prozess) innerhalb einer spezifischen Lebensphase ebenso in den Blick genommen werden wie die sozialen Erwartungen und Anforderungen in ihrer alltagspraktischen 
Bearbeitung und beispielsweise die Wirksamkeit gesellschaftlicher Altersnormen in Unternehmerfamilien analysiert werden (Kontext). Die Analyseebene des Lebenslaufes lenkt den Fokus hingegen stärker auf die Platzierung eines Lebensereignisses im Werdegang der Person (Timing), das Zusammenwirken früherer und aktueller Koordinationsweisen (Prozess) und bestehende institutionelle Kontexte (Wohlfahrtsstaat, soziale Sicherungssysteme) und legt einen Vergleich der Lebenslaufmuster von Familienunternehmern mit dem „Normallebenslauf“ nahe. Schließlich kann die Analyseebene der Biografie Erkenntnisse darüber liefern, ob die Personen den Zeitpunkt der Rollenausübung als angemessen, zu früh bzw. zu spät einschätzen im Sinne einer biografischen Selbstsicht und sich daraus ableitender Zukunftsdeutungen (Timing), wie die Personen über die aktuelle Lebensweise und sich daraus ableitende Lebenspläne reflektieren (Prozess) und inwiefern sich Familienunternehmer in ihren jeweiligen sozialen Kontexten verstrickt sehen bzw. diesen als Herausforderung oder Hindernis für die eigene Entwicklung biografisieren.

Zieht man Timing, Prozess und Kontext als zentrale Kategorien innerhalb der Analyseebenen der Alltäglichen Lebensführung, des Lebenslaufes sowie der Biografie bei einer Untersuchung von Unternehmerfamilien in Betracht, so werden die Querverbindungen der Lebensläufe und Lebensphasen offensichtlich. Es entsteht eine Sensibilität für die Relevanz aktueller Situationsdefinition in später gewählten Anpassungsstrategien, für den Einfluss von Lebensereignissen auf die Leben mehrerer Familienmitglieder und für die unterschiedliche Qualität einer Verbindung von Familie und Unternehmen in der alltäglichen Lebensführung oder der biografischen Selbstsicht.

\subsubsection{Eingrenzung und Präzisierung der Forschungsfrage}

Diese Arbeit reiht sich in die deutschsprachige soziologische Forschung zu Unternehmerfamilien ein, die auf ihr Untersuchungsobjekt aus einer modernisierungstheoretischen Perspektive blickt. Im Unterschied zu einer rein systemtheoretischen Perspektive soll mit den Leitbegriffen Lebenslauf, Biografie und Alltägliche Lebensführung das ,ganze Leben“ der Unternehmerfamilien eingefangen werden. Gemeint ist damit keine kleinteilige Analyse des Alltagsgeschehens, sondern ein Ansatz, der versucht, die alltäglichen Tätigkeiten der Familienmitglieder zur Aufrechterhaltung der Unternehmerfamilie in ihrem zeitlichen Verlauf zu verstehen.

Ausgehend von der Annahme, dass die Familien eigener Art von einer strukturellen Eigenlogik geprägt sind, rückt der Einfluss des Unternehmens auf das Leben und das Beziehungsgefüge in Unternehmerfamilien in den Mittelpunkt. Es soll einerseits untersucht werden, welche konstanten Routinen, Handlungsregeln und Beziehungskonstellationen der Besitz und die Lei- 
tung eines „Familienunternehmens“ für die Lebensführung von Unternehmerfamilien mit sich bringt. Anderseits gilt es, die Handlungs- und Interpretationsspielräume näher zu bestimmen: Wie bewältigen und interpretieren die Familienmitglieder den Einfluss des Unternehmens? In welcher Weise formt das Unternehmen die Lebensläufe von Mitgliedern aus Unternehmerfamilien? Welche Konsequenzen lassen sich für die Beziehung der Familienmitglieder zueinander erkennen? Und schließlich: Inwiefern werden Interpretations- und Beziehungsmuster über die Generationen hinweg tradiert?

Im Zentrum der Analyse steht also nicht so sehr die Genese einer Unternehmerfamilie (Gründung etc.), sondern der Einfluss eines bestehenden Unternehmens auf eine Familie und ihre Reproduktion. Die Handlungsentscheidungen einzelner Familienmitglieder werden in Bezug auf sich ergebende strukturelle Einschränkungen der Handlungswahl reflektiert. Die komplexe Wirklichkeit der Unternehmerfamilien wird nicht auf abstrakte Systemzusammenhänge reduziert. Stattdessen wird der alltägliche, private und spontane Charakter dieser Familien eigener Art durch das Wechselspiel aus Handlungen sowie den sich ergebenden Strukturen beschrieben.

Der Fokus der Analyse liegt auf der Gegenwart, erhebt jedoch den Anspruch, die Geschichte der jeweiligen Familien in ihrem historischen Kontext einzuordnen und Generationeneffekte mitzudenken. Wesentliche Aspekte bilden die Integration der jeweils geltenden gesellschaftlichen Vorstellungen unternehmerischen und familialen Handelns, die Wahl möglicher Vergleichsgruppen und die Positionierung der Unternehmerfamilie gegenüber ihrer Außenwelt. Der Zusammenhalt und die kollektive Identität der Familien eigener Art spielt eine prominente Rolle. Die Rede von „den“ Unternehmerfamilien darf nicht zu dem kurzsichtigen Schluss führen, dass es sich hier um eine homogene Gruppe handele. Vielmehr müssen bei einer Analyse sowohl die Gemeinsamkeiten der Lebenslauf- und Biografisierungsmuster und Praxen der Lebensführung als auch deren Unterschiede thematisiert werden. Die Kategorien Timing, Prozess und Kontext in den Analyseebenen Lebensführung, Lebenslauf und Biografie versprechen einen differenzierten und systematischen Zugang zum Forschungsobjekt, der Aussagen über das individuelle Leben, die Unternehmerfamilie als Kollektiv sowie ihre gesellschaftliche Einbettung ermöglicht. 



\section{Grundzüge der empirischen Vorgehensweise}

\subsection{Methodologische Vorüberlegungen}

Eine methodologische Positionierung im Raum interpretativer bzw. qualitativer Sozialforschung scheint für die vorgestellte Fragestellung angemessen. ${ }^{46}$ Denn es ist die Alltagswelt der Unternehmerfamilien, die in das Zentrum des Interesses rückt. Und es ist die Alltagswelt, die von Individuen und Gruppen als wirklich erfahren wird, die den Gegenstand des interpretativen Paradigmas markiert. ${ }^{47}$ Den theoretischen Ausgangspunkt bildet die Annahme, dass diese Alltagswelt nicht natürlich gegeben ist, sondern erst intersubjektiv entsteht. Durch Erziehung, Sozialisation und Resozialisation internalisieren Mitglieder von Unternehmerfamilien einen kollektiv geteilten Wissensvorrat an Deutungsmustern, Handlungsregeln, Normen etc., die ihnen als gegeben und unabhängig von einer Person erscheinen. In ihrem alltäglichen Umgang mit dem Unternehmen bedienen sie sich dieses Wissensvorrates und tragen interaktiv zu seiner permanenten Erneuerung bei. Die methodologische Überlegung, dass sich Bedeutungen und Regeln sequenziell in interaktiven Prozessen herausbilden und verändern, spiegelt sich in der theoretischen Vorannahme wider, dass Mitglieder aus Unternehmerfamilien gefordert sind, aktiv eine Grenze zwischen Unternehmen und Familie herzustellen. Berger und Luckmann (1998) betonen den Doppelcharakter der Alltagswelt: Einerseits ist sie nur subjektiv zugänglich und erfahrbar, anderseits besitzt sie objektive Faktizität. Es entsteht eine Gleichzeitigkeit von subjektiver und objektiver Erfahrung des sozial konstruierten Alltags. (Berger und Luckmann 1998:20)

Sozialwissenschaftliche Konstruktionen bauen auf solchen Konstruktionen des Alltags auf. (Schütz 1971:68) Die interpretative Sozialforschung48

46 Während die Bezeichnung „qualitative Sozialforschung“ eher auf eine Abgrenzung zu einer „quantitativen“ Methodologie zielt, soll mit der Bezeichnung ,interpretative Sozialforschung“ oder „rekonstruktive Sozialforschung“ (etwa Bohnsack 2010) eher das gemeinsame und konstitutive Element einer Reihe von ähnlichen Ansätzen hervorgehoben werden. Insgesamt hat sich noch kein einheitlicher Begriff für dieses methodologische Paradigma etabliert. Rosenthal 2008:14.

47 Für eine Einführung in die interpretative Sozialforschung siehe etwa Bohnsack 2010; Rosenthal 2008; Flick 2009; Mayring 2002; Lamnek 2006; Przyborski und Wohlrab-Sahr 2008.

48 Die interpretative Sozialforschung sieht sich in der Tradition einer Verstehenden Soziologie (Max Weber), des Symbolischen Interaktionismus, der phänomenologischen Wissenssozio- 
rekonstruiert die Bedingungen, Merkmale und Auswirkungen sozialer Alltagsphänomene in ihrem jeweiligen Verweisungszusammenhang. Insofern steht die qualitative Sozialforschung auf einer gedanklichen Grundlage, die sich an Bedeutungen, Kontexten, Interpretationen, Verstehen und Reflexivität orientiert. Mittels methodisch-kontrollierten Fremdverstehens vollzieht die Forscherin die Bedeutungskonstruktionen und ihre Handlungswirksamkeit in Unternehmerfamilien in ihren verschiedenen Sinnebenen ${ }^{49}$ nach. Jeder einzelne Fall, so die grundlegende Idee, bildet seine Besonderheit in Auseinandersetzung mit dem Allgemeinen aus. Deshalb ist es möglich, durch die Rekonstruktion der Fallstruktur die Verschränkung von Allgemeinem und Besonderem zu erkennen und zu über den Einzelfall hinausweisenden Aussagen zu gelangen. Handlungen gilt es nicht nur deskriptiv zu beschreiben, sondern im Sinne von ,thick descriptions“ (Geertz 1973) auch ihren Bedeutungskontext offen zu legen.

Rekonstruktive Forschung folgt einer „Logik des Entdeckens“ (Rosenthal 2008:14) von Zusammenhängen, Mustern oder Typen, die es erlauben, empirisch-fundierte Theorien über soziale Sachverhalte zu formulieren, und will nicht schon vorhandene Hypothesen prüfen. ${ }^{50}$ Das Postulat der Offenheit (Hoffmann-Riem) in allen Schritten des Forschungsprozesses soll den explorativen Charakter verfestigen. Den verschiedenen Ansätzen interpretativer Verfahren sind darüber hinaus noch einige andere Prinzipien gemein. Genannt werden in diesem Zusammenhang etwa Flexibilität, Explikation oder Kommunikation. (Lüders 2008:634; Hollstein und Ullrich 2003) Die Abgrenzung der einzelnen Begriffe erscheint jedoch unscharf, und es fehlt an einem grundlegenden Konsens sowie einer Verbindlichkeit, wie diese Prinzipien umgesetzt werden können. Lamnek fasst deshalb diese Prinzipien mit Recht nur vorsichtig als „Forschungsprogrammatik“ (Lamnek 2006:21) zusammen.

Die gegenwärtige Diskussion um eine gemeinsame Denkhaltung der qualitativen Sozialforschung kann als Entgegnung auf eine immer lauter werdende Kritik an der Zersplitterung des methodologischen Paradigmas in verschiedene Schulen gelesen werden. Es stehen sich etwa die Grounded Theory (Glaser/Strauss), die Konversationsanalyse (Sacks), die objektive Hermeneutik (Oevermann), die Narrationsanalyse (Schütze), die qualitative

logie und der Ethnomethodologie. Lamnek 2006; Flick 2009; Rosenthal 2008; Przyborski und Wohlrab-Sahr 2008.

49 Die Klassifizierungen und Bezeichnungen solcher Sinnebenen sind über die unterschiedlichen Schulen qualitativer Sozialforschung hinweg uneinheitlich. Hollstein und Ullrich identifizieren drei grundsätzliche Sinnebenen: subjektiver, objektiver und sozialer Sinn. Hollstein und Ullrich 2003.

50 Die Darstellung des Forschungsstandes hat verdeutlicht, dass die vorhandene theoretische und empirische Literatur zu Unternehmerfamilie nur rudimentär entwickelt ist. Insofern wäre jegliche Form der Hypothesenformulierung vorab eher willkürlich. 
Inhaltsanalyse (Mayring), die Diskursanalyse (Foucault) oder die Dokumentarische Methode (Bohnsack) gegenüber. Diese Schulen unterscheiden sich nicht nur in ihrer methodischen Vorgehensweise - hermeneutische, sequentielle und/oder kategorisierende Auswertung von meist verschriftlichter Kommunikation -, sondern setzen auch unterschiedliche erkenntnistheoretische Schwerpunkte. Mit der zunehmenden Verbreitung, Institutionalisierung und Internationalisierung qualitativer Forschung differenzieren sich die Erhebungs- und Auswertungsverfahren immer feiner aus. Auf einer pragmatischen Ebene werden sie in einem bunten Methodenkoffer vereint, der je nach Forschungsfrage eine angemessene Vorgehensweise bereithält. Der positive Effekt ist darin zu sehen, dass sich auf diese Weise eine „qualitative Wende“ (Mayring 1989) vollzog und es gelang, das Image eines bloßen Gegenprogrammes zu quantitativer Forschung allmählich abzustreifen (Esser 2007:351; Kelle 2008; Kelle und Erzberger 2008). Qualitative Forschung sieht sich heute - zumindest in Deutschland bzw. im deutschsprachigen Raum - einem geringeren Legitimationsdruck ausgesetzt (Reichertz 2007). Die starke Konzentration auf die jeweilige Praxis einer Methode drängt jedoch die methodologischen Differenzen in den Hintergrund. Kritiker warnen vor einer ,verstärkten Kanonisierung der Methoden bei gleichzeitiger Zersplitterung und Beliebigkeit“ (Reichertz 2009:Absatz 14), die eine gemeinsame methodologische Diskussion behindere.

Bestandteil der gegenwärtigen Diskussion ist auch der Ruf nach einheitlichen Qualitätsstandards in der interpretativen Sozialforschung (Seale 1999/2007; Lüders 2008:634; Steinke 2007:323f.), die sich aus ihrem Gegenstand ableiten ließen. Als zentrale Norm wird dabei die Nachvollziehbarkeit des Interpretationsprozesses gehandelt. In der gegenwärtigen amerikanischen Diskussion (für einen Überblick siehe Flick 2005) werden die erkenntnistheoretischen Grundlagen des interpretativen Paradigmas wieder belebt. ${ }^{51}$ Im Mittelpunkt steht die Frage, wie die Forscherin Zugang zur sozialen Welt erhält und auf welche Weise sich ihre Ergebnisse darstellen lassen (Roulston 2010; Frost et al. 2010). Es geht also weniger darum, wie sich die alltäglichen Konstruktionen der Wirklichkeit vollziehen, sondern um ihre sozialwissenschaftliche Bearbeitung. Dabei zählt die korrekte Anwendung von Erhebungs- und Auswertungsverfahren weniger als die Grundlagen und die Praxis der Interpretation (Denzin 2000).

Diese Erwägungen zum Stand aktueller interpretativer Sozialforschung bringen methodische Konsequenzen für die Analyse der Alltagswelt von Unternehmerfamilien und den Aufbau dieser Arbeit mit sich. Der Auswahl des

51 Im deutschsprachigen Raum findet sich in der breiten Einführungsliteratur zu diesem Feld (siehe Fußnote oben) meist in der Einleitung ein methodologischer Exkurs. Insgesamt entsteht jedoch der Eindruck, dass diese Diskussion in den 1960er und 1970er Jahren sehr präsent war und heute abgeschlossen ist. 
empirischen Materials kommt eine Schlüsselrolle zu: Es soll die alltäglichen Konstruktionen von Unternehmerfamilien möglichst gut einfangen und ihren Verweisungszusammenhang erhalten. Der interaktive Prozess von Bedeutungszuschreibungen soll sichtbar werden. Diese Auswahl folgt gleichermaßen erkenntnistheoretischen (kann die Forscherin mittels dieser Daten etwas über die soziale Wirklichkeit in Unternehmerfamilien erfahren? Kapitel 5.2) wie auch pragmatischen (Zugänglichkeit der Daten; Kapitel 5.3) Gesichtspunkten. Für die Bearbeitung des empirischen Materials dient die Geisteshaltung von Offenheit und Reflexivität über den gesamten Forschungsprozess hinweg als zentraler Orientierungspunkt. Die Forschungsfrage wird immer wieder in Interaktion mit dem Forschungsobjekt nachjustiert und präzisiert. In einem zirkulären Prozess werden aus dem empirischen Material theoretische Annahmen über den Einfluss des Unternehmens auf die Familie entwickelt. Über die Generalisierbarkeit der Ergebnisse wird ebenso reflektiert wie über ihre Darstellung - beides findet seinen Ausdruck auch in separaten Abschnitten (Abschnitte 6 und 7) in diesem Kapitel. Schließlich wird der allseits geforderten Nachvollziehbarkeit des Interpretationsprozesses insofern Rechnung getragen, als die einzelnen Schritte des Forschungsprozesses und, darin eingeschlossen, auch die Entscheidungen für bestimmte Erhebungs- (Abschnitt 4) und Auswertungsmethoden (Abschnitt 5) detailliert und an einem konkreten Beispielfall dargestellt werden. Auf diese Weise soll der Leser in die Lage versetzt werden, selbst die „Qualität“ der Interpretationsarbeit zu beurteilen.

\subsection{Lebensgeschichten als Zugang zur Alltagswelt}

Die alltägliche Welt der Unternehmerfamilien wird im Rahmen dieser Arbeit über die Lebensgeschichten ihrer Mitglieder erschlossen. Denn um das Erleben des Unternehmenseinflusses verstehen und erklären zu können, ist es notwendig, diesen eingebettet in den Gesamtzusammenhang des Lebenslaufs sowie der biografischen Konstruktionen und der daraus resultierenden Gegenwarts- und Zukunftsperspektiven zu interpretieren. Der Einblick in die Geschichte der Person und ihrer Familie erlaubt es, das interessierende Phänomen in seiner Entstehungs- und Entwicklungsdynamik zu rekonstruieren.

Lebensgeschichtliche Schilderungen treten beispielsweise in Form von Erzählungen, Briefen oder Tagebucheinträgen auf. Das Dargestellte, wie etwa eigenerlebte Situationen, Zukunftsperspektiven oder auch Fiktives, ist geprägt durch die vergangenen Erlebnisse, ihre biografische Bearbeitung sowie die konkrete Interaktionssituation. Der Begriff der Lebensgeschichte mit seinem narrativen Unterton wird an dieser Stelle bewusst verwandt, um von 
den theoriehaltigen Konzepten des Lebenslaufes als Institution (Kohli 1985), des Lebensverlaufs als zeitlicher Abfolge des Lebens (Mayer 1990; Blossfeld und Huinink 2001), der Biografie als reflexiver Konstruktionsleistung des Subjektes (Alheit und Dausien 2000; Rosenthal 1995; Fischer und Kohli 1987), der biografischen Kompetenz (Sackmann 2007:52f.) dafür sowie der alltäglichen Lebensführung zu unterscheiden. Lebensgeschichten werden hier als empirisches Material begriffen, das nach den objektiven Gegebenheiten, den subjektiven Erlebnissen, den Sinnsetzungen und den biografischen Konstruktionen befragt werden kann. Insofern erfolgt diese Arbeit im Sinne einer mit qualitativen Methoden arbeitenden Biografieforschung (biografischen Methode) im Unterschied zu einer mit quantitativen Methoden arbeitenden Lebensverlaufsforschung, die sich vorwiegend mit den "faktischen“ Ereignissen im Lebenslauf beschäftigt.

Bereits eine Reihe von Arbeiten im Rahmen der so genannten Chicago School nutzte umfassend die Erinnerungen von Menschen in Form von biografischen Auskünften als Materialressource zu rekonstruktiven Zwecken. Das lange Zeit ungelöste Problem mit dieser Datenart war aber, welche Aussagekraft die Analyse einer narrativen Lebensgeschichte überhaupt besitzt. Denn die Lebensgeschichte eines Menschen ist eine subjektgebundene Geschichte mit Brüchen und Ungereimtheiten. Manches wird weggelassen, anderes hinzugefügt, was sich vielleicht ganz anders ereignet hat (Bäcker 2008:46). Einen wissenschaftlich akzeptablen Ausweg aus diesem Dilemma eröffnete Mitte der siebziger Jahre Fritz Schütze (etwa 1983 und 1984) mit seiner Annahme der Übereinstimmung des Stromes der vergangenen Erfahrungen mit dem Erzählfluss in der Stegreiferzählung (Homologiethese), die noch heute einen wichtigen und kritisch reflektierten Bezugspunkt der deutschsprachigen Biografieforschung markiert (Fischer-Rosenthal und Rosenthal 1997:410; Hitzler 2002). In ihrer Habilitationsschrift hat sich Gabriele Rosenthal (1995, insbesondere:27-98) dem Verhältnis von Erleben, Erinnern und Erzählen gewidmet. Die Gegenwartsperspektive, so Rosenthal, bedingt die Auswahl der Erinnerungen, die temporalen und thematischen Verknüpfungen von Erinnerungen und die Art der Darbietung der erinnerten Erlebnisse. Das bedeutet, dass im Verlauf des Lebens jeweils neue erinnerte Vergangenheiten entstehen. Im Erinnerungsprozess erscheinen bestimmte Themen relevanter als andere. Durch Begegnungen oder Erlebnisse können sich die Relevanzen verändern und plötzlich zurückliegende Alltagssituationen in einem anderen Licht erscheinen. Die Erlebnisse werden nun in einen anderen Sinnzusammenhang eingebettet. Auf diese Weise formen zuvor zufällige Begebenheiten miteinander verknüpfte Bestandteile oder in anderen Worten ein ,thematisches Feld“. Rosenthal nennt eine Reihe von Faktoren, die solche Reinterpretationen der Vergangenheit und Gegenwart, aber auch der Zukunft, zur Folge haben können: den öffentlichen Diskurs und die gesellschaftliche Entwicklung (z.B. Bild von Unternehmerfamilien vs. Mana- 
gern in der Öffentlichkeit), Veränderungen im Familiensystem (z.B. Erkrankung eines nahen Familienmitgliedes), im Berufsleben (z.B. unerwartete Arbeitslosigkeit) oder einschneidende Widerfahrnisse (z.B. Krankheit). Die Dialektik zwischen Gegenwart und Vergangenheit, wie sie Rosenthal entwirft, beinhaltet auch, dass diese Konstruktionen der Vergangenheit aus der Gegenwart nicht als eine jeweils von der erlebten Vergangenheit losgelöste Konstruktion zu verstehen sind. Vielmehr sind die auf Erinnerungen beruhenden Darstellungen eigenerlebter Erfahrungen durch das Erleben in der Vergangenheit mitkonstituiert.

Die dialektische Beziehung zwischen Erleben, Erinnern und Erzählen bedeutet also unter anderem: „die in der Vergangenheit liegenden Erlebnisse können sich dem Biographen in der Gegenwart des Erinnerns und Erzählens nicht darbieten, wie sie erlebt wurden, sondern nur im Wie ihrer Darbietung“" (Rosenthal 1995:20). In diesem Sinne verweisen Lebensgeschichten auf beide Zeitdimensionen: auf das heutige Leben mit einer spezifischen Vergangenheit und auf das damalige Erleben. Rosenthal weist auch darauf hin, dass selbst fiktive Erzählungen, also erfundene Geschichten, die dazu dienen, Erlebnisse zu verdecken bzw. die eigene Biografie umzuschreiben, ihren Realitätsgehalt in dem Sinne haben, dass sie in ihrem Inhalt und ihrer Struktur auf das zu Negierende verweisen. ${ }^{52}$

Darüber hinaus sind lebensgeschichtliche Darstellungen in unterschiedliche soziale Rahmungen eingebettet, wie etwa informelle alltägliche Zusammenhänge (z.B. Familie oder Freundeskreis) oder formal organisierte Kontexte (z.B. Interview). Diese sozialen Rahmungen sind in modernen Gesellschaften verknüpft mit funktionalen Teilbereichen der Gesellschaft wie z.B. dem Rechtssystem, dem Ausbildungssystem, dem Berufssystem oder der Wissenschaft. Gesellschaftliche, institutionelle und gruppenspezifische Regeln bzw. die Regeln unterschiedlicher Diskurse geben vor, was, wie, wann und in welchen Kontexten thematisiert werden darf und was nicht. Die individuelle Geschichte eines Menschen und die kollektive Geschichte, die subjektiven und die kollektiven Wirklichkeiten, durchdringen sich wechselseitig (Rosenthal 2008:172). Die Lebensgeschichte ist immer beides: ein individuelles und ein soziales Produkt. (Rosenthal 2002a:142) Insofern ist bei der Analyse von Lebensgeschichten zu berücksichtigen, dass die jeweiligen Rahmungen Regeln für die Artikulation biografischer Ereignisse vorgeben und dieser Umstand das Thematisierte wie das Nichtthematisierte mitbestimmt.

Diese methodologischen Überlegungen verdeutlichen die Vorteile, aber auch die Grenzen von Lebensgeschichten als Zugang zur Alltagswelt in Un-

52 Auch Gerd Vonderach 1997 nimmt in seiner Geschichtenhermeneutik Bezug auf die Dialektik von Erinnern, Erzählen und Erleben. Er erweitert den Begriff der Lebensgeschichte um das philosophische Konzept von Wilhelm Schapp. 
ternehmerfamilien. Die biografische Perspektive trennt nicht von vornherein in unterschiedliche Systemwelten oder Funktionsbereiche von Unternehmen und Familie, sondern erhält deren Gesamtheit. Mit dem zeitlichen Fokus auf das ganze Leben wird der Annahme einer lebenslangen Sozialisation des Individuums empirisch entsprochen. Wann genau der Unternehmenseinfluss zu wirken beginnt, wie ausgeprägt dieser in unterschiedlichen Lebensphasen ist und was zentrale Wendepunkte in diesem Prozess sind, all das sind Fragen, die über die Analyse von Lebensgeschichten beantwortet werden. Die biografischen Aufschichtungen von Mitgliedern aus Unternehmerfamilien geben Auskunft über die lebenslange, individuelle Bearbeitung des Sozialen. Mittels Lebensgeschichten wird es möglich, die Prozesse des Unternehmenseinflusses genauso wie die alltägliche Aufrechterhaltung bzw. die immer wieder erneute handlungspraktische Herstellung des Soseins als Unternehmerfamilie zu rekonstruieren. Bei lebensgeschichtlichen Analysen geht es zudem nicht nur um eine Betrachtung der biografischen Selbstdefinitionen von Individuen, sondern auch um die Analyse von Zuschreibungen, die von anderen Personen ausgehen. Im Falle von Mitgliedern aus Unternehmerfamilien ist etwa $\mathrm{zu}$ fragen, welchen Zuschreibungen durch andere diese im Zusammenhang der Firma und in anderen Lebensbereichen ausgesetzt sind und wie sich diese auf ihre Handlungen und biografischen Konstruktionen auswirken. Insofern können die Wechselwirkungen zwischen Fremddefinition und Selbstdefinition in den Blick genommen werden. Darüber hinaus können die Generationenbeziehungen, die Merkmale dieses kollektiven Zusammenhangs ebenso wie generationale Weitergaben interpretativ nachvollzogen werden. Der Analyse wird lebensgeschichtliche Tiefe verliehen, indem Aussagen über die Veränderung des Einflusses in verschiedenen Lebenslagen möglich sind sowie Aussagen über die aktuelle Darstellungsweise dieser sozialen Einheit.

Mit der Wahl von Lebensgeschichten als Zugang zur Alltagswelt von Unternehmerfamilien folgt diese Arbeit auch auf empirischer Ebene den Grundzügen einer Individualisierungstheorie und der Vorstellung, dass sich Biografien als Mittel sozialer Strukturierung breitenwirksam durchsetzen (wie in Kapitel II dargestellt). Die theoretische Vorannahme, dass sich Mitglieder einer Unternehmerfamilie durch deren paradoxe Struktur in einer Situation mit besonderem Handlungs- und Orientierungsbedarf befinden, findet damit auch in der Wahl des Datenmaterials seinen Ausdruck. Aus den Lebensgeschichten der Mitglieder einer Unternehmerfamilie lässt sich rekonstruieren, inwiefern sie sich einem gesellschaftlichen Rechtfertigungsdruck ausgesetzt sehen und welche Lösungsstrategien zu dessen Bewältigung sie entwerfen und aufrechterhalten. 


\subsection{Fallauswahl}

Mit der Entscheidung für einen biografietheoretisch fundierten Forschungsansatz wird gleichzeitig das Forschungsfeld weiter eingegrenzt. Im Mittelpunkt stehen nun die Lebensgeschichten von Unternehmerfamilien und ihren Mitgliedern. Die Forschungsfrage und die angestrebte Vorgehensweise legen gleichsam eine Doppelstruktur des Falles fest: die individuelle Ebene des Lebens der Familienmitglieder einerseits und die kollektive Ebene des Beziehungsgefüges der Familien anderseits.

Zur Analyse von Lebensgeschichten stehen grundsätzlich verschiedene Datenquellen zur Verfügung. Dazu gehören etwa schriftliche Daten wie Autobiografien, Briefe, Tagebucheinträge, Chroniken, aber auch verbale Daten wie Gespräche mit Einzelpersonen oder Gruppen. Im Kontext dieser Untersuchung bieten sich insbesondere letztere als primäre Quelle an. Lebensgeschichtliche Gespräche haben sich nicht nur in der bisherigen Forschungspraxis zum Thema bewährt, sondern sie erlauben es auch, relativ pragmatisch die gegenwärtige Perspektive des Biografen näher zu bestimmen. Die methodologischen Überlegungen haben offen gelegt, dass Lebensgeschichten zu unterschiedlichen Zeitpunkten im Lebenslauf verschieden dargestellt werden. Insofern ist die gegenwärtige Perspektive auf das eigene Leben eine wichtige Basis, um die Aussagen der Gesprächspartner über bestimmte Themenbereiche und Erlebnisse in der Vergangenheit verstehen und erklären zu können. Ein einheitlicher Erhebungszeitraum sowie die Auswahl einer konkreten Lebensphase, in der sich die Gesprächspartner befinden sollen, tragen zur Vergleichbarkeit der Fälle bei. Diese zeitliche Eingrenzung des Forschungsfeldes wurde wie folgt umgesetzt:

Die analysierten Daten wurden in den Jahren 2007, 2008 und 2009 erhoben. Als Besonderheit ist anzumerken, dass im Herbst 2008 die Finanzkrise die deutsche Wirtschaft erfasste. Entsprechend ist das Material vor dem Hintergrund einer allgemeinen Unsicherheit und eher schlechten wirtschaftlichen Entwicklung zu deuten.

Als einheitlicher „Moment“ in den Lebensgeschichten von Unternehmerfamilien wurde der Transfer des Unternehmens an die nächste Generation gewählt. Denn hier trifft man die Familienmitglieder, wie in Kapitel 3.5 herausgearbeitet, in zentralen Phasen ihres Lebenslaufes an: Während die jüngere Generation eine Karriereentscheidung trifft, sieht sich die ältere Generation mit dem Ruhestand konfrontiert. Üblicherweise können solche Übergangsphasen im Lebenslauf als Zeiten der Reflektion über den eigenen Werdegang verstanden werden, die eine biografische Bearbeitung erfordern (siehe etwa Riessman 2008). Darüber hinaus kann Nachfolge als Prozess verstanden werden, in dem die Generationen ihre jeweiligen Rollen wechselseitig anpassen müssen (Handler 1990 spricht von „,mutal role adjustment“). In- 
sofern markiert sie eine „Extremsituation“, aus der sich Schlüsse über die soziale Strukturierung der Generationenbeziehungen in Unternehmerfamilien ziehen lassen. Solche Fälle, in denen keine transgenerationelle Übertragung des Unternehmens erfolgt oder diese Versuche „scheitern“, werden in der Untersuchung nicht weiter betrachtet. An ihnen ließe sich das Ende des Unternehmenseinflusses auf die Familie beobachten und nicht seine Reproduktion. ${ }^{53}$

Zusammenfassend wird das Forschungsfeld auf die Lebensgeschichten von Unternehmerfamilien begrenzt, die aktuell einen Generationentransfer durchleben oder jüngst durchlebt haben. Es gilt, die Merkmale und die Dynamik der spezifischen Konstellation einer Unternehmerfamilie sowie ihre Bewältigung zu erfassen.

Doch wie erhält man Zugang zu solchen Familien, die - wie in der öffentlichen Diskussion oft betont - gerne im Verborgenen bleiben? Aus pragmatischen Gründen wurde der Weg über das Unternehmen gewählt. Entsprechend muss in der Interpretation der Fälle mitgedacht werden, dass die Gesprächspartner sich eher in ihrer Rolle als Unternehmer denn als Privatmenschen angesprochen fühlen (sofern sie hier einen Unterschied machen). Zur weiteren Konkretisierung der Fallauswahl wurde entsprechend auf bewährte Kriterien der Forschung zu Familienunternehmen zurückgegriffen: Das Unternehmen sollte mehrheitlich (mindestens $50 \%$, bei AGs mindestens 25\%) im Besitz einer Familie sein. Mindestens ein Familienmitglied sollte aktiv im Management oder in einem Kontrollgremium des Unternehmens tätig sein..$^{54}$ Die „Übergabe“ des Unternehmens sollte innerhalb der letzten fünf Jahre (ab 2003) erfolgt oder für die nächsten fünf Jahre geplant sein. So wird die „Aktualität“ der Übergabe konkretisiert und ein zeitlicher Horizont abgesteckt. Es wird jedoch den Befragten überlassen, was genau unter einer „Übergabe“ des Unternehmens zu verstehen ist, und damit ihre Deutung dieses Prozesses nicht vorweg genommen.

Trotz dieser Eingrenzungen und Konkretisierungen ist im Lichte der in Kapitel III vorgestellten Literatur das Forschungsfeld sehr heterogen. Ob und wie das Unternehmen an die nächste Generation weitergegeben wird (und

53 Retrospektiv (im Lichte der hier vorgestellten Ergebnisse) bilden solche Familien jedoch eine interessante Vergleichsgruppe. An ihnen ließen sich die beschriebenen Koordinationsaufgaben und Konflikte in Unternehmerfamilien weiter untersuchen. Darüber hinaus knüpft sich als weitere Forschungsfrage an, wie sich die spezifische Lebensweise und die soziale Identität als Unternehmerfamilie auch bei „weichenden Erben“ und „NichtNachfolgern“ auswirkt, also der Einfluss des Unternehmens auch ohne Unternehmen in die nächste Generation wirkt.

54 Astrachan et al diskutieren verschiedene Operationalisierungen von Familienunternehmen und entwickeln eine Skala (F-PEC Scale), durch die der Grad des Familieneinflusses auf das Unternehmen erfasst werden soll. Als zentrales Kriterium heben sie die Dimension der Macht, verstanden als Eigentum, Management und Kontrolle, hervor. Astrachan et al. 2002; Holt et al. 2007; Klein et al. 2005. 
damit der Einfluss des Unternehmens auf die Familie erneuert wird), ist von einer Reihe sozialstruktureller Einflüsse abhängig. Um die Heterogenität des Forschungsfeldes abbilden zu können, wird in einem ersten Schritt ein qualitativer Kriterienkatalog entwickelt, mit dessen Hilfe ein selektives Startsample (Merkens 2007:292; Lamnek 2006:192) erhoben werden kann. Ziel ist es nicht, die Population aller Familienunternehmen repräsentativ abzubilden. Vielmehr soll die Bandbreite sozialstruktureller Einflüsse annäherungsweise erfasst werden, indem die betrachteten Einzelfälle theoretisch relevant erscheinende Merkmale widerspiegeln. Die Kategorisierung ist als vorläufig zu betrachten und, obwohl diese aus der Literatur abgeleitet wurde, im Hinblick auf die Fragestellung doch als relativ willkürlich zu beurteilen. Kurz gesagt, lassen sich die erarbeiteten Kriterien in zwei Gruppen zusammenfassen:

Erstens Faktoren, die das Unternehmen betreffen. Dazu gehören die Branchenzugehörigkeit sowie die Größe des Unternehmens. (Lansberg 1999) (Kapitel 3.2) Beide könnten sich in erheblichem Maße auf den Tätigkeitsbereich und die Ausbildungsvoraussetzungen für Familienmitglieder auswirken, die im Unternehmen aktiv sind. Sie bestimmen den Beruf einiger Familienmitglieder und die Aufgaben, die ihnen im Alltag begegnen. Damit verbunden sind die Ertragskraft und die Zukunftsperspektive des Unternehmens, die sich wiederum auf den Lebensstil der Familie sowie die Attraktivität für die jüngere Generation niederschlagen könnten. Auch die Identifikation mit dem Produkt durch die Familienmitglieder mag von Branche zu Branche variieren. In das Sample einbezogen werden sollten Unternehmen aus den drei Sektoren. Sie sollen in Branchen tätig sein, die eine gewisse Nähe zwischen Familie und Unternehmen erfordern (Familie als Marke und Ressource) und die über eine übertragungswürdige Masse verfügen (deshalb werden EinPersonen-Betriebe ausgeschlossen).

Zweitens Faktoren, die den Nachfolgeprozess betreffen: Eine Reihe von Kriterien können - so die Literatur zu Nachfolge in Familienunternehmen die Ausgestaltung des Nachfolgeprozesses und damit die Tradierung des Unternehmenseinflusses innerhalb der Familie beeinflussen: Wird das Unternehmen vom Gründer in die zweite Generation oder spätere übergeben? Ist die Nachfolge von langer Hand geplant oder durch ein tragisches Lebensereignis verursacht? Wie viele Personen sind aktiv am Nachfolgeprozess beteiligt (Lansberg 1999)? Und in welchem Verwandtschaftsgrad und Geschlechtsverhältnis stehen diese zueinander? (siehe hierzu auch Kapitel 3.5) Für die Auswahl der Fälle stehen keine Häufigkeitsverteilungen der jeweiligen Nachfolgegeschichten zur Verfügung. Insofern ist es schwer zu beurteilen, ob eine Übergabe von beispielsweise einer Mutter auf den Sohn einen üblichen oder einen Extremfall darstellt. Das Sample versucht jedoch anhand dieser inhaltlichen Kriterien eine möglichst große Heterogenität abzubilden.

Jeder Fall berührt alle Auswahlkriterien und deckt bestimmte Konstellationen $\mathrm{ab}$. Vorab wurden lediglich die unterschiedlichen Ausprägungen der 
Kriterien und ihre wünschenswerte Abdeckung formuliert, nicht aber spezielle Fallgeschichten beschrieben.

Abbildung 5: Auswahlkriterien

\begin{tabular}{|c|c|c|}
\hline \multirow[t]{2}{*}{ 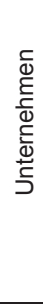 } & Sektor / Branche & $\begin{array}{l}\text { Agrar Beispiel Weinbau/Sektkellerei } \\
\text { Industrie: Beispiele verarbeitendes Gewerbe, insbe- } \\
\text { sondere KFZ-Zulieferer, Metall, Fahrzeugbau, Le- } \\
\text { bensmittel, Umwelttechnik } \\
\text { Dienstleistung: Beispiele private Banken, Unterneh- } \\
\text { mensberater, Gastronomie }\end{array}$ \\
\hline & Unternehmensgröße & $\begin{array}{l}\text { Kleinstunternehmen werden nicht berücksichtigt (Ein- } \\
\text { Mann-Betriebe); KMU; Großunternehmen }\end{array}$ \\
\hline \multirow{4}{*}{ 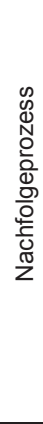 } & Generation & $\begin{array}{l}\text { Übergabe von Gründer auf 2. Generation } \\
\text { Übergabe auf spätere Generation }\end{array}$ \\
\hline & Art der Übergabe & $\begin{array}{l}\text { Nur familieninterne Übergaben } \\
\text { Geplante Übergabe } \\
\text { Plötzliche Übergabe (z.B. durch tragisches Lebenser- } \\
\text { eignis) }\end{array}$ \\
\hline & $\begin{array}{l}\text { Anzahl und Verwandt- } \\
\text { schaftsgrad der Nachfol- } \\
\text { ger }\end{array}$ & $\begin{array}{l}\text { Recycled controlling owner } \\
\text { Sibling partnership } \\
\text { Cousin consortium }^{55}\end{array}$ \\
\hline & Geschlecht & $\begin{array}{l}\text { Beteiligung von Frauen am Generationentransfer in ak- } \\
\text { tiven Rollen. }\end{array}$ \\
\hline
\end{tabular}

Quelle: eigene Darstellung

Die Rekrutierung der gewünschten Fälle erfolgte über sehr unterschiedliche Wege: direkte Anfragen mit Bezug auf Zeitungsartikel; Vermittlungen über Branchenverbände, Unternehmensberater oder Journalisten; persönliche Kontakte oder Aufrufe in Branchenzeitschriften. In einem ersten allgemeinen Telefonat (meist mit dem Sekretariat) wurden die Kriterien für Familienunternehmen sowie die weiteren Merkmale des Kataloges kurz abgefragt. Falls ein Unternehmen den Vorstellungen entsprach, wurden die Mitglieder der Unternehmerfamilie postalisch für ein Gespräch angefragt. Die Rekrutierung verlief relativ problemlos - ganz im Gegensatz zu den geringen Rücklaufquoten, die quantitative Befragungen von Familienunternehmen in der Vergangenheit erzielt haben. Insgesamt wurden auf diese Weise sechs Unternehmerfamilien analysiert. Auf Basis der sich herausbildenden Muster und Typen konnte das selektive Sample evaluiert werden. Im Laufe der Auswertung stellte sich - überraschenderweise - heraus, dass für eine Typisierung des Unternehmenseinflusses auf die Familie die Unternehmenskriterien (also Branche und Größe) eine weniger wichtige Rolle zu spielen scheinen. Zudem

55 Dese Begriffe wurden bereits in Kapitel 3.4.3 (Generation) näher erläutert. 
wurde deutlich, dass die Kriterien, die sich zunächst am Nachfolgeprozess orientieren sollten, in hohem Maße demografische Faktoren der jeweiligen Familienverhältnisse und -geschichten widerspiegeln. Sukzessive wurden sechs weitere Unternehmerfamilie in die Untersuchung aufgenommen. Deren Auswahl orientierte sich nun stärker an den Familienstrukturen (z.B. Kinderzahl, Patch-Work-Familie, Beteiligung von drei Generationen, etc.) sowie an der Besonderheit der Fälle, die nun nicht mehr nur als Nachfolgen im engeren Sinne verstanden werden können (z.B. Rückkauf eines Unternehmens, gemeinsame Gründung durch Eltern und Kinder, Unternehmen im Nebenerwerb etc.). Denn gerade bei der Analyse ungewöhnlicher Fälle können soziale Strukturen besonders deutlich hervortreten (Kelle und Kluge 1999:49). Insgesamt folgt der zweite Teil der Fallauswahl stärker den Forderungen des „theoretical sampling“ (Glaser und Strauss 2009; van Hoonaard 2008:874), bei dem auf Basis der Datenauswertung des jeweiligen Falles entschieden wird, welche Daten als nächste erhoben werden sollen und insofern die entstehende Theorie den Prozess der Datenerhebung kontrolliert.

\subsection{Narrative biografische Interviews}

Im Zusammenhang mit lebensgeschichtlich bezogenen Fragestellungen wird besonders häufig das narrative Interview eingesetzt (Hopf 2007:355), das in den 1970er Jahren von Fritz Schütze im Kontext der „Arbeitsgruppe Bielefelder Soziologen" eingeführt und begründet und seither methodisch und methodologisch weiterentwickelt wurde. (Rosenthal 1995:186-207, 2002b) Es setzt die Forderung nach einer offenen Interviewführung (siehe hierzu die klassischen Aufsätze von Hopf 1978 und Kohli 1978) konsequent um. Die Interviewerin ermuntert ihren Gesprächspartner zur freien Rede (Stegreiferzählung) und überlässt ihm damit die Wahl der Reihenfolge der Gesprächsthemen. (Kohli 1978:7) Sie räumt dem Befragten einen breiten Spielraum der Strukturierung und Äußerung subjektiver Deutungen, Alltagstheorien oder Selbstinterpretationen ein. (Hopf 1978:99) Das narrative Interview soll Erzählungen hervorlocken (Ayres 2008:545), gemeint ist damit eine spezifische „Textsorte“, die Abfolgen von tatsächlichen oder fiktiven Ereignissen in ihrem Zusammenhang wiedergibt und dabei einen hohen Indexikalitäts- und Detaillierungsgrad erreicht.

Schütze geht davon aus, dass solche verdichteten Erzählungen diejenigen sprachlichen Texte sind, die es am ehesten erlauben, die Handlungsabläufe, das Erleben der Befragten und ihre Orientierungsstrukturen nachzuvollziehen (Rosenthal 2008:139). Gelingt es, die Interviewten ins Erzählen und damit ins Erinnern zu bringen, dann fließen in ihre Geschichten nicht nur die Dar- 
stellungen der Handlungen, sondern auch die damit verbundenen Emotionen ein. Zudem werden die so genannten „Zugzwänge des Erzählens“ in Kraft gesetzt. Sie bewirken, dass die Erzählenden versuchen, ihre Geschichten zu Ende zu bringen (Gestaltschließungszwang), zentrale Punkte in nötiger Detailfülle auszuführen (Detaillierungszwang), aber auch, dass sie ihre Erzählung beschränken müssen (Kondensierungszwang). Das Ergebnis ist ein Erzähltext, der die Entwicklung und Wandlung einer Lebensgeschichte und mit ihr der biografischen Identität kontinuierlich darstellt (Schütze 1983:286). Es werden eine Vielzahl von einzelnen Stationen des Lebens geschildert und richtungsweisende Entscheidungen von zum Teil lebenslanger Tragweite in den Blick genommen.

Im Umgang mit dem Interviewmaterial ist zu bedenken, dass sich im Prozess der Erinnerung Relevanzen ändern und bestimmte Ereignisse und Zusammenhänge ausgespart werden, die vielleicht früher einmal wichtig waren. Aktuelle Orientierungen und biografische Vergangenheit stehen in einer Wechselwirkung. (Kohli 1991) Die Zugzwänge des Erzählens wirken zwar auf die (beinahe) Vollständigkeit von Zusammenhängen und plausible Anschlüsse für den Zuhörer hin, können jedoch das Problem nicht gänzlich beseitigen (doch mehr als andere Interviewverfahren). (Przyborski und Wohlrab-Sahr 2008:222) Das unvollständige Wissen und eventuell „,falsche“ Erinnern des Erzählenden ist Gegenstand einer kritischen Auseinandersetzung mit der Güte von Interviewmaterial, die aktuell in der angloamerikanischen Ethnographie wieder belebt wird. (Walford 2007:147f.)

Mit narrativen Interviews, die einzeln mit den Mitgliedern von Unternehmerfamilien geführt werden, kann das Leben der befragten Person in den Blick genommen werden. Die Forscherin signalisiert, dass die Individualität der Familienmitglieder ernst genommen wird und versucht, eine offene Atmosphäre zu schaffen, in der Konflikte thematisiert werden können. Die Befragten erhalten Raum, ihre biografische Selbstsicht zu entfalten. Gleichsam kann eine zu intensive Selbstproduktion den Zugang zu den eigentlich interessanten Themen versperren. Eine Reihe von Arbeiten zu Mehrgenerationenfamilien hat gezeigt, dass sich die einzelnen Interviews in Beziehung zueinander setzen lassen und auf diese Weise Tradierungen zwischen den Generationen sichtbar werden. (Vierzigmann und Kreher 1998; Schallberger 2003; Rosenthal 1999; Rosenthal und Völter 1997) Trotzdem liefern Einzelinterviews Material, in dem nur aus je individueller Perspektive auf die Vorstellungen von Familie und ihre Verstrickungen geschlossen werden kann. Die Gruppendynamik und die kollektive Analyseebene werden in erheblichem Maße von der Forscherin konstruiert.

Insofern stellte sich die Frage, ob solche narrativen Interviews nicht noch mit Familiengesprächen - eine besondere Form des Gruppeninterviews (Bohnsack 2007; Hildenbrand 2005:28ff.) - kombiniert werden sollten. Der Vorteil von Familiengesprächen ist darin zu sehen, dass nicht nur Aussagen 
von einzelnen Personen erhoben werden, sondern ein Eindruck der Familiendynamik entsteht. In den Blick genommen werden können kollektive Orientierungen, Wissensbestände und Werthaltungen. Das Verfahren stößt jedoch an seine Grenzen, wenn es um individuelle Orientierungen und spezifische Aspekte der Handlungspraxis sowie um langfristige Prozessstrukturen geht. Zudem besteht die Gefahr, dass eventuell kontroverse Meinungen oder Einstellungen nicht ausreichend thematisiert werden. Rein forschungspragmatisch sind Familiengespräche zeitlich schwieriger zu koordinieren - was sich insbesondere vor dem Hintergrund eines recht engen Zeitbudgets von Familienunternehmern als Nachteil erweisen kann - und auch die Bereitschaft für ein Gruppeninterview im Familienkreis ist oft geringer. Durch eine Triangulation der Erhebungsmethoden (Rothbauer 2008; Flick 2007) könnten die Vorteile beider genutzt werden. Zudem würde dann je eigenes empirisches Material für die individuelle und kollektive Analyseebene zur Verfügung stehen, was die Ausarbeitung der Theorien auf eine breitere Basis stellt. Das Bild, das Familiengespräche auf kollektiver Ebene vermitteln, erscheint jedoch im Gegensatz zu den lebensgeschichtlichen Daten aus den Einzelinterviews eher statisch. Die Familiendynamik wird nur zu einem bestimmten Zeitpunkt abgebildet und nicht im Zeitverlauf, was eigentlich dem Interesse der Forschungsfrage entspricht. Der gewonnene Mehrwert erscheint deshalb relativ gering und rechtfertigt nicht den erhöhten Aufwand für die Befragten. Aus diesen inhaltlichen und pragmatischen Gründen fiel die Entscheidung gegen zusätzliche Familiengespräche.

Die Interviews führten nach Möglichkeit immer zwei MitarbeiterInnen des Forschungsprojektes „Von Generation zu Generation“56 durch, wobei eine der Forscherinnen das Gespräch leitete und die andere einen Blick auf die Technik, die Zeit und den Gesprächsverlauf hatte. Die Interviewerinnen haben sozialwissenschaftlichen Hintergrund und sind geschult für die Frageund Zuhörtechniken, die ein solches offenes, narratives Interview erfordern. Alle Interviews wurden im Einverständnis der Befragten mittels eines digitalen Diktiergerätes aufgezeichnet.

$\mathrm{Wir}^{57}$ befragten, wenn möglich, mehrere Mitglieder einer Unternehmerfamilie. Die ersten Interviews führten wir jeweils einzeln mit den Übernehmern und Übergebern. Aus ihren Geschichten wurde deutlich, welche weiteren Familienmitglieder relevante Positionen in der Familie wahrnehmen, die

56 Dieses Forschungsprojekt wurde von 2007-2010 an der Freien Universität unter Leitung von Prof. Dr. Martin Kohli durchgeführt und war Teilprojekt des Forschungsverbundes „Generationen der Erbengesellschaft“, der im Rahmen der „Schlüsselthemen der Geisteswissenschaften“" von der VolkswagenStiftung gefördert wurde. Nicole Schmiade und Thomas Treiber haben an der Interviewdurchführung in Deutschland mitgewirkt.

57 Die wechselnden Erzählperspektiven innerhalb dieses Kapitels sind durchaus intendiert und sollen die Rolle der Forscherin im Untersuchungsprozess verdeutlichen. Siehe dazu auch die Anmerkungen zur Darstellungsweise in Abschnitt 7. 
zur Aufrechterhaltung und Wahrung des Unternehmenseinflusses beitragen. Die so ausgewählten Familienmitglieder fragten wir für weitere Interviews an. Die biografischen Gespräche dauerten zwischen einer und vier Stunden und fanden an einem von den Interviewten bestimmten Ort statt: im Besprechungszimmer, „Show-Room“, Büro eines Supermarktleiters, oder im Wohnzimmer. Im Anschluss an das Interview erkundigten sich die Befragten häufig nach dem Werdegang der Forscherinnen (eine typische Reaktion, nachdem man so viel von sich selbst preisgegeben hat) und dem weiteren Verlauf des Forschungsprojektes. Gerne führten uns die Befragten oder ihre Mitarbeiter abschließend durch das Unternehmen und erläuterten die Betriebsabläufe. Der Kontakt zu den Unternehmerfamilien war freundlich, und sie zeigten sich meist sehr offen. Die große Redebereitschaft vieler Befragter vermittelte den Eindruck, dass ein regelrechtes Bedürfnis besteht, seine Geschichte einmal im Ganzen zu reflektieren. Einige Beteiligte betonten nach dem Interview, dass ihnen selbst nun bestimmte Zusammenhänge klarer erscheinen. In einem Fall hatten die Forscherinnen den Eindruck, dass das Interview als Anlass genommen wurde, die Nachfolge innerhalb der Familie zu thematisieren.

Das narrative Interview gliederte sich in drei Abschnitte (Schütze 1983:285ff.): Wir eröffneten das Gespräch immer mit folgender autobiografisch orientierter Aufforderung:

„Wie bereits angedeutet, interessieren wir uns für die Lebensgeschichte von Menschen aus Unternehmerfamilien. Wir möchten Sie bitten, uns die Geschichte Ihres Lebens und Ihrer Familie zu erzählen. Auch die so genannten Kleinigkeiten des beruflichen und familiären Alltags können dabei eine Rolle spielen. Sie können sich so viel Zeit nehmen, wie Sie möchten. Ich werde Sie auch erstmal nicht unterbrechen, nur einige Notizen machen und später darauf zurückkommen.“

Nach dieser sehr offen gehaltenen Erzählaufforderung ${ }^{58}$ (oder nach kurzen Rückfragen) begannen die Interviewpartner, ihre Lebensgeschichte zu erzählen. Die Interviewerinnen hielten sich während dieser Zeit aufmerksam zurück und warteten das Ende der Erzählung ab. Der Befragte wurde zum freien Erzählen ermuntert und sollte in seiner Darstellung möglichst nicht beeinträchtigt werden.

Es folgte der so genannte interne Nachfrageteil, bei dem die Forscherinnen aus ihren Notizen in der Reihenfolge der Erzählung einzelne Themenbereiche wieder aufgriffen und zu vertiefenden Erzählungen aufforderten (Fi-

58 Da die Fragestellung versucht, den Unternehmenseinfluss in einer möglichst breiten Perspektive und in seiner Dynamik zu fassen, wurde die Erzählaufforderung sehr offen gehalten. Die Alternative einer eingegrenzten Erzählaufforderung, die beispielsweise die Nachfolge oder direkt das Verhältnis von Familie und Unternehmen in den Mittelpunkt rückt, birgt auch die Gefahr nur eingeschränkter Analysemöglichkeiten. 
scher-Rosenthal und Rosenthal 1997; Rosenthal 2008:137): „Sie haben erwähnt, dass sie als Kind das Unternehmen als immer präsent wahrgenommen haben, können Sie dazu noch mehr erzählen?“ oder „Können Sie dazu ein Beispiel erzählen?" Die Interviewerinnen orientierten sich bei der Frageformulierung an den Sprachcodes des Interviewten. Rosenthal betont die Notwendigkeit solcher Vertiefungsfragen (Rosenthal 2008:152f.), die es erlauben, tiefer auf Vagheiten, Inkonsistenzen und Lücken einzugehen. Die Antworten liefern wichtiges Datenmaterial, das es erlaubt, die aus der Haupterzählung entwickelten Thesen empirisch zu prüfen und weiter zu detaillieren. Die Forscherin erhält damit die Chance, latente Bedeutungen offen zu legen, die man sonst subsumieren müsste.

Erst im letzten Interviewabschnitt wurde die Reihenfolge der Erzählung durchbrochen und externe - also vom Interviewten nicht selbständig genannte - Themenkomplexe angesprochen. In der Vorbereitungsphase auf die Feldarbeit erarbeiteten wir für diesen Interviewabschnitt einen ausführlichen Gesprächsleitfaden. Dieser umfasste die Felder: Betriebsinformationen, Nachfolge, Familie und Unternehmen sowie Zukunftsplanung und legte einen Schwerpunkt auf die Generationenbeziehungen. Mit dieser Vorgehensweise integrierten wir ein Instrument in das narrative Interview, das üblicherweise bei fokussierten Interviews oder Expertengesprächen Anwendung findet. Der Leitfaden sollte sicherstellen, dass alle „relevanten“ Themenbereiche angesprochen werden, und den Forscherinnen Sicherheit verleihen. In der Erhebungspraxis zeigte sich jedoch, dass wir hier dem Trugschluss der „Leitfadenbürokratie“ (Hopf 1978:101) auferlegen waren und dem Potential des offenen Interviews nicht genügend vertraut hatten. Denn schnell stellte sich heraus, dass die Befragten selbständig einen sehr großen Teil dieser Felder abdeckten. Des Weiteren erzeugte der Leitfaden einen zu massiven Eingriff in die Relevanzstruktur der Befragten und provoziert eine rechtfertigende Haltung. Schließlich erwies sich der Leitfaden auch aus zeitlichen Gründen als unpraktikabel. Aus diesen Gründen begrenzten wir schon nach wenigen Interviews den externen Frageteil auf zwei Aufforderungen, die von den Gesprächspartnern eine Reflektionsleistung erforderte: Die Befragten wurden gebeten, ihre Zukunftsvorstellungen zu schildern und schließlich einen Rat für andere Unternehmerfamilien zu formulieren. Die Antworten markierten meist einen runden, einvernehmlichen Interviewabschluss. Die vollzogene Wende in der Erhebungspraxis hat Konsequenzen für die Auswertung, in der nun die Nicht-Thematisierung von Erlebnissen, Beziehungen und Themenkomplexen zu einem wichtigen Tatbestand für die Interpretation wird.

Nach dem Interview notierten die Forscherinnen den Gesprächseindruck. Detailliert schildertem sie die Umgebung, die Erzählsituation, die Eigenheiten der Person, den Gesprächsablauf und besprochene Inhalte außerhalb der Aufzeichnung. Sie reflektierten über das Verhältnis zu den befragten Personen ebenso wie über die Qualität der Interviewführung. Nicht bei allen Inter- 
views gelang es, die oben skizzierte Struktur durchzuführen. Zudem fertigten die Interviewerinnen ein ad-hoc Memo mit ersten Ideen zu theoretischen Annahmen über die Struktur und die Besonderheiten des Falles an. Von einem zweiten Interview wurde aus zeitlichen Gründen abgesehen. In wenigen Fällen erfolgten nach dem Interview noch Telefonate mit den Befragten, um weitere Aspekte zu vertiefen oder objektive Daten zu ergänzen. Insgesamt führten wir 32 Interviews mit 12 Unternehmerfamilien. Im Anhang sind die einzelnen Fälle in anonymisierter Form kurz skizziert und angegeben, mit welchen Familienmitgliedern gesprochen wurde.

Je nach Neuheitsgehalt der Interviews wurde entschieden, ob eine Transkription erfolgt oder eine Auswertung auf Basis der Tonaufzeichnung genügt (siehe hierzu auch den nächsten Abschnitt). Insgesamt liegen von 25 Interviews vollständige oder teilweise Transkriptionen vor. Die Verschriftlichung geht über eine rein inhaltliche Wiedergabe hinaus: es wird wörtlich transkribiert, mundartlicher Einschlag wird sprachlich geglättet, Pausen und Betonungen werden durch Hervorhebungen im Text kenntlich gemacht. Die angewandten Transkriptionsregeln setzen im Großen und Ganzen die Vorschläge von Kuckartz (Kuckartz 2005:40ff.) um, die sich in der sozialwissenschaftlichen Praxis bewährt haben (siehe hierzu auch die Richtlinien für Gesprächstranskription bei Froschauer und Lueger 2003:224). Auf eine sehr detaillierte Transkription im Sinne der Linguistik wird verzichtet. Hier die Transkriptionszeichen im Überblick: 
Abbildung 6: Transkriptionsregeln

\begin{tabular}{|c|c|}
\hline Transkriptionsregel & Zeichen \\
\hline \multicolumn{2}{|l|}{ es wird wörtlich transkribiert } \\
\hline $\begin{array}{l}\text { mundartlicher Einschlag wird sprachlich geglättet } \\
\text { in Hochdeutsch wieder gegeben; erfolgt ein Wech- } \\
\text { sel von Hochdeutsch zu Mundart oder stechen } \\
\text { einzelne Sätze besonders hervor so werden Be- } \\
\text { ginn und Ende mit Sternchen markiert und in } \\
\text { Klammern spezifiziert }\end{array}$ & $\begin{array}{l}\text { *das war dann eben so üblich* (Mund- } \\
\text { art) }\end{array}$ \\
\hline $\begin{array}{l}\text { Sprache und Interpunktion wird geglättet (Einfügen } \\
\text { von Kommata und Punkten, Ausschreiben von } \\
\text { verkürzten Worten) }\end{array}$ & z.B. statt 'n ein \\
\hline $\begin{array}{l}\text { kurze Pausen werden durch Punkte, längere Pau- } \\
\text { sen (ab } 4 \text { Sekunden) durch Angabe von Sekunden } \\
\text { gekennzeichnet }\end{array}$ & ... bzw. (4) \\
\hline $\begin{array}{l}\text { lautes Sprechen wird nur bei Besonderheit durch } \\
\text { Verwendung von Großbuchstaben markiert }\end{array}$ & UND DAS WAR GUT SO \\
\hline $\begin{array}{l}\text { leises Sprechen wird nur bei Besonderheit durch } \\
\text { Verwendung von Kursivschrift markiert }\end{array}$ & ich wusste dann auch nicht so richtig \\
\hline $\begin{array}{l}\text { betonte Worte werden nur bei Besonderheit durch } \\
\text { Unterstreichung hervorgehoben }\end{array}$ & $\begin{array}{l}\text { und wenn ich das noch mal so machen } \\
\text { sollte }\end{array}$ \\
\hline $\begin{array}{l}\text { schnelles Sprechen wird nur bei Besonderheit } \\
\text { markiert, indem die einzelnen Worte durch Unter- } \\
\text { striche miteinander verbunden werden }\end{array}$ & $\begin{array}{l}\text { wo_soll_ich_denn_da_jetzt_mal_anfan } \\
\text { gen }\end{array}$ \\
\hline $\begin{array}{l}\text { Dehnung von Worten, Flüstern etc. werden nur bei } \\
\text { Besonderheit von Anfang bis Ende durch Stern- } \\
\text { chen markiert und in Klammern gekennzeichnet }\end{array}$ & $\begin{array}{l}\text { *und ab da habe ich dann immer alles } \\
\text { so gemacht wie er wollte* (flüstert) }\end{array}$ \\
\hline $\begin{array}{l}\text { aktives Zuhören der Interviewer wird nur dann } \\
\text { kenntlich gemacht, wenn es den Redefluss der be- } \\
\text { fragten Person unterbricht }\end{array}$ & (mh, aha) \\
\hline $\begin{array}{l}\text { Einwürfe von anderen Personen werden in doppel- } \\
\text { te Klammern gesetzt }\end{array}$ & $((\ldots))$ \\
\hline $\begin{array}{l}\text { Begleiterscheinungen des Sprechens z.B. Lachen, } \\
\text { Seufzen werden in Klammern notiert }\end{array}$ & (lacht) \\
\hline $\begin{array}{l}\text { Unterbrechungen des Interviews werden nur inhalt- } \\
\text { lich wiedergegeben -> wird bei jedem Interview zu- } \\
\text { vor mitgeteilt }\end{array}$ & (Telefonklingeln) \\
\hline \multicolumn{2}{|l|}{$\begin{array}{l}\text { Interviewerinnen werden durch I1 und I2 (bei allen } \\
\text { Interviews gleich), befragte Person durch B ge- } \\
\text { kennzeichnet }\end{array}$} \\
\hline \multicolumn{2}{|l|}{$\begin{array}{l}\text { jeder Sprecherwechsel wird mit einer Leerzeile ge- } \\
\text { kennzeichnet. }\end{array}$} \\
\hline \multicolumn{2}{|l|}{ im Transkript werden 5-Minuten-Marken gesetzt } \\
\hline $\begin{array}{l}\text { Auslassungen im Transkript werden durch Klam- } \\
\text { mern und Angaben über die Länge in Sekunden } \\
\text { markiert }\end{array}$ & (ausgelassen 5) \\
\hline $\begin{array}{l}\text { Unverständlichkeiten und nicht genau verstandene } \\
\text { Passagen werden durch Sternchen und als schwer } \\
\text { verständlich markiert }\end{array}$ & $\begin{array}{l}\text { *das war dann so* (ungenau oder ver- } \\
\text { mutlich) }\end{array}$ \\
\hline
\end{tabular}

Quelle: eigene Darstellung 


\subsection{Biografische Fallrekonstruktion}

Die Auswertung erfolgte im Sinne eines biografischen, fallrekonstruktiven Verfahrens, wie es von Gabriele Rosenthal (1995) vertreten wird. Dieses Verfahren baut auf der von Fritz Schütze entwickelten Narrationsanalyse auf und verbindet sie mit der sequenzanalytischen Vorgehensweise der objektiven Hermeneutik (Ulrich Oevermann). ${ }^{59}$ Während der Auswertung soll die Forschungsfrage vorläufig zurückgestellt und stattdessen die Fallstruktur offen gelegt werden. Es geht nicht einfach nur um eine Beschreibung der Lebensgeschichte, sondern um die Rekonstruktion der Struktur des Falles mit den Regeln ihrer Reproduktion und Transformation. Erst auf dieser Basis werden Bezüge zur Forschungsfrage hergestellt.

Das Besondere an der biografischen Fallrekonstruktion - und der Grund, warum ich mich gerade für dieses Auswertungsverfahren entschieden habe liegt in einer aus der Gestalttheorie von Aron Gruwitsch abgeleiteten Trennung in eine erzählte und erlebte Lebensgeschichte. Rosenthal argumentiert im Sinne eines dialektischen Verhältnisses von Erleben, Erinnern und Erzählen, dass Erzählungen eigenerlebter Erfahrungen sowohl auf das heutige Leben mit der Vergangenheit als auch auf das damalige Erleben dieser verweisen. (Rosenthal 2008:168) Ihr Anliegen ist es deshalb,

„einen Einblick in die Genese und die sequenzielle Gestalt der Lebensgeschichte $[\mathrm{zu}]$ geben sowie Rekonstruktion von Handlungsabläufen in der Vergangenheit und des damaligen Erlebens [zu] ermöglichen und eben nicht nur die Deutungen der untersuchten Person in der Gegenwart offen [zu] legen." (Rosenthal 2008:173)

Rosenthal unterscheidet deshalb eine „erzählte Lebensgeschichte“, die als biografische Selbstpräsentation in der Gegenwart verstanden wird, von einer „erlebten Lebensgeschichte“, die den chronologischen Verlauf der Erlebnisaufschichtung und die biografische Bedeutung des in der Vergangenheit Erlebten $\mathrm{zu}$ fassen sucht. In analytisch getrennten Auswertungsschritten wird die Lebensgeschichte einmal in der Chronologie der Erzählung und einmal in der Chronologie des Erlebens nachvollzogen. Einerseits wird der Frage nachgegangen, in welcher Reihenfolge und in welchen Textsorten die Befragten ihre biografisch relevanten Erlebnisse oder ihre Lebenserzählung präsentieren, und anderseits wird untersucht, wie sich die einzelnen biografischen Erfahrungen in der erlebten Lebensgeschichte chronologisch aufgeschichtet haben; das schließt ausdrücklich die Verwendung weiterer Quellen (Archivmaterial, Berichte, Bilder, etc.) ein. Schließlich werden die jeweiligen

59 Eine ähnliche Verbindung wendet auch Bruno Hildenbrand 2005 in seiner Fallrekonstruktiven Familienforschung an, die auf Familiengesprächen aufbaut. 
Ablaufgestalten von erzähltem und erlebtem Leben prozessorientiert aufeinander bezogen und die Fallstruktur ersichtlich. (Rosenthal und FischerRosenthal 2007:460)

Die biografische Fallrekonstruktion nimmt als Fall zunächst das Leben einzelner Familienmitglieder in den Blick. Jedoch ist es nach abgeschlossener Auswertung möglich, auf die Fallstruktur von anderen sozialen Einheiten wie etwa der Familie zu schließen. (Rosenthal 2008:195) Denn ein transkribiertes Interview repräsentiert auch die jeweiligen Kontexte wie etwa die Interviewsituation, die Milieus bzw. Lebenswelten, denen die Beteiligten angehören, etc. (Oevermann 2000:106) Für die Analyse familiengeschichtlicher Daten hat sich in der Forschungspraxis das Genogramm und dessen sequenzielle Auswertung als ein Hilfsmittel bewährt (Hildenbrand 2005:32ff.; Rosenthal 1999; Völter 2003:48) ${ }^{60}$ und wird deshalb als zusätzlicher Auswertungsschritt integriert. Auf diese Weise kann der Doppelstruktur des Falls, nämlich auf der Ebene des individuellen Lebens sowie auf der Ebene der Familie, die schon in der Forschungsfrage angelegt ist, entsprochen werden.

Anhand der Unternehmerfamilie Treuninger und insbesondere an der Lebensgeschichte von Michael sollen im Folgenden die einzelnen Schritte in diesem Auswertungsprozess (für eine Übersichte siehe z.B. Rosenthal 2008:161-198) erneut begangen und ausführlich dargestellt werden. Dabei steht vor allem die methodische Erläuterung, die zur Transparenz und Nachvollziehbarkeit beiträgt, und weniger der inhaltliche Erkenntnisgewinn im Vordergrund, der in den nächsten Kapiteln seinen Platz findet. Die einzelnen Interviewauswertungen unterscheiden sich in ihrer Tiefe. Nach einer intensiven Auseinandersetzung mit den ersten beiden Unternehmerfamilien (Treuninger, Singermayer) werden die folgenden Fälle zunächst nur globalanalytisch ausgewertet und bei erhöhtem theoretischen Interesse an einem Fall die Auswertung vertieft. Die Auswahl des nächsten Falls folgt nach der vermuteten theoretischen Ergiebigkeit im Vergleich mit dem bisher analysierten empirischen Material. Die Entscheidung, Singermayer nach Treuninger auszuwerten, lag beispielsweise in der Vermutung begründet, einen möglichst großen Kontrast in der Entwicklung der jüngeren Generation sowie dem Generationenverhältnis bei relativer Konstanz der Lebensgeschichte der älteren Generation vorfinden zu können.

Als Hilfsmittel für die Analyse dienten Ausdrucke der Interviewtranskripte, Notizen, Genogramme, Tabellen und Textverarbeitungsprogramme. Qualitative Auswertungssoftware wie etwa MaxQDA oder atlas.ti, die für kategorisierende Verfahren wie die Grounded Theory oder die Inhaltsanalyse entwickelt wurden, erweisen sich als eher unpraktikabel und fanden keine Anwendung. 
Vorab sei noch erwähnt, dass mir in der Auswertung der Interviews meine eigenen lebensgeschichtlichen Berührungspunkte mit Unternehmerfamilien hilfreich sind. Die Nähe zum Forschungsobjekt erlaubt es mir, mich leichter in die jeweiligen Rollen der Familienmitglieder zu versetzen und ihre Äußerungen einzuordnen. Als ebenfalls hilfreich erweist es sich, dass ich selbst die Interviews - bis auf wenige Ausnahmen - geführt habe. Während meiner Auseinandersetzung mit dem Text sind die Interviewsituationen ebenso wie markante parasprachliche Äußerungen noch präsent und können direkt einfließen. Die methodologische Auseinandersetzung mit der Rolle des Forschers kritisiert jedoch zu Recht, dass solche Verstrickungen die Gefahr von blinden Flecken und voreiligen Schlüssen bergen. (z.B. Froschauer und Lueger 2003:104ff.) Um diesem Risiko vorzubeugen, oder es zumindest etwas zu verringern, bin ich gefordert, über meine eigenen Erfahrungen und Wahrnehmungen zu reflektieren. Zudem habe ich mir gezielt Personen unterschiedlicher sozialer Hintergründe als Sparring-Partner in der Auswertung der Fälle gesucht. Am Institut für Soziologie der Freien Universität haben diverse Auswertungsrunden stattgefunden, ich habe an Auswertungsworkshops teilgenommen und mit Studenten, Freunden und Familie Analysen von kleineren Texteinheiten durchgeführt. Diese Bemühungen tragen zu einer größeren Vielfalt in der Lesartenbildung und Thesenformulierung bei. Sie können jedoch das „Risiko“ der Subjektivität der Forscherin nicht ausschalten - und sollen es auch nicht.

\subsubsection{Analyse der biografischen Daten}

Die biografische Fallrekonstruktion beginnt, wie auch die objektive Hermeneutik, mit der Analyse biografischer bzw. objektiver Daten. Zu diesen zählen alle Ereignisse, die kaum von der Interpretation der Erzählenden abhängen, oder anders ausgedrückt solche, die objektivierte und protokollierbare Spuren hinterlassen, wie etwa Geburtsdatum, Anzahl der Geschwister, Ausbildungsdaten, Familiengründung, Wohnortwechsel, Krankheiten, Eigentumsübertragungen, Unternehmensgründung, Unternehmensentwicklung, etc. Diese Daten werden in chronologischer Reihenfolge und unter Ausblendung des Wissens über den weiteren Lebenslauf und seine biografische Deutung durch den Erzählenden (Rosenthal 2008:175) gedankenexperimentell durchgespielt. Dieser Auswertungsschritt zeigt alternative Handlungsmöglichkeiten auf, vor deren Hintergrund es nicht nur gelingt, Lebenslaufentscheidungen nachzuzeichnen, sondern die jeweiligen Handlungsprobleme in ihrem gesellschaftlichen und familialen Kontext zu rekonstruieren. Im Rahmen des gesamten Auswertungsprozesses dient dieser Schritt der Vorbereitung auf die Analyse des erlebten Lebens. Die anfängliche Konzentration auf das Manifeste sowie die gesellschaftliche und familiale Rahmung ermöglicht es, den 
Lebenslauf „vorwärts“ zu interpretieren. Indem die Forscherin die Zeitperspektive der biografischen Selbstpräsentation, die die Lebensgeschichte aus der Gegenwart erzählt, durchbricht, gewinnt sie Distanz zu den biografischen Deutungen der Erzählenden. Diese Distanz lässt es zu, Thesen über das Erleben der Ereignisse und ihre Bedeutsamkeit für den weiteren Lebenslauf (und darin eingeschlossen seine Biografisierung) zu formulieren.

Die Abfolge der Ereignisse im Leben von Michael Treuninger arbeite ich zunächst anhand der Interviews, die mit ihm, seiner Ehefrau und seinem ältesten Sohn geführt wurden, heraus. Als weitere Quellen dienen mir die Firmenchronik, die Website des Unternehmens, Zeitungsartikel über diese Familie und ihr Unternehmen sowie Archivmaterial. Die objektiven Daten sollen in ihren historischen Kontexten ebenso wie vor dem Hintergrund der jeweiligen Lebensphase gedeutet werden, denn beides wirkt sich stark auf die Intensität der Erlebnisse aus. Die Liste der objektiven Daten umfasst deshalb auch gesellschaftspolitisch relevante Daten (z.B. Beginn des Zweiten Weltkrieges, Fall der Mauer), branchenrelevante Daten (z.B. technologische Umbrüche, Globalisierungsbewegungen), ebenso wie Hinweise auf das jeweilige Lebensalter von Michael zu den Lebenslaufereignissen.

Das erste Datum, das bei der Analyse ausgelegt wird, ist das Datum der Geburt. Dieses Ereignis steht in einem spezifischen sozialen Setting, das unter anderem durch die familiale Konstellation, die Vorgeschichte der Familie sowie die historischen Umstände geprägt ist. Dieses „Setting“ der Familie Treuninger kann wie folgt skizziert werden:

Seit Jahrhunderten ist die Familie Treuninger in einer ländlichen Region in Baden-Württemberg ansässig und baut Geräte für den bäuerlichen Bedarf. Sie stehen dem württembergischen Pietismus nahe. Seit den ersten Aufzeichnungen Mitte des 16. Jahrhunderts lassen sich Produktentwicklungen und Patente der Familie nachverfolgen. Mitte des 19. Jahrhunderts fällt die Werkstatt nach einer Reihe von frühen Todesfällen für kurze Zeit in „externe“ Hände: Die Witwe Anna von P. M. Treuninger heiratet Gustav Loeber, der Ende der 1840er Jahre auf Basis der bestehenden Werkstatt und Vertriebskanäle seine eigene Firma gründet. Im Jahr 1870 erfolgt die erste familieninterne Übergabe: Gustav Loebers Stiefsohn Adam Treuninger tritt in das Unternehmen ein (von nun an „Loeber \& Sohn“). Adam forciert die Mechanisierung und baut den Handwerksbetrieb in einen Industriebetrieb um. Er errichtet ein Fabrikgelände mit Wohnung, auf dem die Familie für die nächsten Jahrzehnte ihren Lebensmittelpunkt haben wird. 1903 setzt sich Adam zur Ruhe und sein Sohn Gustav übernimmt in dritter Generation das Unternehmen.

Die Unternehmensentwicklung verläuft während des Ersten Weltkriegs und der Weimarer Republik recht turbulent. Schon früh treten die männli- 
chen Mitglieder der Familie in die NSDAP ein. Ende der 1930er Jahre übergibt Gustav seinen beiden Söhnen Paul und Walter (es gibt noch eine Schwester) das Eigentum und die Leitung der Firma. Michael wird im Jahr 1937 als ältester Sohn von Walter geboren. Die Familie lebt in der großzügigen Wohnung direkt in der Fabrik im Zentrum der Kleinstadt. Seine Mutter ist Hausfrau. Weitere Familienmitglieder in diesem Haushalt sind der Großvater Gustav Treuninger, seine Großmutter, sowie seine Tante Else (väterlicherseits).

Auf Basis der hier knapp dargestellten familiengeschichtlichen Daten lassen sich nun alle möglichen Thesen formulieren, einerseits über das Leben von Michael, andererseits über die Familienkonstellation, in die er hineingeboren wurde. Im Gedankenexperiment werden extensiv die möglichen Bedeutungen des biografischen Datums interpretiert und zu Lesarten verdichtet (Przyborski und Wohlrab-Sahr 2008:253). Gemäß der Sparsamkeitsregel beschränke ich mich jedoch nur auf solche Lesarten, die grundsätzlich möglich sind. Dieser Schritt erfordert methodische Disziplin: Einerseits muss das Wissen über den Fall ständig eingeklammert werden. Aus diesem Grund ist die Interpretation in Gruppen mit ,unbelasteten“ Interpretationsteilnehmern sehr hilfreich. Andererseits muss das Wissen über den Kontext (zu bestimmten Erlebnissen oder historischen Umständen) durch Recherchearbeit erweitert werden. Eine extensive Literatur (z.B. zur Verwicklung von Wirtschaftsunternehmen im NS-Regime oder zu bestimmten Lebensphasen) erschwert die Auswahl relevanter Anregungen und später das Beenden der Suche nach weiterer Literatur (Wenk 2007:73).

Anhand der objektiven Daten lässt sich eine hypothetische Skizze der Unternehmerfamilie Treuninger zum Zeitpunkt der Geburt von Michael entwerfen (sozialer Status, Geschlechterrollen, Erziehungsstil, etc.) und lassen sich auch Gegenpositionen dazu formulieren. An dieser Stelle möchte ich jedoch eine (von vielen) Thesen darüber ausführen, welche Bedeutung die Geburt von Michael für diese Familie haben könnte:

T 1.1: Michael markiert die Begründung der „fünften Generation“. Als Sohn setzt er die männliche Ahnenreihe fort und könnte als (ein) potentieller Nachfolger für das Unternehmen gehandelt werden. Mit Michael wird die Sicherung der Zukunft von Betrieb und Familie verknüpft. Die Reproduktion der Familie erfüllt auch Erwartungen, die sich aus religiösen Überzeugungen oder Regimeanforderungen speisen, und der Familie in diesen Bereichen weitere Anerkennung verschaffen.

Auf der Grundlage dieser Überlegungen können wir nun mehrere Folgehypothesen darüber ableiten, wie sich der folgende Lebenslauf von Michael, seine 
Handlungsmöglichkeiten und biografischen Deutungen, entwickeln können. Es wird ein anschlussfähiger Fortgang entworfen, der die jeweilige Hypothese überprüfbar bzw. plausibel machen könnte.

F. 1.1.1: Michael wird zum Nachfolger erzogen. Sein Vater und sein Großvater wachen über ihn und ein großer Erwartungsdruck lastet auf Michael. Früh wird er in das Unternehmen eingebunden. Gegenüber seinen (eventuellen) jüngeren Geschwistern wird er bevorzugt behandelt. Michael erhält eine gute Ausbildung, doch sein Weg wird maßgeblich von der Familie bestimmt. Er hat wenig Freiräume. Michael wird einen großen Teil des Eigentums erben. Retrospektiv könnte er sein Leben als „schicksalshaft“ oder „naturgegeben“ biografisieren.

F 1.1.2: Michael bricht aus. Sein Vater und sein Großvater wachen über ihn mit strenger Hand und ein großer Erwartungsdruck lastet auf Michael. Michael fühlt sich eingeengt und will diesem vorgezeichneten Weg entkommen. Er bricht mit der Familie und ihrer Lebensweise, sucht sich einen anderen Beruf oder eine Partnerin, die nicht den Vorstellungen der Familie entspricht. Retrospektiv könnte er sein Leben als eine Befreiungsgeschichte darstellen.

F 1.1.3: Michael soll ganz ,normal“ aufwachsen. Seine Eltern wollen ganz bewusst eine Trennung von Unternehmen und Familie leben. Sie brechen mit dem Lebensstil und den Vorstellungen der Großeltern und beziehen eine eigene Wohnung. Michael kommt nicht übermäßig mit dem Unternehmen in Kontakt. Hinsichtlich seiner Ausbildung lassen ihm seine Eltern Wahlmöglichkeiten.

F 1.1.4: Michael wird zum Spielball der Familie. Die Eltern und Großeltern sind sich über die Zukunft von Michael uneinig. Er steht zwischen den Stühlen und versucht, es allen Recht zu machen. Es finden sich Brüche in seinem Lebenslauf, er entwickelt sich zu einem unentschlossenen Mittler.

F 1.1.5: Michael fühlt sich berufen. Michael erkennt das Familienunternehmen als ein erhaltenswertes Projekt an. Er sieht sich in der Tradition seiner Ahnen. Seine Ausbildung absolviert er auf das Unternehmen bezogen. Sein Wunsch ist es, später einmal selbst mit seiner Familie in der Fabrikwohnung zu leben. Er will die Tradition auch an seine Kinder weiter geben. 
Weitere Hypothesen und Folgehypothesen lassen sich beispielsweise über die Beziehung zu bestimmten Familienmitgliedern oder den Umgang mit dem Unternehmen formulieren. Es würde den Rahmen dieses Kapitels sprengen, alle formulierten Hypothesen vorzustellen. Stattdessen möchte ich ein weiteres biografisches Datum vorstellen, um die zunehmende Verdichtung des Interpretationsprozesses zu illustrieren:

Mit dem Kriegsausbruch steigt die Firma in die Rüstungsproduktion ein. Vater, Onkel und die wesentlichen Mitarbeiter ziehen nicht in den Krieg, sondern verbleiben im Unternehmen. Ab dem Jahr 1942 werden bei der Firma „Loeber \& Sohn“ Zwangsarbeiter aus Frankreich und Holland beschäftigt. Während dieser Zeit werden die beiden Geschwister von Michael geboren: Er hat eine Schwester (*1939) und einen Bruder (*1942). Michael wird in die örtliche Volksschule eingeschult. Kurz darauf werden die Wirtschafts- und Industriezentren der Region massiv bombardiert (Linde 2007:43). Eine Bombe trifft das Wohn- und Verwaltungsgebäude der Firma, dabei sterben der Vater und der Onkel von Michael Treuninger.

Wieder bilde ich alle uns möglichen Hypothesen zu dieser neuen Lebenssituation und folgere dann auf die weitere Entwicklung der Familie, auf deren Auswirkungen auf das Erleben von Michael und seine weitere Lebensgeschichte. Bereits gebildete Hypothesen werden immer wieder hinterfragt, gegebenenfalls mitgeführt bzw. verworfen. Es lässt sich formulieren, dass der Tod von Walter und Paul die Familie in Schock versetzt. Die Wohnung und das Unternehmen liegen in Trümmern, ihre Existenz ist gefährdet. Ohne die beiden Brüder fehlt ihnen Orientierung und Halt. Die Treuningers verlieren ihren privilegierten Status, sie sind nun eine von vielen Familien, die Armut erfahren. Michael ist zu diesem Zeitpunkt sieben Jahre alt. Die Trauer um seinen Vater wird von Existenzsorgen überlagert.

Vereinfacht formuliert können unter anderen folgende Hypothesen aufgestellt werden:

T 2.1: Die Leerstelle, die Michaels Vater hinterlässt, wird durch die Witwe auch nach Kriegsende gefüllt. Sie übernimmt eine führende Rolle in der Versorgung der Familie und der Erziehung der Kinder. Sie kämpft für den Wiederaufbau des Unternehmens (nach einer Phase der Demontage und Entnazifizierung). Die Mutter setzt den vom Vater eingeschlagenen Kurs fort. 
F 2.1.1 (zu 1.1.1): Michael wird weiterhin zum Nachfolger erzogen. Die Mutter sieht sich in der Rolle der Platzhalterin 61 und will, dass ihr Sohn so schnell wie möglich in das Unternehmen eintritt. Michael spürt den Erwartungs- und Zeitdruck, der auf ihm lastet. Eine Nachfolge durch eines seiner jüngeren Geschwister scheint unwahrscheinlich. Michael erhält eine praxisnahe Ausbildung, die maßgeblich von der Familie bestimmt wird. Er hat wenige Freiräume. Michael wird einen großen Teil des Eigentums erben. Retrospektiv könnte er sein Leben als „schicksalshaft“ oder „naturgegeben“ biografisieren.

F 2.1.2 (zu 1.1.2): Die Alternative, dass Michael mit der Lebensweise seiner Familie und seinem Weg ins Unternehmen bricht, scheint in dieser neuen Konstellation nur unter einer enormen Kraftanstrengung von Michael möglich.

F 2.1.2 (zu 1.1.5): Michael fühlt sich berufen. Michael erkennt das Familienunternehmen als ein erhaltenswertes Projekt an, das den Wohlstand der Familie und ihren Status sichert. Er will seinen Beitrag dazu leisten und die Erwartungen der Mutter erfüllen. Er sieht sich in der Tradition seiner Ahnen. Seine Ausbildung absolviert er auf das Unternehmen bezogen. Sein Wunsch ist es auch die Fabrikwohnung wieder aufzubauen, dort später einmal selbst mit seiner Familie zu leben.

Als eine Alternative lässt sich folgende Hypothese aufstellen:

T 2.2: Das Unternehmen verbleibt nicht in der Hand der Familie. Die Treuningers verlassen das Fabrikgelände und schlagen sich mit anderen Tätigkeiten durch. Die Familie lebt in bescheidenen Verhältnissen. Michael muss helfen, Einkommen für die Familie zu generieren. Er übernimmt Verantwortung für seine Geschwister und ist emotionale Stütze der Mutter und Großeltern.

F 2.2.1: Der Verlust des Unternehmens kann auch als ein Verlust einer Lebenslaufoption für Michael verstanden werden. Der berufliche Werdegang von Michael ist durch Erwerbsarbeit geprägt. Das Unternehmen hat retrospektiv keine biografische Bedeutung mehr bzw. wird von den Verlusterfahrungen überlagert. 
F. 2.2.2: Michael fühlt sich berufen. Das Unternehmen markiert für Michael ein besseres Leben. Es ist stark mit seinen frühen Kindheitserinnerungen verknüpft, in denen es der Familie gut ging. Er träumt davon, selbst einmal Unternehmer zu werden oder das Unternehmen wieder aufbauen zu können.

Jedes zusätzliche Datum zeigt an, wie sich der Lebensweg von Michael tatsächlich gestaltet, und bestätigt oder falsifiziert damit die generierten Hypothesen. Nur einige wenige kristallisieren sich im Verlauf der Analyse zu empirisch fundierten Fallstrukturhypothesen heraus. Während zu Beginn der Analyse der Horizont an Möglichkeiten fast unendlich erscheint, wird dieser mit Fortschreiten der Analyse immer enger. Nur durch eine ausgiebige Lesartenbildung gelingt es, diese Handlungsräume zu fassen, die Bedeutung der biografischen Daten (die sich oft erst später herausstellt) wahrzunehmen und eventuell zu den latenten Sinnstrukturen vorzudringen, die den Befragten selbst nicht bewusst sind.

Im Fall von Michael bilden sich unter anderem folgende zwei Fallstrukturhypothesen heraus:

Michael wird zum Hoffnungsträger der Familie delegiert: Der Tod des Vaters und des Onkels durch einen Bombenangriff ist ein markanter Punkt in den biografischen Daten Michaels. In der Zeit davor mag Michael eventuell schon als Nachfolger gehandelt worden sein. Doch sein Vater und Onkel hatten das Unternehmen selbst erst übernommen und eine weitere Nachfolge war noch weit entfernt. Ihr plötzliches Ableben reißt jedoch eine große Lücke in die Familie: Die beiden Versorger der Familie und Unternehmensleiter sind nun weg. Der Großvater ist bereits sehr alt und kann das Unternehmen scheinbar nicht mehr leiten. Der Blick fällt auf die Kinder des verstorbenen Sohnes: Michael ist zu diesem Zeitpunkt 7 Jahre alt und gerade eingeschult worden. Seine Schwester ist vier Jahre und sein kleiner Bruder gerade einmal zwei. Michael ist nun nicht mehr nur ein potentieller Nachfolger, sondern könnte als „,einziger Hoffnungsträger" der Familie gesehen werden. Seine Tante übernimmt die Rolle der „Platzhalterin“62, bis Michael soweit ist. Dieses frühe Erlebnis spricht sehr dafür, dass Michael in dieser Zeit mit der Mission der Unternehmensnachfolge betraut wurde. Damit verbunden ist nicht nur ein Moment des Vertrauens, sondern auch einer bleibenden Lo-

62 Das Unternehmen wird durch „externe Manager“ geführt, während seine Tante die persönliche Haftung $(\mathrm{KG})$ übernimmt. 
yalitätsverpflichtung, die bei dem Delegierten ein Gefühl von Wichtigkeit und Sinnhaftigkeit hervorruft. (Siefer 1996:182)

Die folgenden biografischen Daten sprechen dafür, dass sich Michael von nun an in einem ambivalenten Verhältnis von „Sollen“, „Müssen“ oder „Wollen“ befindet. (Siefer 1996:185) Der Druck der verbliebenen Familienmitglieder auf Michael dürfte nicht unerheblich gewesen sein. Gleichsam sprechen die kaum erkennbaren Versuche, aus seinem recht stringenten Berufs- und Familienweg auszubrechen, dafür, dass Michael diese Erwartungen erfüllen wollte oder gar einen Drang entwickelt hat, das Unternehmen in den Schoß der Familie ,zurückzuerobern“. Mit dem Tod der Großeltern (beide 1954) wird Michael bewusst, dass er nun der „Nächste“ in einer Reihe von Unternehmern ist. Er spürt, dass als „einziger Hoffnungsträger" nun ein erhöhter Zeitdruck auf ihm lastet, und beginnt seinen Weg als Nachfolger. Die Delegationshypothese könnte damit begründen, warum Michael zu diesem Zeitpunkt das Gymnasium abbricht und stattdessen eine praktische Ausbildung beginnt. Alternative Lesarten, etwa, dass er aufgrund schlechter Leistungen keine andere Wahl hatte, scheinen zwar möglich, aber eher unwahrscheinlich. Seine Ausbildung zum Nachfolger rundet Michael durch den Besuch der Ingenieursschule ab. Möglicherweise folgt er hier dem Ausbildungsweg seines Vaters oder hört auf den Rat von nahe stehenden Personen. In kürzester Zeit tritt Michael in das Unternehmen ein (er ist Anfang 20) und beginnt, gestaltend zu wirken. Kurz darauf übernimmt er die persönliche Haftung. Doch mit dem Eintritt in das Unternehmen hat Michael seine Mission noch nicht erfüllt. Er verweilt fast 50 Jahre auf seiner Position als Geschäftsführer und manövriert die Firma durch Zeiten des technischen Wandels in die Hände der nächsten Generation, seines ältesten Sohnes Adam (sic!).

Nicht nur Michaels Berufswahl, sondern auch die enge Beziehung, die er zu seiner Herkunftsfamilie pflegt, sowie der Weg der eigenen Familiengründung untermauern die Delegationshypothese. Michael zieht nach seinem Aufenthalt in der Ingenieursschule wieder in die Familienwohnung in der Fabrik ein und lebt hier mit seiner Tante, Mutter und seinen Geschwistern. Er stellt sein privates Leben zunächst zurück. Dennoch scheint Michael die Gründung einer eigenen (Groß-)Familie wichtig. Er wählt, relativ spät, eine Partnerin, die selbst aus einem Haushalt von Freiberuflern stammt, und versucht, mit ihr die Lebensweise seiner Vorfahren fortzuführen. 
Insgesamt scheint das Leben von Michael durch die Delegation Richtung zu erhalten, es verankert sich in einer Kette von Verpflichtungen, welche die Generationen überspannen und familiäre Identität ermöglichen. Die Beauftragung erhält damit eine über-persönliche Bedeutung. Sie erfolgt sehr wahrscheinlich unbewusst, und ihr sinnstiftender Charakter wird nicht reflektiert. Michael bleibt lebenslang an das Familienunternehmen schicksalhaft gebunden. (Siefer 1996:238) Die Grenze zwischen dem eigenen Leben und dem der Familie verschwimmt. Michaels Identität müsste stark in der Geschichte der Familie verankert sein. Aufgrund seiner Delegation könnte Michael seine berufliche Laufbahn später als ,selbstverständlich" biografisieren und weniger als von außen bestimmt verstehen. In seiner Selbstdarstellung könnte er Formeln benutzen wie etwa „Solange ich denken kann....“, „das war schon immer so..." oder „da gab es nichts zu überlegen...“. Dennoch lässt die Delegation zentrale Lebensentscheidungen von Michael als eher hypothetisch wirken. Sie entsprechen nicht dem Ideal einer „freien Wahl“" und könnten in der biografischen Darstellung ein Abwehrverhalten hervorrufen.

Als zweite Strukturhypothese lässt sich formulieren:

Michael wächst zunehmend in die Rolle des Fürsorgers, die sich über sein Leben hinweg konstant erhält. Wieder scheinen der frühe Tod des Vaters und des Onkels Ausgangspunkt zu sein. Michael könnte sich damit nicht nur als ,auserkorener" Nachfolger, sondern gleichsam als „Familienoberhaupt“ verstehen und versuchen, eine besondere Verantwortung zunächst für seine Geschwister und später für seine eigene Familie zu übernehmen. Michael könnte versuchen, den Ausbildungsweg der Geschwister zu beeinflussen. Er könnte sich als „Lastenträger" verstehen, der das schwere Erbe auf sich genommen hat und seinen Geschwistern damit Freiheit schenkt. Michael dürfte nicht bewusst sein, dass er durch seinen Drang, das Unternehmen zu übernehmen, seine Geschwister aus der Nachfolgerolle gedrängt haben könnte. Das Unternehmen liegt in seinem Hoheitsbereich. Die Unterstützung der Familienmitglieder nimmt er als seine Aufgabe an.

In diesem Licht scheint die spätere Konstellation der Verteilung von Eigentum und Leitungsfunktionen plausibel. Michael akzeptiert als „Fürsorger" für die Familie die Rolle seines Bruders als passiver 
Teilhaber. Durch seine unternehmerische Leistung nimmt er eine finanzielle Entbehrung zu Gunsten der Familie seines Bruders auf sich. Eventuell erwartet Michael für seine Taten eine gewisse Anerkennung durch die Familienmitglieder. Ein Versuch des Bruders, die von Michael als gegeben verstandenen Spielregeln zu verletzen beispielsweise der Versuch, operativ im Unternehmen tätig zu werden oder auf seinen Anteilen zu beharren - dürfte großes Konfliktpotenzial in sich bergen.

In analoger Weise übernimmt Michael die Rolle des „Fürsorgers“ für seine eigene, kinderreiche Familie. Er dürfte konstant den größeren Teil des Haushaltseinkommens bestritten haben und war streckenweise gar der „Alleinversorger“ der Familie. Michael scheint ein relativ traditionelles Familienbild zu pflegen, dem seine Ehefrau eher ambivalent gegenüber steht: Die Medizinerin gibt ihren Beruf für die Familie auf. Dennoch versucht sie, sich immer wieder jenseits der Mutterrolle weitere Aufgaben im Unternehmen zu suchen. Michael lässt sie walten. Doch die wechselnden Projekte seiner Frau weisen darauf hin, dass diese keine durchgängige Rolle in Michaels Hoheitsgebiet finden kann.

\subsubsection{Text und thematische Feldanalyse}

Mit diesem Analyseschritt beginnt die intensive Textarbeit. In den Blick geraten die Gegenwart des Interviews und die biografische Selbstpräsentation der Erzählenden. Der Text soll die Frage beantworten: Warum stellt Michael - ob bewusst oder latent gesteuert - sein Leben so und nicht anders da? Die Reflexion über den Erzählmodus einer Textpassage ist der Narrationsanalyse entlehnt. Rosenthal folgt Schütze in der Annahme, dass die Wahl der Darstellung einer Erfahrung nicht zufällig ist. (Rosenthal 1995:210) Ob Michael über den Tod seines Vaters in Form einer Erzählung, Argumentation, Beschreibung, Evaluation oder eines Berichtes spricht, ${ }^{63}$ wird ebenso zum Gegenstand der Interpretation wie die Ausführlichkeit dieser Textpassage oder ihre zeitliche Platzierung in der Interviewabfolge. Die gewählte Selbstpräsen-

63 Die Abgrenzung der einzelnen Textsorten erfolgt nicht dogmatisch. Grob wird unter einer Erzählung die Darstellung einer Ereignisverkettung verstanden, unter einem Bericht eine kurze Darstellung mit Konzentration auf Fakten und unter einer Beschreibung die Darstellung von statischen Situationen, die vor allem Hintergrundinformationen liefern. Argumentationen und Evaluationen verweisen auf den Gegenwartsstandpunkt des Erzählers und enthalten theoretische bzw. wertende Anteile. Für eine genauere Abgrenzung siehe etwa Przyborski und Wohlrab-Sahr 2008:225f. 
tation wurzelt zum einen in der Interaktion zwischen Interviewerin und Interviewten, zum anderen in der Lebensgeschichte des Erzählenden. Ziel ist es, die Regeln für die Genese der biografischen Erzählung und ihre typischen Strukturierungsmuster offen zu legen. (Rosenthal 2008:183) In anderen Worten, die Gestalt des erzählten Lebens, also der Beziehungszusammenhang zwischen den einzelnen Textteilen, soll erschlossen werden.

Zur Vorbereitung auf die sequenzanalytische Auswertung fertige ich eine Gliederung des Interviewtranskriptes an. Jeder Themen-, Textsorten- und Sprecherwechsel leitet eine neue Sequenz (Analyseeinheit) ein. In einer Tabelle (siehe Abbildung 7) erfasse ich diese Sequenzen in ihrer zeitlichen Abfolge, notiere ihre Länge, die Textsorte sowie stichwortartig den Inhalt.

Anhand dieser Übersicht erfolgt dann die sequenzielle Analyse der präsentierten Themen, ebenso wie ihrer temporalen und inhaltlichen Verknüpfungen. Von Sequenz zu Sequenz - und unter Ausblendung des weiteren Interviewverlaufes - formuliere ich Hypothesen als Antwort auf eine Reihe von Fragen (siehe hierzu Rosenthal 2008:187): Warum führt Michael diesen Inhalt an dieser Stelle ein? Weshalb präsentiert er diesen Inhalt in dieser Textsorte? Weshalb spricht er über diesen Inhalt so ausführlich oder so kurz? Was könnte das Thema dieses Inhaltes sein bzw. was sind die möglichen thematischen Felder, in die sich dieses Thema einfügt? Welche Lebensbereiche und welche Lebensphasen spricht Michael an und welche nicht? Ähnlich der Analyse der objektiven Daten formuliere ich am Ende jeder Sequenz mögliche Gesprächsanschlüsse, um mir die Optionen seiner Selbstpräsentation zu vergegenwärtigen.

Bei jeder Sequenz geht es um das Auffinden der inhärenten Verweisungen auf mögliche thematische Felder. (Rosenthal 2008:184ff.) Gemeint ist damit, dass jedes Thema in einen komplexeren Zusammenhang eingebettet ist. Es verknüpft nicht nur unterschiedliche Inhalte, sondern verweist auch auf kopräsente Gegebenheiten, die insgesamt ein thematisches Feld formen. Gerd Vonderach (1997) beschreibt diesen Zusammenhang in seiner Geschichtenhermeneutik sehr plastisch als Rekonstruktion „biographischer Großgeschichten“, also eines geschichtenförmigen Sinnzusammenhangs der Selbstwahrnehmung, in den einzelne Themen eingelassen sind. Im Laufe der Analyse wird deutlich, wie Michael seine Erlebnisse systematisch spezifischen Feldern zuordnet und andere Rahmungen vermeidet. Auch wird deutlich, welche Themen er ausspart. Michael entwirft unter anderem die thematischen Felder „Mein Leben als Unternehmer ist von äußeren Widerfahrnissen geprägt", „Ich habe alleine eine Bürde auf mich genommen und mit vollem Engagement meine Aufgaben erfüllt“ und „Unsere Familiengeschichte als Rahmen meines Lebens“. 
Abbildung 7: Ausschnitt aus der Sequenzübersicht Michael Treuninger

\begin{tabular}{|c|c|c|c|c|c|}
\hline Nr. & Von & Bis & Zahl & Textsorte & Inhalt \\
\hline 1 & $1 / 1$ & $1 / 8$ & 8 & Erzählaufforderung & $\begin{array}{l}\text { I1 bekundet Interesse an Unternehmer- } \\
\text { familien und fordert B auf die Geschich- } \\
\text { te seines Lebens und seiner Familie zu } \\
\text { erzählen. }\end{array}$ \\
\hline 2 & $1 / 9$ & $1 / 25$ & 16 & Aushandlung & $\begin{array}{l}\text { B kündigt erst souverän eine längere } \\
\text { Erzählung an und erkundigt sich dann } \\
\text { doch, welches Vorgehen üblich ist. Er } \\
\text { unterscheidet in zwei Dinge: das eige- } \\
\text { ne Leben und das der Firma. I1 schlägt } \\
\text { vor in der Kindheit zu beginnen. Nach } \\
\text { erneutem Nachfragen bittet I1 darum } \\
\text { beides zu erzählen. }\end{array}$ \\
\hline 3 & $1 / 26$ & $1 / 28$ & 2 & Erzählbeginn & $\begin{array}{l}\text { B beginnt zunächst zögerlich „Hm, } \\
\text {..naja, also." Und kündigt dann an } \\
\text { "ganz persönlich“ zu beginnen. }\end{array}$ \\
\hline 4 & $1 / 28$ & $1 / 28$ & 1 & Bericht & $\begin{array}{l}\text { "Ich bin xy geboren“ -> Beginn wie in } \\
\text { einem Lebenslauf }\end{array}$ \\
\hline 5 & $1 / 28$ & $1 / 29$ & 2 & Beschreibung* & $\begin{array}{l}\text { Ordnet dieses Datum historisch ein: vor } \\
\text { dem } 2 . W K \text {, erste Eindrücke während } \\
\text { der Kriegszeit }\end{array}$ \\
\hline 6 & $1 / 30$ & $1 / 31$ & 2 & Bericht & $\begin{array}{l}\text { „und hab da auch erlebt, .. äh dass } \\
\text { mein Vater als... Geschäftsführer starb } \\
\text { im Krieg und... -> überspringt sechs } \\
\text { Jahre und kommt zum Tod des Vaters; } \\
\text { betont, dass dieser kein Soldat, son- } \\
\text { dern Geschäftsführer war. }\end{array}$ \\
\hline 7 & $1 / 31$ & $1 / 32$ & 2 & Beschreibung* & $\begin{array}{l}\text { Ordnet diese Zeit historisch ein: Prä- } \\
\text { gung durch frühe Nachkriegszeit und } \\
\text { Währungsreform. }\end{array}$ \\
\hline
\end{tabular}

Quelle: eigene Darstellung

In der sequenzanalytischen Arbeit mit dem Interview von Michael hat sich insbesondere die Analyse der Textsorten als ergiebig erwiesen. Beispielsweise zieht sich durch den ersten Teil seiner zweistufigen Haupterzählung ein Rhythmus von wechselnden Berichten und Beschreibungen. Immer wenn Michael etwas zu seinem persönlichen Werdegang berichtet, folgt unmittelbar eine Beschreibung der Unternehmenssituation oder allgemeiner zeithistorischer Umstände. So kommt es, dass diese „Erzählung“ zu einem „kurzen Überblick über das Berufsleben" wird, wie Michael am Ende selbst zusam- 
menfasst und damit verdeutlicht, dass er sich sehr wohl bewusst ist, dass es noch einen anderen Teil des Lebens gibt. Durch die schnellen Wechsel in den Textsorten sowie den Telegrammstil seiner Berichte wirkt Michaels Rede zögerlich und ein wenig abgehackt. Der Rhythmus dieses Interviewabschnittes verweist gleichzeitig auf eine Besonderheit in Michaels biografischem Verständnis: Er nutzt den Bericht als Modus für sein Leben, während er die Beschreibung als Modus für die „Umwelt“, das „Drumherum“ und das „Normale“ einsetzt. Insofern versteht sich Michael als atypisch, doch hat eine genaue Vorstellung davon, was als normal gilt. Michael grenzt „sich“ ab von all dem anderen, kann jedoch ohne das ,andere“ nicht sein. Durch die schnellen Wechsel entsteht der Eindruck, dass Michaels Leben in diesen Elementen verstrickt ist. Sein Leben kann nur verstanden werden, wenn man all diese Umstände berücksichtigt, von denen es abhängt. Es wirken starke Interdependenzen, die Differenzierungen verschwimmen lassen. Unterfüttert man diese These noch mit den jeweils angesprochenen Themen, so begreift Michael sein Leben als geformt von Familie, Unternehmen, geschichtlichen und technologischen Entwicklungen, wobei letztere einen besonderen Stellenwert einnehmen.

Im Laufe der Auswertung verdichten sich solche Regeln des erzählten Lebens von Michael sowie die thematischen Felder zu einer biografischen Gesamtsicht, als ein durchgehendes, nicht unbedingt bewusst intendiertes Präsentationsinteresse. Diese lässt sich wie folgt zusammenfassen:

Michael präsentiert sich als (väterlichen) Experten, der den wesentlich jüngeren Interviewerinnen sein Wissen und sein Lebensmodell in einem positiven Lichte vorstellen möchte. Er zeigt sich als Person, die gerne die Kontrolle übernimmt, dafür jedoch Strukturen und Ordnung benötigt. Darüber hinaus ist seiner Selbstdarstellung eine Ambivalenz zwischen „Erzählen wollen"“ und "Distanz wahren“ zueigen. Michael ist bemüht, sich in das Relevanzsystem der Interviewerinnen einzudenken und ihre Erwartungen zu erfüllen. Dadurch verschließt sich Michael jedoch und kommt nur selten ins Erinnern. Er baut eine Grenze der Privatheit auf, die sich insbesondere im Nachfrageteil deutlich zeigt. Als zu intim scheint Michael insbesondere Fragen zu seiner Herkunfts- und Kernfamilie sowie seiner weiteren Verwandtschaft zu empfinden.

In der ersten Eingangserzählung präsentiert sich Michael verstrickt in zeithistorische Konstellationen. Der Tod seines „Vaters als Geschäftsführer" im Zweiten Weltkrieg hat sowohl im Unternehmen als auch in der Familie eine Lücke hinterlassen. In dieser Situation hat Michael die Mission erhalten, das Unternehmen im Rahmen der Fa- 
milie fortzuführen. Seine Selbstdarstellung ist geprägt von dem Bild eines einsamen Leidenden, der seinen vom Schicksal bestimmten Weg aus freien Stücken und sogar mit Freude erfüllt. Michael ist sich bewusst, dass sein Leben von der Normalität abweicht. Er hat jedoch als stiller Beobachter eine Vorstellung von dieser Normalität gewonnen. In seiner biografischen Sicht wird sein Leben vom Puls der Zeit bestimmt: Technische Neuerungen und zeithistorisches Geschehen spielen eine große Rolle. Kopräsent in seiner Erzählung ist der Einfluss der „Familie“ - zu der er lieber als Kollektiv Bezug nimmt als auf einzelne Familienmitglieder einzugehen -, den Michael jedoch argumentativ aus seiner Selbstdarstellung ausgrenzt. Michael deutet seine Leistungen als Unternehmer immer wieder an, doch gibt sich zurückhaltend. Diese fast schon zur Schau gestellte Bescheidenheit ist wesentliches Element seines Verständnisses des ethischen Unternehmers.

Michael benötigt die Unterstützung der Interviewerinnen, um von dem eigenen Lebenslauf und dem Unternehmen auf die Rahmung seines Lebens überleiten zu können: In seiner biografischen Sicht ist er Teil einer Familientradition, die einen besonderen Anfang hat (Begegnung mit einem Erfinder und Dorfpfarrer), mit einer spezifischen Aufgabe versehen ist (der Gerätetechnik) und es geschafft hat, 220 (ab Begegnung mit Pfarrer) bzw. 160 Jahre (ab Unternehmensgründung) lang an dieser Aufgabe festzuhalten. Michael entwirft eine Theorie über den Unternehmer und seine Familie und betont durch kontinuierliche Verweise auf die eigene Familie bzw. das eigene Unternehmen, dass diese „wirkliche“ Unternehmer, wie viele andere auch, mit allen Höhen und Tiefen sind. Er ordnet sich selbst dieser Gruppe der „Unternehmer“ zu und schöpft daraus seine Identität. Er fühlt sich seiner Familie und seiner Branche verbunden.

Im dem ausgedehnten Nachfrageteil blendet Michael Themen, die quer zu seiner Selbstpräsentation liegen, konsequent aus. Hierzu gehören seine frühe Kindheit, sein Einstieg in das Berufsleben oder einzelne Familienmitglieder. Oder er versucht, Argumente anzuführen, warum diese Wendungen in seinem Leben dennoch in seine Gesamtdarstellung passen, z.B. der Besuch der Ingenieursschule als notwendige Vorbereitung. Besonders intensiv und souverän geht Michael auf jene Themen ein, die den Kern seines Selbstbildes darstellen: den Tod seines Vaters, die Verwaisung von Unternehmen und Familie, seinen Zugang zur Familiengeschichte oder seine Kin- 
der und ihr Zusammenhalt. Erst im Nachfrageteil taucht das Thema der Übergabe an seinen eigenen Sohn Adam zum ersten Mal auf. Michael präsentiert diese aktuelle Nachfolgegeschichte als eine Nebensache in seinem Leben. Der Eintritt seines Sohnes in das Unternehmen ist ein natürlicher und unkomplizierter Vorgang. Mit dieser Übergabe erfüllt Michael einen wesentlichen Teil seiner Mission. Jedoch ist sein eigenes Selbstbild so stark in der eigenen Rolle als Nachfolger verhaftet, dass er die Rolle des Übergebers nur schwerlich in seine biografische Sicht integrieren kann.

In seiner Selbstdarstellung tauchen immer wieder schicksalhafte Momente auf, denen Michael und seine Familie ausgesetzt sind. Die aktiven Momente, die von Michael vollbrachten Leistungen, deutet er hingegen nur kurz an. Sein Leben als Unternehmer ist gerahmt von der Familie und durchsetzt von Widerfahrnissen. So präsentiert er sein Leben zweigeteilt in Unternehmen und Familie. Beide Bereiche folgen ihrem spezifischen Rhythmus, sind jedoch aufs engste miteinander verflochten. Am Ende seiner Rede greift Michael diese Zweiteilung wieder auf und verortet sich mit Blick in die Zukunft auf der anderen Seite: er ist nicht mehr aktiv im Unternehmen verwickelt, sondern gleitet an den Rand, in die Rahmung der Familie.

\subsubsection{Rekonstruktion der erlebten Lebensgeschichte}

Die Text- und thematische Feldanalyse hat einen quellenkritischen Effekt: Sie deckt das Präsentationsinteresse des Erzählenden auf und lässt den Text als ein fast schon strategisches Produkt einer Selbstbeschreibung erscheinen. Die entstehende Skepsis an der „Wahrheit“ der dargestellten Vergangenheit und das Bewusstsein für die Subjektivität der Erzählperspektive bilden eine wichtige Basis für die Rekonstruktion des erlebten Lebens.

In diesem Analyseschritt wird der Text detektivisch auf die Spuren der vergangenen Erlebnisse hin untersucht. In der Chronologie des Lebenslaufes schreitet man von Erlebnis zu Erlebnis und betrachtet dabei die jeweiligen Interviewpassagen. Dabei kann die Sequenzübersicht als Inhaltsverzeichnis wieder verwendet werden. Einige besonders hervorstechende Textstellen werden einer detaillierten Feinanalyse im Sinne der objektiven Hermeneutik unterzogen. Ziel ist es, die latenten Sinnstrukturen des Textes zu entschlüsseln. Die Gefühle, Bewertungen und Wünsche in unterschiedlichen Lebensphasen bzw. bei einschneidenden Erlebnissen sollen aus der Perspektive der jeweiligen Zeit eingefangen werden. 
In dem vorliegenden Interview gelingt diese Rekonstruktion des erlebten Lebens nur bedingt, da Michael während des Interviews eine große Distanz wahrt. Nur gelegentlich gewährt er - zwischen zahllosen Beschreibungen der Zeit und seinen Theorien zum Unternehmer - Einblicke in seine Erinnerungen. Dennoch lässt sich beispielsweise für seine Kindheit und Jugend festhalten:

\section{Kindheit: „Ich bin 1937 geboren...“ (2:7 $)^{64}$}

Aus Michaels Kindheit ist lediglich sein Geburtsjahr bekannt - 1937

- und damit, dass er vor dem Zweiten Weltkrieg geboren wurde. Seine „ersten Eindrücke“ (2:8) habe er „schon in der Kriegszeit“ (2:8) erfahren. Dabei bleibt unklar, was Michael damit meint: Hat er Soldaten gesehen, erinnert er sich an den Rundfunk oder an die Zwangsarbeiter im Unternehmen? Oder spielt Michael hier auf seine Erinnerungen an die Bilder vom Einschlag der Bombe in die Fabrik an (siehe ausführlicher unten)? Im späteren Interview bezeichnet Michael die Erinnerung an diese Zeit als „schwierig, schwierig, schwierig." (11:12) Eine fundierte Aussage über das Erleben der frühen Kindheit ist auf dieser dünnen Datenlage nicht möglich. Michaels Erlebnisse scheinen von anderen überschattet zu sein oder er wollte sie im Rahmen des Interviews nicht thematisieren.

\section{Tod des Vaters: „habe da auch erlebt,... äh dass mein Vater als... Geschäftsführer starb im Krieg“ (2:9)}

Auch über das Erleben von Michael in dieser Zeit/während dieses Ereignisses lassen sich nur wenige Aussagen treffen. Er selbst sagt, er habe nur wenige Erinnerungen an diesen Moment. Doch vergleicht man diese Sätze mit dem restlichen Interview, gibt sich Michael hier erstaunlich offen.

Was ist vorgefallen? Auf den Tag genau kann Michael den Tod seines Vaters benennen. Vielleicht war es das erste Mal, dass Michael Flieger hörte. Sein Vater sorgte dafür, dass alle Familienmitglieder sich im Keller des Gebäudes in Deckung brachten. Michael und die anderen waren bereits im Keller, als der Vater immer noch nach weiteren Familienmitgliedern - wahrscheinlich seinem Bruder, der am gleichen Tag verstirbt - suchte. In diesem Moment schlägt die Bombe in die Fabrik und Wohnung der Familie ein und trifft dabei Michaels Vater. Der zu diesem Zeitpunkt siebenjährige Junge erlebt sind die entsprechende Seite und Zeile aus dem Transkript angegeben. 
diesen Moment als Ausnahmesituation mit verheerenden Konsequenzen, die das Leben der Familie tief greifend verändert. In seiner Erinnerung verknüpft er den Tod seines Vaters mit den Bildern der Zerstörung. Wie das „Zeug rumliegt“ (11:30) ist für ihn eine entsetzliche Erfahrung, an die er noch heute bei Kriegsbildern in den Medien erinnert wird. Die Fetzen seiner Erinnerung sowie die Erzählungen der Anderen, die er im Nachhinein gehört haben muss, formen ein Bild von dieser Situation, dass Michael immer wieder durchlebt. So kommt es, dass der Vater nicht nur einmal, sondern ,immer geguckt" (10:27) hat, dass alle im Keller waren.

\section{Weitere Kindheit: „mehr hatte der halt auch nicht" (13:27)}

Durch den Tod des Vaters ist die Firma verwaist und keiner ist mehr da, der sie führen könnte. Gleichzeitig drückt sich in Michaels Erzählungen seine Trauer über den Verlust des Vaters aus und das Gefühl, dass nun für ihn keiner mehr da zu sein scheint. Wie das „nachher so *war* (gedehnt)..." (10:33) beschreibt Michael als „schon entsetzlich“ (10:32), wobei unklar bleibt, auf welchen zeitlichen Rahmen er sich bezieht. Michael hat in seiner weiteren Kindheit erlebt, was es heißt, Entbehrungen auf sich zu nehmen. Mehrmals sagt er, dass seine Familie „null und gar nichts“ (12:16; 13:13) hatte. Zwar musste die Familie keinen Hunger leiden, aber es hat immer nur gerade so gereicht. Michael erzählt von Streifzügen auf das Land, bei dem sie nach Milch und Eiern gefragt haben. Er erwähnt, dass die Schule für eine zeitlang geschlossen war.

Ein paar Jahre später, Michael ist nun ca. zehn Jahre alt, muss sich die Familie immer noch stark einschränken. Gerne hätten die Kinder Spielzeug gehabt und „da war also schon gar nichts“ (13:24). Abhilfe hat jedoch der Schreiner des Unternehmens geschafft, der für Michael einen Roller baute. Michael erfuhr diesen Roller als besonderes Geschenk, mit dem er wohl viel gefahren ist. Dieser Roller war sein ganzer Stolz. Er sagt „Und ich hatte den... andere hatten den nicht, weil sie keinen Schreiner hatten... äh das war dann schon was.“ (13:29) Michael erfährt durch seine spezielle Familienkonstellation damit nicht nur Entbehrungen, sondern auch ein paar kleinere Privilegien. Gemeinsam mit seinem Bruder entdeckt er mehr und mehr das Unternehmen. Hier toben sie sich aus, spielen Streiche. Gelegentlich stehlen sie dem Schreiner einen Hammer und müssen dafür gerade stehen. Michael beobachtet die Mitarbeiter, manch ei- 
ner hat sich mit ihm unterhalten, andere nicht. Er lernt die Menschen und die Umgebung des Unternehmens kennen, aber nicht den Betrieb in seiner Funktionsweise. Er erlebt diese Zeit spielerisch und als Abenteuer, darüber hinaus verbindet ihn ein starkes Zusammengehörigkeitsgefühl mit seinen Geschwistern. Dennoch spricht Michael von einem gespaltenen Verhältnis zum Betrieb. Möglicherweise spürte Michael Spannungen zwischen der Familie und den Mitarbeitern oder der Fremdgeschäftsführung, die ihn selbst in eine verzwickte Lage brachten.

Ein einschneidendes Erlebnis in Michaels weiterer Kindheit war die Währungsreform. Er bezeichnet dies als logistisches Wunder und war fasziniert von all den Produkten, die über Nacht in den Geschäften präsentiert wurden. Michael erzählt wie „wir“ uns die Nasen platt gedrückt haben. Er erinnert sich an die Hochachtung vor einem 5-DM-Schein, der unglaublich viel wert war. Aber ,der kriegte den schon gar nicht, der hatte 50 Pfennig" (14:8), sagt Michael und beschreibt sich selbst als Kind. Mit dem Geld gönnte er sich einen besonderen Luxus und kaufte sich ein Eis.

Schließlich geht Michael kurz auf seine Konfirmation ein. Auch mit 14 Jahren ging es der Familie finanziell noch nicht so gut wie anderen. Michael hat diesen materiellen Unterschied zu seinen Klassenkameraden wahrgenommen und beschreibt, wie diese ein Sportrad erhalten haben, er hingegen hat sich mit einem alten Fahrrad begnügt. Inwieweit Michael Neid und Eifersucht auf seine Klassenkameraden empfunden hat oder aber diese eher für ihre Konsumorientierung belächelte, bleibt Spekulation.

Michael erlebt in seiner Kindheit eine starke Differenz zwischen den Generationen. Er fühlt sich den Kindern verbunden und grenzt diese gegenüber anderen Familienmitgliedern ab. Beispielsweise beschreibt er seine Tanten als ,fürchterlich alt, entsetzlich alt“ (15:2). Auch seinen Großvater, der mit dem Tod seiner beiden Söhne mit dem Leben abgeschlossen hatte, erlebt Michael als lustlos, deprimiert, alt und gebrochen. Auf Familientreffen haben „wir Kinder keine große Rolle gespielt, nicht?“ (15:4) Und auch sonst lässt sich vermuten, dass Michael wenig Aufmerksamkeit von Seiten der älteren Generation, die sehr mit ihrem eigenen Schicksal beschäftigt war, während seiner Kindheit zuteil war. 


\section{Jugend/Ausbildung: ,das war schon da“6 (7:18)}

Michaels Leben als Oberschüler muss unbeschwert gewesen sein. Es gab wenige Möglichkeiten, aber er war zufrieden mit diesen. Als Jugendlicher verbrachte er seine Zeit gerne im Freibad oder fuhr mit seinem Fahrrad.

Doch dann ging Michael vom Gymnasium ab und begann eine Ausbildung zum Metallbauer (den Tod seines Großvaters im gleichen Jahr thematisiert er nicht). Dieser Übergang in seinem Leben schmerzte Michael sehr und es fiel ihm nicht leicht, sein schönes Leben als Oberschüler aufzugeben. Wie genau es dazu kam, bleibt unklar. Michael war ein Jugendlicher, der sich sehr um seine Familie sorgte. Er sah sich in der Verantwortung, den Betrieb zu übernehmen und war ,eigentlich schon überzeugt, das ist mal deine Aufgabe und ... äh das machst du halt" (7:20-21). Dennoch hatte Michael das Gefühl, sich einer höheren Gewalt zu beugen. „Und da musste ich mich dann fügen ... oder habe mich eigentlich auch gefügt, weil ich eben das Ziel sah, ich will in den Betrieb und alles andere wäre zeitlich kritisch geworden.“ (17:1-3) Wem Michael sich fügen musste, bleibt Spekulation: konkreten Familienmitgliedern oder sich selbst. Den Abgang vom Gymnasium erlebte Michael als Bruch, der ihm einige Optionen versperrte. „Aber das GING NICHT und... WAR auch ein bisschen belastend.“ (17:14-15) Trost lieferte ihm sein geheimer Wunsch zuerst eine Ausbildung zu machen und später noch andere Wege ausprobieren zu können.

Mit dem Schritt in die Ausbildung entfernte sich Michael von seinem jüngeren Bruder. Hier teilte sich der Weg der Geschwister, die ihre Kindheit gemeinsam erlebt haben. Spätestens von nun an wurden die Unterschiede zwischen den beiden deutlich: „Ja, also das war insofern furchtbar einfach, ich war der Älteste... und äh... da es, wie gesagt, eilte, konnte auch nur der Älteste in Frage kommen.“ (23:16-17) Michael fühlte sich in der Verantwortung des Erstgeborenen. Einerseits war er derjenige, dem diese Möglichkeit, das Unternehmen zu übernehmen, eröffnet wurde. Andererseits durfte sein Bruder nun jenes unbeschwerte Leben von Abitur und Studium führen, welches Michael auch gefallen hätte.

Als ebenfalls schwierig erwies sich die Arbeit als Lehrling, bei der sich Michael körperlicher Belastung und langen Arbeitszeiten ausgesetzt sah. Er musste kräftig anpacken und wurde schmutzig. Michael 
unterliefen während der Ausbildung einige Fehler und er wurde angewiesen, sich zu verbessern. Er fühlte sich klein und wahrscheinlich auch ein wenig einsam. Michael durchlebte diese Zeit und das Arbeiten mit seiner „Hand am Arm“ (16:4) intensiv.

In seinem Ausbildungsbetrieb fand er seine ,alten Chefs“ (12:24) als Vorbilder und beobachtete sie bewundernd in ihrem Tun. Michael lernte hier ein unternehmerisches Ethos am lebenden Objekt kennen, dessen zentrale Merkmale Sparsamkeit, Bescheidenheit und Zielstrebigkeit waren, die er nur aus Erzählungen seines Großvaters kannte. Michael wurde nun auch die Funktionsweise eines Unternehmens bewusst. Er zog Vergleiche zwischen dem „eigenen“ Familienunternehmen und seinem Ausbildungsbetrieb und stellte Unterschiede fest: Wir haben veraltete Maschinen und den anderen geht es besser. Er sagt: „das fiel, ... oder ist einem Jungen natürlich sofort auffällig..." (12: 17-18) und ,das hat mich schon geschmerzt als ich das sah" (12:21). Durch diesen Kontrast gewann seine Vorstellung vom eigenen Unternehmen eine andere Dimension, er könnte hier einen Drang zum Erfolg entwickelt haben.

Solche Erwägungen über die Äußerungen von Michael zu den einzelnen Lebensereignissen können nun in Beziehung zu den rein gedankenexperimentell formulierten Thesen aus der Analyse der biografischen Daten gesetzt werden. Einige Hypothesen und Lesarten bestätigen sich nicht, während sich andere erhärten oder ergänzen lassen. Der Vergleich erlaubt es, die Gestalt des erlebten Lebens zu erfassen. Für die vorgestellten Fallstrukturhypothesen im Fall Michael Treuninger lässt sich zunächst feststellen, dass sich die Delegationshypothese bestätigt, jedoch an Dynamik verliert. Die Fürsorgerthese findet hingegen keine weitere empirische Erhärtung. Es ergibt sich zusammenfassend folgendes Bild:

In Michaels erlebtem Leben markieren die Bilder von Krieg und Zerstörung einen zentralen Ausgangspunkt. Diese sind unmittelbar mit dem Tod seines Vaters verknüpft, der nicht nur in der Familie, sondern auch in seiner Funktion als Geschäftsführer im Unternehmen eine Lücke hinterlässt. Die historische Konstellation, das Erleben des Verlustes des Vaters und die im Unternehmen entstehende kritische Situation sind zu einem Ereignis verschmolzen, das den späteren Weg von Michael prägt. Seine Kindheit ist durchzogen von ambivalenten Erfahrungen und gespaltenen Verhältnissen. Das Unternehmen ist für Michael ein Ort des Spielens, an dem er als etwas Besonderes wahrgenommen wird und das ihm Privilegien verschafft. 
Andererseits spürt Michael das gespannte Verhältnis zwischen dem Unternehmen und seiner Familie, die sich in einer ökonomisch schlechten Lage befindet. In der Familie erfährt Michael wenig Aufmerksamkeit und nimmt einen großen Abstand zwischen den Generationen wahr. Er muss sich mit Entbehrungen abfinden. Anderseits gibt ihm der Geschwisterverbund ein Gefühl von Zugehörigkeit und des Zusammenhaltes. Vergleicht Michael diese Konstellation mit dem Leben von anderen, beispielsweise seinen Mitschülern, nimmt er Differenzen wahr. Er wird zum Beobachter seiner sozialen Umwelt, die ihm als ,fremd“ erscheint und bindet sich damit an seine Familie und ihre spezifische Geschichte, die er durch seinen Großvater und das Leben in den historischen Gemäuern erfährt.

Eine besondere Wendung nimmt Michaels Leben mit dem Beginn der Ausbildung zum Metallbauer. Inwieweit Michael die Entscheidung, vom Gymnasium abzugehen, selbst getroffen hat, oder aber von seinen Familienmitgliedern beeinflusst wurde, bleibt weiterhin Spekulation. In der Bewältigung dieser Situation zeigt sich jedoch, dass Michael zu diesem Zeitpunkt bereits als „Hoffungsträger der Familie“ delegiert war. Er selbst sagt: „das war schon da“ (7:18) und meint damit eine innere Verbundenheit zu einem beruflichen Weg ins Unternehmen. Michael kann nicht mehr zwischen „Wollen“ und „Sollen“ unterscheiden, da sich hier beide Dimensionen treffen: Einerseits schmerzt ihn der Abgang vom Gymnasium sehr, und er erlebt diesen als von außen aufgezwungen. Anderseits fühlt sich Michael so stark mit seiner Familie verbunden, dass er ihr seine Loyalität beweisen will. Durch die Ausbildung wird Michael sowohl von seinen Geschwistern als auch von seinen Mitschülern isoliert. Zudem empfindet er die schwere körperliche und zeitlich intensive Arbeit als belastend. Er erfährt Einsamkeit und Andersheit, die in ihm das Bedürfnis weckt, einer von vielen zu sein. Die betriebsleitende Unternehmerfamilie ist für Michael ein wichtiges Vorbild aus seiner sozialen Umwelt. Michael bewundert diese Familie für ihren Arbeitsethos und ihren Erfolg. Dadurch verstärkt sich sein Wunsch danach, selbst den Beruf des „Unternehmers“ zu erwählen und dieser sozialen Gruppe anzugehören. Insgesamt scheint das Leben von Michael durch die Delegation Richtung zu erhalten, es verankert sich in einer Kette von Verpflichtungen, welche die Generationen überspannt und familiäre Identität ermöglicht. Die Beauftragung erhält damit eine über-persönliche Bedeutung. 
Das anschließende Studium erlaubt es Michael, den empfundenen Verlust, den er durch den Abgang vom Gymnasium erlitten hat, wieder auszugleichen. Es wird ihm möglich, den alternativen Lebensentwurf eines Oberschülers bzw. eines Studenten, den er sich während seiner Schulzeit ausmalte, zumindest teilweise mit dem Lebensentwurf des Nachfolgers zu verbinden. Michael erfährt diese Option als ein großes Privileg. Gleichzeitig wird er in dieser Zeit mit den Leben seiner Kommilitonen und ihren Lebensentwürfen konfrontiert. Er erfährt, was alles möglich wäre, und träumt davon, als Jungingenieur nach Frankreich zu gehen. Doch seine Delegation als Nachfolger verdrängt diese Lebensträume, und so erfährt Michael den Einsteig in das Familienunternehmen wiederum als Verlust. Mit diesem Schritt schließen sich eine Reihe alternativer Optionen aus, und Michael ist sich über diesen Umstand bewusst. Gleichzeitig hat er mit diesem Schritt sein Ziel, selbst Unternehmer zu werden, erreicht und die Erwartungen der Familie an ihn erfüllt. In seinem bisherigen Leben hat Michael gelernt, Widerfahrnisse aktiv zu bewältigen. Dafür beobachtet er seine Umwelt höchst sensibel und versucht Signale zu deuten, um rechtzeitig und proaktiv Maßnahmen einzuleiten. Dieses Handlungsmuster ist es, das Michaels Karriere als Unternehmer bestimmt und das es ihm erlaubt, mit dem technologischen Wandel und veränderten Märkten umzugehen. Insofern befindet sich Michael eigentlich in einer passiven und aktiven Rolle zugleich. Immer wieder kommt ein Ereignis, das Michael trifft, ihn schmerzt und dem er ausgesetzt ist. Doch immer wieder schafft es Michael, die Situation zu bewältigen und zieht daraus seine Erfolgserlebnisse.

Michaels Bedürfnis, einer von vielen zu sein, wird durch seine Mitgliedschaft im Kollektiv Unternehmen teilweise gestillt und findet mit der eigenen Familiengründung schließlich ein vorläufiges Ende. Hier fühlt er sich zugehörig, erlebt Zusammenhalt. Hier schöpft er Kraft, um sein Abenteuer als Unternehmer zu leben. Gleichzeitig nimmt der Bedarf an einer Orientierung nach außen ab. Michael braucht keine Vorbilder mehr aus dem sozialen Umfeld. Er ist in seiner gewünschten Rollenkonstellation angekommen und lebt nun seine Vorstellung eines Unternehmers. Michael will ab jetzt selbst ein Vorbild für seine Kinder sein, ein Vorbild, das er sich selbst in seiner Kindheit und Jugend gewünscht hat. Michael freut sich über die Anerkennung seiner Kinder und hofft darauf, dass sie ihm folgen werden, so wie er seinem Vater und Ahnen gefolgt ist. 
Mit dem Älterwerden seiner Kinder stellt sich Michael mehr und mehr in eine Reihe mit den Protagonisten in seiner Familiengeschichte. Wieder nimmt er die Signale, dass sich Adam eventuell für das Unternehmen interessieren könnte und seine Zeit zu Ende geht, frühzeitig wahr. Er räumt ihm - und seinen anderen Kindern - Möglichkeiten ein, das Unternehmen kennen zu lernen, er erzählt von Vorfällen im Unternehmen und wie es ihm damit geht. Später sucht Michael das Gespräch mit seinem Sohn und trifft Vereinbarungen. Gleichzeitig ist sich Michael bewusst, dass er sich nun langsam von seinem Lebensentwurf als aktiver Unternehmer verabschieden muss. Mit der Zeit schwächt auch die Dynamik des Familienverbundes ab: Die Kinder ziehen aus, und der Zusammenhalt wird nicht mehr jeden Tag und im Alltag erfahrbar. Michael fühlt eine Lücke und muss nach Alternativen suchen. Insofern könnte Michaels Engagement im Branchenverband und sein ausgesprochenes Hobby der Familiengeschichte als Versuch gewertet werden, diese beiden parallel laufenden Widerfahrnisse zu bewältigen. In seiner nun kommenden Lebensphase sieht sich Michael als „Spezialist“ (38:20) und unternimmt aktive Schritte, sich als solcher auszuweisen. Darüber hinaus sucht Michael Halt und Zugehörigkeit in der längeren Familiengeschichte. Er wendet sich sozusagen der Gemeinschaft der Ahnen zu.

\subsubsection{Kontrastierung von erzählter und erlebter Lebensgeschichte}

Abschließend soll nun die analytische Trennung zwischen erzähltem und erlebtem Leben hinterfragt werden. Inwiefern unterscheiden sich die jeweils entwickelten Thesen der beiden Zeitperspektiven und in welchem Verhältnis stehen sie zueinander?

Der Kontrast der entwickelten Strukturhypothesen legt nahe, dass Michael in der Situation des Interviews sein typisches Handlungsmuster anwendet: Er versucht, die Signale der Interviewerinnen hinsichtlich ihres Forschungsinteresses zu deuten. Übermäßig stark scheint er sich in die Relevanzstrukturen der Forscherinnen einzudenken und wählt mit Bedacht seine Antworten, die ihre Erwartungen erfüllen sollen. Michael ist bemüht, Struktur in der kontingenten und offenen Interviewsituation (die ihn zunächst etwas aus dem Konzept bringt) zu finden und diese zu bewältigen. Aus dem Interviewkontext heraus könnte sich auch erklären, warum in seiner biografischen Selbstdarstellung der ,väterliche Experte“ so deutlich zu Tage tritt. 
Darüber hinaus lassen sich einige Thesen darüber formulieren, welche biografischen Erfahrungen zu dieser Präsentation in der Gegenwart geführt haben.

Erstens, im erzählten Leben von Michael tritt die Delegationshypothese deutlicher zu Tage als im erlebten Leben. Warum baut er diesen Aspekt als zentrales Thema seiner Selbstpräsentation aus? Der Lebenslauf von Michael sowie sein erlebtes Leben lassen grundsätzlich Raum für alternative Entscheidungen und Optionen: Beispielsweise hätte Michaels Bruder das Unternehmen übernehmen können, hätte der Betrieb verkauft oder einfach nur noch ein wenig länger durch einen Fremdgeschäftsführer geleitet werden können. In Michaels biografischer Darstellung entwickelt er jedoch eine Unweigerlichkeit seines Lebensweges. Ebenso wird in der Rekonstruktion des erlebten Lebens ein möglicher Einfluss von anderen Familienmitgliedern auf die Lebenslaufentscheidungen Michaels deutlich. Diesen familiären Einfluss grenzt Michael in seiner biografischen Darstellung systematisch aus. Michael betont, dass für die Nachfolge kein anderer in Frage kam und ihn aber auch niemand zu diesem Schritt gezwungen habe. Vielleicht versucht Michael auf diese Weise, seine Berufwahl als eine von der Herkunftsfamilie losgelöste Entscheidung zu präsentieren und der Normalerwartung eines eigenständigen Lebensentwurfes zu entsprechen. Michael bezieht einen großen Teil seiner Identität aus der Delegation als Nachfolger. Er spielt mit einer spezifischen Konstellation aus Unternehmersein, Tradition und Familie als Quelle seiner Individualität. Sein besonderer Auftrag hat nicht nur seinem Leben, sondern vor allem seinem biografischen Entwurf davon Struktur verliehen.

Zweitens, in seinem erlebten Leben ist Michael oft klein und macht Fehler. Er muss Situationen bewältigen und aus diesen Erfahrungen lernen. Die Momente des Leids, der Einsamkeit und des Andersseins verblassen in der erlebten Geschichte mit dem Eintritt ins Erwachsenenalter und der Familiengründung. In der biografischen Darstellung gibt sich Michael jedoch über sein Leben hinweg als leidgeprüft. Er wendet die Sprachfiguren des Leidensweges auch noch auf seine Unternehmerkarriere an. Vielleicht sind die frühen Erfahrungen von Tod, Entbehrung und Fremdheit Michael heute noch stark präsent, sodass sie den Grundton seiner Selbstdarstellung angeben. 
Drittens, Michaels neue Rolle als Übergeber wird nur unterschwellig in seiner biografischen Selbstdarstellung deutlich. Zwar spürt Michael die Veränderungen, die mit dem Generationenwechsel einhergehen. Er erkennt die Zeichen des Wandels. Doch er hat das Widerfahrnis Nachfolge noch nicht bewältigt und seinen Rollenwechsel zum Übergeber noch nicht vollzogen. Vor diesem Hintergrund könnte man Michaels Selbstpräsentation als Experte(n) der Familiengeschichte auch so interpretieren, dass er sich gegenwärtig in seiner künftigen Rolle als „Spezialist“ und nicht mehr als Unternehmer übt.

\subsubsection{Rekonstruktion der Familiengeschichte}

Mit der Kontrastierung von erzähltem und erlebtem Leben ist die biografische Fallrekonstruktion von Michael Treuninger vorläufig abgeschlossen. In gleicher Weise interpretiere ich auch die Interviews mit seiner Ehefrau Marcella sowie seinem ältesten Sohn Adam. In diesem Analyseschritt will ich nun die einzelnen Fallrekonstruktionen in Beziehung zueinander setzen. Damit verlasse ich die Fallebene des individuellen Lebens und rekonstruiere die Fallebene der Familie.

Das Genogramm dient als wichtiges Hilfsmittel. (Hildenbrand 2007; Rosenthal 2008: 198; Völter 2003: 48; Hildenbrand 2005:32f.) Es ist eine bildliche Darstellung der Familienkonstellation über mehrere Generationen hinweg, die wesentliche Informationen über die Familie enthält: Namen, Geburts- und Sterbedaten, Heirat, Scheidung, Kinderzahl, etc. Zusätzlich nehme ich in das Genogramm den Besitz von Unternehmensanteilen sowie Tätigkeiten im Unternehmen in leitender Position auf, da sie objektivierte Berührungspunkte mit dem Familienunternehmen darstellen. Der Übersichtlichkeit wegen fertige ich zusätzlich eine Liste mit allen objektiven, biografischen Daten der Familie und ihrer Mitglieder an. Diese ausführliche Liste diente mir bereits bei der Analyse der objektiven Daten als Grundlage. Während ich mich dort auf den jeweiligen Lebenslauf konzentrierte und die Informationen über Eltern und Geschwister nur am Rande einbezog, gilt meine Aufmerksamkeit nun besonders den parallelen Entwicklungen der Familienmitglieder sowie der Handlungslogik des Kollektivs. Insgesamt entsteht ein komplexeres Verlaufsbild als das eines einzelnen Lebenslaufs (trotz seines Verhaftetseins im familialen Kontext). 
Abbildung 8: Genogramm der Familie Treuninger

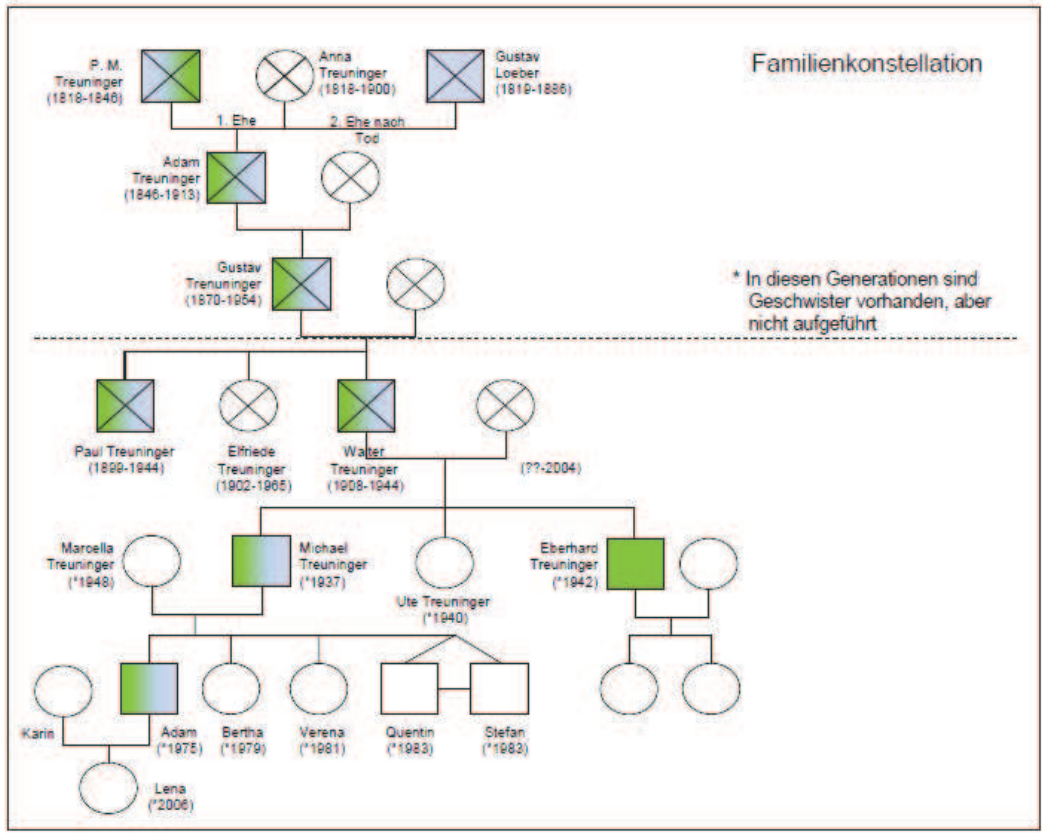

Quelle: eigene Darstellung ${ }^{65}$

Analog zur Analyse der objektiven Daten beginne ich mit der Geburt des ältesten Familienmitgliedes und versuche die Bedeutung dieses Ereignisses für die Familie zu erschließen. Von dort an arbeite ich mich von Ereignis zu Ereignis vor und hinterfrage seine Bedeutsamkeit für diese Familie, anstatt in das Leben einzelner Personen einzutauchen. Dabei formuliere ich vor allem Thesen über die Zusammenhänge dieser Familiengeschichte: Partnerwahl, Heiratsstrategien, Generationenlagen, Namensgebung, Kinderzahl, Berufe, Milieu, Freiheitsspielräume in der Ausgestaltung der Lebensläufe, Ablöseprozesse, Wiederholungen (z.B. in Lebenslaufmustern, Kinderzahl, Namen), die Bedeutsamkeit von Familiensträngen, die Verbindungen zwischen Geschwistern, Eltern und Kindern - diese Ereignisse oder Beziehungen werden gedankenexperimentell durchgespielt. Ersichtlich werden dabei Handlungs- und Lebensweisen sowie wichtige Orientierungsmuster der Familie als eigene soziale Einheit. Zudem gelingt es durch die Arbeit mit dem Genogramm, die Bruno Hildenbrand als „Kunstlehre“ (Hildenbrand 2007:25) be-

65 Erläuterungen zu den verwendeten Zeichen im Genogramm finden sich im Anhang. 
zeichnet, auch solche Familienmitglieder und ihre Rollen stärker in den Blick zu bekommen, die nicht befragt wurden.

Nach der sequenzanalytischen Auswertung der objektiven Daten zur Familie lese ich noch einmal die Interviews sowie meine Auswertungen der einzelnen Fälle quer. Dieser Schritt dient nicht der Überprüfung von Informationen, sondern der Thesenbildung über die Positionierung der Familienmitglieder zueinander. Besondere Aufmerksamkeit widme ich dabei offensichtlichen Widersprüchen, Spannungen sowie latenten Konflikten.

Anhand von zwei Auffälligkeiten im Genogramm der Familie Treuninger möchte ich die Thesenbildung auf Familienebene kurz verdeutlichen:

Erstens, als Michael und Marcella im Jahr 1975 ihren ersten Sohn bekommen, nennen sie ihn nach Michaels Urgroßvater: Adam Treuninger. Diese Wiederholung der Namen sticht besonders hervor, da kein weiteres Kind der beiden nach Personen aus der Familien-, wohl aber aus der Unternehmensgeschichte benannt ist. Warum wählen die jungen Eltern gerade diesen Namen für ihren Erstgeborenen? Lesarten, die über Zufälligkeiten oder Modewellen formuliert werden können, scheinen vor dem Hintergrund der langen Tradition der Familie eher unwahrscheinlich. Michael und Marcella scheinen sich bewusst für den Lebensentwurf einer Unternehmerfamilie entschieden zu haben. Mit der Namensgebung ihres ersten Kindes unterstreichen sie diesen Weg symbolkräftig. Durch diesen Namen wird deutlich, dass sich nicht nur die Familie, sondern auch ihre Geschichte reproduziert. Ebenfalls manifestiert sich in dieser Wahl die Dominanz der männlichen Linie in dieser Familie - die sich auch in den vielen Leerstellen im Genogramm hinsichtlich der Namen und Geburtsdaten der Ehefrauen ausdrückt, die weder aus den Interviews, der Firmenchronik noch weiteren Recherchen hervorgehen.

Aber warum gerade Adam Treuninger und nicht etwa der Name des Firmengründers oder des verstorbenen Vaters von Michael? Vielleicht ist diese Wahl tatsächlich zufällig. Doch bei genauerer Betrachtung werden eine Reihe von Parallelen in den Lebensläufen von Urgroßvater Adam und Michael deutlich: Auch Adam verliert seinen Vater und Onkel in früher Kindheit, wodurch das Unternehmen in externe Hände fällt. Es gelingt Adam, wie Michael später, das Unternehmen in die Blutslinie der Treuningers zurückzuerobern. In ihrer jeweiligen Zeit als Unternehmer durchleben sie große technische Umbrüche (Mechanisierung, Elektronisierung), denen sie mit technischen und organisatorischen Innovationen begegnen. Während Adam das Unternehmen vom Handwerksbetrieb zum Industriebe- 
trieb umbaut, expandiert unter Michaels Führung der regionale Betrieb zu einem internationalen Unternehmen. Auch Adam war, wie Michael, geschichtlich interessiert, seine Neugier galt vor allem dem erfindungsreichen Dorfpfarrer, der die Familie zu ihrer heutigen Tätigkeit brachte. Vielleicht identifiziert sich Michael aufgrund dieser Parallelen besonders mit seinem Urgroßvater, den er nur aus Geschichten seines Großvaters kennt. Michael, dem direkte Vorbilder in seinem Aufwachsen fehlten, könnte Adam zu einer Unternehmerfigur idealisiert haben, für die er große Bewunderung empfand. Vielleicht wünscht sich Michael, dass sein Sohn von diesem Geist beseelt sein mag und die Tradition in diesem Sinne fortsetzen kann. Wenn diese These über die Namensgebung zutreffend ist, dann fügt sich Marcella den Wünschen ihres Mannes. Sie ordnet sich der Tradition unter.

Zweitens, insgesamt bekommen Michael und Marcella fünf Kinder. Auf Adam folgen zwei Mädchen und schließlich noch Zwillingssöhne. Betrachtet man die Ausbildung und berufliche Entwicklung dieser fünf Kinder, so qualifiziert sich theoretisch nicht nur Adam für eine Nachfolge, sondern auch seine jüngere Schwester Verena. Bereits in ihrer Jugend arbeitet sie in den Ferien im Unternehmen mit, sie studiert Betriebswirtschaft und sammelt anschließend Berufserfahrung in England. Drei Lesarten scheinen zunächst wahrscheinlich: Innerhalb der Familie werden, ob nun bewusst oder unbewusst, nur männliche Nachkommen als Nachfolger angesehen. Der Lebensentwurf einer engen Verbindung von Familie und Unternehmen im ländlichen Raum passt nicht zu den Zukunftsvorstellungen der Tochter. Die Geschwister verstehen sich untereinander nicht gut, so dass eine gemeinschaftliche Führung unmöglich erscheint. Um diese Frage zu vertiefen, konsultiere ich noch einmal die Interviewtranskripte.

Hinsichtlich einer Alternative zu seiner Nachfolge für das Unternehmen sagt Adam: „Man hätte es wahrscheinlich verkauft,.. man hätt es verkauft..“ (23:21-22) und weiter „Habe ich... vier, tolle Geschwister also die sind äh.. aber.... die hätten das wahrscheinlich nicht gemacht. Ich glaube nicht dass da einer gesagt hätte, so, das ist was für mich“ (23:27-29). Vor dem Wissen über die biografische Selbstpräsentation Adams, in der sich das Muster des Vaters einer Delegation wiederholt, scheint diese Aussage plausibel. Adam war „eigentümlicher Weise“(3:10) schon immer klar, dass er das Familienunternehmen einmal übernehmen möchte. Er sieht sein Interesse 
an dem Unternehmen schon von Geburt an vorhanden (mit der Namensgebung). In seiner Wahrnehmung sind die Geschwister froh darüber, dass er es macht und keiner von ihnen diesen Weg auf sich nehmen muss. Michael stellt im Interview klar, dass sich unter seinen fünf Kindern sicherlich noch ein anderes gefunden hätte, das die Führung im Unternehmen übernimmt. „Also, bei uns wäre es relativ leicht gewesen zu sagen, dann wird halt ein anderes Kind dann diesen Job übernehmen. ... Also, eine wahrscheinlich, eine große Wahrscheinlichkeit wäre schon gewesen." (40:11) Michael spielt hier auf die relativ hohe Kinderzahl seiner Familie an, ebenso wie seine selbst zugeschriebene Fähigkeit, Interesse an der Familien- und Unternehmenstradition zu erwecken. Aus dieser Aussage lässt sich eine These über die Reproduktionsstrategie von Marcella und Michael ableiten: Eine hohe Anzahl der Kinder erhöht die Chancen der Reproduktion der Familientradition. Der ausgeprägte Wunsch nach einer Großfamilie, der beiden Eheleuten zueigen ist, mag neben der Sehnsucht nach Zugehörigkeit und Zusammenhalt auch von diesem strategischen Element geprägt worden sein. Auf die Frage der Interviewerinnen, ob er ein bestimmtes Kind im Auge habe entgegnet Michael: „DIE FRAGE ist spannend. ... (schnalzt) Ähm, es sind zwei Mädchen, es sind drei Knaben, ... aber äh, also eine studiert Medizin, die wird es wohl nicht sein, aber die Andere ist Betriebswirtschaftlerin, die könnte es. Und ich hätte auch nicht die geringste Hemmung zu sagen, die macht es jetzt"(40:25-26) Es scheint, als ob Michael sich diese Frage im Moment des Interviews zum ersten Mal stellt. Der Reihe nach geht er seine weiteren Kinder durch und bleibt bei Verena stehen. Gegenüber den beiden jungen, weiblichen Interviewerinnen gibt er sich aufgeschlossen. Jedoch scheint diese Option innerhalb der Familie nie durchdacht worden zu sein. Denn: „es stand nicht so an, sagen wir mal so. Dadurch, dass der Adam das immer klar formulierte, war das eigentlich nicht so sehr das Thema." (41:2-3) Adam besetzt damit innerhalb der Familie die ihm zugedachte Rolle des Trägers der Tradition. Gleichsam scheint es innerhalb der Familie eine implizite Vereinbarung zu geben, dass diese Rolle nur von einem Kind ausgefüllt werden kann. Der Zugang für Verena zu diesem Platz innerhalb der Familie und die damit verbundene Anerkennung und Nähe zu den Eltern ist der Tochter verwehrt. $\mathrm{Ob}$ dieser Umstand von ihr als Befreiung - wie von Adam vorgeschlagen - oder als Exklusion erlebt wird, bleibt unklar. Aus Marcellas Perspektive besteht ein enges Verhältnis zwischen Verena und 
ihrem älteren Bruder. Als Adam beginnt, BWL zu studieren, beschließt sie, ihm in dieser Wahl zu folgen. Verena erfährt bei ihren Ferienjobs im Unternehmen jedoch keine Anerkennung. Die weitere Verwandtschaft blickt missbilligend auf ihr Engagement. Marcella sagt:,,weil Frauen gelten da nicht so viel (lacht) und äh die wird doch nicht auch noch kommen wollen."(24:22-23) Aber Verena will nicht. Die Tochter hat „für sich beschlossen, das ist das, was für sie nie in Frage kommen wird, das Unternehmen" (Aus dem Interview mit Marcella 24:11-12) Es lässt sich die These entwerfen, dass Verena ihrem Bruder die Rolle des Nachfolgers überlässt. Sie will nicht in Konkurrenz mit ihm treten oder gegen die männliche Dominanz innerhalb der weiteren Familie ankämpfen müssen. Sie strebt nach einem konträren Lebensentwurf: eine angestellte Tätigkeit, ein sehr großes Unternehmen, Leben in der Großstadt.

Diese Erwägungen verdeutlichen, dass der Lebensentwurf einzelner Familienmitglieder stark von dem Leben und den Vorstellungen der anderen abhängt. Notwendig wird eine Koordination der Lebensläufe nicht nur zwischen Übergeber und Nachfolger, sondern zwischen allen Familienmitgliedern. Interessant scheint darüber hinaus auch die Frage, wie diese Koordination und die Zuteilung der Rollen erfolgt. In dem Wechselspiel aus Handlung und Struktur scheinen die einzelnen Mitglieder kontinuierlich die Lebensvorstellungen der anderen zu beobachten und ihre eigenen implizit daran auszurichten.

\subsection{Theoriebildung und Generalisierbarkeit der Ergebnisse}

Die Lebens- und Familiengeschichten der Treuningers lassen sich nun, da die Fallstruktur offen gelegt ist, im Lichte der Forschungsfrage interpretieren. Die herausgearbeiteten Handlungsregeln, Biografisierungsmuster und Orientierungspunkte stellen an sich schon Verallgemeinerungen dar, denn es wird von der Besonderheit des Falls auf seine allgemeinen Regeln geschlossen. Diese analytischen Schlüsse können nun weiter auf die Frage angewandt werden, welchen Stellenwert das Vorhandensein eines „Unternehmens“ innerhalb dieser Regeln und Muster einnimmt. Ziel ist es, empirisch fundierte Theorien über den Einfluss des Unternehmens auf das Leben und die Beziehungen von Unternehmerfamilien zu formulieren. 
Nach der Auswertung des Falles Treuninger ließen sich drei vorläufige Thesen aufstellen:

Erstens, das Unternehmen sticht besonders stark als Feld beruflicher Aktivität hervor. Die Lebenswege von Michael und Adam werden durch das Unternehmen ähnlich der Erwerbsarbeit in der Normalbiografie strukturiert: Es lässt sich eine Phase der Ausbildung, eine (lange) Phase der Arbeit im Unternehmen und ein Lebensentwurf für das Alter jenseits des Unternehmens erkennen. Der Weg in das Unternehmen erfolgt bei Vater und Sohn in enger Abstimmung mit der Familie und wird als „Mission“ biografisiert. Die Idee, dass das Unternehmen als eine berufliche Option allen Familienmitgliedern offen steht, läuft quer zu den impliziten Handlungsregeln, die sich über mindestens zwei Generationen hinweg institutionalisiert haben. Es gilt, dass nur einer die Rolle des aktiven Unternehmers besetzen kann. Dieser eine ist männlich und wird quasi von der Familie erwählt. Diese Handlungsregeln spiegeln sich in genealogischen Metaphern und der Vorstellung einer Qualifikation qua Blut und Namen in den biografischen Selbstdarstellungen wieder. Das Unternehmersein als Profession wird zu einem wichtigen Bestandteil der Erwachsenenidentität von Adam und Michael. Das gesetzliche Rentenalter oder übliche Vorstellungen über den „Ruhestand“ scheinen hier keine Orientierungsfunktion zu entfalten. Stattdessen führen Vater und Sohn als gewünschten Zeitpunkt für den Austritt die Gesundheit von Michael und damit verbunden seinen Nutzen für das Unternehmen an. Mit einem zweistufigen Alternsbild folgen sie dem Konzept einer Leistungsgesellschaft: Zunächst steht der erfahrene Senior dem Unternehmen unter Führung der jüngeren Generation noch mit Rat und Tat zur Seite. Das Leben jenseits des Unternehmens ist mit Vorstellungen von Pflegebedürftigkeit, Altersschwäche und Nutzlosigkeit verbunden.

Zweitens, der Grad, in dem das Unternehmen auf den Familienalltag wirkt, kann als Resultat einer aktiven Praxis verstanden werden, die das Modell Unternehmerfamilie als ganzheitlichen Lebensentwurf fasst. Die Grenze zwischen Familie und Unternehmen sowie ihre Durchlässigkeit muss aktiv hergestellt werden. Die Familie Treuninger praktiziert zunächst über Generationen einen Lebensentwurf, der eine fast vollständige Aufhebung dieser Grenze vorsieht. Die Fabrikglocke, die den Tagesrhythmus einläutet, kann als metaphorischer Ausdruck für diese Praxis verstanden werden. Mit dem Umzug aufs 
Land und später auch mit der Umsiedlung des Unternehmens an den Stadtrand setzen Michael und Marcella eine etwas stärkere Trennung beider Sphären um. Michael, der während der jährlichen Skiurlaube den Kindern (und auch Marcella) die Familienhistorie als Fortsetzungsgeschichte erzählt, wird zum Sinnbild dieser Praxis. Beiden Entwürfen ist gemein, dass das Unternehmen maßgeblich den Alltag der Familie formt: Es ist ihr zeitlicher, räumlicher und thematischer Anker. Positiv gewendet entsteht so ein Familienleben, das sich durch Regelmäßigkeit, regionale Verwurzelung und ein hohes $\mathrm{Zu}$ sammengehörigkeitsgefühl auszeichnet. Diese idyllische Vorstellung von Familie, die eine emotionale Heimat bietet, hatte für Marcella besondere Attraktivität und war letztendlich der Grund für ihre Partnerwahl. Negativ gewendet entsteht ein Familienleben, das von einem geringen Freizeitkontingent, Immobilität und Enge geprägt wird. Spannungen in der Paarbeziehung, Rivalität zwischen den Geschwistern und Exklusion sind ihre Folgeerscheinungen.

Drittens, die Verbindung von Familie und Unternehmen wird zur Ressource von Individualität. Die Vorstellung eines selbst bestimmten Lebensentwurfes, die sich in den Beschreibungen von einer „freien Wahl“ für dieses spezielle Lebensmodell in den Selbstpräsentationen der Familienmitglieder wieder findet, scheint quer zu den Einflüssen von Strukturen und Handlungsregeln, die sich aus der Verbindung von Unternehmen und Familie ergeben, zu laufen. Die biografische Fallrekonstruktion von Marcella weist jedoch auf ein zentrales Moment hin: In der Orientierungsphase der Jugend gelingt es der angehenden Medizinerin, sich mit ihrem Wunsch nach dem Lebensmodell einer Unternehmerfamilie (in diesem Fall einer landwirtschaftlich tätigen Familie) von ihren Altersgenossen zu differenzieren. Auch Michael und Adam empfinden den Lebensentwurf eines Familienunternehmers im Vergleich mit den peers als individuell. Vor dem Hintergrund einer Gesellschaft, in der die Trennung von Unternehmen und Familie zur Normalität geworden ist, löst sich das Spannungsverhältnis auf: Gerade das Anknüpfen an und das Spielen mit den Traditionsbeständen erscheint als ungewöhnlich und kann das Individuum als einzigartig auszeichnen. Im Kontext von Unternehmerfamilien stellt diese Orientierung an der Generationenlage eine Hürde im Nachfolgeprozess dar: Hier muss der Lebensentwurf des Familienunternehmers, der in der Familie erfahren und als Option erkannt wurde, dem „Individualisierungstest“ der Alters- 
genossen standhalten. Diese These löst jedoch noch nicht die Frage, inwiefern es Familienunternehmern gelingt, sich als von ihren Eltern autonom darzustellen.

Erst der systematische Vergleich dieser vorläufigen Thesen lässt eine theorierelevante Generalisierung von Zusammenhängen zu. Ein solcher Vergleich erfolgt auf zwei Ebenen: Zum einen finden die theoretischen Beschreibungen immer vor dem Vergleichshorizont des Normallebenslaufes (Beruf und Familie) und seiner Destandardisierungs- sowie Pluralisierungstendenzen statt. Die fiktiven Vergleichsgruppen einer modernen Kernfamilie und einer multilokalen Mehrgenerationenfamilie im Gegensatz zu einer vom „Unternehmen beeinflussten" Familie sind wesentlicher Hintergrund der theoretischen Abstraktionen. Zum anderen werden die einzelnen Lebens- und Familiengeschichten im Sinne von kontrastiven Vergleichen in Beziehung zueinander gesetzt. Die Fall übergreifende Analyse beginnt mit der Einzelfallkontrastierung nach dem Prinzip des minimalen und maximalen Vergleichs. Insofern ist die Wahl des nächsten auszuwertenden Falls theoretisch geleitet.

Konkret beziehe ich die Unternehmerfamilie Singermayer als nächstes in die Auswertung ein. Die Anlage dieses Falls weist zunächst einige Parallelen auf: Das Unternehmen befindet sich seit mehreren Generationen in Familienhand. Sven gehört wie Michael der Kriegskindergeneration an, und auch Sven übernimmt sehr früh, ohne Studium, Verantwortung im Unternehmen. Er gründet eine kinderreiche Familie, die etwas abseits des Unternehmens auf dem Land lebt. Seine Ehefrau engagiert sich aktiv im Unternehmen. Ein oberflächliche Blick auf die biografische Selbstdarstellung von Sven lässt auch eine ähnliche Fallstruktur vermuten: Sven biografisiert sein Leben ebenfalls als Mission. Insofern stellt dieser Fall auf der Ebene des individuellen Lebens der älteren Generation einen Minimalvergleich dar. Interessant scheint, dass die jüngere Generation dieses Muster nicht reproduziert. Zwar finden zwei Kinder den Weg in das Unternehmen, scheinen jedoch ganz anderen Handlungslogiken zu folgen: Während die Tochter eher zufällig hineinstolpert, fordert der Sohn seinen Platz aktiv ein. Es herrscht ein offener Konflikt zwischen den Geschwistern. Von diesem starken Kontrast zu der Familie Treuninger auf der Ebene der individuellen Leben der jüngeren Generation erhoffe ich mir, Hinweise auf alternative Handlungsstrategien im Umgang mit dem Unternehmen ebenso wie auf den unterschiedlichen Umgang mit dem Unternehmen innerhalb der Familie zu finden. In ähnlicher Weise werden sukzessive weitere Fälle in die Auswertung einbezogen bzw. erhoben. Im Verlauf des Fallvergleichs entsteht ein Eindruck von den Ähnlichkeiten, Gemeinsamkeiten und Unterschieden der Lebens- und Familiengeschichten. Sie verdichten sich zu typischen Fall übergreifenden Strukturzusammenhängen bzw. Gestaltmustern. (Vonderach 1997:16) Auf dieser Stufe der Forschungsarbeit tritt die Differenziertheit der Fälle notwendig in den 
Hintergrund, nicht aber ihre spezifische Logik und Entwicklungsdynamik. (Przyborski und Wohlrab-Sahr 2008:336) Das empirische Material entledigt sich im Zuge der Abstraktionsarbeit seines Detailreichtums zugunsten von kohärenten Mustern.

Ziel der komparativen Analyse ist eine theoretische Beschreibung der verbindenden und trennenden Elemente der Fälle. Es werden „Typen“ formuliert, die nicht nur verschiedene, oberflächliche Merkmale subsumieren, sondern die Wirkungsmacht und die Regeln des Unternehmenseinflusses erklären. Entlang der Zeitschiene des Lebenslaufes gelingt es, den Einfluss des Unternehmens in seinen unterschiedlichen Erfahrungsgestalten $\mathrm{zu}$ fassen. Relativ feinteilig können für die jeweiligen Lebensabschnitte z.B. typische Wege in das Unternehmen, Elemente des Familienlebens mit dem Unternehmen oder Vorstellungen des Ruhestandes formuliert werden. Diese diachronen Erlebnisse und Bewältigungen überlagern sich innerhalb der Familie und bedürfen einer aktiven Synchronisation durch die Generationen im Zuge von Aushandlungsprozessen, für die sich wiederum typische Themen sowie Verhandlungsweisen beschreiben lassen. Schließlich eröffnet dies den Blick auf die Unternehmerfamilie als Erfahrungs- und Erinnerungsgemeinschaft. So lassen sich nicht nur Thesen über die Unternehmerfamilie in ihrer sozialisatorischen Funktion und über die zukunftsoffene Reproduktion des Unternehmenseinflusses formulieren, sondern auch typische Merkmale herausarbeiten, wie das Unternehmen die Welt der Unternehmerfamilie durchdringt und ein spezifisches Milieu formt.

Die feinteiligen Muster, Normen, Codes, Strategien und Regeln liefern keinen Katalog unterschiedlicher „Arten“ von Unternehmerfamilien. Sie sind vielmehr kleine Bausteine, die zusammengenommen ein analytisches Portrait der Unternehmerfamilie ergeben. In diesem Sinne erlaubt jeder Fall die Genese verschiedener Typen, und umgekehrt kann jeder Fall unterschiedlichen theoretischen Mustern zugeordnet werden. Diese Muster sind insofern idealtypisch als sie über den Einzelfall hinaus weisen und einige Aspekte pointieren. Der Generalisierungsanspruch, der mit den Ergebnissen erhoben wird, ist theoretischer Natur. Die entwickelten Thesen erlauben es nicht, Aussagen über die gesamte Population aller Unternehmerfamilien oder Häufigkeitsverteilungen zu treffen - ein solcher deduktiver Schluss ist auch nicht der Anspruch dieser Arbeit. Stattdessen zielen sie darauf ab, die Unternehmerfamilie besser analytisch fassen zu können und die Regeln ihrer Reproduktion und Handlungen zu verstehen, die von allgemeiner Bedeutung sind (Przyborski und Wohlrab-Sahr 2008:320). 


\subsection{Anmerkungen zur Darstellungsweise}

In der qualitativen Forschungspraxis hat sich ein Berichtsstil für die Ergebnispräsentation herausgebildet, der sich kaum von solchen der quantitativen Forschungspraxis unterscheidet. Die Darstellung qualitativer Ergebnisse scheint sich zu professionalisieren. Eher persönlichere Varianten wie etwa Sozialreportagen oder Forschungstagebücher werden zur fast schon spektakulären Ausnahme und grenzen an populäre Literatur. Problematisch an dieser einheitlich strukturierten Darstellungsweise ist zum einen, dass es nur schwerlich gelingt, den zirkulären Forschungsprozess in ein starres Gerüst zu zwingen. Zwar stellt man - da es den üblichen Konventionen entspricht - die Fragestellung an den Anfang, doch die Forscherin ist sich darüber bewusst, dass die konkrete Fragestellung erst im Laufe (oder fast am Ende) der Forschung so detailreich beschrieben werden kann. Darüber hinaus, und dieser Punkt wurde und wird in der amerikanischen Literatur zur qualitativen Sozialforschung diskutiert (Flick 2005), werden die Ergebnisse mehr und mehr eher als „Fakten“ denn als „Deutungen“, die aus einem Interpretationsprozess hervorgegangen sind, präsentiert. Eine Ergebnispräsentation, die sich auf die entwickelten Theorien konzentriert, trägt zu einer Vereinfachung der Daten auf Kosten der vielen Nuancen und Widersprüchlichkeiten des einzelnen Falls bei. Sicherlich soll ein solches Vorgehen dazu beitragen, die Wissenschaftlichkeit der Ergebnisse zu betonen und sie auf die gleiche Stufe mit den ,findings“ quantitativer Forschung zu stellen. Damit treten jedoch wichtige Unterschiede der erkenntnistheoretischen Grundlagen beider Methodologien in den Hintergrund. Insbesondere die Rolle der Forscherin, die in der qualitativen Forschung eben nicht nur als eine „kontrollierbare“ Variable verstanden wird, sondern als aktive Teilnehmerin, deren lebensweltliches Wissen dem Prozess der Re-Konstruktion der sozialen Wirklichkeit dienlich ist, tritt durch die typische Präsentation in den Hintergrund. (Frost et al. 2010; Flick 2005) Doch an welche Stelle in der konventionellen Gliederung eines Forschungsberichtes passe ich als Forscherin? Ist es überhaupt angebracht, von ,"mir" zu schreiben?

Frost et. al (2010) weisen auf die Ambivalenzen qualitativer Forscher hin, die sich der Besonderheiten des Forschungsprozesses, der Reichhaltigkeit des Materials und ihrer Rolle im Auswertungsprozess bewusst sind und sich dennoch einer konventionellen Ergebnisdarstellung bedienen. Das vorhandene Problembewusstsein der Forscher hat noch keine geeignete Form der Darstellung gefunden. ${ }^{66}$

66 Während Lüders 2004 noch das Fazit zieht, dass diese Diskussion ohne konkrete Ergebnisse hinsichtlich der Darstellungsweise geblieben sind, weisen Frost et al. darauf hin, dass 
So stellt sich auch für mich die Frage, wie ich am besten meine Ergebnisse präsentieren kann. Dieser Satz erweist sich als problematisch, denn ich kann nicht von „meinen“ Ergebnissen sprechen. Diese sind, wie schon mehrfach betont, in einem Interaktionsprozess mit den Befragten, mit weiteren Interviewerinnen, mit meinem Doktorvater, Kollegen und Studenten entstanden. Die vorgestellten Ergebnisse sind ein emergentes Produkt. Sie sind als empirisch erarbeitete Theorien, als plausible Deutungen zu verstehen und nicht als meine subjektive Meinung. Aus diesem Grund wird in der Darstellung der Ergebnisse bewusst nicht eine Erzählperspektive der ersten Person eingenommen.

Die Gliederung dieser Arbeit orientiert sich deshalb an den in der Forschungspraxis geltenden Konventionen. Sie bieten der Verfasserin ein Gerüst, das hilfreich ist, die Gedanken am Ende des Forschungsprozesses zu sortieren und noch einmal die einzelnen Schritte und Ergebnisse zu reflektieren. Zudem wird damit den Erwartungen der Leserschaft entsprochen.

Schlussendlich werden die Ergebnisse nicht in Form von ausführlichen Fallbeschreibungen präsentiert. Die Wiedergabe der jeweils erzählten und erlebten Lebensgeschichte sowie der Familiengeschichten würde zu viel Raum einnehmen und stellt sich auch im Hinblick auf die Anonymität der Befragten als schwierig dar. ${ }^{67}$ Kurze Fallabrisse, Interviewpassagen, Strukturhypothesen und eine Übersicht der Fallgeschichten im Anhang tragen dazu bei, die empirische Fundierung der Ergebnisse zu belegen. Mit dem Verzicht auf eine Darstellung des Facettenreichtums der sozialen Einbettung des einzelnen Falls (die im übrigem auch in Falldarstellungen nur annäherungsweise gelingen kann) wird mehr Raum gelassen für eine ausführliche Schilderung und theoretische Einbettung der Ergebnisse. Eine Konzentration auf die erarbeiteten theoretischen Annahmen, Muster und Typologien soll zudem die Generalisierbarkeit der Ergebnisse (im oben dargelegten Sinne) unterstreichen. Die Hoffnung, die sich damit verbindet, ist, dass auf diese Weise eine breite Basis für eine thematische Diskussion sowie mögliche Anknüpfungspunkte für weitere (hypothesengenerierende und hypothesenprüfende) Forschung geschaffen werden.

Die Ergebnisse dürfen jedoch nicht als objektive Fakten missverstanden werden. Aus diesem Grund wird der Beschreibung des Forschungsprozesses relativ viel Platz eingeräumt. Die ausführliche Schilderung der einzelnen Schritte soll Transparenz und intersubjektive Nachvollziehbarkeit schaffen. (Lüders 2008:634) Damit soll einer zentralen Anforderung an die Qualität der qualitativen Forschung nachgekommen werden - der Leser soll sich

solche zur Zeit im Entstehen sind, wie etwa „,performative“ oder „,performance work“ Frost et al. 2010: 456.

67 Diese Entscheidung bedeutet auch, dass ein großer Teil der geleisteten Auswertungsarbeit nicht dargestellt wird Albertini et al. 2006. 
selbst ein Bild von der Qualität der präsentierten Forschung verschaffen können. In diesem Abschnitt tauche auch ,ich“ und „wir“ auf - diesen Bruch mit der sonst einheitlichen Erzählperspektive setze „ich“ als stilistisches Mittel ein, um die Dynamik des Forschungsprozesses sowie die Rolle der Forscherinnen zu vergegenwärtigen.

\subsection{Zusammenfassung}

Zur Untersuchung der Fragestellung wird eine interpretative Vorgehensweise gewählt, die die Bedingungen, Merkmale und Auswirkungen des Unternehmenseinflusses auf die Unternehmerfamilie rekonstruiert. Besonders geeignet scheint eine biografietheoretisch fundierte Untersuchungsmethode, die davon ausgeht, dass sich subjektive und kollektive Wirklichkeiten wechselseitig durchdringen. (Hildenbrand 1991:257) Insofern lassen sich aus den Lebensgeschichten der Mitglieder einer Unternehmerfamilie sowohl das individuelle Erleben als auch der Generationenzusammenhang rekonstruieren.

Insgesamt werden 32 narrativ-biografische Interviews mit Mitgliedern aus zwölf Unternehmerfamilien geführt. Das heißt, die Befragten werden in jeweils separaten Gesprächen aufgefordert, die Geschichte ihres Lebens und ihrer Familie zu erzählen. Nach der Eingangserzählung werden einige der erwähnten Punkte vertieft und vorbereitete Nachfragen speziell zur Nachfolgeregelung gestellt. Den Fällen ist gemeinsam, dass mindestens ein Familienmitglied die Nachfolge im Unternehmen antritt, sie unterscheiden sich im Hinblick auf Branchenzugehörigkeit, Region und Familiendemografie.

Die transkribierten Interviews werden einzeln fallrekonstruktiv nach Rosenthal ausgewertet. Nach einer Analyse der biografischen Daten erfolgt die Rekonstruktion des erzählten sowie erlebten Lebens. (Fischer-Rosenthal und Rosenthal 1997; Rosenthal 1995, 2008; Rosenthal und Fischer-Rosenthal 2007; Rosenthal 1999) Auf diese Weise entsteht ein Verständnis für die Lebenslaufentscheidungen sowie die biografische Selbstdarstellung der Befragten. Abschließend werden die Interviews der einzelnen Familienmitglieder in Verbindung gesetzt. Hierfür werden mittels Genogrammen zunächst die objektiven Daten der Familiengeschichte rekonstruiert und anschließend die Aussagen der Familienmitglieder zu den jeweiligen Ereignissen und unter Berücksichtung ihrer biografischen Selbstdarstellungen aufeinander bezogen. Die doppelte Anlage des Falles, einerseits auf der Ebene des individuellen Lebens der Befragten und andererseits auf der Ebene der Familie, erlaubt es, den Einfluss des Unternehmens nicht nur auf der Ebene des Lebenslaufes der Familienmitglieder, sondern auch im Hinblick auf die Generationenbeziehungen und die Familie als Erinnerungsgemeinschaft offen zu legen. 
Die Interpretationsarbeit wird exemplarisch an einem Fall dargestellt und auf diese Weise dem Kriterium der Nachvollziehbarkeit qualitativer Forschung entsprochen. Die aus dem empirischen Material generierten Muster und Typen sind als theoretische Verallgemeinerungen zu verstehen. 


\section{Lebenslauf und Lebensführung des Familienunternehmers}

In diesem Kapitel wird der Versuch unternommen, die Leben der befragten Personen nebeneinander zu legen und die typischen Phasen näher zu beschreiben. Auf der einen Seite soll in den einzelnen Lebensphasen verweilt und der Einfluss des Unternehmens auf das Leben der Person herausgearbeitet werden (synchrone Perspektive), auf der anderen Seite gilt es den dynamischen Entwicklungsprozess des Lebenslaufes zu erfassen (diachrone Perspektive). Die Analyse erfolgt auf den Ebenen der alltäglichen Lebensführung, des Lebenslaufes sowie der Biografie, die von Moen herausgearbeiteten Kategorien Timing, Prozess und (personaler und historischer) Kontext werden als Aufmerksamkeitspunkte einbezogen und danach gefragt, inwieweit vorgelagerte Lebensphasen spätere berühren. Der personale Zugang soll zeigen, auf welche Weise sich Unternehmen und Familie verbinden. Der Familienzyklus findet aus einer personalen Perspektive Eingang und unterscheidet sich deshalb von anderen Zyklusmodellen über Unternehmerfamilien (etwa Klein 2000; Lansberg 1999; de Kets Vries 2007), die in junge Unternehmerfamilien, Unternehmerfamilien mit sich autonomisierenden Kindern, eine Phase des Zusammenarbeitens und schließlich (eine) des „Wachwechsels" unterscheiden. Erst im nächsten Kapitel erfolgt eine intensive Betrachtung der Generationenbeziehungen.

\subsection{Das Aufwachsen in Unternehmerfamilien}

Im Folgenden sollen die kindliche und jugendliche ${ }^{68}$ Perspektive auf das Familienunternehmen und die damit verbundenen Besonderheiten der Familie thematisiert werden. Dieser spezifische Blickwinkel kommt der Forderung nach, das Kind und den Jugendlichen nicht mehr „bloß als defizitäres Sozia-

68 Die gebildeten Thesen bedienen sich hauptsächlich jener Interviews, die mit Personen der zweiten und späteren Generation geführt worden sind. Der Ergebnisdarstellung werden keine Altersgrenzen für „Kindheit“ und „Jugend“ zugrunde gelegt, über die ohnehin in der Forschung kein Konsens besteht Schweizer. Herbert 2007:80. Gemeint ist damit die Zeit von der Geburt bis zum Ende der schulischen Ausbildung, die relativ grob mit dem „Aufwachsen in einer Unternehmerfamilie“ umschrieben wird. 
lisationsobjekt zu begreifen, sondern von Geburt an seine bemerkenswerten Kompetenzen hervorzuheben, die es als Subjekt und Experte für die eigene Lebensführung von Anfang hat" (Schweizer. Herbert 2007:54). ${ }^{69}$ Im Vordergrund stehen Aspekte des alltäglichen und außeralltäglichen Lebens ebenso wie der Versuch die kindliche Wirklichkeit einzufangen - so es eben gelingen kann, diese „Sicht des Kindes“ aus der Perspektive des erwachsenen Forschers zu rekonstruieren. Im Rahmen dieser Arbeit lautet die zentrale Frage: Wie erschließen sich Kinder und Jugendliche die Welt des Unternehmens?

\subsubsection{Das Unternehmen als Erlebniswelt}

Den Erinnerungen der Befragten an ihre Kindheit und Jugend ist eine unheimliche Plastizität des Unternehmens zueigen. Sie riechen, schmecken und fühlen das Unternehmen, erinnern sich an Begegnungen mit Mitarbeitern oder an Gespräche der Eltern über die Vorgänge im Unternehmen. Sie erfahren das Unternehmen situationsgebunden im Hier und Jetzt.

In den 1960er Jahren wachsen Wilhelm Fritz und Friedrich Carsten Naumann als die beiden älteren Söhne von Felix Walter und Ottilie auf einem Weingut auf. Das herrschaftliche Gutshaus ist eingebettet zwischen einem kleinen Dorf und den Weinfeldern. Im Keller befindet sich das Kelterhaus, darüber lebt die Familie, auf dem Hof wird eine bunte Mischung an Nutztieren gehalten. Die Jungen erleben das Gelände, auf dem sie sich frei bewegen können, als eine idyllische Umgebung. Ihr Spielplatz ist der Hof, auf dem es nie langweilig wird. ,Viele Nachbarskinder, befreundete Kinder“" (Wilhelm Fritz Naumann, 4:15) kommen vorbei. Die Brüder dürfen auf den Pferden reiten, klauen sich im Hühnerstall Eier und werfen diese übers Dach oder spielen fangen. Gleichsam nehmen sie schon früh die landwirtschaftlich-betrieblichen Abläufe auf dem Weingut wahr. Das Pferd ist eben nicht nur ein Pferd, sondern „Ackergaul“" und „Zugpferd“. Sie erkennen, dass das Weingut für die Eltern eine „Riesenaufgabe“ darstellt, die „,räumlich gesehen und auch zeitlich“ bindet. Die betriebliche Seite gehört für sie einfach ,immer" dazu ${ }^{70}$.

Der Fall Naumann veranschaulicht die große Faszination der Kinder für das Unternehmen als Räumlichkeit, als Ort der Tätigkeit und als Welt der Erwachsenen, der erkundet werden kann oder aber ein Geheimnis bleibt. Sie erinnern sich an ein Gebäude mit vielen Stockwerken und vielen Leuten, an

69 Diese Forderung, das Kind als sozialen Akteur nicht mehr länger nur aus der Perspektive der Eltern, sondern in seinem eigenen Recht zur Geltung kommen zu lassen, markiert einen Paradigmenwechsel innerhalb der Familien- und Kindheitssoziologie Schweizer. Herbert 2007; Honig 1999.

70 Die Zitate stammen aus dem Interview mit Wilhelm Fritz Naumann 2:5ff. und 41:21. 
große Felder oder an interessante Maschinen und einen ausgeprägten Metallgeruch. Konrad Niest sagt: „Es war also alles schon groß und dreckig und laut und hat mächtig nach Öl gerochen." (9:14-15) Das phänomenale Erleben des Zusammenhanges zwischen Unternehmen und Familie der Kinder hat einen vordringlichen und einnehmenden Charakter, der prägende Erinnerungen und Assoziationen hinterlässt.

Der Kontakt mit den Menschen des Unternehmens (Mitarbeitern, Lieferanten, Kunden) markiert im kindlichen Erleben des Unternehmens ein weiteres zentrales Element. Claudia Claas etwa begleitete ihren Vater regelmäBig zu Bankgesprächen oder „Fabrikleute“ wurden zu Hause empfangen. Michael (Vater) und Adam (Sohn) Treuninger beschreiben, wie sie sich von den Werkstattmitarbeitern öfter einen Hammer ,geliehen“ haben und Ärger bekamen, wenn sie ihn nicht rechtzeitig zurücklegten. Das „Spiel“ mit Menschen aus dem Dunstkreis des Unternehmens wird zu einer festen Größe in der kindlichen Lebensführung. Kindern wird im Umgang mit diesen oft fremden Personen deutlich, dass sie als Teil einer Gemeinschaft (der Unternehmerfamilie) wahrgenommen werden, sie fühlen sich verbunden und geschätzt. Christina Lubinski weist in diesem Zusammenhang darauf hin, dass Familienangehörige ihre Namensgleichheit als Symbolisierung ihrer Zugehörigkeit, aber auch als Gebundenheit erleben (Lubinski 2010:123). Mit der Veralltäglichung dieser Begegnungen verstetigen sich die ambivalenten Gefühle über die eigene Rolle und Position. Die Menschen des Familienunternehmens schenken den Kindern Aufmerksamkeit, haben ein offenes Ohr oder etwas Süßes in der Schublade und erweitern auf diese Weise den Kreis an Bezugspersonen ${ }^{71}$. Der Umgang mit diesen Personen wirkt gleichzeitig als Bühne für die Eltern-Kind-Beziehung, als Chance, zusätzliche Aufmerksamkeit von den Eltern zu erhaschen, etwa über ein gutes Benehmen gegenüber Kunden und Lieferanten.

Bei den Naumanns finden regelmäßig große Feste aus verschiedenen Anlässen statt, wobei die Weinlese den Höhepunkt des Jahres markiert. Die ganze Familie versammelt sich, unterstützt von Mitarbeitern und Helfern, um im Weinberg die Trauben zu lesen. Am Abend wird im Kelterhaus ausgelassen mit Wein und Musik gefeiert. Friedrich Carsten erinnert sich:

„Eine meiner allerfrühesten Kindheitserinnerungen ist, dass mein Bruder, mein älterer Bruder und ich mit dem Ohr auf dem Fußboden waren in unserem Kinderzimmer und der Quetschkommodenmusik im Kelterhaus ein Stockwerk darunter, es war nämlich genau darunter, hingebungsvoll gelauscht haben. Wir hatten einen Jugoslawen, Herr *Kolar*, ähm Namen, die sich einfach *eingeprägt haben da-

71 Damit soll nicht behauptet werden, dass sich eine Bindung zur Unternehmerfamilie nur aus solchen Kontakten erklären lässt. 
mals* (lachend), der als Gastarbeiter gearbeitet hat bei meinem Großvater. ... Ja, das war wirklich nur bis ... Anfang der 70er schätze ich mal, da war ich wirklich noch klein, und der spielte sehr schön die Quetschkommode und der machte dann immer Rumtata und ja, das waren so, so Erinnerungen.“ (20:17ff.)

Solche Festivitäten spielen in der Erlebniswelt der Kinder eine herausragende Rolle, denn sie stellen das Nichtalltägliche dar, das sich von der Normalität abhebt. Mit Spannung beobachten sie das Schauspiel, in dem ihnen oft eine repräsentative Rolle zugedacht wird. Die im Alltag erlangten Rollen- und Gruppendefinitionen werden in dieser Ausnahmesituation auf die Spitze getrieben und überlagern sich. Die Kinder fühlen sich als Teil ihrer Familie im Sinne von Verwandtschaft und gehören auch einer größeren Gemeinschaft an, die das Unternehmen „von innen“ kennt. Sie nehmen wahr, dass Mitarbeiter, Kunden, Lieferanten und andere ihnen den Status eines Mitgliedes der Unternehmerfamilie verleihen und sie sich dadurch von ihnen differenzieren. Darüber hinaus erkennen sie eine Grenze zur Erwachsenenwelt, die für sie unüberwindbar ist. Die Unternehmerfamilie präsentiert sich gleichzeitig nach innen und nach außen und unterzieht die kindliche Positionsbestimmung sowie das Bedürfnis der Kinder nach Zugehörigkeit einem Belastungstest.

Das Unternehmen fließt zudem und überwiegend in Form von Erzählungen in die Gestaltung der kindlichen Lebensführung ein: Die Eltern erzählen beim Abendessen von aktuellen Vorgängen im Unternehmen, lustigen Begebenheiten oder besprechen Probleme; beim Sonntagsausflug klingelt das Telefon und Kinder hören das Gespräch mit einem Kunden; am Abend steht ein Mitarbeiter vor der Tür und erzählt von einem dringenden Problem; die Eltern streiten sich darüber, dass die Firma zu viel Zeit in Anspruch nehme oder der Familienurlaub verschoben werden muss; bei einem Firmenjubiläum werden Anekdoten aus alten Zeiten erzählt und über die Unternehmensentwicklung bilanziert; bei einem Besuch in der Firma erklärt ein Mitarbeiter, wie eine bestimmte Maschine funktioniert; die Großeltern berichten davon, wie der Betrieb früher aussah, welche Arbeitsmittel eingesetzt wurden und vielleicht auch von der Gründung des Unternehmens. Die Kinder nehmen das Unternehmen als Organisation, aber vor allem als Bestandteil der erwachsenen Lebensführung, vermittelt über solche Narrative, wahr. Insbesondere (dann,) wenn diese kommunikative Ebene mit der physischen Erfahrung des Unternehmens gepaart ist, sind die Gedanken und Probleme der Erwachsenen für die Kinder greifbar.

An dieser Stelle seien besonders der Moment des Hörens und seine Erlebnisdimension betont. Das Gespräch über das Unternehmen transportiert nicht nur internes Wissen über die Organisation, sondern lässt die Kinder und Jugendlichen auch an der Reaktion der Sprecher teilhaben. In diesem Moment können sie über die Freude oder Wut der Eltern reflektieren, versuchen 
sich in ihre Situation zu versetzen. Auf diese Weise wird das Unternehmen vermittelt über die Eltern Bestandteil ihrer Erfahrungswelt, die sie mit anderen Familienmitgliedern teilen und auf deren Grundlage sie Verhaltensregeln und Rollenzuschreibungen ableiten. Auf die Besonderheiten der Erzählkultur in Unternehmerfamilien sowie die Bedeutung der Familiengeschichte wird an anderer Stelle noch ausführlicher eingegangen. Der Punkt, der hier verdeutlicht werden soll, ist folgender: Das Unternehmen ist ein wesentliches Thema in der familialen Kommunikation.

Mit der Zeit verstehen Kinder und Jugendliche die Tätigkeiten der Erwachsenen und üben sich in diesen. Unter dem Motto „Schau, was ich schon kann" lässt sich die Mitarbeit als weiteres Element im Streben nach Aufmerksamkeit und Anerkennung von den Eltern und auch durch Altersgenossen lesen.

Die Brüder Naumann beginnen im Alter von sieben und acht Jahren vielfältige Aufgaben auf dem Hof zu übernehmen: von Laubarbeiten an den Reben über Pflanzungen bis zum Rebenbiegen oder der Mithilfe bei der Weinlese. Sie möchten „mitmachen“ und „mitbekommen“, was die Erwachsenen so treiben. Wilhelm Fritz macht ,gleich“ mit 16 Jahren den Führerschein, um nun auch Traktor fahren zu können. Dies bereitet ihm ,großen Spaß“ und erfüllt ihn wahrscheinlich auch mit Stolz, da er nun eine Erwachsenenaufgabe übernimmt, die ihn von seinen jüngeren Geschwistern unterscheidet.

Die Brüder Andreas und Oscar Vester haben ,schon mit drei Jahren den ersten Teller getragen und dann ging es so weiter" (Oscar Vester, 1:11). Sie arbeiten am Büffet, bei Veranstaltungen oder unterhalten sich mit den Gästen. Neben dem „Spaß“ an der Arbeit erhoffen sie sich, den Eltern damit nahe zu sein. Die Mitarbeit der Kinder könnte ihnen das Gefühl geben, Bestandteil der Erwachsenenwelt zu sein.

Auch Adam Treuninger verbringt einen Teil seiner Freizeit im Betrieb und arbeitet in den Ferien mit. Er teilt diese Erfahrung mit Schulkameraden und „sogar" seiner Freundin. Adam holt sich einen wichtigen Teil seines Lebens in das Unternehmen hinein. Er steckt Altersgenossen mit seiner Begeisterung für die Tätigkeiten und Möglichkeiten des Betriebes an. Gemeinsam übernehmen sie Marketingaktionen und haben Spaß. Adam berichtet davon, wie sie mit der Telefonanlage der Firma über mehrere Leitungen gleichzeitig für ihre Favoriten in der Radio-Hitparade abgestimmt haben. „Insofern war das ähm mitunter recht heiter." (3:2-3) Adam hat Spaß an diesen Tätigkeiten und kann sich unter seinen Altersgenossen profilieren.

Um ein kurzes Zwischenfazit zu ziehen: Das Unternehmen wirkt in der Phase der Kindheit und Jugend in einer phänomenalen Wucht ein, die bislang in der Literatur m.E. unterschätzt wird. Kinder und Jugendliche erleben das Unternehmen als einen festen Bestandteil der alltäglichen Lebensführung, die nicht in verschiedene funktionale Bereiche zerfällt, sondern eine Einheit bildet. Das Unternehmen ist eine Tatsache in der Art und Weise wie ihre Fami- 
lie als Unternehmerfamilie lebt. Sie erleben das Familienunternehmen als eine Örtlichkeit, begegnen seinen Menschen, feiern besondere Anlässe, lauschen den Erzählungen der Erwachsenen und beobachten ihre Tätigkeiten. Im Vordergrund steht für sie nicht die Herausforderung, Erwartungen unterschiedlicher funktionaler Systeme zu koordinieren, sondern ihre Position in dem Kollektiv der Unternehmerfamilie zu entdecken, zu internalisieren und zu verstehen. Das Unternehmen wird zu einem Vehikel der persönlichen Beziehungen und zum Erlangen von Aufmerksamkeit und Anerkennung. Besonders interessant ist, dass sich die geschilderten Erfahrungen im Bereich der Landwirtschaft potenzieren: Hier wird der Zusammenhang von Familie, Arbeit und Anerkennung unmittelbar greifbar. Das verdeutlichen nicht nur die Interviews mit den im Agrarsektor tätigen Familien, sondern auch jene mit Angehörigen der Kriegskindergeneration, die während der Nachkriegsjahre einige Zeit auf einem Hof verbracht haben.

Schließlich sei auf eine Studie von Eva Schmitt-Rodermund und Elke Schröder ${ }^{72}$ verwiesen, die quantitativ belegt, dass das Unternehmen ein fester Bestandteil der kindlichen Erlebniswelt ist: Nur 4\% der Kinder gehen nie in den Betrieb, $31 \%$ gehen täglich, $22 \%$ mehrfach in der Woche und 18\% mehrfach im Monat einfach so in den Betrieb, beispielsweise, um die Eltern zu besuchen oder sich dort aufzuhalten. Die Studie verweist zudem auf die herausragende Rolle der Mitarbeit im Familienbetrieb. Nur 10\% der Jugendlichen geben an, nie mitzuarbeiten, und der größte Teil (53\%) übernimmt unregelmäßig in den Ferien Arbeiten.

\subsubsection{Die Mitarbeit im Familienunternehmen}

Das Thema Mitarbeit im Familienunternehmen zeigt besonders deutlich, dass in Unternehmerfamilien die Kindheit und Jugend als eine Lebensphase an sich verstanden wird, die mit der Entwicklungsaufgabe verbunden ist, seine Persönlichkeit und Identität zu finden. Das Unternehmen wird zwar als feste Größe in den familialen Alltag integriert und die Teilnahme an Firmenveranstaltungen, Festen, etc. kann einen verpflichtenden Charakter haben, dennoch bleibt das Unternehmen ein Raum, in dem sich das Kind entfalten kann. So beruht Mitarbeit im Unternehmen weitgehend auf Freiwilligkeit, spielerischem Interesse und später im jugendlichen Alter auf der Möglichkeit, sich etwas dazu zu verdienen. Die kulturelle Vorstellung der Kindheit als gesellschaftlich notwendiges und sinnhaftes Moratorium (Schweizer. Herbert

72 Die Studie wurde an der Universität Jena zur Förderung und Entwicklung des Nachwuchses in Familienunternehmen durchgeführt. Insgesamt wurden deutschlandweit 255 Jugendliche aus 155 Unternehmerfamilien getrennt von den Eltern interviewt. Wenn möglich, wurden pro Familie alle Kinder im Alter von 14 bis 21 Jahren berücksichtigt. Das Durchschnittsalter der Befragten lag bei 16,4 Schmitt-Rodermund und Schröder 2009: 13. 
2007:83), also als ein geschützter und institutionalisierter Raum des Lernens und Spielens, die wesentlicher Bestandteil moderner Familienformen ist, hat sich damit auch in Unternehmerfamilien etabliert. Ein Verstoß gegen diese allgemeine Norm zieht pathologische Folgen nach sich. Werden Kinder, wie im Ganzen Haus üblich, als kleine Erwachsene verstanden und die Mitarbeit als selbstverständliche Pflicht der Kinder erachtet, so kann dies als ausgrenzendes Element erlebt und retrospektiv als Ausbeutung bewertet werden, was letztlich zu einem Bruch in der Eltern-Kind-Beziehung führen kann. Folgender Fall verdeutlicht diese These:

Kurz nach der Wende entscheidet sich Familie Übermann dazu, die alten Ländereien der Familie zurückzunehmen und einen eigenen Weinbetrieb zu gründen. Durch das gemeinsame Projekt wird der 13jährige Markus als tatkräftiger Mitarbeiter eng an seine Eltern gebunden, und es bleibt nur wenig Raum für Autonomisierung und Individualisierungsbestrebungen des Heranwachsenden. Er sagt: „meine Eltern haben den „Betrieb gegründet ... und dann 92 habe ich eben aktiv daran teilnehmen dürfen, müssen, können, ... habe ich dann eben Verantwortung übernehmen müssen." (4:31-33) Zwar hat Markus schon als Kind im Betrieb geholfen, doch der Arbeitsaufwand und die Belastung für Markus verstärken sich nun drastisch.

Unter Gleichaltrigen fühlt sich Markus als Außenseiter. Er kann weder von seinen Freizeitaktivitäten oder Urlauben berichten, noch spielt er Fussball in einem Verein. Weder kann er nachmittags fernsehen, noch hat er Taschengeld zur freien Verfügung. Er erinnert sich gut daran, wie er für seinen Großvater Getränke kaufen sollte und dieser ihm nicht einmal das Restgeld schenkte. Markus weiß, dass es auch ein anderes Leben mit mehr Freizeit und materieller Zuwendung gibt. Das Traktorfahren bietet Markus endlich die Gelegenheit, sich durch ein Privileg gegenüber anderen auszuzeichnen. „Da war man schon der Held!“ (3:31) Doch diese kurze Phase der Geltung gleicht nicht das permanente Gefühl des Außenseiters aus. Er sehnt sich nach einem normalen Familienleben und (er sehnt sich) nach mehr materiellem Wohlstand. Markus drängt seine Eltern, größere Anschaffungen zu tätigen, z.B. einen C64 (Computer) zu kaufen. Die Eltern erfüllen ihrem Sohn den Wunsch, verwenden die Spielzeit jedoch als Belohnung für seine Arbeit im Weinberg.

Nach der Realschule nimmt Markus eine Ausbildung zum Elektroinstallateur auf. Er begründet diese Entscheidung damit, dass ein praktischer Beruf immer von Nutzen für den Hof sei. Doch der wichtigste Grund für den Ausbildungsberuf scheint die regionale Nähe und damit die Verfügbarkeit für den elterlichen Betrieb zu sein. Dennoch verwundert es, dass Markus nicht gleich in die Winzerlehre geht. Sein Vater war einer der wenigen Ausbilder in diesem Beruf in der Region. Doch diese Option wird nicht thematisiert.

„Natürlich“ arbeitet Markus auch während seiner Lehrzeit am Wochenende und nach Feierabend in den Weinbergen und auf dem Hof mit. Weiter- 
hin ist für ihn die Mitarbeit verbunden mit Entbehrung und einem Außenseiterdasein. „Wo andere dann eben im Bad gelegen haben usw.“ (28:14) musste Markus arbeiten oder sein Schlafdefizit ausgleichen. Dennoch versucht er, typische Aktivitäten der Jugend in sein Leben zu integrieren, wie etwa Diskobesuche in den frühen Morgenstunden zwischen Weinlese und Weinabfüllen.

Die Arbeit in der Ausbildung und auf dem Hof füllt Markus stark aus. Er entwickelt keine ausgeprägten Hobbies, schwärmt nicht für Stars oder pflegt keine ernsthafte Beziehung. Er sagt: „Ein Mädel hat ja immer Zeit geraubt. ... Mir nicht, aber den Eltern halt, du warst dann eben nicht verfügbar." (30:20-22) Hier deutet sich an, dass die Eltern von Markus auch nicht viel Verständnis für solche Interessen gezeigt hätten, da sie vom Wesentlichen hätten ablenken können und vielleicht als Flausen oder Spinnerein abgetan worden wären.

Die zeitliche Korrelation zwischen der Wendezeit und der Gründung des Unternehmens (das eine bedingt das andere) verknüpfen sich für Markus mit dem Ende seiner Kindheit. Insofern scheint es eine ,gute alte DDR' zu geben, in der er noch relativ behütet war, und eine neue Zeit, in der er zur Verantwortung gezogen wird und hart arbeiten muss. Denn ,alles war zu Ende und alles wurde anders" (2:10-11). Insbesondere vor dem Hintergrund der gesellschaftlichen Veränderungen mag Markus die Lebensweise und die Vorstellung von Familie seiner Eltern als rückständig empfunden haben. Überall, ob in der Schule oder in den Medien, ist nun die Rede von der großen Verfügbarkeit von Waren und vielen Optionen und Möglichkeiten, von denen Markus aber kaum etwas selbst erlebt. Retrospektiv sagt Markus über seine Eltern:

„ich hasse meine Eltern dafür, dass sie mir eigentlich meine Jugend und meine Kindheit verbaut haben. Also, das ... muss ich wirklich klipp und klar sagen, ... dafür hasse ich sie einfach und ... es ist teilweise eben auch kein elterliches Verhältnis, sondern es ist mehr oder weniger eine, na, eine Zweckgemeinschaft gewesen oder ist es heute noch." $(5: 10-14)$

\subsubsection{Kinder als Beobachter der Lebensweise in Unternehmerfamilien}

Ein weiterer zentraler Faktor darin, wie sich die Kinder die Welt des Unternehmens erschließen, ist die Beobachtung der Eltern sowie anderer signifikanter Personen im Umgang mit diesem. Auch die Studie von Eva SchmittRodermund und Schröder (2009) hebt neben der Mitarbeit im Familienunter- 
nehmen die prägende Rolle der Eltern in der Entwicklung der Kinder zu Unternehmern hervor. Gespräche mit den Eltern und Praktika (überwiegend in „fremden“ Betrieben) seien die beiden wichtigsten Quellen in der Auseinandersetzung der Heranwachsenden mit ihrer Berufswahl. Die Kinder nehmen die Eltern als unternehmerische Vorbilder wahr. Von ihnen erfahren sie über den Unternehmensalltag, sehen die berufliche Belastung und die zeitliche Einschränkung der Eltern. Christina Erdmann (1999) hat in ihrer Studie von Vätern und Söhnen zur Nachfolgebereitschaft in Unternehmerfamilien ein Modell über diese Art der stillen Beobachtung entwickelt (Erdmann 1999:211). In diesem Modell stehen sich die Persönlichkeiten von Vater und Sohn gegenüber. Der Vater wirkt auf den Sohn über erzieherisches Handeln ein. Der Sohn nimmt diese Handlungen und darüber hinaus den Vater in seinen unterschiedlichen Rollen wahr. Das Unternehmen wirkt auf das Kind über die Präsentation durch die Eltern ebenso wie über die (Nicht-) Anwesenheit in der Familie. Erdmann hebt besonders den Punkt unterschiedlicher Prioritätensetzungen hervor und unterscheidet in eine Unternehmens- und eine Familienorientierung. ${ }^{73}$

Ihre These der Kinder als stille Beobachter erhärtet sich in dem dieser Arbeit zugrunde liegenden Material. Die exemplarischen Fallbeschreibungen von Adam Treuninger und den Geschwistern Claas unterstreichen die Wirkung des Unternehmens auf die Kinder in Abhängigkeit von der Präsentation durch die Eltern:

Während seiner Jugendjahre beobachtet Adam die berufliche Tätigkeit seiner Eltern. Er hat eine Vorstellung von ihrem Alltag und ihren Aufgaben, er weiß, was sie umtreibt und welche Entscheidungen sie treffen. Diese Offenheit zwischen den Eltern und den Kindern nimmt Adam positiv wahr. Er kann das bereitwillige Erzählen seiner Eltern auf sich beziehen und sich über ihr Vertrauen freuen. Er darf teilhaben und er darf mitreden. In Adams Wahrnehmung schimpfen die Eltern selten über das Unternehmen, sondern er sieht sie meist zufrieden mit ihrer Tätigkeit. Seine Mutter und ihren Aufgabenbereich nimmt er als unstet war. Sie hat eine Leidenschaft für Innovationsprojekte und eine besondere Fähigkeit, Dinge zu erkennen, die nicht rund laufen. Er bewundert seine Mutter für diese Fähigkeit und erkennt das Unternehmen als „Frucht meiner Eltern“ (29:11) an. Dennoch scheint die Bindung zu seinem Vater besonders stark. Für Adam verkörpert sein Vater den typischen Unternehmer, er ist der Treuninger, der in der Ahnenlinie steht. Adam erlebt die beruflichen Erfolge seines Vaters mit, insbesondere die Produktinnovationen, die er in den 80er Jahren einführt. Für diese Taten scheint er seinen Vater zu bewundern, fast schon heldenhaft zu verehren.

73 Auf diesen Aspekt der unterschiedlichen Orientierungen der Eltern in ihrer alltäglichen Lebensführung wird unter Absatz 3 in diesem Kapitel noch ausführlicher eingegangen. 
Während der frühen Kindheit von Cornelius, Claudia und Carsten Claas brennt der Betrieb zweimal. Sie erfahren die Sorgen der Eltern und erleben dieses Unglück emotional mit. Das Unternehmen ist ein Ort der Katastrophe. Darüber hinaus nimmt das Unternehmen die Eltern, insbesondere den Vater, stark in Anspruch. Die Kinder erleben den Betrieb als Konkurrenz um die Aufmerksamkeit der Eltern in zeitlicher und emotionaler Hinsicht. Sie finden keinen Platz in der Welt der Eltern. Zwar „dürfen“ die Kinder viel im Unternehmen spielen und die Eltern regelmäßig begleiten, dennoch bleiben sie als Zuschauer außen vor. Claudia Claas sagt: „Wir haben als Kinder hier viel gespielt. Ich hab das äh als Kind sag ich mal, nicht so richtig alles realisiert. Aber später habe ich gemerkt, dass wir als Kinder ziemlich allein gelassen worden waren." ${ }^{\circ 4}$ Der schwierige Charakter des Vaters und seine Anwendung häuslicher Gewalt verschärfen die Situation noch weiter. Der Vater ist zwar der bewundernswerte Gründer und Macher, der über Einfluss, Ansehen und Macht verfügt. Gleichzeitig scheint er in emotionaler Ferne. Das Unternehmen wird in dieser Konstellation in extremer Weise zu einem Spielfeld des Strebens nach Aufmerksamkeit und Anerkennung.

Es zeigt sich, dass die Eltern die Erfahrungen des Kindes mit dem Ort des Betriebes filtern und damit eine mehr oder weniger große Faszination beeinflussen können. Die Einzigartigkeit des Betriebes entsteht im Zuge der alltäglichen Lebensführung der Unternehmerfamilie. Die Kinder beobachten die Handlungen und Äußerungen der Familienmitglieder über oder im Zusammenhang mit dem Unternehmen und leiten daraus seine Bedeutsamkeit ab. (siehe hierzu auch Erdmann 1999:199) Auch wenn Erdmann meiner Ansicht nach die Dimension der physischen Erfahrung des Unternehmens und seiner Mitarbeiter etwas unterschätzt, bestätigen und erweitern die hier durchgeführten Fallanalysen ihre These. Das Beobachtungsverhältnis kann nicht nur für eine Vater-Sohn-Beziehung in Anspruch genommen werden, sondern auf Eltern-Kind-Beziehungen im Allgemeinen erweitert werden. Zudem lässt sich die Wahrnehmung des Unternehmens als Teil der elterlichen Persönlichkeit und Lebenswelt sowie des erzieherischen Handelns noch um die Rollenzuschreibungen ergänzen, die Eltern für ihre Kinder innerhalb der Unternehmerfamilie vorsehen. Insgesamt prägt dieser Komplex aus Präsentation und Wahrnehmung die Einschätzung des Eltern-Kind-Verhältnisses aus Sicht der Kinder maßgeblich.

Eine weitere Beobachtung lässt sich ebenfalls in die These der Kinder als stille Beobachter integrieren: Die meist egalitär denkenden Kinder nehmen unterschiedliche Lebenslagen war, verstehen und akzeptieren diese aber nicht immer (Schweizer. Herbert 2007:418). Gerade im Vergleich mit Gleichaltrigen, der eine ausgeprägte Alltagsrelevanz für Kinder und Jugendliche besitzt,

74 Das Interview von Claudia Claas wurde nicht transkribiert, entsprechend können keine Seitenzahlen und Zeilennummer angegeben werden. 
erkennen sie die vom Unternehmen ausgehenden Arbeitsverpflichtungen, Freizeit- und Konsummöglichkeiten, aber auch unterschiedliche Bildungschancen. Sie erkennen, dass die Lebensweise als Unternehmerfamilie ihnen eine spezifische gesellschaftliche Position zuweist und damit sozial strukturierend wirkt. Die Geschichte vom Roller, die Michael Treuninger erzählt, ist ein beredtes Zeugnis dafür:

Familie Treuninger verliert durch einen Bombeneinschlag während des Zweiten Weltkrieges beide Söhne und Geschäftsführer. Die Lücke in der Generationenfolge stellt für die Familie ein existenzielles Problem dar. Ein externer Geschäftsführer übernimmt mit nur mäßigem Erfolg den operativen Betrieb während der Wirtschaftswunderjahre. Die Familie erfährt den für die Nachkriegszeit typischen Konsumverzicht. Als jedoch die Wirtschaft beginnt zu florieren, ist Familie Treuninger immer noch gezwungen, sich stark einzuschränken. Michael erkennt, dass es ihnen im Vergleich zu anderen Kindern schlechter geht. Er sieht die vielen bunten Spielzeuge in den Läden und wünschte, er könnte auch ein solches besitzen. Aber er sagt: „da war also schon gar nichts"(13:24). In dieser Situation bereitete ihm der Schreiner des Betriebes ein kostbares Geschenk - er baute Michael einen Roller. Stolz erzählt er: „Und ich hatte den... andere hatten den nicht, weil sie keinen Schreiner hatten... äh das war dann schon was.“ (13:28-29)

Kinder gewinnen einen Eindruck der wirtschaftlichen Situation der Familie anhand von Geschenken, Urlauben und des Lebensstils der Eltern. In dem Bewusstsein, dass das Unternehmen die ökonomische Lebensgrundlage der Familie maßgeblich gestaltet, schlussfolgern sie auf den wirtschaftlichen Erfolg des Unternehmens. In den Begegnungen mit Gleichaltrigen (Mitschülern, Freunden, Verwandten) vergleichen sie die eigene wirtschaftliche Situation und Lebensführung mit den anderen. Das Erleben von Andersartigkeit oder gar Ausgrenzung (das sowohl aus einer ökonomisch privilegierten als auch einer benachteiligten Stellung resultieren kann) kann zu einer defensiven Haltung gegenüber dem Unternehmen, das als Verursacher oder Schuldiger ausgemacht wird, führen.

Zusammenfassend und die bisherigen Studien erweiternd kann festgehalten werden, dass Kinder nicht nur eine Vorstellung vom „Beruf“ der Eltern entwickeln, sondern von der facettenreichen Verknüpfung von Unternehmen, Familie und sozialem Status, in anderen Worten von der Art und Weise des Lebens der Eltern als Unternehmerfamilie.

\subsubsection{Berufliche Orientierung und Identitätsfindung}

Wie sich in den eben angestellten Überlegungen schon andeutet, markiert das Unternehmen im Zuge der beruflichen Orientierung und Identitätsfindung, also der zentralen Entwicklungsaufgabe von Heranwachsenden, einen we- 
sentlichen Fluchtpunkt. In der Pubertät beginnen sich Jugendliche kritisch mit den ihnen zugedachten Rollenzuschreibungen und der Lebensweise der Eltern auseinanderzusetzen. Konkret lassen sich drei Bereiche benennen, wie das Unternehmen diese Reflexionsarbeit der Jugendlichen in Unternehmerfamilien beeinflusst:

(1) Die Heranwachsenden haben eine mehr oder weniger konkrete Vorstellung eines Unternehmens entwickelt, die als Maßstab für weitere berufliche Erfahrungen dienen kann. So können bei Praktika oder einer Berufsausbildung Vergleiche angestellt und die betrieblichen Abläufe noch besser durchdrungen werden. Einen solchen Vergleichsmaßstab können sich Jugendliche aus Familien, in denen die Eltern abhängig erwerbstätig sind, oft erst während ihrer ersten beruflichen Erfahrungen aneignen.

(2) Die Wahrnehmung der Persönlichkeit der Eltern und anderer Familienmitglieder ist eng mit dem Unternehmen verknüpft. Es ist Bestandteil ihrer Identität und lässt sich nicht ausklammern. Eine kritische Auseinandersetzung mit den Eltern beinhaltet deshalb immer auch eine Reflexion über deren Rolle und Leistungen im Unternehmen.

(3) Das Unternehmen erweitert und verändert die Rollenzuschreibungen, die Eltern ihren Kindern entgegenbringen. Heranwachsende aus Unternehmerfamilien sehen sich damit nicht nur mit geschlechtstypischen Vorstellungen hinsichtlich Bildung, Beruf und Familie konfrontiert, sondern sie haben auch ein Gespür dafür entwickelt, welchen Platz ihre Eltern für sie im Unternehmen und innerhalb der Gemeinschaft der Unternehmerfamilie (ob bewusst oder unbewusst) vorsehen. Im Zuge ihrer Identitätsfindung gilt es also, sich auch mit dieser Rolle auseinanderzusetzen.

Der Fall Anabella Singermayer veranschaulicht den Prozess einer kritischen Auseinandersetzung mit der eigenen Familie und der Rolle des Unternehmens sowie die dabei möglicherweise auftretenden Unsicherheiten, Abwehrtendenzen und Problemlagen:

Anabellas Jugendjahre, in denen sie sich von der Familie abwendet, stehen im Zeichen der Rebellion. Sie empfindet ihren konservativen und wohlhabenden familiären Hintergrund als „Belastung“ (2:34) und „Makel“ (2:34) und spürt fortwährend den „Schatten von Singermayer" (3:1) über ihrer eigenen Existenz. Ihre hervorgehobene Rolle als einziges Mädchen neben drei Brüdern wird für sie nun zum Problem. Wähnte sie sich im Kindesalter noch „unter vieren“ (25:21), werden jetzt im Vergleich zu ihren Brüdern andere, 
geschlechtsbezogene Erwartungen bzw. Lebensentwürfe an sie herangetragen. Diese sind von einem eher klassisch-konservativen Frauenbild geprägt. Anabella spürt, dass innerhalb der Familie für sie keine funktional tragende Rolle vorgesehen ist, wohingegen ihre drei Brüder als mögliche Nachfolger betrachtet werden. ,... weil wenn ich dann später mal verheiratet bin und Kinder habe, dann könnte ich ja immer noch irgendwie eine Filiale führen. Also, .. *so viel wurde mir damals mir dann auch zugetraut* (ironisch)." (2:20-22) beschreibt sie die Sichtweise der Familie, mit der sie als Jugendliche konfrontiert wird. Diese wohlgemeinte Sicherheitsoption im Lebenslauf interpretiert Anabella als Entsagung von Vertrauen in die eigene funktionale Bedeutung. Sie wendet sich von der Familie ab und stellt sich ein Stück weit außerhalb dieses sie belastenden Kontexts.

Eine eigene, klar definierte Rolle im Sinne eines „role making“ entwickelt Anabella jedoch nicht. Insbesondere während der Pubertät scheint sich Anabella vor allem über die Negation der Familie und des Unternehmens zu definieren. Sie schwimmt mit dem Fluss der Dinge und passt sich hiermit auch dem vorherrschenden, eher ablehnenden Zeitgeist an. Sie nimmt die Familie als „reich“ (13:22) und in einer gewissen Art und Weise als ,ausge-

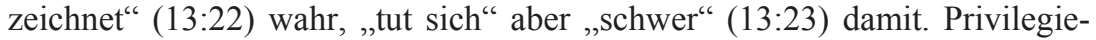
rung ist für sie eine negative Empfindung, da sie von der Normalität abweicht und ausgrenzt. Mit der Konzeption des Erbens kann sie nicht viel anfangen, will vom Erbe nicht profitieren: „Kraft Familie und kraft Amt oder kraft äh, kraft Geburt und Name wollte ich nie was haben“" (2:27-28), Anabella will lieber ,aus eigener Kraft arbeiten“ (3:4). Jedoch setzt Anabella diese Einstellung nicht in die Tat um. Sie scheint in der Schule nicht besonders erfolgreich zu sein, und auch auf außerschulische Aktivitäten wie z.B. ehrenamtliches Engagement gibt es keine Hinweise. Sie entwickelt keine klaren beruflichen Vorstellungen. Sie hat „keinen Plan“ (3:8), hat „keine klaren Berufswünsche“ (15:8) und das Gefühl, dass sich ihr „Leben einfach so weiter entwickelt" (3:12). Die Vorstellungen über die berufliche Zukunft reichen von „handwerklich kreativen“ (15:9) Tätigkeiten bis hin zu sozialer Arbeit mit Kindern und Jugendlichen. Anabella wünscht sich viele Kinder.

Anabella verharrt in einer grundlegenden Protesthaltung und stellt den Machern der Unternehmerfamilie eine kreatives, laissez-faire' entgegen. Mit ihren Worten und ihrer Einstellung will sie zeigen, dass sie nicht auf die Familie angewiesen ist, indirekt bestärkt sie ihre Familie jedoch in der Sichtweise, ihr keine tragende Rolle im Familienprojekt zuzutrauen. Von außen betrachtet erscheint Anabellas Verhalten sicherlich als sehr subtile Form des Protestes und nicht außergewöhnlich. Es zeigt jedoch innerhalb der Familie seine Wirkung. Anabella gilt als schwieriges Kind. Besonders der Großvater kann Anabellas Verhalten nicht nachvollziehen. Er spricht jahrelang nicht mit ihr und enterbt sie schließlich. 
Nach dem Abitur arbeitet Anabella eine Weile im unternehmenseigenen Lager und zieht schließlich an das andere Ende der Republik. Beides kann als Versuch gewertet werden, sich der Familie weiter zu entziehen. Die ungelernte, maskuline Tätigkeit im Unternehmen stellt einen Gegenpol zu der weiblich besetzten/konnotierten Filialleitung dar, die ihre Eltern für sie vorgesehen hatten. Mit ihrem Wegzug schafft Anabella schließlich ein räumliches Äquivalent zu ihrer emotionalen Distanz gegenüber der Familie. Auch in der Ferne verfolgt Anabella keinen bestimmten Weg, sie will sich vielmehr klar werden, was sie machen will, sich „sortieren“ (15:23). Sie jobbt zunächst, während sie mit dem Beginn eines einjährigen Studiums generale liebäugelt.

Dieser Fall stellt den Prozess der Reflexionsarbeit und Identitätsentwicklung beispielhaft dar und verdeutlicht, wie sich dieser auf das Beziehungsgefüge innerhalb der Familie, auf schulische Leistungen und Berufsvorstellungen auswirken kann. Kinder und Jugendliche werden nicht in erster Linie von Normen angetrieben, sondern suchen vielmehr Normalitäten und Normalisierung, sie suchen unentwegt Vermittlungsstrategien, gangbare Übergänge und Abstimmung heterogener Ansprüche (Schweizer. Herbert 2007:253). Insofern hat oder kann alles Konsequenzen auf die Beziehungsstruktur haben. Jedoch muss betont werden, dass dieser Prozess grundsätzlich ergebnisoffen ist - also nicht immer in einer Einstellung des ,in den Tag hinein Lebens' münden muss. Im Fall Claas beispielsweise findet eine Rebellion der Kinder ganz im Geiste der 1968er Bewegung statt. Die Jugend positioniert sich gegen den konservativen Patriarchen und strebt nach Unabhängigkeit in wirtschaftlicher wie in finanzieller Hinsicht. Alle drei Kinder ergreifen einen unternehmensfernen Beruf und versuchen auf diese Weise Distanz zu ihrem Vater und dem Unternehmen zu schaffen. Im Fall Treuninger hingegen nimmt Adam die ihm zuteil gewordene Rollenzuschreibung als Nachfolger an und integriert diese in seine Identität. Auf den Aspekt der Berufswahl wird noch einmal näher in Kapitel 6.2 (Wege in das Familienunternehmen) eingegangen.

\subsubsection{Fazit: Unternehmen als familialer Raum}

Das Bild der Kindheit und Jugend in einer Unternehmerfamilie ist ganz bewusst etwas vereinfachend und polarisierend dargestellt. Dies soll zum einen den kindlichen, pragmatischen Maßstab der Weltbeschreibung und zum anderen den verklärten Charakter der Erzählungen einfangen. Deutlich geworden ist, dass Kinder eine Vorstellung davon entwickeln, was es heißt, eine Unternehmerfamilie zu sein, eine kindliche Theorie vom Leben in einer Unternehmerfamilie. Diese ist maßgeblich von der physischen Erfahrung des Unternehmens als Örtlichkeit, dem Umgang mit den Menschen aus dem Un- 
ternehmen, Erzählungen über das Unternehmen, der wirtschaftlichen Situation der Familie, der aktiven Mitarbeit im Unternehmen sowie der stillen Beobachtung des Unternehmens als Teil der elterlichen Lebensführung geprägt. Kinder nehmen die Bedeutung wahr, die Erwachsene dem Unternehmen beimessen und machen ihre eigenen Erfahrungen im Umgang mit diesem. Das Unternehmen wird zu einem Bestandteil der Alltagswirklichkeit von Kindern und Jugendlichen, die in einer Unternehmerfamilie aufwachsen. Sie gewinnen ganz selbstverständlich einen Wissensvorrat über das Unternehmen, internalisieren Rollenzuschreibungen und greifen auf Generationen und Institutionenbeziehungen zurück. Damit fließen die Erfahrungen mit dem Unternehmen in das Netz der Verarbeitungs- und Bewältigungsmuster ein, mit dem Kinder ihre gesellschaftliche Umwelt deuten (Schweizer. Herbert 2007:410). Sie setzen ihre eigene Situation zu jener von Großeltern, Eltern und Gleichaltrigen in Bezug, um ihre biografischen Erwartungen zu präzisieren und ihre eigene Lebensgeschichte zu verstehen (Schweizer. Herbert 2007:428). Das Unternehmen erhält auf diese Weise einen festen Platz in ihrer Lebensführung und ihrer Identitätsentwicklung. Dabei kann das Unternehmen wie im Fall der älteren Naumann Brüder eher positiv, aber auch wie im Fall der Geschwister Claas eher negativ besetzt sein.

Auch Sabine Klein stellt fest, dass Kinder in den frühen Jahren ein implizites Bild davon entwickeln, wie das Unternehmen in die Familie hineinragt und was es für die Familie bedeutet (Klein 2000:80). In diesen Familien eigener Art sind frühe Einblicke in das Unternehmen möglich, die im Falle einer abhängigen Erwerbstätigkeit der Eltern nicht möglich sind. Es zeigt sich, dass für Kinder aus Unternehmerfamilien das Unternehmen zu einem erweiterten familialen Raum wird, und zwar nicht im Sinne einer Betriebsfamilie, bei der Angehörige des Unternehmens nun auch als Familienmitglieder angesehen werden, sondern als Örtlichkeit und Thema familialen Handelns.

Inwiefern sich die Phase der Kindheit in Unternehmerfamilien im Sinne der Familiensoziologie wandelt, lässt sich im Rahmen dieser Studie schwerlich beurteilen. Weder über die Rolle der berufstätigen Mutter noch über die fortschreitende Institutionalisierung der Kindheit lassen sich zuverlässige Aussagen treffen. Ebenso wenig können Pluralisierungstendenzen der familialen Lebensweisen oder ein Festhalten an der klassischen Unternehmerehe mit einer mithelfenden Ehefrau erfasst werden. Solche Ergebnisse kann nur eine quantitative Untersuchung von Unternehmerfamilien liefern, die sicherlich wünschenswert ist.

Anhand der Fallauswertungen lässt sich jedoch sehr deutlich zeigen, dass das kulturelle Bild, dass Kinder die Aufgabe haben, ihr eigenes Glück zu finden, in Unternehmerfamilien weit verbreitet ist. Die Mitarbeit im Unternehmen steht unter der Norm der Freiwilligkeit, und das gesetzliche Verbot der Kinderarbeit ist eine Selbstverständlichkeit. Davon unabhängig ist die Prä- 
senz der Kinder im Unternehmen als, Ort des Spielens' zu betrachten, deren Häufigkeit in hohem Maße von der Einstellung der Eltern abhängt. Darüber hinaus ist die Mitarbeit der Kinder als Mechanismus zur Erlangung elterlicher Aufmerksamkeit strukturell angelegt. Ein Wandel dieser Rolle des Unternehmens in Bezug auf die Kindheit lässt sich anhand der Interviews, die Schlaglichter auf die Zeit seit Bestehen der Bundesrepublik werfen, nicht feststellen. ${ }^{75}$ Es lassen sich also in dieser Hinsicht keine Spuren des Ganzen Hauses feststellen. Im Gegenteil, eine Missachtung dieser Normen zur Kindheit kann psycho-soziale Krisen nach sich ziehen.

Zudem entsteht der Eindruck, dass solche modernen Lebensformen, die mit verschiedenen Aufenthaltsorten der Kinder einhergehen (z.B. Patchworkfamilien oder Mehrgenerationenfamilien) die Wahrscheinlichkeit einer (emotionalen) Distanz zum Unternehmen erhöhen. Die physische, alltägliche Erfahrung des Unternehmens kann sich dadurch reduzieren. Es wird den Eltern leichter möglich, eine Grenze zwischen Unternehmen und dem privaten Leben zu organisieren. Zudem kann das Unternehmen als Auslöser für z.B. eine Scheidungserfahrung stilisiert und damit negativ belegt werden.

\subsection{Wege in das Familienunternehmen}

In einer alterschronologischen Darstellung des Lebenslaufes würde sich an die Phase der Kindheit und Jugend nun der Übergang in das Erwachsenenalter anschließen. Wie Konietzka herausarbeitet, handelt es sich dabei um eine Statuspassage, in der zentrale Lebenslaufentscheidungen getroffen werden, „die teilweise langfristige Bindungswirkung mit irreversiblem Charakter besitzen" (Konietzka 2010:107). In dieser richtungweisenden Lebenszeit werden in einem relativ begrenzten Zeitraum viele biografische Entscheidungen getroffen, zu denen die Wahl von Ausbildung und Beruf, die Partnerwahl und die Entscheidung über eine Familiengründung zählen. In der Terminologie von Glen Elder (1985b) stellt der Übergang in das Erwachsenenalter ein Verlaufsmuster dar, also eine sich über einen längeren Zeitraum erstreckende Sequenz von Ereignissen.

Dieses Verlaufsmuster zeichnet sich dadurch aus, dass junge Erwachsene allmählich in den Status wirtschaftlicher und sozialer (sowie psychischer) Unabhängigkeit von den Eltern „hineinwachsen“ und zunehmend Erwachsenenrollen übernehmen (Konietzka 2010:108). Die Ereignisse im frühen Erwachsenenalter wie der Auszug, der Erwerbseintritt, die Gründung einer Paargemeinschaft und Elternschaft sind keine notwendigen Stufen in der Le-

Wohl aber ein Wandel der Erziehungsideale wie in Kapitel 7 dargestellt wird. 
benslaufentwicklung, sondern eher Hinweise auf einen Zuwachs an ökonomischer oder sozialer Unabhängigkeit vom Elternhaus. Aus diesem Grund scheint es angemessen, die einzelnen demografischen Ereignisse dieser dichten Lebensphase als „transition markers“ (Konietzka 2010:112; Shanahan 2000) zu bezeichnen. Insbesondere im Lichte einer Diskussion über pluralisierte Familienformen wird eine langfristige Paarbeziehung oder die Familiengründung als Kriterium für das „Erwachsensein“ immer fragwürdiger. Darüber hinaus verdeutlicht die Generationenforschung, dass das herrschende Familienregime und die sozialpolitischen Rahmenbedingungen in Deutschland eine durchgreifende ökonomische und soziale Verflechtung auch zwischen erwachsenen Kindern und ihren Eltern vorsehen, welche gegenseitigen Versorgungsverpflichtungen der Generationen einen lebenslangen Horizont verleiht (Born und Krüger 2001; Kohli und Szydlik 2000). Dennoch lässt sich festhalten, dass gemeinhin derjenige als „erwachsen“ gilt, der sich auf dem Arbeitsmarkt etabliert und einen eigenen Haushalt gegründet hat, man also in einen ökonomischen und einen sozialen Pol der Verselbständigung differenzieren kann (Konietzka 2010:114).

In dem Streben nach wirtschaftlicher und sozialer Unabhängigkeit von den Eltern stellen auch junge Erwachsene aus Unternehmerfamilien keine Ausnahmen dar. Die Mitglieder dieser Familien eigener Art haben die gesellschaftlichen Individualisierungsnormen internalisiert und verstehen den Auszug aus dem Elternhaus, den eigenen Haushalt und ein elternunabhängiges Einkommen als zentrale Meilensteine in dem Abnabelungsprozess von den Eltern. Gleichzeitig müssen sie sich zu dem Kontinuitätsanspruch des Familienunternehmens positionieren, der eine enge Bindung der Generationen aneinander vorsieht. Das Unternehmen beeinflusst also die Bedingungen, unter denen sich junge Erwachsene aus Unternehmerfamilien von ihren Eltern autonomisieren. Die Analyse zeigt, soviel sei vorab gesagt, dass besonders dann, wenn in der Zeit des Aufwachsens das Leben mit dem Unternehmen als eine gute Lebensweise erfahren wurde, sich dieses zu einem lebenslangen Bezugspunkt in der beruflichen und familialen Lebenslaufgestaltung entwickeln kann. Die typischen „transition markers“ für den Status des Erwachsenseins werden eingereiht in einen größeren Bezugsrahmen, dienen als Demonstrationen einer Entscheidung gegen das Familienunternehmen oder als Rechtfertigung nach außen und innen für einen selbständigen Lebensentwurf trotz der Entscheidung für den Lebensstil einer Unternehmerfamilie. Es kann behauptet werden, dass sich die Fragen des "Erwachsenwerdens“ und der „Unabhängigkeit" von den Eltern in Unternehmerfamilien unter anderen Vorzeichen stellen.

Die Darstellung der Ergebnisse erfolgt deshalb ausgerichtet an dem Fluchtpunkt des Unternehmens und geht auf Motivlagen und strukturelle Interdependenzen ein. In diesem Kapitel werden mögliche „Wege in das Fami- 
lienunternehmen“, im nächsten Kapitel die Familiengründung näher beschrieben.

\subsubsection{Berufswahl, Unternehmensgründung und Nachfolge}

Die Wahl eines bestimmten Berufes steht in der Regel am Anfang des beruflichen Werdegangs. Während ihrer letzten Schuljahre sehen sich Jugendliche dem Druck ausgesetzt, ihre Berufsvorstellungen zu konkretisieren und eine entsprechende Ausbildung zu ergreifen, die sie auf ihr Berufsleben vorbereitet. Die Forschung zum zeitlichen Verlauf von Berufswahlprozessen zeigt, dass es sich bei der Wahl eines Berufes um eine „,big decision“ handelt: eine Entscheidung mit sehr weitreichenden Konsequenzen für den weiteren Lebensweg (Sackmann 2007:130). Walter Heinz u.a. beschreiben die Berufswahl als einen Anpassungsprozess an realistische Möglichkeiten, in den Ratschläge von Bezugspersonen, eigene Neigungen, schulische Leistungen und die antizipierte Arbeitsmarktlage eingehen (Heinz et al. 1987). Es ist der Komplexität der Entscheidungssituation geschuldet, dass nicht alle Handlungsalternativen gleichzeitig erwogen werden können, sondern dass zeitlich strukturiert mit eingeschränkter Rationalität einzelne Wahlentscheidungen geprüft und erst bei ihrem Scheitern weitere Möglichkeiten in Betracht gezogen werden (Sackmann 2007:131 siehe dazu auch Schimank 2005:195ff.). Sukzessive erfolgt eine Rollenkonkretisierung, in deren Verlauf immer mehr Berufe als nicht wählbar ausgeschlossen werden (Sackmann 2007:131). Bei der Berufswahl, wie bei jeder anderen Lebenslaufentscheidung, ist das Individuum eingebunden in institutionelle Kontexte und abhängig von Figurationen mit anderen Akteuren (Sackmann 2007:129). Im Laufe der Zeit werden strukturelle Effekte personalisiert und in die individuelle Identität integriert. So entsteht eine Balance zwischen gesellschaftlichen Anforderungen und individueller Motivation.

Im Sinne eines dreigliedrigen, institutionalisierten Lebenslaufes (Kohli), bei dem Arbeit als wichtiger Strukturgeber von Biografie und Lebenslauf fungiert, vollzieht sich mit dem Übergang vom Bildungs- in das Beschäftigungssystem der Beginn einer neuen Lebenslaufphase. Die empirische Lebenslaufforschung zeigt, dass sich nach dem Berufseinstieg zwanzig bis fünfzig Jahre der Erwerbstätigkeit im mittleren Lebensalter anschließen. Die Beschäftigungsdauer ist in Deutschland, auch wenn jüngst leicht rückläufige Zahlen $\mathrm{zu}$ beobachten sind, üblicher Weise relativ lang (Sackmann 2007:138). Darüber hinaus lässt sich eine "normale" intergenerationale Mobilität und eine stark reduzierte intragenerationale Mobilität beobachten (Sackmann 2007:139). In anderen Worten, bezogen auf die Volkswirtschaft findet ein Austausch der Mitarbeiter meist entlang der Generationenfolge statt: Ältere Menschen gehen in den Ruhestand, und ihr Arbeitsplatz wird 
durch junge Menschen ersetzt. Das heißt auch, dass sich die Karriereschritte neben der eigenen Leistung über das Alter und die Länge der Betriebszugehörigkeit bestimmen und "Abkürzungen" nur schwer möglich sind. Mit diesem Umstand ist eine relative Karrieresicherheit verbunden, aber auch zähe Aufstiegschancen (Blossfeld 1990; Sackmann 2007:143) verdeutlicht die verlaufsprägende Wirkung der Einstiegsbedingungen im deutschen Erwerbssystem, in dem sich ein Anfangsnachteil (z.B. durch Arbeitslosigkeit) nur schwerlich kompensieren lässt. Die empirische Lebenslaufforschung schreibt darüber hinaus individuellen Faktoren (etwa Persönlichkeit, familiale Ereignisse, Krankheit), institutionellen Faktoren und Gelegenheitsstrukturen (Konjunkturzyklus, Lehrstellenangebot) eine verlaufsprägende Wirkung zu.

Im vorangegangenen Kapitel konnte gezeigt werden, dass junge Erwachsene aus Unternehmerfamilien - so denn ein Unternehmen in ihrer Kindheit und Jugend in der Familie präsent war - über ein intimes Wissen über das Unternehmen verfügen, das ihnen als zentraler Vergleichsmaßstab in der Berufswahl und der damit verbundenen Rollenkonkretisierung dienen kann. Sie haben ein Verständnis über den Ablauf der Prozesse im Unternehmen und die darin vertretenen Berufsgruppen. Sie verfügen über ein mehr oder weniger detailliertes Wissen über die Arbeit der Eltern im Unternehmen. Im Hinblick auf das Thema Berufswahl ist es jedoch fraglich, inwiefern es sich bei der Führung eines Familienunternehmens um einen „Beruf“ mit konkreten Qualifikationsanforderungen und einem spezifischen Tätigkeitsfeld handelt (auf diesen Punkt komme ich in Kapitel 6.2.4 noch einmal zu sprechen). Die eingeschlagenen Ausbildungen (etwa eine handwerkliche Lehre oder ein BWL-Studium) sind generell auch für andere Optionen und insbesondere für den Weg in eine abhängige Beschäftigung offen. Die unternehmensfremde Berufswahl lässt sich aufgrund von Neigungen oder frühen Erfahrungen mit dem Lebensstil der Unternehmerfamilie interpretieren. Der tatsächliche Berufseintritt sagt jedoch wenig darüber aus, ob man später vielleicht doch den Weg ins Unternehmen einschlägt. Berufliche Entscheidungen wirken im Raum von Unternehmerfamilien weit weniger langfristig und irreversibel.

Die Entrepreneurshipforschung beschäftigt sich mit einem sehr ähnlich gelagerten Phänomen. Auch hier sind die üblichen Prozesse der „Berufswahl“" kaum zu vergleichen mit den Abwägungen, die zu einer Unternehmensgründung führen. Timmons (1999) setzt als Ausgangspunkt die marktliche Gelegenheit, das schumpeterianische Erkennen eines unternehmerischen Handlungsfeldes. Der Gründungsprozess wird ferner in kreativer Weise von einer aufmerksamen Unternehmersperson (Kirzner) bzw. einer entrepreneurial group (Ruef) vorangetrieben, die sich einer gegebenen Infrastruktur (Kapital, Wissen, Kontakte) bedienen kann und durch diese eingeschränkt wird. Ein kleiner Strang innerhalb der überwiegend amerikanischen Entrepreneurshipforschung beschäftigt sich mit der „entrepreneurial career“ und stellt fest, dass die Gründung eines Unternehmens als eine Abkürzung auf der Kar- 
riereleiter, als ein direkter Weg zu mehr Wohlstand und einer erhöhten Arbeitszufriedenheit verstanden wird (Douglas und Shepherd 2002; Dyer 1994; Schjoedt und Shaver 2007). Schwierige Startbedingungen oder geringe Aufstiegschancen lassen sich durch den Wechsel in eine unternehmerische Laufbahn kompensieren. Diese Verbindung des „Unternehmerseins“ mit einer Vorstellung des guten Lebens spiegelt sich auch in der Einschätzung von Jugendlichen wider, die zu einem sehr hohen Prozentsatz eine Selbständigkeit als erstrebenswert erachten (so eine Studie der Bertelsmannstiftung aus dem Jahr 2007).

Innerhalb dieses Forschungszweiges ist dem familialen Hintergrund der Unternehmer (meist als Selbständige operationalisiert) viel Aufmerksamkeit geschenkt worden. Matthews \& Human (2004) stellen zusammenfassend fest, dass die Ressourcen der Familie und der Vorbildcharakter von Familienmitgliedern einen deutlichen Effekt auf die Gründungswahrscheinlichkeit ausüben, dieser Einfluss jedoch eher als indirekt zu verstehen sei, über eine intergenerational tradierte Einstellung gegenüber diesem Karriereweg. Blanchflower \& Oswald (1998) zeigen in ihrer Studie, wie sich die Wahrscheinlichkeit einer Unternehmensgründung durch den Erhalt einer Erbschaft bzw. eines Geldgeschenkes erhöht. Falter (2007) deutet die hohe Gründungsneigung von Kindern aus Selbständigenhaushalten im Wesentlichen als einen Sozialisationseffekt. Der positive Effekt intergenerationeller Transfers, sowohl in materieller wie in immaterieller Weise, scheint im Hinblick auf eine Unternehmensgründung unbestritten. Zellweger und Nordqvist (2010) nehmen diese Zusammenhänge zwischen Unternehmensgründung und familialem Hintergrund auf und formen den Begriff des ,transgenerational entrepreneurship“.

Wenn sich Familienmitglieder für eine Nachfolge entscheiden, dann wählen sie gleichzeitig eine, unternehmerische Karriere' und eine spezifische Organisation (Lubinski 2011:5). Insofern können für den Weg ins Familienunternehmen einige der Faktoren in Anspruch genommen werden, die innerhalb der Gründungsforschung als lebenslaufprägend erarbeitet wurden. In beiden Fällen haben wir es nach dieser „Berufswahl“ mit einem Unternehmer zu tun. Für diese „Wahl“ lässt sich keine Altersspanne bestimmen. Unternehmer kann man immer werden, ob mit Anfang 20, wie im Fall Leopold Clemens, oder mit über 60, wie im Fall Felix Walter Naumann. Dennoch kann man Nachfolge und Gründung nicht einfach gleichsetzen, da ihnen unterschiedliche strukturelle Voraussetzungen zu Grunde liegen. Bei der Nachfolge muss kein neues Unternehmen gegründet, sondern ein bestehendes angeeignet werden. Dieser Unterschied macht sich vor allem in der gesellschaftlichen Wahrnehmung deutlich. Gilt die Gründung eines Unternehmens als Ausdruck von Selbstverwirklichung, Individualität und Mut, so ist die Nachfolge Ausdruck von Nachahmung, Tradition und Bindung. Während sich die Problemlage der Gründer um Bürokratie, Risiko und Finanzierung 
rankt, ist der Nachfolger vor allem mit Ablösungsprozessen und Machtverschiebungen beschäftigt. Die Nachfolge lässt sich als eine unmittelbare und langwierige Variante der intergenerationalen Mobilität in einer Organisation verstehen, die sich sonst volkswirtschaftlich auf dem Arbeitsmarkt beobachten lässt.

In einer Länder übergreifenden Befragung von Studierenden aus Unternehmerfamilien nehmen Thomas Zellweger, Philipp Sieger und Frank Halter (2011) gezielt die drei Berufsoptionen der eigenen Unternehmensgründung, der Nachfolge oder einer abhängigen Beschäftigung ins Visier. In ihrer Analyse setzen sie die Karriereabsichten in Bezug zu einschlägigen Persönlichkeitsmerkmalen der Befragten. Entgegen der traditionellen EntrepreneurshipForschung stellen sie fest, dass eine persönliche Eignung zum Unternehmer diesen Weg noch lange nicht attraktiv erscheinen lassen muss. Als Reihenfolge der Wahlmöglichkeiten zeigt sich, dass eine Unternehmensgründung der Nachfolge und diese wiederum einem abhängigen Beschäftigungsverhältnis vorgezogen wird, also entsprechend der möglichen Selbstbestimmtheit geordnet wird. Jedoch scheinen die anvisierten Berufswege stark kontextabhängig zu sein. So bevorzugen die deutschen Unternehmerkinder eher die abhängige Beschäftigung vor der Nachfolge.

Im Unterschied zu der Nachfolgeabsicht im jugendlichen Alter soll im Folgenden der Weg in das Familienunternehmen als ein spezifischer Fall der Berufswahl sowie der Unternehmensgründung in den Mittelpunkt gerückt werden. Nachfolge wird weder als Managementaufgabe (wie in der Forschung zu Familienunternehmen üblich) noch unter dem Aspekt des Vermögens betrachtet, sondern als ein Verlaufsmuster (trajectory), und der Eintritt in das Unternehmen als ein darin eingebetteter Übergang im Lebenslauf verstanden: als eine Richtungsentscheidung der Berufsbiografie. Insofern reiht sich dieser Abschnitt in die bestehenden lebenslaufsoziologischen Arbeiten zu Nachfolge in Familienunternehmen ein, die durchweg auf Individualisierungskonflikte verweisen. ${ }^{76}$ Es wird danach gefragt, was dazu führt, dass man sich irgendwann für einen Eintritt in eine verantwortliche Position im Familienunternehmen entscheidet. Die Betrachtungen berücksichtigen die frühe Erziehung und Sozialisation, Berufswahlprozesse und ein Berufsleben vor dem Familienunternehmen. Das Interesse gilt dabei den zugrundeliegenden strukturellen Faktoren und ihrer biografischen Bearbeitung. Die Darstellungen der Wege ins Familienunternehmen beruhen im Wesentlichen auf den Rekonstruktionen des erlebten Lebens sowie den sich abzeichnenden Fall-

76 Auf diese Studien wurde bereits in Kapitel 3 ausführlich eingegangen. Hier noch einmal der Verweis auf einige zentrale Arbeiten: Aldrich, Kim 2007; Bäcker, 2008; Bohler, Hildenbrand 1997; Büttner 2007; Davis, Taguiri 1989; Down, 2006; Dumas, Dupuis, Richer, St.Cyr 1995; Dunn 1999; Erdmann 1999; Handler 1992; Jayawarna, Rouse, Macpherson 2007; Lambrecht 2008; Moen 1998; Timmermann, Vonderach 1993. 
strukturen. In einem zweiten Schritt werden diese mit den biografischen Selbstdarstellungen der Befragten kontrastiert und ein dominantes narratives Muster offen gelegt. Außer Acht gelassen werden typische Laufbahnen, die in alternative Berufe oder Tätigkeitsfelder führen.

\subsubsection{Typische Wege ins Familienunternehmen}

Aus dem dieser Arbeit zugrunde liegenden Interviewmaterial lassen sich vier konsistente Muster kondensieren, welche Wege in das Familienunternehmen eingeschlagen werden. Die dargestellten Typen idealisieren die gefundenen Merkmale und Motivlagen, insofern finden sie sich in keinem Fall in Reinform. ${ }^{77}$

\subsubsection{Das Familienunternehmen als Zuflucht}

Das erste Lebenslaufmuster angehender Familienunternehmer lässt sich anhand der Merkmale professionelle Desorientierung, unerwartete Lebensereignisse und geringe Karrieremöglichkeiten charakterisieren.

Nachdem sich Anabella Singermayer einige Monate mit Gelegenheitsjobs durchgeschlagen hat (für ihre Vorgeschichte siehe Kapitel 6.1), wird sie schwanger. Die Beziehung scheint wenig tragfähig, deshalb beschließt die Rebellin wieder näher an die Familie zu rücken. Die Verantwortung für ihr Kind verändert Anabella: Sie sehnt sich nun nach einem „ordentlichen Platz in dieser Gesellschaft" (3:21). Sie nimmt das Angebot ihres Vaters an, eine Ausbildung zur Handelsfachwirtin in dem Familienunternehmen auf Teilzeitbasis zu absolvieren. Die Ausbildung ist pragmatisch orientiert; „Passion, Überzeugung, Lust“" (4:13) bringt sie für ihre Ausbildung nicht auf. Wohl fühlt sich Anabella dann, wenn sie etwas "wie alle anderen auch" (4:21) macht, z.B. das „normale Wanderpraktikum“ (4:20). Im Verlauf der Zeit verliert sie die „Befremdung“ (4:30) gegenüber dem Unternehmen. Anabella ist ihren Eltern für die Unterstützung in der Kinderbetreuung dankbar. Ihre drei Brüder messen ihrer Ausbildung keine große Bedeutung bei und fühlen sich nicht ernsthaft in ihren jeweiligen Rollen bedroht. Als Anabellas Mutter aufgrund eines Unfalls für einige Wochen im Unternehmen ausfällt, übernimmt Anabella „unausgesprochen klar eigentlich“ (5:2) vorübergehend ihren Aufgabenbereich. Sie rutscht dadurch zum ersten Mal in eine verantwortliche

77 Eine erste Skizze der Typologie zu den Wegen in das Familienunternehmen, ihre strukturellen Einflussfaktoren ebenso wie erste Gedanken zu einem narrativ der freien Wahl wurden in dem Aufsatz Stamm, Marchese 2011 vorgestellt. In diesem Beitrag werden die Ergebnisse des deutschen und italienischen Interviewmaterials verglichen werden, das im Rahmen des Projektes „Von Generation zu Generation“ erhoben wurde. 
Position für das die Familie so stark prägende Unternehmen, zum ersten Mal hat sie das Gefühl ,ich mache was Sinnvolles und ich werde gebraucht“ (5:8). Dieser Zuspruch verändert Anabellas Sicht auf das Unternehmen: ,irgendwas hat sich verändert in meiner Selbst-, eigenen Wahrnehmung" (5:2021). Die Tradition der Familie und des Unternehmens bedeutete für sie lange Zeit „Belastung“ (13:16) und „Makel“ (13:16), ,meterdicker Staub“ (5:21), der sie bedrückte und ihre eigene Entwicklung erschwerte. In dieser Zeit - so scheint es - beginnt Anabella erstmals das Unternehmen als etwas „Schönes und Wertvolles" (5:30) zu empfinden. Der Wandel der vormals intensiven, aber relativ wenig nachhaltigen Ablehnung gegenüber der Familie und dem Familienunternehmen spiegelt sich in dem Verhältnis zu ihrem Großvater wieder: Anabella lehnte ihn nicht als Menschen, sondern als Repräsentanten des patriarchalischen, konservativen und männlichen Unternehmens ab. Rückblickend beschreibt sie ihn als ,großen, starken, tollen Mann“ (14:6), der aber ein „Nichtschätzer von Frauen“ (11:34) war.

Ausgelöst durch den Zuspruch der Familie und ihrem Gestaltungsspielraum im Unternehmen entwickelt Anabella ein anderes Selbstverständnis und eine andere Perspektive auf das Unternehmen. Die Firma muss ihrem Empfinden nach „,in die Zukunft geführt werden“ (5:31). „Und .. ich will es gerne machen“ (6:3). Sie attestiert sich und ihren Brüdern, nie richtig „Herzblut" (5:33) für das Unternehmen entwickelt zu haben - die Nachfolge des Unternehmens ist für Anabella kein lange entwickelter Wunsch, den sie nun gegenüber der Familie artikulieren würde; das „Ganze“ (6:5) wird vielmehr erst nach und nach ,wach“" (6:5).

Im Verlauf der nächsten Monate nehmen die Dinge ihren gewohnten Lauf: Die Mutter beginnt wieder zu arbeiten, Anabella kehrt auf ihren Platz zurück. Der Vater scheint Anabellas Einstellungswandel zu spüren und lässt sie fortan eigenverantwortlicher und projektorientierter arbeiten. Anabella empfindet eine ,mehr oder weniger unausgesprochene“ (6:14) Klarheit zwischen sich und ihrem Vater. Der Vater ist es auch, der Anabellas Nachfolgeaspirationen expliziert. Anabella schildert ausführlich wie ihr Vater sie scheinbar beiläufig - fragt: „Nicht wahr, das machst du schon weiter?“ (6:22) Sie scheint diesen Moment sehr bewusst wahrzunehmen. Anabella willigt in die Nachfolge ein. Einige Zeit darauf wird auf Grund einer steuerrechtlichen Änderung eine Art Familientisch einberufen, in dessen Rahmen erörtert wird, wer in das Unternehmen einsteigen möchte und wer Kompensationsleistungen erhält. „Und da kam nichts” (7:17). Für Anabella scheint alles klar: Ein Bruder wird Arzt, ein anderer Bruder Tierarzt - und der dritte Bruder will außen vor bleiben. „Da war es klar, Anabella macht es“ (7:18).

Dieser typische Weg in das Familienunternehmen nimmt seinen Anfang in einer abwehrenden oder wenig interessierten Haltung der Heranwachsenden am Unternehmen. Während ihrer Kindheit haben sie zwar eine Vorstellung vom Betrieb entwickelt, sehen jedoch in diesem Raum aufgrund der 
Rollenzuschreibungen der Eltern, mangelnden Selbstvertrauens oder schlicht einer anderen Lebensvorstellung keine Zukunft für sich. Trotzdem fehlt es an einem alternativen Lebensplan, der für sie einen sinnvollen Platz in der Gesellschaft definiert. Diesen Heranwachsenden der zweiten oder einer späteren Generation mangelt es an einer beruflichen Qualifikation und Kompetenz oder sie sehen sich einem angespannten Arbeitsmarkt ausgesetzt. In jedem Fall sind ihre Chancen auf eine berufliche Karriere gering. Sie müssen sich mit unerfüllten Träumen und Unterbrechungen im Lebenslauf arrangieren. Das Familienunternehmen bietet ihnen eine Möglichkeit, ihre Lebenschancen und den beruflichen Status zu verbessern. Hier lassen sich flexible Arbeitszeiten oder eine längere Ausbildungsphase ebenso realisieren wie ein praktischer statt ein akademischer Zugang zu dem jeweiligen Tätigkeitsfeld. Das Unternehmen ist ein Ort der Zuflucht, ein sicherer Hafen, der den Desorientierten eine Richtung im Leben verleiht.

Am Anfang dieses Weges in das Familienunternehmen steht meist ein Wendepunkt im Leben. Im Fall von Anabella Singermayer war dies zunächst die Schwangerschaft, später der Unfall der Mutter. In einem anderen Fall (Hagemann) ist es die plötzliche Arbeitslosigkeit aufgrund der turbulenten Wendejahre. Die zuvor rebellischen Kinder und Jugendlichen, oder aber bereits selbständigen Erwachsenen werden nun damit konfrontiert, in einem engen Verhältnis zur Familie und zum Unternehmen zu leben. Sie müssen für sich einen Weg finden, wie sie mit den Eltern arbeiten können, sich gegenüber ihren Geschwistern positionieren und sich der Aufgabe stellen, diese große Wende im Lebensweg in ihre Biografie zu integrieren.

\subsubsection{Das Familienunternehmen als Mission}

In seiner qualitativen Analyse von Lebensläufen von Familienunternehmern fragt Thomas Siefer (1996) danach, wie Eltern ihre Kinder dazu bewegen können, die Nachfolge anzutreten. Die Antwort, die er darauf gefunden hat, beschreibt er in seinem Konzept der Delegation (ursprünglich vorgestellt von Helm Stierlin) - ein Ansatz, den ich anhand des empirischen Materials bestätigen und erweitern kann.

Delegation ist ein anerkennender Vorgang, in dem die Eltern ihre Kinder mit einer Mission betrauen. Charakteristisch für diesen Prozess ist ein lang anhaltendes Verpflichtungsgefühl der Delegierten gegenüber den Sendern (Siefer, 1996, p. 181). Das Konzept der Delegation beinhaltet eine Dimension von Vertrauen, Obligation, persönlicher Bedeutung und Loyalität, oder kurz ausgedrückt: ein verbindliches und ethisches Element. Die Mission verleiht dem Leben der Delegierten einen Sinn und transzendiert die Familienidentität. Zugleich verwickeln sie sich in eine Abhängigkeit der Verbindung aus Familie und Unternehmen. Die Beauftragung findet oft implizit und 
unreflektiert statt. Nachfolger müssen die Nachfolge als ihr Schicksal auf sich nehmen, während sowohl Eltern als auch Kinder die Fiktion aufrechterhalten, dass die Qualifikation alleine den Einstieg in das Unternehmen bestimmt. Büttner verweist darauf, dass konkreter als andere Bedingungen sozialer Herkunft die Nachfolgedesignation ein Lebensprogramm beinhaltet, mit dem sich der Nachfolgekandidat biografisch auseinandersetzen muss. (Büttner 2007:41) Die Delegation steht häufig in Verbindung zu frühen Erlebnissen der Nachfolger mit dem Übergeber, einem beeindruckenden Familienmitglied oder der ,Aura' des Unternehmens. Der Nachfolger hat den Eindruck, in einer Linie mit den Unternehmern der Familiengeschichte zu stehen - ihre Aufgabe ist vorbestimmt und einzigartig.

Die Dimensionen des Wollens einerseits und des Müssens andererseits können kaum auseinander gehalten werden (Siefer 1996:185). Entsprechend scheint die Nachfolge als „Berufswahl“ oder „Karriereentscheidung“ einen hypothetischen Charakter zu haben. Das Familienunternehmen wandelt sich in eine kollektive Aufgabe mit einem ausgeprägten Zugehörigkeits- und Gemeinschaftsgefühl. Auch wenn der Eintritt in das Unternehmen von langer Hand geplant ist und eine logische Konsequenz des bisherigen Weges zu sein scheint, markiert dieser Schritt einen Übergang im Lebenslauf des Nachfolgers. Zur gleichen Zeit erleben die Eltern wahrhaftig die Kontinuität der Familiengeschichte und erkennen, dass ihre Arbeit weitergeführt wird. Zudem sehen sie sich mit dem Rücktritt aus der ersten Reihe des Familienunternehmens konfrontiert.

Indizien für die Fähigkeiten des Nachfolgers werden oft schon in einem sehr frühen Alter ,erkannt“, und die Heranwachsenden bewegen sich auf einem direkten Weg ins Unternehmen. Jeder Schritt erfolgt in enger Abstimmung mit den Eltern: Die Ausbildung bzw. das Studium wird sorgfältig gewählt, frühe praktische Erfahrung in der Branche oder in verwandten Unternehmen wird gesammelt und ein Plan für die gemeinsame Zeit im Unternehmen erarbeitet. Die Nachfolger treten in einem relativ jungen Alter in das Unternehmen ein und bekommen Verantwortung übertragen.

Der Fall Michael Treuninger, wie er bereits in Kapitel V beschrieben wurde, veranschaulicht diesen typischen Weg in das Unternehmen fast schon in idealer Form. In dieser Familie reproduziert sich das Lebenslaufmuster des Unternehmens als „Mission“ auch in der jüngeren Generation mit Adam. Als weiteres illustratives Beispiel sei an dieser Stelle auf den Fall von Leopold Clemens verweisen, der im Kapitel VII dargestellt wird. In Erweiterung zu Siefer möchte ich vorschlagen, dass die Betrauung mit der Mission, das Familienunternehmen fortzuführen, auch sozusagen als ,zweite Wahl“" erfolgen kann:

Sven Singermayer (Anabellas Vater) wird ebenfalls im Laufe des Zweiten Weltkrieges geboren. Er wächst außerhalb der Stadt und fern vom Unternehmen auf einem Einödhof auf. Er genießt die Freiheit und das unbeschwer- 
te Leben, erlebt eine „wunderbare Kindheit“ (1:21). Mit Kriegsende kehrt sein Vater aus dem Krieg zurück, sein Großvater, ein Anhänger des NSSystems, ist ein gebrochener Mann, der aus Resignation einige Teile des Unternehmens arglos verschenkt. Sven nimmt die Unterschiede zwischen seinem Vater und Großvater deutlich wahr: auf der einen Seite der resignierte Großvater, der versucht, nach außen den Schein der wohlhabenden, privilegierten Familie zu wahren; auf der anderen Seite der Vater, der geprägt ist von seiner Erfahrung als Soldat und sich um einen Neuanfang in Unternehmen und Familie bemüht. Mit zehn Jahren wechselt Sven auf ein Klosterinternat, da keine Oberschule in der Nähe vorhanden ist. Die fünf Jahre dort sind für Sven schwierig. "Irgendwo es ... war nicht meine Welt" (2:9). Schließlich kann Sven auf ein Gymnasium in der Stadt des Firmensitzes ziehen. Mit dem Umzug und dem gemeinsamen Alltag kommt auch das Unternehmen in seinen Erfahrungskreis. Seine älteste Schwester heiratet und zieht weg, der ältere Bruder beginnt in einer Brauerei zu arbeiten, an der das Familienunternehmen Aktien hält, der jüngere Bruder interessiert sich für das Unternehmen und beginnt dort eine Ausbildung. Sven sagt: ,ich hatte mich überhaupt nicht für die Firma interessiert" (2:22); doch die Eltern versuchen ihn ein wenig in diese Richtung zu drängen: Sie würden gerne zwei Kinder im Unternehmen sehen, einen Kaufmann und einen Techniker. Sven sollte der Kaufmann werden, doch er sieht eher seinen älteren Bruder in dieser Rolle. „Außerdem fand ich es auch schon als Jugendlicher besser, wenn das einer macht" (2:32) Sven fühlt sich der Traditionsfamilie nicht zugehörig, sieht sich eher als Außenseiter. Die zu vergebenden Rollen empfindet er bereits durch andere Familienmitglieder besetzt. Sven blickt auf seinen Vater und sieht, dass er nicht die Möglichkeit hatte, seine Interessen auszuleben. In seinen Augen hat er unter dem Einfluss des patriarchischen Vaters (Svens Großvater) gelitten. Er will seinen Weg nicht wiederholen. Svens Interessen liegen in der Liebe zur Natur. Deshalb entscheidet er sich ,auf dringendes Abraten meines Vaters“ (2:34) zunächst für ein Studium der Biologie und schwenkt kurz darauf aber auf Medizin um.

Dann ereilt die Familie ein tragisches Ereignis: Der jüngere Bruder verunglückt tödlich. Die Familie ist unter Schock, besonders die Mutter leidet unter dem Verlust des jüngsten Kindes, zu dem sie ein sehr enges Verhältnis hatte. Auch Sven trauert um seinen Bruder und sieht die Lücke, die nun innerhalb der Traditionsfamilie entsteht. Gemeinsam fährt die Familie im Sommer in den Urlaub. Dort bittet der Vater Sven und seinen Bruder zu sich. Das Hotelzimmer ist abgedunkelt und mit Kerzen beleuchtet. Der Vater übergibt den beiden einen Siegelring mit dem Familienwappen und fragt, ob sich einer von beiden vorstellen könnte, in das Unternehmen einzusteigen. Der Bruder antwortet zuerst und verneint. Sven entscheidet sich spontan, es zu versuchen. Sven erlebt diesen Moment als sehr feierlich. Retrospektiv ist ihm jedoch nicht klar, warum er so und nicht anders geantwortet hat (eine 
Frage, die ihn zeitlebens begleitet). Sven hat die Möglichkeit, nun Teil der Traditionsfamilie, der Gemeinschaft der Unternehmerfamilie zu werden. Er empfindet tiefe Bewunderung für seinen Vater, will nicht, dass er auch als gebrochener Mann endet wie der Großvater. Sven tritt sein Medizinstudium nicht an, sondern beginnt stattdessen die Ausbildung im Unternehmen: „das war alles im Prinzip schon vorbereitet gewesen für meinen Bruder" (4:30) Sven beginnt seine Arbeit im Familienunternehmen zwar mit der Befürchtung, die Erwartungen des Vaters nicht erfüllen zu können, aber mit der Gewissheit, einen schicksalhaften Weg zu beschreiten. Im Laufe seiner langjährigen Tätigkeit als Firmenführer entwickelt er eine unternehmerische Intuition und fühlt sich der Familienhistorie stark verbunden.

Während der ursprünglich Delegierte seine Mission nicht erfüllen und den angedachten Weg nicht beschreiten kann, etwa aufgrund von Tod, Krankheit oder anderen unerwarteten Lebensereignissen, sehen sich die anderen Familienmitglieder plötzlich mit der unerfüllten Mission konfrontiert, das Unternehmen weiterzuführen. Selbst wenn bereits andere berufliche Vorstellungen entwickelt oder sogar schon Karrieren beschritten worden sind, kann in diesem Moment eine Kursänderung erfolgen. Der Delegierte (zweiter Wahl) tritt den direkten Weg ins Unternehmen an. Diese Familienunternehmer weisen die gleiche emotionale Verbundenheit mit dem Unternehmen und der Familie auf und sehen sich dem gleichen hypothetischen Charakter ihrer Berufswahl ausgesetzt.

\subsubsection{Das Familienunternehmen als Quelle von Privilegien}

Der dritte idealtypische Weg ins Familienunternehmen gleicht in der Abfolge der Lebenslaufereignisse und ihrer relativen Gradlinigkeit dem „Familienunternehmen als Mission“. Jedoch liegt diesem Weg eine völlig andere Lebensorientierung und Werteinstellung zugrunde.

Andreas und sein älterer Bruder Oscar wachsen in einer Gastronomiefamilie auf, die zunächst einen Tennisclub und später ein Hotel-Restaurant betreibt. Als Andreas fünf Jahre alt ist, kommt sein Vater bei einem Verkehrsunfall ums Leben. Seine Mutter sorgt die folgenden drei Jahre alleine für die Kinder und übernimmt diverse Jobs in der Branche. Schließlich lernt sie Florian Wagner kennen, der selbst Pächter von Veranstaltungslokalitäten und Restaurants ist, und geht mit ihm eine langjährige Partnerschaft ein. Die Brüder müssen aufgrund der beruflichen Aktivitäten der Eltern auf ein Internat und sind jede zweite Woche zu Hause. Sie verbringen viel Zeit im Restaurant und helfen regelmäßig mit. Andreas entwickelt eine selbstbewusste Persönlichkeit mit einer materiellen Orientierung und stellt gerne seine Leistungsfähigkeit in Wettbewerben unter Beweis. Zwischen ihm und seinem Bruder herrscht ein ausgeprägter Konkurrenzkampf. Für Andreas ist das Aufwach- 
sen in einer Gastronomiefamilie mit vielen Freiheiten verbunden: ,in einer Gastronomenfamilie, wo keiner da ist, dann macht man einfach das, worauf man Lust hat" (4:32-33).

Nach seinem Fachabitur finanzieren die Eltern Andreas ein Studium an einer privaten Universität. Sein Berufsziel ist es, Hoteldirektor zu werden, denn „Gastronomie habe ich keine Lust darauf. Ich habe keine Lust 14, 15, 16 Stunden am Tag zu arbeiten und dafür kein Geld zu verdienen.“ (22:3334) „Das wollte ich mir nicht immer so antun.“(6:29) „Ein Hoteldirektor hat im Gegensatz zu dem .. Selbständigen den Vorteil, dass er ein, ein gesichertes Einkommen hat und auch vom finanziellen Status her gesichert ist und trotzdem alle Entscheidungen hat.“ (8:25-27) Andreas wünscht sich eine große Karriere, die ihm viel Geld einbringt bei einem möglichst geringen Arbeitseinsatz. Nach dem Grundwehrdienst arbeitet Andreas für kurze Zeit in einer E-Business Firma, anschließend in einer Reihe von namhaften Hotels, und nimmt schließlich eine Stelle im Veranstaltungsbereich einer Großbank an. Während seiner Berufskarriere arbeitet Andreas immer wieder für einige Monate in den Betrieben der Eltern. Nebenbei absolviert er die Prüfung zum Restaurantfachmann, seine Ausbildereignungsprüfung und nimmt regelmäßig an Branchenwettbewerben teil. Er legt Wert auf einen lückenlosen Lebenslauf und zahlreiche Zertifikate. Andreas arbeitet nur dort, wo er einen Fortschritt für seine Karriere erkennen kann und er Spaß an der Arbeit hat. „Es ging mir echt nur um den, um den Schritt eben nach oben zu kommen, einen Schritt höher zu kommen“" (11:4) Das Netzwerk der Eltern sowie ihre finanziellen Mittel ermöglichen ihm ein Leben in Freiheit, in dem er sich auf jene Dinge konzentrieren kann, die ihm Spaß bereiten.

In seiner letzten Anstellung ist Andreas mit den beruflichen Perspektiven und den zwischenmenschlichen Beziehungen unglücklich. Gleichzeitig streben die Eltern an, einen Gastronomiebetrieb zu kaufen, und Andreas entschließt sich dazu, seine Stelle zu kündigen und neben seinem Bruder und seinen Eltern als vierter Geschäftsführer einzusteigen. Diese biografische Wende $^{78}$ geht mit einem Wandel seiner Lebensführung einher: Sein stabiles Leben mit hohem Einkommen wird eingetauscht gegen hohes finanzielles

78 Die Gründe für diesen biografischen Wandel werden in der Rivalität zwischen den Brüdern vermutet. Zwar versucht Andreas in seiner biografischen Selbstdarstellung eine Narration aufzubauen, die sich rein auf die Abkehr von der und dann auf die Wiederbesinnung zur Gastronomie stützt, jedoch legt die Rekonstruktion des erlebten Lebens eine andere Variante nahe: Hinter den etwas naiv anmutenden Berufsplänen von Andreas könnte das Gefühl von Zurücksetzung verborgen sein. Andreas weiß, dass er alles von seinen Eltern haben kann und nutzt diesen Umstand gerne aus (z.B. für sein privates Studium) - als Nachfolger jedoch scheint sein älterer Bruder vorgesehen zu sein. Seit ihrer gemeinsamen Zeit im Internat versucht sich Andreas mit Oscar zu messen - eine spielerische Rivalität. Alles, was Oscar hat, möchte Andreas auch und noch etwas mehr. Erst mit dem Eintritt in die Rheingauer Mühle werden beide Brüder vor den Eltern gleichberechtigt. Andreas „erkauft“ sich diese Gleichstellung jedoch mit der Aufgabe einer Vielzahl von Freiheiten. 
Risiko und lange Arbeitszeiten. Andreas, für den das Unternehmen der Familie immer ein Quell von Privilegien und Geld war, muss sich nun diesen Lebenswandel - den er als Jugendlicher als nicht erstrebenswert beurteilte - aneignen. Er sagt: ,ganz ehrlich, ich glaube, wenn ich zweieinhalb Millionen hätte auf den Tisch legen können, um das Ganze zu kaufen, hätte ich wahrscheinlich was anderes mit den zweieinhalb Millionen gemacht“ (9:29-30).

Jene Mitglieder aus Unternehmerfamilien, die sich unter dieses Muster fassen lassen, sehen das Familienunternehmen als einen Schlüssel zu einem guten Leben. Sie möchten in Wohlstand leben, einen hohen sozialen Status genießen und in einer angenehmen Atmosphäre arbeiten. Diese Familienunternehmer können mit einem hohen Arbeitspensum umgehen, sie sind als „Familienunternehmer“" sozialisiert, aber sie wollen dafür mit einem guten Einkommen, flexiblen Arbeitszeiten, Reputation und Entscheidungsfreiheit belohnt werden. In anderen Worten: Die oberste Priorität liegt nicht notwendigerweise auf der Kontinuität des Familienunternehmens und der Erfüllung einer Generationen überspannenden Mission, stattdessen stellen diese Familienunternehmer ihr eigenes Glück vor das der Gemeinschaft der Unternehmerfamilie. Das Unternehmen wird für den persönlichen Gewinn genutzt und als Quelle von Privilegien verstanden.

Diese Gruppe von Familienunternehmern beginnt schon früh ihren familiären Hintergrund zu ihrem Vorteil zu nutzen, was jedoch nicht als rein strategisches Handeln interpretiert werden darf. Sie genießen es, sich durch das Unternehmen auszuzeichnen, und sehen ihren Status als ein Mittel, das ihnen Individualität verleiht. Das Familienunternehmen stellt eine Gemeinschaft dar, in der man bekannt und akzeptiert ist, und ebenso eine Möglichkeit, das Taschengeld aufzubessern. Das Netzwerk der Unternehmerfamilie ist eine wichtige Ressource für Kontakte, Jobs und gemeinnützige Tätigkeiten. Die Eltern zahlen für eine private Universität, eine Wohnung oder bieten andere finanzielle Unterstützung an. In ihrer Lebenslaufgestaltung verfügen diese Familienunternehmer über die Gewissheit, dass das Unternehmen immer eine Option für sie ist, was es ihnen erlaubt, sich frei durch ihr berufliches Leben zu bewegen. Kann eine Anstellung ihren hohen Ansprüchen nicht gerecht werden, ist etwa der Spaß- oder Lernfaktor nicht ausreichend, können sie den Arbeitsplatz wechseln, ohne ernsthaft Arbeitslosigkeit zu fürchten.

Der Eintritt in das Unternehmen erfolgt als natürliches Ereignis, sobald der adäquate Zeitpunkt erreicht ist (etwa bei Unzufriedenheit mit der letzten Anstellung). Die Nachfolger erwarten von ihren Eltern, die notwendigen Schritte bereits vorbereitet zu haben und ihnen einen eigenen Aufgabenbereich und Verantwortung zu übergeben. Sie fordern ihr Recht innerhalb des Familienunternehmens und in der Beziehung mit dem Übergeber. Abschließend sei noch erwähnt, dass Angehörige dieser Gruppe nicht unbedingt eine langfristige Perspektive verfolgen. Sie leben für den Moment und ihre Freu- 
de am Leben. Dieser Weg ins Unternehmen erfüllt damit am ehesten das Klischee, das man mit der Redewendung ,von Beruf Sohn“"verbindet. ${ }^{79}$

\subsubsection{Das Familienunternehmen als emotionale Heimat}

Diesem letzten Typus ist eine lebenslaufstrukturierende Sehnsucht nach "Heimat" zueigen. Dieser folkloristisch anmutende Begriff ist bewusst gewählt, um die emotionale Komponente dieser Lebenslauforientierung zu betonen:

Malte Niest und seine zwei Jahre jüngere Schwester wachsen in den 1980er Jahren in Berlin auf. Ihre Mutter ist Lehrerin und ihr Vater Konrad Geschäftsführer und später auch Teilinhaber eines industriellen Familienunternehmens in zweiter Generation. Als Malte vier Jahre alt ist, stirbt sein Großvater, der als Firmenpatriarch stark in das Leben seines Vaters eingriff. Malte hat keine eigenen Erinnerungen an den Unternehmensgründer. Der Vater ist aus eigener Erfahrung darum bemüht, die Familie als geschützten Raum zu erhalten, und klammert die Vorgänge im Unternehmen aus dem Familienleben systematisch aus. Die Kinder wissen zwar um den Arbeitsort des Vaters, er hat aber „nie erzählt, dass das seine Firma ist zum Beispiel“ (4:27). Das Unternehmen ist nicht Thema von Tischgesprächen, kein Ort des kindlichen Spielens. Malte hat kein großes Interesse an der Schule. Er widmet sich lieber der Freizeit, fährt Mofa und spielt Tennis.

Mitte der 1990er Jahre verlässt Maltes Mutter überraschend die Familie. Dieses Ereignis markiert eine starke Zensur im Leben von Malte, seiner Schwester und seines Vaters. Sie sind unter Schock und fühlen sich unvollständig. Einige Monate später lernt Konrad eine neue Partnerin kennen, die sehr kleine Kinder in die Beziehung mitbringt. Sowohl Malte als auch seine Schwester sind mit dieser neuen Beziehung des Vaters nicht einverstanden. Sie ziehen sich vom Vater zurück, sind rebellisch, nutzen ihre Freiheiten aus.

Im Jahr darauf fährt Malte seine Vespa zu Schrott und braucht nun Geld für eine neue Maschine. Sein Vater schlägt ihm diesen Wunsch aus - stattdessen bietet er ihm an, sich im Unternehmen etwas dazu zu verdienen. Die ersten Tage seiner Ferienarbeit sind Malte noch fest in Erinnerung: Jeder weiß, dass er der Sohn des Chefs ist. Malte begegnet dieser ungewohnten Aufmerksamkeit und seinem plötzlichen Sonderstatus mit ambivalenten Gefühlen: Die subjektiv wahrgenommene Distanz zwischen ihm und den Mitarbeitern ist ihm unangenehm, und zugleich fühlt er sich geehrt. „Keiner wusste wie er mit mir umgehen soll.“ (6:9) Mit der Zeit entspannt sich das Ver-

79 In seiner Typologie von Nachfolgern, die Müller 2008 aus seiner Untersuchung der Dreierkonstellation aus der Zusammenarbeit zwischen Mitarbeitern, Nachfolgern und Übergebern entwickelt, sieht er neben dem „Rebell“ und dem „strebsamen Lehrling“ auch den Typ „,von Beruf Sohn vor". 
hältnis, und Malte fühlt sich wohl, übernimmt gerne Sonderschichten und Urlaubsvertretungen. Malte erfährt im Unternehmen soziale und monetäre Anerkennung für seine Person und sein Engagement. Durch seine Tätigkeit im Unternehmen kommt Malte erstmals in Berührung mit dem Erbe seiner Familie, insbesondere seines Großvaters. Seine Großmutter, die selbst noch aktiv im Unternehmen arbeitet, beginnt ihm von ihrem Mann zu erzählen und stellt zunehmend Parallelen zwischen den beiden fest. Malte ist fasziniert von dem Charakter des Großvaters und stilisiert ihn zu einem guten Unternehmer, einem Vorbild, zu dem man aufsehen kann.

Ein weiteres Jahr später heiratet Konrad seine neue Partnerin, woraufhin Malte und seine Schwester von zu Hause ausziehen und sich eine Wohnung im gleichen Stadtteil nehmen. Malte ist zu dieser Zeit 16 Jahre alt. Er fühlt, dass es in dieser neuen Familie keinen Platz mehr für ihn gibt, er treibt sich viel herum, stellt Unsinn an. Das Wissen darum, im Unternehmen mit seiner Arbeit Geld verdienen zu können, gibt ihm das Vertrauen, auch ohne Schule gut durchs Leben zu kommen. Zwischen seiner familialen und unternehmensbezogenen Erfahrungswelt besteht ein starker Gegensatz: Während er in der Patchworkfamilie vereinsamt, fühlt er sich im Unternehmen (und der Großelterngeneration) aufgenommen und zugehörig. Malte sieht sich in einer Linie mit seinem Großvater, der eins ist mit dem Unternehmen.

Nach seinem Abitur, das schlechteste der Schule, und seinem Wehrdienst beginnt Malte Wirtschaftsingenieurwesen zu studieren. Durch die Erlebnisse im Unternehmen „War mein Interesse eigentlich dafür geweckt, ehrlich gesagt“" (9:27-28). Malte weiß nun, wohin er gehört. Mit seiner Studienwahl versucht Malte vielleicht seinem Vater, der das gleiche Fach an der gleichen Universität studierte, zu signalisieren, dass er Interesse an einer Nachfolge hätte. Konrad will seinem Sohn jedoch die ,freie' Berufswahl lassen und schließt unbewusst die Option einer Nachfolge aus. Malte lässt sich davon nicht beirren und legt großes Engagement an den Tag: Er beginnt ein Pflichtpraktikum im Unternehmen, lernt Fremdsprachen und absolviert mehrere Auslandspraktika in der Branche. Er lernt immer mehr über die Geschichte des Unternehmens, erfährt mehr Details zu seinem Großvater und bekommt einen Überblick über die strategische Aufstellung des Unternehmens und seine Anforderungen. „Ich höre es von meiner Großmutter oder von meinem Vater ,Da bist Du wie der Opa'.“(11:18-19) Malte identifiziert sich immer mehr mit dem Unternehmen. Er sieht es als seine Lebensaufgabe an, das Erbe des Großvaters weiter zu führen. Je stärker sich Maltes Leidenschaft für das Unternehmersein und seine Branche entfaltet, desto weniger bedeutet es ihm, viel Geld zu besitzen. Er differenziert sich immer mehr von seinem materiell orientierten Freundeskreis. Gegen Ende seines Studiums wird Maltes Vater ein wichtiger Ansprechpartner für ihn. Gemeinsam planen sie den Eintritt ins Unternehmen. 
In Maltes biografischer Selbstdarstellung bedient er sich eines Determinations-Narratives. Der erste Kontakt mit dem Unternehmen, das ihm bis dahin unbekannt war, habe Richtung und Sinn in sein Leben gebracht. Er habe seine Handlungen gänzlich an einer Reflektion über die Bedürfnisse des Unternehmens ausgerichtet. Dieser Weg ist ihm quasi genetisch über seinen Großvater in die Wiege gelegt worden. Im Interview wird Malte mit dieser strategischen Selbstdarstellung konfrontiert und entgegnet: „, nein, also ... das, das klingt jetzt zu geplant, nein.“ (24:27-28) Während Malte sich gerne als ,guten Familienunternehmer' darstellt - zu dem eben auch eine gewisse strategische Weitsicht, eine Generationengeschichte und ein Narrativ von Berufenheit gehört - gibt er zu erkennen, dass es hier einen Unterschied zu seinen tatsächlichen Erlebnissen gibt. Der Weg ins Unternehmen ist für Malte weniger bewusst als emotional geprägt. Das Unternehmen erlebt er als einen Ort der Anerkennung und des Zusammenhaltes. Das „Wir-Gefühl“ des Unternehmens scheint ihm, der durch die Scheidung der Eltern in der Familie keinen rechten Platz mehr hat, eine Heimat zu bieten.

Dieser typische Lebensweg in das Familienunternehmen zeichnet sich durch ein Aufwachsen in einer Familie ohne Unternehmensanschluss aus. Dies kann bedeuten, dass beide Eltern abhängig erwerbstätig oder freiberuflich tätig sind (wie im Fall Marcella Treuninger) oder eben eine strikte Trennung der beiden Bereiche praktiziert wird (wie im Fall Malte Niest). In der Familie und eventuell auch im Freundeskreis fühlen sich die Kinder durch Scheidungserfahrung, häufige Ortswechsel oder einen großen Altersabstand vereinsamt. Sie sehnen sich nach Geborgenheit, festen Strukturen oder einer bedeutsamen Rolle in der Familie. Durch ein Schlüsselerlebnis kommen sie mit dem Unternehmen und der Option, Teil dieser Gemeinschaft zu sein, in Kontakt. Die Immobilität des Unternehmens, seine festen Strukturen und die emotionale Nähe zur Familie verleihen dem Unternehmen eine „heimatliche“ Qualität. Familienunternehmer dieses Typs nehmen das Unternehmen als persönliches Projekt an und zeigen volles Engagement. Sie stellen das Ziel eines Fortbestandes des Unternehmens an oberste Stelle, jedoch weniger die Kontinuität des Familieneinflusses.

\subsubsection{Strukturelle Einflussfaktoren}

Aus diesen idealtypischen Beschreibungen der unterschiedlichen Wege in das Familienunternehmen leiten sich einige strukturelle Faktoren ab, die maßgeblich die Lebenslaufentscheidungen von allen Mitgliedern in Unternehmerfamilien beeinflussen. Diese gliedern sich grob in drei Gruppen: sozioökonomische, emotionale und generationelle Faktoren. 


\subsubsection{Fähigkeiten, Lebenslage und Gelegenheitsstruktur}

Zunächst lässt sich festhalten, dass in der beruflichen Lebensplanung von Mitgliedern aus Unternehmerfamilien die üblichen Determinanten der Berufswahl wirksam sind. Zu diesen zählen die Fähigkeiten der Person, die Lebenslage der Familie und die wirtschaftlichen Gelegenheitsstrukturen. Mit Bourdieus (2006) kapitaltheoretischem Blick auf die Berufswahl ${ }^{80}$ lässt sich etwa argumentieren, dass die Mitglieder einer Unternehmerfamilie im Laufe ihrer Sozialisation einen „Unternehmerhabitus“ annehmen, also bestimmte Werte, Fähigkeiten und Einstellungen zu Beruf und Familie internalisieren, die einen „feinen Unterschied“ im Vergleich zu anderen Schichten oder Milieus ausmachen. Die Attraktivität der Mitglieder aus Unternehmerfamilien auf dem Arbeitsmarkt leitet sich einerseits aus der Ausstattung mit den verschiedenen Kapitalsorten (ökonomisches, kulturelles und soziales) sowie anderseits aus der lokalen Wirtschaftsstruktur, der allgemeinen Wirtschaftslage, Politik und der historischen Entwicklung einer Branche ab. Durch die Lebenslage ihrer Eltern können Mitglieder aus Unternehmerfamilien einen Startvorteil auf dem Arbeitsmarkt verbuchen: sei es durch ein vermitteltes Einstellungsset, die Finanzierung einer guten Ausbildung oder durch Netzwerkkontakte. Zugleich haben sie ein intimes Wissen über die Lebensführung eines Unternehmers erworben, eine Tätigkeit mit einem relativ hohen Berufsprestige, und sich eine Meinung über die Attraktivität dieser Lebensweise gebildet. ${ }^{81}$

\subsubsection{Emotionale Verbundenheit}

Ein zweiter, nicht zu unterschätzender Faktor ist die emotionale Verbundenheit mit dem Familienunternehmen, die sich mit dem Ansatz des ,psychological ownership“82 näher beschreiben lässt: Die Familienmitglieder empfinden, dass sie Mühe, Zeit und psychologische Energie in das Unternehmen investiert haben und deshalb ein intimes Wissen über dieses besitzen. Die Dauer der Verbundenheit bestimmt die Stärke des gefühlten Eigentumsverständnisses, das jenseits von rechtlichen und wirtschaftlichen Grenzen operiert.

80 Die Idee eines Transfers der Kapitaltheorie Bourdieus auf den Untersuchungsgegenstand der Familienunternehmen ist nicht neu. Auch Lubinski greift auf diesen Ansatz zurück Lubinski 2010: 175ff.

81 Interessanterweise wird ein hohes Einkommen nicht als Grund für die Wahl eines Weges in das Familienunternehmen genannt. Ein solches Argument würde quer stehen zu der üblichen Präsentation des „Familienunternehmertums“ (siehe Abschnitt 4 in diesem Kapitel).

82 Dieses Konzept wurde von Pierce et al. entwickelt Pierce et al. 2001, 2003 und schon mehrfach auf Familienunternehmen und ihre Mitarbeiter angewandet Lubinski 2009; Zellweger und Astrachan 2008; Bernhard und Jaskiewicz 2011; Bernhard 2011; Bernhard und Jaskiewicz 2011; Lubinski 2010. Siehe hierzu auch Kapitel 3.4.4. 
Ein solches „psychologisches Eigentum“ mag zu einem höheren Engagement von Familienmitgliedern im Unternehmen führen, zu persönlicher Aufopferungsbereitschaft, der Neigung, Risiko und Verantwortung auf sich zu nehmen, ebenso wie dem Widerwillen, das Unternehmen zu teilen, oder Ambivalenzen im Umgang mit Veränderung. Das Unternehmen als Besitzobjekt hilft dabei, die eigene Identität zu definieren, diese auszudrücken und sie über die Zeit hinweg aufrecht zu erhalten (Bernhard 2011:32). Als gefühlte Besitzer finden diese Familienmitglieder einen Ort, an dem sie Anerkennung erfahren, an dem sie ihren Einfluss zur Geltung bringen können und an dem sie sich zu Hause fühlen. Eigentumsgefühle prägen die Anforderungen an den eigenen Lebensweg, mit denen eine persönliche Auseinandersetzung stattfinden muss (Lubinski 2010:116).

\subsubsection{Generationale Faktoren}

Schließlich sollten als wesentlicher Faktor - einer unvollständigen Liste - die Generationenbeziehungen in Unternehmerfamilien angeführt werden. Diese bestimmen sich zunächst aus der Beziehung zu den Eltern, die von einer Spannung zwischen Autonomisierungsbestrebungen und Nähe gekennzeichnet ist. Die Eltern beobachten die beruflichen Vorstellungen und Fähigkeiten ihrer Kinder genau. In Unternehmerfamilien spielen Eltern eine aktive Rolle in der Lebenslaufgestaltung der Kinder. Als Ratgeber, Bezugsperson oder als Vermittler von Kontakten nehmen sie direkten Einfluss auf die berufliche Weichenstellung. Zudem zeigt sich, dass die berufliche Orientierung der Geschwister direkten Einfluss auf die Lebenslaufentscheidungen nehmen kann. Sie mögen sich selbst in einem konstanten Wettbewerb wähnen oder in einer Linie möglicher Nachfolger verstehen. Sie müssen ihren Weg finden und gleichzeitig den der anderen berücksichtigen, was die Leben der Geschwister in einer direkten Art und Weise aneinander bindet, die ungewöhnlich für andere familiale Lebensformen ist. Schließlich ist die Interaktion mit Gleichaltrigen bzw. die Integration in die eigene Altersklasse ein weiteres Merkmal, das die Lebenslaufentscheidungen in Unternehmerfamilien beeinflusst. Auf dieser horizontalen Ebene der Generationenbeziehungen vergleichen junge Familienunternehmer ihre berufliche Orientierung mit anderen. Dieser Prozess scheint in diesem Forschungszusammenhang erwähnenswert, da er sich in einen Legitimationstest der Nachfolgeaspirationen wandeln kann und deshalb einen kritischen Moment für den Fortbestand des Familienunternehmens markiert. Zusammenfassend entfaltet das Streben nach Aufmerksamkeit und Anerkennung innerhalb der Familie und im Generationenverbund, also die hier stattfindenden Rollenzuweisungen und -verhandlungen, in Unternehmerfamilien eine in hohem Maße lebenslaufprägende Wirkung. Hier deutet sich an, dass das in der Kindheit festgestellte Beobachtungsverhältnis zwischen 
Eltern und Kindern lebenlang anhält. Dieser Aspekt wird in Kapitel VII, das die Generationenbeziehungen in den Mittelpunkt rückt, noch einmal aufgegriffen und als Indizienanalyse der Lebensläufe beschrieben.

\subsubsection{Fazit}

Diese strukturellen Einflussfaktoren sind der spezifischen Konstellation einer Unternehmerfamilie und ihrem generationalen Setting geschuldet. Überraschenderweise lassen sich die genannten Merkmale konsistent aus allen betrachteten und nach Relevanz ausgewählten Fällen herausarbeiten. Dieser Umstand lässt sich als Indiz für die Wirkmächtigkeit der Verbindung von Familie und Unternehmen interpretieren, die kontextbezogene Faktoren in die zweite Reihe verweist. Weder die Größe des Unternehmens noch die Branche, eine ländliche vs. eine urbane Umgebung oder wirtschaftliche Krisenzeiten scheinen den Weg in das Familienunternehmen so stark zu prägen wie die emotionale Verbundenheit mit diesem sowie die permanenten generationalen Aushandlungsprozesse. Das Unternehmen erhält in der beruflichen Orientierung der Mitglieder aus Unternehmerfamilien einen lebenslang wirksamen Horizont. Sie stehen mit ihrer ganzen Person hinter diesem Prozess. Es gibt keinen Tag, der nicht für den Weg in oder aus dem Unternehmen entscheidend sein könnte. Hildenbrand geht sogar soweit zu behaupten, dass es sich bei dem Generationswechsel (in der Landwirtschaft) nicht um eine reflexiv-universalistische Berufsorientierung oder rationale Entscheidungsfindung handle, sondern vielmehr um ein Prinzip des „Dran-Seins“. Die strukturellen Kräfte, die sich hier entfalten, scheinen jeden „Entscheidungsfreiraum“ zu erdrücken. Im Rahmen dieser Studie muss diese starke These jedoch etwas relativiert werden. Zu groß sind die vorgefundenen Umbrüche und überraschenden Wendungen in den Lebensläufen der Befragten, um von einer eindeutigen Umsetzung von Erbfolgeregelungen zu sprechen. Das „DranSein" umschreibt jedoch sehr gut die vorgefundene Gefühlslage und die impliziten Regelungen zwischen den Generationen. Nachfolge lässt sich am ehesten als komplexe Entscheidung für einen bestimmten Lebensstil beschreiben. Erst die Tätigkeit im Unternehmen und die zunehmende Selbstidentifikation mit der Rolle des Unternehmers führen dazu, dass nun Innovationspotenziale und unternehmerische Handlungsspielräume erkannt werden. In diesem Sinne ist Nachfolge also nicht als Unternehmensgründung zu verstehen, sondern als Rollenaneignung. Das Familienunternehmen verleiht dem Lebenslauf von Familienunternehmern Struktur und Kontinuität. Jedoch erfordern, wie Regina Wenk (2007) herausstellte, die materiellen und emotionalen Verstrickungen der Mitglieder einer Unternehmerfamilie miteinander und mit dem Unternehmen in hohem Maße biografische Arbeit. 
Abschließend soll noch der Frage nachgegangen werden, inwiefern bei der Betrachtung der eingeschlagenen Wege in das Familienunternehmen ein Gender-Aspekt berücksichtigt werden sollte, es also typisch weibliche und typisch männliche Nachfolgen gibt. Jäckel (2010) stellt in ihrer Untersuchung der Nachfolge von Töchter in Unternehmerfamilien eine dominante „Plan-B Symbolik“ (Jäkel-Wurzer 2010:84) fest. Erst dann, wenn keine männlichen Nachfolger vorhanden sind oder sich diese im Laufe der Zeit als ungeeignet oder unwillig herausstellen, werde die weibliche Nachfolge unausweichlich. Es ist bezeichnend, dass in der oben vorgenommenen Darstellung der typischen Wege in das Familienunternehmen der einzige weibliche Fall tatsächlich einer solchen Plan-B-Symbolik folgt. Nach Jäkel-Wurzer konzipiert sich der Möglichkeitsrahmen von Töchtern in Unternehmerfamilien besonders stark aus der komplexen Zusammensetzung aus familialer Position, familialen Erwartungshaltungen, Identitätszuschreibungen und dem darauf ausgerichteten familialen Normen- und Wertesystem. Trotzdem ist es eine Frage der Häufigkeit, wie oft eine Nachfolgerin über direkte Betrauung mit einer Mission ${ }^{83}$ oder über Umwege ${ }^{84}$ in das Familienunternehmen findet, diese kann im Rahmen dieser Arbeit nicht geklärt werden. Eine zugespitzte, strukturelle Enge für Töchter aus Familienunternehmen findet sich hingegen in dem hier untersuchten Interviewmaterial wieder. Jäkel-Wurzer beschreibt sehr treffend, wie Töchter eine spezifische Dilemma-Situation zwischen einer empfangenen Delegation und dem Geschlechterrollenarrangement erleben. Sie sehen sich unter Druck, ihre Delegation nachträglich zu legitimieren (Jäkel-Wurzer 2010:453f.) und sind in diesem Bestreben in besonderem Maße von der Bestätigung durch den Vater abhängig. Töchter aus Unternehmerfamilien seien deshalb gefordert, diese rigide Bedingung mit der Entwicklung ihrer persönlichen Identität zu koordinieren (Jäkel-Wurzer 2010:455f.). Schließlich beobachtet Jäkel-Wurzer eine einseitig überzogene Idealisierung des Vaters, die eine abwesende Väterlichkeit kompensiert.

83 In der hier entwickelten Typik „das Familienunternehmen als Mission“, Jäkel-Wurzer spricht von der ,legitimen Nachfolgerin“.

84 In der hier entwickelten Typik etwa ,das Familienunternehmen als Mission - zweite Wahl“ oder „das Familienunternehmen als Zuflucht“, Jäkel-Wurzer arbeitet die Typen der „Ersatzspielerin“, der „Steigbügelhalterin“ und der „Platzhalterin“ aus. Diese Varianten stellen Wege dar, bei denen die Tochter im Schatten des männlichen Nachfolgers steht und in einer außerordentlichen Solidaritätskonstellation zwischen den Geschwistern ihrem Bruder zuarbeitet und ihn unterstützt. 


\subsubsection{Der Eintritt ins Unternehmen als biografische Herausforderung}

Der Blick auf die biografische Selbstdarstellung der Mitglieder aus Unternehmerfamilien erweist sich als äußert interessant. Trotz der herausgearbeiteten Selbstverständlichkeiten und Zwangsläufigkeiten im Leben der Familienunternehmer findet sich ein starkes Narrativ der ,freien Entscheidung“ für diesen Lebensweg. In seiner Befragung von Familienunternehmern beschreibt Breuer (2009:286) ein fast identisches Bild: Auch seine Interviewpartner stellen beharrlich in Abrede, dass es eine elterliche bzw. vorgängerseitige Ausübung von Übernahmezwang auf sie gegeben habe. Immer wieder betonen sie, dass sie über ihre berufliche Zukunft frei entscheiden können und die Eltern höchstens als Ratgeber oder Impulsgeber in diesem Entscheidungsprozess gewirkt hätten. Der Eintritt in das Familienunternehmen, der aus einer strukturlogischen Analyse heraus als familiärer Aushandlungsprozess beschrieben werden kann, wird also in den Selbstdarstellungen der Betroffenen zu einer individuellen Entscheidung stilisiert. Im Folgenden soll dieses dominante narrative Muster der Selbstpräsentation, das unabhängig von dem tatsächlich eingeschlagenen Weg rekonstruiert werden kann, näher beschrieben und analysiert werden.

Die Familienunternehmer der zweiten oder einer späteren Generation beschreiben die Aktivität oder Nicht-Aktivität im Familienunternehmen aus einer Ich-zentrierten Perspektive. Es werden rationale Gründe für oder wider einen Eintritt in das Familienunternehmen abgewogen und schließlich eine Entscheidung in diesem Prozess der Berufswahl getroffen. Die schlagenden Argumente für einen Weg ins Familienunternehmen lassen sich in drei Kategorien zusammenfassen:

Aus den Selbstdarstellungen der Familienunternehmer gewinnt man erstens den Eindruck, dass diese sich zu ,ihrem' Produkt bzw. ,ihrer' Branche hingezogen fühlen, egal ob die Familie industrielle Güter oder landwirtschaftliche Erzeugnisse vertreibt. Die spezifische Leidenschaft für ein Produkt oder eine Branche - die fast schon willkürlich erscheint - wird als ein Grund für die Berufswahl angeführt, lässt sich jedoch auch als Bestandteil einer Rhetorik des „guten Unternehmers“ interpretieren. Wendy Handler (1992:290) stellt ebenfalls fest, dass die Mehrheit ihrer Befragten beteuert, auch dann in der gleichen Branche tätig sein zu wollen, wenn es das Familienunternehmen nicht gäbe.

Als weiteres Hauptargument für die Attraktivität einer Karriere als Familienunternehmer wird Selbstbestimmtheit angeführt. In ihren Alltagsbeschreibungen verweisen die Befragten sowohl auf eine hohe Arbeitsbelastung als auch auf ein Verschwimmen der Grenzen zwischen Arbeit und Freizeit, setzen diesen aber flexible Arbeitszeiten, die Möglichkeit, Privates in 
den beruflichen Alltag zu integrieren ebenso wie einen hohen Entscheidungsfreiraum in unternehmerischen Belangen entgegen. Wenn sie von ihrer Berufswahl sprechen, betonen sie zudem die Möglichkeit, kreativ und innovativ zu sein.

Die dritte Kategorie der Argumente für einen Eintritt in das Familienunternehmen kreist um die Übernahme von Verantwortung für das Unternehmen und seine Mitarbeiter in einem zum Teil relativ jungen Alter. Die Interviewpartner stellen das Familienunternehmen als eine Möglichkeit dar, einige Schritte auf der Karriereleiter zu überspringen und sich statt mit Vorgesetzten nun unmittelbar mit gesellschaftlichen und ökonomischen Entwicklungen auseinanderzusetzen. Darüber hinaus bildet ein Gefühl der Verbundenheit mit früheren Generationen einen wichtigen Topos in ihren Selbstdarstellungen. Sie demonstrieren eine ausgeprägte Sorgfaltspflicht im Umgang mit dem Unternehmen, das sie von ihren Vorfahren erhalten haben und nun lediglich zeitweise verwalten.

Alle diese Argumente schlagen vor, dass sich das "Ich" bewusst für einen spezifischen beruflichen und privaten Lebensweg entschieden hat. Typisch für die Darstellung des weiteren Verlaufs nach dieser richtungweisenden Entscheidung ist die starke Betonung von Eigeninitiative und Leistungsbereitschaft. Die Familienunternehmer erzählen von lehrreichen Erfahrungen, Qualifikationen, unternehmerischen Erfolgen und der erforderlichen Sensibilität im Umgang mit den Eltern. Die Verbindung von Familie und Unternehmen wandelt sich in ein relativ stabiles Muster des alltäglichen Lebens. Es wird von einem Ensemble an Wertorientierungen, Interpretationen, Handlungen und Interaktionen begleitet. Der Lebenslauf wird von den Individuen als freiheitlich stilisiert und erhält einen holistischen, bedeutungsvollen Charakter. Das Narrativ der ,freien Wahl“" markiert den Beginn einer Selbstdarstellung als Unternehmer und ist damit wesentlich an der Begründung einer neuen Rollenidentität beteiligt. Die Biografie eines „Familienunternehmers“ wirkt identitätsbildend und differenzierend (Bäcker 2008; Down 2006).

Das Narrativ der rationalen Entscheidung findet sich nicht nur in den Interviews mit den Nachfolgern der zweiten oder einer späteren Generation, sondern auch in den Gesprächen mit anderen Familienmitgliedern. Aus dem Interviewmaterial mit der übergebenden Generation lässt sich das Pendant dazu kondensieren: Während die einen eine freie Entscheidung betonen, stellen die anderen die offene Wahlsituation in den Vordergrund.

Das Unternehmen wird als eine Option im Lebenslauf der Kinder erzählt, die von Geburt an besteht, aber nicht notwendigerweise ergriffen werden muss. In ihren Präsentationen versuchen die Eltern, ihre Kinder von Pflichtgefühl und Druck zu befreien. Sie argumentieren gegen die Wahrnehmung der Nachfolge als strukturell und familial determinierten Weg und betonen stattdessen, dass das Familienunternehmen nur eine Karrieremöglichkeit unter vielen biete. Sven Singermayer etwa sagt: 
"Ich habe immer ... klar signalisiert, ... ich freue mich, wenn einer von euch in die Firma geht, aber ihr müsst aus innerem Entschluss in die Firma gehen. ... Selbständigkeit ist kein leichtes Brot und ... ihr müsst mit Herzblut so was machen, weil sonst wird es nichts. Und wenn keiner in die Firma geht, das habe ich auch signalisiert, bricht mein Herz nicht. Es hat alles seinen Rhythmus, auch ein Unternehmen ist ein lebendiger Organismus." (24:27-33)

Den Höhepunkt der elterlichen Darstellung der Berufswahl ihrer Kinder und des damit verbundenen Eintritts in das Familienunternehmen markiert eine Frage, die meist von der älteren Generation gestellt wird: Willst Du meine Position im Unternehmen übernehmen? Auf diese Weise wird die ,Freiheit der Entscheidung' symbolisch auf die Spitze getrieben. Eine solche Frage kann als stilistischer Kunstgriff in der Selbstdarstellung der Eltern verstanden werden. Sie soll den Wunsch nach einer innerfamilialen Nachfolge ebenso wie ihr Zutrauen in die Leistungsfähigkeit der jüngeren Generation zum Ausdruck bringen und gleichzeitig die endgültige Entscheidungsgewalt in das Hoheitsgebiet der jüngeren Generation legen.

Diese Analyse der Selbstpräsentationen von Familienunternehmern soll nicht die Kräfte und Verpflichtungen, die in Unternehmerfamilien wirken, verleugnen. Der Eintritt in das Familienunternehmen mag sehr wohl eine extern geformte Statuspassage darstellen. Der interessante Aspekt ist weniger darin zu sehen, ob wir auf eine formal ,freie“ bzw. ,rationale“ Wahl oder einen „selbst bestimmten“ Lebensweg blicken, sondern in der Tatsachte, dass dieser Schritt retrospektiv als solcher dargestellt und in die eigene Biografie integriert wird. Eltern stellen sich nicht als autoritäre Instanz dar, die über das Leben der Kinder bestimmt. Nachfolger betonen ihre Autonomie und argumentieren gegen ein Stereotyp des ,ins gemachte Nest Setzens“. Narrative Muster, die sich auf Tradition berufen, scheinen nicht als legitim erachtet zu werden. Beide Generationen bringen ihre Vorstellungen von Nachfolge in Einklang mit den kulturellen Skripten von Erfolg, Individualität, Selbstbestimmtheit und Autonomie. In diesem Sinne scheint das Narrativ der freien Wahl Familienunternehmern eine Möglichkeit zu bieten, die normativen Erwartungen, die an einen modernen Lebensentwurf gestellt werden, zu erfüllen.

Trotz des klaffenden Widerspruchs zwischen den strukturellen Faktoren und Zwangsläufigkeiten im Lebensweg von Familienunternehmern und dieser Betonung der freien Wahl in ihrer Selbstdarstellung ist es mit Verlaub zu kurz gegriffen, wie andernorts vorgeschlagen, von einer „Metapher der freien Wahl“ (Stamm und Marchese 2011) oder einem „Freiwilligkeitsmythos“ (Altmeier 2005:56) zu sprechen. Beide Begrifflichkeiten schlagen eine nur scheinbar freie und individuelle Entscheidung vor und unterschätzen die Handlungswirksamkeit dieses biografischen Musters ebenso wie die Wirk- 
mächtigkeit der darin integrierten gesellschaftlichen Norm zur Individualisierung, die auch in Unternehmerfamilien Anwendung findet. Die Rede von einem Mythos oder einer Metapher unterstellt einen hohen Grad an Selbsttäuschung. Aber es reicht nicht aus, den eigenen Lebensweg als eine freie Entscheidung „zu verkaufen“. Die biografische Deutung der eigenen beruflichen Laufbahn als Ergebnis einer persönlichen Wahl bedarf des faktischen Erlebens, sonst ist keine nachhaltige Identitätsbildung möglich. In anderen Worten: In dem Prozess des stetigen Ineinandergreifens von Strukturen, die sich aus der Verbindung von Unternehmen und Familie ergeben, und ihrer biografischen Bearbeitung muss der Weg ins Unternehmen als ein innerer Entschluss gefasst werden. Als solcher wird er in einem familialen Aushandlungsprozess ermöglicht und respektiert.

In der Familie Singermayer wird im Zusammenhang mit der Berufswahl vom „,inneren Ergreifen“ (z.B. Sven Singermayer, 26:30) gesprochen. Diese Begrifflichkeit verdeutlicht, dass es gelingen muss, sich ein bestehendes Projekt zueigen zu machen, aktiv „Ja“ zu einer bestimmten Art und Weise des Lebens zu sagen. Besonders deutlich wird diese emotionale Dimension der „freien Wahl“" in folgendem Zitat von Anabella Singermayer: „Und ich habe diese Frage genau verstanden in ihrer ganzen Scheinbarkeit und also da erinnere ich mich eben auch daran, weil das war einfach auch ähm .. ganz klar lebensprägend, das war mir auch klar. Und mir war auch ganz klar, wenn ich jetzt „Ja“" sage und, dass ich nur „Ja“" sagen kann und will, aber dass dieses „Ja“ einfach eine neue Dimension aufmacht. Weil bisher war ich verantwortlich für mein Leben und das meines Sohnes .. und .. ähm .. mit der Entscheidung die Firma, „Ja“ zur Firma zu sagen letztendlich dann, was damals noch weiter weg war, aber die Firma zu übernehmen, das ist kein, kein Paarjahresjob, das ist ein „Ja“ fürs Leben, so und so habe ich das ungefähr auch empfunden." (6:23-31)

Die Intensität des Erlebens dieser freien Entscheidung spiegelt sich deutlich in den geführten Interviews wider. Sehr detailreich erinnern sich die Befragten an diesen Moment, in dem ihnen bewusst wurde, dass sie nun den Weg ins Unternehmen einschlagen werden. Hingegen sind die Erinnerungen an den sonst so bedeutenden ersten Arbeitstag in einem neuen Beruf oder einem neuen Unternehmen sehr blass oder gar nicht vorhanden.

Das biografische Muster der ,freien Wahl“ kann als eine Rechtfertigung für den Lebensweg in das Familienunternehmen verstanden werden. Es ist eine Strategie, wie die internalisierten Ideale und Normen einer individualisierten Gesellschaft mit der Kontinuität der Familientradition in Einklang gebracht werden können. Es ist eine Antwort auf ihnen entgegengebrachte Stereotypen, denn einen anderen Beruf zu ergreifen und sich gegen das Familienunternehmen zu entscheiden, entspricht scheinbar eher der gesellschaftlichen Normalerwartung als die Nachfolge anzutreten. Mit der „Entscheidung“ für das Unternehmen sehen sich die Mitglieder aus Unternehmerfamilien 
dem Verdacht ausgesetzt, keinen eigenständigen Lebensentwurf zu entfalten. Gleichzeitig birgt dieser „Ausnahmeweg“ auch das Potenzial, sich von seinen Altersgenossen zu differenzieren und darin Individualität zu finden. Das Narrativ der „freien Wahl“ nutzt dieses Potenzial und versucht, die kritische Lesweise ihrer Biografie zu entkräften.

Die Schlussfolgerung von Regina Wenk (2007), die sie aus ihrer Analyse der Nachfolgeentscheidung der eintretenden Generation zieht, kann hier nur bekräftigt werden:

„In den Lebensgeschichten ist weniger eine Kluft zwischen Familienbetrieb und Individualisierung bedeutsam, als vielmehr ihre Individualisierung im und mit dem Familienbetrieb. Eine biografische Erklärung der Übernahme oder Ablehnung eines Familienbetriebes zeigt diese - weder in den untersuchten landwirtschaftlichen Betrieben, noch in Betrieben aus anderen Branchen - auch nicht als Ausdruck nach wie vor unhinterfragt wirkender Automatismen, sondern es ist eher eine „Entselbstverständlichung traditioneller Vorstellungen" zu beobachten, die sich in der reflexiven Aneignung der Möglichkeit ausdrückt, die der Familienbetrieb den NachfolgerInnen im Zuge ihrer biografischen Arbeit bietet.“ (Wenk 2007:219). ${ }^{85}$

Die hier vorgestellten Ergebnisse erweitern die Studie von Wenk insofern, als sie eine Antwort darauf anbieten, wie sich diese biografische Arbeit konkret gestaltet. In ihrem Versuch, eine zeitgemäße Selbstdarstellung und Weltsicht zu konstruieren, greifen sie auf einen Code persönlicher Entwicklung und Emergenz zurück und ordnen ihr Leben teleologisch auf einen bestimmten biografischen Fluchtpunkt hin. Dabei dient ihnen das leistungsorientierte Skript einer individualisierten Gesellschaft als Richtschnur. Jenseits allein ökonomischer Motivationen oder einer unreflektierten Weiterführung der Familientradition sind Familienunternehmer bemüht, einen individuellen Nutzen aus dem Betrieb zu ziehen. Diese individuellen Argumente für das Familienunternehmen münden in ein Narrativ der ,freien und rationalen Entscheidung", das die Selbstdarstellungen der Familienunternehmer durchzieht. Darüber hinaus wurde gezeigt, dass trotz aller strukturellen Vorgaben und der Komplexität und Langfrist des Weges in das Unternehmen dieser auch als eine „freie Entscheidung“ empfunden werden muss. Dabei kommt dem Zusammenspiel der Präsentationen innerhalb der Familie eine große Bedeutung zu. Passen die Darstellungen der beteiligten Generationen nicht zusam-

85 In ähnlicher Weise argumentiert auch Dunn 1999, die Erkenntnisse aus der systemischen Familienforschung auf Unternehmerfamilien überträgt. Sie zeigt, wie die persönlichen, familialen, unternehmerischen und eigentumsbezogenen Entwicklungsphasen in Einklang gebracht werden müssen. Hinsichtlich der Phase des Erwachsenenalters weist sie darauf hin, dass ein „become his own man“ sich mit der jeweiligen Aufgabe im Unternehmen realisieren lassen muss - und ansonsten die Nachfolge scheitert Dunn 1999: 48. 
men, wird der Boden für Konflikte, Misskommunikation und einen erhöhten Erwartungsdruck bereitet. ${ }^{86}$

\subsubsection{Warum ,entscheiden “ sich Familienunternehmer für das Unternehmen?}

Bei einer Antwort auf diese Frage ist, folgt man der hier entfalteten Argumentation, in zwei Ebenen zu differenzieren: Einerseits lassen sich strukturelle Faktoren in Unternehmerfamilien beobachten, die in hohem Maße die berufliche Laufbahn und vor allem aber den Eintritt in das Familienunternehmen prägen. Diese Handlungsstrukturen und -zwänge scheinen sich aus der Verbindung von Unternehmen und Familie sowie ihrem generationellen Kontext abzuleiten. Sie formen ein konstantes, fast a-historisches Muster. Andererseits lässt sich eine narrative Argumentationsebene identifizieren, die eine biografische Bearbeitung dieser Strukturen verdeutlicht. Die in der biografischen Selbstdarstellung aufgeführten ,guten Gründe“ für einen Eintritt in das Familienunternehmen dienen dazu, den eigenen Lebensweg als ,sinnvoll“" zu begreifen und zu rechtfertigen. In ihnen spiegeln sich aktuelle und kulturelle Vorstellungen des modernen Lebens wider. Die Konstellation der Unternehmerfamilie muss Platz für eine Identität bieten, die sich aus den jeweils geltenden gesellschaftlichen Skripten speist.

Aus dem empirischen Material sind zunächst vier typische Lebenslaufmuster in das Familienunternehmen abgeleitet und beschrieben worden. Das Familienunternehmen mag als Zufluchtsort, als Mission, als Ressource oder als emotionale Heimat Orientierung im Lebenslauf bieten. In dem Interviewmaterial deutet sich an, dass es noch weitere Idealtypen der Nachfolge geben mag, sich diese jedoch nicht ausreichend fundieren lassen. In der Konsequenz darf die vorgestellte Typologie keine Vollständigkeit für sich in Anspruch nehmen. ${ }^{87}$ Sie dient jedoch als Grundlage für einen zweiten Abstraktionsschritt, in dem jene strukturellen Faktoren benannt werden, die auf Mit-

86 Die Metapher der freien Wahl wird hier als dominantes narratives Muster der Selbstpräsentationen von Familienunternehmern vorgestellt, das einen Rückgriff auf die kulturellen Skripte von Individualität und Leistung idealisiert. Die Ausnahmen zu diesem Regelfall wurden in einem interkulturellen Vergleich von deutschen und italienischen Interviews mit Unternehmerfamilien deutlich. Hier tritt das Individuum hinter dem Kollektiv der Familie zurück. Der Eintritt in das Unternehmen wird als logische Folge biografisiert, die keiner weiteren Erklärung oder Begründung bedarf. Dieses Muster scheint vor allem dann Anwendung zu finden, wenn eine starke Orientierung an der Familie und weniger an Gleichaltrigen erfolgt. Diese Thesen sind näher in dem Aufsatz von Stamm/Marchese 2011 ausgeführt.

87 Besonders bemerkenswert erscheint, dass sich diese Typen auch konsistent aus Interviews mit italienischen Unternehmerfamilien ableiten lassen, wie der Aufsatz von Stamm/Marchese 2011 darlegt. 
glieder der Unternehmerfamilie einwirken und bestimmen, welcher berufliche Weg eingeschlagen wird. Neben den in der Berufswahl üblichen Faktoren von Fähigkeiten, Lebenslage und Gelegenheitsstrukturen scheinen in Unternehmerfamilien die emotionale Bindung an das Unternehmen sowie die Generationenbeziehungen die Lebenslaufentscheidungen nachhaltig zu beeinflussen. Die lebenslauftheoretische Perspektive legt dabei die Komplexität und die Langfrist des Weges in das Familienunternehmen offen.

Die Ausführungen zu den strukturellen Faktoren, die einen Eintritt in das Familienunternehmen bedingen, unterstreichen das hohe Maß an biografischer Arbeit, das diesen Prozess begleitet. Unabhängig davon, welcher Weg nun beschritten wird, stellt die „Entscheidung“ für das Familienunternehmen eine biografische Herausforderung dar. Mit dem Narrativ der freien Wahl ist eine Strategie beschrieben worden, wie der Code zur Individualisierung mit der Kontinuität des Familienunternehmens in Einklang gebracht werden kann. Die ausscheidende Generation betont, dass das Familienunternehmen nur eine Option unter vielen sei. Die jüngere Generation präsentiert die Verbindung aus Unternehmen und Familie als einen spezifischen Lebensstil, für den sie sich bewusst entschieden habe.

Schließlich wird die These vertreten, dass in dem permanenten und alltäglichen Wechselspiel aus Handlungs- und Deutungsmustern das Narrativ einer ,freien Entscheidung“ des faktischen Erlebens bedarf, um eine nachhaltige Identitätsbildung zu ermöglichen.

Als Randnotiz sei erwähnt, dass ein solches, doppelseitiges Verständnis einer „freien Entscheidung“ für oder wider einen Weg in das Familienunternehmen Konsequenzen für zukünftige Untersuchungen von Nachfolgemotiven nach sich zieht: Die direkte Frage „Warum haben Sie sich für eine Nachfolge entschieden?" lässt Antworten auf einer narrativen Ebene der Selbstdarstellung erwarten. Um hingegen zu den strukturellen Faktoren vorzudringen, scheint es notwendig, darüber hinaus die eingeschlagenen Lebenswege $\mathrm{zu}$ analysieren.

\subsection{Familiales Handeln in Unternehmerfamilien}

Neben dem beruflichen Lebensweg markiert die eigene Haushalts- und Familiengründung den zweiten wesentlichen Strang im Übergang zum Erwachsenenalter. Interessanterweise wird dieser zentrale Lebensbereich weit weniger ausführlich von den Befragten thematisiert als ihre beruflichen Entscheidungen. Die Wahl des Lebenspartners, der Kinderwunsch und seine Umsetzung sind Themen, die offensichtlich hinter einer Grenze der „Privatheit" liegen und nicht notwendigerweise Bestandteil der Selbstpräsentation als Familien- 
unternehmer sind. Erst im Zuge von immanenten und externen Nachfragen gehen die Interviewpartner näher auf die Umstände des Kennenlernens oder ihren Familienalltag ein. Trotzdem bestätigt sich auch hier die These, dass es sich bei der Entscheidung für oder wider das Familienunternehmen um eine Entscheidung für oder wider ein spezifisches Lebensmodell, nämlich jenes als Unternehmerfamilie handelt. Mitglieder aus Unternehmerfamilien entwickeln eine ideale Vorstellung davon, wie in ihrem Leben Familie und Unternehmen verbunden sein sollten, und richten ihr familiales Handeln daran aus.

\subsubsection{Paarbildung}

Die Partnerwahl unterliegt einem starken kulturellen Wandel und kann als Ausdruck der Individualisierung verstanden werden. In vorindustriellen Zeiten wurden vor allem in Adelsfamilien und dort, wo Besitz und Vermögen zu vererben waren, Ehen arrangiert. Die Auswahl des Partners oder der Partnerin $^{88}$ oblag also der Familie und beinhaltete Verhandlungen zwischen den Herkunftsfamilien über Besitz- und Erbschaftsverhältnisse ebenso wie über eine Mitgift (Nave-Herz 2006:120). Ein anderer Teil der Ober- und Mittelschichten trat in das Kloster oder kirchliche Ämter ein. Darüber hinaus waren insbesondere in den unteren Gesellschaftsschichten rigide Heiratsverbote in Kraft. Die typische Verknüpfung der Ehe mit der Gründung eines ökonomisch selbständigen Haushaltes führt zu einem relativ späten Heiratsalter und einem hohen Anteil an Ledigen in der Gesellschaft (Sackmann 2007:161). Dieses als European Marriage Pattern bekannte Muster löst sich im Laufe des 19. Jahrhunderts auf und wird durch das bürgerliche Ideal der Liebesehe abgelöst. ${ }^{89}$

Die Vorstellung einer unbeschränkten, völlig freien Partnerwahl, deren einziger anerkannter Grund für eine langfristige Bindung an einen Partner in der romantischen Liebe zu sehen ist, hat sich in unserem Kulturkreis durchgesetzt. Eine Ehe aus ökonomischen oder religiösen Gründen wird hingegen als traditionell oder nicht legitim eingestuft. Die Familienforschung zeigt jedoch, dass auch das freie Partnerwahl-Modell bestimmten „Zwängen“ folgt, die allerdings weit weniger offensichtlich und den Betroffenen häufig nicht bewusst sind (Nave-Herz 2006:131). Einerseits bestimmen internalisierte soziale Normen die Wahl des Partners (beispielsweise hinsichtlich Altersdifferenz oder Größe). Andererseits bestimmen die Verfügbarkeit von Personen auf dem „Heiratsmarkt“ und die Möglichkeiten sowie Wege des Kennenler-

88 Im Folgenden wird die kürzere, maskuline Form des „Partners“ verwandt, dies schließt jedoch ausdrücklich beide Geschlechter ein.

89 Für weiterführende Literatur zur Partnerwahl in historischer Perspektive siehe etwa Möhle 2001. 
nens in erheblichem Maße, wer mit wem eine dauerhafte Beziehung eingeht.${ }^{90}$ Die empirische Forschung spricht dabei Freunden und Bekannten als Vermittler und Ratgeber eine erhebliche Rolle zu, während der Einfluss der Verwandten nur noch eine nachrangige Bedeutung hat (Klein und Lengerer 2001:283). Wie Nave-Herz zusammenfasst, verspricht die romantische Liebe dem Einzelnen die Freiheit, die Chance und die Legitimation, über seine sozialen Grenzen hinweg eine private Beziehung zu Personen anderer sozialer Zugehörigkeit einzugehen, entpuppt sich aber - unter soziologischen Gesichtspunkten - als neues Regulierungsinstrument zur Stabilisierung der gesellschaftlichen Schichten (Nave-Herz 2006:131).

Die Partnerwahl ist empirisch betrachtet als ein mehrstufiger Entscheidungsprozess zu interpretieren, der jederzeit abgebrochen werden kann und bei dem am Anfang keineswegs sicher ist, wie lange die Beziehung anhalten wird. (Hill und Kopp 2001:28) Der Kontaktaufnahme folgt das erste Treffen, dann die Wiederverabredung und so weiter. Die Partnerwahl wird als erste Stufe der Paarbildung verstanden (und in diesen Begriff integriert siehe etwa Sackmann 2007:160ff. oder Burkart 2008:175). Nach dem Kennenlernen sind nach vorliegenden Erkenntnissen die Faktoren ,wahrgenommene“ und „,vermutete“ Ähnlichkeiten von Bedeutung (Nave-Herz 2006:134). Diese bewirken eine Idealisierung des Partners und eine verklärte Betrachtung der Beziehung sowie das Gefühl ,zusammenzupassen“. Schließlich setzt ein Angleichungsprozess ein, der von der Fähigkeit zur Kommunikation, zur Empathie und zur Anpassung bestimmt wird (Nave-Herz 2006:134). Die Partner investieren in ihre Beziehung und gemeinsame Projekte, sie bilden eine geteilte Lebensvorstellung aus. Durch die zunehmende emotionale und materielle Verstrickung wird das Gefühl, in eine Beziehung eingebunden zu sein, ebenso wie der gegenseitige Verpflichtungscharakter stärker (Hill und Kopp 2001:30).

In seinen historischen Untersuchungen konnte Jürgen Kocka zeigen (siehe Kapitel III), dass bereits in der industriellen Unternehmerfamilie das Ideal der Liebesehe weit verbreitet war. Für die Unternehmerfamilien der Gegenwart ist die freie Partnerwahl zu einer Selbstverständlichkeit geworden. Aus dem Interviewmaterial kann darüber hinaus die These entwickelt werden, dass durch das Aufwachsen mit einem Unternehmen und/oder der beruflichen Orientierung am Unternehmertum die Suche nach einem potenziellen Lebenspartner um eine wesentliche Voraussetzung erweitert wird. Es geht nicht nur um die Attraktivität einer Person, ihr Alter oder ihre Bildung. Gesucht werden eine Passung in der Vorstellung vom eigenen Leben und die

90 Zur Erklärung, wer mit wem eine Beziehung eingeht, existiert eine große Zahl an Partnerwahltheorien (mit einem Schwerpunkt auf Austauschtheorien) und daran anknüpfenden Stufenmodellen über die Entwicklung von Partnerschaften. Für einen Überblick siehe etwa Hill und Kopp 2006 oder Nave-Herz 2006. 
darin eingeschlossene Verbindung von Familie und Unternehmen. Oscar Vester beispielsweise wünscht sich: „dass sich das ein oder andere dann auch privat .. mal ergibt, wo dann auch, aber muss schon irgendwo jemand sein, der auch mit einsteigen kann dann irgendwann." Diese Lebensvorstellung beinhaltet nun einen spezifischen Grad an Durchlässigkeit zwischen dem privaten, familialen Bereich und der beruflichen Verstrickung der Familienmitglieder im Unternehmen. Dies kann sowohl bedeuten, dass der Wunsch entwickelt wird, der zukünftige Partner solle sich aktiv am Geschehen im Unternehmen beteiligen und selbst aus einer Unternehmerfamilie stammen, oder aber auch, dass der zukünftige Partner einen entsprechenden Ausgleich zum Betrieb bieten und einen anderen familialen Hintergrund mitbringen solle. Gerade der Wunsch nach einem Partner mit einem ähnlichen Erfahrungshintergrund, so lässt sich angesichts der geringen Anzahl an Unternehmerfamilien vermuten, begrenzt die Auswahl an potenziellen Partnern erheblich - und sie wird noch geringer in solchen Branchen, in denen das Unternehmen den Rhythmus des Alltages wesentlich bestimmt, wie etwa in der Gastronomie oder Landwirtschaft. In jedem Fall geht es um eine $\mathrm{Zu}$ kunftsvorstellung über die eigene Lebensführung, die sich aus den eigenen Erfahrungen sowie Idealvorstellungen vom Unternehmersein speist und die eine aktive Konstruktion der Grenze zwischen Arbeiten und Leben, zwischen Unternehmen und Familie beinhaltet. Der Begriff der „Grenzarbeit", der in der systemischen Forschung zu Familienunternehmen entwickelt wurde, ist in diesem Zusammenhang, wenn auch seinem ursprünglichen Kontext entfremdet, tragfähig.

Erwähnenswert scheint zudem die Bedeutung der Eltern bei der Partnerwahl von Mitgliedern in Unternehmerfamilien, die sich für einen Weg in das Familienunternehmen entschieden haben. Zwar suchen die Eltern nicht den Partner für ihren Nachfolger aus, aber sie spielen in mehrerer Hinsicht eine gewichtige Rolle. Erstens, die von den Eltern vorgelebte Verbindung von Unternehmen und Familie kann ein wesentlicher Fluchtpunkt in der eigenen Vision der Lebensführung sein. Zweitens, die Heranwachsenden nehmen die Wünsche und Vorstellungen der Eltern bewusst wahr und versuchen einen Partner zu finden, der diesen entspricht - oder gerade nicht. Drittens, die Eltern sind als Übergeber des Unternehmens, die vielleicht noch aktiv im operativen Geschäft beteiligt sind oder nahe an dem Unternehmen leben, ein wesentlicher Teil der Lebensführung, und deshalb wird ein positives Verhältnis zwischen den Eltern und dem zukünftigen Partner angestrebt.

Zusammenfassend wird hier die These vertreten, dass die an sich freie Partnerwahl in Unternehmerfamilien neben den üblichen Selektionskriterien über eine spezifische Vorstellung der Grenze zwischen Familie und Unternehmen sowie die aktive Rolle der Eltern in der Lebensführung von Nachfolgern zusätzlich begrenzt und erschwert wird. Dadurch erfolgt die Paarbildung bei Mitgliedern aus Unternehmerfamilien nicht unbedingt strategischer 
oder bewusster als bei anderen Personen, sie wird lediglich voraussetzungsreicher.

Im Hinblick auf eine familieninterne Nachfolge und damit auf die Reproduktion der Unternehmerfamilien wird die Partnerwahl zu einem Risikofaktor, wie der Fall Naumann verdeutlicht:

Nach dem Tod seines Vaters übernimmt Felix Walter Naumann mit tatkräftiger Unterstützung seiner Frau die Führung des in Familienbesitz befindlichen Weingutes im Nebenerwerb. Hauptberuflich arbeitet er weiterhin in der gleichen Branche als abhängig Beschäftigter im öffentlichen Dienst. Seine Söhne Wilhelm Fritz und Friedrich Carsten wachsen als Nachfolger für den Betrieb auf, ihre Nachfolgeversuche scheitern allerdings an der Vorstellung des Vaters, nach seiner Pensionierung den Traum vom Unternehmer auszuleben. Weder Wilhelm Fritz noch Friedrich Carsten sind gewillt, so lange auf die ,Thronfolge“ zu warten, und gehen ihre eigenen Wege. Der Ältere eröffnet eine Anwaltskanzlei in der Großstadt und heiratet noch dazu eine Frau, die sich ein Leben in räumlicher Dichte mit den Schwiegereltern nicht vorstellen kann. Felix Walter Naumann sagt über seine Schwiegertochter: „für ein Weingut gehört eine tüchtige Frau, die diesen Job macht. Andere Frauen haben andere Vorzüge, also (lacht) äh wir haben immer gesagt äh .. diese Schwiegertochter ist für hier, für den Betrieb eigentlich nicht, nicht geboren, kann sie ja nichts dazu, liebenswerter Mensch." (11:5-8) Der Jüngere heiratet eine Landwirtin adeliger Abstammung, und die beiden betreiben gemeinsam eine Landwirtschaft in Ostdeutschland.

Die beiden jüngeren Kinder der Familie, Ullmann und Claudia, werden lange Zeit nicht als Nachfolger für das Weingut gehandelt. Ullmann entscheidet sich schließlich für ein kinderloses Leben in der Großstadt und scheint damit ganz aus dem Rennen. „So, jetzt kommt der Clou, jetzt haben wir die Tochter vergessen." (Felix Walter Naumann, 12:1-2) Claudia hingegen, ihres Zeichens in der Verlagsbranche tätig, heiratet Conrad, der selbst in einer Brauereifamilie groß geworden ist. Er ist es, der Interesse am Weingut und der Lebensart der Familie äußert und letztlich Claudia den Anstoß dazu gibt, eine Nachfolge anzustreben. Mit diesem Mann als ,Trumpf im Ärmel' qualifiziert sich nun Claudia auch als Frau für die Position der Nachfolgerin. Ihr Vater sagt:

„Und die junge Frau hat sich also doch dann bereit erklärt .. die Last und die Lust, ich wiederhole es noch mal, die Last und die Lust .. dieses Betriebes zu übernehmen, .. hat Gott sei Dank auch einen Mann geheiratet, der dazu passt, der zwar, der kommt aus einer Brauerei-Familie, spielt aber *keine Rolle* (lachend) (lacht), aber der sage mal, die Kultur mitbringt, die ein solcher Betrieb auch braucht.“ (12:9-14). 


\subsubsection{Der Übergang in die Elternschaft}

Der interkulturell vergleichende „Value-of-Children“-Ansatz (u.a. Nauck 2001; Nauck und Trommsdorff 2010; Nauck 2011) versucht, ausgehend von austauschtheoretischen und familienökonomischen Prämissen das weltweit unterschiedliche, generative Verhalten von Eltern (also ob und wie viele Kinder eine Familie bekommt) mittels der Nutzenerwartungen, die Eltern an ihre Kinder stellen, zu erklären (Nave-Herz 2006:193). Dabei wird in drei Dimensionen unterschieden: Eltern erwarten von ihren Kindern einen ökonomisch-utilitaristischen Nutzen etwa in Form von frühen Beiträgen der Kinder zum Familienhaushalt durch Mithilfe oder Kinderarbeit sowie Hilfe und materielle Unterstützung im Alter; ein psychologischer Nutzen kann aus der Stärkung der innerfamilialen Beziehung oder der Freude, die Kinder aufwachsen zu sehen, entstehen; und schließlich erwarten sich Eltern durch Kinder einen Statusgewinn, Kompetenz in der Elternrolle sowie die Weiterführung des Familiennamens, also einen sozial-normativen Nutzen. Die Erhebungen im Rahmen der „Value-of-Children-Studies“ zeigen, dass sich mit dem zunehmenden technischen und industriellen Entwicklungsgrad eines Landes der Wert von Kindern für ihre Eltern verschiebt. In der Haushaltsökonomie besitzen Kinder einen ökonomischen Wert für die Eltern als Arbeitskräfte und sichern die Altersversorgung der Eltern. In modernen, wohlfahrtsstaatlichen Gesellschaften werden diese Werte unwichtiger, dafür gewinnt die psychologische, emotionale Nutzendimension an Bedeutung: Kinder stellen eine emotionale Bereicherung des Lebens der Eltern dar, sie sind Sinnstifter und Glücksbringer. Während bei einem primär ökonomischen Nutzen von Kindern mit einer steigenden Kinderzahl auch der Nutzen steigt, wird bei einem emotionalen Nutzen der Kinder bereits mit wenigen Kindern das Nutzenmaximum erreicht, das danach nicht mehr steigt (Sackmann 2007:171). Bevölkerungswissenschaftler sind sich einig, dass der Rückgang der Geburten in Europa sowohl im internationalen Vergleich als auch im Zuge des historischen Modernisierungsprozesses von diesem Funktionswandel von Kindern in der Familie und durch eine Veränderung des Selbstverständnisses der Eltern ausgelöst wurde (Nave-Herz 2007:33). Als einen wesentlichen Faktor für die Entfaltung des Funktionswandels identifizieren die Forscher ein zuverlässiges Versicherungssystem gegen Lebensrisiken und für das Alter (Nave-Herz 2006:193). Insofern hat die von Kohli beschriebene Institutionalisierung des Lebenslaufes einen wesentlichen Anteil an der Verschiebung des Wertes von Kindern (Sackmann 2007:172).

Der „Value-of-Children“-Ansatz liefert eine plausible, makrosoziologische Erklärung für die Familiengründung und -erweiterung einer Gesellschaft, wird aber erst durch eine Ergänzung um eine lebenslauftheoretische Position auch auf einer individuellen Ebene aussagekräftig. Wenn es darum 
geht, die Entscheidung zur Familiengründung zu erklären, so scheint dem Zeitpunkt im Leben sowie rivalisierenden Wertevorstellungen große Bedeutung zuzukommen (Burkart 2008:221). Kann die Familiengründung „geplant" werden, so warten Paare meist das Ende der Ausbildungsphase sowie das Erreichen einer ökonomischen Unabhängigkeit von der Herkunftsfamilie ab. Die erwartete emotionale Bereicherung der Beziehung durch ein Kind sowie Erwägungen zur Vereinbarkeit von Familie und Beruf scheinen schließlich ausschlaggebend zu sein. Die Entscheidung für oder gegen ein zweites Kind ist aufgrund der nun überschaubaren Folgen einfacher zu fällen (Sackmann 2007:173). Die Ursachen für das in Deutschland immer noch dominante Zwei-Kind-Ideal werden darin gesehen, dass zum einen der Wunsch nach einem Spielgefährten für das erste Kind und zum anderen nach einer Verlängerung der Elternzeit besteht (Dornseiff und Sackmann 2003). Hinsichtlich der Geburt dritter Kinder weisen empirische Untersuchungen einen Einkommenseffekt derart nach, dass in reicheren Familien häufiger dritte Kinder anzutreffen sind (Sackmann 2007:175).

Wendet man diese Erkenntnisse auf Unternehmerfamilien an, lassen sich einige theoretische Erwartungen ableiten: Aufgrund der charakteristischen Verbindung von Familie und Unternehmen lässt sich vermuten, dass neben der emotionalen Dimension Kinder in Unternehmerfamilien stärker unter ökonomisch-utilitaristischen und sozial-normativen Nutzenerwartungen betrachtet werden. Selbst wenn sich die Vorstellung einer behüteten Kindheit, wie oben gezeigt, weitgehend durchgesetzt hat, stellen die erwachsenen Kinder potentielle Arbeitskräfte und vor allem Nachfolger für das Unternehmen dar. Durch sie kann die Tradition erhalten, der Name fortgeführt und die Kontinuität des Familienprojektes gesichert werden. Eltern könnten erwarten, dass sich ihr solidarisches Bündnis mit den Kindern auch auf den Bereich des Unternehmens erstreckt: Sie stehen in Notsituationen oder in Krankheitsfällen als Arbeitskräfte und Denkmalpfleger zur Verfügung. Darüber hinaus könnte ein Versicherungsnutzen durch die Kinder in Unternehmerfamilien noch präsent sein, denn als Selbständige können Unternehmer für ihre Lebensrisiken und das Alter privat vorsorgen und sind nicht automatisch Bestandteil des wohlfahrtsstaatlichen Sicherungssystems. Geht man von diesen Nutzenerwartungen aus, so lässt sich vermuten, dass Unternehmer in langfristigen Beziehungen seltener kinderlos bleiben. Des Weiteren lässt sich die These formulieren, dass die Kinderzahl in diesen Familien eigener Art höher ist als in anderen Familien, gleichwohl mit einer zunehmenden Kinderzahl das Risiko von Konflikten oder einer Erbteilung steigt.

In seiner Arbeit über den Zusammenhalt der Unternehmerfamilie greift Kormann (Kormann 2011:83ff.) einige dieser Gedanken auf, ohne jedoch einen direkten Bezug zur „Value-of-Children“-Forschung herzustellen. Er betont, dass die Familiengründung ein wichtigeres Fortbestandsmerkmal als die Institution der Ehe darstellt. Unter dem Abschnitt „Verantwortung für die 
Generationenfolge" führt er aus, wie sich der Wunsch nach Nachkommen insbesondere bei Gründer-Unternehmern mit dem Wunsch nach einem Nachfolger verbinde. Wenn eine Partnerschaft kinderlos bleibt, so Kormann, sei häufig zu beobachten, dass ein Unternehmer ein weiteres Mal heirate. Wenn eindeutig keine leiblichen Abkömmlinge zu erwarten seien, blieben noch die Alternative einer Adoption oder aber die Einbeziehung von anderen Verwandten (Neffen, Nichten). Mit Kormann ließe sich also die These einer relativ hohen Kinderzahl in Unternehmerfamilien unterstreichen. Mehr noch, Kormann erhebt die Mehr-Kind-Familie zu einem normativen Ideal der Unternehmerfamilie, das es mit pragmatischen Mitteln zu unterstützen gilt: „Da es im Interesse der Institution Familienunternehmen liegt, dass es eine Gesellschafterfamilie gibt, stellt sich die Frage, auf welche adäquate Weise das Unternehmen den Wunsch nach Kindern so gut wie möglich unterstützen kann. Dies kann zunächst wohl nur durch finanzielle Unterstützung geschehen." (Kormann 2011:84)

Vor diesem Hintergrund sind die Ergebnisse der hier vorliegenden Studie umso überraschender. Keines der befragten Mitglieder aus Unternehmerfamilien rekurriert in seinen Erzählungen auf ökonomische oder sozialnormative Gründe für die Entscheidung ein erstes, zweites oder ein weiteres Kind zu bekommen. In den Selbstdarstellungen der Unternehmer ist also keineswegs eine strategische Nachkommenplanung zu entdecken, wie dies die Ausführungen Kormanns oder ein Rekurs auf die „Value-of-Children“Forschung vermuten ließen. Im Gegenteil, die Familiengründung wird als ein Ausdruck und Ergebnis der Paarbeziehung verstanden und nicht in Bezug zum Unternehmen und der Nachfolgefrage gestellt. Zumindest auf einer narrativen Ebene scheint das Unternehmen die Erwägungen zur Reproduktion weit weniger zu beeinflussen als die Partnerwahl und die Paarbildung. Wie lässt sich dieser Befund einordnen?

- Eine Interpretationsmöglichkeit wäre die Tabuisierung dieses Themenkomplexes. Unternehmerfamilien vermeiden es, ihre Nutzenerwartungen an ihre Kinder jenseits der emotionalen Dimension zu thematisieren, um nicht in Abweichung zu dem gesellschaftlich anerkannten Rechtfertigungsmuster unter Legitimationsdruck zu geraten.

- In einer zweiten Lesart könnten Unternehmerfamilien als ein Milieu begriffen werden, in dem Elternschaft noch weitgehend eine natürliche Selbstverständlichkeit ist und nicht das Ergebnis eines Entscheidungsprozesses, der ein Abwägen von Argumenten dafür und dagegen einschließt.

- Eine weitere Lesart ist darin zu sehen, dass die jungen Familienunternehmer selbst tatsächlich keine Verbindung zwischen der Familiengründung und einer zukünftigen Nachfolge herstel- 
len, da sie selbst biografisch noch zu sehr in die eigene Nachfolgegeschichte verstrickt sind.

Aus den Erzählungen zu der frühen Familienphase lassen sich einige Merkmale herausarbeiten, die die dritte Lesart stützen: Einerseits befinden die Betroffenen sich im Übergang zum Erwachsenenalter und haben schon einige „transition markers“ umgesetzt: Die Ausbildungsphase ist abgeschlossen, erste berufliche Erfahrungen gesammelt, ein eigener Haushalt ist gegründet, und sie stehen in einer festen Partnerschaft. Trotzdem sind sie im Hinblick auf den Weg ins Unternehmen in ihrer Rolle als „Kind“ und nachfolgende Generation mit den „Eltern“ als übergebende Generation stark eingebunden. Sie sind vielleicht selbst gerade erst Nachfolger geworden oder spielen mit dem Gedanken, die Nachfolge anzutreten - da liegt eine Übergabe an die eigenen Kinder noch in weiter Ferne. Sobald sich ein Kind in der jungen Unternehmerfamilie ankündigt, wird der abstrakt vorhandene Wunsch nach der Kontinuität des Familienprojektes lauter. Die jungen Eltern sehen sich erstmals selbst in der Position der „Alten“, der „Übergebenden“, und beginnen, ihre Kinder in den Horizont der Unternehmerfamilie einzubeziehen. In den Familien Treuninger oder Clemens symbolisiert sich dieses wachsende Bewusstsein in der Namensgebung der Kinder, mit der sie teilweise direkt an die historische Familiengemeinschaft anschließen. Im Fall Anabella Singermayr und Claudia Nentzik geben die eigenen Kinder einen wesentlichen Anstoß, sich eine Nachfolge überhaupt vorstellen zu können. Claudia Nentzik sagt: ,also mit Sicherheit, wenn ich keine Kinder gehabt hätte, hätte ich es, ähm, von daher war das Kinder kriegen sicherlich auch nochmal ne Weichenstellung dafür, dass man gesagt hat man kann sich so nen Weingut und so ne Tradition eben automatisch besser vorstellen." (56:30-32) Insofern öffnen die Kinder den biografischen Horizont der Eltern, dieser schlägt allerdings nicht unbedingt in eine strategische Nachkommensplanung um, denn auch bei der Entscheidung für ein zweites oder späteres Kind wird nicht im Sinne eines größtmöglichen Nachfolgepools argumentiert.

Aus dem Interviewmaterial und den Lebensläufen der Mitglieder aus den Unternehmerfamilien ergeben sich darüber hinaus zwei zugegebenermaßen sehr subjektive und nicht verallgemeinerbare Eindrücke: Erstens scheint häufig eine Parallelität von einschneidenden Ereignissen in Familie und Unternehmen zu bestehen, etwa eine Hochzeit im gleichen Jahr wie der Unternehmenseinstritt. ${ }^{91}$ Zweitens, in Unternehmerfamilien, die sich für Kinder entscheiden, sind Mehrkindfamilien häufig vertreten. Mit Blick auf die konkreten Fälle scheint als Erklärung hierfür ein multi-kausaler Ansatz aus religiö-

91 Gleiches bemerkt auch Dunn 1999, verheiratete Murray 2003, in ihren qualitativen Studien: „The holistic approach in this research found that nodal family life-cycle events (significant birthdays, marriages, deaths, when to start a family, serious illness) almost always coincide with and had a profound affect on transition activity in the business." Murray 2003:31. 
sen Motiven, finanzieller Ausstattung, Unterstützung durch Verwandte und der langfristigen Möglichkeit zum Wiedereinstieg im Unternehmen plausibel.

Es ist das Ergebnis dieser Analyse, dass die Vorstellung einer ökonomisch oder sozial-normativ motivierten Elternschaft in Unternehmerfamilien wie diese etwa durch Kormann (2011) vertreten wird, der sich im übrigen lediglich auf selektive historische Beispielfälle und Medienberichterstattung stützt, als in der Forschung zu Familienunternehmen gängiges Stereotyp angezweifelt werden kann. Zur Klärung, ob sich Unternehmerfamilien in ihrem reproduktiven Verhalten von anderen Familien unterscheiden, wäre eine quantitative Untersuchung sehr wünschenswert, die der Verkettung von Lebenslaufereignissen sowie den unterschiedlichen Familienformen Beachtung schenkt.

\subsubsection{Familienalltag}

In dem Abschnitt zur Partnerwahl habe ich herausgearbeitet, dass die Vorstellung vom Leben mit dem Unternehmen, die ausdrücklich auch die Bereiche Freizeit und Familie einschließt, einen wesentlichen Faktor in dem Paarbildungsprozess von Familienunternehmern darstellt. Mit der Gründung einer eigenen Familie gilt es nun, aktive Grenzarbeit zwischen den beiden Sphären zu praktizieren. Nicht nur in der Paarbeziehung, sondern auch im Umgang mit den eigenen Kindern müssen Familienunternehmer immer wieder aufs Neue bestimmen, wie stark das Unternehmen den Familienalltag beeinflusst. Im Folgenden wird zunächst ausführlich die alltägliche Lebensführung der Familie Treuninger beschrieben. Dieser Fall illustriert nicht nur, wie die getroffenen Tätigkeitsarrangements als Produkt eines permanenten Aushandlungsprozesses verstanden werden können, sondern sich die Grenzen zwischen Unternehmen und Familie über die Zeit hinweg verschieben. Im Anschluss wird eine Typologie zur aktiven Grenzarbeit in Unternehmerfamilien entwickelt und abschließend die Bedeutsamkeit des Familienalltags für den Lebenslauf der Familienunternehmer eingeordnet.

\subsubsection{Aktive Grenzarbeit in der Unternehmerfamilie Treuninger}

Marcella studiert auf Wunsch ihres Vaters Medizin. In ihrer Studienzeit entwickelt sie den Wunsch, einen Mann zu heiraten, der selbst kein Arzt ist, und eine Familie mit vier Kindern zu gründen. Sie sehnt sich nach festen Strukturen, einem örtlichen Mittelpunkt in ihrem Leben und Geborgenheit ,weil das fühlte ich .. vielleicht ein bisschen zu kurz gekommen in unserem Wanderleben“ (12:8-9). In ihrer biografischen Selbstsicht drückt sich eine Grundstimmung innerhalb der Herkunftsfamilie aus, die nach einer intensiven Phase mit vielen Umzügen, selbst in einer relativ stabilen Zeit an einem Ort im- 
mer noch Bestand hat. Marcellas Wunsch impliziert zugleich eine Kritik an ihren Eltern, zu denen sie in dieser Zeit in einem spannungsreichen Verhältnis steht: Sie beginnt eine spezifische Vorstellung von „Familie“ zu entwickeln, der ihre Eltern nicht mehr gerecht werden können. Insofern ist dieser Wunsch nicht nur Ausdruck eines tief empfundenen Bedürfnisses nach Halt und Heimat, sondern auch einer Rebellion gegen die Eltern. Sie beginnt, sich emotional von ihren Eltern zu lösen und einen eigenen Lebensentwurf zu entwickeln.

In dieser Zeit stolpert Marcella über eine Anzeige in der FAZ, an deren Überschrift und Inhalt sie sich noch sehr gut erinnert: „Warten auf den Zufall, wir könnten alt dabei werden“" (10:15-16); mit ihren 24 Jahren fühlt sich Marcella von dieser Anzeige angesprochen, was die Dringlichkeit ihres Wunsches unterstreicht. Der Rest der Anzeige ist so formuliert, dass eigentlich Väter oder aber Frauen in ihrer Funktion als Tochter angesprochen werden. „Und äh das hat mich irgendwie gereizt“ (10:21) Die Anzeige trifft ihr Erleben zu diesem Zeitpunk. Sie entschließt sich, kritisch auf diese Anzeige $\mathrm{zu}$ antworten und trägt mit einem Unbekannten ihren Autonomisierungskampf aus, den sie mit dem Vater selbst nicht eingeht. Der Unbekannte antwortet ihr und sie treffen sich. Sie ist eine von fünf oder sechs Damen, die er besucht.

Die Begegnung mit Marcella war für Michael Treuninger, der schon früh das Familienunternehmen übernommen hat, abenteuerlich, aufregend und amüsant, eben „total anders“ (Michael Treuninger, 30:15). Die Frau aus einem anderen Kulturkreis mit einer hohen Bildung, die nicht aus einem Unternehmermilieu stammt, hatte, so Michael, ,mit der, einem schwäbischen Unternehmen auch *nichts* (Mundart) am Hut gehabt. Ist schon klar, aber (80:00) das hat sich, ...“(30:17-19) geändert. Michael folgt Marcellas Einladung, sie auf einer mehrwöchigen Reise mit ihren Freunden zu besuchen. Die Reise markiert für beide den Beginn der Beziehung. „So haben wir uns dann eigentlich kennen gelernt" (Marcella Treuninger, 11:6).

Nach zwei Jahren heiraten die beiden. Während des Interviews betont Marcella mehrmals den Altersunterschied von zehn Jahren, der in ihrer Wahrnehmung von Michael eine große Rolle zu spielen scheint. Er gehört einer anderen Generation und einer anderen Erfahrungswelt an. Trotzdem ist beiden das Bedürfnis gemein, sich im Leben zu verankern und eine große Familie zu gründen.

Mit der Heirat zieht Marcella in die Fabrikwohnung ein, in der schon Michaels Großeltern lebten und die nach dem Krieg nur notdürftig renoviert wurde. Marcella beschreibt die Lebensumstände ohne großen Enthusiasmus, die Wohnung sei einfach, kahl und vorläufig (gewesen). Doch zu dieser Zeit gibt Marcella wenig auf solch „äußere Dinge“ (18:11). „Mein Mann hatte mir erklärt, er ist in diesem Haus geboren, er wird in dem Haus sterben .. und .. dann habe ich mir gedacht, ihm aber damals nicht gesagt, und ich werde 
hier nicht sterben. (lacht)“ (15:1-2) und später ,und mein Mann hing halt dran,... insofern kam eine Alternative sowieso nicht in Frage“ (18:24-25). Offensichtlich haben Michael und Marcella andere Vorstellungen von ihrem Leben und der Nähe zum Unternehmen. Doch Marcella hat Verständnis für die Bindung ihres Mannes an diese Räumlichkeiten und folgt seinem Willen. Und schließlich ist die Lage der Wohnung „erstmal praktisch“ (18:15), denn beide können zu Fuß zu ihrer Arbeit im Krankhaus bzw. im Unternehmen gehen.

Nach der Geburt des ersten Sohnes beginnt Marcella nach acht Wochen Baby-Pause wieder zu arbeiten. Sie praktiziert eine moderne Mutterrolle ganz nach dem Vorbild ihrer eigenen Mutter. Die Versorgung des Kindes übernimmt Marcellas Schwiegermutter. Über das Verhältnis von Marcella zu Michaels Mutter ist wenig bekannt. Scheinbar hat diese im gleichen Haushalt wie das junge Paar gelebt und könnte eine Belastung für die Beziehung bedeutet haben. Marcella erkennt, dass Adam ein großer Wunsch der Schwiegermutter ist, die sich mit Freude um den Kronprinzen der Familie kümmert. Möglicherweise distanziert sich Marcella deshalb ein wenig von ihrem eigenen Sohn. Sie könnte ihn eher als einen Teil von Michaels Familie als ihrer eigenen empfunden haben. Diese Distanz drückt sich auch in Marcellas Sprache aus, wenn sie von „dem Baby“ und „dem Kind“ (18:33) statt von ihrem Sohn oder Adam spricht. Vielleicht versucht sie damit auch die Situation zu versachlichen, da es ihr emotional nicht leicht fällt, dieses Wunschkind der Obhut ihrer Schwiegermutter zu überlassen.

Marcella erzählt eine interessante Passage: „als unser Ältester im Kinderwagen lag und ich mit dem spazieren ging, dann .. hat man also reingeguckt in den Kinderwagen: „Ach, ist ja ein *Junge*“ (Mundart) und „Ach, wie nett", und „Der wird doch später sicher mal Chef werden!“ (lacht) Das hat mich so, also ein bisschen .. gelöchert. Ich dachte, das ist so *dämlich* (lachend). (lacht)"(16:12-17). Die Vorstellung der Delegation für einen Beruf findet Marcella befremdlich. Zum einen reflektiert sie ihre eigene Berufswahl in dieser Zeit kritisch. Sie erlebt es als problematisch, dass sie sich nicht selbst für ihren Weg entschieden hat. Zum anderen sieht sie als „Externe“ auch die negativen Seiten des „Unternehmerdaseins“. Sie kennt andere Lebensentwürfe und will ihrem Sohn diese - für Marcella ungewohnte - Nähe zum Unternehmen ersparen. Diese Einstellung Marcellas ist zudem Ausdruck ihres inneren Protestes. Sie sagt etwas später: „Und deswegen dachte ich, na, diesen einen von tausenden verschiedenen Berufen, den kann ich ja schon mal für ihn *ablehnen* (lachend). (lacht)“(16:25-26).

Die Arbeit als Stationsärztin im örtlichen Krankenhaus wird für Marcella zunehmend belastend. Schließlich gibt sie diese Arbeit auf und ,saß dann zu Hause“ (3:18). In dieser Redewendung klingt bereits die empfundene Langeweile, Nutzlosigkeit und auch ein wenig Resignation an. In dieser Zeit wird ihr die Nähe zum Unternehmen immer bewusster. Sie beschreibt, wie 
sie die Wäsche im Hof aufhängt und Adam dort spielt. Immer abends läutet eine große Glocke, und die Mitarbeiter gehen nach Hause. Anschließend kehrt Ruhe ein. Marcella nimmt zunehmend an dem Ablauf im Betrieb teil, sie nimmt die Post und Anrufe entgegen. Sie ist nur wenige Tage oder Wochen „nur“ Mutter und beginnt tagsüber das Büro zu besuchen. Das Unternehmen bietet ihr spannende Aufgaben und die Möglichkeit, ihrer Schwiegermutter ein wenig aus dem Weg zu gehen, die ,auch sehr hing an dem Kind" (18:33). Als die Buchhalterin zufällig krank wird, übernimmt Marcella ihre Aufgaben. „So dass ich das Unternehmen von Seiten dieses Skeletts, der sich verdichtet in der Buchführung... kennen lernte..." (3:33-34) Marcella studiert die Anatomie des Unternehmens und beginnt, dieses als einen krankenden Organismus zu begreifen. Sie sieht organisatorische und personelle Schwachpunkte und ,da habe ich halt zugegriffen, was ähm natürlich mit Gegenreaktionen verbunden war (lacht)“ (19:10). Marcella vergleicht sich mit einem Katalysator, der widrige Umstände ans Tageslicht befördert. Ihr ist bewusst, dass sie das bestehende Sozialgefüge stört. Marcella empfindet sich selbst „nicht immer als sozial verträglich“ (21:30) und versucht deshalb, im Hintergrund zu wirken. Doch es scheinen gerade diese Gegenreaktionen zu sein, die sie reizen.

In den folgenden Jahren arbeitet Marcella „intensiv“ (15:29) im Unternehmen mit. Das Paar bekommt im Abstand von jeweils drei Jahren zwei Töchter. Die Kinder spielen im Hof des Unternehmens, besuchen Mitarbeiter, verbringen Zeit bei ihr im Büro oder sind bei der Schwiegermutter. Marcella beschreibt diese Zeit als positiv, sie ist mittendrin. Auch Michael findet diesen Lebensentwurf attraktiv. Seine Kinder streifen gelegentlich durch den Betrieb und jeder kennt sie. Michael begrüßt es, ,wenn sie äh... da mal einen Blick drauf werfen, ... denn irgendwann braucht man ja mal einen Nachfolger und wenn die sich... das ansehen, ist schon gut. ..." (Michael Treuninger, (35:28-29) Doch die Wohnsituation wird zunehmend beklemmend. Das Unternehmen wächst, und der ehemals grüne Hof wird zu einem Parkplatz. Diese Beschreibung könnte zudem sinnbildlich für das ausufernde Unternehmen sein, dass immer mehr in die Privatsphäre der Familie eindringt. Marcella drängt darauf umzuziehen, doch Michael will bleiben. Sie finden als Kompromiss ein Ferienhaus im Grünen.

Mit der Einschulung Adams zieht die Familie schließlich in das Ferienhaus auf dem Land um. Das kleine Dorf, mit nicht einmal fünfzig Einwohnern, scheint Marcella die geeignete Umgebung für das Aufwachsen ihrer Kinder. Mit der Geburt der beiden Zwillinge konzentriert sich Marcella schließlich voll auf ihre Mutterrolle und ist nur noch sporadisch im Unternehmen tätig. In dieser katholischen Gegend fällt die Familie mit der großen Kinderzahl, es sind nun fünf, nicht weiter auf. Marcella lebt in dieser Zeit ihren Traum vom guten Leben auf dem Land und empfindet die Zeit mit ihren Kindern als sehr beglückend. Während sie bereits acht Wochen nach der Ge- 
burt des ersten Sohnes wieder zu arbeiten begann, spricht sie nun davon, dass ein Kind sehr von der Anwesenheit seiner Mutter profitiert. Marcella findet Gefallen an der Rolle der „traditionellen“ Mutter und Hausfrau. Erst als die beiden jüngsten in den Kindergarten kommen, beginnt Marcella wieder vormittags und gelegentlich nachmittags im Unternehmen tätig zu werden. Die räumliche Trennung zum Unternehmen lässt Michael zu Hause nicht zum bloßen Privatmann werden. Er kann die Tür zum Geschäft nicht hinter sich verschließen. Er erzählt der Familie, insbesondere den Kindern von seinen guten und schlechten Erlebnissen. „Und die nehmen auch heute Anteil, also wenn wir ... beisammen sind, dann ... berichte auch aus der Firma und erzähle was oder die fragen dann auch, also das geht locker. ... Also, es ist denen keineswegs egal, ... was die Eltern tun, ... sondern die nehmen da schon Anteil. ...“ (36:16-19) Michael trägt das Unternehmen via Erzählungen in die Familie und ist erfreut über das Interesse seiner Kinder an ihm und seiner Arbeit.

Nach etwa sieben Jahren werden die Lebensumstände in der Ferienwohnung jedoch wiederum zu eng für die heranwachsende Familie. Erneut verabschieden sie sich von diesem wohnlichen Provisorium und sie ziehen zurück in die Stadt, in der auch das Unternehmen ansässig ist. Hier werden die beiden Zwillinge eingeschult. Mit der räumlichen Nähe zum Unternehmen verstärkt sich erneut die Intensität, in der die Familie mit dem Betrieb in Berührung kommt. Wie schon bei ihrem ersten Arbeitsbeginn sucht sich Marcella gegen Widerstand einen neuen Arbeitsplatz in der Firma. „Und da gab es kein Angebot oder Arbeitsplatz für mich, wie mir auch bedeutet wurde sehr deutlich. (lacht)" (Marcella Treuninger, (4:21-24) Ganz im Sinne der schöpferischen Zerstörung bricht Marcella alte Strukturen von innen heraus auf, um Wandel einzuläuten. Sie führt neue Arbeitsmethoden ein, ,damit ein Bruch mit der Vergangenheit stattfinden konnte. (lacht)“ (5:15-16) In den verschiedenen Arbeitsbereichen, in denen sie tätig wird, führt sie Systembrüche herbei, bis sie selbst von den geschaffenen neuen Aufgaben überfordert ist, diese Stelle durch einen neuen Mitarbeiter besetzt und zu neuen Taten aufbricht.

Die Kinder nehmen die beruflichen Verstrickungen ihrer Eltern wahr und erleben ihr begrenztes Zeitkontingent. Marcella versucht ihr Engagement im Beruf mit den Kindern zu teilen. Während der Ferien verbringen die Kinder die meiste Zeit im Unternehmen. Insbesondere Adam zeigt großes Interesse an den Betriebsabläufen und Prozessen. Im Gegensatz zu dem Leben auf dem Land ist Marcella nun wieder zu ihrer alten Auffassung der Mutterrolle zurückgekehrt. Sie ist fast täglich im Unternehmen, und die Abwesenheit der Mutter verschafft den Kindern eine Reihe von Freiheiten, aber sie haben „teilweise auch darunter gelitten“ (22:28), was besonders für die ältere der beiden Töchter gilt. Den Sonntag zelebriert Michael als einen echten freien Tag. Alleine geht er morgens in die Kirche und einen Tag lang nicht in 
den Betrieb. Einmal im Jahr an Weihnachten unternimmt die Familie einen gemeinsamen Winterurlaub. Die Skiwanderungen der familialen Gemeinschaft sind für Michael eine „Quelle der Freude“ (Michael Treuninger, 33:9). Er teilt mit Begeisterung dieses Erlebnis mit seinen Kindern. Die Tatsache, dass seine Kinder diese Art des Familienurlaubs fortführen, erfüllt Michael mit Bestätigung. Er sagt: „Und siehe da, die Kinder haben das auch übernommen, obwohl es sehr mühsam ist, wenn man also rauflaufen muss ist ja nicht so schön als in den Lift reinsetzen, ... aber die tun es auch.“(33:6-10)

\subsubsection{Eine Typologie der aktiven Grenzarbeit}

Wie dieser Fall verdeutlicht, ist die gelebte Grenze zwischen Familie und Unternehmen das Ergebnis eines stetigen Verhandlungs- und Verarbeitungsprozesses von eigener Lebenserfahrung und den Wünschen des Partners. Durch das Familienunternehmen entsteht eine soziale und räumliche Nähe sowohl zum Betrieb als auch zu anderen Familienmitgliedern, die für alle Beteiligten ein erträgliches Maß einnehmen muss. Die Entscheidung für das Leben als Unternehmerfamilie ist auch an eine Entscheidung für einen bestimmten Wohnort gekoppelt. Die Grenze zwischen Familie und Unternehmen kann sowohl über die Zeit hinweg als auch von Familie zu Familie auf unterschiedliche Art und Weise gelebt und konstruiert werden.

Aus der Perspektive der subjektorientierten Soziologie (siehe Kapitel IV, etwa Voß 1991) erscheint die jeweils praktizierte Form der aktiven Grenzarbeit zwischen Unternehmen und Familie als unterschiedliche, aber je für sich relativ stabile Arrangements von Tätigkeiten. In Unternehmerfamilien finden sich also verschiedene Formen der alltäglichen Lebensführung, die ein harmonisches Ganzes bilden. Sie werden über Routinen, Gewohnheiten und gegenseitige Absprachen aufrechterhalten. Die Tiefenstruktur der jeweiligen Lebensführung bestimmt sich im Wesentlichen daraus, ob und wie die Grenze zwischen Unternehmen und Familie konstruiert wird.

Aus dem Interviewmaterial lassen sich die drei Typen „Verschmelzung“, „Festung“ und „Zwei Kerne“ kondensieren. Als Ausgangsidee für die Typenbildung diente der Vorschlag von Erdmann (1999), in eine Familien- vs. Unternehmensorientierung der Eltern zu differenzieren. Eng damit zusammen hängt die Frage, welchen Tätigkeiten im Alltag Priorität eingeräumt wird. Das Interviewmaterial schlägt hingegen drei idealtypische Gruppen vor, wie die aktive Grenzarbeit erfolgt. Im weiteren Verlauf der Typenbildung dienten deshalb die Ergebnisse von Sue Birley et al. zur Einstellung von Eigentümerunternehmern als wesentlicher Orientierungspunkt. In einer Pilotstudie haben Sue Birley, Dennis Ng und Andrew Godfrey (1999) in einer nicht-repräsentativen, quantitativen Befragung Eigentümerunternehmern in Großbritannien knapp 50 Aussagen über das Leben mit einem Familienun- 
ternehmen und insbesondere zu der Rolle von Kindern in einem Familienunternehmen vorgelegt und danach gefragt, inwiefern sie mit diesen Meinungen übereinstimmen oder nicht. ${ }^{92}$ Die Forscher identifizieren drei Cluster mit einem in sich konsistenten Antwortverhalten, die sich in einer weiteren Studie (Birley 2001) bestätigen, die anhand eines gekürzten Fragebogens gezielt die Einstellungen von Eigentümerunternehmern in 16 Ländern in Europa, Nordamerika und Japan miteinander vergleicht. Während die Arbeiten von Sue Birley et al. auf einer Einstellungsebene verweilen, dringt das hier vorliegende Interviewmaterial zu der alltäglichen Lebensführung von Unternehmerfamilien vor. Beide Analyseebenen sind jedoch aufs engste miteinander verflochten. Im Folgenden wird deshalb die von Sue Birley et al. erarbeitete Typologie näher vorgestellt und um die Ebene der Tätigkeiten ergänzt.

\subsection{Typ 1: Verschmelzung}

Die erste Gruppe besteht aus Familien, die das Unternehmen als einen natürlichen Raum der Familie verstehen. Birley et al. (1999:605) fassen als „The Family Rules Group“ und später als „Family In Group“ (2001:69) solche Personen zusammen, die überzeugt davon sind, dass das Unternehmen leistungsfähiger und robuster ist, wenn Familienmitglieder beteiligt sind. Ein Nachfolger ist vorzugsweise aus den Reihen der Familie zu rekrutieren, und die ausscheidende Generation sollte, so lange sie möchte, beratend zur Seite stehen. Auch das Eigentum ist ausschließlich an Familienmitglieder zu übertragen. Sie finden, dass Kinder bereits in einem jungen Alter in das Unternehmen einbezogen werden und Verantwortung dafür übernehmen sollten. Es ist wichtig, dass die Kinder ein Interesse an den Produkten und Märkten des Unternehmens entwickeln. In der Lebensführung dieser Unternehmerfamilien verschwimmen die Grenzen zwischen Unternehmen und Familie fast gänzlich. Die sonst als zwei Sphären gedachten Bereiche verschmelzen. Leopold Clemens betont mehrfach, dass in ihrer Familie „Firma auch gelebt wird“. Bei Familie Vester ist das Restaurant ein selbstverständlicher familialer Raum, hier treffen sie Freunde, frühstücken oder streiten sich. Das zeitliche Kontingent für „Freizeit" im klassischen Sinn ist sehr begrenzt. Auch Bianca Carstens lebt mit ihrer Familie dieses Modell. Sie sind findet es praktisch, ihr Baby mit ins Büro zu nehmen, nutzt eine flexible Zeiteinteilung und begrüßt es, dass auch ihr Mann einen Job im Familienunternehmen übernimmt. In der Zeit, als Familie Treuninger noch in der Fabrikwohnung lebt und Marcella sich eine Aufgabe im Betrieb sucht, praktizieren sie diese Art

92 In diesem Zusammenhang sei betont, dass in der Pilotstudie die Aussage „Children should be allowed to choose not to join the business“ über alle Gruppen hinweg die höchste $\mathrm{Zu}$ stimmung erhielt Birley 1999:604. Dieses Ergebnis ist kompatibel mit der von mir aufgestellten These über ein Narrativ der freien Wahl (siehe Abschnitt 2.4). 
des Familienalltags, der nicht mehr in unterschiedliche Orte und Themen für Familie und Unternehmen differenziert.

\subsection{Typ 2: Festung}

Die von Birley et al. als „Family Out-Group“ (1999:605) bezeichnete Personengruppe vertritt in vielen der oben beschriebenen Punkte eine diametral gegensätzliche Einstellung. Darüber hinaus sind sie der festen Meinung, dass Kinder auf keinen Fall in einem jungen Alter Anteile am Unternehmen erhalten sollten und diese auch später? nicht unbedingt in gleichem Verhältnis bekommen müssen. Aus ihrer Sicht sind nicht alle Familienmitglieder automatisch berechtigt, finanzielle Zuwendungen aus dem Unternehmen zu erhalten oder dort eine Anstellung zu finden. Auch im Alltag versucht diese Personengruppe, eine strikte Grenze zwischen Unternehmen und Familie zu leben. Dies äußert sich beispielsweise in der Wohnsituation der Familie (ein Häuschen im Grünen) und dem Versuch, innerhalb der Familie das Unternehmen nicht zu thematisieren, sondern ,voll für die Familie“ da zu sein. Sven Singermayer beispielsweise sagt: „dadurch dass ich nicht hier gewohnt habe bin ich auch nicht am Wochenende in die Firma gegangen und ich habe mir auch keine Arbeit mit nach Hause genommen. ... Ähm, lange eigentlich ... konnte ich ganz gut auch abschalten." Mit dem Umzug aufs Land schafft Familie Treuninger nicht nur eine physische Distanz (Graben) zwischen Unternehmen und Familie, sondern auch der Familienalltag richtet sich nun zeitlich und thematisch nicht mehr an dem Fluchtpunkt des Unternehmens aus. Die Familie wird, ganz dem bürgerlichen Idealbild folgend, zu einem Raum der Privatheit und Regeneration.

\subsection{Typ 3: Zwei Kerne}

Die letzte Gruppe schließlich bezeichnen Birley et al. (2001:603f.) bildhaft als Jongleure von Familie und Unternehmen. Diese Personen vertreten keine extreme Meinung in die eine oder andere Richtung, sondern versuchen, eine Balance zwischen Familie und Unternehmen zu halten. Sie halten es nicht für notwendig, dass kleine Kinder bereits Kontakt zum Unternehmen (z.B. zu Kunden) oder ein Interesse an dessen Produkten und Märkten entwickeln. Die Frage einer Nachfolge in der Unternehmensleitung ist grundsätzlich offen und auch externe Personen vorstellbar. Falls sich Kinder für den Eintritt ins Unternehmen entscheiden, sollten sie ganz unten anfangen und eine auf das Unternehmen zugeschnittene Ausbildung erhalten. Dieser Personenkreis vertritt darüber hinaus die Meinung, dass der Transfer von Anteilen am Unternehmen nicht mit dem Tod von Familienmitgliedern gekoppelt sein sollte. Die Lebensführung dieser Unternehmerfamilien zeichnet sich durch eine 
vorhandene, aber durchlässige Grenze zwischen Unternehmen und Familie aus. In der Familie Hagemann etwa sind Gespräche über den Supermarkt zwischen Vater und Sohn alltäglich, doch die Mutter interveniert, wenn dieses Thema überhand nimmt. In der Familie Treuninger findet sich diese Art der aktiven Grenzarbeit zu Zeiten des Rückzuges in die Stadt und der erneuten Arbeitsaufnahme von Marcella im Unternehmen. Unternehmen und Familie bilden nun eine große, verschwimmende Grauzone. Jedoch haben sich einerseits ein Unternehmensbereich und andererseits ein Familienbereich institutionalisiert, die strikt voneinander getrennt werden. Beispiele hierfür sind etwa Familientage oder Abendessen, bei denen nicht über das Unternehmen gesprochen werden soll.

\subsubsection{Fazit: Die lebensprägende Bedeutung des Familienalltags}

Dieses Kapitel hat deutlich illustriert, inwiefern die Vorstellung vom Leben als Unternehmerfamilie das familiale Handeln von Familienunternehmern beeinflusst. Zentrale Ergebnisse sind: Erstens, die Partnerwahl für Familienunternehmer wird durch die zusätzlichen Kriterien der Passung für den Lebensstil als Unternehmerfamilie sowie die Nähe zu den Eltern voraussetzungsreich. Zweitens, der Kinderwunsch scheint eine private Frage zu sein, die außerhalb der Reichweite des Unternehmens verhandelt wird. Die biografische Verhaftung in der eigenen Nachfolgerrolle wurde als ein Grund dafür benannt und daran anschließend gezeigt, inwiefern die Geburt eines Kindes die biografische Perspektive von Familienunternehmern in Richtung Kontinuität des Familienprojektes jenseits der eigenen Generation öffnet. Schließlich wurden die Typen „Verschmelzung“, „Festung“ und „Zwei Kerne“ als unterschiedliche Formen der alltäglichen Lebensführung von Unternehmerfamilien entwickelt, die eine spezifische Ausprägung von aktiver Grenzarbeit beinhalten.

Mit Blick auf den bisher beschrittenen Lebensweg von Familienunternehmern und ganz im Sinne der von Moen vorgeschlagenen ProzessKategorie kommt der jeweils gewählten Form des Familienalltags eine Schlüsselfunktion zu: Sie bestimmt maßgeblich, in welchem Ausmaß ein Kind mit dem Unternehmen in Kontakt kommt und dieses als familialen Raum begreift. Sie wird zu einem Orientierungspunkt in der Partnerwahl, der beruflichen Karriere ebenso wie in der Ausgestaltung des eigenen alltäglichen Familienlebens.

Birley et al. gehen in ihren Studien auch auf die Frage ein, wie sich die Mitgliedschaft in einem Cluster erklärt. Entgegen ihren Erwartungen spielt dabei weder die Länderzugehörigkeit noch die Branche oder die Größe des Unternehmens (und zumindest in der Pilotstudie auch nicht die Position in der Generationenfolge) eine Rolle (Birley et al. 1999:606). Stattdessen stel- 
len die Forscher heraus, und dieses Ergebnis deckt sich mit meinen Beobachtungen aus dem Interviewmaterial, dass der Lebensweg und die Anzahl der anderen im Unternehmen aktiven Familienmitglieder die Einstellung zur gelebten Grenze zwischen Unternehmen und Familie beeinflussen. Birley et al. stellen fest, dass die Mitglieder, der „Family Rules Group“ eher das Unternehmen direkt nach der Schule übernehmen oder gründen und hier mehrere Familienmitglieder tätig sind. Die Mitglieder der „Family Out Group“ hingegen haben zunächst fünf oder mehr Jahre in einem anderen Unternehmen gearbeitet, und oft sind keine anderen Familienmitglieder zeitgleich im Unternehmen tätig. Leider liegen keine Ergebnisse darüber vor, inwiefern die Partner mit ihren Meinungen übereinstimmen oder die Geburt von Kindern das Einstellungsset verändert.

Es scheint also ein starkes Wechselverhältnis zwischen dem in der Kindheit erfahrenen Familienalltag, der eigenen Lebenserfahrung sowie dem letztlich selbst praktizierten Familienalltag zu bestehen. Aus dem Interviewmaterial lässt sich zeigen, dass die Art und Weise, wie Eltern die Grenze zwischen Unternehmen und Familie vorleben, nicht notwendigerweise tradiert wird, die Kinder jedoch darüber reflektieren und diese Lebensweise als Ausrichtungspunkt für ihr eigenes Leben nutzen. Adam Treuninger etwa sagt:

„Also, ich habe mir so überlegt, aber das ist jetzt wirklich nur so aus dem Bauch gesprochen... Vielleicht mach ich es wie meine Eltern... ich erzähl ihr viel, so was passiert, was ich mache, so_was_Papa_macht, aber ich werd sie nicht irgendwie in die Richtung schubsen oder lenken oder so. So hätte ich es jetzt mal versucht (lacht).“

Bereits in Kapitel 6.1 wurde festgestellt, dass Kinder die Art und Weise der Lebensführung ihrer Eltern beobachten und wahrnehmen. Nun kann diese These spezifiziert werden: Der jeweils praktizierte Typ der Lebensführung bestimmt maßgeblich die Erfahrungen eines Kindes mit dem Unternehmen. Die Reflexion über die eigene Familie beinhaltet immer auch eine Auseinandersetzung mit ihrer Art der aktiven Grenzarbeit. Diese implizite oder reflexive Auseinandersetzung ist es, und nicht unbedingt die jeweilige Form des Familienalltages, die eine wesentliche Rolle in der Lebenslaufgestaltung von Mitgliedern aus Unternehmerfamilien spielt. ${ }^{93}$

93 In der Konsequenz müsste eine quantitative Überprüfung dieser These nicht nur in Betracht ziehen, mit welcher Wahrscheinlichkeit etwa ein Kind, das in einer Unternehmerfamilie des Typs „Festung“ aufgewachsen ist, in das Familienunternehmen eintritt, sondern auch, wie dieses Kind diese Form der alltäglichen Lebensführung wahrgenommen und bewertet hat. 


\subsection{Unternehmersein}

Der innere Entschluss, einen Weg in das Familienunternehmen zu beschreiten, markiert, wie oben dargestellt, den Beginn einer „Karriere“ als Unternehmer und legt den Grundstein für die Ausbildung einer unternehmerischen Identität. Die Erzählungen und Beschreibungen der Gesprächspartner über ihr Leben als Unternehmer weisen unwahrscheinlich starke Parallelen auf. Das direkte Ringen mit den Märkten, technische Innovationen, der Umgang mit den Mitarbeitern oder auch Gedanken über eine angemessene Schnittstelle zwischen Familie und Unternehmen sind Elemente einer gemeinsamen Erfahrungswelt. Zudem zeigt sich, dass das „Unternehmersein“ den Kern der biografischen Selbstdeutungen der Befragten bildet. Aus der Reflektion über ihren Lebensweg und ihre Fähigkeiten, aber auch über gesellschaftliche Stereotype zum Unternehmer und unternehmerische Vorbilder haben sie eine eigene Theorie entwickelt, was es bedeutet, ein „Familienunternehmer“ zu sein. Diese dient als Richtschnur in ihrem Handeln und als narrativer Bezugspunkt in ihrer Selbstpräsentation. Geht man wie Eva-Maria Bäcker (2008) davon aus, dass Unternehmen und Familie als zwei getrennte Systeme $\mathrm{zu}$ betrachten sind, leisten Familienunternehmer in einer funktional ausdifferenzierten Gesellschaft eine spezielle Identitätsarbeit: „Sie ,spielen“ Unternehmer in allen Lebensbereichen und -phasen (außer in der Kindheit). Sie verhalten sich selbst im Privatleben als Rollenhandelnde gemäß jener Strukturen, die einer ,Unternehmerrolle“ entsprechen.“ (Bäcker 2008:221). Die bisherige Analyse der einzelnen Lebensphasen im Rahmen dieser Arbeit zeigt hingegen, dass die praktizierte Grenzarbeit zwischen Unternehmen und Familie und die sich daraus ergebende Lebensführung einen zentralen $\mathrm{Be}-$ standteil der Rolle als Familienunternehmer bilden. Sie sind „Berufene“, leben das Unternehmertum mit jeder Faser ihres Seins.

In der Interviewsituation werden die Befragten eben in ihrer Rolle als „Familienunternehmer" angesprochen. In ihrer Präsentation können sie auf eingeübte Elemente der Selbstdarstellung zurückgreifen, die es fast unmöglich machen, in der biografischen Fallrekonstruktion zwischen einer ,Rhetorik des guten Unternehmers" und dem Erleben dieser Lebenszeit zu differenzieren. Der durchdringende und gar allumfassende Charakter des „Unternehmerseins" erinnert in vielen Aspekten an den calvinistischen Unternehmer Max Webers, der seine Lebensführung einem spezifischen Arbeitsethos unterwirft. Die Konsistenz dieses biografischen Musters überrascht vor dem Hintergrund der Allgegenwärtigkeit des Unternehmertums in der modernen, globalen Marktwirtschaft und einer individualisierten Leistungsgesellschaft. Die „beruflichen Aufgaben“ der Unternehmer verschieben sich (wie von Schumpeter prognostiziert) in Richtung angestellter Manager und Kapitalbesitzer. Zugleich kann das unternehmerische Einstellungsset beliebig internali- 
siert und praktiziert werden, etwa innerhalb von Organisationen (,Intrapreneurship' siehe etwa Pinchot 1985), von jedem, der seine Arbeitskraft auf dem Arbeitsmarkt feilbietet ('Arbeitskraftunternehmer' Voß und Pongratz 1998) und sogar von wohltätigen Organisationen oder sozialen Aktivisten ('Social Entrepreneurship' etwa Faltin 2008).

Im Folgenden sollen die typischen Elemente des „Unternehmerseins“ ausgehend von den narrativen Selbstdarstellungen der Familienunternehmer rekonstruiert werden, wobei das soziologische Konzept der Profession der Ergebnisdarstellung Struktur verleiht. Zunächst wird die These entwickelt, dass Familienunternehmer sich auf einer narrativen Ebene als Profession darstellen, in einem zweiten Schritt werden mögliche Gründe hinterfragt, welchen Zweck Familienunternehmer damit verfolgen könnten.

\subsubsection{Typische Elemente in unternehmerischen Selbstdarstellungen}

In der Soziologie spricht man immer dann von einer Profession, wenn sich eine Berufsgruppe, die sich meist auf eine spezifische Art von Wissen und Dienstleistung spezialisiert hat, durch ein professionelles Wertesystem, eine institutionalisierte Ausbildung und exklusive Mitgliedschaftsregeln auszeichnet. Mediziner und Richter erfüllen die Kriterien einer Profession fast schon in Reinform. Innerhalb der Forschung zu Professionen wird in den letzten Jahrzehnten vermehrt darüber diskutiert, ob nicht auch andere Berufsgruppen als Profession zu bezeichnen sind, wie etwa Lehrer, Universitätsprofessoren aber auch das komplexe Feld der sozialen Arbeit. ${ }^{94}$ Es kursieren diverse Listen, die versuchen abzugrenzen, wer nun den Status einer Profession für sich in Anspruch nehmen kann und wer die Kriterien nicht erfüllt. Wie sich an diesem Diskurs ablesen lässt, werden gegenwärtige Veränderungen der Berufskategorien und -strukturen im Sinne einer zunehmenden Professionalisierung interpretiert.

Die Schwierigkeiten, eine Unternehmensgründung oder eine Nachfolge als „Berufswahl“ zu fassen (siehe hierzu Abschnitt 2) deuten bereits an, wie problematisch es ist, Unternehmer als Beruf, geschweige denn als eine Profession zu bezeichnen. Wirft man einen Blick in den aktuellen Katalog (2010) der Berufsbezeichnungen der Bundesagentur für Arbeit, findet man zwar unter der Nummer 71104 die Unternehmer/innen, die sich diese Position mit den Unternehmensmanager/innen teilen, jedoch gibt es keinen einheitlich definierten Tätigkeitsbereich für diese Berufsgruppe. Ebenso fehlt es an einer institutionalisierten Ausbildung oder einer Qualifikation zum Unter-

94 Siehe hierzu etwa das Schwerpunktheft der FQS Dausien et al. 2008 oder beispielhaft die Artikel von Williams 2008 oder Carr 2000. 
nehmer. Weder ein Studium der Betriebswirtschaftslehre noch eine duale Ausbildung im Unternehmen führen schließlich zu dem Titel „Unternehmer“. Erst in den letzten Jahren entstehen zunehmend Studiengänge, die sich auf die Führung von kleinen und mittleren Unternehmen konzentrieren. Trotzdem bleibt es fraglich, ob ein „Unternehmerhabitus“ oder eine „Unternehmerpersönlichkeit" überhaupt durch eine berufliche Ausbildung erworben werden können oder vielmehr Produkt der familialen Sozialisation oder gar einer genetischen Disposition sind. Schließlich finden sich in Deutschland zwar einige Verbände für Unternehmer (etwa der Bund Junger Unternehmer, Arbeitgeberverband, etc.), jedoch ist der Zugang grundsätzlich offen. Die Bezeichnung „Unternehmer" ist nicht geschützt und an keine verbindlichen Kriterien der guten Praxis geknüpft, auch wenn sich in den letzten Jahren vermehrt freiwillige Selbstverpflichtungen zur Einhaltung ethischer Verhaltenskodizes in (großen) Unternehmen finden. Unternehmer sind auch dahingehend besonders, als sie immer zugleich Mitglied mehrerer Berufsgruppen sind: Sie sind Bauern, Zimmerer oder Ingenieure UND Unternehmer. Von einer geforderten „occupational closure“ kann also keine Rede sein.

Trotzdem erweist sich das Konzept der Profession (im Folgenden vor allem Evetts 2003) als geeignet, die Selbstdarstellungen der Familienunternehmer zu analysieren und zu sortieren, denn sie nehmen auf narrativer Ebene einige der markanten Kriterien einer Profession für sich in Anspruch:

Typische Elemente in den Beschreibungen der unternehmerischen Tätigkeit meiner Interviewpartner sind Markteroberungen, Produktinnovationen, Standortverlagerungen oder andere strategische Unternehmensentscheidungen. Familienunternehmer vermarkten ein spezifisches Produkt oder eine Dienstleistung, organisieren die dafür notwendigen Mittel und führen ihre Mitarbeiter. Dafür erwarten sie, in Form von Gewinnen oder eines Unternehmerlohns ein Einkommen zu erzielen. Sie sehen sich der Herausforderung ausgesetzt, mit Unsicherheiten und den Anforderungen der Märkte ebenso wie permanentem Wandel umzugehen. Sie müssen kontinuierlich Risiken bewerten.

Die Leitung eines Unternehmens, so der allgemeine Tenor, sei nicht irgendein Projekt, sondern vergleichbar mit einer gemeinsamen Fahrt durch die mal ruhigen, mal stürmischen Meere. Die Zeiten des Aufruhrs sind vor allem durch technologische Umbrüche oder aber durch die Veränderungen des globalen Wirtschaftens bestimmt. Das Kollektiv des Unternehmens wird von diesen Umwälzungen ,erwischt“ (Michael Treuninger, 2:33), und es gilt Lösungen zu finden. Es ist die Aufgabe der Familienunternehmer, ,wieder die Umsteuerung hinzukriegen“ (3:1). Sensibel analysieren sie Trends, Veränderungen auf den Märkten und nehmen Signale aus der Umwelt wahr. Sie beobachten, probieren und beschreiten neue Wege und lernen auf diese Weise, die unterschiedlichen Situationen zu bewältigen. Erforderlich dafür sind Kreativität, Pioniergeist und Innovationen. Das berufliche Leben der Famili- 
enunternehmer wird fast schon wie ein Kriminalfilm dargestellt, der mit den entsprechenden Geräuschen hinterlegt ist: ,und der Tipp kam, ... (ploppt mit den Lippen) ... der kam von einem (schnalzt) Bürohändler“" (27:24-25).

Für diese Tätigkeit ist in den Augen der Familienunternehmer ein praktisches und technisches Wissen von Nöten, das hauptsächlich direkt über unternehmerisches Handeln und weniger über eine akademische Ausbildung angeeignet werden kann. In ihren Selbstdarstellungen betonen die Familienunternehmer (besonders jene einer zweiten oder einer späteren Generation) deshalb die praktische Orientierung ihrer Ausbildung, Lehrjahre oder „Übungsstücke“ (Adam Treuninger, 25:20), die sie für diese spezielle Tätigkeit qualifizieren. Adam Treuninger etwa geht ausführlich auf sein eigenes Unternehmen ein, das er neben seiner Tätigkeit im Familienunternehmen betreibt und hier wichtige Erfahrungen für seine späteren Aufgaben als alleiniger Geschäftsführer sammelt. Auch Timmo Hagemann stellt sein unternehmerisches Können durch eine weitere Filiale des Supermarktes unter Beweis. Darüber hinaus sei die Eignung für das Familienunternehmertum immer auch mit persönlichen Eigenschaften verbunden, allen voran der Bereitschaft, Wagnisse auf sich zu nehmen und Rückgrat in harten Entscheidungen zu beweisen.

Es lässt sich festhalten, dass Familienunternehmer in ihren Selbstdarstellungen mit anderen Familienunternehmern eine spezifische Erfahrungswelt, ein Selbstverständnis, ein spezialisiertes Wissen ebenso wie eine Art und Weise, Probleme wahrzunehmen, teilen. Gemäß der Definition von Hughes (wie in Evetts 2003:401 zitiert) verfügen sie damit über eine Berufsidentität. Die Betonung ihrer Aktivitäten als Risikomanager verleiht Familienunternehmern einen professionellen Charakter: "Professionals are extensively engaged in dealing with risk, with risk assessment and, through the use of expert knowledge, enabling customers and clients to deal with uncertainty." (Evetts 2003:397)

\subsubsection{Das professionelle Wertesystem des Familienunternehmertums}

Das „Unternehmersein“ der Familienunternehmer geht jedoch über die bloße unternehmerische Aktivität hinaus. In ihren Selbstdarstellungen verweisen die Familienunternehmer durchgehend auf eine außergewöhnlich hohe Arbeitsbelastung und die Notwendigkeit zur Flexibilität und Leistungsbereitschaft: „das Unternehmen nimmt ja eigentlich auch, sagen wir mal $90 \%$ unserer Zeit in Anspruch. Also jetzt, für mich war es zum Beispiel so, ich habe ... Donnerstag bis nachts um zwei eine Veranstaltung gehabt, Freitag bis um zwei. Dann war ich in Weimar noch am Samstag, am Samstagabend wieder eine Veranstaltung bis Sonntagmorgens um zwei. Und Sonntagmittag stand 
dann hier ein Lieferant vor der Tür bis abends um sechs, das heißt, ich habe an diesem ganzen Wochenende null Freizeit gehabt und das über viele Wochen schon, ja?" (Gabriel von Moline, 46:21-28) Familienunternehmer verstehen sich selbst als Leistungsträger und wollen anhand dieser Leistung gemessen und beurteilt werden. Sie streben danach, aktiv zum Erfolg des Unternehmens, seiner Geschichte und der Branchenentwicklung beizutragen. Sie sind gefordert, eine drückende Verantwortung, die sich aus der FamilienUnternehmens-Konstellation ergibt, in ihren Alltag zu integrieren: ,man sollte nicht vergessen, Unternehmer heißt, etwas unternehmen, das heißt 24 Stunden am Tag, sieben Tage die Woche. Und Sie gehen mit den Gedanken schlafen ... oder Sie gehen mit den Gedanken ins Bett, ob Sie dann schlafen können ist eine andere Frage, aber Sie gehen mit den Gedanken ins Bett und Sie stehen mit den Gedanken auf und den Verantwortlichkeiten und den Sorgen." (Malte Niest, 49:9-13) Die Verbindung von Familie und Unternehmen ist es auch, die Familienunternehmer mit einem „normativen Überbau“ ausstattet. Ihr unternehmerisches Handeln erfolgt entlang ethischer Grundsätze und innerhalb moralischer Grenzen. Der ,gute Familienunternehmer“ gibt das verdiente Geld nicht maßlos aus, übt sich stattdessen in (einer zur Schau gestellten) Bescheidenheit und reinvestiert in das Unternehmen. Familienunternehmer erzählen davon, wie sie sich selbst ein geringes oder gar kein Gehalt bezahlen, in schlechten Zeiten den Betrieb subventionieren oder einen privaten Kredit aufnehmen, um Investitionsprojekte voranzutreiben. Betont wird eine langfristige Perspektive in Unternehmensentscheidungen sowie nachhaltige Unternehmensführung.

Aus den Selbstdarstellungen der Familienunternehmer lässt sich ein hohes Pflichtgefühl gegenüber den Mitarbeitern kondensieren. Sie haben eine außerordentlich starke innere Bindung an ,ihren“ Betrieb und „kümmern sich um ihre Mannschaft“. Sie zahlen gute Löhne, legen Wert auf ein gutes Arbeitsklima und zollen der Arbeitsleistung der Mitarbeiter Respekt. Die unternehmerische Tätigkeit baut für sie auf der Einlösung eines Gegenseitigkeitsprinzips auf, bei dem Mitarbeiter und Unternehmer Einsatz- und Leistungsbereitschaft zeigen. In diesem Zusammenhang sei auf die instruktive Arbeit von Kothoff (1990) über die „Soziale Welt kleiner Betriebe“ verwiesen, in der das „Dritte“ in der Beziehung zwischen Mitarbeitern und Unternehmer in den Fokus gerät und das gemeinschaftstiftende Potenzial der unternehmerischen Tätigkeit beschrieben wird. Familienunternehmer fühlen sich des Weiteren in ihrer Region verankert. Sie profitieren von der Infrastruktur und den Mitarbeitern und wollen einen Beitrag zu Lebenszufriedenheit und Wohlstand in ihrem nächsten Umfeld leisten. Als Beleg für die Wahrnehmung dieser Verantwortung für die Region führen Familienunternehmer ihr ehrenamtliches Engagement an: Familie Clemens betreibt eine Bürgerstiftung, Familie Vester ist im Branchenverband tätig, Michael Treuninger beteiligt sich an einem Branchenmuseum für seine Stadt, Familie Hagemann veranstaltet Feste 
zu wohltätigen Zwecken, etc. Schließlich legen die Selbstdarstellungen der Familienunternehmen ein starkes Gefühl der Verantwortlichkeit gegenüber früheren und zukünftigen Generationen offen.

Dieser Bezug zu Generationen wurde von Christina Lubinski (2010) (siehe auch Kapitel 3.2.6) als wesentliches Element der Selbstdarstellungen von Familienunternehmern hervorgehoben. Ihre Selbstpräsentationen nehmen die Gestalt einer „Generationenerzählung“ an, die meist einer Schöpfungsgeschichte gleicht. Dieses narrative Muster geht in der Regel von der Person eines Gründers und den besonderen Umständen der Gründung aus, schreibt dann den einzelnen Generationen je spezifische Perioden zu und behandelt Firmen- sowie Familiengeschichte gleichermaßen (Lubinski 2010:139). Mittels solcher „Generationenerzählungen“ situieren sich Familienunternehmer in einem größeren Kontinuum und lassen das Verhältnis von Familie und Unternehmen zum Thema eines intergenerationellen Deutungsprozesses werden. Auch Simon Down (2006) entdeckt in seiner Analyse der Unternehmeridentität dieses narrative Muster und nutzt dafür den Begriff der „relational narratives“, er ergänzt diese um ,generational narratives“, die auf eine narrative Identifikation mit der gesellschaftlichen bzw. historischen Generation verweisen. Familienunternehmer verstehen sich in einer horizontalen Linie als Teil einer gleichaltrigen Unternehmergeneration, die ein spezifisches Wissen über unternehmerisches Handeln, Managementmethoden und zeithistorische Umstände, mit denen verfahren werden muss, teilt. Durch diesen Bezug zur gesellschaftlichen Generation entsteht in den Narrativen der Familienunternehmer ein künstlicher Antagonismus zwischen den ,jungen Innovatoren' und den ,alten Experten' (Down 2006:57).

Mit Julia Evett lässt sich zusammenfassend argumentieren, dass Familienunternehmer über ein ,professional value system“ verfügen. Bescheidenheit, Sparsamkeit, Leistungs- und Risikobereitschaft, Loyalität gegenüber den Mitarbeitern, öffentliches Engagement, Familiensinn und Verantwortungsbewusstsein sind Bestandteile dieses Wertekanons, der als eine Konstruktionsleistung zu verstehen ist. Folgendes Zitat verbildlicht noch einmal die wesentlichen Punkte dieses professionellen Wertesystems:

„,ich lebe für das, was ich hier tue und ich liebe das ... mit vollem Einsatz und für mich ist ... die Freude über einen Auftrag nie mit dem Gedanken so viel Geld verdienen wir daran verbunden, weil ich mache das einfach gerne. Das ist mein Leben, das ist mein, das macht mir Freude. [ausgelassen ] mir das Geschäft an sich Spaß, Verantwortung zu tragen, ein Unternehmen zu entwickeln, (zieht die Luft ein) Arbeitsplätze ... zu schaffen oder vielleicht auch mal in schwierigen Zeiten zu halten.“ (Malte Niest, 11:7-17) 
In dem professionellen Werteset der Familienunternehmer verquicken sich eigene Einstellungen, Entlehnungen aus den klassischen Theorien zum Unternehmer ${ }^{95}$, Bezüge zu aktuellen Managementtrends sowie Entgegnungen auf gesellschaftliche Stereotype. In anderen Worten: Hier vermischen sich öffentlich zugängliche Narrative einschließlich ihrer evaluativen und normativen Komponenten mit einer mehr oder weniger ausgefeilten biografischen Selbstsicht (Down 2006:103). Die Analyse zeigt, dass die Familienunternehmer nicht nur eine Rolle spielen, sondern auch in einem interpretativen Verhältnis zu dieser Rolle stehen.

Schließlich beinhaltet die Selbstpräsentation der Familienunternehmer eine klare Verortung ihrer Position innerhalb der Gesellschaft. In den Selbstdarstellungen der Befragten wird deutlich, dass sie als Firmenlenker, Entscheidungsträger und Innovatoren eine zentrale Funktion im Produktionsprozess und Wirtschaftsgefüge für sich in Anspruch nehmen. Sie besitzen Macht über die Arbeitsplätze, die sie schaffen und erhalten, Steuern, die sie zahlen, ihre Netzwerkbeziehungen und ihre Verwicklungen in Branchenverbände oder die lokale Politik. In ihren Narrativen betonen Familienunternehmer ihre gehobene Position innerhalb ihrer Gemeinde (Dorf oder Stadt), ihre gute Reputation in der Region oder Branche ebenso wie Neider ihres Erfolges. Sie sehen einen hohen sozialen Status für sich selbst. In ihren Selbstdarstellungen differenzieren sie sich aktiv von Investoren, Angestellten, aber ganz besonders von den ,gierigen Managern“ - ein Umstand, der vor dem Hintergrund der Finanzkrise und des damit einhergehenden Diskurses über Gier interpretiert werden muss. Familienunternehmer fühlen sich einem Kollektiv zugehörig, das für seine eigenen Kriterien einer guten und erfolgreichen Praxis verantwortlich zeichnet. Insofern entfaltet Familienunternehmertum eine integrierende und zur gleichen Zeit sozial differenzierende Wirkung. Ein Familienunternehmer zu sein, kann als Mitgliedschaft in einer spezifischen sozialen Gruppe verstanden werden, die sich selbst von anderen Berufs- und Professionsgruppen unterscheidet. Auf einer individuellen Ebene dient diese Vorstellung vom Unternehmer als ein Referenzpunkt in der Ausbildung personaler Identität und bietet Orientierung im Lebenslauf. Sie mag dazu beitragen, die eigene Individualität gerade im Vergleich zu Altersgenossen zu bestimmen. Auf einer Gruppenebene konstituiert die Vision vom Familienunternehmer eine elitäre Unternehmergemeinschaft, die eine kollektive Identität teilt.

Zusammenfassend lässt sich festhalten, dass sich in den Selbstpräsentationen von Familienunternehmern folgende Elemente eines „Professionalismus" finden lassen: ein Bündel an typischen Tätigkeiten, die eine sehr spezifische Art des Wissens und Fähigkeiten erfordern ebenso wie eine typische

95 So betont Max Weber etwa die asketische Lebensweise oder Kirzner die Bereitschaft, Risiken auf sich zu nehmen. 
Lebensführung, die über die reine „unternehmerische Tätigkeit“ hinausgeht; ethische Grundsätze und Vorstellungen der guten unternehmerischen Praxis, die sich im Laufe der familialen und beruflichen Sozialisation produzieren und reproduzieren; eine professionelle Selbstsicht, die sie aus ihrer Position an der Schnittstelle unterschiedlicher institutioneller Bereiche und ihrem Beitrag zu einer Leistungsgesellschaft ableiten. Darüber hinaus passt Familienunternehmertum gut zu modernen Ideen der Professionalität, in denen Messbarkeit und Leistungsindikatoren fundamentale Bedeutung erhalten haben. Der Erfolg von Familienunternehmern kann in Form von Gewinn, Anzahl der Mitarbeiter oder der Dauer des Bestehens erfasst werden. Anhand dieser Merkmale lässt sich also durchaus argumentieren, dass Familienunternehmer als eine „Profession“ bezeichnet werden können.

\subsubsection{Fazit: Die Identitätsschaffende und Sozialdifferenzierende Wirkung des Familienunternehmertums}

Wesentlich interessanter als die Antwort auf die Frage, ob Familienunternehmer nun als eine Profession aufgefasst werden können oder nicht, ist, warum diese soziale Gruppe in ihren Selbstpräsentationen Professionalität für sich in Anspruch nimmt. Julia Evetts betont, dass ein professionelles Wertesystem dazu beitrage, Vertrauen zu bilden, Risiken zu reduzieren, sowie die Qualität der eigenen Leistung hervorzuheben. Der Status einer „Profession“ erleichtere es, die Reproduktion von Macht zu legitimieren und wirke als starker Motivationsfaktor. Überträgt man diese Thesen auf Familienunternehmer, scheint es sehr plausibel, warum es so attraktiv ist, sich als ,professionell" darzustellen. Auf diese Weise gelingt es ihnen, sich von anderen Gruppen (insbesondere den Managern) abzuheben, was als wichtiger Mechanismus in der Ausbildung einer individuellen Identität zu verstehen ist (Down 2006:23). Die Generationenerzählung im vertikalen wie im horizontalen Sinne erlaubt es ihnen, die Koordinaten für ihre soziale Position zu bestimmen und ein unternehmerisches Selbstverständnis zu entwerfen und zu erhalten (Down 2006:109). In Abgrenzung zu der familialen und gesellschaftlichen Generation konstruieren sie eine antagonistische oder deviante Gruppe der „Anderen“. Gleichzeitig konstruieren sie sich ein Kollektiv, zu dem sie in einem gemeinschaftlichen Verhältnis stehen. Beide Mechanismen der Generationenerzählungen sind wichtige Ressourcen darin, sich selbst als Familienunternehmer zu denken. Jenseits der individuellen Ebene erhöht eine Selbstpräsentation als ,professionell“ den Marktwert des Unternehmens (Stichwort Markenbildung) sowie die politische Stellung der Familienunternehmer und mag sich motivierend auf Mitarbeiter und zukünftige Nachfolger auswirken. Auf einer Makro-Ebene kann das professionelle Wertesystem der Familienunternehmer dazu beitragen, ausufernden Wettbewerb zu begrenzen 
und Kooperation voranzutreiben. Zudem wird es mit Autorität, einem hohen sozialen Status und Privilegien belohnt (Evetts 2003:400), wie sich etwa anhand des Ausnahmestatus' von Familienunternehmen in der Erbschaftssteuerregelung ablesen lässt. Folgt man Evett weiter, ließe sich Familienunternehmertum als eine Art der „Professionalisierung von innen“ (Evetts 2003:409) verstehen. Familienunternehmern gelingt es, normative Aspekte und Diskurse dafür zu nutzen, eine Berufsidentität zu etablieren, eine hohe Reputation gegenüber Kunden und Geschäftspartnern aufzubauen und ihren Sonderstatus mit selbst regulierter Verantwortlichkeit in Verhandlungen mit dem Staat zu sichern und aufrechtzuerhalten.

\subsection{Familienunternehmer im „Alter“}

Besonders interessant stellt sich schließlich die Phase des „Alters“ bei Familienunternehmern dar. In der Familienunternehmensforschung wird der Rückzug aus dem Familienunternehmen meist unter der Perspektive des Ruhestandes beschrieben. Das häufig dargestellte Festhalten am Familienunternehmen erscheint dann als dysfunktional - dem Familienunternehmer gelingt es nicht, die gesellschaftliche Norm umzusetzen. Im Folgenden sollen zunächst einige einschlägige Studien zum Ruhestand des Familienunternehmers vor dem Hintergrund der allgemeinen soziologischen und gerontologischen Forschung vorgestellt und eingeordnet werden. Anschließend wird der Begriff Ruhestand im Hinblick auf Familienunternehmer kritisch diskutiert. Aus dem Interviewmaterial werden schließlich zwei Modelle des Rückzuges aus dem Familienunternehmen herausgearbeitet und dem Typ des „Ruhestandes“ einen leistungsorientierten, graduellen Rückzug entgegen gestellt.

\subsubsection{Der Ruhestand von Familienunternehmern in der Forschung}

Die gerontologische Forschung weist in aller Deutlichkeit darauf hin, dass für die Definition des „Alters“ oder einer ,alternden Gesellschaft“ die chronologische Altersgrenze von meist 60 oder 65 Jahren nur wenig mit biologischen oder psychischen Prozessen zu tun hat, sondern vielmehr mit dem Übergang vom Erwerbsleben in den Ruhestand (Kohli 2000:I/16). Empirisch betrachtet erreicht ein wachsender Teil der Bevölkerung das Rentenalter, geht auch tatsächlich in den Ruhestand und das in meist besserer gesundheitlicher Verfassung und materieller Absicherung als frühere Kohorten. Das „Alter“ kann heute nicht mehr bloß als „Restzeit“ verstanden werden, sondern stellt eine strukturell klar abgrenzbare Lebensphase bzw. einen ,selbst- 
verständlichen und eigenständigen Teil der Normalbiographie“ (Kohli 2005:13) dar. Mit Blick auf die historische Entstehung der Altersphase (etwa Kohli 1992) wird in diesem Zusammenhang auch von einem Strukturwandel des Alters gesprochen (etwa Clemens \& Backes 1998). Es ist also Teil der „Normalerwartung“ an einen Lebenslauf, dass die Person zu einem spezifischen Zeitpunkt aus dem Erwerbsleben ausscheidet und alternative Pläne biografisch entwirft und umsetzt, wie sie die gewonnene Zeit nutzt und sich weiterhin am sozialen Leben beteiligen wird, etwa in Form von sozialem oder politischem Engagement, der Betreuung von Enkelkindern, Nachbarschaftshilfe (sog. produktive Tätigkeiten) oder durch die zeitliche Ausdehnung von Gewohnheiten (Künemund 2005:277).

Nach Kohli erfüllt die Altersgrenze dabei mehrere Funktionen (Kohli 2000:I/16): Sie markiert den Zeitpunkt des Austrittes aus dem (,regulären“) Erwerbsleben, bestimmt den Zugang zu bestimmten Leistungen der Sozialversicherungssysteme, schafft Orientierung für die subjektive Gliederung und Planung des Lebens und liefert ein Kriterium für den legitimen und erfolgreichen Abschluss des Erwerbslebens. Sackmann (2008:151) beschreibt den Übergang in den Ruhestand als einen ,institutionellen Aushandlungsprozess". Denn sowohl der Zeitpunkt der Verrentung als auch ihre Gestaltung wird vom Individuum in Auseinandersetzung mit den institutionellen Rahmenbedingungen entschieden. Er betont dabei die individuellen Handlungsspielräume zur Gestaltung dieses Prozesses, die trotz seiner institutionellen Rahmung durch Altersnormen und die Sozialversicherungssysteme bestehen. Die Bindung an den Beruf, Anreize eines frei gewählten Alltags, das Gefühl, sein Lebenswerk getan zu haben, das Einkommen, der aktuelle und prognostizierte Gesundheitszustand, das betriebliche Umfeld oder die Abstimmung mit dem Ehepartner oder Freunden sind Faktoren, die in die biografische Gestaltung des Übergangs einfließen (Sackmann 2008:148). In der empirischen Forschung lässt sich eine Tendenz dahingehend beobachten, dass Personen mit einer hohen Bildung und/oder einem geringen Einkommen später aus dem Erwerbsleben ausscheiden.

Das steigende Lebensalter sowie die Tendenz zu einem frühzeitigen $\mathrm{Ru}-$ hestand (beides sind große Themen gegenwärtiger Forschung) tragen wesentlich dazu bei, dass sich die Altersphase weiter ausdehnt und sowohl für die eigene biografische Gestaltung dieser Lebenszeit als auch für die sozialstaatliche Finanzierung des „Lebensabends“ an Gewicht gewinnt. Besonders vor dem Hintergrund sich verschärfender Systemprobleme (Finanzierung der Sozialversicherungssysteme oder Allokation von Arbeitskräften), aber auch hinsichtlich eines individualisierten Wertekanons werden Altersgrenzen kritisch diskutiert. Die nun schon seit einigen Jahren lauter werdende Forderung nach „Flexibilisierung“ meint, dass die Personen zukünftig eigene Wege des Übergangs vom Erwerbsleben in den Ruhestand finden sollen, was sowohl den Zeitpunkt, den Umfang als auch die Art und Weise der beruflichen Tä- 
tigkeit vor dem Ausstieg betrifft. Der Charakter des Übergangs in den Ruhestand als „institutioneller Aushandlungsprozess“ würde dann noch wesentlich stärker zum Tragen kommen. Studien aus Ländern, die schon vor Jahren ein festes Rentenalter abgeschafft haben (wie etwa die USA) zeigen jedoch, dass die ,normalen“ Altersgrenzen weiterhin eine hohe Verhaltenswirksamkeit und Orientierungsfunktion beibehalten.

Mit der kontinuierlich ansteigenden Lebenserwartung fallen Ruhestand und Alter in den individuellen Deutungen der Personen zunehmend auseinander (Kohli 2005:18). Das Ausscheiden aus dem Erwerbsleben wird als Kriterium dafür, sich selbst als ,alt“ zu fühlen oder zu bezeichnen, immer schwächer. In der Diskussion steht deshalb eine weitere Differenzierung in die ,jungen Alten“, deren Lebensführung sich durch eine selbst bestimmte und nicht durch Erwerbsarbeit geprägte Lebensführung auszeichnet, sowie in die ,alten Alten“ (oldest old), deren Lebensführung immer stärker durch ihre psychische und physische Beeinträchtigung geprägt wird (Dunkel et al 2001). Empirisch steigt in einem Lebensalter von 75 bis 80 Jahren das Risiko der Gebrechlichkeit erheblich an. Es handelt sich hierbei aber nicht um eine institutionalisierte Altersgrenze, die eine weitere strukturell abgrenzbare Lebensphase einläutet (Kohli 2005:14).

Die wesentliche Besonderheit der „Altersphase“ von Familienunternehmern, verstanden als jene längerfristige Lebensphase, die sich in unterschiedlichen Teilabschnitten um den Rückzug aus dem Unternehmen (und dabei vor allem aus der operativen Tätigkeit) rankt, ist in einer hohen Pluralität der vorfindbaren Lebenslaufmuster $\mathrm{zu}$ sehen, die teilweise konträr $\mathrm{zu}$ dem „Normallebenslauf“" stehen. Dieses Thema wird innerhalb der Familienunternehmensforschung intensiv diskutiert. Jeffrey Sonnfeld stellt unter dem Titel „A Hero's Farewell: What happens when CEOs retire“ vier plakative und psychologisch ausgerichtete Typen vor, die später von Lansberg (1999) aufgegriffen wurden und weite Beachtung gefunden haben:

Die "Monarchen" verweigern sich einem Rückzug aus dem Unternehmen gänzlich. Sie sind unfähig, sich von ihrem Lebensprojekt des Familienunternehmens und seiner Kontinuität zu verabschieden und entwerfen keine alternativen Handlungspläne. Ihr Tun wird von Ängsten (vor dem Tod, vor der Sterblichkeit, vor Misserfolg etc.) getrieben, und sie weisen oft eine narzisstische Persönlichkeit auf. Diese Typen von Familienunternehmern gestalten ihre Lebensführung in einer Art und Weise, die Abhängigkeiten entstehen und bestehen lässt, sowohl innerhalb der Familie als auch im Unternehmen. Sie sind nicht gewillt, Autorität abzugeben oder Informationen zu teilen. Ein „Monarch“ regiert bis an sein Lebensende oder bis er durch „Palastrevolution" - etwa durch den Aufsichtsrat, Mitarbeiter, Familienmitglieder oder externe Investoren - unter Zwang aus dem Amt entfernt wird.

Die "Generäle" sind ebenfalls entschlossen im Amt zu bleiben, verfolgen allerdings eine weniger offensive Strategie. Es fällt ihnen schwer, sich von 
ihrem Lebensprojekt des Familienunternehmens und seiner Kontinuität zu lösen und alternative Handlungspläne zu entwerfen. Dennoch sind sie bereit, einige Teilaspekte ihrer Tätigkeit aufzugeben und auf diese Weise ihre Autorität zu wahren. Sie mögen selbst die Führung des Unternehmens übergeben, jedoch stets einen wachen Blick auf die Handlungen der Nachfolger richten. In Zeiten der Krise stehen sie allzeit abrufbereit, um die Macht wieder an sich zu nehmen (und den eigenen Kindern zu entreißen). Sie fürchten den Tod und die Sterblichkeit und versuchen ihrem eigenen Verfall zu entfliehen.

Die "Botschafter" übertragen die Macht am Unternehmen an die folgende Generation, verbleiben jedoch im Unternehmen und definieren sich selbst darin eine neue Rolle etwa als „Berater“ oder „Repräsentant“. Sie sind aufmerksam gegenüber den Erwartungen und Bedürfnissen der Nachfolger und fördern ihre Karriere im Unternehmen. Unterstützt von ihrem Lebenspartner planen sie für eine Zeit nach der Tätigkeit im Unternehmen. Als „Botschafter" sind sie erfüllt von einer grundlegenden Zufriedenheit mit ihrem Beitrag zu dem Erfolg und der Geschichte des Unternehmens. Sie wünschen sich in irgendeiner Art und Weise mit dem Unternehmen verbunden zu bleiben.

Die "Gouverneure" schließlich sind in der Lage, ihre Verbindung zum Familienunternehmen gänzlich aufzugeben und sich neuen Herausforderungen zu widmen (etwa ein neues Unternehmen oder soziales Engagement). Sie übertragen die Verantwortung an die folgende Generation, gehen auf ihre Bedürfnisse sensibel ein und verstehen sich als Förderer der Karriere und persönlichen Entwicklung ihrer Kinder. Auf Basis einer hohen Zufriedenheit mit der eigenen Leistung gelingt es ihnen, alternative Lebenspläne zu entwerfen. Sie wünschen sich, die Leinen zu kappen und zu neuen Ufern aufzubrechen. „Gouverneure“ widmen einen großen Teil ihrer Zeit externen Aktivitäten.

Diese eingängige Typologie zeichnet ein relativ differenziertes Bild über unterschiedliche Wege des Rückzuges aus dem Familienunternehmen, der sich auch die im Rahmen dieser Arbeit erhobenen Fälle leicht zuordnen lassen. Gleichzeitig operiert diese Typologie in hohem Maße normativ. Aus der starken Betonung eines Zusammenhanges zwischen Nachfolge und Rücktritt leitet sich ein Bewertungsmaßstab über die Zuträglichkeit der unterschiedlichen Typen für den Generationswechsel ab. „Monarchen“ und „Generäle“ verhinderten eine offene Kommunikation und strategische Planung der Machtübergabe an die jüngere Generation und schadeten damit dem Unternehmen. „Botschafter" und „Gouverneure“ hingegen verließen ihr Amt in Würden und verstünden es, die Entfaltung der Nachfolger und ihres Innovationsgeistes zu fördern (Landsberg 1999:266). Zudem fußt diese Einteilung auf der gesellschaftlichen Vorstellung eines „Normallebenslauf“ und wertet das Verharren der Familienunternehmer in ihrer Position als Abweichung von der „Normalerwartung“, irgendwann in den Ruhestand zu gehen. Kets de Vries (2003) spricht etwa von dem „retirement syndrome“. Die vier Typen 
unterscheiden sich nach dem Grad ihres Verhaftetseins mit dem „Amt“ als Firmenleiter und ihrer Identität als Familienunternehmer. Lediglich der Typ des „Gouverneurs“ kann mit dem „Ruhestand“ eines abhängig Erwerbstätigen verglichen werden. Allen anderen Beschreibungen ist eine Unfähigkeit $\mathrm{zu}$ eigen, sich von dem Unternehmen zu lösen, Verantwortung abzugeben und eine neue Sinn- und Lebensperspektive zu entwickeln.

Eine Reihe von weiteren Studien identifiziert die (psychologische) Fähigkeit des Loslassens als wichtige Entwicklungsaufgabe in der Beschäftigung mit der individuellen Alterung und als wesentliches Kriterium für den Rückzug aus dem Familienunternehmen (z.B. Ibrahim, Saufani, Lam 2001; Levinson, Wofford 2000; Baumgartner 2009; Breuer 2009). Fabian Bernhard (2011) schlägt vor, das Konzept des psychologischen Eigentums als Theorie zur Erklärung des schwierigen Lösungsprozesses anzuwenden. ${ }^{96}$ Auf diesen Ansatz wurde bereits in Kapitel 2.3.2 verweisen, als Eigentumsgefühle und die starke Identität mit dem Unternehmen als struktureller Faktor für einen Weg in das Familienunternehmen identifiziert wurden. Bernhard verweist in dem Zusammenhang mit dem Ruhestand des Unternehmers vor allem auf die negativen Effekte des psychologischen Eigentumes (Bernhard 2011:33f.). ${ }^{97}$ Die starke Bindung an das Unternehmen, die in einer früheren Lebensphase $\mathrm{zu}$ einer hohen Motivation und Aufopferungsbereitschaft beigetragen hat, mag sich im Laufe des Alterungsprozesses zu einer Obsession mit dem Unternehmen, einer Unwilligkeit, den Besitz zu teilen und dem starken Bedürfnis nach exklusiver Kontrolle entwickeln. Diese negativen Seiten der Eigentumsgefühle treten besonders dann zum Vorschein, wenn das Besitzobjekt zu verschwinden oder außer Reichweite zu gelangen droht, wie dies im Ruhestand der Fall wäre. Eine solche Veränderung bedroht den Kern der Unternehmeridentität und kann Stress, Frustration und ein Gefühl der Nutzlosigkeit hervorrufen. Bernhard beschreibt weiter, dass Familienunternehmer entsprechend mit einem abwehrenden Verhalten reagieren und in extremen Fällen zur Sabotage der Besitzübergabe oder zu Selbstmordgedanken neigen.

Unbestritten trägt die Anwendung des Ansatzes des psychologischen Eigentums auf die Lebensphase des Alters von Familienunternehmen zu einem besseren Verständnis des Rückzugsprozesses und vor allem der starken Bindung des Familienunternehmers an sein Projekt bei. Allerdings wird aus dieser Perspektive das Weiterarbeiten des Familienunternehmers über die gesellschaftlich institutionalisierte Altersgrenze von 60-65 Jahren hinweg als ein „dysfunktionaler Effekt“ übermäßig starker Eigentumsgefühle interpretiert und damit als pathologisch bewertet. Im Folgenden möchte ich unter Rückgriff auf den Ansatz der „Institutionalisierung des Lebenslaufes“ eine

96 Genauer gesagt, wendet Bernhard diesen Ansatz auf Eigentümerunternehmer der ersten Generation an Bernhard 2011:27f.

97 Er bezieht sich dabei im Wesentlichen auf einen Aufsatz von Dirks et al 1996. 
weitere Deutung des „Alterns“ von Familienunternehmern vorschlagen, die Raum für das Konzept des psychologischen Eigentums lässt, aber weitaus weniger normativ argumentiert. Um es kurz vorweg zu nehmen: Aus dem empirischen Material kann ein alternatives Orientierungsmuster zum „Normallebenslauf" kondensiert werden, das sich in seinen Grundzügen an einer Leistungsgesellschaft ausrichtet und eine Erfolgskultur inkorporiert.

\subsubsection{Eine Kritische Diskussion des Begriffes Ruhestand}

Zunächst sei angemerkt, dass sich meiner Meinung nach der Begriff „Ruhestand" nur bedingt als Bezeichnung für die Altersphase von Familienunternehmern eignet:

Erstens, das Konzept des Ruhestandes ist sehr stark an abhängige Erwerbsarbeit und ihren institutionellen Kontext gekoppelt. Der Familienunternehmer kann und muss den Zeitpunkt und die Art und Weise seines beruflichen Rückzuges „,frei“ wählen, also unabhängig von einem gesetzlichen Rentenalter, von gewerkschaftlichen Vorgaben oder betrieblichen Altersnormen. Diese potentielle Freiheit in der Gestaltung des Überganges wird von anderen Faktoren gerahmt und bestimmt, zu denen neben jenen, die Sackmann herausgearbeitet hat (z.B. Bindung an den Betrieb, Gefühl, sein Lebenswerk getan zu haben, siehe oben), auch Gesellschaftsverträge, Familiennormen, die Kreditvergabepolitik der Banken oder die Abstimmung mit anderen Familienmitgliedern zählen.

Zweitens, der übliche Nexus aus Ende der Lohn- bzw. Gehaltszahlung und Beginn der Rentenzahlung ist ebenfalls keine Selbstverständlichkeit in Unternehmerfamilien. Zwar besteht die Möglichkeit, freiwillig in die gesetzlichen Alterssicherungssysteme einzuzahlen, doch die „Unternehmerrente“ speist sich meist aus privater Vorsorge und dem Unternehmen selbst. ${ }^{98} \mathrm{Be}-$ sonders dann, wenn ein Familienmitglied das Unternehmen übernehmen möchte, kann sich die finanzielle Absicherung des Alters der ausscheidenden Generation kompliziert gestalten und zu einem kritischen Moment der Nachfolge entwickeln (kann die jüngere Generation eine Betriebsrente erwirtschaften oder einen Kaufpreis bezahlen?). In diesem Zusammenhang sagt Bianca Carstens über ihren Vater, der schließlich das Familienunternehmen ohne ihr Wissen verkauft hat:

98 Für einen Überblick der Finanzierung des Ruhestandes von Familienunternehmern sei an dieser Stelle noch einmal auf den Artikel von Layer 2010 verwiesen. Die Unwägbarkeiten der sozialen Absicherung des Familienunternehmers im Alter sind ein in der Forschung stark unterbeleuchtetes Thema, das an dieser Stelle leider nicht weiter aufgegriffen werden kann. 
„Es war immer so die Frage, was macht eigentlich mein Vater, wenn er mal nicht mehr arbeitet. Nicht zuletzt auch finanziell. Na diese Absicherung, so. Also er kommt noch nicht aus dieser Generation, die heute so extrem um Vorsorge bemüht ist. Die versicherte Generation, junge Leute, die mit 30 darüber nachdenken wie ihre Rente aussieht. Das Konzept war meinem Vater vollkommen fremd. Auch mit 70 hat er sich da noch keine großen Gedanken darum gemacht. Das führt aber auch dazu, dass er überlegt hat: Wie weiter? Und ich hab gesagt, ich übernehm den Laden - Du ziehst Dich raus. Und dann wird das schon gehen. Ne Art von Betriebsrente oder was auch immer, das wird schon funktionieren. Ob er mir nicht getraut hat. Wer weiß es."

Drittens, „Ruhestand“ ist gesellschaftlich - und gerade in Deutschland - mit dem Austausch der Belegschaft verbunden: „Alte“ Mitarbeiter werden über den Arbeitsmarkt durch ,junge“ Mitarbeiter ohne große zeitliche Verzögerung ersetzt. Doch gerade in Unternehmerfamilien darf Ruhestand nicht mit Nachfolge gleichgesetzt werden. Der Eintritt des Nachfolgers und der Rückzug des Übergebers können, müssen aber nicht notwendigerweise in einem zeitlichen oder gar inhaltlichen Zusammenhang stehen. Sicherlich stehen sich die beiden Generationen in einem Aushandlungsprozess gegenüber, und die Erwartungen und Wünsche des einen fließen als Koordinaten in die Lebenslaufgestaltung des anderen ein. Trotzdem folgt der Weg in das Familienunternehmen einer gänzlich anderen Logik als der Weg aus dem Familienunternehmen - erst beide Übergänge zusammen formen die Nachfolge. Beide Teile der Nachfolge berühren sich darin, dass die Rahmenbedingungen zu klären sind, unter welchen Führungsaufgaben, Entscheidungsbefugnisse und Anteile übertragen werden, gehen aber nicht darin auf. Bei dem Rückzug aus dem Unternehmen geht es, wie Laske treffend schreibt,

„um sehr viel mehr: Es geht um einen möglichen Abschied von Macht, Geld, Einfluss und Ehre, um Familiengeschichte und -geschichten, um Absicherung und Versicherungen, um Ängste und Emotionen, Liebe und Anerkennung, Mythenbildung und Denkmalpflege, um Einsamkeit und Nähe, Eitelkeiten, Konflikte und vieles andere mehr" (Laske 1998:12 zitiert in Bäcker 2008:232).

Die existentiellen Fragen nach der Bedeutung des eigenen Lebens und seines Fortganges stehen beim Rückzug aus dem Unternehmen im Vordergrund. Deshalb plädiere ich für eine stärkere Trennung zwischen der Bereitschaft, das Unternehmen an die jüngere Generation zu übergeben, und der Bereitschaft, seinen Platz im Unternehmen zu verlassen. In unseren Interviews sind wir durchgängig einem großen Interesse der älteren Generation begegnet, die Kinder in das Unternehmen zu integrieren und ihnen nicht nur Verantwortung, sondern auch Besitzanteile zu übertragen. Ungeachtet dessen verfügen die Interviewpartner über äußerst unterschiedliche Vorstellungen über ihr 
Leben im Alter und darin eingeschlossen über die Reduktion der eigenen beruflichen Tätigkeit.

Folglich geht der Begriff „Ruhestand“ von anderen institutionellen und strukturellen Rahmenbedingungen aus als sie im Fall von Unternehmerfamilien vorliegen. Der Rückzug aus dem Unternehmen gestaltet sich hier nicht nur wesentlich stärker als Aushandlungsprozess, sondern auch die potentiellen Orientierungspunkte sind vielfältiger. Es gilt, individuelle Bedürfnisse mit den Erwartungen anderer Familienmitglieder und der Belegschaft ebenso wie mit gesellschaftlichen Altersnormen zu koordinieren. Im Vergleich zu einem abhängig Erwerbstätigen kommt in diesem Prozess den generationalen Verwicklungen ein höheres Gewicht zu, wohingegen die gesellschaftlichen Altersnormen weniger stark mit faktischen Altersgrenzen und Sanktionen (z.B. Abschlag in der Höhe der Rentenzahlung bei Frühverrentung) belegt sind. In ähnlicher Weise stellt auch Büttner (2007:30) fest, dass Nachfolger in Familienunternehmen ihre beruflichen Lebenspläne nicht in erster Linie an den gesellschaftlichen „Normalbiografien“ orientieren können.

\subsubsection{Inkorporation einer Leistungskultur}

In den biografischen Selbstdarstellungen der Übergeber taucht kein spezifisches Alter als legitime Grenze der Arbeitsphase auf, selten werden andere Familienmitglieder oder etwa der Beginn einer Lebensversicherung als Kriterium für den Beginn dieser Lebensphase erwähnt. Stattdessen bestimmt sich das Ende der Wirkungszeit im Unternehmen allein durch den Nutzen der Person für das Unternehmen, der sich an dem Maßstab der körperlichen Gesundheit und Leistungsfähigkeit orientiert:

„Es war absehbar, dass irgendwann irgendwas geschehen musste. Und ehe ich also, hätte konstatieren müssen, ich überblicke nicht mehr alles. Mir fallen die Sachen aus den Fingern. Also dann habe ich gedacht, lieber so, nicht?" (Erwin Carstens) ${ }^{99}$

„Ich sage immer, nun ja, so lange ich kann, mache ich das. Aber natürlich nicht mehr in dem Maße, weil eben doch die Kräfte nachlassen und so weiter." (Beate Übermann, 39:18-20)

„Ja, ich kann natürlich, wir wissen nicht, wie viele Jahre mir noch oder Wochen oder Monate beschieden sein werden, aber so lange ich es kann, mache ich es.“ (Felix Walter Naumann, 10:10-12)

99 Das Interview mit Erwin Carstens wurde nicht transkribiert. Entsprechend können keine Seitenzahlen und Zeilennummern angegeben werden. 
Dieser überdeutliche Bezug zur eigenen körperlichen Fitness in den Selbstdarstellungen der Familienunternehmer verschmilzt mit den bisher aus anderen Lebensphasen herausgearbeiteten typischen narrativen Mustern zu einem kohärenten Bild. Mit dem „Entschluss“, den Weg in das Unternehmen zu beschreiten, geben Familienunternehmer gegenüber ihren Familienmitgliedern und auch gegenüber ihrer Belegschaft ein Leistungsversprechen ab. Sie verpflichten sich dem Kontinuitätsanspruch des Projektes „Unternehmerfamilie“, der Sicherung von Arbeitsplätzen und unternehmerischem Innovationsgeist. Im Laufe ihrer beruflichen Bewährung verinnerlichen sie ein meritokratisches Denken, das sich an den Erfolgsmaßstäben der Marktwirtschaft einerseits sowie der institutionalisierten Erfolgskultur der Unternehmerfamilie andererseits orientiert. Insofern setzen Unternehmerfamilien die gesellschaftlich um sich greifende Pflicht zum Erfolg, wie sie von Sighard Neckel (2008) beschrieben wird, konsequent um. Umsatz, Gewinn, Mitarbeiterzahl, aber auch die Dauer des Bestehens, Mitarbeiterzufriedenheit etc. werden als Erfolgsindikatoren nicht nur in der Bewertung der Mitarbeiter, sondern auch des eigenen beruflichen Wirkens angesetzt. Die soziale Kontrolle durch andere Familienmitglieder und die Familienhistorie verstärkt den permanenten Erfolgsdruck. Die Phase der Leistungserbringung des Familienunternehmers gestaltet sich, zumindest auf der Ebene der Selbstdarstellung, als körperlich und geistig fordernd. Die hohe Zahl an Arbeitsstunden, persönliche Verfügbarkeit, die Notwendigkeit, sich immer wieder technischen Neuerungen anzupassen und gar proaktiv zu handeln, etc. verlangen den Familienunternehmern ein hohes Maß an Leistungsvermögen und körperlicher Fitness ab. Aus Anlass von Firmenjubiläen, des Eintritts des Nachfolgers oder aufgrund runder Geburtstage resümieren Familienunternehmer über ihren eigenen beruflichen Werdegang und stellen in ihren biografischen Zukunftsplänen Nützlichkeitserwägungen über ihre weitere Schaffenszeit an. Dabei lassen sich zwei diametral gegensätzliche Einstellungen zum Rückzug aus dem Unternehmen aus dem Interviewmaterial kondensieren:

Die erste Gruppe möchte zu einem Zeitpunkt des gerade beginnenden Leistungsverfalles ausscheiden und auf diese Weise innerhalb des Unternehmens keinesfalls peinlich oder belastend wirken. Konrad Niest etwa sagt: , „ich muss für mich ... das Gefühl haben mein Gehalt wert zu sein, ... ja? ... Ist natürlich immer, immer schwer festzustellen, ob das nun wirklich die, die letzten 100 Euro dann auch noch äh verdient sind, aber ... äh ... ich will also eigentlich hier nicht als Sozialmaskottchen rumlaufen, sondern als, als jemand, der ... * $\mathrm{pf}^{*}$ (Luft ausblasend) was Positives zum Unternehmensergebnis beiträgt. ... Und wenn ich das Gefühl habe (schlägt mit etwas auf den Tisch) es ist nicht mehr so, ... ja, dann würde ich schon ganz gern ausscheiden." (35:27-33) Den Familienunternehmern dieser Gruppe ist gemein, dass sie die restliche zur Verfügung stehende Zeit noch anders nutzen möchten: ,,ich hätte eigentlich so viele Dinge, die ich noch machen wollte, ... äh näm- 
lich noch viele Bücher lesen und_und äh wir kriegen nächste Woche wieder einen Hund. Mal mit dem Hund spazieren gehen, solche Dinge und auch mal .. tagsüber mal sagen, okay, ich lege mich in meinem Garten und mach mal den Faulen, also Langeweile bekäme ich nicht.“" (Florian Wagner, 36:20-24) Diese Gruppe entwirft also eine biografische Vorstellung ihrer Altersphase, die sich stark an jener des institutionalisierten Lebenslaufes orientiert. Das Ende der Berufstätigkeit ist dabei ein ebenso fester Bestandteil wie der Entwurf eines alternativen Lebensplanes. Auch wenn in den biografischen Selbstdarstellungen für den Zeitpunkt dieses Überganges kein chronologisches Alter benannt wird, wäre es interessant, eine eventuelle Orientierung an der institutionalisierten Altersgrenze quantitativ zu prüfen. Folglich legen die Fallauswertungen und auch die oben vorgestellte Typologie nahe, dass es sich beim „Ruhestand“ nur um eine Variante handelt, wie Familienunternehmer ihre Verbindung zum Familienunternehmen beenden bzw. die Phase des Alters gestalten.

Die zweite Gruppe geht hingegen von einer graduellen Veränderung der Tätigkeiten entsprechend ihres Leistungsvermögens aus und sieht auch in repräsentativen und sozialen Funktionen eine Nützlichkeit. Barbara Vester etwa hat ,immer gesagt, irgendwann mache ich dann hier nur noch den Grüßaugust. [ausgelassen] Dann latsche ich durch das Restaurant und mache die Honneurs äh, ob mir das reicht, das weiß ich noch nicht, aber das ist so das, was ich mir mal vorstellen könnte, dass man sich so langsam immer mehr rausklinkt, dass man dann sagt, okay, ... man arbeitet halt mal nur noch 3 Tage die Woche oder an bestimmten Stellen oder äh, ich glaube ein bisschen Gästekontakt, das brauche ich schon noch.“ (51:5-13) Der Wunsch, möglichst lange im Unternehmen aktiv zu arbeiten und dort ,seinen Platz“ zu behalten, ist hier sehr stark ausgeprägt. Eine Vorstellung des „Ruhestandes“ existiert nicht. Einer unserer Gesprächspartner hat das Unternehmen gar erst in seinem „Ruhestand“, also nach der altersbedingten Entlassung aus seiner Erwerbstätigkeit, übernommen. Die Tätigkeiten der alternden Familienunternehmer hören nicht gänzlich auf, sondern sie verschieben sich. Sie sind meist noch mit einem großen Gewicht im Unternehmen versehen, wie etwa bei der Betreuung von zentralen Kunden, von ganzen Produktionsstandorten oder aber in Beratungsfunktion bei strategischen und finanziellen Entscheidungen. Damit wird der Rückzug aus dem Unternehmen zu einem gleitenden Ende. In dieses Bild passt, dass Kim und DeVaney (2003) in ihrer Untersuchung eine relativ hohe Zahl an Familienunternehmern feststellen, die sich ein Altersteilzeitmodell vorstellen können. Diese „Unruhe“ oder Aktivität ist nicht nur Ausdruck von körperlicher Fitness und „Spaß“ an der Arbeit, sondern eben auch Ausdruck des Wunsches, weiterhin gebraucht zu werden und etwas bewirken zu können. Das Deutungsmuster einer Leistungsgesellschaft, in der man nicht zum alten Eisen gehören möchte, ist hier Fluchtpunkt der biografischen Lebensplanung für das Alter. 
Interessanterweise begegnet man in den Interviews mit den Nachfolgern dem narrativen Gegenstück zu dieser Orientierung an einer Leistungsgesellschaft. Malte Niest sagt über seinen Vater: „Er soll nie aufhören, (schlagendes Geräusch) er soll nie aufhören, er soll immer seinen Arbeitsplatz haben.“ (46:15-16) und Andreas Vester äußert sich in ähnlicher Weise über seine Eltern: „ICH HOFFE, also ich werde meine Eltern mit Sicherheit nicht hier rausschmeißen, sondern wenn sie da unterstützend tätig sind und der Herr Wagner ist, wie gesagt, der, eigentlich der kreative Kopf hier. Und so lange er einen kreativen Kopf hat, soll er das auch weitermachen und sich um diese Sachen kümmern.“ (33:31-34) Dieser ausgeprägte Wunsch der nachfolgenden Generation, dass die Eltern noch länger im Unternehmen tätig sein sollen, verwunderte zunächst in der Fallauswertung. Als Lesarten wurden etwa mangelndes Selbstvertrauen oder auch eine fehlende Autonomisierung von den Eltern in Betracht gezogen. Jedoch erhält dieser Wunsch hinsichtlich der These eines alternativen, leistungsorientierten Fluchtpunktes in der Lebenslaufgestaltung von Familienunternehmen eine andere Bedeutung. Die Nachfolger bringen mit diesem narrativen Muster ihre Anerkennung für die Erfahrung und Leistungsfähigkeit der Eltern zum Ausdruck. Zugleich geben sie zu erkennen, dass für sie so lange ein Platz im Unternehmen reserviert ist, bis diese Leistungsfähigkeit nachlässt. Damit soll nicht behauptet werden, dass Nachfolger und Übergeber immer die gleiche Vorstellung vom Rückzug aus dem Familienunternehmen teilen.

Die Konsequenz einer solchen Lebenslauforientierung ist zunächst darin zu sehen, dass keine Entlastung durch institutionelle Altersgrenzen erfolgt. Der Zeitpunkt des „Leistungsnachlasses“ für den allmählichen Übergang aus dem Ruhestand erfordert ein hohes Maß an Reflexivität und Flexibilität, sowohl in der Gestaltung des eigenen Lebenslaufes als auch in der Gestaltung der Generationenbeziehungen. Das Fehlen einer fixen Altersgrenze erlaubt ein größeres Entfaltungspotenzial individueller Vorstellungen und Persönlichkeit inklusive ihrer ,dunklen“ Seiten wie etwa übergebührlichen Eigentumsgefühlen. Es erweist sich als Herausforderung für beide Generationen, die Übertragung der Verantwortung mit dem Bedürfnis, weiterhin gebraucht und gefragt zu werden, in Einklang zu bekommen. Vor dem Hintergrund sowohl einer hohen emotionalen Verbundenheit mit dem Unternehmen als auch dem Ideal einer Leistungsgesellschaft als biografischer Fluchtpunkt scheint der Wunsch nach einem graduellen Rückzug plausibel. Ebenso wird es nicht notwendig, die eigene Identität mit neuen Inhalten zu füllen. Die rigorose Identitätsbehauptung des „Unternehmerseins“ und seine anhaltende Aktivität können, folgt man der Kontinuitätsthese, auch im Alter zu einer hohen Lebenszufriedenheit führen und den Prozess des geistigen und körperlichen Verfalls abfedern (Bäcker 2008; Moen 1998). 


\subsubsection{Fazit: Das Ruhestandsmodell und das Leistungsmodell}

Es wird also vorgeschlagen, den Rückzug aus dem Familienunternehmen entlang der beiden Orientierungspunkte des „Normallebenslaufes“ in der Arbeitsgesellschaft („Ruhestand-Modell“) einerseits und eines voll flexiblen Überganges in der Leistungsgesellschaft (,Leistungs-Modell“) andererseits zu charakterisieren. Die „Schwierigkeiten des Loslassens“ stellen sich in diesem Lichte nicht notwendigerweise als Unfähigkeit oder als pathologisch dar, sondern als eine spezifische Strategie, mit dem Ende der Leistungskraft und der Tatsache der eigenen Sterblichkeit emotional zu verfahren (so etwa auch Dunn 1999:47-48), als ein Ausdruck inkorporierter Erfolgskultur.

Abschließend stellt sich die Frage, welche Faktoren die Ausgestaltung der Altersphase entlang dieser zwei Pole - „Ruhestandmodell“" vs. „Leistungsmodell“" - beeinflussen. Folgt man dem Erklärungsansatz des psychologischen Eigentums, so spielen insbesondere die Wirkdauer im Unternehmen sowie die besonderen Umstände des Eintrittes in das Unternehmen (Aneignung des Besitzobjektes) eine große Rolle darin, wie sich später der Lösungsprozess von dem Unternehmen gestaltet. In den vorliegenden Fällen zeichnet sich noch ein weiterer Faktor ab: Es besteht eine relativ hohe Kongruenz zwischen der alltäglichen Lebensführung in der Phase des „Unternehmerseins" sowie der Art und Weise des Rückzuges aus dem Familienunternehmen. Der Familienunternehmer, der seinen Alltag nach dem Typ „Festung" organisiert, lebt eine strikte Trennung zwischen Unternehmen und Familie, ähnlich einem abhängigen Beschäftigungsverhältnis. Es scheint plausibel, dass sich deshalb auch der Rückzug aus dem Unternehmen an den typischen Übergängen im Erwerbsleben orientiert. Jene Familienunternehmer, die ihren Alltag eher nach dem Typ „Verschmelzung“ organisieren, praktizieren zeitlebens die Ideale einer wirtschaftlichen Erfolgskultur. So ist es nur konsequent, dass sich solche Familienunternehmer auch im Alter ganz an ihrer Leistungsfähigkeit orientieren. Kurzum, es wird vorgeschlagen, dass die Art der Lebensführung in der Phase des Unternehmerseins die biografischen Lebenspläne für das Alter beeinflusst und sich in ihrer konkreten Ausgestaltung als handlungswirksam erweist. Auf diese Weise entsteht eine Pfadabhängigkeit der Lebenswege von Familienunternehmern.

\subsection{Biografische Selbstbestimmung?}

In diesem Kapitel ist einmal hypothetisch der Lebensweg eines Familienunternehmers von der Kindheit bis ins Alter beschritten worden. Die aus den vorliegenden Fallanalysen kristallisierten Lebensphasen und Lebensthemen 
wurden sowohl in der Breite der alltäglichen Lebensführung als auch in der Tiefe des Lebenslaufes und der Biografie näher beschrieben, wobei in den jeweiligen Lebensbereichen verschiedene Varianten und Faktoren der Alltagsgestaltung sowie biografischer Orientierungspunkte identifiziert werden konnten. Strukturelle Unterschiede in der Gestaltung der Lebensführung und des Lebenslaufes zwischen landwirtschaftlichen, industriellen oder dienstleistungsorientierten Betrieben und auch im Hinblick auf die Größe des Unternehmens erscheinen im Hinblick auf die sich ergebenden zahlreichen Parallelen vernachlässigbar klein.

Dargestellt wird in idealisierender Weise ein kontinuierlicher und quasi maximaler Einfluss des Unternehmens auf das Leben eines „Familienunternehmers", wohl wissend, dass das Unternehmen nicht immer in allen Lebensphasen präsent ist (z.B. bei Unternehmensgründung mit erwachsenen Kindern) und sich der Einfluss nicht bei allen Mitgliedern einer Unternehmerfamilie in gleicher Intensität entfaltet. Gleichsam scheint keine ExitOption aus der Unternehmens-Familien-Konstellation $\mathrm{zu}$ bestehen, denn selbst, wenn sich ein Familienmitglied für einen anderen Beruf und ein anderes Lebensmodell entscheidet, bleibt das Kollektiv der Unternehmerfamilie im Erfahrungsbereich dieser Person etwa über Erzählungen der anderen Familienmitglieder (Großeltern, Eltern, Geschwister), über Familientische zur Erbschaftsregelung usw. Sogar in einem extremen Fall bewusster Abkehr von der Unternehmerfamilie gilt es, diese Lebenslaufentscheidung biografisch zu integrieren. Die Ergebnisdarstellung legt die Art und Weise offen, wie das Unternehmen in den verschiedenen Lebensphasen wirken kann:

Das Unternehmen wird zu einem Bestandteil der Alltagswirklichkeit von Kindern und Jugendlichen, die in einer Unternehmerfamilie aufwachsen. Diese ist maßgeblich von der physischen Erfahrung des Unternehmens als Örtlichkeit, dem Umgang mit den Menschen aus den Unternehmen, Erzählungen über das Unternehmen, der wirtschaftlichen Situation der Familie, der aktiven Mitarbeit im Unternehmen sowie der stillen Beobachtung des Unternehmens als Teil der elterlichen Lebensführung geprägt. Kinder nehmen die Bedeutungen wahr, die Erwachsene dem Unternehmen beimessen und machen ihre eigenen Erfahrungen im Umgang mit diesem. Sie gewinnen ganz selbstverständlich einen Wissensvorrat über das Unternehmen, internalisieren Rollenzuschreibungen und greifen auf Generations- und Institutionsbeziehungen zurück. Damit fließen die Erfahrungen mit dem Unternehmen in das Netz der Verarbeitungs- und Bewältigungsmuster ein, mit dem Kinder und Jugendliche ihre gesellschaftliche Umwelt deuten. Sie entwickeln eine kindliche Theorie vom Leben einer Unternehmerfamilie, die Konsequenzen für ihre eigene Identitätsentwicklung, berufliche Orientierung und biografische Lebensplanung mit sich bringen. Deutlich geworden ist vor allem die Wucht der „Erlebniswelt“ Unternehmen, in der weniger die Koordination unterschiedlicher funktional differenzierter Lebensbereiche im Vordergrund steht, 
als ein kontinuierliches Streben nach Aufmerksamkeit und die Suche nach der eigenen Rolle innerhalb der Unternehmerfamilie; insofern ist das Unternehmen in dieser Lebensphase fester Bestandteil des familialen Raumes. Das Aufwachsen mit einem Familienunternehmen ist kein Garant für eine starke Bindung an das Familienprojekt. Aus einer sozialisationstheoretischen Perspektive lässt sich sicherlich feststellen, dass Kinder und Jugendliche in dieser Zeit unternehmerische Werte und Wissen internalisieren, die ihnen in ihrem späteren beruflichen Werdegang (und eventuell auch der Partnerfindung) zu Gute kommen können. Betont werden soll im Rahmen dieser Arbeit vor allem, dass Kinder und Jugendliche eine Vorstellung davon entwickeln, was es heißt, eine Unternehmerfamilie zu sein und damit auch davon, ob sie dieses Lebensmodell in ihrer eigenen Lebensplanung reproduzieren wollen oder nicht.

Eben diese Feststellung wiederholt sich in der Analyse der Wege in das Familienunternehmen, die sich am ehesten als „Entscheidung“ für ein spezifisches Lebensmodell charakterisieren lässt. Aus dem empirischen Material wurden zunächst vier typische Lebenslaufmuster in das Familienunternehmen abgeleitet und beschrieben. Das Familienunternehmen mag als $\mathrm{Zu}-$ fluchtsort, als Mission, als Ressource oder als emotionale Heimat Orientierung im Lebenslauf bieten. In einem zweiten Abstraktionsschritt wurden jene strukturellen Faktoren benannt, die auf Mitglieder der Unternehmerfamilie einwirken und bestimmen, welcher berufliche Weg eingeschlagen wird. Neben den in der Berufswahl üblichen Faktoren von Fähigkeiten, Lebenslage und Gelegenheitsstrukturen scheinen in Unternehmerfamilien die emotionale Bindung an das Unternehmen sowie die Generationenbeziehungen die Lebenslaufentscheidungen nachhaltig zu beeinflussen. Diese Handlungsstrukturen und -zwänge formen ein konstantes, fast a-historisches Muster. Der dritte Analyseschritt widmete sich der biografischen Bearbeitung dieser Strukturen und identifizierte, unabhängig davon, welcher Weg nun beschritten wird, ein einheitliches narratives Muster der „freien Wahl“. Die in der biografischen Selbstdarstellung aufgeführten ,guten Gründe“ für einen Eintritt in das Familienunternehmen dienen dazu, den eigenen Lebensweg als ,sinnvoll“ zu begreifen und zu rechtfertigen. In ihnen spiegeln sich aktuelle und kulturelle Vorstellungen des modernen Lebens wider. Die Konstellation der Unternehmerfamilie muss Platz für eine Identität bieten, die sich aus den jeweils geltenden gesellschaftlichen Skripten speist. Mit dem Narrativ der freien Wahl wurde damit eine Strategie beschrieben, wie der Code zur Individualisierung mit der Kontinuität des Familienunternehmens in Einklang gebracht werden kann. Die ausscheidende Generation betont, dass das Familienunternehmen nur eine Option unter vielen sei. Die jüngere Generation präsentiert die Verbindung aus Unternehmen und Familie als einen spezifischen Lebensstil, für den sie sich bewusst entschieden habe. Abschließend wurde die These entwickelt, dass in dem permanenten und alltäglichen Wechselspiel aus Hand- 
lungs- und Deutungsmustern das Narrativ einer ,freien Entscheidung“ des faktischen Erlebens bedarf, um eine nachhaltige Identitätsbildung zu ermöglichen. Der Eintritt in das Familienunternehmen markiert einen Übergang im Lebenslauf der Familienunternehmer, der in das Verlaufsmuster der Nachfolge eingebettet ist. Hinsichtlich der von Moen vorgeschlagenen Kategorie des „Timings“ von Übergängen im Lebenslauf lässt sich eine weitere Besonderheit hervorheben: Der Eintritt in das Familienunternehmen kann - wie auch eine Unternehmensgründung - theoretisch zu fast jedem Zeitpunkt im Leben erfolgen und muss deshalb als eine zumindest unterschwellig lebenslange Option verstanden werden. Der konkrete zeitliche Auslöser bestimmt sich aus der Synchronisation der Lebensläufe und Lebensentwürfe der Familienmitglieder.

Auch im Hinblick auf familiales Handeln konnte der Einfluss des Unternehmens offen gelegt werden: Bei der Paarbildung stehen Zukunftsvorstellungen über die eigene Lebensführung im Vordergrund, die sich aus den persönlichen Erfahrungen sowie Idealvorstellungen zum Unternehmersein speisen und die eine aktive Konstruktion der Grenze zwischen Arbeit und Leben, zwischen Unternehmen und Familie beinhalten. Es wurde die These entwickelt, dass die an sich freie Partnerwahl in Unternehmerfamilien durch die zusätzlichen Selektionskriterien einer sehr speziellen biografischen Vorstellung des Lebensmodelles Unternehmerfamilie und einer aktiven Rolle der Eltern in der Lebensführung von Nachfolgern voraussetzungsreicher ist. Bezogen auf eine familieninterne Nachfolge und damit auf die Reproduktion der Unternehmerfamilie wird die Partnerwahl zu einem Risikofaktor. Hinsichtlich des Überganges in die Elternschaft konnte die These vertreten werden, dass in Unternehmerfamilien der Kinderwunsch nicht im Sinne einer strategischen Nachfolgeplanung zur Erhöhung der Zahl potentieller Übernehmer erfolgt. Stattdessen scheint die Frage, ob und wie viele Kinder ein Paar bekommt, sich aus den auch in anderen Familien wirksamen Faktoren abzuleiten. Als Besonderheit erwähnenswert ist jedoch, dass Kinder den biografischen Horizont der Eltern in Richtung einer Kontinuität des Familienprojektes öffnen. Als dritter Bereich des familialen Handelns wurde schließlich der Familienalltag näher analysiert. In der alltäglichen Lebensführung des Paares und der jungen Familie zeigt sich, inwiefern die biografischen Entwürfe und Vorstellungen des Lebensmodelles einer Unternehmerfamilie handlungspraktisch umgesetzt werden. Familienunternehmer müssen immer wieder aufs Neue bestimmen, wie stark das Unternehmen den Familienalltag beeinflusst, müssen aktive Grenzarbeit leisten, die als Ergebnis eines stetigen Verhandlungs- und Verarbeitungsprozesses von persönlicher Lebenserfahrung, den Wünschen des Partners und Gelegenheitsstrukturen verstanden werden kann. Das Familienunternehmen erhöht die soziale Dichte in Unternehmerfamilien hinsichtlich einer sozialen und räumlichen Nähe sowohl zum Betrieb als auch zu anderen Familienmitgliedern. Aus dem Interviewmaterial wurden in An- 
lehnung an die Typenbildung von Sue Birley drei idealtypische Gruppen der praktizierten Grenzarbeit herausgearbeitet: Der Typ „Verschmelzung“ unterscheidet nicht in ein Unternehmen hier und Familie da. Der Typ „Festung“ praktiziert hingegen eine strikte Trennung beider Bereiche ähnlich einem abhängigen Erwerbsverhältnis. Der Typ „Zwei Kerne“ schließlich bildet eine intermediäre Form der Grenzarbeit. Das Interviewmaterial zeigt deutlich, dass Kinder die Art und Weise, wie ihre Eltern die Grenze zwischen Unternehmen und Familie vorleben, wahrnehmen und darüber reflektieren. Die Tradierung der Lebensweise einer Unternehmerfamilie erfolgt nicht unhinterfragt. Die Reflektion über die Form der praktizierten Grenzarbeit fließt als Fluchtpunkt oder Abgrenzungsfolie in die Ausrichtung des eigenen Lebens ein. Im Sinne der „Process“ Kategorie von Phylis Moen konnte der zentrale Stellenwert der jeweiligen Form des Familienalltags für die Leben der Familienmitglieder herausgearbeitet werden.

Die folgende Lebensphase des Unternehmerseins beschreibt eine relativ stabile Zeit der Tätigkeit im Unternehmen und der konkreten Umsetzung des Lebensmodelles Unternehmerfamilie. In den Selbstdarstellungen der Familienunternehmer zu dieser Lebensphase treten unwahrscheinlich starke Parallelen zu Tage. Mittels des soziologischen Konzeptes der Profession wurde die These entwickelt, dass sich Familienunternehmer als professionell darstellen und damit eine spezifische Strategie verfolgen. Sie beschreiben ein Bündel an typischen Tätigkeiten, die eine sehr spezifische Art des Wissens und Fähigkeiten erfordern ebenso wie eine typische Lebensführung, die über die reine „unternehmerische Tätigkeit“ hinausgeht. Teil ihrer Lebensführung ist eine hohe Arbeitsbelastung, die Notwendigkeit zur Flexibilität und Leistungsbereitschaft. Familienunternehmer vertreten ethische Grundsätze und Vorstellungen der guten unternehmerischen Praxis, die sich im Laufe der familialen und beruflichen Sozialisation produzieren und reproduzieren. Die Eckpfeiler dieses professionellen Wertesystems bilden Bescheidenheit, Sparsamkeit, Leistungs- und Risikobereitschaft, Loyalität gegenüber Mitarbeitern, öffentliches Engagement, Familiensinn und Verantwortungsbewusstsein. Zudem verfügen sie über eine klare Vorstellung von ihrer Position innerhalb der Gesellschaft (eine professionelle Selbstsicht), die sie aus ihrer Position an der Schnittstelle unterschiedlicher institutioneller Bereiche und ihrem Beitrag zu einer Leistungsgesellschaft ableiten. Auf diese Weise gelingt es ihnen, sich von anderen Gruppen (insbesondere den Managern) abzuheben, was als wichtiger Mechanismus in der Ausbildung einer individuellen Identität $\mathrm{zu}$ verstehen ist. In Abgrenzung zu der familialen und gesellschaftlichen Generation konstruieren sie eine antagonistische oder deviante Gruppe der „Anderen“. Gleichzeitig konstruieren sie sich ein Kollektiv, zu dem sie in einem gemeinschaftlichen Verhältnis stehen. Beide Mechanismen der Generationenerzählungen sind wichtige Ressourcen darin, sich selbst als Familienunternehmer zu denken. Jenseits der individuellen Ebene erhöht eine 
Selbstpräsentation als ,professionell“ den Marktwert des Unternehmens (Stichwort Markenbildung) sowie die politische Stellung der Familienunternehmer und mag sich motivierend auf Mitarbeiter und zukünftige Nachfolger auswirken. Auf einer Makro-Ebene kann das professionelle Wertesystem der Familienunternehmer dazu beitragen, ausufernden Wettbewerb zu begrenzen und Kooperation voranzutreiben. Die Analyse zeigt deutlich den durchdringenden und allumfassenden Charakter der Rollenausübung als Familienunternehmer.

In der Altersphase lässt sich feststellen, dass Familienunternehmer sich anderen institutionellen und strukturellen Rahmenbedingungen ausgesetzt sehen als abhängige Erwerbstätige und sich deshalb der Begriff „Ruhestand“ als Bezeichnung für diese Lebensphase nur bedingt eignet. Der Rückzug aus dem Unternehmen gestaltet sich als Aushandlungsprozess mit einer Vielfalt an potentiellen Orientierungspunkten, in dem individuelle Bedürfnisse mit den Erwartungen anderer Familienmitglieder und der Belegschaft ebenso wie mit gesellschaftlichen Altersnormen koordiniert werden müssen. Im Vergleich zu einem abhängig Erwerbstätigen kommt in diesem Prozess den generationalen Verwicklungen ein höheres Gewicht zu, wohingegen die gesellschaftlichen Altersnormen weniger stark mit faktischen Altersgrenzen und Sanktionen (z.B. Abschlag in der Höhe der Rentenzahlung bei Frühverrentung) belegt sind. Die Art und Weise, wie sich nun der Rückzug aus dem Unternehmen gestaltet, ist vielfältig und unterscheidet sich zum Teil drastisch von den Normalerwartungen an einen Lebenslauf. In der Literatur ist die (psychologische) Fähigkeit des Loslassens als wichtige Entwicklungsaufgabe in der Beschäftigung mit der individuellen Alterung und als wesentliches Kriterium für den Rückzug aus dem Familienunternehmen identifiziert und mit dem Konzept des psychologischen Eigentums theoretisch untermauert worden. Aus dem Interviewmaterial wurde die ergänzende These entwickelt, dass der Rückzug aus dem Familienunternehmen entlang zweier Orientierungspunkte des „Normallebenslaufes“ in der Arbeitsgesellschaft (,Ruhestand-Modell") einerseits und eines voll flexiblen Überganges in der Leistungsgesellschaft (,Leistungs-Modell“) andererseits erfolge. Aus dieser Perspektive stellen sich die „Schwierigkeiten des Loslassens“ weniger als Unfähigkeit oder als pathologisch dar denn als eine spezifische Strategie, mit dem Ende der Leistungskraft und der Tatsache der eigenen Sterblichkeit emotional zu verfahren.

Diese kurze Zusammenfassung verdeutlicht das Wechselspiel aus alltäglicher Lebensführung und Erfahrung, biografischer Konstruktion und ihrer Umsetzung bzw. Revision über das Leben hinweg. Der Einfluss des Unternehmens auf die Familie in vorgelagerten Lebensphasen mag die biografische Selbstsicht inklusive biografischer Zukunftspläne beeinflussen und sich, selbstverständlich immer in Abhängigkeit von Gelegenheitsstrukturen und strukturelle Begrenzungen, auf die Gestaltung nachgelagerter Lebensphasen 
auswirken, bis dahin, dass sich bestimmte Pfadabhängigkeiten im Leben von Familienunternehmern beobachten lassen. Die Vorstellung einer Unternehmerfamilie als ein spezifisches Lebensmodell, das eine aktive Konstruktion des Verhältnisses von Unternehmen und Familie beinhaltet, bestätigt sich in allen Lebensphasen und verdichtet sich zu einer Fall übergreifenden Strukturhypothese.

Insgesamt können wir festhalten, dass das Unternehmen den Lebenslauf der Familienunternehmer maßgeblich beeinflusst. Es ist die Verbindung von Familie und Unternehmen (nicht unbedingt das Unternehmen an sich), die einen Orientierungsrahmen für das Leben, eine bestimmte Sequenzialität im Lebenslauf sowie einen Fluchtpunkt in der Ausbildung von Identität schafft. Es gibt eine Phase „Vor“, „im“ und „ausscheidend“ aus dem Unternehmen, auch wenn die Grenzen der einzelnen Lebensphasen ebenso wie ihr „Timing" im Lebenslauf weniger stark institutionalisiert erscheinen. Das Programm eines „Normallebenslaufes“ lässt sich nur bedingt auf die Familien eigener Art übertragen. Grund dafür scheint vor allem die besondere Qualität der Tätigkeit im Unternehmen zu sein, die sich nur schwer einfangen lässt mit den üblichen Begriffen der „Berufswahl“, der „Profession“ oder des „Ruhestandes“, die auf der Vorstellung abhängiger Erwerbsarbeit und ihren flankierenden Sozialversicherungssystemen sowie gesellschaftlichen Altersnormen fußen. Der Einfluss des Unternehmens scheint die Flexibilität in der Ausgestaltung des Lebenslaufes und der Lebensführung zu erhöhen und die Einflusskraft der institutionalisierten Vorgaben des Normallebenslaufes zumindest teilweise zu reduzieren. Das Unternehmen schafft flexible Strukturen hinsichtlich der Ausgestaltung und Finanzierung einzelner Lebensphasen sowie der Ordnung der Lebensphasen über den Lebenslauf hinweg. Zwar stehen auch Unternehmerfamilien nicht außerhalb der Gesellschaft mit ihrem spezifischen Wohlfahrtssystem, einer gegebenen Infrastruktur, kulturellen Vorgaben usw., dennoch scheint es in ihrem Leben eine außergewöhnlich hohe potentielle Eigenbestimmtheit zu geben. ${ }^{100}$

Familienunternehmer stehen vor der Aufgabe, die entstehenden Freiräume aktiv zu gestalten. Sie müssen eine Grenze zwischen Arbeit und Leben aktiv konstruieren und haben dabei die Möglichkeit, die Vereinbarkeit beider Bereiche und ihre Zeiteinteilung zu optimieren. Andererseits ist dafür eine hohe gestalterische Leistung erforderlich. Hinzu kommt, dass Familienunternehmer diese Freiräume immer nur innerhalb des unternehmerfamilialen Kontextes gestalten und mit Sinn erfüllen können - insofern wird die poten-

100 Erste Gedanken zu der biografischen Selbstbestimmung von Familienunternehmen habe ich gemeinsam mit meinen Kollegen des Forschungsprojektes „Von Generation zu Generation“ auf dem 34. DGS Kongress „Unsichere Zeiten“ in Jena vorgestellt und sie sind in dem anschließenden Tagungsband veröffentlicht worden Stamm et al. 2009a. Seither ist diese Idee kritisch beleuchtet und weiter entwickelt worden. 
tielle Eigenbestimmtheit begrenzt. Ein möglicher Einstieg in das Familienunternehmen, der Zeitpunkt des Einstieges, die Übertragungen von Unternehmensanteilen, die Rollen einzelner Personen innerhalb der Unternehmerfamilie uvm. sind das Ergebnis einer Koordination der Lebensphasen und direkter Aushandlungen unter den Familienmitgliedern. Kennzeichnend für ein solch direktes Generationenverhältnis sind vielerlei emotionale Ambivalenzen und Oszillationen im Verlauf einer langjährigen gemeinsamen Familiengeschichte (Breuer 2009:163). Entsprechend fließen in diese Aushandlungen die je spezifischen Vorstellungen der Familienmitglieder zur Kontinuität des Unternehmens, ihrer Rolle innerhalb der Unternehmerfamilie ebenso wie gesellschaftliche Deutungen und Regelungen von Alter, Altersgrenzen und Generationen ein.

Das sehr spezielle generationale Setting einer Unternehmerfamilie stellt für die Autonomisierungs- und Individualisierungsbestrebungen der Familienmitglieder eine Herausforderung dar und erfordert ein erhöhtes Maß an biografischer Arbeit (Wenk 2007). Der Familienunternehmer ist gefordert, die bestehenden Strukturen sowie die Ergebnisse der Aushandlungen von seinem Ich aus zu strukturieren und ein verzeitlichtes Selbst zu konstruieren. Insofern wird der Familienunternehmer zu einem besonderen Unternehmer seiner eigenen Biografie (Down 2006:5). Die Analyse zeigt, wie diese Individualisierungsaufgabe in allen Lebensphasen erhalten bleibt. Die lebenszeitlich unterschiedlichen Bedürfnisse nach Autonomie, Aufstieg, Unabhängigkeit oder Nützlichkeit sind immer im Kontext der Unternehmerfamilie zu befriedigen (Handler 1992:292). Für den Weg in das Familienunternehmen ist das Narrativ der freien Wahl als eine solche Strategie vorgeschlagen worden; das Verständnis der Unternehmerfamilie als eine spezifische Art und Weise der Lebensführung, als ein Lebensstil und auch die Darstellung des Familienunternehmertums als professionell können als weitere Antworten auf diese Individualisierungsherausforderungen gelesen werden. Diesen Strategien ist gemein, dass sie in starkem Maße mit „Abgrenzungsmetaphern“ operieren. Sie rufen antagonistische Bilder in der Form von ,die Jungen und die Alten“, „Tradition und Innovation" sowie „Unternehmer und Angestellte" hervor, die dazu beitragen können, die eigene Individualität von anderen Gruppen abzugrenzen. Die Schwierigkeit der biografischen Selbstbestimmtheit in Unternehmerfamilien ist darin zu sehen, dass diese keine Selbstverständlichkeit darstellt und einen lebensprägenden Rechtfertigungsdruck hervorruft. Die Selbstpräsentationen von Familienunternehmern versuchen, den Code der Individualisierung sowohl mit den gesellschaftlichen Vorgaben als auch mit den spezifischen Strukturen einer Unternehmerfamilie zu versöhnen. 


\section{Familiale (Generationen-)Beziehungen in Unternehmerfamilien}

Es ist unmöglich, den Lebenslauf von Menschen aus Unternehmerfamilien zu betrachten, ohne dabei auf das Verhältnis der Generationen einzugehen. Die Darstellungen in dem vorangegangenen Kapitel haben deutlich gezeigt, wie eng die Leben der jeweiligen Familienmitglieder verbunden sind, viel enger als dies in der Forschung mit dem Begriff ,linked lives“" (Elder 1985a; Elder 1995) beschrieben wird. Das Unternehmen wird zum Gegenstand familialer Kommunikation und zum Ring familialer Annerkennungskämpfe. Im Folgenden sollen nun diese Begegnungen der Generationen unter Einfluss des Unternehmens explizit zum Gegenstand der Analyse werden. Zunächst werden dabei bereits erarbeitete Erkenntnisse zusammengefasst und systematisch die verschiedenen Dimensionen der Generationenbeziehungen in Unternehmerfamilien beschrieben. Der zweite Abschnitt widmet sich den spezifischen Koordinationsproblemen zwischen den Generationen, die sich in diesen Familien eigener Art ergeben und in einem direkten Aushandlungsprozess zu lösen sind. Der dritte Abschnitt wirft schließlich die Frage auf, auf welche Art und Weise diese Aushandlungen stattfinden und Lösungsstrategien entworfen werden. In einem Resümee tritt die Unternehmerfamilie als generationale Gemeinschaft hervor.

\subsection{Dimensionen der Generationenbeziehungen}

Im Gegensatz zu dem personalen Zugang zum Leben von Unternehmerfamilien des vorangegangenen Kapitels stehen im Folgenden die Begegnungen der Generationen im Vordergrund. Es ist nahe liegend, in diesem Zusammenhang von Generationen ${ }^{101}$ in ihrem familialen Sinn zu sprechen, also das

101 Der Begriff Generation(en) ist in den letzten Jahren schillernd und inflationär gebraucht worden. Mit der Genealogie und Zirkulation des Begriffes „Generation“ im Hinblick auf semantische Verschiebungen, den Einsatz in historischen Debatten und verschiedenen Forschungsfeldern sowie seiner Reichweite haben sich unter anderem Parnes, Vedder und Willer 2008 auseinander gesetzt. Für einen Eindruck von der Interdisziplinarität dieses Forschungsgegenstandes sei auf den Sammelband von Künemund und Szydlik 2009 verwiesen. Im Rahmen dieser Arbeit werde ich mich vor allem auf die sozialwissenschaftliche Fassung von Generationen stützen. 
Verhältnis von Großeltern, Eltern und Kindern zu betrachten, die jeweils Glieder in einer genealogischen Verbindungs- bzw. Abstammungslinie (lineage) darstellen. Im Laufe des Lebens kann das Individuum die verschiedenen Abstufungen durchlaufen und je verschiedene generationale Rollen annehmen, gleichzeitig verbleibt es in einem konstanten Altersabstand und Verhältnis zu den anderen Familienmitgliedern (z.B. als Kind von xy), zeichnet sich also durch eine spezifische Generationenzugehörigkeit aus (Künemund und Szydlik 2009:9). Die folgende Analyse rückt den Einfluss des Unternehmens auf solche familialen Generationenbeziehungen in den Mittelpunkt. Es sollen Antworten darauf gegeben werden, wie sich im Kontext einer Unternehmerfamilie die familialen Generationen aufeinander beziehen und voneinander abgrenzen, auf welche Weise sich familiale Solidarität entfaltet und, eng damit verbunden, welche Herausforderungen und Konfliktlinien auftreten.

Davon unterschieden werden muss eine zweite Analyseebene, die sich in der Generationenforschung, insbesondere angestoßen durch die Arbeiten von Mannheim, etabliert hat (siehe etwa Szydlik 2000; Kohli und Szydlik 2000 oder Künemund und Szydlik 2009): Dabei handelt es sich um Altersgruppen, in der Demografie spricht man von Kohorten, die spezifische soziale und historische Erfahrungen teilen, ein Bewusstsein für diesen gesellschaftlichen Zusammenhang entwickelt haben und als öffentlicher Akteur auftreten. Kohli und Syzdlik (2000:7f.; ausführlich Szydlik 2000:19ff.) unterscheiden diese weiter in eine Verbundenheit über politische Einstellungen und Erfahrungen (politische Generation), spezifische Lebensorientierungen und -einstellungen (kulturelle Generation), oder über ökonomische Chancen und Risiken im Leben (ökonomische Generation). Die typischen Eigenschaften einer gesellschaftlichen Generation differenzieren sie dauerhaft von nachfolgenden oder vorangegangenen Generationen. Das Konzept der gesellschaftlichen Generation wird in der Analyse von Unternehmerfamilien ausdrücklich mitgedacht, steht jedoch nicht im Zentrum des Interesses.

Dieser Abschnitt nimmt seinen Ausgangspunkt in der soziologisch geprägten familialen Generationenforschung. In einer kurzen Skizze werden zentrale Ergebnisse und Modelle eingeführt und einige wertvolle Anhaltspunkte für die Analyse herausgestellt. Im Anschluss erfolgt eine Beschreibung der verschiedenen Beziehungskonstellationen in Unternehmerfamilien, die wesentliche Ergebnisse aus Kapitel VI unter dem Aspekt der familialen Generationen zusammenfasst, pointiert und ergänzt. In einem zweiten Schritt werden weitere Generationenverständnisse in Betracht gezogen und in Beziehung zu der Dynamik zwischen den familialen Generationen in Unternehmerfamilien gesetzt. Es zeigt sich dabei, so viel kann vorab festgehalten werden, dass sich das Unternehmen auf alle sozialen Beziehungen innerhalb der Unternehmerfamilie auswirkt und damit ein neues Feld für Solidarität und Zusammenhalt, aber auch für Konflikt und Exklusion schafft. Im Lichte 
der überwiegend deskriptiven Präsentation der Ergebnisse erfolgt eine kritische Auseinandersetzung mit der eingangs kurz skizzierten Forschung zu familialen Generationen und bestehenden methodischen Differenzen, die in den Vorschlag mündet, Unternehmerfamilien als Figurationen (Elias) zu fassen, die sich über die direkte Qualität ihres Verweisungszusammenhanges auszeichnen.

\subsubsection{Soziologische Generationenforschung}

Die soziologische Forschung zu familialen Generationen hat sich in den letzten Jahrzehnten vor allem an Parsons These über die Isolierung der Kernfamilie abgearbeitet und diese mannigfach empirisch widerlegt. Es konnte gezeigt werden, dass auch zwischen erwachsenen Kindern und ihren Eltern ein weiterhin starker Generationenzusammenhalt besteht (etwa Szydlik 2008:110; Künemund und Szydlik 2009:11), größere Konflikte sind dagegen eher selten; wenn sie jedoch auftreten, droht ein Auseinanderleben der Generationen und in letzter Konsequenz der Beziehungsabbruch (etwa Kohli und Szydlik 2000). In Deutschland zeichnet sich der dominante Beziehungstyp familialer Generationen durch gegenseitige Hilfeleistungen, große emotionale Verbundenheit und häufigen Kontakt aus (Kohli et al. 2005a:204f.). Diese Erkenntnisse sind zentral für die Beurteilung der Beziehungsqualität in Unternehmernehmerfamilien und können als Vergleichsmaßstab dienen.

Die im Rahmen dieser Arbeit geleistete Analyse der Generationenbeziehungen in Unternehmerfamilien operiert jedoch auf einer anderen Ebene. Zwar lassen sich aus den Interviews Informationen über finanzielle und andere Unterstützungsleistungen, Erbschaften, Kontakthäufigkeiten und Wohnortentfernung gewinnen, diese sind jedoch nicht verallgemeinerbar und deshalb nicht besonders aussagekräftig. Vielmehr zielt die qualitativmethodische Anlage der Arbeit darauf ab, die „Qualität“ der Generationenbeziehungen aus der Perspektive des Erlebens zu fassen.

In diesem Zusammenhang erweisen sich einige theoretische Modelle und Grundbegriffe der Generationenforschung als hilfreich. Das von Bengston et al. (etwa 1976; 1990; 2001) entwickelte Modell trägt der Vielschichtigkeit der Generationenbeziehungen Rechnung und hat sich zu einem wesentlichen Bezugspunkt der internationalen Forschung entwickelt. Sie differenzieren in sechs verschiedene Arten von Solidarität zwischen familialen Generationen (Steinbach 2008:116): Strukturelle Solidarität umfasst den genealogischen Aufbau der Familie, die Anzahl der Familienmitglieder; assoziative Solidarität verweist auf die Häufigkeit und Muster der Interaktion; affektive Solidarität drückt sich im Grad der wechselseitigen Gefühle aus; normative Solidarität umfasst die Verbundenheit mit familialen Rollen und einem Verpflichtungsgefühl gegenüber den Familienmitgliedern; konsensuelle Solidarität 
meint die Übereinstimmung mit Werten, Einstellungen und Weltsichten zwischen einzelnen Familienmitgliedern; funktionale Solidarität schließlich umfasst die Unterstützungsleistungen und den Austausch von Ressourcen zwischen Familienmitgliedern. Szydlik kritisiert an dieser Einteilung, dass sie nicht zwischen „dem Potential für Solidarität und Solidarität an sich“ (Szydlik 2000:36) unterscheide. Die Kategorien der strukturellen, normativen und konsensuellen Solidarität stellen eher Möglichkeiten dar, auf denen sich Solidarität ausbilden kann, aber nicht muss. Szydlik hingegen versteht als wesentliche Elemente von Solidarität ein spezifisches aufeinander bezogenes Verhalten und ein Gefühl der Zusammengehörigkeit. Diese werden durch die verbleibenden Kategorien von funktionaler, assoziativer und affektiver Solidarität hinreichend beschrieben. Darauf aufbauend entwickelt Szydlik (2000) ein allgemeines theoretisches Modell zur Erklärung eines mehr oder weniger ausgeprägten Generationenzusammenhalts, das drei Ebenen (Individuum, Familie und Gesellschaft) sowie vier Faktorengruppen beinhaltet (Opportunitäts-, Bedürfnis-, familiale und kulturell-kontextuelle Strukturen). Die Qualität und Gestalt intergenerationaler Beziehungen hängen demnach von den individuellen Möglichkeiten, Erwartungen, Wünschen und Bedürfnissen der einzelnen daran beteiligten Personen ab (Opportunitäts- und Bedürfnisstrukturen), den Familienstrukturen, die sich im Laufe der Sozialisationsgeschichte der Familie ausbilden, und den gesellschaftlichen Rahmenbedingungen und kulturellen Vorstellungen (Künemund und Szydlik 2009:12).

Lüscher und Pillemer (Lüscher 2002; Lüscher und Pillemer 1998; Pillemer und Lüscher 2004; Lüscher 2004) kritisieren an solchen Solidaritätsmodellen, dass sie sich zu einseitig auf Zusammenhalt und die "harmonische“ Seite der familialen Generationenbeziehungen konzentrieren und dabei Konflikte vernachlässige. Sie prägen den Begriff der Generationenambivalenzen, der davon ausgeht, dass familiale Generationenbeziehungen per se mit unauflösbaren inneren Konflikten und Polaritäten verbunden sind. Diese Ambivalenzen teilt Lüscher in zwei Pole: Einerseits treffen in Familien Reproduktion und Innovation aufeinander, anderseits stehen die Familienmitglieder zwischen Annäherung und Distanzierung. Gerade die Ähnlichkeiten zwischen Eltern und Kindern haben ein hohes Potenzial der Gemeinsamkeiten und Identifikation, das in zunehmendem Alter zur Distanzierung führen kann (Lüscher 2000:151).

Bengston hat in Reaktion auf das überzeugende Konzept der Generationen-ambivalenzen sein Modell noch um die siebte Dimension „Konflikt“" erweitert. Auch Szydlik zeigt auf, wie sich in seinem Modell Ambivalenzen erfassen lassen. Er gibt allerdings zu bedenken, dass Konflikt nicht den Antipoden zu Solidarität bilde, sondern dieser in Autonomie zu sehen ist.

Folgende Punkte können auf die Analyse der Generationenbeziehungen in Unternehmerfamilien übertragen werden: 
- Ein enges Verhältnis zwischen den Generationen als Vergleichsmaßstab (Kohli, Künemund)

- Die Vielschichtigkeit der Solidaritätsbeziehungen (Bengston)

- Eine Differenzierung zwischen den strukturellen Voraussetzungen für Solidarität und ihrer handlungspraktischen Umsetzung (Szydlik)

- Die Rolle von Konflikten als Teil der Generationenambivalenz (Lüscher).

Dieser Erkenntnisgewinn der bestehenden Forschung fließt als Aufmerksamkeitspunkte in die Betrachtung der unterschiedlichen Beziehungskonstellationen in Unternehmerfamilien ein und wird am Ende des Kapitels noch einmal explizit aufgegriffen.

\subsubsection{Familiale (Generationen-)beziehungen}

\subsubsection{Paarbeziehung}

Dauerhafte Paarbeziehungen ${ }^{102}$ von Familienunternehmern (etwa in Form einer Ehe, Lebenspartnerschaft oder einer eheähnlichen Gemeinschaft) gewinnen durch das Unternehmen zusätzliche Dynamik. Dieses ist Thema der partnerschaftlichen Kommunikation und eröffnet beiden Partnern spezifische Rollen und Tätigkeiten im Unternehmen wie im häuslichen Bereich. Die Beschreibungen der Partnerwahl und Familiengründung legen offen, wie das Unternehmen ein fester Bestandteil einer gemeinsamen Lebensplanung wird, einer geteilten Vorstellung vom Leben miteinander und mit dem Unternehmen. Gerade die räumliche Immobilität, regionale Verwurzelung und Tradition der Unternehmerfamilie verleihen einem solchen gemeinschaftlichen Lebensentwurf Richtung und kommen einem Wunsch nach stabilen Verhältnissen entgegen. Wer welche Aufgabe innerhalb einer Paarbeziehung erfüllt, ist das Ergebnis eines kontinuierlichen und niemals abgeschlossenen Aushandlungsprozesses, in den sowohl persönliche, familiale als auch gesellschaftliche Vorstellungen von einer Partnerschaft einfließen. Die jeweils getroffenen Arrangements, die sich in der alltäglichen Lebensführung ausdrücken, variieren im Hinblick auf das Geschlechterrollenverständnis ebenso wie die Gleichberechtigung der Partner. Sie reichen von dem Modell eines „traditionellen Unternehmers“, in dem der männliche Unternehmer die Rolle des Hauptverdieners und Familienoberhauptes übernimmt und seiner Partnerin eine unterstützende Funktion im häuslichen Bereich zukommt, bis hin zu

102 Auch wenn die Paarbeziehung üblicherweise nicht unter einer Betrachtung von Generationenbeziehungen Erwähnung findet, würde ein wesentlicher Aspekt übersehen werden, wenn diese Beziehungskonstellation aus der Analyse ausgeschlossen wird. 
einem Modell „egalitärer Partnerschaft“, in dem beide Partner im Unternehmen verantwortungsvolle Aufgaben übernehmen, sich die Hausarbeit teilen und sich als gleichberechtigte Partner gegenüber treten. Das jeweilige Arrangement trägt das Potenzial in sich, das Unternehmen zu einem gemeinsamen Projekt der Partner werden zu lassen, in dem sie Verbundenheit erleben und Erfüllung finden, birgt allerdings auch die Risiken einer zu starken zeitlichen Belastung der Beziehung ebenso wie einer nicht übereinstimmenden Rollendefinition, die zu einer zunehmenden Entfremdung der Partner und Unzufriedenheit mit der Beziehung führen können.

Aus den Fallanalysen ergibt sich zudem eine besondere Schwierigkeit hinsichtlich der Integration des ,,angeheirateten“ Partners in das Lebensmodell der Unternehmerfamilie. Innerhalb der Paarbeziehung lassen sich meist ein beheimateter und ein fremder Partner identifizieren. Von letzterem wird eine hohe Anpassungsfähigkeit an räumliche, familiale und betriebliche Strukturen erwartet. Der hinzukommende Partner taucht in die Lebensweise des Familienunternehmers und (der Geschichte) seiner Familie ein, muss sich diese aneignen und kann sie nur graduell verändern. In diesem Prozess investiert der Partner viel Zeit und Energie in das Projekt „Unternehmerfamilie“ und ist gefordert, diese neue Identität mit vorhergehenden Lebenserfahrungen und Selbstvorstellungen in Einklang zu bringen. Mit dem Ansatz des „psychological ownership“ lässt sich behaupten, dass diese eintretenden Partner im Laufe der Zeit Eigentumsgefühle gegenüber dem Unternehmen entwickeln, die aber meist nicht ihrem rechtlichen Status entsprechen. Innerhalb jener Paarbeziehungen, die eine alltägliche Lebensführung gemäß dem Typ „Festung“ praktizieren, erscheinen diese Integrationsprobleme weniger gewichtig.

Diese Dynamik der Paarbeziehung unter Einfluss des Unternehmens lässt sich anhand von Marcella und Michael Treuninger eingängig veranschaulichen. Die Ausführungen zu diesem Fall in Kapitel 6.3.3 verdeutlichen die Rollenkonstanz Michaels in der Paarbeziehung und beruflichen Tätigkeit sowie die starken Schwankungen im Leben von Marcella, die sich phasenweise ganz auf die Erziehung der Kinder und die häusliche Arbeit konzentriert und dann wieder einen Platz im Unternehmen einnimmt. Marcella sucht aktiv - auch entgegen den Vorstellungen ihrer Schwiegermutter - eine Tätigkeit, eine sinnvolle Rolle innerhalb des Unternehmens, das klar im Hoheitsgebiet Michaels liegt. Sie blickt mit der nötigen Distanz auf eingefahrene Muster innerhalb des Unternehmens und versucht, Innovation in den organisatorischen Prozessen (nicht allerdings in den Produkten) voranzutreiben. Während sie am Anfang diese Umbrüche hart „erkämpft“, scheinen sich im Laufe der Zeit bestimmte Rollen - vor allem vor den Mitarbeitern - zu institutionalisieren: „Wir haben da äh sicher auch ein, ein Ergänzungsspiel getrieben" (Marcella Treuninger, 22:13). Marcella ist die Rebellin, die Veränderung und Umdenken einfordert. Michael fungiert als ihr Gegenspieler, der 
sich auf die Seite der Mannschaft schlägt. Er versucht den Betriebsfrieden zu wahren und steht in den Augen der Mitarbeiter für Verlässlichkeit ebenso wie für Kontinuität. Diese Art der gemeinsamen Arbeit im Unternehmen bietet viel Reibungsfläche für die Beziehung zwischen Michael und Marcella. Einerseits belasten die Diskussionen über solche Veränderungsprozesse, in denen beide inhärent gegnerische Positionen einnehmen, die Beziehung phasenweise sehr stark. Andererseits werden sie durch ihr Ergänzungsspiel aneinander gebunden, und Marcella empfindet dies als eine ,sehr besondere, erfüllende Zeit“ (34:3-4), in der sie Erfolgserlebnisse teilen. Der mehrfache und kontinuierliche Versuch Marcellas, eine funktionale Rolle für das Unternehmen auszufüllen, kann als Streben nach einer egalitären Partnerschaft gelesen werden. Marcella fühlt ihren Beitrag zum Erfolg des Unternehmens nicht genügend anerkannt und wertgeschätzt, sieht sich nicht als legitimen Teil des Familienprojektes. Michael hingegen, der selbst eine Lebensweise der Unternehmerfamilie erfahren hat, in der Frauen nicht in die Belange des Unternehmens eingreifen, versteht diese Gewährung eines Gestaltungsspielraumes für Marcella im Unternehmen bereits als eine solche Wertschätzung und Ausdruck einer gleichberechtigten Partnerschaft. Marcella fühlt sich in ihren Vorhaben von ihrem Mann zu wenig unterstützt. Retrospektiv rät sie anderen Frauen davon ab, in die Mitarbeit im Unternehmen ,hineinzurutschen" - und kann damit meinen, einen Versuch zu unternehmen, die Rollenvorstellungen zu ändern.

\subsubsection{Eltern und ihre Kinder}

In Kapitel VI ist bereits mehrfach der direkte Charakter der Eltern-KindBeziehung in Unternehmerfamilien als kontinuierlicher Aushandlungsprozess angesprochen worden. Im Folgenden werden noch einmal pointiert die zentralen Merkmale dieser Beziehung unter dem Einfluss eines Unternehmens zusammengefasst und einige Aspekte ergänzt.

Gerade in Bezug auf das Aufwachsen in einer Unternehmerfamilie konnte gezeigt werden, inwiefern das Unternehmen Bestandteil eines familialen Raumes wird. In seiner Örtlichkeit, mit seinen Menschen und seinen Geschichten durchdringt das Unternehmen die kindliche Erlebniswelt. Zugleich nehmen die Kinder die Lebensweise einer Unternehmerfamilie mit all ihren Sorgen und Privilegien vermittelt über die Eltern wahr. Es ist der spezifischen Struktur der Unternehmerfamilie geschuldet, dass das Unternehmen zu einem Vehikel im Ringen um die Aufmerksamkeit der Eltern werden kann und somit zu einem Mittler in der Eltern-Kind-Beziehung. Für Kinder ist weniger die Koordination unterschiedlicher Systemwelten (Unternehmen und Familie) bedeutsam als das Auffinden ihrer funktionalen Rolle innerhalb der Gemeinschaft der Unternehmerfamilie. Aus dem Wechselspiel zwischen 
Funktionszuschreibung durch die Eltern (Fremdzuschreibung) und dem eigenen Rollenverständnis der Kinder (Selbstzuschreibung) entspringt eine Dynamik, die nicht nur das Verhältnis zwischen Eltern und Kindern, sondern auch ihre jeweiligen Lebensläufe maßgeblich prägt. ${ }^{103}$ Im Umgang mit dem Unternehmen - und insbesondere an dem Beispiel der Mitarbeit im Familienunternehmen als freiwillige Option - symbolisieren sich zudem die inkorporierten Verständnisse der Eltern von Kindheit als eine eigenständige Lebensphase, der die Entwicklungsaufgabe des Kindes, zu einer selbständigen Persönlichkeit zu werden, zueigen ist.

Des Weiteren konnte in der bisherigen Arbeit gezeigt werden, dass die Autonomisierungsbestrebungen heranwachsender Kinder in Unternehmerfamilien unter anderen Vorzeichen stehen, besonders dann, wenn sie früh einen Weg in das Familienunternehmen einschlagen. In dem Entwurf eines eigenständigen Lebensplans müssen sich Kinder stärker als in anderen Familien üblich zu ihren Eltern positionieren, wobei die familiale Generation als Abgrenzungsmetapher zwischen den „Alten“ und den „Jungen“ Differenzierung ermöglicht. Das Narrativ einer „freien Wahl“" wurde als Generationen übergreifende Strategie herausgearbeitet, wie die Reproduktion der Unternehmerfamilie mit der gesellschaftlichen Forderung nach Individualität und Autonomie in Einklang gebracht werden kann.

Folgendes Fallbeispiel soll veranschaulichen, wie das Unternehmen in der Beziehung von Eltern zu ihren erwachsenen Kindern als zusätzlicher familialer Raum fungiert:

Bianca Carstens ${ }^{104}$ arbeitet bereits während ihrer Schulzeit im Musikverlag ihres Vaters Erwin mit und beginnt dort direkt nach dem Abitur eine Ausbildung. Ihre Eltern haben sich bereits vor einigen Jahren scheiden lassen, und ihr Vater ist mit einer zwanzig Jahre jüngeren Frau liiert, die ebenfalls im Unternehmen tätig ist. Biancas älterer Bruder ist Bundeswehrpilot und zeigt kein berufliches Interesse am Verlagshaus. Nach erfolgreicher Ausbildung reist Bianca um die Welt, absolviert diverse Praktika und Jobs. Schließlich beginnt sie, Anglistik und Musik in Berlin zu studieren, und schließt mit sehr gutem Erfolg ab. Kurz darauf ereilt sie ein Anruf ihres Vaters, der sagt: ,ich solle doch kommen. Er stünde bis über beide Ohren in Arbeit und er würde mich brauchen." In Anbetracht der alternativen Möglichkeiten, die Bianca zu diesem Zeitpunkt offen stehen, zögert sie zunächst.

103 Der Begriffe „Rolle“ wird hier als ein konsistentes Erwartungsbündel verstanden, dass sich weniger aus einem funktionalen System ableitet (wie in der systemtheoretischen Herangehensweise an Familienunternehmen vorgeschlagen), sondern sich aus der strukturellen Eigenlogik von Unternehmerfamilien, also gerade aus der Verbindung von Unternehmen und Familie, ergibt.

104 Der Fall Carstens ist nicht transkribiert. Die hier aufgeführten Zitate sind ausschnittsweise Verschriftlichungen der Audioaufnahme. Entsprechend können keine Seitenzahlen und Zeilennummer angegeben werden. 
„Ich hab das aber trotzdem gemacht. Dann hab ich mich aber natürlich ganz furchtbar mit meinem Vater angelegt. Der natürlich nicht nur weiß wie man es macht, sondern immer alles besser weiß und es besser macht. Der dem Neuen gegenüber sicherlich aufgeschlossen ist, aber das nicht immer so zu vermitteln weiß. Nach einem halben Jahr hab ich gesagt, pass mal auf, warum hast du mich überhaupt geholt, wenn Du mich nicht haben willst? Dann hatten wir einen schönen Show-Down. Und danach gings. Da hat es mal so richtig geknallt. Wir hatten die Fronten geklärt. Und ab da durfte ich dann auch, also da hatte ich dann eben auch so ein paar Bereiche, wo er mir nicht mehr reingeredet hat. Und ab da gings."

Wenige Zeit später wird Bianca von ihrem damaligen Freund schwanger und beendet die Beziehung. 1996 lernt Bianca ihren heutigen Ehemann, den Industriefachwirt Jan kennen, die beiden bekommen noch zwei weitere Söhne. Jan steckt für die Karriere seiner Frau zurück. Er schlägt berufliche Chancen aus, um am Wohnort der Familie verweilen zu können, und übernimmt für einige Zeit die Kindererziehung. Die Familie kauft sich 2000 ein kleines Haus in der Nähe des Musikverlages. Bianca sieht sich in der Tradition einer Medienfamilie und bezieht daraus ihre Identität. Ihre Familie hat Teil an ihrer Tätigkeit im Unternehmen, sie nimmt die Kinder mit ins Büro und kann das positive Potenzial freier Zeiteinteilung für sich nutzen. Ihr Vater hat für sie und ihre Arbeit eine große Vorbildfunktion, sie begreift ihn als einen Gegenspieler, an dem man sich reiben und von dem man lernen kann.

„Aber dann kam er irgendwann zu mir und fragt mich, und das war auch eine ehrliche Frage, also das war nicht eine rhetorische Frage, sondern sagte eben wirklich, pass mal auf, das ist so und so, ich wäre bereit zu verkaufen, was sagst Du dazu? ... Und da habe ich ähm, war ich erstmal beleidigt ... und hab gedacht, was soll ich denn dazu, was ist das denn für eine Frage, verkauf ich meinen Mann, verkauf ich meine Kinder, also was ist das denn für eine blöde Frage. Hab ich nicht gesagt, aber vom Gefühl her war das so. Ich war richtig gehend beleidigt, weil vom Gefühl her war das so. Ist doch eigentlich überhaupt keine, kann man überhaupt nicht fragen so was.“

Allein mit der Vorstellung, das gemeinsame Familienprojekt verkaufen zu können, gibt der Vater deutlich zu erkennen, dass er eine gänzlich andere Vorstellung vom Unternehmen und der Bedeutung davon vertritt, was es heißt, eine „Unternehmerfamilie“ zu sein. Im Gegensatz zu seiner Tochter verschreibt er sich zwar als Unternehmer dem Erfolg der Organisation, trennt diese aber stärker von der Familie. Erwin, der ein eher konservatives Frauenbild besitzt, traut Bianca die Fortsetzung seines Werkes und die zusätzliche Belastung der Finanzierung seines Ruhestandes nicht zu. Sein Selbstbild ist geprägt von der Idee des Unterstützers, der es seiner Tochter ermöglicht hat, trotz der aus seiner Sicht ,nicht ganz einfachen“ familiären Verhältnisse Karriere zu machen. Erwin sagt, ,Das war 2002, da hatte ich bereits das reife Alter von 70 erreicht. Und irgendwo dachte man sich schon, wie wird das wer- 
den, wie wird das gehen. Und die Überlegungen, die ich ihnen gerade vorgeführt habe, Bianca mit drei Kindern und dann dieser riesen Laden mit den Belastungen, mit den Arbeitsbelastungen. Das gab einem schon zu denken, nicht. Ja, deswegen war das Angebot eben durchaus interessant nicht." Ganz auf einer Linie mit seiner biografischen Gesamtsicht stellt Erwin in den Verkaufsgesprächen die Bedingung, dass ein Arbeitsplatz für ihn und seine Tochter im Unternehmen auch weiterhin garantiert werden müsste.

Dieser Fall verdeutlicht einige Besonderheiten der Generationenbeziehung zwischen Eltern und ihren erwachsenen Kindern in Unternehmerfamilien: In materieller Hinsicht wird zunächst evident, dass das Einkommen und Vermögen der Familie eng mit der Tätigkeit im Unternehmen und dem Besitz von Betriebsanteilen verknüpft ist. Die Leistung, die einzelne Familienmitglieder im Unternehmen erbringen, steht in einem direkten Verhältnis zu dem Wohlstand der anderen Familienmitglieder (etwa in Form von Gewinnausschüttungen oder des Wertes der Unternehmensanteile). Vor allem im Übergang zum Ruhestand kann es zu einem unmittelbaren Generationenvertrag zwischen Eltern und Kindern kommen, nämlich dann, wenn sich die Rente überwiegend aus dem Unternehmen generieren soll. Solche impliziten oder expliziten Generationenverträge zwischen Familienmitgliedern sind von dem Generationenvertrag als ordnungspolitische Figur der gesetzlichen Rentenversicherung grundsätzlich verschieden (siehe etwa Kohli/Szydlik 2000:10 oder Künemund/Szydlik 2009, Kohli/Künemund 2005), enthalten aber ebenfalls die beiden Komponenten eines finanziellen Transfers sowie der Moral, durch seine Arbeit ein Recht auf Versorgung im Alter zu erwerben (Leisering 2000:67). Damit einher gehen nicht nur finanzielle Risiken, sondern auch ein stärkeres Abhängigkeitsverhältnis als dies in anderen Familien üblich ist. Im Fall Carstens wollte sich Erwin nicht auf einen solchen privaten Generationenvertrag mit seiner Tochter einlassen. $\mathrm{Zu}$ groß schien ihm die Bürde, die er damit Bianca auflastet, und vielleicht war ihm das daraus entstehende Geflecht aus Abhängigkeiten und Verpflichtungen zu eng.

Auf der Ebene von Lebensläufen und Biografien lässt sich zudem festhalten, dass getroffene Richtungsentscheidungen, Wendepunkte oder Krisen im Leben eines Familienmitgliedes sich unmittelbar auf die Lebenslaufgestaltung und biografische Selbstsicht der anderen Familienmitglieder auswirken können, wie dies im Fall Carstens evident wird. Gerade im Hinblick auf den Beruf scheint die Beziehung zwischen Eltern und ihren erwachsenen Kindern in Unternehmerfamilien besonders. Eltern dienen hier weit über die Übergangsphase ins Erwachsenenalter hinaus als berufliche Ratgeber und Fluchtpunkt in der eigenen Tätigkeit. Die Fallauswertungen legen nahe, dass in Unternehmerfamilien direkte Eingriffe in die berufliche Karriere der Kinder jederzeit möglich sind, etwa in Form von Hilfestellungen, über Netzwerkkontakte oder als Arbeitsplatzgeber. Die Solidargemeinschaft einer Unternehmerfamilie schließt ausdrücklich die berufliche Sphäre ein - und das teilwei- 
se sogar ungeachtet der beruflichen Qualifizierung für eine Tätigkeit im Unternehmen: In Zeiten der Krise oder eines hohen Arbeitsaufkommens bitten die Eltern um die Mithilfe der Kinder (etwa für das Weinfest in der Familie Naumann oder eben wie im Fall Carstens der Eintritt nach dem Studium), umgekehrt bieten sie ihren Kindern in einer beruflichen Notsituation einen Arbeitsplatz im Unternehmen an.

Besteht ein Arbeitsverhältnis zwischen den Generationen, intensiviert sich der direkte Charakter der Eltern-Kind-Beziehung noch einmal: Es erhöht sich die Kontakthäufigkeit, die Anzahl der gemeinsamen Themen und zu treffenden Entscheidungen sowie die finanzielle Verbundenheit (Abhängigkeit der Einkommen von der Leistung des Anderen; Finanzierung des Ruhestandes). Neben der räumlichen Nähe entsteht eine ausgesprochene soziale Dichte, die für beide Seiten aushaltbar sein muss. In der Familienunternehmensforschung wird in diesem Zusammenhang gerne darauf verwiesen, dass sich Eltern und ihre erwachsenen Kindern nun auch als Ausbilder und Lehrling, als Chef und Angestellter oder als gleichberechtigte Geschäftspartner gegenüber stehen. Garcia-Alvarez und Lopez Sintas (2002) sprechen deshalb von einer betrieblichen Sozialisation, die exklusiv nur den ,auserwählten“ Nachfolgern zuteil wird. Es erfolgt ein Transfer von Wissen und Kontakten zwischen den Generationen, der nicht immer reibungslos verläuft. Wie der Fall Carstens verdeutlicht, können hier unterschiedliche Arten des Arbeitens, der Mitarbeiterführung und der Lebenslaufführung aufeinanderprallen. Zugleich eröffnet die starke Polarisierung von Innovation vs. Tradition, von „junger Rebell“ vs. „alter Hase“ eine Möglichkeit, sich vom jeweils anderen abzugrenzen und Individualität für sich in Anspruch zu nehmen.

Im Umgang von Eltern und Kindern im Unternehmenskontext sei, so Handler (Handler 1992:294ff.), ein gegenseitig respektvolles Miteinander unverzichtbar. Beide Generationen müssen ihre Rollen, im Sinne ihrer Funktion im Unternehmen, wechselseitig aufeinander abstimmen und auf diese Weise die Verantwortung für das Unternehmen übertragen. Die Fallauswertungen legen nahe, dass gerade das Arbeitsverhältnis zwischen Eltern und ihren erwachsenen Kindern aus Unternehmerfamilien von der oben skizzierten Dynamik aus Fremd- und Selbstzuschreibung von funktionalen Rollen innerhalb des Kollektives der Unternehmerfamilie geprägt ist.

\subsubsection{Geschwisterbeziehungen}

Das Unternehmen bietet Kindern aus Unternehmerfamilien eine gemeinsame, geteilte Erfahrungswelt. Die Geschwister verbindet das Spiel auf dem Betriebsgelände, Besuche der Eltern im Büro, Firmenfeiern, Ferienarbeit oder das Wissen um die beruflichen Verstrickungen der Eltern. Als „Eingeweihte“ sind sie aneinander gebunden und können eine Koalition gegenüber ihren El- 
tern oder Altersgenossen eingehen. Gleichzeitig stehen sie sich als Konkurrenten um die Aufmerksamkeit der Eltern gegenüber. Die Fallauswertungen zeigen, ebenso wie jene von Regina Wenk (2007), dass von klein auf die Rollenzuschreibungen der Eltern sowie die eigenen Rollendefinitionen das Verhältnis der Geschwister maßgeblich beeinflussen. Ungleiche Chancen der Kinder in dieser Hinsicht entfalten eine langfristige Wirksamkeit in ihrer biografischen Gesamtsicht (Wenk 2007:210) und haben einen differenzierenden Effekt auf die Geschwisterbeziehung. Eine bereits besetzte Rolle mag dazu führen, dass ein Geschwisterteil einen alternativen Lebensplan entwirft oder aber in einen erbitterten Konkurrenzkampf eintritt. Diese Rollendefinitionen werden auch im Umgang von erwachsenen Geschwistern miteinander stetig aktualisiert und eine Balance in den Anerkennungsverhältnissen der Familie angestrebt.

In der Familiengeschichte Singermayer (siehe auch die Kapitel 6.1.4 und 6.2.2.1) wiederholt sich in zwei aufeinander folgenden Generationen eine Konkurrenzsituation zwischen den Geschwistern, die in beiden Fällen zu einer Teilung des Unternehmens führt. Sven Singermayer hat den Weg in das Familienunternehmen anstelle seines tödlich verunglückten jüngeren Bruders angetreten. Als er bereits einige Zeit mit der Führung des operativen Geschäftes betraut ist, tritt sein älterer Bruder, der eigentlich im Brauereigewerbe tätig war, mit der Bitte an ihn heran, nun auch in das Familienunternehmen einzutreten. Das Verhältnis zu seinem Bruder gestaltet sich schon lange schwierig, dennoch sieht er seinen Bruder, genau wie sich selbst auch, berechtigt dazu, einen Anteil am Familienunternehmen zu erhalten. Er entscheidet sich dafür, eine Sparte als eine rechtlich unabhängige Unternehmung auszugliedern, die dann sein Bruder erhält und leitet. Unter Svens fünf Kindern entwickelt nur Anabella über Umwege ein ausgeprägtes Interesse am Unternehmen. Sie ist bereits seit einigen Jahren im operativen Geschäft tätig. Auf einem Familientisch wurden die Ansprüche auf das Unternehmen klar geregelt, woraufhin Anabella einige Anteile und ihre Geschwister Kompensationszahlungen erhielten. Zwei Jahre später tritt Anabellas Bruder an den Vater heran und bittet darum, nun doch auch in das Familienunternehmen einsteigen zu können. Sven fühlt sich an die Situation mit seinem Bruder erinnert. Er will den Familienfrieden wahren und freut sich über das Interesse seines Sohnes. Sven stimmt ohne großes Zögern dem Wunsch des Sohnes zu, obgleich ihm das schwierige Verhältnis zwischen den Geschwistern bekannt ist. Er teilt das Unternehmen erneut in zwei Sparten, die jedoch noch über einige gemeinsame Abteilungen (Verwaltung, Logistik) miteinander verbunden bleiben. Auf dem 160. Firmenjubiläum verkündet Sven feierlich diese neue Nachfolgeregelung, zur Überraschung der Mitarbeiter und auch Anabellas. Diese fühlt sich erneut - wie schon in ihrer Kindheit - in ihrer funktionalen Rolle innerhalb der Unternehmerfamilie bedroht, ist verletzt (davon), nur als Beobachterin an diesen Änderungen teilzuhaben. Die Vor- 
stellung, aus dem Unternehmen als „rundes Ganzes“ einen Teil herauszuschneiden, bereitet ihr nicht nur aus ökonomischer Perspektive Sorgen, sondern erschüttert auch ihre erst spät gefundene Identität als Teil eines Ganzen. In langen Verhandlungen mit Beratern werden Regeln für die Zusammenarbeit der Geschwister festgelegt. Sven junior hält sich jedoch nicht an diese Regeln und greift seine Schwester an. Schließlich kündigt Anabella in Abstimmung mit ihrem Vater dem eigenen Bruder das Mietverhältnis in der Firmenzentrale. Daraufhin werden die Sparten endgültig rechtlich getrennt. Der Kontakt zum Bruder und Sohn bricht ab. Sven sagt: „Es tut mir leid, es tut mir weh, äh aber auf der anderen Seite ist, ich weiß, es war die richtige Entscheidung" (51:15-16)

In Unternehmerfamilien lässt sich ein weites Solidaritätsverständnis zwischen den Geschwistern beobachten, das sich aus der engen Verbundenheit des Einkommens und Vermögens der Familie sowie der Tätigkeit im Unternehmen ableiten lässt. Es wird durch Verzicht einerseits und Fürsorge andererseits charakterisiert. Markus Übermann etwa sieht sich gegenüber seiner Schwester verantwortlich: „Weil dann einen doch schon Leute darauf ansprechen und man steht dann eben als Bruder halt ... äh nicht so toll da, wenn einem dann gesagt wird, hast du denn keinen Job für deine Schwester im Betrieb?“ (15:28-30) In der Beziehung zu seiner Schwester treibt Markus einerseits die Angst vor übler Nachrede durch das Umfeld, andererseits die Angst davor, sich ausgerechnet von seiner Schwester zu entfremden, jenem Mitglied seiner Herkunftsfamilie, zu dem er die engste emotionale Beziehung unterhält. Er befürchtet, dass irgendwann die Lebensweisen und auch der materielle Wohlstand immer weiter auseinander klaffen. Der so aufkeimende Neid könnte sich nicht nur zu einem Konflikt zwischen den Geschwistern auswachsen, sondern auch seinen Status und sein Werk bedrohen. Insofern versucht Markus auf den Lebensweg seiner Schwester einzuwirken. Als großer, fürsorgender Bruder überredet er sie zu einer Weiterbildung und ist froh, als sie einen passablen Job annimmt.

\subsubsection{Großeltern}

Aus den vorliegenden Fallanalysen lassen sich (leider) nur sehr wenige Erkenntnisse über die Bedeutung der Großeltern in Unternehmerfamilien ableiten. Gerade im Hinblick auf die Mehrgenerationenfamilie als potentielle Vergleichgruppe für die Familien eigener Art wäre zusätzliche Forschung in diese Richtung wünschenswert: Wie gestalten sich etwa die Unterstützungsleistungen der Großeltern in Unternehmerfamilien mit kleinen Kindern? In welcher Weise werden innerhalb von Unternehmerfamilien Pflegeleistungen übernommen? Oder gibt es Unternehmensübergaben, die direkt von der Großeltern- auf die Enkelgeneration stattfinden? 
Die gezielte Analyse der Fallgeschichte auf die Rolle der Großeltern zeigt jedoch, dass die Familienmitglieder stark zwischen solchen Großeltern, die Bestandteil des Kollektivs Unternehmerfamilie sind, und solchen, die außerhalb dieser Grenze liegen, differenzieren. Während letztere Einblicke in ein anderes Leben gewähren, einen neutralen Ort der Erholung, des Ausgleichs und der Freizeit bieten, symbolisiert sich in den Großeltern des Familienunternehmens die Tradition und Kontinuität des gemeinsamen Projektes. Gerade von den Großvätern, die selbst einmal die Geschicke des Unternehmens geleitet haben, geht eine große Autorität aus. Sie sind unternehmerische Vorbilder, gatekeeper der Familienkultur und Vertreter einer ,alten“ Lebensweise und (eines/ihres) Wertesets. Die räumliche Nähe zu den Großeltern (die aus der Immobilität des Familienunternehmens resultiert) führt zu einer Vertrautheit mit dieser Vorgeneration. Es ist darüber hinaus auffällig, dass sich die Enkel ihrer genealogischen Verbindung mit diesen Großeltern der Unternehmerfamilie sehr bewusst sind.

Diese Ergebnisse decken sich mit einer Studie von Valerie King und Glen Elder (1995), die für Bauernfamilien ein engeres Verhältnis zwischen Großeltern und Kindern feststellen, als dies in anderen Familien üblich ist. Sie betonen die besondere Nähe zu den Großeltern väterlicherseits, die sich in der Wohnortnähe, Kontakthäufigkeit und einer tiefen Qualität der Beziehung niederschlägt. Diesen Umstand führen sie zum einen auf die ländliche Region zurück, zum anderen auf den spezifischen Kontext von Bauernfamilien, deren Lebensführung von einer höheren wechselseitigen Abhängigkeit der Familienmitglieder geprägt sei (King und Elder 1995:174). Andere familiale Konstellationen (etwa Großeltern-Eltern oder Eltern-Kinder) würden sich weit weniger stark von anderen Familien unterscheiden (King und Elder 1995:175).

\subsubsection{Weitere Verständnisse von Generation}

\subsubsection{Familiale Generation mit historischer Tiefe}

„Was wollen wir jetzt machen?“ (3:24) fragt Michael Treuninger die Interviewerinnen zu Beginn des zweiten Erzählabschnitts und verdeutlicht damit sein Bedürfnis nach einem weiteren thematischen Input. Mit der Ankündigung ,Jetzt wird es also doch ein bisschen historisch“ (3:31) geht er der Aufforderung nach, die Geschichte seiner Familie zu erzählen. Als Anfangspunkt der Familienhistorie verweist Michael weder auf ein spezifisches Familienmitglied noch auf die Gründung des Unternehmens. Stattdessen erzählt er detailreich von einem Zusammentreffen seiner Familie, die seit Jahrhunderten Dorfschmiede waren, mit einem erfindungsreichen Dorfpfarrer, die in der Hinwendung zur Gerätetechnik resultierte. Seine anschließende Erzählung 
folgt ganz dem Muster einer Generationenerzählung, wie sie Lubinski (2010:113ff. und Kapitel 3.2.5 in dieser Arbeit) beschreibt: In der Form einer Schöpfungsgeschichte erzählt Michael, wie seither die einzelnen aufeinander folgenden Generationen die Verbindung von Familie und Unternehmen fortgesetzt haben, mit ihren jeweiligen zeithistorischen Umständen verfahren sind und dabei das Unternehmen weiterentwickelt und zum Erfolg geführt haben. Michael präsentiert sich als einen Teil der Familientradition, die einen besonderen Anfang hat und mit einer spezifischen Aufgabe versehen ist. Auf diese Weise bringt er zum Ausdruck, dass man ihn nur als einen Teil dieses Kollektivs begreifen kann. Auf die Nachfragen der Interviewerinnen, wie Michael Zugang zu der Familiengeschichte erhalten habe, antwortet er ausführlich. Er betont sein ausgesprochenes historisches Interesse, das er mit seinem Urgroßvater teile. Schon als Junge war er fasziniert von den alten Familienstücken in der Fabrikwohnung oder den Geschichten des Großvaters. Seit den 1990er Jahren, zum 150-jährigen Firmenjubiläum, habe er sich dann intensiver mit der Firmenchronik beschäftigt. Hier habe er „Werkzeuge“ gefunden, wie etwa extreme Sparsamkeit, Verzicht, Kündigungen oder Umstrukturierungen, die dem Unternehmer in Zeiten des Wandels hilfreich sein können. Die restliche Interviewauswertung und auch die Erzählungen anderer Familienmitglieder zeigen: Michael hat nicht nur ein ausgeprägtes historisches Interesse für die Daten und Anekdoten seiner Familiengeschichte, sondern gibt sie auch gerne zum Besten. Bei Besuchen von Geschäftspartnern wie auch auf den winterlichen Skiwanderungen mit der Familie erzählt Michael von den Eigenarten und Errungenschaften der Vorfahren wie in einem Fortsetzungsroman. Die „Familie als Kontinuum“ kann als fester Bestandteil von Michaels Selbstverständnis gesehen werden. Michael besitzt nicht nur ein enormes Wissen üer diese Geschichte, sondern hat den verschiedenen Wendungen auch emotionale Bewertungen zugeschrieben. Damit „durchlebt“" Michael eine Zeit, die weit vor seiner Geburt liegt: Die Umbrüche durch die Eisenbahn, die Weimarer Republik oder der Beginn des Dritten Reiches werden zu schillernden Bestandteilen seiner Lebensgeschichte, die bis in die Gegenwart Auswirkungen auf seine Identität und sein Selbstbild haben. „Sie ist immer da“ sagt er über diese Vorgeschichte und verweist auf die Kontinuität dieser „Frühzeit“ oder dieser „Wurzeln“ über sein Leben hinweg. Durch sie drückt sich Michaels Wunsch nach Zugehörigkeit, Struktur und einem festen Platz aus. So wird er, trotz des hohen Theoriegehalts seiner Geschichten, zum ,autobiographischen Erzähler“.

Dieser Fall führt anschaulich vor, inwiefern eine Tradition der Verbindung von Unternehmen und Familie den familialen Generationenbeziehungen historische Tiefe verleihen kann. Ausgelöst durch Sammelstücke, Jubiläen oder Umbrüche im Unternehmen oder der Familie erzählen sich die Familienmitglieder (über) die Geschichte ihrer Vorfahren, wobei den Großvätern in der Tradierung der Familienhistorie eine Schlüsselfunktion zukommt. Die- 
se Erzählungen stellen aktive Konstruktionsleistungen der Familienmitglieder dar, die in der Gegenwart verankert sind und bestehende Sinnstiftungsangebote aufgreifen und verändern. Familiengeschichte vermittelt zentrale Werte und Grundhaltungen hinsichtlich ethischen Unternehmertums oder einen „Kompass“ in unruhigen Zeiten. Zudem finden sich hier Hinweise auf zu vergebende Rollen innerhalb der Unternehmerfamilie, die Praxis der gelebten Verbindung von Unternehmen und Familie ebenso wie auf die Bedeutsamkeit des Familienprojektes. Die Historie als Teil des Familiengedächtnisses spiegelt die kollektive Identität der Erzählgemeinschaft einer Unternehmerfamilie wider. Gleichsam bietet sie Anknüpfungspunkte für die individuelle Identitätsbildung und Sinngebung. Jedes Familienmitglied hat seine eigene Form der Aneignung dieser Geschichte und erzählt sie in Nuancen oder gar in großen Teilen anders. Familiengeschichte wirkt deshalb nicht nur vergemeinschaftend, sondern kann auch als Abgrenzungsmerkmal in der Ausbildung von Individualität genutzt werden.

Wie im Fall Michaels scheinen noch weitere Interviewpartner ein sehr enges Verhältnis zur Familiengeschichte zu unterhalten, sie gar als Verlängerung der eigenen Lebensgeschichte zu begreifen. Sicherlich tragen zu einem solch innigen Verhältnis eine Vertrautheit mit der Familiengeschichte von Kindheit an sowie eine lange Wirkdauer im Unternehmen und damit einige Jahre der eigenen Geschichtsschreibung bei. Ein weiterer Faktor sticht jedoch als wesentlich hervor: die Praxis des Erzählens. Indem die Familienmitglieder selbst zu Erzählern der Familiengeschichte werden, begeben sie sich in das „Jetzt“ einer narrativ geteilten Wirklichkeit (Rosenthal 2007:457). Sie tauchen ein in die Erlebnisdimension der Geschichte, die es ihnen erlaubt, diese in ihrer Emotionalität und Faktizität zu erfahren. In diesem Sinne könnte die Erzähltheorie erklären, wie aus der Kraft von Erzählungen das Gefühl einer eigenen erlebten Erfahrung entsteht und verinnerlicht wird.

Der Begriff der Generation verbindet sich in solchen Familiengeschichten mit einer genealogischen Dimension, die eine Fiktion der Dauerhaftigkeit des Familienprojektes aufrechterhält und überzeitliche, natürlich gegebene (biologisch verankerte) Legitimität vermittelt (Lubinski 2010:152). Hinter die generationelle Periodisierung treten Zeiten des Übergangs, der Unsicherheit und der Rückschritte zurück. Es wird ein sozialer Erfolgsdruck aufgebaut, der auf den gegenwärtigen und zukünftigen Generationen lastet. Lubinski formuliert als Schattenseite der positiven Sinnstiftungspotentiale, die sich aus der Familiengeschichte ableiten lassen, das unbewusste Gefühl, „mit kollektiven Erwartungen konfrontiert zu sein, selbst wenn diese nicht ausdrücklich artikuliert werden“ (Lubinski 2010:113). Folgendes Zitat von Anabella Singermayer unterstreicht den sozialen Druck einer erweiterten Solidargemeinschaft, der sich aus der Familiengeschichte ableiten lässt: ,so eine Zeit, wo ich wirklich physisch im Rücken diese Vorgeneration gespürt habe. Und hier und einfach auch so diese .., du, du musst .. das schaffen, also 
da ist keine Option, .. sondern also ich kann nicht hier dieses Schiff nach über 150 Jahren an die Wand fahren. Da schauen welche zu, da haben welche ihre ganze Lebensenergie, Zeit, Geld rein gesteckt und ähm also das war [ausgelassen] am Anfang meiner Entscheidung, also der ausgesprochenen Entscheidung, war die Verantwortung für die Vergangenheit die größte, gröBer als die nach vorne." $(12: 5-11)$

Zusammenfassend soll an dieser Stelle die These formuliert werden, dass sich die biografische Selbstsicht von/bei Mitgliedern aus Unternehmerfamilien nach hinten in die Geschichte der Familie und nach vorne in die Zukunft ihrer Nachfahren erweitert. Die Kernfamilie teilt eine gemeinsame Welt der Wir-Beziehungen mit den vergangenen und künftigen Generationen. Auf diese Weise wird die Gemeinschaft der Unternehmerfamilie generationsübergreifend verlängert, und ihre kollektive Identität erhält eine historische Tiefe.

\subsubsection{Gesellschaftliche Generation}

Die Bedeutung der Generationen im Sinne des Mannheimschen Verständnisses einer Alterskohorte mit geteiltem Generationenbewusstsein ist in Bezug auf Unternehmerfamilien bislang gänzlich unerforscht und damit bleibt, wie hier behauptet werden soll, ein zentraler Faktor in der Lebensplanung dieser Familien eigener Art unerkannt. Aus den Ausführungen zum Lebenslauf lässt sich ableiten, dass sich Familienunternehmer ihren Altersgenossen, mit denen sie gemeinsam die Schichten des Lebens in einer spezifischen zeithistorischen Generation durchlaufen, verbunden fühlen. Sie erkennen Parallelen zwischen sich und den anderen „Kriegskindern“, „,68ern“ oder der „Wendegeneration" - die Interviewpartner nutzten teilweise selbst solche Generationenbezeichnungen - mit denen sie ein kollektives Bewusstsein teilen. Die gesellschaftliche Generation bietet jenseits der Familie eine Möglichkeit der Verortung und ein Gefühl von Zugehörigkeit oder in den Worten von Heinz Bude einen „Wir-Bezug“ (Bude 2000).

Diese Gemeinschaft einer horizontalen Generation stellt eine lebenslang vorhandene Referenzgruppe in der Reflexion über das eigene Leben dar. Der Blick zur Seite informiert über andere Lebensmodelle und Lebenswege und schafft Distanz zu den eigenen Verstrickungen zwischen Familie und Unternehmen. Die Altersgenossen sind zentrale Impulsgeber und Maßstab für die eigene Lebensplanung. Erst im Vergleich mit Mitschülern, anderen Auszubildenden, Kommilitonen oder Arbeitskollegen treten die als selbstverständlich erlebten Besonderheiten, die sich aus der Verbindung von Familie und Unternehmen ergeben, zum Vorschein.

Kinder und Jugendliche können in ihrem Streben nach „Normalität“" und „Zugehörigkeit“ diese Besonderheiten der Familien eigener Art als belastend 
empfinden, man denke beispielsweise an Anabella Singermayer, die den Wohlstand ihrer Eltern als unerträglich empfindet, oder daran, wie Markus Übermann unter der Mitarbeit im Familienunternehmen leidet (siehe Kapitel 6.1). Mit ansteigendem Individualisierungsdruck trägt das Familienunternehmen das Potenzial in sich, zu einem Alleinstellungsmerkmals unter den ,peers“ zu werden: Markus Übermann erkennt bei seiner Rückkehr auf den elterlichen Hof - nach einer Zeit der Ausbildung im Süden Deutschlands - mit ein wenig Genugtuung, dass aus seinen Schulkameraden, die früher so viel hatten (z.B. Fußballspielen, Freizeit, Computer) in seinen Augen ,noch nichts Richtiges und noch nichts Ganzes“" (21:26) geworden ist. Im Gegensatz zu ihnen wird er das Familienunternehmen übernehmen und modernisieren, zudem gelingt es ihm etwas später, mit seiner sieben Jahre älteren Partnerin seine Vorstellung einer Familie, die enge emotionale Bande unterhält, umzusetzen. Neider dieses Erfolges nimmt Markus billigend hin, sie sind für ihn sogar ein positives Zeichen dafür, nun auf dem richtigen Pfad zu wandeln.

Die horizontale Generation erzeugt sozialen Druck: Hier konzentrieren sich gesellschaftlich geltende Normen und werden als Forderung nach einem eigenständigen Lebensentwurf laut. Durch die Orientierung an den Altersgenossen gerät das Lebensmodell einer Unternehmerfamilie auf den Prüfstand. Familienunternehmer mögen sich im Ergebnis unter Rechtfertigungsdruck sehen, alternative Lebenspläne entwickeln oder sich in ihrem Lebensentwurf als individuell bestätigt fühlen. Hinsichtlich einer familieninternen Nachfolge als Reproduktion der Unternehmerfamilie wird der Vergleich mit den Altersgenossen damit zu einem Risikofaktor.

Aus dem Interviewmaterial lässt sich ableiten, dass die gesellschaftliche Generation nicht über alle Lebensphasen hinweg in gleicher Intensität auf das Leben der Familienunternehmer einwirkt, sondern gerade in Zeiten von Statuspassagen ihre orientierende Wirkung entfaltet. In diesen Übergangsprozessen im Leben der Familienmitglieder übernimmt die Gruppe der Gleichaltrigen eine wichtige Funktion. Unter den Altersgenossen werden, wie Ansgar Weymann herausarbeitet, „die für den jeweiligen neuen Lebensabschnitt notwendigen Rollen gelernt, wird soziale und personale Identität gefestigt, werden altersangemessene Kooperation, Autorität und soziale Verantwortung geübt" (Weymann 2000:39). Während dieser Zeit werden die familiär geprägten Generationenbeziehungen von den gesellschaftlichen Generationenverhältnissen überformt.

Schließlich könnte man auch danach fragen, inwiefern etwa die „Generation Wirtschaftswunder" im Vergleich zu einer "Generation Golf" verschiedene Verständnisse hinsichtlich des Lebens in einer Unternehmerfamilie unterhält. Der Eindruck, der sich aus den vorliegenden Fallanalysen ergibt, ist, dass die strukturelle Eigenlogik der Unternehmerfamilie mit den sich ergebenden Lebensmodellen einen Generationeneffekt überwiegt. Lediglich im 
Bereich der Führungsstile lassen sich generationentypische Managementmoden vermuten. Zudem zeichnet sich ein Wandel der Erziehungsideale (siehe hierzu auch Absatz 3.1) und Geschlechterrollen in Unternehmerfamilien ab.

\subsubsection{Der Zusammenhang von vertikalen und horizontalen Generationenbeziehungen in Unternehmerfamilien}

Die vorangehende Analyse rückt insbesondere die familialen Generationenbeziehungen in Unternehmerfamilien in den Mittelpunkt. ${ }^{105}$ Es zeigt sich deutlich, dass das Unternehmen die Beziehungen zwischen den Partnern, Eltern und Kindern, Geschwistern sowie Großeltern und Enkel durchdringt:

Materielle Ebene: Das Einkommen und Vermögen der Familie steht in einem engen Zusammenhang mit der Tätigkeit im Unternehmen sowie dem Besitz von Betriebsanteilen. Daraus leitet sich ab, dass der Wohlstand einzelner Familienmitglieder nicht nur von dem Erfolg des Unternehmens, sondern auch von der Leistung anderer Familienmitglieder abhängt. Hinsichtlich der Finanzierung des Ruhestandes können Eltern mit ihren erwachsenen Kindern einen privaten Generationenvertrag eingehen. Der Transfer des Eigentums ist eingebettet in ein komplexes Geflecht aus Abhängigkeiten, Verpflichtungen und Loyalität.

Lebensläufe und Biografien: Das Unternehmen wird zu einem Fluchtpunkt in der eigenen Lebenslaufgestaltung. Getroffene Richtungsentscheidungen, Wendepunkte oder Krisen in den Leben eines Familienmitgliedes können sich unmittelbar auf die Lebenslaufgestaltung und biografische Selbstsicht der anderen Familienmitglieder auswirken. Insofern sind die Leben der Mitglieder einer Unternehmerfamilie noch stärker aneinander gebunden als dies der Begriff ,linked lives“ (Elder 1975, 1985b, 1998) vermittelt.

Kommunikation: Auf einer kommunikativen Ebene bindet das Unternehmen als gemeinsames Thema bzw. als gemeinsames Projekt die Familienmitglieder aneinander. Sie teilen ein intimes Wissen über die Organisation, das es ihnen erlaubt, jederzeit ein Gespräch über das Unternehmen zu führen. Dieses gemeinsame Thema kann sogar dazu führen, die familialen Beziehungen auf einer emotionalen Ebene zu verstärken. Jannik Lösch etwa sagt: „So fing eigentlich auch eine ganz tolle Freundschaft an zwischen mir und meinem Vater, weil er auf einmal schätzen lernte, was ich kann. Und ich auch schätzen lernte, was er da aufgebaut hat." Gleichzeitig manifestiert sich in anderen Fällen (etwa der Familie Claas) sehr deutlich, dass ein solches gemeinsames Thema nicht alleine ausreichen kann, um ein konfliktreiches Verhältnis zwischen den Generationen auszugleichen.

105 Eine erste Skizze dieser Gedanken findet sich bereits in einem Beitrag zum Sozialwissenschaftlichen Journal. Stamm 2009. 
Kollektive Identität: Der Kontinuitätsanspruch der Unternehmerfamilie tritt als konstitutives Element dieser Gemeinschaft hervor. Die familialen Generationen werden zu Verbündeten in einem gemeinsamen Projekt mit einer spezifischen kollektiven Identität, deren Bindungskraft sich über das ganze Leben erstrecken kann.

Dieser Kern der Generationenbeziehungen wird durch die Analyse in zwei Richtungen erweitert (siehe Abbildung 9). Auf der vertikalen Achse verlängert sich der zeitliche Horizont der familiären Generationenbeziehungen in beide Richtungen. Über den Bezug zu einer ,erlebten' und verinnerlichten Unternehmens- und Familiengeschichte, gewinnt die Vergangenheitsdimension an Tiefe. Über den Wunsch der Fortsetzung durch die Nachfahren wird der Zukunftshorizont erweitert.

Auf der horizontalen Achse gerät der Bezug zur gesellschaftlichen Generation, also jenen Personen, die historische Ereignisse in ähnlichem Lebensalter erfahren, in den Blick. Die Orientierung an der gesellschaftlichen Generation, die für alle Familien üblich scheint, entpuppt sich für die Nachfolge in Unternehmerfamilien als eine wichtige. Hier muss das Lebensmodell Unternehmerfamilie, das der Familienunternehmer als Option für sein Leben entwirft, dem ,Individualisierungstest' der Altersgenossen standhalten. In anderen Worten, das Familienunternehmen muss einen individuellen Lebensentwurf nicht nur innerhalb der Kernfamilie, sondern auch im Vergleich zu den peers zulassen.

Abbildung 9: Vertikale und Horizontale Generationenbeziehungen

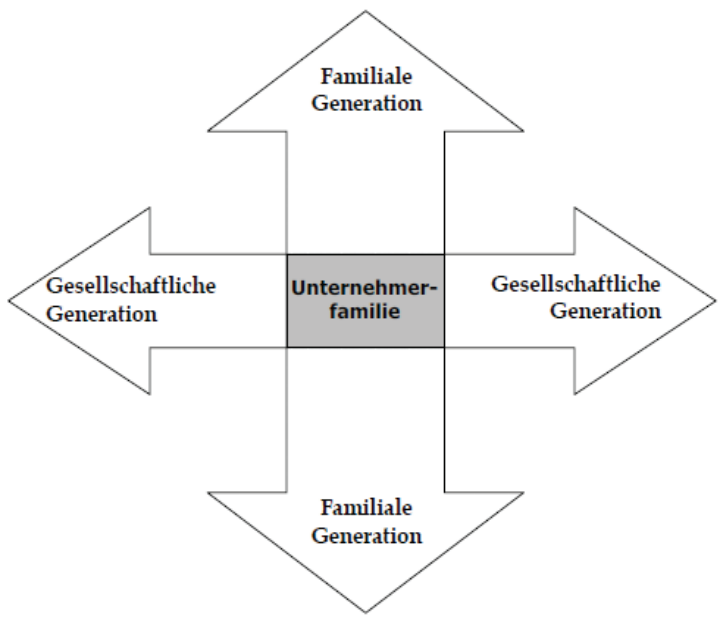

Quelle: eigene Darstellung 


\subsubsection{Unternehmerfamilien als Figuration}

Die Quintessenz der bisherigen Analyse lautet: Die Besonderheit der Generationenbeziehungen in Unternehmerfamilien besteht weniger in der Intensität der Beziehung als in ihrem ,direkten Charakter“. Was damit konkret gemeint ist, soll im Folgenden erläutert werden:

Die gegenwärtige Forschung zu Generationenbeziehungen zeigt sehr deutlich, dass ein enges, vielschichtiges Verhältnis zwischen den Generationen besteht. Dass diese über Vermögen, Eigentum, Lebensläufe, Verpflichtungen und Emotionen miteinander verbunden sind und in einem Austauschverhältnis stehen, stellt an sich noch keine Besonderheit dar. Es kann wohl kaum behaupten werden, dass Unternehmerfamilien in einer strukturellen, assoziativen oder affektiven Solidaritätsdimension eine Ausnahme bilden (Bengston). Auch hinsichtlich der anderen Dimensionen können höchstens kleinere Unterschiede vermutet werden: Mit Blick auf Unterstützungsleistungen und den Austausch von Ressourcen lässt sich auf den besonderen Charakter des Eigentums an einem Unternehmen als einem persönlichen $\mathrm{Ob}-$ jekt verweisen (siehe Breuer 2009) (funktionale Solidarität), und auch eine starke Verbundenheit mit familialen Rollen und ein ausgeprägtes Verpflichtungsgefühl gegenüber den Familienmitgliedern scheinen das Generationenverhältnis in Unternehmerfamilien zu bestimmen (normative Solidarität). Eventuell lässt sich auch ein ausgeprägtes meritokratisches Denken über die Generationen hinweg feststellen (konsensuelle Solidarität). Daraus lässt sich die Hypothese ableiten, dass sich in einer quantitativen, vergleichenden Untersuchung der Unterstützungsleistungen, Gefühlshaltungen oder gemeinsamen Aktivitäten von Unternehmerfamilien und etwa Familien, die ihr Einkommen aus einer abhängigen Erwerbstätigkeit generieren, nur geringfügige Unterschiede in den Generationenbeziehungen finden lassen.

Die methodische Herangehensweise dieser Arbeit nähert sich jedoch der "Qualität" der Generationenbeziehungen in diesen Familien eigener Art aus einer anderen Perspektive, nämlich jener der persönlichen und kollektiven Erfahrung. Dabei erweist sich die theoretische Unterscheidung in strukturelle Vorraussetzungen für Solidarität und ihre handlungspraktische Umsetzung (Szydlik) als gewinnbringend:

Der Einfluss des Unternehmens auf die Unternehmerfamilie verändert die strukturellen Voraussetzungen für die Begegnungen der Generationen. Ihre Tätigkeit als Unternehmer platziert sie in einen höchst individualisierten Kontext der Absicherung für Lebensrisiken. Aus dem Kontinuitätsanspruch der Unternehmerfamilie leitet sich eine Norm zur Fortsetzung des Familienprojektes ab. In diesem normativen Raum werden die Lebensläufe und Biografien der einzelnen Familienmitglieder nicht nur in Bezug zu einer potentiellen Karriere im Familienunternehmen gesetzt, sondern werden auch in hö- 
herem Maße abhängig von den Wegen der anderen Familienmitgliedern, bis dahin, dass direkte Eingriffe in die Lebenslaufgestaltung zugelassen werden. Das Eigentum am Familienunternehmen verlangt nach Wertschätzung, Aneignung und Verzicht der verschiedenen Familienmitglieder und schafft ein komplexes Geflecht aus Verpflichtungen.

Die einzelnen Familienmitglieder sind nun gefordert, sich mit diesen spezifischen strukturellen Voraussetzungen in Unternehmerfamilien auseinander zu setzen. In einer ungewöhnlich direkten Weise gilt es einen Nachfolger zu bestimmen, Zeitpunkte für den Eintritt in das Unternehmen zu koordinieren, das Eigentum zu übertragen, Generationenverträge auszuhandeln, die eigene Identität zu bestimmen, etc. Die Begegnung der Generationen erhält dadurch den Charakter einer unmittelbaren Aushandlung in der sich die Familienmitglieder als Verbündete und Gegner gegenüber stehen.

Diese Art der direkten Verhandlung innerhalb von Unternehmerfamilien erhöht das Potenzial für Ambivalenzerfahrungen (Lüscher). Auf einer persönlichen Ebene können die Familienmitglieder gleichzeitig emotionale Verbundenheit und kognitive Übereinstimmung einerseits, Rollendifferenzen und Unterschiedlichkeit andererseits erfahren. Auf einer institutionellen Ebene oszilliert die Unternehmerfamilie zwischen der Reproduktion des Familienprojektes sowie der Erneuerung der Gemeinschaft durch jede weitere Generation. Diese Gegensätze im Fühlen, Denken, Handeln, Wollen und der Beziehungsgestaltung, die zeitweise oder dauernd als unlösbar interpretiert werden (Lüscher 2000:62), wohnen allen Generationenbeziehungen konstitutiv inne. Indem in Unternehmerfamilien die Generationen nun aber ihre Beziehungen und Verbindungen direkt aushandeln (müssen), werden diese Ambivalenzen unmittelbar und intensiv erfahren. Die Auswertungen der Interviews zeigen deutlich, dass Unternehmerfamilien ein hohes Potential für aufeinander bezogenes Verhalten ebenso wie ein Gefühl der Zusammengehörigkeit innewohnt und sich in gleichem Maße die Konfliktlinien zwischen den Generationen brisanter gestalten. Ferner zeigt sich, dass das üblicherweise bestehende Potenzial der Gemeinsamkeiten und Identifikationen zwischen Eltern und ihren Kindern durch das gemeinsame Familienprojekt steigt und gleichzeitig ein hohes Maß an Distanzierungs- und Autonomisierungsarbeit erforderlich wird. Das Unternehmen ist gleichzeitig Thema und Ort dieser Aushandlungsprozesse.

Diese Besonderheiten der Generationenbeziehungen in Unternehmerfamilien lassen sich adäquater in einer figurationssoziologischen Terminologie, wie sie von Norbert Elias geprägt wurde, beschreiben. Elias nutzt den Begriff der Figuration für das sich wandelnde Muster, das Individuen in ihren sozialen Beziehungen ausbilden. Die Interdependenz der Individuen formt einen komplexen Verflechtungszusammenhang, in dem sich eine Hierarchie der Beziehungskonstellationen ausbilden kann (Elias 2006:173). Jede Figuration zeichnet sich durch ein spezifisches Spannungsgefüge aus und folgt einer 
spezifischen Form. Im Figurationsprozess fluktuiert das Spannungsgleichgewicht, und die Machtbalancen verschieben sich (Elias 2006:174). Elias zufolge können jegliche Interdependenzketten als Figurationen beschrieben werden, z.B. Kinder im Kindergarten, ein Fußballspiel, Familien, Dörfer, Nationen etc. Eine Figurationssoziologie will nun die Eigentümlichkeiten der Figurationen und weniger die Interdependenzkette an sich analysieren.

Der Verflechtungszusammenhang einer Unternehmerfamilie kann entsprechend als eine Figuration verstanden werden. Die Individuen sind dabei mit der Figuration untrennbar und ineinander verwoben (Elias 1998:88). Trotzdem besitzt sowohl das einzelne Familienmitglied eine relative Autonomie gegenüber der Unternehmerfamilie als auch die Unternehmerfamilie eine relative Autonomie von den einzelnen Familienmitgliedern (Elias 1998:88). Dieser beidseitige Freiheitsspielraum ermöglicht es dem Individuum, sich von der Unternehmerfamilie abzulösen, und umgekehrt der Unternehmerfamilie, sich von einzelnen Familienmitgliedern zu trennen, ohne dabei die spezifische Figuration zu beenden. Die Unternehmerfamilie als Figuration organisiert sich damit zu einem Teil selbst, folgt einer eigenen Logik. Elias beschreibt in diesem Zusammenhang eine Logik der Beharrung sowie eine Logik des Wandels als zwei gegenläufige Selbstregulierungstendenzen von Figurationen, die stark an die institutionellen Pole der Reproduktion und Innovation in dem Konzept der Generationenambivalenzen von Lüscher erinnern.

Tritt ein neues Mitglied der Unternehmerfamilie bei (etwa durch Geburt oder Heirat), wird es Teil einer spezifischen Symbolwelt einer schon vorhandenen Figuration (Elias 1998:87). Im Laufe der Zeit erfolgt eine Wissensübertragung von einer zur anderen Generation. Die Form der Figuration, das vollzogene Machtspiel, folgt einem Entwicklungsprozess und muss als dynamisch verstanden werden. Ein besonderes Augenmerk legt Elias nun auf die geltenden Spielregeln der Figuration. Dabei wird die Analyse der sozialen Beziehungen auf eine Ebene der kollektiven Erfahrung gehoben. Im Fall von Unternehmerfamilien lässt sich die Form der Figuration als ein unmittelbarer Aushandlungsprozess der Individuen beschreiben. Dieser Perspektive folgend, rücken die weiteren Abschnitte zunächst den Gegenstand (der Aushandlungen) und schließlich die Strategien der Aushandlung(en) in den Mittelpunkt.

Es ist unschwer zu erkennen, dass sich die herausgestellten Besonderheiten der Generationenbeziehungen in Unternehmerfamilien nur schwerlich mit den bestehenden Modellen, Begriffen und Methoden der gegenwärtigen Generationenforschung fassen lassen. Insofern versteht sich diese Arbeit auch als methodische Weiterentwicklung der Generationenforschung und zeigt das Potenzial qualitativer Herangehensweisen und eines figurationssoziologischen Ansatzes für ein tieferes Verständnis der Formen und Deutungsmuster von Generationen auf. 


\subsection{Koordinierungsaufgaben in Unternehmerfamilien}

Der Blick auf den kontinuierlichen Aushandlungsprozess in Unternehmerfamilien, der sich im Kontext einer Nachfolgeregelung zuspitzt, verdeutlicht drei zentrale Koordinierungsaufgaben: die Positionsbestimmung der Individuen in Familie und Gesellschaft, die Abstimmung der Lebensläufe zwischen den Familienmitglieder und schließlich den Übergang des Eigentums und des Vermögens. ${ }^{106}$ Diese drei Leitthemen der intergenerationellen Beziehungen sollen im Folgenden näher erläutert werden.

\subsubsection{Positionsbestimmungen in Familie und Gesellschaft}

Rainer Claas ${ }^{107}(* 1923)$ dient als Soldat im Zweiten Weltkrieg. Nach seiner Rückkehr heiratet er, 1949 wird sein erster Sohn Cornelius geboren. Als arbeitsloser Maurer beschließt Rainer 1951, sich mit dem Recycling von Altpapier und Kleidung selbständig zu machen. Das Unternehmen bezieht ein Ruinengrundstück und verwertet dort Materialien, die Rainer und seine Frau mit Pferd und Wagen in der Stadt einsammeln. In den folgenden Jahren werden Claudia und Carsten geboren. Die drei Kinder wachsen sehr eng mit dem Unternehmen verbunden auf. Die Eltern sprechen täglich von den betrieblichen Vorgängen, und die Kinder verbringen viel Zeit dort. Carsten erinnert sich, dass ,eigentlich die Firma immer im Vordergrund stand, so in der Familie. Ja meine Eltern drehten sich letztendlich da drum."

Rainer nimmt besonders häufig seine „Püppi“ - Tochter Claudia - mit ins Unternehmen, zu Kunden oder Bankgesprächen. Die beiden haben eine relativ enge Beziehung zueinander. Claudia sagt von sich selbst: „Ich bin eine Vater-Tochter gewesen“. Das Verhältnis ihrer Brüder zum Vater gestaltet sich hingegen konfliktreich: Der ältere Bruder Cornelius leidet unter den cholerischen Anfällen des Patriarchen und wird häufig verprügelt. Der über sechs Jahre jüngere Carsten erlebt den Vater schon wesentlich gemäßigter, jedoch „missbraucht“, so die Worte Carstens, ihn seine Mutter als Puffer in der schwierigen Beziehung zu ihrem untreuen Ehemann. Carsten erfährt die schlechte Frau-Mann-Beziehung seiner Eltern von Seiten seiner Mutter und ergreift ihre Partei. Er verachtet seinen ,unmoralischen“ Vater, der einen strengen Kommando-Ton pflegt. Die Mutter behandelt ihre Söhne bevorzugt und blickt mit Eifersucht auf die gute Beziehung zwischen Vater und Tochter. Die Familie sozialisiert die beiden Söhne als Nachfolger für das inzwi-

106 Die beiden letztgenannten bezeichnet Simon 2002b als Paradoxien.

107 Der Fall Claas wurde nicht transkribiert. Bei den folgenden Zitaten handelt es sich um eine ausschnittsweise Verschriftlichungen der Audioaufnahme. Entsprechend können keine Seitenzahlen und Zeilennummern angegeben werden. 
schen stark gewachsene Unternehmen, während Claudia aufgrund ihres Geschlechts für ein solches Amt ausscheidet. Ihr Vater kommuniziert ihr klar: „Die Jungs sollten möglichst in die Firma kommen. Und Du, sagt er, du kannst natürlich auch in die Firma kommen, aber Du musst dann im Büro arbeiten, weil Frauen können da ja nicht irgendwas, so richtig Verantwortung, aber Büro ist doch auch wichtig. (lacht) so." (Claudia Claas)

Cornelius engagiert sich in seiner Jugend in der links-politischen Szene und versucht, auf diese Weise Distanz zum Vater zu schaffen. Er trägt den Geist der 1968er-Bewegung in die Familie hinein. Claudia hat das Gefühl, innerhalb der Familie eine vermittelnde Funktion zu erfüllen und die einzige zu sein, die dem Patriarchen die Meinung sagen kann. Sie sucht die offene Konfrontation mit ihrem Vater. Die Rebellion der beiden älteren Geschwister endet mit dem Rauswurf aus dem Elternhaus. Der älteste Sohn versucht sich von nun an immer wieder mit unternehmerischen Projekten, während die Schwester Kinderkrankenschwester lernt und nebenbei ihr Abitur nachholt. Carsten absolviert zunächst eine Lehre zum Speditionskaufmann und legt dann das Wirtschaftsabitur auf dem zweiten Bildungsweg ab. Er fühlt sich der Mutter verpflichtet und bleibt als Mediator zwischen den Eltern relativ lange zu Hause wohnen.

Unter den Geschwistern besteht ein starkes Gemeinschaftsgefühl. Während und nach dem Auszug aus dem Elternhaus unterstützen sie sich gegenseitig, lassen sich beieinander wohnen und tauschen sich regelmäßig aus. Sie teilen eine gemeinsame Erfahrungswelt der Unternehmerfamilie, die sich durch die starke Präsenz des Unternehmens im Alltag, die dominante Persönlichkeit des Vaters sowie die schwierige Beziehung der Eltern zueinander auszeichnet. Sie wissen um die jeweilige Position des anderen in dieser Leidensgemeinschaft. Zudem teilen sie ein Bewusstsein dafür, dass die Eltern das Unternehmen aus einer Situation der Armut für sie als folgende Generation aufgebaut haben und dieses Werk nur dann sinnvoll ist, wenn sie das Projekt weiterhin in Familienhand erhalten.

Mitte der 70er Jahre läuft Cornelius' ${ }^{6}$ Unternehmen sehr schlecht, und er entschließt sich, nun die Nachfolge im Familienunternehmen anzutreten. Die gemeinsame Arbeit mit dem Vater gestaltet sich weiterhin sehr konfliktreich. Der strenge Vater ist oft mit der Leistung seines Sohnes unzufrieden. Einige Zeit darauf verweigert Carsten den Wehrdienst und teilt seinen Eltern mit: „Ich werde nicht in die Firma einsteigen. So, ich mach das nicht. Waren dann sehr enttäuscht, weil sie irgendwie auf mich setzten." Damit steht Cornelius zunächst als einzige Zukunftsoption für das Familienunternehmen fest. Carsten arbeitet nach seinem Studium der Sozialpädagogik als Sozialarbeiter. Im Laufe der Zeit mäßigen sich seine linkspolitischen Einstellungen. Claudia absolviert ihr Studium der Psychologie erfolgreich und nimmt anschließend eine Anstellung in diesem Bereich auf. 
Mitte der achtziger Jahre kommt es zu einem großen Konflikt zwischen Cornelius und seinem Vater hinsichtlich unternehmerischer Entscheidungen. Cornelius kündigt daraufhin sein Arbeitsverhältnis und gründet mit einer Abfindung vom Vater erneut sein eigenes Unternehmen. Einige Monate später ruft Rainer seinen jüngeren Sohn Carsten bei der Arbeit an: „Du musst das jetzt überlegen, ob Du hier kommst, oder. Ich werde immer älter hier. Die Arbeit ist immer mehr geworden. Entweder Du machst das jetzt oder ich verkauf oder nehme einen Teilhaber rein, oder so." Carsten hat Verständnis für die Perspektivlosigkeit des Vaters. Ihm ist bewusst, dass der Vater mit diesem Anruf ein Drohszenario aufbaut: Ein Verkauf oder ein Teilhaber würde zur Aufgabe des Familieneinflusses auf das Unternehmen führen und damit den normativen Kontinuitätsanspruch des Familienprojektes unterlaufen. Carsten bespricht diese Situation eingehend mit Freunden und entschließt sich dafür, die Nachfolge im Unternehmen anzutreten. Mittlerweile kann er sich eher mit der Rolle eines Unternehmers identifizieren. Zudem hat er Distanz zu der Zeit des Aufwachsens in der Unternehmerfamilie gewonnen. Er versteht den Weg in das Familienunternehmen als Chance, noch einmal ein anderes Verhältnis zu seinem Vater aufzubauen. Die Zusammenarbeit zwischen den beiden gestaltet sich erwartungsgemäß konfliktreich. Rainer übt großen Druck auf seinen Sohn aus, will ihn kontrollieren. Carsten fühlt sich an die Strukturen seiner Kindheit erinnert, und ihm fällt es schwer, sich gegen den Vater durchzusetzen. Gemeinsam legen Vater und Sohn der Mutter nahe, ihre Tätigkeit in der Buchhaltung niederzulegen. Als Carsten drei Jahre später schwer erkrankt, wird ihm die Dringlichkeit bewusst, seine Situation zu verändern. Er droht dem Vater mit seiner Kündigung, wenn er sich nicht aus dem operativen Geschäft zurückziehe und nur noch als Eigentümer fungiere. Der Vater willigt ein, installiert aber einen Beirat aus Wirtschaftsberater, Bankdirektor und Steuerberater, die den Sohn von nun kontrollieren sollen. Carsten gelingt es, ein kooperatives Verhältnis zu diesem Beirat aufzubauen. Das Verhältnis zwischen Carsten und seinem Vater entspannt sich.

Während dieser Zeit spricht ein Mitgesellschafter des Vaters Claudia darauf an, ob sie nicht in einem Schwesterunternehmen arbeiten wolle. Sie nimmt die Stelle im Dunstkreis des Familienunternehmens, aber außerhalb der direkten Kontrolle des Vaters und ohne in Konkurrenz mit ihrer Mutter zu treten an, wird etwas später sogar dort Geschäftsführerin. Carsten holt schließlich seine Schwester - gegen den Willen des Vaters - in das Hauptunternehmen. Die Geschwister teilen sich die Sparten auf, die später in rechtlich selbständige Organisationen umgewandelt werden. Claudias Lebenspartner Peter wird Assistent ihres Vaters. Der älteste Bruder Cornelius geht in der Zwischenzeit mit seiner Firma erneut insolvent und gründet ein weiteres Unternehmen.

Auf dem Weg zu einer Weihnachtsfeier stirbt der mittlerweile 77jährige Rainer, der bereits drei Bypässe gelegt bekam, an einem Herzinfarkt. Dieser 
Tod des Vaters kommt für die Familie, die selbst noch so sehr darin verstrickt ist, sich zu ihm zu positionieren, überraschend. Jedes der drei Kinder erbt ein Drittel an der Holding des Unternehmens, die Mutter erhält eine Betriebsrente. Daraufhin will Cornelius erneut in das Familienunternehmen einsteigen. Carsten und Claudia verhindern über eine Auszahlung des Bruders gemeinsam diesen Schritt. Als Schutz vor der „Bedrohung“ des Familienprojektes durch die unternehmerische Unfähigkeit des Bruders gehen sie entgegen der geschwisterlichen Gemeinschaft eine neue Zweierallianz ein.

Die ausführliche Darstellung der Fallgeschichte der Unternehmerfamilie Claas macht deutlich, dass das Unternehmen als Bühne verstanden werden kann, auf der die konfliktreichen Familienverhältnisse und unterschiedlichen Vertrauens- und Missbrauchskonstellationen aufgearbeitet werden können. Die Nachfolgegeschichte ist hier als ein Abarbeiten an einer schwierigen Eltern-Kind-Konstellation zu interpretieren. Das Unternehmen bietet den Ring, in dem die erwachsenen Kinder ihrem Vater begegnen und versuchen, neue Machtverhältnisse zu schaffen. Sie teilen ein starkes Wir-Gefühl, eine große Loyalität gegenüber dem Familienprojekt. Die Bestimmung der eigenen Position innerhalb der Unternehmerfamilie richtet sich in extremer Weise an Gründerfigur und Vater Rainer aus. Dieser Fluchtpunkt der eigenen Identität ist bei Claudia und Carsten so stark ausgeprägt, dass beide nur rudimentäre Zukunftspläne entwickeln, obwohl sie bereits über 55 Jahre alt sind. Auffällig an ihren Selbstdarstellungen ist darüber hinaus, dass typische Merkmale der Präsentation als Unternehmer fehlen. Sie berichten wenig über ihre beruflichen Erfolge und die Entwicklung des Unternehmens. Im Vordergrund steht vielmehr die Position der Familienmitglieder zueinander, ähnlich einem Schachspiel.

Gerade in der Zeit des Aufwachsens in einer Unternehmerfamilie, in der ein Umgang mit dem Unternehmen alltäglich und selbstverständlich ist, wird, wie auch Lubinksi beobachtet (2010:118), die Grundlage für eine individuelle Verortung innerhalb der Generationen übergreifenden Gemeinschaft gelegt. Im Laufe des Lebens wird diese Positionsbestimmung mit Blick auf die Bewegungen der signifikant Anderen innerhalb der Unternehmerfamilie, aber auch mit Blick auf die gesellschaftliche Generation biografisch reflektiert und nachjustiert. Daraus leitet sich ein komplizierter Intergruppenprozess ab, der die Koordination der einzelnen Positionen erforderlich macht. Es entsteht eine spezifische Binnenstruktur und Außengrenze der Unternehmerfamilie als Kollektiv, die nicht mit der Familie als Verwandtschaftsnetz identisch ist, sondern sich über die Teilhabe bzw. Nichtteilhabe am Familienprojekt definiert und hierfür spezifische Mitgliedschaftsregeln produziert, die im Laufe der Zeit veränderbar und neubesetzbar sind. Insofern entsteht die Unternehmerfamilie als eine „Wir-Gruppe“ (Elias 1990; Berger, Luckmann 1989), die daran arbeitet, eine spezifische Präsentation aufrecht zu erhalten, und damit einen Familienauftrag erfüllt. 
Die hier skizzierte Koordinationsaufgabe der Positionsbestimmung lässt sich mit Hilfe des so genannten „Family FIRO“-Modells analytisch feiner beschreiben. Die Psychologen Doherty and Colangelo $(1984 ; 1991)$ wenden die bereits in den 1950er Jahren von Schutz (1958) vorgestellte Theorie zur Analyse von interpersonaler Orientierung in Gruppeninteraktionen (fundamental interpersonal relations orientation $=$ FIRO) auf Familien an. Dieses Modell hat breite Anerkennung gefunden und ist in zahlreichen Studien empirisch angewandt worden (etwa Parr 2000). Danes et al. (2002; 2004; 2005) haben schließlich diesen Ansatz auf Unternehmerfamilien übertragen. Im Zentrum steht die These, dass sich familiale Interaktion an drei zentralen Orientierungspunkten ausrichtet: dem Bedürfnis nach Inklusion, Kontrolle sowie Affektivität. Inklusion definiert, wer Teil der Unternehmerfamilie ist und wer nicht, die Art und Weise, in der Familien den Mitgliedern spezifische Funktionen zuweisen, sowie das Verhältnis zwischen Unternehmerfamilie und Außenwelt (Danes et al. 2002). Diese Dimension umfasst im Wesentlichen Mitgliedschaftsregeln und Machtstrukturen, ein Gefühl der Verbundenheit ebenso wie geteilte Bedeutungszuschreibungen und Werte. Kontrolle umfasst die Praktiken des Konfliktmanagements, der Einflussstrategien und Verhandlungsweisen in den intergenerationalen Positionsbestimmungen. Diese Dimension verweist darauf, wie sich Macht und Einfluss innerhalb der Familie auf Konflikte und Konfliktlösung auswirken. Es wird dabei in dominante, kooperative oder reaktive Verhaltensweisen der Familienmitglieder unterschieden, die in den inhaltlichen Verhandlungen zu Tage treten. Die letzte Dimension der Affektivität bzw. Intimität beschreibt eine wechselseitige emotionale Verbundenheit, das Teilen von Gefühlen und Verletzlichkeit mit anderen Familienmitgliedern. Diese Dimensionen stehen in einem sequentiellen Verhältnis zueinander. Fragen der Inklusion (Mitgliedschaft, Grenzbestimmung) werden gefolgt von Fragen nach Kontrolle und Macht und schließlich dem Bedürfnis nach einer tief empfundenen Gemeinschaft. Doherty et al. (1991) stellen die Hypothese auf, dass sich die Familie in unterschiedlichen Lebensphasen in verschiedener Intensität mit den drei Bedürfnissen nach Inklusion, Kontrolle und Intimität beschäftigt.

Danes et al. schlagen vor, die dritte Kategorie im Raum von Unternehmerfamilien als „Integration“ zu bezeichnen (Danes et al. 2002:33). Sie betonen dabei die intermediäre Position zwischen dem gefühlsarmen System des Unternehmens und dem gefühlsbetonten System der Familie, wobei die jeweils unterschiedlichen Zielvorstellungen zu einem harmonischen Ganzen balanciert werden sollen. Diese analytische Trennung in zwei funktionale Bereiche mit unterschiedlichen Erwartungshaltungen und Ansprüchen zeigt sich in den Fallauswertungen jedoch weit weniger ausgeprägt als die Erweiterung des Unternehmens zu einem familialen Raum und als Mittler in der emotionalen Beziehung zwischen Eltern und Kindern. Aus diesem Grund soll hier an dem ursprünglichen Begriff der Affektivität festgehalten werden. 
Mit Hilfe des Family FIRO-Modells lässt sich das Verhältnis zwischen (erwachsenen) Kindern und ihren Eltern in Unternehmerfamilien und vor allem die Koordinierungsaufgabe der Positionsbestimmung wie folgt beschreiben: Die einzelnen Familienmitglieder streben nach Inklusion in die Gemeinschaft der Unternehmerfamilie, die über spezifische Mitgliedschaftsregeln und eine Außengrenze verfügt. Diese sind gleichsam Gegenstand und Produkt des intergenerationalen Aushandlungsprozesses, in dem die Funktionszuweisungen und Funktionsselbstsichten zur Disposition gestellt werden und die einzelnen Akteure unterschiedliche Einflussstrategien und Lösungsansätze verfolgen (Kontrolle). Die Gruppe teilt eine kollektive Identität, an der sich die Beziehungen zwischen den Mitgliedern ausrichten, die nach einem tief empfundenen Gemeinschaftsgefühl streben (Affektivität).

Auf diese Weise tritt auch das Konfliktpotenzial, das dieser Koordinierungsaufgabe innewohnt, zu Tage: Es kann Uneinigkeit über die Besetzung der Positionen bestehen, die einen starken Konkurrenzkampf oder Vakanzen nach sich zieht. Ebenso können sich einzelne Familienmitglieder aus der Gemeinschaft exkludiert fühlen und so ein geringeres Maß an Bindung und Solidarität aufweisen.

\subsubsection{Koordination der Lebensläufe}

Die zweite Herausforderung für die Generationenbeziehungen in Unternehmerfamilien besteht in der starken Notwendigkeit, die Lebensläufe der Mitglieder in zeitlicher, biografischer und betrieblicher Hinsicht zu koordinieren. Dieser Umstand tritt als wesentliches Muster in allen Falldarstellungen auf und verdeutlicht sich zumeist an mehreren Zeitpunkten im Lebenslauf der Mitglieder einer Unternehmerfamilie, besonders stark jedoch beim Eintritt in das Familienunternehmen. An dieser Stelle seien nur einige wenige Beispiele kurz skizziert:

Konrad Niest studiert in den 1970er Jahren, wie auch sein jüngerer Bruder, Wirtschaftsingenieurwesen. Bereits in den Ferien ist er im Familienunternehmen tätig und tritt dort unmittelbar nach seinem Studienabschluss eine leitende Stelle an. Sein Vater ist zu diesem Zeitpunkt 71 Jahre alt. Konrad sagt: „Nach dem Studium, Studienabschluss habe ich hier gleich angefangen. ... Äh wäre vielleicht gar nicht so verkehrt gewesen, wenn ich mal irgendwo was anderes (schnipst mit den Fingern) äh, ... äh geschaut hätte, aber äh mein Vater war relativ äh alt. [ausgelassen 1]... na dann überlegt man sich schon wie es mit der Biologie so weitergeht.“ (3:30-4:3)

Im Alter von 51 Jahren erkrankt Beate Übermann an Krebs. Sie wird operiert, verbringt einige Wochen im Krankenhaus und anschließend auf Kur. Ihr Sohn Markus (25 Jahre), der zu dieser Zeit auf einem Weingut in Süddeutschland arbeitet und eine Fachschule für Weinbautechnik absolviert, 
tritt den direkten Weg in den elterlichen Betrieb an. In diesem Fall war ein Eintritt des Sohnes in das Familienunternehmen bereits angelegt, die Krankheit der Mutter gab lediglich den Anstoß für die Übergabe. Sohn und Mutter sehen sich nun schon zu einem frühen Zeitpunkt in ihrem Leben mit der Führungsverantwortung einerseits und einer Rolle in der zweiten Reihe andererseits konfrontiert.

Als Florian Wagner (Anfang 50) und Barbara Vester (Mitte 50) endlich den Zuschlag für das Gelände der „Pfälzer Mühle“ von der Stadt erhalten und sie hier ihren Traum eines eigenen Gastronomiebetriebes umsetzen wollen (sie sind bereits Pächter von weiteren Restaurants), blickt die Bank kritisch auf das Alter der Unternehmer. Für die Kreditvergabe erhalten sie die Auflage, eine klare Nachfolgeregelung mit ihren Söhnen zu treffen. Oscar, der seine Zukunft schon lange im Familienunternehmen sieht, bekennt sich $\mathrm{zu}$ den unternehmerischen Risiken. Überraschenderweise kündigt auch Andreas seine Festanstellung und steig in das Familienprojekt ein.

In diesen Beispielen wird offensichtlich, wie die Erwerbs- und Familienbiografien der Mitglieder aus Unternehmerfamilien ineinander greifen, wobei in der Fallauswertung der Eindruck entsteht, dass das Leben der Eltern stärkeren Einfluss auf die Kinder ausübt als umgekehrt. Die Ungleichzeitigkeit der Lebensphasen zwischen den familialen Generationen bedarf einer zeitlichen Abstimmung, damit das Kollektiv der Unternehmerfamilie eine Fortsetzung finden kann. Die Lebensplanungen der einzelnen Familienmitglieder können durcheinander geraten oder sich der zeitliche Umfang einer Lebensphase verschieben. So kann etwa die Familiengründung mit Blick auf das Familienunternehmen hinausgezögert oder der Rückzug aus dem Unternehmen früher angetreten werden.

Der Umstand, dass historische oder konkrete familiale Situationen nicht nur innerhalb des individuellen Lebenslaufes bedeutsam sind, sondern auch die Leben der Familienmitglieder beeinflussen, ist von Glen Elder (1995) als „linked lives“ beschrieben worden. So erhält etwa eine längere Arbeitslosigkeit, eine Scheidung oder eine Krankheit eines Familienmitgliedes eine intergenerationale Qualität. Die Besonderheit der Unternehmerfamilie besteht also nicht darin, dass ihre Lebensläufe miteinander verbunden sind, sondern in einer spezifischen Qualität der Verbindung. Das Unternehmen als langfristiges Projekt wirkt kontinuierlich auf die Unternehmerfamilie ein und verstärkt damit die Intensität des wechselseitigen Einfluss von Lebenslaufereignissen und -entscheidungen der jeweiligen Familienmitglieder. Diese direkte Qualität der Generationenbeziehungen macht es erforderlich, die jeweiligen Lebensvorstellungen und Lebenspläne miteinander abzustimmen.

Die Akteure sind gefordert, diese unmittelbare Verwicklung ihrer Leben in ihre biografische Selbstsicht zu integrieren, dies betrifft sowohl die direkt Beteiligten als auch jene Familienmitglieder, die einen Teil ihrer Identität aus der Gemeinschaft der Unternehmerfamilie beziehen. Sie sehen sich dabei von 
Seiten der Unternehmerfamilie (auch in ihrem historisch verlängerten Verständnis) einem Erwartungsdruck, von Seiten der gesellschaftlichen Generation einem Individualisierungsdruck ausgesetzt, zu dem sie sich positionieren und rechtfertigen müssen. Insofern bestätigt sich hier die in der Forschungsliteratur hinlänglich beschriebene Aufgabe, einen eigenständigen Lebensentwurf innerhalb der Unternehmerfamilie zu entwerfen (siehe hierzu Kapitel 3.4.2), die ein hohes Maß an biografischer Arbeit erforderlich macht.

Auf betrieblicher Seite wirkt sich diese Koordinierung der Lebensphasen erheblich auf die strategische Positionierung des Unternehmens aus, etwa hinsichtlich der Führungs- und Eigentümerstruktur, des Timings größerer Investitionen oder der Vermarktung als „Familienunternehmen“. Eventuell können innerhalb der Unternehmerfamilie institutionalisierte Vorstellungen von der Führung des Familienunternehmens nicht mehr aufrechterhalten werden.

Dieser Prozess der Lebenslaufkoordination in Unternehmerfamilien birgt ein nicht unerhebliches Potential an Identitätskonflikten, Verwerfungen zwischen den Generationen und betrieblichen Risiken, die in letzter Konsequenz eine Reproduktion der Unternehmerfamilie scheitern lassen können.

\subsubsection{Materielle Transfers}

Die Fallauswertungen legen nahe, dass in Unternehmerfamilien ein hohes Bewusstsein für die gesellschaftlichen Vorstellungen des materiellen Transfers herrscht (siehe z.B. (Kohli 1999; Kohli et al. 2005b; Albertini et al. 2007; Light und McGarry 2004): Der Fluss von Geldvermögen erfolgt zu Lebzeiten aus altruistischen Motiven. Die Generationen greifen sich wechselseitig in unsicheren und prekären Phasen des Lebens finanziell unter die Arme, wobei eine ,ungerechte“, aber legitime Verteilung zwischen den Geschwistern möglich ist. Im Erbgang jedoch sollen alle Kinder zu gleichen Teilen berücksichtigt werden (Gleichbehandlungsgrundsatz).

Mit dem Transfer von Unternehmensanteilen an die Kinder wird nicht nur eine Einkommensquelle, sondern auch ein verbrieftes Mitspracherecht am Unternehmen übertragen. Unternehmerfamilien sehen sich mit der Notwendigkeit konfrontiert, eine Regelung für die Führungs- und Eigentumsverhältnisse zu schaffen, die dem Unternehmen zuträglich ist. In den Fallauswertungen zeichnet sich ein hoher Konsens darüber ab, dass diejenigen Familienmitglieder, die im Unternehmen operativ tätig sind, auch die (Haupt)Anteile an diesem besitzen sollten. Den Hintergrund für diese Maxime bilden drei Annahmen: Erstens, mit der Mehrheit der Eigentumsanteile verfügen diese Familienmitglieder auch über die Entscheidungsgewalt im Unternehmen, was die Führung dessen erleichtern oder konfliktärmer gestalten kann. Zweitens, mit seiner Tätigkeit im Unternehmen begibt sich dieses Fa- 
milienmitglied in eine höhere berufliche sowie finanzielle Abhängigkeit und trägt das unternehmerische Risiko. Drittens, es besteht die Gefahr, dass jene Familienmitglieder, die sich nicht beruflich im Unternehmen engagieren, dieses lediglich als Teil ihres Vermögensportfolios begreifen und entsprechend einer Handlungslogik folgen, die weniger stark die emotionalen Aspekte der Familientradition berücksichtigt oder ausdauernd in Zeiten der Krise verfährt.

In der Familie Naumann entzündet sich genau an diesem Punkt ein langwieriger Erbstreit: Werner und Amalia Naumann erben überraschend von ihrer Großmutter das Weingut im Rheingau - und erstmals in der Familiengeschichte wird der Besitz unter Geschwistern aufgeteilt. Während Werner in fünfter Generation tatsächlich auf dem Gut lebt und arbeitet, zieht seine Schwester Amalia in eine andere Stadt, in der sie als Ärztin tätig ist. Sie hält sich weitgehend aus dem Alltagsgeschäft heraus. Innerhalb von Werners Familie ist ,ganz klar“", dass die Eigentumsaufteilung zwischen den Geschwistern - „die Konstruktion“ - etwas Unnatürliches ist, „eine furchtbare, schlechte und ... sehr gefahrbringende Konstellation" (Wilhelm Fritz Naumann, 11:15-16), eine ,ganz schlechte Lösung“ (11:23), die ,immer, immer wie ein Damoklesschwert über dem, über dem Weinbaugut" (11:2324) hing.

Als Anfang der 1980er Jahre Werner stirbt, gehen seine Anteile vollständig an seinen Sohn Felix Walter über. „Nicht mal drei Wochen nach seinem Tode“(Wilhelm Fritz Naumann, 12:3) kommt „die andere Seite“ und erklärt, das Gut nicht mehr in der bisherigen Konstellation weiterführen zu wollen. In Vertretung für den anderen Familienstamm unterbreitet ein wohlhabender Vetter aus Amerika der Familie das Angebot, ihren Anteil auszulösen und fortan das Gut mit einem externen Betriebsleiter zu führen. Felix Walter erzählt: „Das war, war ziemlich tief in der Magengrube, nicht, so eine Ankündigung“ und das hat ihn ,natürlich ZUTIEFST ERSCHROCKEN, allertiefst erschrocken.“ (28:19-20) Das rationale Denken des einen Familienstammes in Kategorien von „Verzinsung“, „Prozent" und „,sehr nüchtern und sehr betriebswirtschaftlich" (Wilhelm Fritz Naumann, 15:12) steht dem tief empfundenen ,ideellen Wert“ des Gutes für den anderen Familienstamm als eine spezifische Lebensweise entgegen. Das Unverständnis richtet sich vor allem gegen die Kinder der (Groß)-Tante, ,immerhin auch Vettern und Cousinen, nicht?" (Wilhelm Fritz Naumann, 34:14). Gerade die Befürchtung, dass sich „,irgendjemand“ Fremdes, d.h. eine dritte Partei das Gut „schnappen“ könnte und die Familie nicht „,dagegen...angehen“ könne, ist Anlass zur Sorge. Der Verlust des Weinguts für die nachfolgenden Generationen wäre für die Familie von Felix Walter der schlimmste anzunehmende Fall.

Unter großen finanziellen Anstrengungen und einer beruflichen Doppelbelastung aus einer abhängigen Vollzeitbeschäftigung und einer Teilzeittätigkeit im Weingut kauft Felix Walter den anderen Familienstamm aus. „Es 
hat funktioniert. JA, das war (lacht) eigentlich die Krise des Lebensweges oder eine der Krisen, ja, wenn man vom Bombenkrieg mal absieht und so was alles, mhm.“ (Felix Walter Naumann, 28:25-27) In der Konsequenz vermittelt Felix Naumann seinen Kindern klar, dass es fortan nur noch einen Erben des Weingutes geben kann (in diesem Fall seine Tochter Claudia) und verlangt von seinen Kindern Einsicht, dass ein Hauptteil des Vermögens in diesem Gut gebunden ist und entsprechende Ausgleichszahlungen ,selbstverständlich" nicht oder nur in geringerem Maße erfolgen können. Der Kontinuitätsanspruch der Unternehmerfamilie schließt die Liquidierung des Weingutes, das nur ,auf dem Papier vielleicht toll äh und wertvoll ist“" normativ aus - und unterbindet damit auch die Möglichkeit einer anderen Variante der „gerechten“ Aufteilung unter den Kindern. Felix Walter Naumann kommuniziert diese Erbstrategie offen: „Das Testament ist bekannt in der ganzen Familie, ich habe da Kopien allen 4 Kindern gegeben, nicht, nichts hinterm Rücken.“" (49:31-32)

Die Koordinierungsaufgabe des materiellen Transfers besteht nicht nur in der Balance zwischen der normativen Vorstellung einer Gleichbehandlung der Kinder im Erbgang und einer für das Unternehmen zuträglichen Regelung der Führungs- und Eigentümerstruktur (wie etwa von Simon 2002b oder Stamm 2009 herausgestellt), sondern muss auch Werte zum Umgang mit dem Familienerbe berücksichtigen, die innerhalb der Unternehmerfamilie bestehen. Diese an sich anspruchsvolle Aufgabe kann zusätzlich erschwert werden, wenn überproportional viel Vermögen im Unternehmen steckt (Hildenbrand 2002), die Finanzierung des eigenen Lebensabends mit dem Unternehmen verbunden ist oder die Konstellation einer Patchwork-Familie vorliegt. Die Falldarstellungen zeigen, dass die Übergebenden angesichts dieser „Gerechtigkeitsfrage, die sich aber nicht lösen lässt" (Michael Treuninger, 42:32-33) bzw. der „etwas komplizierteren Aufgabe“ (Konrad Niest, 8:2) in einen inneren Konflikt geraten, der oft in eine „ungerechte“ Erbstrategie mündet. Insofern bestätigen und erweitern die Ergebnisse der hier vorliegenden empirischen Studie die in der Forschungsliteratur bereits vorhandenen Ansätze zu materiellen Transfers (siehe hierzu Kapitel 3.5).

Als ein wesentlicher Knackpunkt bei der Übertragung des Familienvermögens erweist sich eben die „Bewertung“ des Unternehmens, die sich an betriebswirtschaftlichen Faktoren ebenso wie der ideellen Wertschätzung bemisst. Die Arbeiten von Thomas Zellweger (Zellweger und Astrachan 2008) weisen in diesem Zusammenhang daraufhin, dass bei einer hohen emotionalen Bindung ein Familienunternehmer bei einem Verkauf an eine externe Person dazu neigt, den Wert zu hoch anzusetzen. Die Falldarstellungen legen nahe, dass bei einer familieninternen Übergabe eher ein niedriger Wert angesetzt wird. Sven Singermayer etwa begründet dieses Vorgehen wie folgt: „weil ich sage, wenn ich eine Immobilie habe, da brauche ich wenig tun, da brauche ich keinen persönlichen Einsatz leisten. Ich kriege Mieten, äh 
ich mache die Hausverwaltung selber oder vergebe sie, aber es ist ein Einkommen, aber das Firmenvermögen muss ich täglich neu erringen sozusagen. Das fordert meine ganze Energie und Aufmerksamkeit und Zeit und wenn einer ein Haus oder Immobilie oder was immer äh äh erbt, dann kann er nebenbei einen anderen Beruf machen und hat aber trotzdem ein Einkommen." (46:34-47:6)

Im Kontext der Unternehmerfamilie scheinen beide Varianten der Erbverteilung mit einem hohen Konfliktpotenzial verbunden zu sein: Im Fall einer ungleichen Verteilung können sich gerade die weichenden Erben, die sich in Verzicht und Rückhaltung üben sollen, als ungerecht behandelt fühlen. Umgekehrt mögen sich die Haupterben in einer Fürsorgepflicht gegenüber ihren Geschwistern wähnen. Es ist möglich, dass sich im Laufe der Zeit die Lebenswelten und der Wohlstand der Geschwister erheblich auseinander entwickeln und so Anlass zu Missgunst und Neid werden können (Wenk 2007:209ff.). Zudem manifestiert sich in der faktischen Eigentumsregelung eine Exklusion aus dem engsten Kreis der „Protagonisten“ der Familiengeschichte, die den persönlichen Eigentumsgefühlen nicht entsprechen und so $\mathrm{zu}$ Verletzungen und Identitätskrisen führen kann. Aber auch im umgekehrten Fall einer ungefähren Gleichverteilung der Anteile am Unternehmen können Konflikte entstehen, wie der Fall Naumann verdeutlicht hat. Die im Unternehmen tätige Person kann sich von den Geschwistern kontrolliert und „ihrer“ Einnahmen beraubt fühlen. Es entsteht eine zwanghafte Solidarität zwischen den Geschwistern, die als Last empfunden werden und auf lange Sicht zu Streit führen kann.

Diese Ausführungen verdeutlichen, dass für ein Verständnis der Koordinationsaufgabe des materiellen Transfers und der damit verbundenen Konfliktlinien auch die Geschwisterbeziehungen jenseits einer Ungleichbehandlung durch die Eltern in Betracht gezogen werden müssen - eine Forderung, die bereits Regina Wenk (2007) formulierte. In diesem Zusammenhang scheint der Begriff der „Reziprozität“, der für familiale Beziehungen im Allgemeinen in Anspruch genommen wird, besonders aufschlussreich. Der Austausch von Gaben kann innerhalb der Familie mit einer Verpflichtung zu einer äquivalenten Gegengabe verknüpft sein. Die Norm der Reziprozität, wie Hollstein und Bria (1998:8) herausarbeiten, beschränkt sich nicht auf den Austausch materieller Güter, sondern kann alles umfassen, etwa auch Dienstleistungen, Kognitionen oder Emotionen. Mit Bezug auf die Anthropologin Gloria Wentowski (1981) unterscheiden sie in ,balancierte“ und ,generalisierte" Reziprozität. Erstere betrifft kleinere Gaben in kurzen Abständen, die oft in einem ökonomisch äquivalenten Maße erwidert werden, zweitere verweist auf eine Gabe, deren Erwiderung nur langfristig, wenn überhaupt erwartet wird, aber mit einem tiefen Verpflichtungsgefühl einhergeht. Hollstein und Bria schlussfolgern, dass die Ausdehnung der zeitlichen Perspektive Konsequenzen für die Form und den Inhalt der Reziprozität mit sich bringt. 
Bezogen auf Unternehmerfamilien könnte man nun nicht nur den materiellen Transfer der Eigentumsanteile von Eltern auf die Kindern, sondern auch den Verzicht der Geschwister auf das Erbe oder zumindest den Unternehmensteil davon als eine Gabe verstehen. Ein Ende der (Austausch)Beziehung zwischen den Geschwistern wird, wenn sie der Norm einer generalisierten Reziprozität folgen, nicht antizipiert. Der Moment der erwarteten Rückgabe verschiebt sich auf unbestimmte Zeit in die Zukunft, und auch eine in ökonomischem Sinne äquivalente Gegenleistung wird nicht erwartet (Hol1stein und Bria Gina 1998:12). Stattdessen orientiert sich diese Gabe an dem Vertrauen, dass die erhaltene Hilfe später einmal zurückgegeben werden kann und sich an die situativen Bedürfnissen und Ressourcen beider Seiten anpasst, also etwa Unterstützung in Zeiten von Not erfolgt.

Zusammenfassend lässt sich festhalten, dass der Geber des materiellen Eigentums am Familienunternehmen in einen inneren Konflikt gerät, in dem es darum geht, widerstreitende Normen zu koordinieren. Der Nehmer auf der anderen Seite findet sich in einem komplexen Geflecht aus Erwartungen und Verpflichtungen wieder, die sich aus dem Kontinuitätsanspruch des Familienprojektes sowie der Versorgungspflicht der Eltern im Ruhestand und der Geschwister bei Bedürftigkeit ableiten.

Die Art und Weise, wie der materielle Transfer schließlich erfolgt, also etwa in Form einer Schenkung, eines Erbes oder einer Stiftung, scheint letztlich nur ein Umsetzungsproblem zu sein. Es geht vielmehr darum, die Koordination zwischen den Familienmitgliedern zu ,verhandeln“. Dabei kann der faktische Transfer als ein Endprodukt gesehen werden oder aber (wie im Fall Clemens dargestellt in Abschnitt 3.1) instrumentalisiert werden. Vor diesem Hintergrund erklärt sich auch, warum etwa die Reform der Erbschaftssteuer, die in den Jahren 2008-2009, in denen die Interviews durchgeführt wurden, trotz ihrer großen Präsenz in der öffentlichen Diskussion für die Erwägungen der Befragten nur eine sehr geringe Rolle spielte.

\subsection{Verhandlungsweisen}

Die bestehenden Koordinierungsaufgaben in Unternehmerfamilien werden nicht ausgelagert (wie dies z.B. im gesellschaftlichen ,Generationenvertrag' der Fall ist), sondern unmittelbar verhandelt. Nachdem nun der Gegenstand der generationellen Aushandlungen näher bestimmt ist, sollen im Folgenden die Verhandlungsweisen in den Blick genommen werden. Die Antwort, es handle sich um einen etappenweisen subtilen Prozess, ist sicherlich korrekt, jedoch höchst unbefriedigend. Aus den Falldarstellungen lassen sich drei Modi kondensieren, die als typische Herangehensweisen in diesem Aushand- 
lungsprozess gelesen werden können: eine Art Pädagogik der Nachfolge, eine Indizienanalyse der Lebensläufe und schließlich explizite Kommunikation, die sich in Form einer spezifischen Gesprächs- und Erzählkultur, von Fragezeremonien sowie eng daran anschließend Familiennarrationen ausdrücken kann. Diesen drei Verhandlungsmodi ist gemein, dass sie gangbare Strategien beschreiben, wie die Koordinierungsaufgaben, die sich Unternehmerfamilien stellen, unter den Bedingungen moderner Gesellschaften gelöst werden können. Sie stellen jedoch typische Muster und keine „Erfolgsrezepte" dar.

\subsubsection{Pädagogik der Nachfolge}

Inwiefern kann die „Erziehung“ der Kinder in Unternehmerfamilien dazu beitragen, die bestehenden Koordinationsaufgaben zwischen den Generationen zu lösen bzw. zu vereinfachen? Diese Frage ist eng daran gekoppelt, ob eine „Erziehung zur Nachfolge“ möglich ist.

Die Antwort auf diese Frage scheint vom jeweiligen historischen Kontext abhängig zu sein. Christina Erdmann weist darauf hin (im Folgenden Erdmann 1999:43), dass in den 1960-70er Jahren eine Erziehung zum Nachfolger in Unternehmerfamilien als selbstverständlich angesehen wurde. Den Eltern diente der Traditionserhalt als zentraler Maßstab in der Vorbereitung des Nachfolgers, der qua Geburt für dieses Amt bestimmt war. In den 1980er Jahren bahnt sich dann die Auflösung dieser Selbstverständlichkeit an. Nachfolge wird nun mehr als Entscheidung verstanden, auf die die Eltern hauptsächlich motivierend einwirken können. Trotzdem werden die ,sich einfach so ergebenden sozialisatorischen Momenten im Heranwachsen eines Unternehmernachkommens“ (Erdmann 1999:218) durch „,die aktive Gestaltung (mehr oder weniger bewusst) vorgenommener erzieherischer Handlungen“ (ebd.) flankiert. Die Studie von Eva Schmitt-Rodermund und Elke Schröder (2009) charakterisiert den Erziehungsstil gegenwärtiger Unternehmerfamilien anhand der Kriterien elterliche Wärme, Förderung von Eigenverantwortung und Unabhängigkeit sowie Erzählungen über das Familienprojekt (Schmitt-Rodermund und Schröder 2009:29). Und auch in einer Reihe von Beiträgen zur Sozialisation in Unternehmerfamilien (etwa Lubinski 2011 oder Kormann 2011:87ff.) besteht ein großer Konsens darüber, dass sich eine Maxime der Selbständigkeit der Kinder durchgesetzt hat, die Eltern in einen Zwiespalt bringt, einerseits die Kinder jenseits von Druck und Traditionserhalt ihren eigenen Lebens- und Berufsweg finden zu lassen, ihnen andererseits den Wunsch nach Kontinuität des Familienprojektes nahe zu bringen. Breuer geht sogar soweit zu behaupten, dass ,ein Familienbetrieb [..] heutzutage eher verkauft oder stillgelegt [wird], als dass ein Nachkomme gegen sei- 
nen Willen zur Übernahme bzw. Weiterführung verpflichtet oder gezwungen wird.“ (Breuer 2009:276).

Insofern folgt die Erziehung in Unternehmerfamilien dem gesellschaftlichen Wandel von Erziehungsvorstellungen, wie diese in familiensoziologischen Studien für die letzten Dekaden beschrieben worden sind. Auch hier lässt sich eine Abkehr von traditionellen Erziehungszielen wie Gehorsam und Sauberkeit und eine stärkere Betonung der Eigeninitiative feststellen (etwa Nave-Herz 2007; Schweizer. Herbert 2007:128ff.). Dem Individuum wird eine größere Handlungsmacht über das eigene Leben zugeschrieben. Die Autorität des Familienoberhauptes sowie direkte Vorgaben nehmen in den Erziehungshandlungen der Eltern $\mathrm{ab}$ und werden durch eine wertvermittelnde Funktion ersetzt.

In den Fallauswertungen spiegelt sich dieser Wandel der „Erziehung“ in Unternehmerfamilien ebenso wie die gegenwärtige Norm zur Selbständigkeit der Kinder sehr deutlich wider. Anhand der Unternehmerfamilie Clemens soll diese Entwicklung dargestellt und die These entwickelt werden, dass die zeitgenössische „Pädagogik“ der Nachfolge dazu beiträgt, einen Rahmen für die Positionsbestimmung in Familie und Gesellschaft zu schaffen, einen selbständigen Lebensentwurf zuzulassen und den materiellen Transfer pädagogisch aufzuladen, und dass sie auf diese Weise zur Lösung der Koordinationsaufgaben beitragen kann.

Als Conrad Clemens, der das Unternehmen bereits in dritter Generation führt, 1928 relativ jung (er ist noch keine 60 Jahre alt) verstirbt, fehlt eine klare Erbregelung. Die Anteile gehen gemäß den gesetzlichen Bestimmungen an seine drei Söhne über. Die beiden jüngeren treten in den Betrieb als kaufmännische und technische Leiter ein. Der Älteste hingegen wird Gynäkologe und finanziert seinen hohen Lebensstandard aus den Unternehmensanteilen. Seine Brüder sehen sich zum Schutz des Unternehmens dazu gezwungen, ihn gegen eine großzügige Altersvorsorge auszulösen. Aus dieser Erfahrung heraus verständigen sich die beiden verbleibenden Brüder in Gesprächen und Briefen früh darüber, wer das Unternehmen in der nächsten Generation einmal leiten solle: Sie legen sich auf Leopold fest ${ }^{108}$. Mit 19 Jahren übertragen sie ihm die Mehrheit der Anteile am Unternehmen und signalisieren mit diesem Schritt deutlich, dass sie ihr Vertrauen in ihn setzen. Oscar, Leopolds Vater, und sein Bruder wollen einer erneuten ,plötzlichen“ Übergabe vorbeugen, die ihnen selbst eine große finanzielle und persönliche Bürde im Konflikt mit dem Bruder auferlegt hat.

Leopolds Familie lebt bürgerlich in der Kleinstadt, in der auch das Unternehmen angesiedelt ist. Die Kinder besuchen die örtlichen Schulen und sind im Verein engagiert. Leopold erlebt den Aufstieg und die Expansion des

108 Der Werdegang von Leopold Clemens bis zum Eintritt in das Unternehmen wurde bereits in Kapitel 6.2.2.2 als Beispiel für eine Nachfolge als Mission vorgestellt. 
Unternehmens zu Zeiten des Wirtschaftswunders und ist stolz auf seinen Vater und die Erfolgsgeschichte der Familie. Am Ort wird die Fabrik immer präsenter, und sein Vater ist politisch engagiert. Die Familie genießt hohes Ansehen. Jeder weiß um den Hintergrund der Clemens-Nachkömmlinge. Leopold lernt, mit diesem sozialen Status, aber auch mit der damit verbundenen sozialen Kontrolle umzugehen. Er wird Klassen- und später Schulsprecher, er ist engagiert im Sportverein und Chef vom Dienst der Schülerzeitung. Mit 14 Jahren beginnt er in den Ferien im Unternehmen zu arbeiten. Die Geschwister erhalten von der Firmensekretärin Sprachunterricht. Leopold erkennt, dass sein Vater ihnen einen möglichst guten Start ins Berufsleben ermöglichen will. Er schätzt diese Bemühungen und versucht die Möglichkeiten zu nutzen. Er sagt:,,der Eine nutzt es intensiv, der Andere weniger intensiv, der Eine hat es begriffen worauf es ankommt.." (5:18)

Mit der klaren Delegation Leopolds zum Nachfolger während seines Abiturs enden alle alternativen Pläne, die er für sein Leben entworfen hatte, wie etwa Theologie zu studieren. Jeder Schritt seines „beruflichen Werdegangs" (9:12) (also der Weg vom Abitur bis zum Eintritt ins Unternehmen) ist mit dem Vater/der Familie abgestimmt und dient dem Ziel einer möglichst guten Vorbereitung auf ein späteres Amt im Unternehmen. Leopold wiederholt (fast exakt) den Weg des Vaters: Nach einer Banklehre verbringt er zwei Jahre in England und den USA. Er übernimmt dann die Leitung eines neu gekauften Unternehmens in Brasilien, bis er schließlich in die Kleinstadt zurückkehrt und im Hauptsitz des Unternehmens tätig wird. Leopold kann mit den vorgegebenen Strukturen umgehen. Die Auslandserfahrung, insbesondere die Zeit in Brasilien, scheint ihn für ein verpasstes Studium zu entschädigen. Leopold verschreibt sich dem Projekt der Fortführung des Unternehmens, so dass Wollen und Sollen ineinander übergehen.

Mit dem Eintritt in das Familienunternehmen ändert sich für Leopold das Berufsleben grundlegend. Er arbeitet nun nicht mehr auf das große Ziel der Übernahme hin. Er hat Entscheidungsfreiheit und muss Verantwortung tragen. Mit Ina findet Leopold eine Partnerin, die ihn in seinem Tun versteht und unterstützt. Die beiden setzen ihren Wunsch nach einer Großfamilie um. Jedes der Kinder erhält Namen aus der Familiengeschichte.

Leopold ist ein aktiver Vater: Er wickelt die Babys, macht sie morgens fertig für den Tag und spielt ausgiebig. Familie wird für ihn zu einem wichtigen Ausgleich zum Unternehmen. Er legt Wert auf ein enges, emotionales Verhältnis zwischen den Familienmitgliedern. In seinem Verständnis als Bürger dieser Stadt schickt Leopold seine Kinder in den örtlichen Kindergarten und Sportverein. Sie sollen sich frei bewegen können. In der Zwischenzeit ist das stark gewachsene Unternehmen in der Stadt überall präsent (diverse Gebäude, Schilder, Arbeitsplätze, etc.). Insofern wiederholt und verstärkt sich für die Kinder, was Leopold selbst erlebte: Sie unterscheiden sich durch ihre Bekanntheit und ihren sozialen Status von anderen Kindern. Sie 
erfahren Privilegierung und soziale Kontrolle. Mit dem Unternehmen kommen die Kinder über Anekdoten aus der Familiengeschichte sowie Besuche bei „Papa im Büro“ in Kontakt. Sie sind bei Firmenfesten dabei, und gelegentlich holt sie der Fahrer vom Kindergarten oder der Schule ab.

Als seine Kinder älter werden, versucht Leopold, jene Punkte in der Erziehung, die er selbst als positiv erlebt und auch ganz bewusst wahrgenommen hat, an seine Kinder weiterzugeben, Selbstanteile in ihnen zu deponieren. Dazu zählen: Förderung von Sprachen, sozialem Engagement, sportlichen Aktivitäten, Gemeinschaftssinn und Vertrauen sowie Leistung. Leopold passt diese Grundpfeiler der Erziehung an seine Zeit an: Ganz bewusst entscheidet sich Leopold auch dafür, die Kinder auf ein örtliches Gymnasium zu schicken und die Vereinskultur zu nutzen: In ihrer Freizeit sind die Kinder mit Leistungssport (Schwimmen, Turnen), Musik, Schüleraustausch und Reisen ausgelastet. Ab ca. elf Jahren müssen die Kinder jeden Sommer für 4-6 Wochen ein Sommercamp im Ausland besuchen. Die Mutter sagt dazu: „Die sollten früh lernen sich... selbst zu sehen ... mal abgenabelt von dem Namen [Clemens] und hier ... kennen sie alle in den Schulen, .. jeder kennt sie. Einfach dort ... bewertet zu werden oder ... ja, anerkannt zu werden als eigenständige Person.“ (Ina Clemens, 16:5-8) Ina und Leopold legen Wert auf gemeinsame Erlebnisse wie Urlaube, Spieleabende und ein gemeinsames Essen am Sonntagabend ,und dann wurde, am Tisch wurde all das ausgesprochen, was sich während der Woche angestaut hat". (Ina Clemens, 21:19-20)

Während eines Familienurlaubs befragt Leopold seine jugendlichen Kinder, ob sie sich eine Nachfolge im Unternehmen vorstellen könnten. Sie wissen um den Wunsch des Vaters, dass das Unternehmen in Familienhand bleiben soll, sind jedoch von diesem Vorstoß überrascht. Leopold will seinen Kindern vermitteln, dass ihnen die Option einer Karriere im Familienunternehmen offen steht. Er will sicherstellen, dass sie diesen Weg in ihrer $\mathrm{Zu}-$ kunftsplanung bedenken. Ob sie sich schließlich für die Nachfolge qualifizieren, läge in ihrer Hand, nämlich in ihrer Gestaltung des beruflichen Lebenslaufes. Diese Erziehungsvorstellung Leopolds steht in einem starken Kontrast zu seiner eigenen Erfahrung, in der Vater und Onkel den Nachfolger bestimmten und den Lebensweg konkret vorgaben. Leopolds Kinder hingegen sollen sich selbst als Nachfolger ausweisen.

Gemäß dem Interesse, das die Kinder am Unternehmen bekunden, beginnt Leopold (und parallel auch seine Brüder) mit der Eigentumsübertragung in Schenkungspaketen. Die Anteile werden unter den Kindern ungleich verteilt. Diese ungleiche Verteilung zu Lebzeiten begründet er wie folgt: ,,... der soll Nachfolger werden, deshalb hat er mehr, (klopft auf den Tisch) ... dafür kriegt er die Pflicht für euch zu sorgen, sollte euch was passieren ... auferlegt" (Leopold Clemens, 26:15-15)

Leopold versucht, seinen Kindern für die Ausgestaltung ihres beruflichen Lebenslaufs Empfehlungen zu geben: Selbständigkeit und Auslandser- 
fahrung werden zu wichtigen Maximen. Seine jüngere Tochter sagt „mein Vater hat schon von, .. immer sehr stark propagiert, dass wir ins Ausland gehen sollen, von klein auf. Und ich glaube unsere Jungs haben sich da immer am schwersten mit getan mit dem Gedanken und ich und meine Schwester fanden das eigentlich von Anfang an immer einen Reiz." (Julia Clemens, 14:14-16) Die beiden Schwestern, und ganz besonders Andrea, beginnen, Maßstäbe im Geschwisterverbund zu setzen. Sie gehen mit 16 Jahren ins Ausland und schließen dort ihr Abitur ab. Sie zeigen Leistungsbereitschaft und streben nach Selbständigkeit. Die anderen Geschwister ziehen nach. Sie tauschen sich aus, diskutieren miteinander, welche Schritte im Lebenslauf nun sinnvoll wären. Mit Wohlwollen beobachtet Leopold, wie seine Kinder die Philosophie des Vaters verinnerlichen und weiterleben. „Also ich habe es wieder weitergegeben, das was bei mir eigen ist, ist bei denen auch eigen, teilweise haben sie es selbst getan. Sie sind nur noch konsequenter gewesen." (Leopold Clemens, 17:18-19)

Parallel $\mathrm{zu}$ dieser Entwicklung institutionalisiert Leopold die Jahreshauptversammlung des Unternehmens zum „Familientag“. Jedes Familienmitglied, das Anteile am Unternehmen hält und mindestens 18 Jahre alt ist, erhält eine gleichberechtigte Stimme. Als Vorstandsvorsitzender verliest Leopold den Jahresbericht, und zentrale strategische Entscheidungen werden angesprochen. Zudem ist jedes Familienmitglied dazu aufgerufen, über die Entwicklung seines Lebens im letzten Jahr zu berichten. Zusätzlich treffen sich die fünf Geschwister einmal im Jahr mit ihren Cousinen auf privater Basis.

Zwischen den Geschwistern ist klar, dass mindestens einer von ihnen im Unternehmen aktiv mitarbeiten möchte. Julia sagt: „Was wünscht man sich eigentlich mehr als für ein Unternehmen zu arbeiten, mit dem man sich identifizieren kann, auf das man stolz ist“ (9:20-21). Andrea sagt: „Also ich kenne keinen also meiner Geschwister, die irgendwie jetzt sagen: Oh nein, hör mir damit auf und am liebsten würde ich sofort ausbezahlt werden und ich will meine Ruhe haben.“ (37:6-8) Und Julia gibt zu bedenken „Auf der anderen Seite ist natürlich die Angst, .. ähm weil man einfach weiß, was der eigene Vater geleistet hat und man sich das natürlich selber nicht zutraut in der Form“ (9:30-32) Die Geschwister halten sich gegenseitig über ihre Absichten auf dem Laufenden und beraten, wer den Vater als Vorstandsvorsitzenden einmal beerben wird. Der älteste Bruder gibt mit seinem Lebenslauf am deutlichsten seine Ambitionen auf dieses Amt zu erkennen.

Es lässt sich festhalten, dass sich in der Familie Clemens über drei Generationen hinweg der Modus einer frühen Eigentumsübertragung institutionalisiert hat. Leopold kommt in diesem Prozess eine wichtige Funktion zu: In dem Versuch, sein stark vorstrukturiertes Leben zu biografisieren, greift er auf ein scheinbar natürlich gewachsenes Wertefundament zurück. Auf diese Weise gelingt es ihm, seinen Weg in das Unternehmen zu legitimisieren und 
seine Individualität zu behaupten. Jeder Schritt seiner Ausbildung, beginnend mit der Eigentumsübertragung, verfolgt das Ziel einer bestmöglichen Vorbereitung auf ein Amt im Unternehmen und der Weiterführung eines größeren Projektes. Innerhalb dieses ganz besonderen normativen Raumes macht sein Lebensweg Sinn. Mit Bourdieu lässt sich sagen, dass sich der Familienbesitz nicht auf bloße Kontinuität beschränkt, „,vielmehr trägt er praktisch zu deren moralisch-geistiger Reproduktion bei, d.h. zur Weitergabe von Werten, Tugenden und Kompetenzen, welche die legitime Zugehörigkeit" (Bourdieu (1987:137) zu der Familiendynastie begründen. Es bestätigt sich Bourdieus These: Es gibt kein materielles Erbe, das nicht auch kulturelles Erbe ist (Bourdieu 1987:136).

Als Leopold selbst Vater wird, abstrahiert er diese Wertvorstellung und entwickelt eine Art „Pädagogik“ der Nachfolge. Leopold wandelt sich von einem jungen Mann, der sein Leben in einen größeren Zusammenhang einordnet, zu einem Lehrer einer bestimmten transgenerationalen Übertragungsphilosophie. Sein Bestreben, klare Botschaften zu vermitteln, wird auch in seiner Selbstpräsentation mehr als deutlich. Durch diese Abstraktionsleistung scheint es möglich, die Ideen zur transgenerationalen Übertragung zu kodifizieren und Mechanismen zu schaffen, diese auch in Zukunft aufrechtzuerhalten (z.B. Familientreffen). Die Institutionalisierung der Nachfolgepädagogik wird zu Leopolds eigentlichem Projekt. Folgende Regeln sind Bestandteil der „Nachfolgephilosophie“ der Familie Clemens: Erstens, als Unternehmerfamilie müsse Unternehmen auch in der Familie praktiziert werden. Zweitens, der Transfer von Eigentum müsse zu einem frühen Zeitpunkt stattfinden. Die nicht-aktiven Mitglieder müssen Verzicht üben, während die Nachfolger einer Versorgungspflicht für die anderen nachkommen sollen. Drittens, Bescheidenheit, Offenheit, Bodenständigkeit und Leistungsbereitschaft sind zentrale Werte, die ein Familienmitglied für die Trägerschaft von Verantwortung qualifizieren. Viertens, die Autonomie der Kinder von den Eltern wird eingefordert. Jeder muss in der Lage sein, sich seinen Lebensunterhalt selbst zu finanzieren. Fünftens, die potentiellen Nachfolger müssen sich anhand ihres Lebenslaufes auszeichnen und Leistung erbringen.

Durch diese Philosophie wird die Eigentumsübertragung pädagogisch aufgeladen. Es geht nicht nur darum, ein verbrieftes Mitspracherecht am Unternehmen, ein Anrecht auf Gewinnausschüttung etc. an die nächste Generation zu übergeben. Mit dem materiellen Transfer des Eigentums wird der Druck auf die nächste Generation erhöht, sich mit dem Unternehmen und seiner Bedeutung für die Familie auseinanderzusetzen. Die Familienmitglieder geraten in eine paradoxe Situation: Als Eigentümer sind sie nun gefordert, sich dieses Eigentum auch wirklich anzueignen. Die Vorgänger überreichen den Nachfolgern ein recht fertiges Geschenk. Es liegt nun an der neuen Generation, daraus Identität zu beziehen, sich einer Gemeinschaft zuzurechnen. Die auf diese Weise erzwungene oder zumindest nicht selbst gewählte 
Verbindung mit dem Unternehmen drängt die Beschenkten dazu, sich dieses nun freiwillig anzunehmen.

Die Regeln der Nachfolge integrieren die normativen Erwartungen einer funktional differenzierten und individualisierten Gesellschaft: Unternehmen und Familie werden als zwei Bereiche anerkannt. Die Autonomisierung von den Eltern sowie eine selbständige Gestaltung des Lebenslaufes werden als Maxime übernommen. Auf diese Weise gelingt es, Tradition mit Moderne zu verbinden. Ina fasst dies wie folgt zusammen: „Und ich denke, das haben sie bei ihrem Vater gesehen, eindeutig, dass Tradition nicht ein Hindernis ist, dass man sie bejahen kann, aber trotz alledem sich der Zukunft öffnet.“

Auch die anderen Fallauswertungen legen nahe, dass in Unternehmerfamilien über die „Pädagogik“ der Nachfolge die Bedingungen für eine „erfolgreiche" Nachfolge transgenerational übertragen werden - mit erfolgreich ist in diesem Fall der Fortbestand der Verbindung von Unternehmen und Familie gemeint. Die institutionalisierte Forderung nach Autonomie der Kinder gekoppelt mit einem Gemeinschaftsgefühl sowie einem ausgeprägten meritokratischen Denken formen die zentralen Elemente dieser Pädagogik. Es wird nicht mit Zwang ein Weg vorgegeben, sondern bestimmte Werte und Bedingungen vermittelt, über die eine Reproduktion der Gemeinschaft möglich wird. Dieser Bedingungskatalog kann als Antwort von Unternehmerfamilien auf die Voraussetzungen moderner Gesellschaften verstanden werden. Er schafft Orientierung in der Eltern-Kind-Beziehung, entfaltet gemeinschaftsstiftende Kraft und stellt die Lebensweise als Unternehmerfamilie als möglichen Zukunftsentwurf vor. Wenn man so mag, wenden die Unternehmerfamilien den Habermas'schen Trick der Formalpragmatik an.

Durch die erzieherische Übermittlung der Bedingungen einer Nachfolge wird gleichsam das Feld für eine Positionsbestimmung innerhalb der Familie enger abgesteckt, Anknüpfungspunkte für einen eigenständigen Lebensentwurf geschaffen und im Fall Clemens der materielle Transfer für diese Zwecke instrumentalisiert. Mit dem Transfer von Bedingungen wird die transgenerationale Übergabe jedoch voraussetzungs- und risikoreich. Inwiefern die ideelle und emotionale Aneignung, die Bejahung der Bedingungen erfolgt, bleibt ungewiss. Die Übergeber sehen sich der Gefahr ausgesetzt, dass ihr Projekt scheitert - wie der Fall Naumann an anderer Stelle eindrucksvoll zeigt. Der Übergabeprozess ist als vernünftiges Erkennen und emotionales Aneignen des Erbes zu beschreiben.

Als eine Randnotiz sei am Ende dieses Abschnittes noch eine kurze anekdotische Beobachtung erlaubt: Immer wieder sind wir bei den Besuchen in den Unternehmerfamilien und auch in ihren Erzählungen einem weiteren, tierischen Familienmitglied begegnet. Es scheint, als ob in diesen Familien eigener Art besonders gerne Hunde (und auch Pferde) gehalten werden. Dieser sicher aufgrund der geringen Fallzahl sehr selektive Eindruck bekommt vor dem Hintergrund der „Erziehung“ in Unternehmerfamilien eine gewisse 
Plausibilität, wie uns auch Leopold Clemens nach Abschluss der Tonaufzeichnung erklärt. Die Frage, ob er einen Hund habe, bejahrt er leise lächelnd. Er macht eine kurze Pause und reflektiert dann darüber, dass man einen Hund wunderbar als Erziehungsmittel zur Langfristigkeit und Verantwortung einsetzen könne. Unternehmer hätten deshalb in der Regel Hunde oder Pferde, aber keine Katzen.

\subsubsection{Indizienanalyse der Lebensläufe}

In einer Studie zur Zufriedenheit mit dem Generationswechsel stellen Sharma et al. (2003, bzw. in ihrer Vorläuferstudie Sharma et al. 2001) fest, dass beide Seiten über eine unterschiedliche Wahrnehmung dieses Prozesses und seiner zentralen Kriterien verfügen. Während die Übergeber angeben, dass ihre Zufriedenheit mit dem Generationswechsel von der Bereitschaft und dem Engagement des Nachfolgers beeinflusst werde, stellen Nachfolger heraus, dass ihre Zufriedenheit eher von der Bereitschaft der Eltern zum Rückzug aus dem Unternehmen beeinflusst werde. Beide Parteien messen der eigenen Motivlage eine geringere Bedeutung zu. (Sharma et al. 2003:668) Diese Ergebnisse unterstreichen eine zentrale Erkenntnis aus den Fallauswertungen: Die Mitglieder einer Unternehmerfamilie beobachten und reflektieren permanent über die Aktivitäten des oder der anderen und richten daran eigene Lebenslaufentscheidungen und Identitätsdefinitionen aus. Der hohe Informationsgrad der Familienmitglieder über die Werdegänge und Lebensvorstellungen der signifikant Anderen innerhalb der Familie ist sehr auffällig.

Mit Blick auf die Nachfolge in Familienunternehmen schlägt Breuer vor $^{109}$, diesen Prozess der kontinuierlichen Beobachtung als beidseitiges „Sammeln und Bilanzieren von Geeignetheits-Indizien“ (Breuer 2009:291) zu bezeichnen. Vorgänger und Nachfolger setzen Aspekte des jeweils anderen, der eigenen Person, sowie des Unternehmens und der allgemeinen Umstände in Verhältnis zueinander und gelangen zu einer labilen, wandelbaren Einschätzung, die eng mit der Frage verknüpft ist, ob man sich selbst bzw. sein Kind sich für die Position des Nachfolgers qualifiziert. Dieser Prozess endet in Breuers Theorie der Übergänge mit dem Aufbrechen des verdeckten Charakters des beidseitigen Indizien-Sammelns, indem die Vereinbarungen über die Unternehmensnachfolge nunmehr offiziell erfolgen - quasi ein „Explizitheits-Switch" umgelegt wird. (Breuer 2009:292) Indem er diese Art der „Nicht-Kommunikation“ (Pfannenschwarz 2006:261) zu einem wesentlichen Kommunikationsmodus in Unternehmerfamilien erhebt, verleiht Breuer der allgemeinen Forschungsmeinung - und auch seiner eigenen Beobachtung -,

109 Wobei Breuer lediglich Nachfolgen im Typ „recycled controlling owner“ betrachtet, also Übergaben von einem Eigentümerunternehmer an nur ein Kind. 
dass innerhalb dieser Familien das Nachfolge-Thema verdrängt oder vermieden wird, einen neue, andere Bedeutung. Die Implizitheit der intergenerationallen Aushandlungen bedingt sich durch die Generationendifferenz zwischen Eltern und Kindern, in der Eltern eine Bahnungsrolle einnehmen, weit bevor die Kinder den Status von gleichrangigen Aushandlungspartnern erlangen, ebenso wie durch die soziokulturelle Tradition der Abgrenzung der Belange von Eltern und Kindern. Gleichzeitig gibt es gute Gründe, diese „Aushandlungen“ hinsichtlich der eigenen Rolle im Nachfolgeprozess nicht anzusprechen: Denn so bleibt das Ergebnis deutungsoffen, ein „Umentscheiden“ ist auf beiden Seiten ohne Image-Schaden leichter möglich, spätere zentrale Lebenslaufentscheidungen (wie etwa die Ausbildung oder die Partnerwahl) können abgewartet werden, und auch die Gefahr von Konflikten scheint kleiner. (Breuer 2009:291)

Breuers Konzept erweist sich für die Analyse von Unternehmerfamilien und ihren Verhandlungsweisen als äußert tragfähig. Fraglich ist lediglich, ob der Fokus auf die Interaktion zwischen Vorgänger und Nachfolger nicht zu einer unnötigen Engführung führt. Denn in Erweiterung zu Breuer kann auf Basis der Fallauswertungen ein solcher Modus der kontinuierlichen Beobachtung auch für andere Konstellationen innerhalb der Unternehmerfamilie und dabei insbesondere zwischen Geschwistern in Anspruch genommen werden. Eine wechselseitige Indizienanalyse der Lebensläufe stellt sich als ein lebenslange Art und Weise, wie Familienmitglieder über ihre Positionen innerhalb der Unternehmerfamilie verhandeln, heraus.

Die Eltern in Unternehmerfamilien beobachten von klein auf die Talente, Merkmale und Zukunftsvorstellungen ihrer Kinder. Indizien für ein Interesse am Familienprojekt werden hinsichtlich ihres Alters mehr oder weniger ernst genommen: „die Vorliebe für Aktivitäten auf dem Betriebsgelände, die Beschäftigung mit Materialien aus der betrieblichen Produktion, die Begeisterung für eine Begleitung des Vaters bei Kundenbesuchen, bei Auslieferungsfahrten o. Ä.“ (Breuer 2009:293). Diese frühen Begegnungen der Kinder mit dem Unternehmen können später Gegenstand von Familienanekdoten sein, die belegen, dass eine Affinität für das Familienunternehmen schon seit jeher bestand. Zudem kann das Geschlecht der Kinder die Positionszuschreibungen der Eltern beeinflussen. Auch in späteren Lebensphasen bleibt das kontinuierliche Beobachtungsverhältnis aufrechterhalten. Die Eltern analysieren schulische Erfolge, die Ausbildung, berufliche Schritte und die Partnerwahl im Hinblick auf das Glück des Kindes ebenso wie auf eine potentielle Reproduktion der Unternehmerfamilie. Florian Wagner blickt mit Sorge auf die vielen beruflichen Wechsel seines Sohnes Oscar und sieht darin ein Zeichen für Unbeständigkeit. Sven Singermayer und auch Erwin Carstens interpretieren die Position ihrer Töchter als alleinerziehend bzw. in einer Patch-WorkFamilie als zusätzliche Belastung, die mit beruflichen Herausforderungen nur schwer vereinbar ist. In der Familie Clemens werden eine akademische und 
trotzdem praxisnahe Ausbildung sowie Auslandserfahrung zu festen Größen in der Beurteilung, ob sich ein Kind für eine verantwortungsvolle Position im Familienunternehmen qualifiziert. In der Familie Claas wird die unternehmerische Leistung des Sohnes beständig bewertet und beobachtet und führt schließlich dazu, dass der Sohn das Familienunternehmen wieder verlässt.

Umgekehrt blicken die Kinder auf die Handlungen, Lebensweise und Zukunftsvorstellungen ihrer Eltern. Die frühen Begegnungen mit dem Unternehmen und das Einführen in die „Geheimnisse“ der Familienkunst und tradition können als Signal für Anerkennung und Zutrauen gewertet werden. Als Friedrich Carsten Naumann beispielsweise von seinem Großvater in die Buchführung eingeweiht wird, findet er dies ,interessant, also es war irgendwie natürlich auch das Wichtig-Genommen-Werden, das ErnstGenommen-Werden“" (13:29-30). Wie bereits in Kapitel VI ausgeführt, werden Kinder zu Beobachtern der Lebensweise der Eltern, ihrer Sorgen und beruflichen Verwicklungen, auf deren Basis sie selbst eine Theorie davon ausbilden, was es heißt, eine Unternehmerfamilie zu sein. Als Erwachsene analysieren sie zudem die Fähigkeiten der Eltern als Unternehmer und schätzen die wirtschaftliche Tragfähigkeit des Unternehmens in Zukunft ein. Zudem sind sie sich des Alters ihrer Eltern und ihres Gesundheitszustandes sehr bewusst, auf dessen Grundlage sie zu antizipieren versuchen, in welcher Weise und wie lange sich die Eltern im Familienprojekt engagieren wollen und können.

Abbildung 10: Indizienanalyse der Lebensläufe

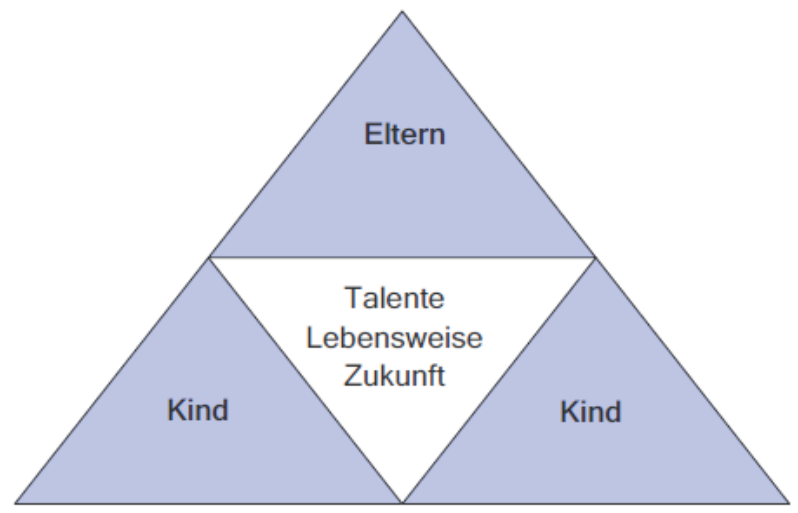

Quelle: eigene Darstellung

Schließlich beobachten sich die Kinder untereinander. Sie ermitteln, welche Talente und Neigungen ihre Geschwister besitzen, welche Erwartungshal- 
tung die Eltern ihren Geschwistern entgegenbringen, welche Zukunftsvorstellungen sie von ihrem Leben entwickeln. Ihre Einschätzung kann dazu führen, dass nach anderen Interessensfeldern gesucht und einige Rollen innerhalb der Unternehmerfamilie für sich selbst ausgeschlossen werden. Friedrich Carsten Naumann etwa sagt: ,,im Zweifel wird es ja doch mein älterer Bruder übernehmen. Also, diese Prägung war vielleicht schon da, dass ich eben doch nur „der Zweite“" war. Ähm ... und dann immer so die Überlegung,... $[.$.$] wie kriegt man das eigentlich gebacken, dann irgendwie in einer$ weniger schönen Umgebung ... äh irgendwie ... das Leben zu machen?“ (21:26-28) Innerhalb der Geschwistergemeinschaft von Unternehmerfamilien kann sich der Kontinuitätsanspruch als eine zentrale Handlungsregel manifestieren, nämlich in einer Einigkeit darüber, dass mindestens ein Geschwisterteil das Familienprojekt fortführt. Claudia Nentzik beschreibt dies wie folgt: ,weil immer so'n bisschen das Spiel war, wenn, BEVOR'S KEINER MACHT, MACHT'S EINER. Aber dann war ja die Frage sozusagen wer fängt den Ball jetzt auf (klopft dabei auf Tisch)“ (35:10-13) Im Fall Naumann treten die Geschwister schließlich in einen intensiven Austauschprozess, da sich die beiden älteren Söhne aus dieser Verantwortung verabschieden wollen und doch eine Regelung gefunden werden muss. „Ja, ... so dass dann eigentlich äh *pf* (pustend) der Geschwisterkreis sich glaube ich viel eher einig war als mein Vater, dass wir gesagt haben, das macht [Claudia], ist doch, passt doch." (Friedrich Carsten Naumann, 17:19-20)

Diese kontinuierliche Indizienanalyse der Lebensläufe erfolgt mit dem Fluchtpunkt einer Fortsetzung des Familienprojektes und hält ein Leben lang an, also auch nach der Entscheidung für einen Weg in das Familienunternehmen. In diesem Kommunikationsmodus werden ähnlich dem Prinzip der „Stillen Post" innerhalb der Unternehmerfamilie, von den Akteuren unbewusst und unverstanden, die jeweiligen Positionen koordiniert. Es entsteht ein empathisches Verständnis für den Lebensweg, die Aufgaben und Belastungen der signifikant Anderen innerhalb der Familie, das dazu beiträgt Gelegenheiten, aber auch Unwägbarkeiten einschätzen zu können.

\subsubsection{Gesprächs- und Erzählkultur}

Als letzte Kategorie der Verhandlungsweisen soll nun die explizite Kommunikation zwischen den Mitgliedern einer Unternehmerfamilie in den Blick genommen werden. Diese lässt sich in drei Gruppen einordnen: alltägliche Gespräche und Unterhaltungen, Fragezeremonien und schließlich Familiennarrative. 


\subsubsection{Alltägliche Gespräche}

Im Rahmen der Analyse der alltäglichen Lebensführung von Unternehmerfamilien wurde in Kapitel VI vorgeschlagen, die ,praktizierte Grenzarbeit“ in Unternehmerfamilien als eine veralltäglichte Konstruktionsleistung zu verstehen, mit der das Ausmaß, in dem das Unternehmen die Lebensführung der Familie durchdringt, bestimmt wird. Die aus dem empirischen Material und der Forschungsliteratur kondensierten Typen dieser Grenzarbeit beinhalten jeweils unterschiedliche Kommunikationsstile, also Arten, wie im Alltag über das Unternehmen gesprochen und erzählt wird. Für den Typ ,Verschmelzung" ist das Unternehmen ein selbstverständlicher Bestandteil der Alltagskommunikation, und umgekehrt werden familiale Belange im Unternehmen thematisiert. Auf kommunikativer Ebene wird keine Grenze zwischen diesen Sphären gezogen. Der Typ „Zwei-Kern“ hingegen konstruiert einen Kern an betrieblichen und familialen Themen, die nur innerhalb der jeweiligen Sphäre zu thematisieren sind. Außerhalb dieses Kerns findet sich eine große Bandbreite an Themen, die unproblematisch überall besprochen werden können. Schließlich praktiziert der Typ „Festung“ eine strikte Trennung von Themen, die alle Familienmitglieder einer Unternehmerfamilie in einem familialen Rahmen diskutieren, und solchen, die nur im Unternehmen involvierte Familienmitglieder in einem betrieblichen Rahmen diskutieren. Eine Vermischung der Themenbereiche wird möglichst vermieden. Der jeweilige Kommunikationsstil dieser drei Typen ist eng an die Konstruktion der Grenze zwischen Familie und Unternehmen gekoppelt. In der alltäglichen Lebensführung verstetigen sich diese Regeln einer Unternehmerfamilie, wer und in welcher Art und Weise über welche Themen sprechen darf und sollte. Sie werden Teil eines gemeinsamen Erfahrungswissens und Bestandteil der jeweiligen Vorstellung davon, was es heißt, eine Unternehmerfamilie zu sein.

\subsubsection{Fragezeremonien}

Von dieser alltäglichen Kommunikation heben sich solche Gespräche ab, die von den Familienmitgliedern sehr detailreich erzählt werden können: unter Angabe von Ort und Datum des Gespräches, einer Beschreibung der herrschenden Atmosphäre und einer Schilderung des Gesprächsablaufes. Sie formen ein Ereignis an sich. Diese besonderen Momente markieren Knotenpunkte der biografischen Selbstsicht der einzelnen Familienmitglieder sowie der Präsentation der Unternehmerfamilie als Gemeinschaft. Auf individueller Ebene haben sie oft lebensprägenden Charakter, auf familialer Ebene stellen sie Wendepunkte der Familiengeschichte dar. Umgekehrt scheint diese Art der außeralltäglichen Kommunikation mit ebenso ungewöhnlichen Ereignis- 
sen innerhalb der Unternehmerfamilie in Zusammenhang zu stehen. Dieser Modus expliziter Kommunikation muss als Ausnahme und Besonderheit verstanden werden, die längst nicht allen Fallgeschichten zu eigen ist. Sehr eindringlich ist in diesem Zusammenhang die Situation in der Familie Singermayer, als der Vater nach dem Tod des designierten Nachfolgers seine beiden verbleibenden Söhne in feierlicher Stimmung fragt, ob sie sich vorstellen könnten seinen Weg einzunehmen (ausführlich siehe hierzu Kapitel 6.2.2.2). Ein weiterer, etwas außergewöhnlicher, aber sehr illustrativer Fall ist die Geschichte des Gabriel von Moline:

Familie von Moline besaß einst eine Reihe von Ländereien und Unternehmen in den Ostgebieten. Im Zweiten Weltkrieg wurde sie vertrieben und fing in Westdeutschland von vorne an. Gabriel, der jüngste Sohn, wird erst in den 1950er Jahren geboren und kennt die Vorgeschichte der Familie nur aus Erzählungen. Kurz nach der Wende besucht ihn sein Vater: „Und dann sagte er: Pass mal auf, eh wir jetzt irgendwie über andere Dinge diskutieren, geh mal bitte in den Keller, hol uns mal einen richtig guten Rotwein und dann setzt du dich mal mit mir ins Arbeitszimmer. Mein Vater war zu dem Zeitpunkt, mein Vater war Baujahr 07, also kann man sich ausrechnen, war schon ein paar Tage älter. Er war 83, aber war im Kopf sehr hell und sehr fit und sagte: So, setz dich mal hierhin und lass uns erst mal einen Wein trinken. Das kam mir ungewöhnlich vor, weil früher war das immer so, wenn er gesagt hat, geh mal in den Keller, hol mal einen Rotwein, hieß das so ungefähr ... du hast wieder was angestellt, äh wir wollen jetzt *ohne Beteiligung deiner Mutter das unter Männern durchdiskutieren* (lachend), ja? Und äh, nein, das war eigentlich ganz anders an dem Tag, sondern er sagte: Hier, guck mal, habe ich eine kleine Liste, das sind Unternehmen, die uns mal gehört haben. Ich glaube nicht, dass wir was wiederkriegen, ja, [ausgelassen 1] aber wenn du Spaß dran hast, also du hast eigentlich die optimalen Möglichkeiten. Du hast Liquidität, du hast schon beruflich was bewegt, du weißt wie die Firmen $\mathrm{zu}$ strukturieren sind, du müsstest das eigentlich mal dir genau angucken. Und ... ich wünsche mir 14 Tage Urlaub und diese 14 Tage, bitte, bitte fahr mal [ausgelassen 2] [dorthin] und guck dir alles an. Und dann habe ich meinem [ausgelassen 3] [Chef] gesagt: Lieber, guter Mann, ... ich will für 14 Tage [ausgelassen 4] [dorthin]." (15:2-21)

Diese Momente sind in den Erinnerungen der Befragten lebendig und stärker präsent als etwa der erste Arbeitstag oder Tode von Familienangehörigen. Diesen außeralltäglichen Gesprächen, wie sie sich in den Interviews mit den Unternehmerfamilien darstellen, ist eine spezifische Form zueigen. Im Mittelpunkt steht immer eine Frage, die von den Fragenden sehr bewusst gestellt und in ein zeremonielles Setting platziert wird. In einer solchen Fragezeremonie wird die „Fiktion“ einer freien Entscheidung ebenso wie die Norm eines eigenständigen Lebensentwurfes auf die Spitze getrieben. Der wissenschaftliche Beobachter verweist zu Recht auf die herrschenden Zwän- 
ge und strukturellen Begrenzungen. Der erlebende Akteur hingegen erfährt einen Moment der gefühlten Entscheidungsmacht.

\subsubsection{Familiennarrative}

Solche Fragezeremonien können im Laufe der Zeit Bestandteil von Familiennarrativen werden - dem dritten Modus der expliziten Kommunikation, der sich für Unternehmerfamilien beschreiben lässt. Sie erzählen davon, wie sich die Gruppe der Unternehmerfamilie konstituiert hat, welche Herausforderungen sie gemeistert hat oder welche Eigenschaften dieses Kollektiv auszeichnen. Derartige Narrative sind Bestandteil des Familiengedächtnisses und wesentliches Element in der Außendarstellung von Unternehmerfamilien.

Familiennarrative können die Historie der Unternehmerfamilie thematisieren und die Form von Generationenerzählungen annehmen, wie sie Christina Lubinski beschrieben hat (siehe hierzu auch die Ausführungen in Kapitel 3.2.5). Solche Entwicklungsgeschichten, die ihren Anfang in dem Zusammentreffen von Familie und Unternehmen als Projekt nehmen und dann entlang generationaler Sequenzen die Leistungen und Entbehrungen der jeweiligen Unternehmerfamilie erzählen, stellen ein zentrales Erzählmuster in diesen Familien eigener Art dar. Dennoch dürfen Familiennarrative nicht mit Generationenerzählungen gleichgesetzt werden. In dem empirischen Material treten Gründungsmythen und Familienpräsentationen als weitere Form der geteilten Narrative in Familien hervor.

Ein anschauliches Beispiel für einen solchen Gründungsmythos zeigt sich in den Erzählungen der Familie Vester-Wagner: Florian Wagner ist Pächter mehrerer Gastronomiebetriebe, die er gemeinsam mit seiner Lebensgefährtin Barbara Vester betreibt. Barbaras Söhne aus erster Ehe arbeiten von klein auf immer wieder in den Restaurants und bei Veranstaltungen mit. Oscar sieht seine Zukunft in den Familienbetrieben, während Andreas eine Festanstellung in dieser Branche bevorzugt. Alle vier sind in Branchenverbänden, Florian zusätzlich in der Kommunalpolitik engagiert. Die Familie interessiert sich für das Gelände der „Pfälzer Mühle“ und kann dieses nach jahrelangen Verhandlungen mit der Stadt schließlich erwerben. Alle vier Familienmitglieder werden zu gleichberechtigten Geschäftspartnern. Ihre Erzählungen vom Kaufprozess, der gemeinsamen Finanzierung, dem Umbau und schließlich der Eröffnung weisen nicht nur inhaltlich, sondern auch in der Erzählweise viele Parallelen auf. Diese Gründungsgeschichte, die sie offensichtlich schon viele Male erzählt haben, stellt ein markantes Element des Familiengedächtnisses dar. In ihr verschmelzen (sich) die Leben der Familienmitglieder und konstituieren eine Gemeinschaft von neuer Qualität. Der vorher eher lose Verbund der Familienmitglieder, die eine Leidenschaft für 
die Gastronomie teilen, erhält mit der Gründung eine vertragliche, finanzielle und personelle Verbindlichkeit, die in diesem Gründungsmythos ihren symbolischen Ausdruck findet. Trotz der vielen Gemeinsamkeiten erzählt jedes der vier Familienmitglieder diese Geschichte in Nuancen anders:

Florian beschreibt diesen Prozess sehr ausführlich und lang. Er betont die Schwierigkeiten, Mühseligkeiten und Anstrengungen dieser Zeit. Der Hörer erhält den Eindruck, dass Florian ein Getriebener der politischen Verwicklungen sei, denen er trotz seiner gastronomischen Kompetenz etwas machtlos gegenüber steht. Zunächst scheitern die Kaufversuche daran, dass der Bürgermeister abgewählt wird, dann daran, dass ein höheres Angebot abgegeben wurde und jemand „Verrat" betrieben habe. Der Kauf durch eine dritte Partei kommt jedoch aufgrund fehlender Solvenz nie zustande. Das Objekt steht wieder über Monate hinweg leer. Über diese erneute Zeit des Stillstandes sagt Florian: „Dann haben wir gewartet und ... dann sagt meine Frau, Mensch, ruf doch noch mal an. Sag ich, nein, ich will eigentlich gar nicht anrufen, die sollen auf uns zukommen, wenn sie was wollen. $\mathrm{Na}$ ja, WIE FRAUEN SIND, sie hat keine Ruhe gegeben, dann hat sie dann selbst die Bürgermeisterin mal angerufen, sich mit ihr mal irgendwo getroffen." (14:13-16) Nach einer weiteren Zeit des Wartens, bis die Bindung der Stadt an einen Makler ausgelaufen ist, hätten sie erneut verhandelt und mit einem erhöhten Angebot schließlich den Zuschlag erhalten. Interessant an Florians Präsentation des Gründungsmythos erscheint, dass er in der neu entstehenden Gemeinschaft zu vereinzeln droht. War er vorher derjenige, der das Ruder in der Hand hält und mit kreativen Ideen den Betrieb gestaltet, ist er nun einer unter vieren, der sich selbst mit diesen neuen Machtverhältnissen noch nicht biografisch arrangiert hat.

Barbaras Darstellung des Gründungsmythos folgt zunächst der Geschichte Florians. Den Moment ,ihres“ Eingreifens in die Geschehnisse jedoch betont sie wesentlich stärker: „Und dann habe ich zu meinem Mann gesagt, er soll dann doch noch mal nachfragen ... und ja, er hatte mal gesagt er fragt, aber das hat er glaube ich dann doch nicht gemacht. ... Und irgendwann habe ich so lange gedrängelt, wir müssen jetzt endlich mal eine Entscheidung treffen und noch mal hören, was da wird und da hat er zu mir gesagt, wenn du glaubst, du kannst es besser, dann mach es doch. Und dann habe ich nur noch mal nachgefragt und habe gesagt: ,Darf ich?'... ,JA, MACH DOCH, mach doch.' Und daraufhin habe ich die Bürgermeisterin angerufen und habe gefragt, ob ich mit ihr mal reden könnte und Termin ausgemacht und ... habe ihm aber davon nichts gesagt." (19:2-10) Von Frau zu Frau spricht Barbara mit der Bürgermeisterin und legt ihre ehrlichen Absichten dar. Die Bürgermeisterin gibt zu erkennen, dass noch Verhandlungsmöglichkeiten bestehen. Barbara erzählt weiter: ,und dann ... sind wir Montagabend alle zusammen ... essen gegangen. ... Und dann habe ich gesagt, ja, ich habe noch eine Überraschung, ... so und so sieht es aus und wir sind wieder im Boot" (19:27-29) - 
„Und ... da kam von meinem Mann gar keine Reaktion. ... Irgendwo, ... nein, also ich fand es ein bisschen enttäuschend. ... Die Jungs haben gesagt, ja, okay, ist fein, ist okay. Und wir haben da relativ schweigend noch gegessen, dann über andere Dinge gesprochen ... und ganz spät abends da kam er dann und hat sich dann mehr oder weniger entschuldigt, ... weil das hätte er erstens mir nicht zugetraut und zweitens ... war er einfach so platt und er freut sich doch sehr und hat sich dann bedankt. (lacht) ... Ja, und dann ist das Ganze halt ins Rollen gekommen." (19:31-20:4) Es zeigt sich deutlich, dass Barbara in ihrer Initiative den Anstoß für das Gelingen des Projektes sieht. Sie will Florian ihre Fähigkeiten unter Beweis stellen und fordert Gleichberechtigung in diesem neuen Projekt ein.

Sohn Oscar, der inzwischen Betriebsleiter in einem der Pachtbetriebe ist, verfolgt die Geschehnisse mit Spannung und ist sich darüber bewusst, dass dieses Projekt auch große Auswirkungen auf seine Zukunft haben kann. Wie auch bei Barbara erfolgt seine Erzählung des Gründungsmythos entlang der Linien Florians, doch nimmt dann noch eine dritte Wendung. Über die Zeit des erneuten Stillstandes sagt er: „Und äh .. daraufhin habe ich meine Mutter dann noch mal angesetzt, sie soll mal mit der Bürgermeisterin hier reden von Frau zu Frau, weil bei manchen Sachen mein Vater auch einfach .. zu langsam ist für uns. (lacht) .. Und das war ein sehr gutes Gespräch dann, daraufhin wurden die Verhandlungen dann wieder neu aufgenommen." (15:18-21) Oscar sieht sich in einer vermittelnden Position zwischen seinen Eltern und schreibt sich selbst in diesem Prozess eine gestaltende Rolle zu. Er fordert einen gleichberechtigten Platz neben seinen Eltern ein, tut sich jedoch mit der Integration seines Bruders in diese Gemeinschaft schwer.

Andreas schließlich, dessen aktives Engagement in dem Familienprojekt erst nach dem Zuschlag beginnt, fasst die Gründungsgeschichte relativ kurz und neutral zusammen. Er unterbricht seine Erzählung mit den Worten: „Wobei ich da jetzt wirklich auch gar nicht mit dringehangen habe oder ähnliches, weil ich ja ganz normal gearbeitet habe. ..." (18:11-12) Dafür ergänzt er an anderer Stelle Details, die in den Erzählungen seiner Familienmitglieder nicht erwähnt werden, wie etwa die Situation der Zuschlagserteilung.

Über die Erzählung des Gründungsmythos entsteht die Unternehmerfamilie Vester-Wagner als Erzählgemeinschaft mit einer spezifischen Gruppenidentität. Gleichzeitig nutzen die einzelnen Familienmitglieder diesen narrativen Raum, um sich von den anderen zu differenzieren und ihre eigene Position zu bestimmen. Diese Art des Familiennarratives kann als Art und Weise verstanden werden, wie Familienmitglieder über spezifische Vergangenheitsdeutungen, Rollenzuschreibungen und Zukunftsvorstellungen verhandeln.

Der Fall Clemens zeigt, dass diese Form der Familiennarrationen sich nicht zwangsläufig auf die Geschichte der Unternehmerfamilie beziehen muss, sondern auch die alltägliche Lebensführung zum Gegenstand machen 
kann. Den Interviews mit Leopold, Ina, Andrea und Julia Clemens ist die Präsentation „Wir sind ganz normal!“ gemein. Andrea Clemens eröffnet ihre Eingangserzählung mit den Worten: ,und ich betone eigentlich immer, dass ich aus einer Kleinstadt komme oder wenn die Leute fragen, weil mich das sehr geprägt hat. Ich glaube, das hat ähm nicht nur mich, sondern auch meine Geschwister, die ganze Familie geprägt, weil wir dadurch ähm..., obwohl wir eben eine Unternehmerfamilie sind... total normal aufgewachsen sind.“ (1:24-29) Auch ihre Mutter Ina betont: „Also wir leben ja ganz normal, ihr lebt ja nicht hinter hohen Mauern“ (26:13). Bemerkenswert an dieser Normalität ist, dass sie überhaupt thematisiert werden muss. Diese Familie definiert sich über eine Abgrenzung zu den Besonderheiten einer Unternehmerfamilie, die sich aus ihrer strukturellen Eigenlogik sowie dem Reichtum dieser speziellen Familie ergeben. Sie fühlen sich einer Wertegemeinschaft zugehörig, die im Einklang mit den ,normalen“ Erwartungen einer Gesellschaft ist und sich trotzdem implizit differenziert.

\subsubsection{Die Unternehmerfamilie als Erzähl- und Erinnerungsgemeinschaft}

Über die kommunikative Selbstthematisierung in alltäglichen Gesprächen, Fragezeremonien und Familiennarrativen schafft und erhält sich letztlich die Unternehmerfamilie mit ihrem generationsübergreifenden Anspruch als Erzähl- und Erinnerungsgemeinschaft. Auf dieser Grundlage können sich ein starkes Zugehörigkeitsgefühl und eine ausgeprägte Bindung an das Familienprojekt ausbilden. Diese Erzählungen liefern den Individuen Anknüpfungspunkte für ihre eigene, individuelle Biografie, definieren ihre spezifische Funktion und Bedeutung innerhalb der Unternehmerfamilie. Das Gespräch im Kreis der Familie liefert, wie auch schon Angela Keppler (1995) zeigte, einen Raum, in dem familiale Werte, Grundhaltungen und Deutungen verhandelt werden können. Handlungen der einzelnen Familienmitglieder werden in diesen Narrativen bewertet, Rollenprofile geschärft und Erinnerungen aufrechterhalten und umgedeutet (Lubinski 2010:121). Mit der Zeit bilden sich in jeder Familie ein bestimmter Kommunikationsstil sowie Familiennarrative aus, die Routine in den Erzählungen und Erzählweisen schaffen. So treten die Generationen in eine direkte Auseinandersetzung miteinander ein, in der die jeweiligen Vorstellungen, Deutungen und Persönlichkeiten aufeinandertreffen und man sich auf gemeinsame Interpretationen und Grenzdefinitionen einigt. Im Rahmen der Gesprächskultur einer Unternehmerfamilie können Funktionsbestimmungen erfolgen, werden Wendepunkten in den Lebensläufen der Familienmitglieder symbolhaft aufgeladen, können die Generationen für gegenseitiges Verständnis werben - etwa für eine ungleiche Verteilung des Erbes -, und können die Außengrenzen dieser Gemeinschaft geschärft werden. 


\subsection{Das Kollektiv der Unternehmerfamilie}

Der Dreischritt dieses Kapitels lautet: Erstens, das Unternehmen durchdringt die verschiedenen Beziehungskonstellationen zwischen den Mitgliedern einer Unternehmerfamilie. Das Unternehmen als Thema der familialen Kommunikation, die Verbundenheit der Lebensläufe sowie finanzielle Abhängigkeiten schaffen sehr spezifische Voraussetzungen für die Begegnungen der Generationen und für die Entfaltung von Solidarität und Konflikt. Die Unternehmerfamilie bildet einen spezifischen Verweisungszusammenhang, eine Figuration, die die Form eines unmittelbaren Aushandlungsprozesses annimmt. Zweitens, Unternehmerfamilien haben in diesem Prozess eine Reihe von Koordinierungsaufgaben zu bewältigen, zu denen vor allem die Positionbestimmung in Familie und Gesellschaft, die Synchronisation der Lebensläufe sowie der materielle Transfer gehören. Dieser aktive und direkte Aushandlungsprozess läuft quasi lebenslänglich und zumeist eher subtil. Bettina Völter sprach in dem Zusammenhang von mehrgenerationalen Familien und ihrer kollektiven Identität einmal vom „Beziehungstanz“ zwischen den Familienmitgliedern. ${ }^{110}$ Diese Bezeichnung bietet sich im Kontext von Unternehmerfamilien in besonderem Maße an und verbildlicht den direkten Generationenbezug. Drittens, Unternehmerfamilien wenden spezifische Verhandlungsweisen in diesen Aushandlungsprozessen an, folgen, wenn man in diesem Sprachbild bleiben möchte, also einer charakteristischen Choreografie. Die wesentlichen Elemente dieser wurden als Pädagogik der Nachfolge, Indizienanalyse der Lebensläufe und schließlich als Gesprächs- und Erzählkultur beschrieben.

Im Lichte dieser Ergebnisse tritt die Unternehmerfamilie sehr deutlich als Erinnerungs- und Erzählgemeinschaft hervor. Das aus der Verbindung von Unternehmen und Familie entstehende Kollektiv kann als soziales Konstrukt verstanden werden, das, so Lubinski, ,insbesondere über Erzählungen aufgebaut und perpetuiert wird" (Lubinski 2010:130). Die Generationen sind Akteure eines Deutungsprozesses, der das Verhältnis von Familie und Unternehmen thematisiert. Dieser Befund ist anschlussfähig an die Forschung zu Familiendiskursen (z.B. Vierzigmann und Kreher 1998; Rosenthal 1995; Völter 2003). Folgt man etwa dem interaktionellen Ansatz von Rosenthal (1995; 1997), so lässt sich ferner festhalten, dass sich erst im Dialog zwischen den Generationen zentrale Werte (Selbständigkeit, meritokratisches Denken) oder Vorstellungen zur Lebensführung etablieren, diese also nicht

110 Diesen Begriff nutzte Bettina Völter im Rahmen ihres Vortrages „Beziehungstänze. Lebensgeschichten als Hinweise auf transgenerationale Interaktionen“ auf der Tagung „Transgenerationale Übertragungen“, die vom 5.-6. November 2010 im Zentrum für Literatur- und Kulturwissenschaften in Berlin stattfand. 
bloß von einer an die nächste Generation übertragen werden, sondern als gemeinsame Bedeutungskonstruktionen $\mathrm{zu}$ verstehen sind, die gleichzeitig bestimmte Differenzierungsmerkmale (etwa alt vs. jung, verschiedene Führungsstile etc.) transportieren. Der langfristige soziale Konstruktionsprozess zwischen den Generationen durchdringt die individuellen Überzeugungen und Lebensskripte der Familienmitglieder und trägt $\mathrm{zu}$ einer andauernden narrativen Fortschreibung der Familiengeschichte bei.

Es wäre jedoch zu kurz gegriffen, das Kollektiv der Unternehmerfamilie ausschließlich im Sinne eines konstruktivistischen Ansatzes analytisch fassen zu wollen. Die Ergebnisse heben deutlich die phänomenale und kontraktuelle Seite der Generationenverbundenheit hervor. Die Unternehmerfamilie formt eine Erfahrungsgemeinschaft, die eine besondere Lebenswelt bewohnt, ebenso wie eine Zweckgemeinschaft, die durch , harte Faktoren' wie etwa Eigentum, Beruf, finanzielle Vorsorge für die Eltern etc. aneinander gebunden ist. Sie bildet ein Netzwerk an sich überlagernden Lebensläufen, das den Austausch zwischen den Generationen ermöglicht und auch erforderlich macht.

Aus dem Zusammenspiel dieser verschiedenen Dimensionen einer Unternehmerfamilie eröffnen sich Chancen für das familiale Gemeinschaftsgefüge (Büttner 2007:23). Als Familien eigener Art bilden sie eine kollektive Identität, spezifische Mitgliedschaftsregeln und eine Außengrenze aus, die Anknüpfungspunkte für die individuelle Identität bilden, ein Zusammengehörigkeitsgefühl ebenso wie solidarische Handlungen zwischen den Generationen und Familienmitgliedern befördern können (Büttner 2007:33; Simon 2002a:42f.; Kocka 1979:123ff.). Gleichzeitig verschärfen sich in einer solchen Gemeinschaft die Risiken der Exklusion, Rivalität, Entfremdung und Ungleichheit. Jede der aufgeführten Konfliktlinien, die mit den Koordinationsaufgaben einer Unternehmerfamilie einhergehen, stellt die Reproduktion der Unternehmerfamilie in Frage. In letzter Konsequenz kann das Familienprojekt daran scheitern, dass es nicht gelungen ist, einen selbständigen Lebensentwurf innerhalb der Unternehmerfamilie zu entwerfen, dass keines der Familienmitglieder sich mit der Rolle des Nachfolgers identifizieren kann, dass keine Regelungen zum materiellen Transfer gefunden werden kann etc. Insofern geben die hier erarbeiteten Ergebnisse einige Hinweise auf das Ende der Unternehmerfamilie als Kollektiv, liefern jedoch, und das war auch nicht die Intention dieser Arbeit, keinen vollständigen Katalog. ${ }^{111}$ Es lässt sich jedoch festhalten, dass sich durch den direkten Charakter der Generationenbe-

111 An dieser Stelle sei noch einmal an das Sampling dieser empirischen Arbeit erinnert: Den ausgewählten Fällen ist gemein, dass sich mindestens ein Kind dazu entschlossen hat, den Weg in das Familienunternehmen anzutreten. Eine in diesem Sinne erfolgreiche Nachfolge darf nicht automatisch mit einer intakten Familie gleichgesetzt werden. Die Darstellungen präsentieren die Begegnungen der Generationen ausgewogen und benennen entstehende Konfliktfelder deutlich. Trotzdem erweist sich die Nichtfortsetzung der Unternehmerfamilie als Leerstelle, die in der Beurteilung der Ergebnisse mit bedacht werden sollte. 
ziehungen in Unternehmerfamilien die Generationenambivalenzen potenzieren. 



\section{Schluss}

Es ist das Ziel dieses letzten Kapitels, die entwickelten Thesen aus dem theoretischen ersten Teil der Arbeit in Beziehung zu den Ergebnissen der qualitativ-empirischen Untersuchung, die im zweiten Teil vorgestellt wurden, zu setzen. Im Zentrum stehen zunächst die strukturelle Eigenlogik von Unternehmerfamilien einerseits sowie die sich ergebenden Handlungsmöglichkeiten im Kontext einer (post-)modernen Gesellschaft andererseits. Es gilt das theoretisch entwickelte Modell über den wechselseitigen Einfluss von Familienunternehmen und Unternehmerfamilien im Lichte der empirischen Ergebnisse kritisch zu prüfen und zu überarbeiten. Schließlich wird die Frage aufgeworfen, in welchem Verhältnis die empirischen Ergebnisse, die einer analytischen Perspektive auf das „Ganze Leben“ folgen, zu den in der Familienunternehmensforschung prominenten systemtheoretischen Ansätzen stehen. Insbesondere soll hier diskutiert werden, ob der Einfluss des Unternehmens auf die Unternehmerfamilie kolonialisierend wirkt (Habermas). In einem abschließenden Ausblick werden die empirischen und analytischen Befunde der Arbeit im Hinblick auf ihren Beitrag zur soziologischen Forschung hinterfragt, die Einschränkungen benannt und schließlich anknüpfende Forschungsfelder und -fragen herausgestellt.

\subsection{Strukturen und Handlungen in Unternehmerfamilien}

Es ist der Anspruch der vorliegenden Arbeit, den Einfluss des Unternehmens auf das Beziehungsgefüge in Unternehmerfamilien zu untersuchen und damit die Besonderheiten dieser Familien eigener Art offen zu legen.

Der erste Teil der Arbeit näherte sich diesem Ziel über analytische Mittel: Anhand der idealtypischen Vorstellungen des Ganzen Hauses, der modernen Kernfamilie und der Multilokalen Mehrgenerationenfamilie wurden gedankenexperimentelle Annahmen über die Eigenarten von Unternehmerfamilien formuliert und auf diese Weise unterschiedliche Vergleichsmaßstäbe für diese Familienform benannt. Im Anschluss erfolgte eine ausführliche Diskussion der bestehenden Literatur zu Unternehmerfamilien, die eine intensive Auseinandersetzung mit der sozialhistorischen Forschung ebenso wie mit den gegenwärtig prominenten systemtheoretischen Ansätzen beinhaltete. Es konnte die These entwickelt werden, dass sich über die Verbindung von 
Unternehmen und Familie in Unternehmerfamilien ein struktureller Kern formt, der sich über die Zeit hinweg als relativ stabil erweist, aber dennoch genügend Handlungsspielraum lässt, um die jeweils geltenden gesellschaftlichen Normvorstellungen hinsichtlich unternehmerischen und familialen Handelns zu integrieren. In diesem Zusammenhang wurde das UFFU-Modell des wechselseitigen Einflusses von UnternehmerFamilien und FamilienUnternehmen vorgestellt. Ausgehend von dem in der Familienunternehmensforschung anerkannten Messinstrument der F-PEC Skala, die den Einfluss der Familie auf das Unternehmen in den Dimensionen Macht, Erfahrung und Kultur misst, wurde eine Ordnungsmatrix über den Einfluss des Unternehmens auf die Familie (also in umgekehrter Richtung) erstellt. Diese diente einerseits dazu die aktuellen Forschungsergebnisse zu Unternehmerfamilien zu sortieren, kann aber anderseits auch als erster systematischer Versuch gelesen werden, die strukturelle Eigenlogik von Unternehmerfamilien zu erfassen.

Der zweite Teil dieser Arbeit machte die Bedingungen einer Unternehmerfamilienkonstellation ebenso wie ihre Bewältigung im Kontext moderner Gesellschaften zum Gegenstand einer qualitativen Untersuchung. Anknüpfend an die Idee, das „Ganze Leben“ in den Blick zu nehmen, wie diese in den Ansätzen des Institutionalisierten Lebenslaufes (Kohli) sowie der Alltäglichen Lebensführung (Voß, Kudera) vertreten wird, wurde mittels narrativ biografischer Interviews mit Mitgliedern aus Unternehmerfamilien die Verbindung von Unternehmen und Familie in der Tiefe des Lebens ebenso wie in der Breite des Alltags thematisiert. Die fallrekonstruktive Analyse verdeutlichte den Einfluss des Unternehmens auf den Lebenslauf, die Generationenbeziehungen und die soziale Identität einer Unternehmerfamilie. Der Weg durch den hypothetischen Lebensweg eines Familienunternehmers von der Kindheit bis ins Alter arbeitete das Spannungsverhältnis zwischen einer hohen potentiellen Selbstbestimmtheit der Familienunternehmer und ihrer Begrenzung durch die Gemeinschaft der Unternehmerfamilie heraus. Letztere wurde im Sinne einer Figuration als interdependenter Verweisungszusammenhang mit einem direkten Aushandlungscharakter beschrieben, der mittels einer Pädagogik der Nachfolge, einer Indizienanalyse der Lebensläufe und Familiennarrativen über die Positionierung der Familienmitglieder im Kollektiv, die Synchronisation der Lebensläufe und den materiellen Transfer verhandelt.

Die jeweiligen Ergebnisse wurden bereits an anderer Stelle ausführlich vorgestellt und zusammengefasst worden. ${ }^{112} \mathrm{Im}$ Folgenden soll nun ein Brückschlag zwischen den beiden Teilen dieser Arbeit geleistet werden. Zunächst gilt es, die entwickelte Ordnungsmatrix des UFFU-Modells im Lichte

112 Der interessierte Leser sei an dieser Stelle auf die jeweils letzten Abschnitte eines Kapitels verwiesen, die über die beschrittenen Analysewege sowie die präsentierten Ergebnisse resümieren. 
der empirisch-qualitativen Ergebnisse zu überdenken. Dabei steht das Spannungsverhältnis aus Handlungen und Strukturen im Fokus.

\subsubsection{Noch einmal: Die strukturelle Eigenlogik von Unternehmerfamilien}

Das in Kapitel III entwickelte UFFU-Modell hat sich in seiner grundlegenden Logik und seinem Aufbau als äußerst hilfreich für die empirische Analyse und das methodische Design dieser Arbeit erwiesen. Im Lichte der Ergebnisse erhärten sich einige der im Modell getroffenen Annahmen, andere jedoch bedürfen einer Revision oder Detaillierung.

Das UFFU-Modell erfasst zunächst den wechselseitigen Einfluss von Familienunternehmen und Unternehmerfamilie in der Dimension „Macht“. Während die Familie auf das Unternehmen über die Kanäle Eigentum, Management und Kontrolle einwirke, beeinflusse das Unternehmen die Einkommensquellen, Lebensläufe sowie Ämter und Privilegien der Familienmitglieder. Diese Dimension umfasst damit die „harten“ Faktoren der jeweiligen sozialen Einheit, sie wirken gerade in Bezug auf die Familie sozialstrukturierend. In der empirischen Analyse erhärtet sich der Eindruck, dass das Unternehmen die materielle Basis für den Wohlstand und das Einkommen der Unternehmerfamilie und für die private Vorsorge für Lebenslaufrisiken bildet. Darüber hinausgehend zeichnet sich ein komplexes Geflecht von materiellen Abhängigkeiten zwischen den einzelnen Familienmitgliedern ab. Dies zeigt sich am eklatantesten, wenn sich ein Familienmitglied zur Ruhe setzen möchte und die private Vorsorge nicht zur Finanzierung des Lebensabends ausreicht. ${ }^{113}$ Ein zentraler Aspekt, der in dem aus der Literatur entwickelten Modell unterbelichtet bleibt, ist jener der Vererbung. Der Besitz eines Unternehmens beeinflusst in erheblichem Maße die gewählte Erbschaftsstrategie in Unternehmerfamilien (siehe hierzu ausführlich Kapitel 7.2.3). Es ist ein Ergebnis dieser Arbeit, dass der materielle Transfer nicht nur als normativer Konflikt des Erblassers zu begreifen ist, der zwischen den gesellschaftlichen Normen einer Gleichbehandlung der Kinder, der Liquidität des Unternehmens ebenso wie in der Familie institutionalisierten Erbschaftsregeln abwägen muss. Vielmehr stellt sich der materielle Transfer als ein

113 Auch in der politischen Diskussion im Winter/Frühjahr 2011/12 erhält das wachsende Problem eines steigenden Altersarmutsrisikos bei Selbständigen zunehmend Gewicht, wie sich an dem Vorstoß der Arbeitsministerin von der Leyen für eine Pflichtversicherung der Selbständigen zeigt. Politisch erreicht werden soll hier eine Gleichstellung der Selbständigen mit abhängigen Erwerbstätigen im doppelten Sinne: Erstens, die Vorstellung eines verdienten Ruhestandes als dritte Lebensphase soll auch Selbständigen finanziell ermöglicht werden (siehe hierzu Kapitel 6.5.2). Zweitens, diese Finanzierung soll unabhängig vom Unternehmen und der Familie erfolgen. 
zentrales Thema in einem intergenerationellen Aushandlungsprozess dar. Eine markante Rolle kommt in diesem Prozess dem Verzicht einiger Familienmitglieder zu, der als generalisierte Reziprozität beschrieben wurde und als Ausdruck von Solidarität gegenüber dem Unternehmen und dem Kollektiv der Unternehmerfamilie verstanden werden kann. Kritisch hingegen sollte die Kategorie Ämter und Privilegien überdacht werden. Die empirische Analyse zeigte deutlich, dass Eigentumsgefühle, Verantwortung und Loyalität gegenüber dem Unternehmen nicht erst aus der aktiven Tätigkeit im Unternehmen entstehen, sondern sich aus dem Kontinuitätsanspruch des Unternehmens selbst ableiten lassen (siehe unten). Ebenso wenig kann behauptet werden, dass das Unternehmen als Quelle von Privilegien und Ressourcen (z.B. Netzwerkkontakte zur Vermittlung eines Praktikums) zu einer grundlegenden Struktur in Unternehmerfamilien zählt. Deutlich wurde dieser Umstand unter anderem in der Formulierung der verschiedenen Nachfolgetypen (siehe Kapitel 6.2.2).

Der im UFFU-Modell aufgeführte Einfluss des Unternehmens auf den Lebenslauf der Mitglieder einer Unternehmerfamilie ebenso wie die bestehenden Forschungsergebnisse in diesem Bereich werden durch die empirische Analyse bestätigt, systematisiert und ergänzt. Der ganzheitliche Ansatz zum Leben in einer Unternehmerfamilie widmete sich dem Einfluss des Unternehmens in verschiedenen Lebensphasen, stellte Zusammenhänge zwischen den Lebensläufen der Familienmitglieder heraus und beschrieb sich ergebende Pfadabhängigkeiten. Der Unternehmenseinfluss wirkt strukturierend auf die Lebenslaufgestaltung in Unternehmerfamilien. Dies drückt sich nicht in einem einheitlichen Muster der Lebenswege aus, ganz im Gegenteil hob diese Arbeit unterschiedliche Positionen innerhalb der Unternehmerfamilie hervor (z.B. Nachfolger oder weichender Erbe) und charakterisierte verschiedene Typen etwa der Wege in das Familienunternehmen oder des Alters eines Familienunternehmers. Trotzdem kann eine Reihe von Punkten hinsichtlich der Lebenslaufgestaltung generell für Unternehmerfamilien in Anspruch genommen werden: Erstens, durch den Einfluss des Unternehmens entsteht eine stärkere Verbundenheit der Lebensläufe zwischen den Familienmitgliedern als dies üblicherweise der Begriff ,linked lives“ ausdrückt. Zweitens, das Unternehmen bleibt eine lebenslange berufliche Option für die Mitglieder aus Unternehmerfamilien, selbst wenn diese sich für andere Berufskarrieren entschieden haben. Drittens, in Unternehmerfamilien herrscht ein Modus der permanenten Indizienanalyse der Lebensläufe als ein Mittel zur erforderlichen Synchronisation der Lebensläufe. Viertens, in Unternehmerfamilien ist ein hohes Maß an biografischer Arbeit nötig, um diese strukturellen Merkmale mit den gesellschaftlichen Idealen der Autonomisierung und Individualisierung in Einklang zu bekommen. Es konnte gezeigt werden, dass sich ein „Narrativ der freien Wahl“ als Rechtfertigungsmuster nach au- 
ßen und innen in den biografischen Erzählungen von Familienunternehmern etabliert hat.

Die Stoßrichtung der zweiten Dimension „Generation“ erweist sich ebenfalls als tragfähig. Durch die empirische Analyse lassen sich die strukturellen Besonderheiten von Unternehmerfamilien im Hinblick auf diese zeitliche und dynamische Perspektive nun konkretisieren: In Form eines Familienprojektes wird das Unternehmen zu einem konstitutiven Element der Unternehmerfamilie als einer interdependenten Gemeinschaft, die sich von dem Verwandtschaftsnetz unterscheidet und sich über die Teilhabe am Familienprojekt definiert. Die empirische Arbeit bestätigt die theoretisch angenommene Ausdehnung einer zeitlichen Perspektive sowohl nach hinten in die Unternehmens- und Familiengeschichte als auch nach vorne in den Wunsch der Fortführung des Familienprojektes. Die Identität der Unternehmerfamilie ist mit ihrer Entstehungsgeschichte eng verbunden und kann ohne weiteres mehrere Generationen umspannen. Dieses zeitliche Kontinuum manifestiert sich vor allem auf der Ebene eines geteilten Familiengedächtnisses und ist von der jeweiligen biografischen Perspektive der Familienmitglieder zu unterscheiden. Es wurde deutlich, dass sich der Zugang zur Familienhistorie ebenso wie der Wunsch nach Kontinuität im Laufe des Lebens verändert (beispielsweise durch die Geburt eines Kindes). Gleichwohl trat der von Jäkel-Wurzer beschriebene Kontinuitätsanspruch des Familienprojektes in den hier vorliegenden Fällen als unhintergehbare Norm hervor, die maßgeblich die Mitgliedschaftsregeln und Außengrenzen der Unternehmerfamilie bestimmt. Das jeweilige Engagement oder Nicht-Engagement einzelner Familienmitglieder im Unternehmen steht unter einem Rechtfertigungsdruck gegenüber der historischen, gegenwärtigen und zukünftigen Unternehmerfamilie. Ergänzend bot diese Arbeit Einblicke in ein horizontales Generationenverhältnis. Mitglieder von Unternehmerfamilien fühlen sich gleichzeitig, in Phasen des Übergangs mehr als in anderen, mit ihren Altersgenossen als gesellschaftliche Generation verbunden. Dieser Blick zur Seite erlaubt ihnen, sich im familialen Generationenkontinuum zu positionieren und im Sinne von „die Alten“ und „die Jungen“ abzugrenzen. Gleichsam fungiert der Vergleich mit den Altersgenossen als Individualisierungstest, der riskante Auswirkungen für die Fortsetzung des Familienprojektes mit sich bringen kann. Insofern wird das Unternehmen in der Tat ein konstitutives Element in der Wahrnehmung von Zeit in Unternehmerfamilien, das Lebenslaufentscheidungen und -orientierungen beeinflusst.

Die dritte Dimension des wechselseitigen Einflusses ist mit dem Schlagwort „Kultur“ besetzt. Ganz entgegen der magereren und widersprüchlichen Literaturlage lieferte das hier gewonnene empirische Material einen reichhaltigen Einblick in die unternehmerische Kultur in Unternehmerfamilien. Diese wird im Wesentlichen von einem meritokratischen Denken getragen, das sich etwa in der Maxime der Selbständigkeit und Leistungsfähigkeit in der Kin- 
deserziehung äußert oder als Orientierungspunkt für die Gestaltung des Ruhestandes fungieren kann. Des Weiteren konnte ein professionelles Wertesystem von Familienunternehmern identifiziert werden. Ein sehr spezifisches Tätigkeitsbild, Standards des guten Unternehmertums sowie eine Vorstellung von ihrem sozialen Status ebenso wie von Gegenbildern (z.B. Manager) markieren die Kernelemente in der biografischen Selbstdarstellung der Familienunternehmer als professionell, die gleichzeitig sozial differenzierend und vergemeinschaftend wirkt. Der Blick auf die Lebensführung in Unternehmerfamilien legte eine kontinuierliche Grenzdefinition zwischen Unternehmen und Familie nahe, die sich in unterschiedlichen Arrangements ausdrückt. Die Typen „Verschmelzung“, „Zwei Kerne“ und „Festung“ können als drei Ausprägungen von praktizierter Kultur in Unternehmerfamilien verstanden werden. Mehr oder weniger starke Eigentumsgefühle sind hingegen nur ein Aspekt der unternehmerischen Kultur in Unternehmerfamilien, der sich jedoch vor allem innerhalb der Generationenbeziehungen und der impliziten familialen Positionsbestimmung auswirkt.Das im Lichte der empirischen Ergebnisse überarbeitete Modell lässt sich wie folgt zusammenfassend darstellen:

Abbildung 11: Überarbeitetes UFFU-Modell

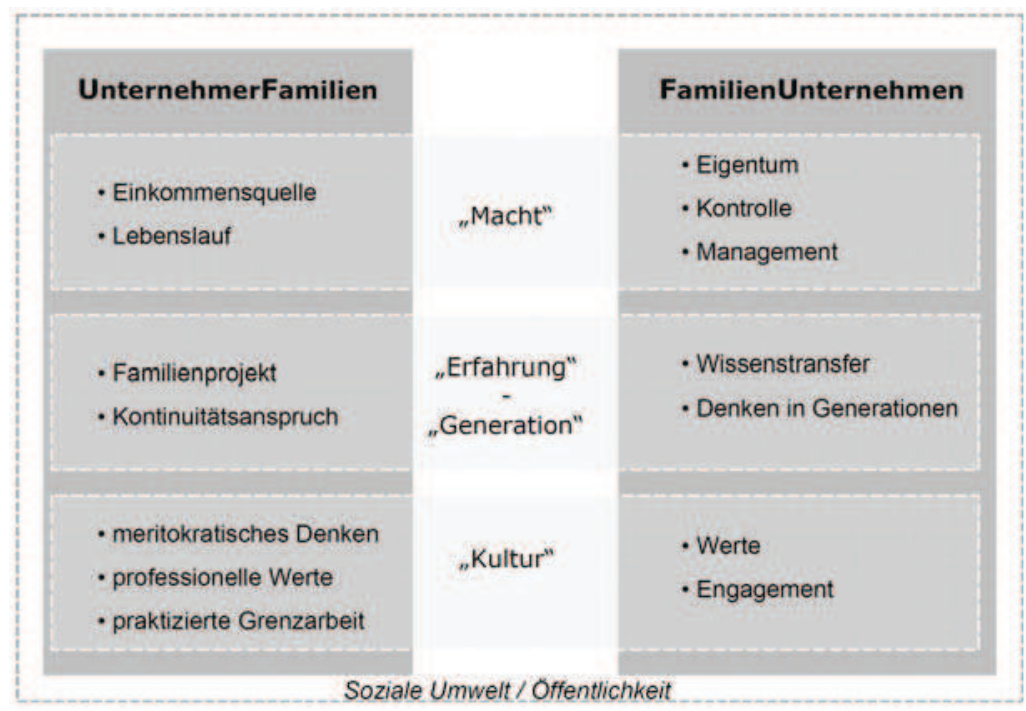

Quelle: eigene Darstellung 


\subsubsection{Handlungsspielräume und Strategien in Unternehmerfamilien}

Der Zusammenhang und die Grenzen von Handlungen und Strukturen sind ein genuin soziologisches Thema und eine komplexe Debatte, in die an dieser Stelle nicht eingestiegen werden soll (siehe hierzu etwa Giddens 1997; Wohlrab-Sahr 1997 oder Schimank 2000). Trotzdem ist es unerlässlich, nachdem nun die wesentlichen strukturellen Komponenten von Unternehmerfamilien beschrieben worden sind, auch auf die Möglichkeiten der aktiven Gestaltung im Raum dieser Familien eigener Art zu sprechen zu kommen. In der empirischen Analyse wurde mehr als deutlich, dass das Unternehmerfamiliesein zwar von den Individuen als eine spezifische Art des Lebens verstanden und praktiziert wird, sich dessen konkrete Ausgestaltung (Lebensstil) jedoch erheblich unterscheiden kann. Diese emergenten Elemente finden sich auf den drei Analyseebenen Lebenslauf, Biografie und Lebensführung, wie sie in dieser Arbeit abgeleitet und angewandt wurden:

Die Mitglieder in Unternehmerfamilien gestalten ihren Lebenslauf selbst. Als Akteure ihres Lebens verfügen sie über das kreative Potenzial, selbst gewählte Wege einzuschlagen und wieder zu verlassen. Die institutionalisierende Wirkung des Lebenslaufes ist dabei kaum zu übersehen, dieser wird als verzeitlichtes Ablaufprogramm verstanden. Jedoch entfaltet der Normallebenslauf in Unternehmerfamilien nur bedingt seine Kraft. Dieser kann muss aber nicht - als ein Orientierungsmuster der Lebenslaufgestaltung angesehen werden. In solchen Fällen strukturiert das Unternehmen das Leben der Familienunternehmer in die üblichen drei Phasen der Ausbildung, Berufsausübung und des Ruhestandes. Diese Arbeit hat darüber hinaus ein alternatives Lebenslaufprogramm identifiziert, eines, das sich an den Leitideen einer Leistungsgesellschaft orientiert. In diesen Fällen gliedert sich das Leben der Familienunternehmer in Form einer Kette aufeinander folgender und ineinander verschachtelter Projekte. Der Akteur wählt sich diese Projekte entsprechend seiner Leistungsfähigkeit und nach strategischen Erwägungen aus: etwa ein kleines eigenes Unternehmen, um wichtige unternehmerische Erfahrungen zu sammeln, die Neuausrichtung des Familienunternehmens, um die Kontinuität des Familienprojektes zu gewährleisten, oder schließlich die Übernahme eines Beratungsmandates für das Familienunternehmen, um Platz für nachfolgende Generationen zu schaffen und gleichzeitig Wissen in der Organisation zu halten etc. Als wesentlicher Grund für die erhöhte Flexibilität in der Ausgestaltung des Lebenslaufes wurde die besondere Qualität der Tätigkeit als Unternehmer identifiziert, die sich nur schwer einfangen lässt mit den üblichen Begriffen der „Berufswahl“, der „Profession“ oder des „Ruhestandes“, die auf der Vorstellung abhängiger Erwerbsarbeit und ihren flankierenden Sozialversicherungssystemen sowie gesellschaftlichen Alters- 
normen fußen. In anderen Worten, die strukturelle Eigenlogik einer Unternehmerfamilie erhöht in diesem Fall sogar das kreative Potenzial der Lebenslaufgestaltung, da Familienunternehmer ihre „Tätigkeit“ entweder als „,normale" Arbeit oder aber im Sinne einer projektförmigen Leistungserbringung interpretieren können. Gleichzeitig werden diese potenziellen Freiräume durch die strukturelle Eigenlogik der Unternehmerfamilien begrenzt, denn die individuelle Lebenslaufgestaltung ist in hohem Maße in das Generationenverhältnis eingebettet und bedarf einer Koordination und direkten Aushandlung mit anderen Familienmitgliedern.

Auf der Ebene der Lebensführung wird dieser doppelte Charakter der strukturellen Eigenlogik in Unternehmerfamilien ebenfalls deutlicht: Die Unternehmens-Familien-Konstellation sieht vor, dass jede Unternehmerfamilie eine aktive Grenzarbeit lebt und damit selbst definieren kann, welches Tätigkeits- und Zeitarrangement sie wählt (wie oben bereits erwähnt, wurden in dieser Arbeit drei Typen der aktiven Grenzarbeit vorgestellt). Diese gestalterische Leistung geht mit der Möglichkeit einher, die Vereinbarkeit beider Bereiche und ihre Zeiteinteilung zu optimieren und einen Lebensstil zu wählen, der der eigenen biografischen Vorstellung entspricht. Gleichzeitig entsteht aus der Unternehmens-Familien-Konstellation eine Vielzahl an Einschränkungen für die tatsächliche Ausgestaltung der Lebensführung, die sich vor allem aus der Immobilität des Unternehmens ebenso wie dem generationalen Setting ableiten.

Aus der strukturellen Eigenlogik der Unternehmerfamilie ergibt sich schließlich die Notwendigkeit, in erhöhtem Maße biografische Arbeit zu leisten. Die Mitglieder aus Unternehmerfamilien sind gefordert, sich mit den Gestaltungsmöglichkeiten des Lebenslaufes und der Lebensführung selbstreflexiv auseinanderzusetzen und eine konsistente Selbstsicht zu entwerfen. In diesem Prozess stehen nur bedingt anwendbare Orientierungsmuster zur Verfügung. Gleichzeit stellt das Kollektiv der Unternehmerfamilie für die Autonomisierungs- und Individualisierungsbestrebungen der Familienmitglieder eine Herausforderung dar. Es gilt die Ergebnisse der Aushandlungen in allen Lebensphasen in das biografisch entworfene Selbst zu integrieren. In dem empirischen Material wurde eine Reihe von Narrativen identifiziert, die aufzeigen, auf welche Weise Familienunternehmer diese biografische Arbeit umsetzen. Die Schwierigkeit der biografischen Selbstbestimmtheit in Unternehmerfamilien ist darin zu sehen, dass diese keine Selbstverständlichkeit darstellt und einen lebensprägenden Rechtfertigungsdruck hervorruft. Als weiteres zentrales Ergebnis lässt sich festhalten, dass die biografische Arbeit im Austausch mit den anderen Familienmitgliedern erfolgt. Diese stehen in einem komplexen Beratungs- und Beobachtungsverhältnis zueinander.

Die Handlungsfreiräume in der Gestaltung des Lebenslaufes, der Lebensführung und der Biografie sind es, die Raum dafür lassen, dass sich eine Unternehmerfamilie zu den jeweils geltenden gesellschaftlichen Vorstellun- 
gen positionieren kann und muss. Auf diese Weise fließt der Geist eines Netzwerkkapitalismus, wie er von Boltanksi und Chiapello beschrieben wurde, ebenso in (post-)moderne Unternehmerfamilien ein wie individualisierte Ansprüche an die Lebenslaufgestaltung oder pluralisierte Formen familialen Handelns. Durch die Integration dieser zeitgenössischen gesellschaftlichen Ideale setzen sich Unternehmerfamilien zugleich den typischen Modernisierungskonflikten und Spannungen aus. Sie stehen unter Individualisierungsdruck, müssen einen erhöhten Koordinationsbedarf, den eine flexible Tätigkeit typischerweise mit sich bringt, bewältigen. Darüber hinaus beinhaltet die Figuration Unternehmerfamilie ein erhebliches Potenzial für Rivalitäten zwischen den Familienmitgliedern, für Exklusion sowie für materielle Benachteiligung. Die Positionsbestimmung innerhalb einer Unternehmerfamilie ist mit emotionalen, sozialen und auch materiellen Konsequenzen verknüpft.

Der Blick auf die entstehenden Handlungsspielräume innerhalb von Unternehmerfamilien entlarvt auch einen wesentlichen Aspekt der Nachfolge. Diese ist weit davon entfernt, ein unilinearer Prozess zu sein, bei dem „etwas" übertragen wird. In dieser Arbeit wurde Nachfolge vielmehr als ein Verlaufsmuster beschrieben, mit dem Ein- und Austritt ins Familienunternehmen als zentralen Übergängen. In dieser Vorstellung verquicken sich erneut strukturelle Zwänge mit aktiven Elementen der Aneignung und Entscheidung. So können beispielsweise Delegationen angenommen oder zurückgewiesen werden. Die Kausalitäten der Übergabe können nur innerhalb des Kollektivs der gesamten Unternehmerfamilie erfasst werden, gehen also über eine reine Vorgänger-Nachfolger-Beziehung hinaus.

\subsection{Unternehmerfamilien als kolonialisierte Lebenswelt?}

In Abgrenzung zu den in der Familienunternehmensforschung prominenten systemtheoretischen Ansätzen wählte diese Arbeit einen analytischen Zugang zum „Ganzen Leben“ in Unternehmerfamilien. Die vorliegende qualitativempirische Untersuchung knüpfte an die Theorien zur Institutionalisierung des Lebenslaufes ebenso wie an die Alltägliche Lebensführung an. Dieser Perspektivenwechsel verschob den Fokus von widerstreitenden Systemlogiken und Rollenkonflikten auf eine Analyse der Selbstverständlichkeiten, der gelingenden Kommunikation und des Alltags in Unternehmerfamilien. Auf diese Weise kann ein umfassendes und empirisch fundiertes Gegengewicht $\mathrm{zu}$ der organisationslastigen Familienunternehmensforschung geschaffen werden.

Es stellt sich nun die theoretische Frage, in welchem Verhältnis die beiden Zugangswege zum Untersuchungsobjekt Unternehmerfamilie stehen. 
Ihnen ist eine Reihe von modernisierungstheoretischen Annahmen gemeinsam, allen voran die Vorstellung einer sich zunehmend funktional differenzierenden Gesellschaft. Während eine systemtheoretische Perspektive selbstreferentielle Systeme beobachtet, die strukturell aneinander gekoppelt sind, versucht ein Zugang zum „Ganzen Leben“, die Zusammenhänge der verschiedenen Lebens- und Tätigkeitsbereiche zu verstehen. Der Einfluss des Unternehmens auf die Unternehmerfamilie wird in systemtheoretischen Kategorien als ein dauerhaftes Hineinragen der rationalen Systemlogik des Unternehmens in das emotionale System Familie gefasst, wodurch die Kommunikation in Unternehmerfamilien paradoxie- und konfliktanfälliger wird. Der Einfluss des Unternehmens auf die Unternehmerfamilie wird in den Kategorien Lebenslauf, Biografie und Lebensführung zu einem selbstverständlichen Muster der Alltagspraxis, zu einem Tätigkeitsarrangement, das im Laufe des Lebens wandelbar (ist) und Gegenstand von Reflexion ist. In Unternehmerfamilien bildet sich eine Vorstellung vom Leben als Unternehmerfamilien heraus, die eine Grenzdefinition von Unternehmen und Familie ebenso beinhaltet wie eine Positionierung gegenüber gesellschaftlichen Normvorstellungen.

Versucht man nun beide Ansichten miteinander zu verbinden, ergibt sich folgende These: Innerhalb der Unternehmerfamilie konstituiert sich eine intersubjektive Ordnungsperspektive, die den Einfluss des Unternehmens als rationales System und die sich daraus ergebenden Ambivalenzen und Konflikte auf ein erträgliches $\mathrm{Ma} ß$ reduzieren ${ }^{114}$ und in ein wandelbares Alltagsarrangement übersetzen kann. Erhellend für eine weitere Diskussion dieser Überlegung ist die Vorstellung einer „Kolonialisierung der Lebenswelt“, wie sie von Jürgen Habermas vorgestellt wurde.

In seiner Theorie des kommunikativen Handelns beschreibt Habermas (1982a; 1982b) Gesellschaft als Differenz von Lebenswelt und System (Palazzo 2002:36). Mit seinem Lebensweltbegriff knüpft Habermas insofern an Husserl an, als damit ein implizites Wissen gemeint ist, das den kommunikativ handelnden Individuen als Hintergrund ihres Handelns immer vorhanden, ihnen jedoch meistens nicht bewusst ist. Die Lebenswelt manifestiert den Bezugspunkt des Wissens, sie ist das Reservoir von Selbstverständlichkeiten und unerschütterlichen, nicht hinterfragten Überzeugungen (Treibel 2000:167). Habermas wendet diesen Begriff jedoch intersubjektiv: Die Lebenswelt ist der Ort, an dem sich Sprecher und Hörer begegnen, an dem sich die soziale Reproduktion von Gesellschaft vollzieht. Wenn kommunikativ Handelnde sich über Themen, Ziele, Pläne, den normativen Rahmen usw. verständigen, bleibt ihnen ihre Lebenswelt im Rücken. Sie besteht aus drei strukturellen Komponenten: Mit „Kultur“ bezeichnet Habermas den Wis-

114 In ähnlicher Weise beschreibt Schweizer die „Lebenswelt“ und ihre Funktionen. Schweizer, Herbert 2007:409f. 
sensvorrat der Kommunikationsteilnehmer. „Gesellschaft“ beschreibt die legitime Regelung sozialer Zugehörigkeiten, die soziale Integration und die Ausbildung von Solidarität. „Persönlichkeit“ meint jene Kompetenzen, die ein Subjekt sprach- und handlungsfähig machen. (Habermas 1982b:209) In der Lebenswelt befinden sich Werte, Glaubensgrundsätze, kulturelle Gemeinsamkeiten, aber auch Institutionen wie die Familie, die für die Erziehung neuer Gesellschaftsmitglieder hauptverantwortlich ist, oder die gesellschaftliche Öffentlichkeit. Als Systeme hingegen bezeichnet Habermas selbstreferentielle Bereiche des erfolgsorientierten bzw. strategischen Handelns (im Gegensatz zum kommunikativen Handeln), die über generalisierte Steuerungsmedien bestimmte gesellschaftliche Aufgaben erfüllen und zur materiellen Reproduktion der Gesellschaft beitragen. Habermas identifiziert insbesondere zwei solcher Systeme (Degele und Dries 2005:240): Die marktregulierte Ökonomie koordiniert die Versorgung der Gesellschaft mit Gütern über das Steuerungsmedium Geld. Der sich durch Steuern finanzierende Staat koordiniert die gesellschaftliche Sicherheit über das Medium Macht. In dieser theoretischen Perspektive treffen in Unternehmerfamilien also Familien als Institutionen der Lebenswelt und Unternehmen als Gebilde des ökonomischen Systems aufeinander.

Habermas beschreibt, wie sich im Laufe des Modernisierungsprozesses drei wesentliche Schritte vollzogen (Linkenbach 1986:32): die Dezentrierung der Weltbilder und damit die Rationalisierung des Handelns, die historische Entkoppelung von System und Lebenswelt, die Komplexitätssteigerung der gesellschaftlichen Systeme und ihre Rückwirkungen auf die Lebenswelt. Blickt Habermas nun auf die Bruchstelle zwischen System und Lebenswelt, stellt er fest, dass die jeweiligen Grenzen fluide und wandelbar sind. Die Beziehung der beiden Bereiche wird über die entsprechenden Austauschmedien vermittelt, etwa im Fall von Staat und Öffentlichkeit über das Medium Macht oder im Fall von Ökonomie und Familie über das Medium Geld. Problematisch ist nun nicht die Tatsache, dass System und Lebenswelt nach verschiedenen Regeln operieren oder sich in einem sozialevolutionären Prozess auseinanderdifferenzieren, sondern der Moment, in dem die monetären und administrativen Steuerungsmechanismen in das lebensweltlich strukturierte Geschehen regulierend und normierend eingreifen wollen (Degele und Dries 2005:243ff.). Ein solches übergebührliches Eindringen eines Systems in die Lebenswelt führt dazu, dass die interaktiven Abstimmungsprozesse an Kraft verlieren (Voß 1991:53f.) und die Lebenswelt dysfunktional wird (Habermas 1982b:522). Dieses bedrohliche Szenario bezeichnet Habermas als Kolonialisierung.

Bezogen auf Unternehmerfamilien lässt sich entsprechend formulieren, dass die Familie mit dem Unternehmen über das Austauschmedium Geld verbunden ist. Über die Eigentumsanteile und die Tätigkeit im Unternehmen bezieht die Familie Einkommen aus Gewinnen, Privatentnahmen und Lohn. 
Die Ergebnisse der empirischen Analyse verdeutlichen jedoch, dass sich die Beziehung zwischen Unternehmen und Familie nicht in solchen monetären Steuerungsmechanismen erschöpft. Der Einfluss des Unternehmens ist umfassender und wirkt in alle drei strukturellen Komponenten der Lebenswelt ein: Es wird Bestandteil des Wissensvorrates und der Wertvorstellungen der Familienmitglieder (Kultur), bestimmt die legitimen Regelungen sozialer $\mathrm{Zu}$ gehörigkeit, die Ausbildung von Solidarität (Gesellschaft) und wird Teil der individuellen Identität und biografischen Selbstsicht (Persönlichkeit). Folglich könnte eine Unternehmerfamilie als kolonalisierte Lebenswelt beschrieben werden, in der sich kommunikatives Handeln nicht entfalten kann. Jedoch vermag eine solche Deutung nicht zu erklären - ebenso wenig wie ein rein systemtheoretischer Ansatz -, wie sich Unternehmerfamilien dennoch reproduzieren.

Die ursprüngliche Kolonialisierungsthese entwickelt Habermas in seinen späteren Schriften mit Blick auf die Zukunft der Demokratie in Europa zu einer Öffnung-Schließung-Dynamik weiter. Diese lässt sich ohne weiteres auf den Bereich der Unternehmerfamilie übertragen. Habermas spricht Gruppen wie Familienverbänden, religiösen Gemeinschaften oder Staaten die Fähigkeit zu, sich gegenüber ihren Umwelten zu öffnen oder zu schließen (Habermas 1998:125). Von systemischen Netzwerken geht eine sprengende Kraft aus, die Gesellschaften als intuitiv geschlossenen Gebilden einem wachsenden Pluralismus aussetzt, der soziale Bindungen, soziale Herkunft und Traditionen lockert und so einen Formwandel in Gang setzt, bis sich die Gesellschaft auf erweitertem Niveau wieder schließt (Habermas 1998:126). Öffnung charakterisiert Habermas als zweideutige Erfahrung, die von Impulsen durch neue Märkte, Kommunikationsmittel, Verkehrswege oder kulturelle Vernetzungen ausgeht. Öffnung bedeutet für die Individuen erhöhte Kontingenz, die ein Spannungsverhältnis zwischen Freiheit und Desintegration hervorruft. „Kurzum, die Entbindung aus einer stärker integrierten Lebenswelt entlässt die Einzelnen in die Ambivalenz wachsender Optionsspielräume“" (Habermas 1998:127). Die Gefahr eines Liberalisierungsschubes besteht darin, dass ohne eine rechtzeitige Schließung Entfremdungs- und Anomieerfahrungen einsetzen. Im Gegensatz dazu charakterisiert Habermas Schließung als dreidimensionale Reorganisation mit einem erweiterten Horizont. Die erste Dimension umfasst eine neue Form der Identität im Sinne eines Selbstbewusstseins. Die zweite Dimension stellt die Selbstbestimmung dar, also die Autonomie der Individuen und der Gesellschaft. Die dritte Dimension bildet letztlich die Selbstverwirklichung im Sinne einer individuellen Ausgestaltung des persönlichen Lebens (Habermas 1998:127). Im Rahmen einer Öffnung werden alle diese Dimensionen in ihrer alten Form bedroht und damit die Gesellschaft aus dem Gleichgewicht gebracht. Erst eine Schließung und damit ein Lernprozess, der in eine neue Definition von Identität, Autonomie und Individualität mündet, stellt die Balance wieder her. 
Der beschriebene Mechanismus aus Öffnung und Schließung präzisiert das Verhältnis von System und Lebenswelt. Die Gründung des Familienunternehmens kann mit der Entstehung eines Netzwerkes verglichen werden, das in den jeweiligen Familien einen Formwandel in Gang setzt. Durch den Einfluss des Unternehmens stellt die Familie nicht automatisch auf die Vorzeichen von Geld und Profit um. Stattdessen öffnet sich die Lebenswelt der Familie dem Unternehmen, was für die Familienmitglieder im Kontext moderner Gesellschaften die ambivalente Erfahrung von Desintegration und Freiheit mit sich bringt. Im Prozess der Schließung bildet sich das Kollektiv der Unternehmerfamilie aus, mit einer eigenen sozialen Identität, definierten Mitgliedschaftsregeln und Außengrenzen und einer spezifischen Alltagspraxis. Die Unternehmerfamilie kann damit als erweiterte, geschlossene Lebenswelt beschrieben werden, die im Zeitverlauf und Generationenwechsel immer wieder einer Öffnung-Schließung-Dynamik unterliegt. Die Reproduktion der Unternehmerfamilie ist entsprechend als eine erfolgreiche Schließung zu beschreiben. Misslingt die Reorganisation der intersubjektiv geteilten Lebenswelt, wird diese vom Unternehmen kolonialisiert - mit all seinen dysfunktionalen, pathologischen und letztlich destruktiven Konsequenzen. Die Grenzen zwischen Schließung auf erweitertem Niveau und Kolonialisierung sind fließend.

Das von Habermas leider nur in Ansätzen entwickelte Konzept einer Öffnung-Schließung-Dynamik als Erweiterung der Kolonialisierungsthese, das die Verbindung von System und Lebenswelt präzisiert, erweist sich als kompatibel mit den empirischen Ergebnissen dieser Arbeit und weiteren qualitativen Studien in diesem Bereich. Es unterstreicht die Fähigkeit des Kollektives Unternehmerfamilie, sich mit wandelnden Umweltfaktoren wie etwa Moden unternehmerischen Handelns, familialen Vorstellungen, Altersnormen, etc. auseinanderzusetzen und diese mit ihrer strukturellen Eigenlogik in Einklang zu bringen. Gleichzeitig werden die dafür notwendigen Anstrengungen benannt. Folgendes Zitat von Daniela Jäkel-Wurzer, die eine eigene Begrifflichkeit von Öffnung und Schließung im Kontext von Unternehmerfamilien entwickelte, unterstreicht noch einmal diesen dynamischen Charakter der Familien eigener Art - und verleiht zugleich ihrer Begrifflichkeit eine zusätzliche theoretische Tiefe:

„So kann die Unternehmerfamilie nicht als System gefasst werden, welches eingebettet in die Verbindlichkeit eines Anspruchs auf Privatheit seine spezifische Dynamik entwickelt. Sie ist vielmehr eine über die besondere Bezogenheit auf ein Unternehmen ,erweiterte Gruppe', welche über eine jeweils individuelle Logik der Öffnung und Schließung verschiedene Formationen einer ,Choreografie' annimmt, welche in einem dynastischen Verlauf über Generationen immer wieder aufgegriffen und verändert wird. Jede folgende Generation gestaltet ihre eigenen Bewegungen, welche abhängig von einer gelingenden Balance von Entwicklung und Tradition darüber entschei- 
det, ob die Unternehmerfamilienchronik fortgeschrieben wird.“ (JäkelWurzer 2010:12f.)

Ein weiterer Aspekt der Öffnung-Schließung-Dynamik entspricht den in dieser Arbeit vorgestellten Ergebnissen: Die erweiterte Lebenswelt der Unternehmerfamilie umfasst ganz explizit Unternehmenskomponenten. Das Unternehmen findet sich damit eben nicht mehr nur als systemischer Einfluss auf die Familie wieder, sondern wird ein fester Bestandteil der Alltagsarrangements, die erinnert, erzählt und erlebt werden. Insofern verweist die Betrachtung der Unternehmerfamilie durch die hier diskutierte theoretische Perspektive auf die lebensweltliche Dimension des Unternehmens - ein Aspekt, der in der wirtschafts- und organisationssoziologischen Forschung eher unterbelichtet ist.

\subsection{Ausblick}

Durch den holistischen und umfassenden Ansatz der vorliegenden Arbeit gelingt es, das bestehende Wissen zu Unternehmerfamilien zu systematisieren und durch eine empirische Untersuchung zu detaillieren. Sehr deutlich wurden dabei bestehende Forschungslücken benannt und auf Anknüpfungspunkte für weitere Forschung hingewiesen. Einige viel versprechende Anschlussmöglichkeiten seien an dieser Stelle kurz zusammengefasst, bevor auf den Beitrag dieser Arbeit zum wissenschaftlichen Diskurs sowie zur Beratungspraxis eingegangen wird.

Die theoretisch geleitete Auswahl der Fälle für die empirische Untersuchung sah einen Fokus auf solche Unternehmerfamilien vor, in denen mindestens ein Familienmitglied die Nachfolge im Familienunternehmen antritt und sich damit die Unternehmerfamilie reproduziert. Die Risiken und Koordinationsaufgaben, die sich aus den Lebensläufen und insbesondere aus den Generationenbeziehungen in Unternehmerfamilien ableiten, geben dennoch Hinweise darauf, aus welchen Gründen eine solche Nachfolge scheitern kann. Eine qualitative Studie, die nun gezielt solche Fälle in Betracht zieht, in denen sich die Unternehmerfamilie eben nicht reproduziert, würde eine ertragreiche Kontrastfolie bieten. Sie würde die vorgestellten kritischen Momente der Nachfolge (vor allem Positionierung, materieller Transfer, Synchronisation der Lebensläufe, Individualisierungstest im Vergleich mit der horizontalen Generation) auf ihre Plausibilität prüfen und eventuell weitere Punkte identifizieren. Gewinnbringend wären darüber hinaus qualitative Arbeiten, die sich stärker auf einzelne Lebensphasen konzentrieren oder einem ländervergleichenden Design folgen. 
Sehr deutlich formuliert worden ist die Notwendigkeit, die Kenntnisse zu Unternehmerfamilien auch auf einer verallgemeinernden, quantitativen Basis zu erweitern. Wünschenswert wären einerseits deskriptive Befunde zu Unternehmerfamilien, die etwa Einkommenshöhen, Haushaltsgrößen, Familienformen, Bildung und Lebenswege offen legen. Andererseits bietet die vorliegende Arbeit eine große Anzahl an Hypothesen, die mit multivariaten Analysen getestet werden könnten, etwa hinsichtlich des Übergangs in den Ruhestand, der Generationenbeziehungen oder der Wertvorstellungen in Unternehmerfamilien. Die schwierige Frage einer geeigneten Vergleichsgruppe wurde im Zuge der vorliegenden Arbeit über einen Bezug zu unterschiedlichen Idealen familialen Handelns gelöst. Gleichzeitig geben die empirischen Ergebnisse einige Hinweise darauf, zu welchen Gruppen sich Unternehmerfamilien selbst in Beziehung setzen: So kontrastieren sie sich etwa in Bezug auf Werte und Professionalität mit angestellten Managern. Für eine Untersuchung des Lebensstils von Unternehmerfamilien bietet sich hingegen ein Vergleich von Unternehmerfamilien mit Arbeitnehmern an, wobei bedacht werden muss, dass sich innerhalb von Unternehmerfamilien unterschiedliche Muster der alltäglichen Lebensführung herausbilden, die sich durchaus an der Vorstellung eines Normalarbeitsverhältnisses orientieren können. Schließlich verspricht ein Vergleich von Unternehmerfamilien mit der Gruppe der Freiberufler (etwa Ärzte, Rechtsanwälte, etc.) fruchtbare Ergebnisse im Hinblick auf die Gestaltung des Ruhestandes.

Neben diesen methodischen Anknüpfungspunkten für weitere Forschung ließen sich die Ergebnisse dieser Arbeit auch noch theoretisch erweitern: Eine stärkere Einbettung von Unternehmerfamilien in Individualisierungstheorien könnte Erkenntnisse darüber liefern, wie Unternehmerfamilien mit dem Spannungsverhältnis aus Selbst- und Fremdzuschreibungen verfahren oder als Unternehmer der eigenen Biografie agieren. Der herausgearbeitete strukturelle Kern ebenso wie die Handlungsfreiheiten in Unternehmerfamilien scheinen darüber hinaus geradezu prädestiniert für eine Analyse im Sinne der Gidden'schen Strukturationstheorie, wie sie etwa im Bereich des strategischen Managements Eingang gefunden hat (etwa Ortmann und Sydow 2001), die eine ertragreiche Gegenposition zu den vorherrschenden systemtheoretischen Ansätzen bieten könnte. Schließlich könnten etwa eine stärkere Anbindung der Ergebnisse zur Kindheit in Unternehmerfamilien an die pädagogische bzw. erziehungswissenschaftliche Literatur oder die Rahmung der beschriebenen Generationenbeziehungen durch beziehungswissenschaftliche Erkenntnisse tiefere Einblicke in einzelne Teilbereiche dieser Arbeit liefern.

Solche empirischen Analysen bzw. theoretischen Erweiterungen würden den eingeschlagenen Kurs konsequent weiterführen. Diese Arbeit leistet einen Beitrag dazu, den Einfluss des Unternehmens auf den Lebenslauf, die Generationenbeziehungen sowie die soziale Identität in Unternehmerfamilien besser zu verstehen und analytisch fassen zu können. Sie bietet ein komple- 
xes Verständnis der Lebensführung in Unternehmerfamilien und zeigt, dass hier in der Tat ein erhebliches Maß an aktiver Grenzarbeit notwendig wird, wie dies in dem Konzept des „Arbeitskraftunternehmers“ angenommen wird. Die vorliegende empirische Studie nahm gezielt eine andere Tätigkeitsform als Erwerbsarbeit ins Visier. Dabei bestätigte sie die Wirkungsmacht des Normallebenslaufes und entdeckte gleichzeitig ein alternatives Orientierungsmuster der Lebenslaufgestaltung. Ein ausgeprägtes meritokratisches Denken sowie die Inkorporation einer Leistungskultur setzen nicht die Verzeitlichung des Lebens außer Kraft, lassen allerdings die scharfen, alterschronologischen Grenzen zugunsten einer Idealisierung der eigenen Leistungsfähigkeit verblassen. Selbst in dieser sehr speziellen Gruppe der Unternehmerfamilien findet sich damit ein Nebeneinander unterschiedlicher Arbeitskrafttypen und Lebenslaufprogramme. Letztlich bereichert diese Arbeit auch die soziologische Generationenforschung. Mit ihrer qualitativen Herangehensweise gerät nicht so sehr das Solidaritätsverhältnis in Unternehmerfamilien in den Blick als vielmehr Voraussetzungen für dieses. Zudem wurde eine Verbindung der Begriffe Figuration und Generation vorgeschlagen. Insgesamt lässt sich diese Arbeit nicht nur unmittelbar dem wachsenden Forschungsfeld des Family Business Research zuordnen, sondern leistet einen Beitrag zur familien-, lebenslauf-, generations-, und wirtschaftssoziologischen Forschung.

Abschließend sei erwähnt, dass diese Arbeit auch eine Vielzahl von Gedankenanstößen für die Beratungspraxis bietet. Mit dem vorgestellten UFFUModell sowie der detaillierten Darstellung der empirischen Ergebnisse verknüpft sich die Hoffnung, dass Unternehmerfamilien nicht länger auf das $\mathrm{Zu}$ sammenspiel zweier konträrer Systemlogiken reduziert werden. Vielmehr soll dazu ermuntert werden, die Unternehmen-Familien-Konstellation in ihrer Komplexität zu verstehen und dabei insbesondere die lebensweltliche Dimension des Unternehmens zu berücksichtigen. 


\section{Literaturverzeichnis}

Abel, Wilhelm. 1953. Das Generationenproblem in der Bauernfamilie. Die Landfamilie-Schriftenreihe für Ländliche Sozialfragen, 59-68.

Albert, Mathias. 1999. Die Neue Weltwirtschaft. Entstofflichung und Entgrenzung der Ökonomie. Frankfurt a.M.: Suhrkamp.

Albertini, Marco; Kohli, Martin und Vogel, Claudia. 2006. Transfers of time and money among elderly Europeans and their children: common patterns-different regimes? Berlin.

Albertini, Marco; Kohli, Martin und Vogel, Claudia. 2007. Intergenerational Transfers of Time and Money in European Families: Common Patterns-Different Regimes? Journal of European Social Policy 17, 319-334.

Aldrich, Howard E, und Cliff, Jennifer E. 2003. The Pervasive Effects of Family on Entrepreneurship: Toward a Family Embeddedness Perspective. Journal of Business Venturing 18, 573-596.

Alheit, Peter, und Dausien, Bettina. 2000. Die biographische Konstruktion der Wirklichkeit. Überlegungen zur Biographizität des Sozialen. In Biographische Sozialisation. Der Mensch als soziales und personales Wesen, Bd. 17, Hrsg. Erika M. Hoerning und Peter Alheit, 257-283. Stuttgart: Lucius \& Lucius.

Amable, Bruno. 2003. The Diversity of Modern Capitalism. New York: Oxford University Press.

Aronoff, Craig E. 1998. Megatrends in Family Business. Family Business Review XI, $181-185$.

Aronoff, Craig E, und Ward, John L. 1994. Beware of the Family-Business Paradox. Nation's Business 82, 80.

Astrachan, Joseph; Klein, Sabine und Smyrnios, Kosmas. 2002. The F-PEC Scale of Family Influence: A Proposal for Solving the Family Business Definition Problem. Family Business Review 15, 45-58.

Ayres, Lioness. 2008. Narrative Interviews. In The Sage Encyclopedia of Qualitative Research Methods, Hrsg. Lisa M. Given, 545. Los Angeles, Calif: Sage Publications.

Bäcker, Eva M. 2008. Identitätsverständnisse von Familienunternehmern. Eine empirische Studie. Univ, Diss. Hagen, 2007. 1. Aufl. Göttingen: Sierke.

Barach, Jeffrey A, und Ganitsky, Joseph B. 1995. Successful Succession in Family Business. Family Business Review 8, 131-155.

Barca, Fabrizio und Becht, Marco. Hrsg. 2001. The Control of Corporate Europe. New York: Oxford University Press.

Baumgartner, Bernhard. 2009. Familienunternehmen und Zukunftsgestaltung. Schlüsselfaktoren zur erfolgreichen Unternehmensnachfolge. 1. Aufl. Wiesbaden: Gabler. 
Baus, Kirsten. 2007. Die Familienstrategie. Wie Familien ihr Unternehmen über Generationen sichern. 2. Aufl. Wiesbaden: Gabler Verlag; Gabler.

Beck, Ulrich. 1983. Jenseits von Stand und Klasse? In Soziale Ungleichheiten, Hrsg. Reinhard Kreckel, 35-74. Göttingen: Schwartz.

Beck, Ulrich. 1986. Risikogesellschaft. Auf dem Weg in eine andere Moderne. 1. Aufl, Erstausg. Frankfurt am Main: Suhrkamp.

Beck, Ulrich, und Beck-Gernsheim, Elisabeth. 1990. Das ganz normale Chaos der Liebe. Erstausg, 1. Frankfurt am Main: Suhrkamp.

Becker, Gary S, und Pies, Ingo. 1996. Familie, Gesellschaft und Politik- die ökonomische Perspektive. Tübingen: Mohr.

Beckert, Jens. 2008. Inherited Wealth. New Jersey: Princton university press.

Beck-Gernsheim, Elisabeth. 1983. Vom "Dasein für andere" zum Anspruch auf ein Stück "eigenes Leben". Individualisierungsprozesse im weiblichen Lebenszusammenhang. Soziale Welt, 307-340.

Beck-Gernsheim, Elisabeth. 1986. Von der Liebe zur Beziehung? In Die ModerneKontinuitäten und Zäsuren, Hrsg. Johannes Berger, 209-233. Göttingen: Schwartz.

Behringer, Luise. 1998. Lebensführung als Identitätsarbeit. Der Mensch im Chaos des modernen Alltags. Frankfurt am Main/New York: Campus.

Bengston, Vern L. 2001. Beyond the Nuclear Family. The increasing importance of multigenerational bonds. Journal of Marriage and Family 63, 1-16.

Bengston, Vern L; Olander, E.B und Haddad, A. A. 1976. The "Generation-Gap" and Aging Family Members: Toward a Conceptual Model. In Time, Roles, and Self in Old Age, Hrsg. J. F. Gubrium. New York: Human Sciences Press.

Berger, Peter L und Luckmann, Thomas. 1998. Die gesellschaftliche Konstruktion der Wirklichkeit. 43. bis 44.Tausend. Frankfurt/Main: Fischer.

Bernhard, Fabian. 2011. Psychological Ownership in Family Business Research. Three Essays on Antecendts and Consequences. Lohmar: EUL Verlag.

Bernhard, Fabian, und Jaskiewicz, Peter. 2011. Ownership Perceptions in Family Businesses. Psychological difficulties of the retiring manager. In Doing Succession in Europe. Generational transfers in family businesses in comparative perspective, Hrsg. Isabell Stamm, Peter Breitschmid und Martin Kohli, 215-242. Zürich: Schulthess Verlag.

Bertaux, Daniel und Bertaux-Wiame, Isabelle. 1981. Life Stories in the Bakers' Trade. In Biography and Society. The life history approach in the social sciences, Hrsg. Daniel Bertaux, 169-189. London: Sage Publications.

Bertram, Hans. 2000. Die verborgenen familiären Beziehungen in Deutschland. Die multilokale Mehrgenerationenfamilie. In Generationen in Familie und Gesellschaft, Hrsg. Martin Kohli und Marc Szydlik, 97-121. Opladen: Leske + Budrich.

Bird, Barbara; Welsch, Harold; Astrachan, Joseph H. und Pistrui, David. 2002. Family Business Research: The Evolution of an Academic Field. Family Business Review $15,337-350$. 
Birley, Sue. 1986. Succession in the Family Firm: The Inheritor's View. Journal of Small Business Management 24, 36-43.

Birley, Sue. 2001. Owner-Manager Attitudes to Family and Business Issues. A 16 Countries Study. Entrepreneurship: Theory \& Practice 26, 63-76.

Birley, Sue; NG, Dennis und Godfrey, Andrew. 1999. The Family and the Business. Long Range Planning 32, 598-608.

Björnberg, Äsa und Nicholson, Nigel. 2007. The Family Climate ScalesDevelopment of a New Measure for Use in Family Business Research. Family Business Review 20, 229-246.

Blanchflower, David und Oswald, Andrew. 1998. What Makes an Entrepreneur? Journal of Laber Economics 16, 26- 60.

Blossfeld, Hans-Peter. 1990. Berufsverläufe und Arbeitsmarktprozesse. In Lebensverläufe und sozialer Wandel, Hrsg. Karl U. Mayer, 118-145. Wiesbaden: Westdeutscher Verlag.

Blossfeld, Hans-Peter und Huinink, Johannes. 2001. Lebensverlaufsforschung als sozialwissenschaftliche Forschungsperspektive. Themen, Konzepte, Methoden und Probleme. BIOS 14, 5-31.

Boch, Rudolf. 1999. Unternehmensnachfolge in Deutschland ein historischer Rückblick. Zeitschrift für Unternehmensgeschichte 44, 164-171.

Bögenhold, Dieter und Fachinger, Uwe. 2010. Mikro-Selbständigkeit und Restrukturierung des Arbeitsmarktes. Theoretische und empirische Aspekte zur Entwicklung des Unternehmertums. In Prekäres Unternehmertum. Unsicherheiten von selbstständiger Erwerbstätigkeit und Unternehmensgründung, 1. Aufl, Hrsg. Andrea D. Bührmann und Hans J. Pongratz, 61-84. Wiesbaden: VS Verl. für Sozialwissenschaften.

Bohler, Karl F. und Hildenbrand, Bruno. 1997. Landwirtschaftliche Familienbetriebe in der Krise, Bd. 3. Münster: Lit Verlag AG.

Bohnsack, Ralf. 2007. Gruppendiskussion. In Qualitative Sozialforschung. Eine Einführung. Rororo Rowohlts Enzyklopädie, Bd. 55654, 5. Aufl, Hrsg. Uwe Flick, 369-383. Reinbek bei Hamburg: Rowohlt-Taschenbuch-Verl.

Bohnsack, Ralf. 2010. Rekonstruktive Sozialforschung. Einführung in qualitative Methoden, Bd. 8242. 8, durchges. Aufl. Opladen: Budrich.

Boltanski, Luc und Chiapello, Ėve. 2003. Der neue Geist des Kapitalismus. Konstanz: UVK-Verl.-Ges.

Born, Claudia und Krüger, Helga. 2001. Das Lebenslaufregime der Verflechtung. Orte, Ebenen und Thematisierungen. In Individualisierung und Verflechtung. Geschlecht und Generation im deutschen Lebenslaufregime, Hrsg. Claudia Born und Helga Krüger, 11-26. Weinheim/München.

Bourdieu, Pierre. 2006. Die feinen Unterschiede. Kritik der gesellschaftlichen Urteilskraft. 1. Aufl. (17. Nachdruck). Frankfurt am Main: suhrkamp taschenbuch wissenschaft.

Breuer, Franz. 2000. Vorgänger und Nachfolger. Weitergabe von/in Betrieben und Organisationen als sozialwissenschaftliches Phänomen. Gruppendynamik und Organisationsberatung, 451-483. 
Breuer, Franz. 2009. Vorgänger und Nachfolger. Weitergabe in institutionellen und persönlichen Bezügen. Göttingen: Vandenhoeck \& Ruprecht.

Budde, Gunilla-Friederike. 1994. Auf dem Weg ins Bürgerleben. Kindheit und Erziehung in deutschen und englischen Bürgerfamilien 1840-1914. Göttingen: Vandenhoeck \& Ruprecht.

Bührmann, Andrea D. und Pongratz, Hans J. 2010. Prekäres Unternehmertum. Einführung in ein vernachlässigtes Forschungsfeld. In Prekäres Unternehmertum. Unsicherheiten von selbstständiger Erwerbstätigkeit und Unternehmensgründung, 1. Aufl, Hrsg. Andrea D. Bührmann und Hans J. Pongratz, 7-24. Wiesbaden: VS Verl. für Sozialwissenschaften.

Burkart, Günter. 2008. Familiensoziologie. Konstanz: UVK-Verlagsgesellschaft.

Burkart, Günter und Kohli, Martin. Hrsg. 1992. Liebe, Ehe, Elternschaft. Die Zukunft der Familie. München: Piper.

Büttner, Philip. 2007. Die Bindungskraft des Familienunternehmens. Eine soziologische Untersuchung seiner Bestandsvoraussetzungen und intergenerationalen Kontinuität. Saarbrücken: VDM Verlag Dr. Müller.

Carr, David. 2000. Education, Profession and Culture: Some Conceptual Questions. British Journal of Educational Studies 48, 248-268.

Casillas, Jose und Acedo, Francisco. 2007. Evolution of the Intellectual Structure of Family Business Literature: A Bibliometric Study of FBR. Family Business Review $20,141-162$.

Chittoor, Raveendra und Das, Ranjan. 2007. Professionalization of Management and Succession Performance: A Vital Linkage. Family Business Review 20, 65-79.

Chrisman, James J.; Chua, Jess H. und Steier, Lloyd. 2005. Sources and Consequences of Distinctive Familiness: An Introduction. Entrepreneurship: Theory \& Practice 29, 237-247.

Chua, Jess H; Chrisman, James J. und Sharma, Pramodita. 1999. Defining the Family Business by Behavior. Entrepreneurship: Theory \& Practice 23, 19-39.

Churchill, Neil C, und Hatten, Kenneth J. 1987. Non-Market-Based Transfers of Wealth and Power: A Research Framework for Family Businesses. American Journal of Small Business 11, 51-64.

Cliff, Jennifer E.; und Jennings, P. D. 2005. Commentary on the Multidimensional Degree of Family Influence Construct and the F-PEC Measurement Instrument. Entrepreneurship Theory and Practice 29, 341-347.

Colli, Andrea. 2003. The History of Family Business, 1850-2000. Cambridge UK New York: Cambridge University Press.

Colli, Andrea und Rose, Mary B. 2003. Family Firms in Comparative Perspective. In Business History Around the World, Hrsg. Franco Amatori, 339-352. Cambridge.

Craig, J. und Moores, K. 2005. Balanced Scorecards to Drive the Strategic Planning of Family Firms. Family Business Review 18, 105-122.

Danes, Sharon M.; Rueter, Martha A.; Kwon, Hee-Kyung und Doherty, William. 2002. Family FIRO model. An application to family business. Family Business Review 15, 31-43. 
Danes, Sharon M.; Haberman, Heather R. und McTavis, Donald. 2005. Gendered Discourse about Family Business. Family Relations 54, 116-130.

Danes, Sharon M. und Lee, Yoon G. 2004. Tensions Generated by Business Issues in Farm Business-Owning Couples. Family Relations 53, 357-366.

Das Erste. 2010. Tatort im Visier der Zuschauer. http://www.daserste.de/tatort/beitrag_dyn uid,5tcmwk8npmvi6hxv $\sim \mathrm{cm}$.asp

(Stand: 6. Dezember 2010).

Dausien, Bettina; Hanses, Andreas; Inowlocki, Lena und Riemann, Gerhard. 2008. The Analysis, Self-Reflection and Shaping of Professional Work. Forum Qualitative Sozialforschung/Forum Qualitative Research 9.

Davis, J. A. und Tagiuri, R. 1989. The Influence of Life Stage on Father-Son Work Relationships in Family Companies. Family Business Review, 47-74.

Degele, Nina und Dries, Christian. 2005. Modernisierungstheorie. Eine Einführung. Paderborn ;, München: Fink.

DeMassis, Alfredo; Chua, J. H. und Chrisman, J. J. 2008. Factors Preventing IntraFamily Succession. Family Business Review 11, 17.

Denzin, Norman K. 2000. The Practices and Politics of Interpretation. In Handbook of Qualitative Research, 2. Auflage, Hrsg. Norman K. Denzin und Yvonna S. Lincoln, 897-922. London: Sage Publications.

Doherty, W.J, N. Colangelo und Hovander, D. 1991. Priority Setting in Family Change and Clinical Pratice. The family FIRO Model. Family Process 30, $227-$ 240.

Doherty, W.J.und Colangelo, N. 1984. The Family FIRO Model. A modest proposal for organizing family treatment. Journal of Marital and Family Therapy 10, 1929.

Dornseiff, Jann-Michael und Sackmann, Reinhold. 2003. Familien-, Erwerbs- und Fertilitätsdynamiken in Ost- und Westdeutschland. In Partnerschaft und Familiengründung. Ergebnisse der dritten Welle des Familien-Survey, Hrsg. Walter Bien und Jan H. Marbach, 309-348. Opladen: Leske + Budrich.

Douglas, Evan J. und Shepherd, Dean A. 2002. Self-Employment as a Career Choice. Attitudes, Entrepreneurial Intentions, and Utility Maximization. Entrepreneurship: Theory \& Practice 26, 81-90.

Down, Simon. 2006. Narratives of Enterprise. Crafting entrepreneurial self-identity in a small firm. Cheltenham, UK: Edward Elgar.

Dumas, Colette; Dupuis, Jean P.; Richer, Francine und St.-Cyr, Louise. 1995. Factors That Influence the Next Generation's Decision to Take Over the Family Farm. Family Business Review 8, 99-120.

Dunemann, Mark und Barrett, Rowena. 2004. Family Business and Succession Planning. Review of the literature: Monash University, Faculty of Business and Economics.

Dunn, Barbara. 1999. The Family Factor: The Impact of Family Relationship Dynamics on Business-Owning Families During Transitions. Family Business Review 12,4155 . 
Durkheim, Emile. 2011 c1921. The Conjugal Family. http://www.sociosite.net/topics/texts/durkheim.pdf (Stand: 5. September 2011).

Dyck, Bruno; Mauws, Michael; Starke, Frederick A. und Mischke, Gary A. 2002. Passing the Baton: The Importance of Sequence, Timing, Technique and Communication in Executive Succession. Journal of Business Venturing 17, 143-162.

Dyer, Gibb. 1994. Toward a Theory of Entrepreneurial Careers. Entrepreneurship Theory and Practice 19, 7-21.

Dyer, Gibb. 2003. The Family: The Missing Variable in Organizational Research. Entrepreneurship Theory and Practice 27, 401-416.

Dyer, W. G. JR und Sànchez, M. 1998. Current State of Family Business Theory and Practice as Reflected in Family Business Review 1988-1997. Family Business Review 11, 287-295.

Elder, Glen H. 1974. Children of the Great Depression. Social Change in life experience. Chicago: Chicago University Press.

Elder, Glen H. 1975. Age Differentiation and the Life Course. Annual Review of Sociology, 165-190.

Elder, Glen H, Hrsg. 1985a. Life Course Dynamics. Trajectories and Transitions, 1968-1980. Ithaca: Cornell University Press.

Elder, Glen H. 1985b. Perspectives on the Life Course. In Life Course Dynamics. Trajectories and Transitions, 1968-1980, Hrsg. Glen H. Elder, 23-49. Ithaca: Cornell University Press.

Elder, Glen H. 1995. The Life Course Paradigm: Social Change and Individual Development. In Examining Lives in Context. Perspectives on the Ecology of Human Development. APA Science Volumes, Hrsg. Phyllis Moen, Glen H. Elder und Kurt Lüscher, 101-140. Washington: American Psychological Association.

Elder, Glen H. 1998. The Life Course as Developmental Theory. Child Development $69,1-12$.

Elias, Norbert. 1998. Figuration. In Grundbegriffe der Soziologie, 5. verb. \& erw. A, Hrsg. Bernhard Schäfers, 87-90. Leverkusen: Leske + Budrich.

Elias, Norbert. 2006. Etablierte und Außenseiter, Bd. 4. Frankfurt am Main: Suhrkamp.

Engelmann, Sabine; Leßmann, Christian und Schmalholz, Heinz. 2005. Unternehmensnachfolge im sächsischen Mittelstand. Dresden: ifo, Institut für Wirtschaftsforschung Dresden.

Erdmann, Christina. 1999. Unternehmer und Nachfolger-Die Entstehung von Nachfolgebereitschaft. Wiesbaden: Der Deutsche Universitäts-Verlag.

Erhardt, Martin. 2001. Nachfolge im Familienunternehmen-eine steuerliche Analyse. Sternenfels: Verl. Wissenschaft und Praxis.

Esser, Hartmut. 2007. Soll das denn alles (gewesen) sein? Anmerkungen zur Umsetzung der soziologischen Systemtheorie in empirische Forschung. Soziale Welt $58,351-358$.

Evetts, Julia. 2003. The Sociological Analysis of Professionalism. Occupational change in the modern world. International Sociology 18, 395-415. 
Faccio, Mara und Lang, Larry H. P. 2002. The Ultimate Ownership of Western European Corporations. Journal of Financial Economics 65, 365-395.

Fallgatter, Michael. 2004. "Entrepreneurship". Konturen einer jungen Disziplin. Schmalenbachs Zeitschrift für betriebswirtschaftliche Forschung (ZfbF) 56, $23-$ 44.

Falter, Jean-Marc. 2007. Entrepreneurship and Intergenerational Links in Switzerland. Labour 21, 121-134.

Faltin, Günter. 2008. Social Entrepreneurship. Definitionen, Inhalte, Perspektiven. In Social Entrepreneurship. Unternehmerische Ideen für eine bessere Gesellschaft, Hrsg. Braun und French, 25-46. Rostock.

Finish Ministry of Industry and Trade. 2006. Family Entrepreneurship-Family Enterprises as the Engines of Continuity, Renewal and Growth-Intensiveness 7. Helsinki: Finish Ministry of Industry and Trade.

Fischer, Erica und Ladwig-Winters, Simone. 2008. Die Wertheims. Geschichte einer Familie, Bd. 62292. 2. Aufl. Reinbek bei Hamburg: Rowohlt-Taschenbuch-Verl.

Fischer, Wolfram und Kohli, Martin. 1987. Biographieforschung. In Methoden der Biographie- und Lebenslaufforschung, Hrsg. Wolfgang Voges, 25-49. Opladen: Leske + Budrich.

Fischer-Rosenthal, Wolfram und Rosenthal, Gabriele. 1997. Warum Biographieanalyse und wie man sie macht. Die Deutsche Schule, 405-427.

Fleming, Q. J. 2000. Keep the Family Baggage Out of the Family Business. Avoiding the seven deadly sins that destroy family buisness. New York: Fireside.

Flick, Uwe. 2005. Qualitative Research in Sociology in Germany and the US-State of the Art, Differences and Developments. Forum Qualitative Sozialforschung / Forum Qualitative Research 6, Art. 23.

Flick, Uwe. 2007. Triangulation in der qualitativen Forschung. In Qualitative Sozialforschung. Eine Einführung. Rororo Rowohlts Enzyklopädie, Bd. 55654, 5. Aufl, Hrsg. Uwe Flick, 309-318. Reinbek bei Hamburg: Rowohlt-Taschenbuch-Verl.

Flick, Uwe. 2009. An Introduction to Qualitative Research. 4th ed, rev, expand. and updated. Los Angeles: SAGE.

Flören, Roberto H. 2002. Crown princes in the clay. An empirical study on the tackling of succession challenges in Dutch family farms. Assen: Van Gorcum.

Freund, Werner. 2004. Unternehmensnachfolgen in Deutschland. Neubearbeitung der Daten des IfM. In Jahrbuch zur Mittelstandsforschung, Hrsg. ifm-Institut für Mittelstandsforschung Bonn, 59-88.

Froschauer, Ulrike und Lueger, Manfred. 2003. Das qualitative Interview. Zur Praxis interpretativer Analyse sozialer Systeme, Bd. 2418. Wien: WUV.

Frost, Nollaig; Nolas, Sevast M.; Brooks-Gordon, Belinda; Esin, Cigdem; Holt, Amanda; Mehdizadeh, Leila und Shinebourne, Pnina. 2010. Pluralism in Qualitative Research. The impact of different reserachers and qualitative approaches on the analysis of qualitative data. Qualitative Research 10, 441-460.

Gallo, Miguel A. 1998. Ethics in personal behavior in family business. Family Business Review 11, 325-336. 
Gantzel, Klaus J. 1962. Wesen und Begriff der mittelständischen Unternehmung. Köln, Opladen: Westdeutscher Verlag.

Garcia-Alvarez, Ercilia; Lopez-Sintas, Jordi und Gonzalvo, Pilar S. 2002. Socialization Patterns of Successors in First- to Second-Generation Family Businesses. Family Business Review 15, 189-203.

Garcia-Alvarez, Ercilia und Lopez Silanes, Florencia de. 2006. Founder-successor's Transition: A Model of Coherent Value Transmission Paths. In Handbook of Research on Family Business, Hrsg. Panikkos Z. Poutziouris, Kosmas X. Smyrnios und Sabine Klein, 237-252. Cheltenham UK Northampton MA.

Gartner, William B. 1989. "Who is an Entrepreneur?" Is the Wrong Question. Entrepreneurship Theory and Practice, 47-68.

Geertz, Clifford. 1973. Thick Description: Toward an Interpretive Theory of Culture. In The Interpretation of Cultures. Selected Essays, Hrsg. Clifford Geertz, 3-30. New York: Basic Books.

Geissler, Rainer und Meyer, Thomas. 2008. Die Sozialstruktur Deutschlands. Zur gesellschaftlichen Entwicklung mit einer Bilanz zur Vereinigung. 4. Auflage. Wiesbaden: VS Verlag für Sozialwissenschaften.

Gestrich, Andreas. 2010. Geschichte der Familie im 19. und 20. Jahrhundert. 2. Aufl. München: Oldenbourg.

Giddens, Anthony. 1997. Die Konstitution der Gesellschaft. Grundzüge einer Theorie der Strukturierung. 3. Aufl. Frankfurt/Main [u.a.]: Campus-Verl.

Glaser, Barney G. und Strauss, Anselm L. 2009. The Discovery of Grounded Theory. Strategies for qualitative research. 4. paperback printing. New Brunswick: Aldine.

Gottschall, Karin und Voß, Gerd G. 2003. Entgrenzung von Arbeit und Leben. Zur Einleitung. In Entgrenzung von Arbeit und Leben. Zum Wandel der Beziehung von Erwerbstätigkeit und Privatsphäre im Alltag, Hrsg. Karin Gottschall und Gerd G. Voß, 11-33. München und Mering: Rainer Hampp Verlag.

Grabka, Markus; Westerheide, Peter ; Hauser, Richard und Becker, Irene. 2007. Integrierte Analyse der Einkommens- und Vermögensverteilung. Abschlussbericht zur Studie. Lebenslagen in Deutschland - Armuts- und Reichtumsberichterstattung der Bundesregierung.

Gräf, Dennis und Krah, Hans. 2010. Tatort. Ein populäres Medium als kultureller Speicher. Univ, Diss.--Passau, 2009, Bd. 1. Marburg: Schüren.

Granovetter, Mark. 1985. Economic Action and Social Structure: The Problem of Embeddedness. The American Journal of Sociology 91, 481-510.

Habbershon, Timothy G, M. Williams und MacMillan, Ian C. 2003. A Unified Systems Perspective of Family Firm Performance. Journal of Business Venturing, 451-465.

Habbershon, Timothy G. und Williams, M. 1999. A Resource-Based Framework for Assessing the Strategic Advantage of Family Firms. Family Business Review, 125. 
Habermas, Jürgen. 1982a. Theorie des kommunikativen Handelns. Handlungsrationalität und gesellschaftliche Rationalisierung, 1. Band. 2. Auflage. Frankfurt am Main: Suhrkamp.

Habermas, Jürgen. 1982b. Theorie des kommunikativen Handelns. Zur Kritik der funktionalistischen Vernunft, 2. Band. 2. Auflage. Frankfurt am Main: Suhrkamp.

Habermas, Jürgen. 1998. Die nationale Konstellation und die Zukunft der Demokratie. In Die postnationale Konstellation. Politische Essays, Erstausg, 1, Hrsg. Jürgen Habermas, 91-169. Frankfurt am Main: Suhrkamp.

Hahn, Alois. 1988. Biographie und Lebenslauf. In Vom Ende des Individuums zur Individualität ohne Ende, Hrsg. Hanns-Georg Brose und Bruno Hildenbrand, 91105. Opladen: Leske + Budrich.

Hall, Peter und Soskice, David. Hrsg. 2001. Varieties of Capitalism. the insitutional foundations of comparative advantage. New York.

Hamilton, Eleanor. 2006. Whose Story is it Anyway? Narrative accounts of the role of women in founding and establishing family businesses. International Small Business Journal 24, 253-271.

Handler, Wendy C. 1990. Succession in Family Firms. A mutual role adjustment between entrepreneur and next-generation family members. Entrepreneurship: Theory \& Practice, 37-51.

Handler, Wendy C. 1992. Succession Experience of the Next Generation. Family Business Review 5, 283-307.

Hartmann, Michael. 1996. Topmanager. Die Rekrutierung einer Elite. Frankfurt/Main: Campus-Verl.

Hartmann, Michael. 2008. Elitesoziologie. Eine Einführung, Bd. 2. 2, korrigierte Aufl. Frankfurt/Main: Campus-Verl.

Haubl, Rolf und Daser, Bettina. 2006. Familiendynamik in Familienunternehmen. Warum sollten Töchter nicht erste Wahl sein? Frankfurt am Main.

Hauser, H.-E; Kay, R. und Boerger, S. 2010. Unternehmensnachfolgen in Deutschland 2010 bis 2014. Schätzung mit weiterentwickeltem Verfahren. IfM-Materilien 198. Bonn.

Heck, Ramona K. Z.; Danes, Sharon M.; Fitzgerald, Margaret A.; Haynes, George W.; Jasper, Cynthia R.; Schrank, Holly L.; Stafford, Kathryn und Winter, Mary. 2006. The Family's Dynamic Role Within Family Business Entrepreneurship. In Handbook of Research on Family Business, Hrsg. Panikkos Z. Poutziouris, Kosmas X. Smyrnios und Sabine Klein, 80-105. Cheltenham UK Northampton MA.

Heck, Ramona K. Z.; Jasper, Cynthia R.; Stafford, Kathryn; Winter, Mary und Owen, Alma J..2000. Using a Household Sampling Frame to Study Family Businesses: the 1997 National Family Business Survey. Advances in entrepreneurship, firm emergence, and growth: Databases for the study of of entrepreneurship 4, 229287.

Heinz, Walter; Krüger, Helga; Rettke, Ursula; Wachtveitl, Erich und Witzel, Andreas. 1987. Hauptsache eine Lehrstelle. Weinheim: Deutscher Studien Verlag. 
Henning, Friedrich-Wilhelm. 1995. Die Industrialisierung in Deutschland. 9, Aufl. Paderborn ;, München ; Wien ; Zürich: F. Schöningh.

Hettlage, Robert. 1992. Familienreport. Eine Lebensform im Umbruch. Originalausg. München: C.H. Beck.

Hildebrandt, Eckart; Kirschbaum, Almut; Pfahl, Svenja und Wotschack, Philip. 2009. Zeit auf der hohen Kante. Langzeitkonten in der betrieblichen Praxis und Lebensgestaltung von Beschäftigten. Berlin: Ed. Sigma.

Hildenbrand, Bruno. 1991. Fallrekonstruktive Forschung. In Handbuch qualitative Sozialforschung. Grundlagen, Konzepte, Methoden und Anwendung, Hrsg. Uwe Flick, Ernst von Kardorff, Heiner Keupp, Lutz von Rosenstiel und Stephan Wolff, 256-260. München: Psychologie Verlagsunion.

Hildenbrand, Bruno. 2002. Familienbetriebe als "Familien eigener Art...". In Die Familie des Familienunternehmens. Ein System zwischen Gefühl und Geschäft, Hrsg. Fritz B. Simon, 115-144. Heidelberg: Carl-Auer-Systeme Verlag.

Hildenbrand, Bruno. 2005. Fallrekonstruktive Familienforschung. Anleitungen für die Praxis, Bd. 6. 2. Aufl. Wiesbaden: VS Verl. für Sozialwiss.

Hildenbrand, Bruno. 2007. Einführung in die Genogrammarbeit. 2. Aufl. Heidelberg: Carl-Auer-Systeme-Verl.

Hill, Paul B. und Kopp, Johannes. 2001. Strukturelle Zwänge, partnerschaftliche Anpassung oder Liebe - einige Überlegungen zur Entstehung enger affektiver Beziehungen. In Partnerwahl und Heiratsmuster. Sozialstrukturelle Voraussetzungen der Liebe, Hrsg. Thomas Klein, 11-34. Opladen: Leske + Budrich.

Hill, Paul B. und Kopp, Johannes. 2006. Familiensoziologie. Grundlagen und theoretische Perspektiven. 4, überarbeitete Auflage. Wiesbaden: VS Verlag für Sozialwissenschaften.

Hitzler, Ronald. 2002. Sinnrekonstruktion. Zum Stand der Diskussion (in) der deutschsprachigen interpretativen Soziologie. Forum Qualitative Sozialforschung / Forum Qualitative Research 3, Art. 7.

Hoffmann-Nowotny, Hans-Joachim. 1995. Die Zukunft der Familie - Die Familie der Zukunft. In Familie der Zukunft. Lebensbedingungen und Lebensformen, Hrsg. Uta Gerhardt, Stefan Hradil, Doris Lucke und Bernhard Nauck, 325-348. Opladen: Leske \& Budrich.

Hollstein, Betina und Gina, Bria. 1998. Reziprozität in Eltern-Kind-Beziehungen? Theoretische Überlegungen und empirische Evidenz. Berliner Journal für Soziologie, 7-22.

Hollstein, Betina und Ullrich, Carsten. 2003. Einheit trotz Vielfalt? Zum konstitutiven Kern qualitativer Forschung. Soziologie 32, 29-43.

Holt, Daniel T.; Rutherford, Matthew W. und Kuratko, Donald F. 2007. The F-PEC Scale of Family Influence: A Refinement. Academy of Management Proceedings, 1-6.

Honig, Michael-Sebastian. 1999. Entwurf einer Theorie der Kindheit. Die generationale Ordnung. Frankfurt am Main: Suhrkamp.

Hopf, Christel. 1978. Die Pseudo-Exploration. Überlegungen zur Technik qualitativer Interviews in der Sozialforschung. Zeitschrift für Soziologie 7, 97-115. 
Hopf, Christel. 2007. Qualitative Interviews - ein Überblick. In Qualitative Sozialforschung. Eine Einführung. Rororo Rowohlts Enzyklopädie, Bd. 55654, 5. Aufl, Hrsg. Uwe Flick, 349-359. Reinbek bei Hamburg: Rowohlt-Taschenbuch-Verl.

Huinink, Johannes und Wagner, Michael. 1998. Individualisierung und die Pluralisierung von Lebensformen. In Die Individualisierungs-These, Hrsg. Jürgen Friedrichs, 85-106. Opladen: Leske + Budrich.

Ibrahim, A.B.; Soufani, K. und Lam, J. 2001. A Study of Succession in a Family Firm. Family Business Review 14, 245-258.

ifm - Institut für Mittelstandsforschung Bonn. 2007. Die volkswirtschaftliche Bedeutung der Familienunternehmen. Bonn: Institut für Mittelstandsforschung im Auftrag der Stiftung Familienunternehmen.

Jaffe, Dennis T. und Lane, Sam H. 2004. Sustaining a Family Dynasty. Key issues facing complex multigenerational business- and investment-owning families. Family Business Review 17, 81-98.

Jäkel-Wurzer, Daniela. 2010. Töchter im Engpass. Eine fallrekonstruktive Studie zur weiblichen Nachfolge in Familienunternehmen. Univ, Diss. u.d.T.: Töchter im Engpass der Nachfolge-Jena, 2010, eine fallrekonstruktive Studie zur weiblichen Nachfolge in Familienunternehmen. 1. Aufl. Heidelberg: Auer.

James, Harold. 2005. Familienunternehmen in Europa. Haniel, Wendel und Falk. 1. Aufl. München: Verlag C.H. Beck; Beck.

Jaskiewicz, Peter und Klein, Sabine. 2007. The impact of goal alignment on board composition and board size in family businesses. Journal of Business Research 60, 1080-1089.

Jaskiewicz, Peter; Gonzalez, V.; Menendez, S. und Schiereck, D. 2005. Long-run IPO Performance Analysis of German and Spanish Family-owned Businesses. Family Business Research 18, 179-202.

Jungbluth, Rüdiger. 2006. Die Oetkers. Geschäfte und Geheimnisse der bekanntesten Wirtschaftsdynastie Deutschlands, Bd. 61594. Vollst. Taschenbuchausg, 1. Aufl. Bergisch Gladbach: Bastei Lübbe.

Jurczyk, Karin und Lange, Andreas. Hrsg. 2009. Vaterwerden und Vatersein heute. Neue Wege, neue Chancen! Gütersloh: Verlag Bertelsmann Stiftung.

Jurczyk, Karin und Rerrich, Maria S. Hrsg. 1993. Die Arbeit des Alltags. Beiträge zu einer Soziologie der alltäglichen Lebensführung. Freiburg: Lambertus.

Kaelble, Hartmut und Spode, Hasso. 1990. Sozialstruktur und Lebensweise deutscher Unternehmer 1907-1927. Scripta Mercaturae 24, 132-178.

Kaufmann, Jean-Claude. 2005. Schmutzige Wäsche. Ein ungewöhnlicher Blick auf gewöhnliche Paarbeziehungen. [2. Aufl.]. Konstanz: UVK-Verl.-Ges.

Keese, Detlef. 2002. Geschlechtstypische Nachfolgeprobleme in kleinen und mittleren Unternehmen. Wirtschaftspsychologie, 34-38.

Kelle, Udo. 2008. Die Integration qualitativer und quantitativer Methoden in der empirischen Sozialforschung. Theoretische Grundlagen und methodologische Konzepte. 2. Aufl. Wiesbaden: VS Verl. für Sozialwiss.

Kelle, Udo und Erzberger, Christian. 2008. Qualitative und quantitative Methoden: kein Gegensatz. In Qualitative Forschung. Ein Handbuch. Rororo Rowohlts En- 
zyklopädie, Bd. 55628, 6, durchges. und aktualisierte Aufl, Orig.-Ausg, Hrsg. Uwe Flick, Ernst von Kardorff und Ines Steinke, 299-309. Reinbek bei Hamburg: Rowohlt-Taschenbuch-Verl.

Kelle, Udo und Kluge, Susann. 1999. Vom Einzelfall zum Typus. Fallvergleich und Fallkontrastierung in der qualitativen Sozialforschung, Bd. 4. Opladen: Leske + Budrich.

Kellermanns, F. W.; Eddleston, K. A. ; Barnett, T. und Pearson, A. 2008. An Exploratory Study of Family Member Characteristics and Involvement. Effects on entrepreneurial behavior in the family firm. Family Business Review 21, 1-14.

Kets de Vries, Manfred. 2007. Family Business on the Couch. A psychological perspective.

Kieser, Ueli. 2011. Wie können Familienunternehmer ihren Ruhestand finanzieren? In Doing Succession in Europe. Generational transfers in family businesses in comparative perspective, Hrsg. Isabell Stamm, Peter Breitschmid und Martin Kohli, 325-346. Zürich: Schulthess Verlag.

Kim, Haejeong und DeVaney, Sharon A. 2003. The Expectation of Partial Retirement Among Family Business Owners. Family Business Review 16, 199-210.

King, Valerie und Elder, Glen H. 1995. American Children View Their Grandparents. Linked lives across three rural generations. Journal fo Marriage and Family 57, $165-178$.

Klein, Sabine. 2000. Familienunternehmen- Theoretische und empirische Grundlagen. 1. Auflage. Wiesbaden: Gabler Verlag.

Klein, Sabine. 2004. Familienunternehmen: Theoretische und empirische Grundlagen (2. überarb. Aufl.). Wiesbaden: Gabler.

Klein, Sabine; Astrachan, Joseph H. und Smyrnios, Kosmas X. 2005. The F-PEC Scale of Family Influence. Construction, validation, and further implication for theory. Entrepreneurship Theory and Practice 29, 321-339.

Klein, Thomas und Lengerer, Andrea. 2001. Gelegenheit macht Liebe - die Wege des Kennenlernens und ihr Einfluss auf die Muster der Partnerwahl. In Partnerwahl und Heiratsmuster. Sozialstrukturelle Voraussetzungen der Liebe, Hrsg. Thomas Klein, 265-286. Opladen: Leske + Budrich.

Klett, David. 2005. Zwischen Kompetenz und Herkunft - zwischen Gleichheit und Selektion. Paradoxe Anforderungen an Familienunternehmen und ihre Unternehmensfamilien. Univ, Diplomarbeit: Witten-Herdecke. 1. Aufl. Heidelberg: Verl. für System. Forschung im Carl-Auer-Verl.

Kocka, Jürgen. 1975. Unternehmer in der deutschen Industrialisierung. Göttingen: Vandenhoeck \& Ruprecht.

Kocka, Jürgen. 1979. Familie, Unternehmer und Kapitalismus. An Beispielen aus der früheren Deutschen Industrialisierung. Zeitschrift für Unternehmensgeschichte 24, 99-135.

Kocka, Jürgen. 1994. Entrepreneurship in Late-Comer Country: The German Case. In Business Elites. The International Library of Critical Writings in Business History, Hrsg. Youssef Cassis, 241-282. Aledershot. 
Kocka, Jürgen. 2002. Unternehmer in Deutschland seit 1945. Schriften der Stiftung Bibliothek des Ruhrgebiets 8.

Kohli, Martin. 1978. "Offenes" und "geschlossenes" Interview. Neue Argumente zu einer alten Kontroverse. Soziale Welt, 1-25.

Kohli, Martin. 1981. Wie es zur "biographischen Methode" kam und was daraus geworden ist. Ein Kapitel aus der Geschichte der Sozialforschung. Zeitschrift für Soziologie 10, 273-293.

Kohli, Martin. 1985. Die Institutionalisierung des Lebenslaufs: Historische Befunde und theoretische Argumente. Kölner Zeitschrift für Soziologie und Sozialpsychologie, 1-29.

Kohli, Martin. 1986. Gesellschaftszeit und Lebenszeit: Der Lebenslauf im Strukturwandel der Moderne. In Die Moderne - Kontinuitäten und Zäsuren, Hrsg. Johannes Berger, 183-208. Göttingen: Schwartz.

Kohli, Martin. 1991. Lebenslauftheoretische Ansätze in der Sozialforschung. In Neues Handbuch der Sozialforschung, Hrsg. Klaus Hurrelmann, 303-317. Weinheim und Basel: Beltz.

Kohli, Martin. 1992. Lebenslauf und Lebensalter als gesellschaftliche Konstruktionen: Elemente zu einem Vergleich. In Zwischen den Kulturen? Die Sozialwissenschaften vor dem Problem des Kulturvergleichs, Sonderband 8, Hrsg. J. Matthes, 283-303. Göttingen: Schwartz.

Kohli, Martin. 1993. Altersgrenzen als Manövriermasse? Das Verhältnis von Erwerbsleben und Ruhestand in einer alternden Gesellschaft. In Innovation und Beharrung in der Arbeitspolitik, Hrsg. B. Strümpel und M. Dierkes, 177-208. Stuttgart: Schäffer-Poeschel.

Kohli, Martin. 1994. Institutionalisierung und Individualisierung der Erwerbsbiographie. In Riskante Freiheiten, Hrsg. Ulrich Beck und Elisabeth Beck-Gernsheim, 219-244. Frankfurt/M.

Kohli, Martin. 1999. Private and Public Transfers Between Generations: Linking the Family and the State. European Societies, 81-104.

Kohli, Martin. 2000. Altersgrenzen als gesellschaftliches Regulativ individueller Lebenslaufgestaltung: ein Anachronismus? Zeitschrift für Gerontologie und Geriatrie $33, \mathrm{I} / 15-\mathrm{I} / 23$.

Kohli, Martin. 2004. Intergenerational Transfers and Inheritance: A Comperative View. In Intergenerational Relations Across Time and Place, Hrsg. Merill Silverstein, 266-289. New York.

Kohli, Martin. 2007. The Institutionalization of the Life Course: Looking Back to Look Ahead. Research in Human Development, 253-271.

Kohli, Martin und Künemund, Harald. 2001. Intergenerational Transfers and Inheritance: What Motives for Giving?

Kohli, Martin und Szydlik, Marc. Hrsg. 2000. Generationen in Familie und Gesellschaft. Opladen: Leske + Budrich.

Kohli, Martin; Künemund, Harald; Motel-Klingebiel, Andreas und Szydlik, Mark. 2005a. Generationenbeziehungen. In Die zweite Lebenshälfte. Gesellschaftliche Lage und Partizipation im Spiegel des Alters-Survey, 2. erweiterte Auflage, Hrsg. 
Martin Kohli und Harald Künemund, 176-211. Wiesbaden: VS Verl. für Sozialwissenschaften.

Kohli, Martin; Kühnemund, Harald und Lüdicke, Jörg. 2005b. Family Structure, Proximity and Contact. In Health, Aging and Retirement in Europe. First Results from SHARE, 164-170. Mannheim.

Konietzka, Dirk. 2010. Zeiten des Übergangs. Sozialer Wandel des Übergangs in das Erwachsenenalter. Wiesbaden: VS Verlag für Sozialwissenschaften.

Kormann, Hermut. 2011. Zusammenhalt der Unternehmerfamilie. Verträge, Vermögensmanagement, Kommunikation. Berlin, Heidelberg: Springer-Verlag Berlin Heidelberg.

Kotthoff, Hermann und Reindel, Josef. 1990. Die soziale Welt kleiner Betriebe. Göttingen: Verlag Otto Schwartz \& Cor.

Kuckartz, Udo. 2005. Einführung in die computergestützte Analyse qualitativer Daten. 1. Aufl. Wiesbaden: VS Verl. für Sozialwiss.

Kudera, Werner und Voß, Günter Gerd, Hrsg. 2000. Lebensführung und Gesellschaft. Beiträge zu Konzept und Empirie alltäglicher Lebensführung. Opladen: Leske + Budrich.

Künemund, Harald und Mücke, Beate. 1990. Betriebsübergaben im Berliner Handwerk. Arbeitsbericht Nr. 25. Berlin.

Künemund, Harald und Szydlik, Marc. Hrsg. 2009. Generationen. Multidisziplinäre Perspektiven. Wiesbaden: VS Verlag für Sozialwissenschaften.

Lambrecht, Johan. 2005. Multigenerational Transition in Family Businesses. A new explanatory model. Family Business Review 18, 267-282.

Lamnek, Siegfried. 2006. Qualitative Sozialforschung. Lehrbuch. 4, vollst. überarb. Aufl, [Nachdr.]. Weinheim: Beltz PVU.

Landes, David S. 2006. Dynasties. Fortunes and misfortunes of the world's great family businesses. New York, NY: Viking.

Lansberg, Ivan. 1999. Succeeding Generations. Realizing the dream of families in business. Boston: Harvard Business School Press.

LaPorta, Rafael; Lopez Silanes, Florencia de und Shleifer, Andrei. 1998. Corporate Ownership Around the World. Harvard Institute of Economic Research Paper No. 1840. Campbridge: Harvard Institute of Economic Research.

Lauterbach, Wolfgang. 2004. Die multilokale Mehrgenerationenfamilie. Zum Wandel der Familienstruktur in der zweiten Lebenshälfte. Würzburg: Ergon.

Lauterbach, Wolfgang und Shanahan, Michael J. 1998. Die Modernisierung des Agrarsektors: Berufliche Kontinuität und Wandel in Familienbetrieben. Berliner Journal für Soziologie 8, 53-72.

Le Breton-Miller, Isabelle; Miller, Danny und Steier, Lloyd P. 2004. Toward an Integrative Model of Effective FOB Succession. Entrepreneurship: Theory \& Practice 28, 305-328.

Lester, Richhard H. und Cannella, Albert A. JR. 2006. Interorganizational Familiness. How family firms use interlocking directorates to build community-level social capital. Entrepreneurship: Theory \& Practice 30, 755-775. 
Levinson, Harry und Wofford, Jerry C. 2000. Approaching Retirement as the Flexibility Phase. Academy of Management Executive 14, 84-95.

Light, Audrey und McGarry, Kathleen. 2004. Why Parents Play Favorites: Explanations for Unequal Bequests. The American Economic Review, 1669-1681.

Lindner, Erik. 2008. Die Reemtsmas. Geschichte einer deutschen Unternehmerfamilie, Bd. 5235. München: Piper.

Linkenbach, Antje. 1986. Opake Gestalten des Denkens - Jürgen Habermas und die Rationalität fremder Lebensformen. München: Wilhelm Fink Verlag.

Lubinski, Christina. 2009. "Was ich habe, bin ich": Psychologisches Eigentum und Gesellschafterkultur in dem Düsseldorfer Familienunternehmen Bagel, ca. 1960 bis 2005. In Familienunternehmen im Rheinland im 19. und 20. Jahrhundert. Netzwerke - Nachfolge - soziales Kapital. Schriften zur rheinisch-westfälischen Wirtschaftsgeschichte, Bd. 47, Hrsg. Susanne Hilger und Ulrich S. Soenius. Köln: Stiftung Rheinisch-Westfälisches Wirtschaftsarchiv.

Lubinski, Christina. 2010. Familienunternehmen in Westdeutschland. Corporate Governance und Gesellschafterkultur seit den 1960er Jahren. Univ, Diss.Göttingen, 2009, Bd. 21. München: Beck.

Lubinski, Christina. 2011. Succession in Multigenerational Family Firms. An explorative study into the period of anticipatory socialization. Electronic Journal of Family Business Studies 5.

Lüders, Christian. 2008. Herausforderungen qualitativer Forschung. In Qualitative Forschung. Ein Handbuch. Rororo Rowohlts Enzyklopädie, Bd. 55628, 6, durchges. und aktualisierte Aufl, Orig.-Ausg, Hrsg. Uwe Flick, Ernst von Kardorff und Ines Steinke, 632-643. Reinbek bei Hamburg: Rowohlt-TaschenbuchVerl.

Lüscher, Kurt. 2000. Die Ambivalenz von Generationenbeziehungen - eine allgemeine heuristische Hypothese. In Generationen in Familie und Gesellschaft, Hrsg. Martin Kohli und Marc Szydlik, 138-161. Opladen: Leske + Budrich.

Lüscher, Kurt. 2002. Intergenerational Ambivalence: Further Steps in Theory and Research. Journal of Marriage and the Family 64, 585-593.

Lüscher, Kurt. 2004. Conceptualizing and Undercovering Intergenerational Ambivalence. In Intergenerational Ambivalences. New perspectives on parent-child relations in later life, Hrsg. K. Pillemer und Kurt Lüscher, 23-62. Oxford: Elsevier.

Lüscher, Kurt und Pillemer, K. 1998. Intergenerational Ambivalence: A New Approach to the Study of Parent-Child Relations in Later Life. Journal of Marriage and the Family 60, 413-425.

Matthews, Charles und Human, Sherrie. 2004. Family Background. In Handbook of Entrepreneurial Dynamics. The process of business creation, Hrsg. William B. Gartner, 94-103. Thousand Oaks, Calif.: SAGE.

Mayer, Karl U. Hrsg. 1990. Lebensverläufe und sozialer Wandel. Wiesbaden: Westdeutscher Verlag.

Mayring, Philipp. 1989. Die qualitative Wende. Grundlagen, Techniken und Integrationsmöglichkeiten qualitativer Forschung in der Psychologie. In Bericht über 
den 36. Kongreß der Deutschen Gesellschaft für Psychologie in Berlin, Hrsg. Wolfgang Schönpflug, 306-313. Göttingen: Hofgrefe.

Mayring, Philipp. 2002. Einführung in die qualitative Sozialforschung. Eine Anleitung zu qualitativem Denken. 5, überarb. und neu ausgestattete Aufl. Weinheim: Beltz-Verl.

Meadows, Donella H. 1972. The Limits to growth. A report for the Club of Rome's project on the predicament of mankind. New York: Universe Books.

Merkens, Hans. 2007. Auswahlverfahren, Sampling, Fallkonstruktionen. In Qualitative Sozialforschung. Eine Einführung. Rororo Rowohlts Enzyklopädie, Bd. 55654, 5. Aufl, Hrsg. Uwe Flick, 286-298. Reinbek bei Hamburg: RowohltTaschenbuch-Verl.

Miller, Danny und Le Breton-Miller, Isabelle. 2005. Managing for the Long Run. Lessons in competitive advantage from great family business. Boston: Havard Business School Press.

Mitterauer, Michael. 1980. Der Mythos der vorindustriellen Großfamilie. In Familie und Gesellschaftsstruktur. Materialien zu den sozioökonomischen Bedingungen von Familienreformen. Suhrkamp Taschenbuch Wissenschaft, Bd. 244, 2. Aufl, 7.-10. Taus, Hrsg. Heidi Rosenbaum, 38-63. Frankfurt/M.: Suhrkamp.

Moen, Phyllis. 1998. A Life Course Approach to the Entrepreneurial Family. In The Entrepreneurial Family, Hrsg. Ramona Heck, 16-29. Massachussettes.

Möhle, Sylvia. 2001. Partnerwahl in historischer Perspektive. In Partnerwahl und Heiratsmuster. Sozialstrukturelle Voraussetzungen der Liebe, Hrsg. Thomas Klein, 57-76. Opladen: Leske + Budrich.

Müller, Volkwin. 2008. Nachfolgertypen und Rollenkonflikte im Nachfolgeprozess von Familienunternehmen. Eine empirische Untersuchung. 1. Aufl. Mering ;, München: Hampp.

Nauck, Bernhard. 2001. Der Wert von Kindern für ihre Eltern. 'Value of Children' als spezielle Handlungstheorie des generativen Verhaltens und von Generationenbeziehungen im interkulturellen Vergleich. Kölner Zeitschrift für Soziologie und Sozialpsychologie 53, 407-435.

Nauck, Bernhard. 2011. Wert der Kinder und Generationensolidarität. In Familie, Bindungen und Fürsorge. Familiärer Wandel in einer vielfältigen Moderne, Hrsg. Hans Bertram und Nancy Ehlert, 329-348. Opladen: Barbara Budrich Verlag.

Nauck, Bernhard und Trommsdorff, Gisela. 2010. Value of Children: A Concept for Better Understanding Cross-Cultural Variations in Fertility Behavior and Intergenerational Relationships. Journal of Cross-Cultural Psychology, 637-651.

Nave-Herz, Rosemarie. 2006. Ehe- und Familiensoziologie. Eine Einführung in Geschichte, theortische Ansätze und empirische Befunde. 2. Aufl. Weinheim: Juventa-Verl.

Nave-Herz, Rosemarie. 2007. Familie heute. Wandel der Familienstrukturen und Folgen für die Erziehung. 3, überarb. und erg. Darmstadt: Wiss. Buchges.

Neckel, Sighard. 2008. Flucht nach vorn. Die Erfolgskultur der Marktgesellschaft. 1. Aufl. Frankfurt am Main: Campus. 
Nordqvist, Mattias und Zellweger, Thomas M. Hrsg. 2010. Transgenerational Entrepreneurship. Exploring growth and performance in family firms across generations. Cheltenham, UK ;, Northampton, MA: Edward Elgar.

o.A. 2011. Frauen an der Macht. http://www.tagesspiegel.de/wirtschaft/karriere/frauen-an-dermacht/1952664.html (Stand: 7. Mai 2011).

Oevermann, Ulrich. 2000. Die Methode der Fallrekonstruktion in der Grundlagenforschung sowie der klinischen und pädagogischen Praxis. In Die Fallrekonstruktion. Sinnverstehen in der sozialwissenschaftlichen Forschung. SuhrkampTaschenbuch Wissenschaft, Bd. 1459, 1. Aufl, Hrsg. Klaus Kraimer, 58-159. Frankfurt am Main: Suhrkamp.

Olson, P.D.; Zuiker, V.S.; Danes, Sharon M.; Stafford, Kathryn; Heck, Ramona und Duncan, K.A. 2003. The Impact of the Family and the Business on Family Business Sustainability. Journal of Business Venturing 18, 639-666.

Ortmann, Günther und Sydow, Jörg. Hrsg. 2001. Strategie und Strukturation. Strategisches Management von Unternehmen, Netzwerken und Konzernen. 1. Aufl. Wiesbaden: Gabler.

Palazzo, Guido. 2002. Die Mitte der Demokratie. Über die Theorie deliberativer Demokratie bei Jürgen Habermas. Baden-Baden: Nomos-Verlag.

Parnes, Ohad; Vedder, Ulrike und Willer, Stefan. 2008. Das Konzept der Generation. Eine Wissenschafts- und Kulturgeschichte. 1. Auflage. Frankfurt am Main: suhrkamp taschenbuch wissenschaft.

Parr, Patricia E. 2000. The Family FIRO Model: Exploring Relationship Needs of "At-Risk" Families. The American Journal of Family Therapy 28, 255-264.

Parsons, Talcott. 1954. The Kinship System of the Contemporary United States. In Essays in Sociological Theory, rev. ed, Hrsg. Talcott Parsons, 177-196. New York: The Free pr.

Peukert, Rüdiger. 2007. Zur aktuellen Lage der Familie. In Handbuch Familie, 1. Aufl, Hrsg. Jutta Ecarius, 36-56. Wiesbaden: VS Verl. für Sozialwissenschaften.

Pfannenschwarz, Armin. 2006. Nachfolge und Nicht-Nachfolge in Familienunternehmen, 1: Ambivalenzen und Lösungsstrategien beim familieninternen Generationswechsel. Heidelberg: Carl-Auer Verlag.

Pieper, Torsten. 2007. Zusammenhalt in Unternehmerfamilien. EQUA-Schriftenreihe. Herrsching: EQUA-Stiftung.

Pieper, Torsten und Klein, Sabine. 2007. The Bulleye - A Systems Approach to Modeling Family Firms. Family Business Review 20, 301-319.

Pierce, Jon L.; Kostova, Tatiana und Dirks, Kurt T. 2001. Towards a Theory of Psychological Ownership in Organizations. Academy of Management Review 26, 298-310.

Pierce, Jon L.; Kostova, Tatiana und Dirks, Kurt T. 2003. The State of Psychological Ownership. Integrating and extending a century of research. Review of General Psychology 7, 84-107.

Pillemer, K. und Lüscher, Kurt. Hrsg. 2004. Intergenerational Ambivalences. New perspectives on parent-child relations in later life. Oxford: Elsevier. 
Pinchot, Gifford. 1985. Intrapreneuring. Why you don't have to leave the corporation to become an entrepreneur. 2. Auflage: Berrett-Koehler Publishers.

Pongratz, Hans J. 2003. Die Heterogenität von Erwerbsorientierungen in der Perspektive der Arbeitskraftunternehmer-These. In Entgrenzung von Arbeit und Leben. Zum Wandel der Beziehung von Erwerbstätigkeit und Privatsphäre im Alltag, Hrsg. Karin Gottschall und Gerd G. Voß, 125-148. München und Mering: Rainer Hampp Verlag.

Porter, Michael E. 1990. The Competitive Advantage of Nations. New York: The Free Press.

Priemel, Kim C. 2007. Flick. Eine Konzerngeschichte vom Kaiserreich bis zur Bundesrepublik. Göttingen: Wallstein Verlag.

Projektgruppe "Alltägliche Lebensführung", Hrsg. 1995. Alltägliche Lebensführung. Arrangements zwischen Traditionalität und Modernisierung. Opladen: Leske + Budrich.

Przyborski, Aglaja und Wohlrab-Sahr, Monika. 2008. Qualitative Sozialforschung. Ein Arbeitsbuch. München: Oldenbourg.

Reichertz, Jo. 2007. Qualitative Sozialforschung - Ansprüche, Prämissen, Probleme. Erwägen - Wissen - Ethik 18, 1-14.

Reichertz, Jo. 2009. Die Konjunktur der qualitativen Sozialforschung und Konjunkturen innerhalb der qualitativen Sozialforschung. Forum Qualitative Sozialforschung /Forum Qualitative Research 10, Art. 29.

Reif, Heinz. 1999. Adel im 19. und 20. Jahrhundert, Bd. 55. München: R. Oldenbourg.

Riessman, Catherine K. 2008. Narrative Methods for the Human Sciences. Los Angeles: Sage Publ.

Roberts, R.E.L. und Bengston, Vern L. 1990. Is Intergenerational Solidarity a Unidimensional Construct? A second test of a formal model. Journal of Gerontology $45,12-20$.

Rogers, Elizabeth D.; Carsrud, Alan L. und Krueger, Norris F. 1996. Chiefdoms and Family Firm Regimes: Variations on the Same Anthropological Themes. Family Business Review 9, 15-27.

Rosenblatt, Paul C.; Mik, Leni; Anderson, Roxanne Marie und Johnson, Patricia A. 1985. The Family in Business. Understanding and dealing with challenges entrepreneurial families face. 1. edition. San Francisco Calif.: Jossey-Bass.

Rosenthal, Gabriele. 1995. Erlebte und erzählte Lebensgeschichte. Gestalt und Struktur biographischer Selbstbeschreibungen. Frankfurt/Main: Campus-Verl.

Rosenthal, Gabriele. 1999. Der Holocaust im Leben von drei Generationen. Familien von Überlebenden der Shoah und von Nazi-Tätern. 3, korrigierte Aufl. Gießen: Psychosozial-Verl.

Rosenthal, Gabriele. 2002a. Biographische Forschung. In Qualitative Gesundheitsund Pflegeforschung. Verlag Hans HuberProgrammbereich Gesundheit, 1. Aufl, Hrsg. Doris Schaeffer und Gabriele Müller-Mundt, 133-147. Bern: Huber.

Rosenthal, Gabriele. 2002b. Biographische-narrative Gesprächsführung. Zu den Bedingungen heilsamen Erzählens im Forschungs- und Beratungskontext. Psycho- 
therapie und Sozialwissenschaften. Zeitschrift für qualitative Sozialforschung, 204-227.

Rosenthal, Gabriele. 2008. Interpretative Sozialforschung. Eine Einführung. 2, korrigierte Aufl. Weinheim: Juventa-Verl.

Rosenthal, Gabriele und Fischer-Rosenthal, Wolfram. 2007. Analysen narrativ biographischer Interviews. In Qualitative Sozialforschung. Eine Einführung. Rororo Rowohlts Enzyklopädie, Bd. 55654, 5. Aufl, Hrsg. Uwe Flick, 456-468. Reinbek bei Hamburg: Rowohlt-Taschenbuch-Verl.

Rosenthal, Gabriele und Völter, Bettina. 1997. Erinnern an die Verfolgungsvergangenheit in ostdeutschen Drei-Generationen-Familien nach der Wende 1989. psychosozial 20, 27-44.

Rothbauer, Paulette M. 2008. Triangulation. In The Sage Encyclopedia of Qualitative Research Methods, Hrsg. Lisa M. Given, 892-894. Los Angeles, Calif: Sage Publications.

Roulston, Kathryn. 2010. Considering Quality in Qualitative Interviewing. Qualitative Research 10, 199-228.

Ruef, Martin. 2010. The Entrepreneurial Group. Social identities, relations, and collective action. Princeton, NJ: Princeton University Press.

Sackmann, Reinhold. 2007. Lebenslaufanalyse und Biografieforschung. Eine Einführung. Wiesbaden: VS Verlag für Sozialwissenschaften/GWV Fachverlage GmbH Wiesbaden.

Schäfer, Michael. 2007. Familienunternehmen und Unternehmerfamilien - Zur Sozial- und Wirtschaftsgeschichte der sächsichen Unternehmer 1850-1940. München: C.H. Beck Verlag.

Schallberger, Peter. 2003. Identitätsbildung in Familie und Milieu. Zwei mikrosoziologische Untersuchungen. Frankfurt a. Main/New York: Campus.

Scherger, Simone. 2007. Destandardisierung, Differenzierung, Individualisierung. Westdeutsche Lebensläufe im Wandel. 1. Aufl. Wiesbaden: VS Verlag für Sozialwissenschaften.

Schimank, Uwe. 2000. Handeln und Strukturen. Einführung in die akteurtheoretische Soziologie. Weinheim: Juventa-Verlag.

Schjoedt, L. und Shaver, K.G. 2007. Deciding on an Entrepreneurial Career. A test of the pull and push hypotheses using the panel study of entrepreneurial dynamics data. Entrepreneurship: Theory \& Practice 31, 733-752.

Schmidt, Uwe und Moritz, Marie-Theres. 2009. Familiensoziologie. Bielefeld: transcript Verlag.

Schmitt-Rodermund, Eva und Schröder, Elke. 2009. Innerfamiliäre Nachfolge in Unternehmen. Studie zur Förderung und Entwicklung des Nachwuchses in Familienunternehmen. Jena: Friedrich Schiller Universität Jena.

Schneider, Norbert F. 2002. Zur Lage und Zukunft der Familie in Deutschland. Gesellschaft - Wirtschaft - Politik, 511-544.

Schneidewind, Uwe. 1998. Die Unternehmung als strukturpolitischer Akteur. Kooperatives Schnittmengenmanagement im ökologischen Kontext. Marburg: Metropolis-Verl. 
Schüßler, Jörn; Märkt, Jörg; Guder, Gerhard; Freund, Werner und Kayer, Gunter. 2004. Unternehmensnachfolge in Hamburg. Hamburg: IfM Bonn.

Schütz, Alfred. 1971. Gesammelte Aufsätze I. Das Problem der sozialen Wirklichkeit. Den Haag: Nijhoff.

Schutz, W.C. 1958. FIRO: A three dimensional theory of interpersonal behavior. New York: Rinehart.

Schütze, Fritz. 1983. Biographieforschung und narratives Interview. Neue Praxis, 283-293.

Schütze, Fritz. 1984. Kognitive Figuren des autobiographischen Stegreiferzählens. In Biographie und soziale Wirklichkeit. Neue Beiträge und Forschungsperspektiven, Hrsg. Martin Kohli und Günther Robert, 78-117. Stuttgart: J.B. Metzlersche Verlagsbuchhandlung.

Schweizer. Herbert. 2007. Soziologie der Kindheit. Verletztlicher Eigen-Sinn. Wiesbaden: VS Verlag für Sozialwissenschaften.

Seale, Clive. 1999 /// 2007. The Quality of Qualitative Research. Reprinted. London: Sage Publications; SAGE.

Seeghitz, Nicolette. 2000. Nachfolgeproblematik in mittelständischen Familienunternehmen - ein interdisziplinäres Handlungsfeld. Fürth.

Shanahan, Michael J. 2000. Pathways to Adulthood in Changing Societies. Annual Sociological Review, 667-692.

Sharma, Pramodita. 2004. An Overview of the Field of Family Business Studies: Current Status and Directions for the Future. Family Business Review 17, 1-36.

Sharma, Pramodita; Chrisman, James J.; Pablo, Amy L. und Chua, Jess H. 2001. Determinants of Initial Satisfaction With the Succession Process in Family Firms. A conceptual model. Entrepreneurship: Theory \& Practice 25, 17-36.

Sharma, Pramodita; Chrisman, James J. und Chua, Jess H. 2003. Predictors of Satisfaction With the Succession Process in Family Firms. Journal of Business Venturing 18, 667-687.

Siefer, Thomas. 1996. "Du kommst mir später mal in die Firma!". Psychosoziale Dynamik von Familienunternehmen. Heidelberg: Carl-Auer-Systeme Verlag und Verlagsbuchhandlung GmbH.

Simon, Fritz B. 2002a. Besonderheiten der Familiendynamik. In Die Familie des Familienunternehmens. Ein System zwischen Gefühl und Geschäft, Hrsg. Fritz B. Simon, 35-54. Heidelberg: Carl-Auer-Systeme Verlag.

Simon, Fritz B, Hrsg. 2002b. Die Familie des Familienunternehmens. Ein System zwischen Gefühl und Geschäft. Heidelberg: Carl-Auer-Systeme Verlag.

Simon, Fritz B.; Wimmer, Rudolf und Groth, Torsten. 2005. Mehr-GenerationenFamilienunternehmen. Erfolgsgeheimnisse von Oetker, Merck, Haniel u. a. 1. Aufl. Heidelberg: Carl-Auer.

Sonfield, Matthew C, und Robert N. Lussier. 2004. First-, Second- and ThirdGeneration Family Firms: A Comparison. Family Business Review 17, 189-202.

Sonnenfeld, Jeffrey. 1988. The Hero's Farewell - What Happens When CEOs Retire. New York: Oxford University Press. 
Stafford, Kathryn; Duncan, Karen A.; Dane, Sharon und Winter, Mary. 1999. A Research Model of Sustainable Family Business. Family Business Review XII, 197208.

Stamm, Isabell. 2009. Generationenbeziehungen in Unternehmerfamilien. Sozialwissenschaftliches Journal XII, 23-39.

Stamm, Isabell; Schmiade, Nicole und Kohli, Martin. 2009a. Familienunternehmer als Pioniere biographischer Selbstbestimmung? In Unsichere Zeiten. Aushandlungen des 34. Kongresses für Soziologie in Jena, Hrsg. Hans-Georg Soeffner, CDROM. Wiesbaden.

Stamm, Isabell; Schmiade, Nicole und Kohli, Martin. 2009b. Von Generation zu Generation: Der Nachfolgeprozess in Familienunternehmen. In Familienunternehmen im Rheinland im 19. und 20. Jahrhundert. Netzwerke - Nachfolge - soziales Kapital. Schriften zur rheinisch-westfälischen Wirtschaftsgeschichte, Bd. 47, Hrsg. Susanne Hilger und Ulrich S. Soenius, 177-187. Köln: Stiftung RheinischWestfälisches Wirtschaftsarchiv.

Stamm, Isabell und Lubinski, Christina. 2011. Crossroads of Family Business Research and Firm Demography. A critical assessment of family business survival rates. Journal of Family Business Strategy 2.

Stamm, Isabell; Breitschmid, Peter und Kohli, Martin. Hrsg. 2011. Doing Succession in Europe. Generational transfers in family businesses in comparative perspective. Zürich: Schluthess Verlag.

Stamm, Isabell und Marchese, Elisa. 2011. Succession as Transition in the Life Course. In Doing Succession in Europe. Generational transfers in family businesses in comparative perspective, Hrsg. Isabell Stamm, Peter Breitschmid und Martin Kohli, 243-266. Zürich: Schluthess Verlag.

Stavrou, Eleni T. und Swiercz, Paul M. 1998. Securing the Future of the Family Enterprise. A model of offspring intentions to join the business. Entrepreneurship Theory and Practice, 19-39.

Steier, Lloyd. 2001. Next-Generation Entrepreneurs and Succession. An exploratory study of modes and means of managing social capital. Family Business Review 14, 259-276.

Steinbach, Anja. 2008. Intergenerational Solidarity and Ambivalence: Types of Relationships in German Families. Journal of Comparative Family Studies 89, 115127.

Steinke, Ines. 2007. Gütekriterien qualitativer Forschung. In Qualitative Sozialforschung. Eine Einführung. Rororo Rowohlts Enzyklopädie, Bd. 55654, 5. Aufl, Hrsg. Uwe Flick, 319-331. Reinbek bei Hamburg: Rowohlt-Taschenbuch-Verl.

Stiftung Familienunternehmen. 2009. Die volkswirtschaftliche Bedeutung von Familienunternehmen. München.

Szydlik, Marc. 2000. Lebenslange Solidarität? Generationenbeziehungen zwischen erwachsenen Kindern und ihren Eltern. Opladen: Leske + Budrich.

Szydlik, Marc. 2008. Intergenerational Solidarity and Conflict. Journal of Comparative Family Studies 39, 97-114. 
Tagiuri, Renato und Davis, John. 1996. Bivalent Attributes of the Family Firm. Family Business Review 9, 199- 208.

Timmermann, Hajo und Vonderach, Gerd. 1993. Milchbauern in der Wesermarsch: Eine empirisch-soziologische Untersuchung, Bd. 8. 1. Aufl. Bamberg: Wissenschatliche Verlags-Gesellschaft.

Tooze, J. A. 2007. Ökonomie der Zerstörung. Die Geschichte der Wirtschaft im Nationalsozialismus. 1. Aufl. München: Siedler.

Treibel, Annette. 2000. Einführung in die soziologischen Theorien der Gegenwart. Opladen: Leske \& Budrich.

Trotha, Trutz von. 1990. Zum Wandel der Familie. Kölner Zeitschrift für Soziologie und Sozialpsychologie, 452-473.

Uhlaner, Lorraine M. 2006. Business Family as a Team: Underlying Force for Sustained Competetive Advantage. In Handbook of Research on Family Business, Hrsg. Panikkos Z. Poutziouris, Kosmas X. Smyrnios und Sabine Klein, 125-144. Cheltenham UK Northampton MA.

van Hoonaard, Will C. d. 2008. Theoretical Sampling. In The Sage Encyclopedia of Qualitative Research Methods, Hrsg. Lisa M. Given, 874. Los Angeles, Calif: Sage Publications.

Vera, Carolina F. und Dean, Michelle A. 2005. An Examination of the Challenges Daughters Face in Family Business Succession. Family Business Review 18, 321-345.

Veszelits, Thomas. 2005. Die Neckermanns. Licht und Schatten einer deutschen Unternehmerfamilie. Frankfurt/Main: Campus-Verl.

Vierzigmann, Gabriel und Kreher, Simone. 1998. Zwischen den Generationen - Familiendynamik und Familiendiskurse in biographischen Erzählungen. Berliner Journal für Soziologie, 23-37.

Völter, Bettina. 2003. Judentum und Kommunismus. Deutsche Familiengeschichten in drei Generationen. Techn. Univ, Diss.-Berlin, 2000. Opladen: Leske + Budrich.

Vonderach, Gerd. 1997. Geschichtenhermeneutik. In Sozialwissenschaftliche Hermeneutik, Hrsg. Ronald Hitzler und Anne Honer, 165-190. Opladen: Leske + Budrich; Utb.

Voß, Gerd Günter 1991. Lebensführung als Arbeit. Über die Autonomie der Person im Alltag der Gesellschaft. Stuttgart: Enke.

Voß, Gerd Günter 2000. Das Ende der Teilung von "Arbeit und Leben"? An der Schwelle zu einem neuen gesellschaftlichen Verhältnis von Betriebs- und Lebensführung. In Lebensführung und Gesellschaft. Beiträge zu Konzept und Empirie alltäglicher Lebensführung, Hrsg. Werner Kudera und Günter Voß Gerd, 309-339. Opladen: Leske + Budrich.

Voß, Gerd Günter und Pongratz, Hans J. 1998. Der Arbeitskraftunternehmer. Eine neue Grundform der Ware Arbeitskraft? Kölner Zeitschrift für Soziologie und Sozialpsychologie 50, 131-158.

Voß, Gerd Günter 2001. Der Arbeitskraftunternehmer. Ein neuer Typus von Arbeitskraft und seine sozialen Folgen. Arbeit und Region 43. Bremen: Universität Bremen, ZWE. 
Walford, Geoffrey. 2007. Classification and Framing of Interviews in Ethnographic Interviewing. Ethnography and Education 2, 145-157.

Ward, John L. 1987. Keeping the Family Business Healthy. How to plan for continuing growth, profitability, and family leadership. 1st ed. San Francisco Calif.: Jossey-Bass.

Ward, John L. 2004. Perpetuating the Family Business: 50 Lessons Learned form Long-Lasting, Successful Families in Business. Hampshire, New York: Palgrave Macmillan.

Ward, John L. und Aronoff, Craig. 1996. Just What is a Family Business? In Family Business Sourcebook II. A guide for families who own businesses and the professionals who serve them : covering succession planning, growth, financial issues, management, psychological issues, women in the family business, the younger generation, and other issues family business face, Hrsg. Craig Aronoff, 2-3. Mariette: Business Owner Resources.

Weber, Max. 1958. Wirtschaftsgeschichte. Abriss der universalen Sozial- und Wirtschaftsgeschichte. In Aus den nachgelassenen Vorlesungen, 3. überarbeitete Auflage, Hrsg. S. Hellmann und M. Palyi. Berlin: Dunker \& Humblot.

Weber, Max. 1988. Vorbemerkungen. In Gesammelte Aufsätze zur Religionssoziologie, [Photomech. Nachdr. d. 1920 ersch. Erstaufl.], 9, Hrsg. Max Weber, 1-17. Tübingen: Mohr.

Welzer, Harald; Moller, Sabine; Tschuggnall, Karoline; Jensen, Olaf und Koch, Torsten. 2005, c2002. Opa war kein Nazi. Nationalsozialismus und Holocaust im Familiengedachtnis. 5. Auflage. Frankfurt am Main: Fischer Taschenbuch Verlag.

Wenk, Regina. 2007. Generationswechsel in Familienbetrieben als biographische Arbeit. http://webdoc.sub.gwdg.de/diss/2007/wenk/wenk.pdf (Stand: 26. November 2010).

Weymann, Ansgar. 2000. Sozialer Wandel, Generationsverhältnisse und Technikgenerationen. In Generationen in Familie und Gesellschaft, Hrsg. Martin Kohli und Marc Szydlik, 36-58. Opladen: Leske + Budrich.

Whitley, Richard. 2002. Divergent Capitalisms. Oxford: Oxford University Press.

Wiechers, Ralph. 2006. Familienmanagement zwischen Unternehmen und Familie. Zur Handhabung typischer Eigenarten von Unternehmensfamilien und Familienunternehmen. Heidelberg: Carl-Auer Verlag.

Wiedemann, Andreas und Layer, Bertram. 2011. Vorsorge für Familienunternehmer und Sicherung des Familienvermögens in Deutschland. In Doing Succession in Europe. Generational transfers in family businesses in comparative perspective, Hrsg. Isabell Stamm, Peter Breitschmid und Martin Kohli, 347-359. Zürich: Schluthess Verlag.

Williams, Kevin. 2008. Troubling the Concept of the 'Academic Profession' in 21st Century Higher Education. Higher Education 56, 533-544.

Wohlrab-Sahr, Monika. 1992. Institutionalisierung oder Individualisierung des Lebenslaufs? Anmerkungen zu einer festgefahrenen Debatte. BIOS, 1-19. 
Wohlrab-Sahr, Monika. 1997. Individualisierung. Differenzierungsprozeß und Zurechnungsmodus. In Individualisierung und Integration. Neue Konfliktlinien und neuer Integrationsmodus?, Hrsg. Ulrich Beck und Peter Sopp, 23-36. Opladen: Leske + Budrich.

Wortman, Max. 1994. Theoretical Foundations for Family-Owned Business: A Conceptual and Research-Based Paradigm. Family Business Review 7, 3-27.

Zahra, S. und Sharma, P. 2004. Family Business Research: a Strategic Reflection. Family Business Review 17, 331-346.

Zellweger, Thomas. 2007. Time Horizon, Costs of Equity Capital, and Generic Investment Strategies of Firms. Family Business Review 20, 1-15.

Zellweger, Thomas; Sieger, Philipp und Halter, Frank. 2011. Should I Stay or Should I Go? Career Choice Intentions of students with family business background. Journal of Business Venturing, 1-16.

Zellweger, Thomas und Astrachan, Joseph. 2008. On the Emotional Value of Owning a Firm. Family Business Review XXI, 347-363. 


\section{Anhang: Falldarstellungen}

Im Folgenden wird als Fall die Familiengeschichte anhand der objektiven Daten dargestellt, die sich aus den Gesprächen sowie zusätzlichen Recherchen ableiten lassen. Das „Firmenprofil“" enthält die wesentlichen Eckdaten des Unternehmens zum Zeitpunkt des Interviews. Der Text skizziert in chronologischer Reihenfolge die Entwicklungsgeschichte des Falls, wobei den Aktivitäten von Familienmitgliedern im Unternehmen sowie den Eigentumsverhältnissen besondere Aufmerksamkeit zukommt. Die jeweilige Familienkonstellation wird mittels eines Genogrammes veranschaulicht. Alle Fälle werden anonymisiert präsentiert. Um sie unkenntlich zu machen, werden den Personen und Firmen andere Namen gegeben, Orts- und Branchenbezeichnung oft verallgemeinert und einzelne Merkmale umgeschrieben, sofern sie sich als nicht wesentlich für die Struktur des Falles erwiesen haben. (vgl. Rosenthal 2005:96).

Abbildung A 1: Erläuterung der im Genogramm verwendeten Symbole

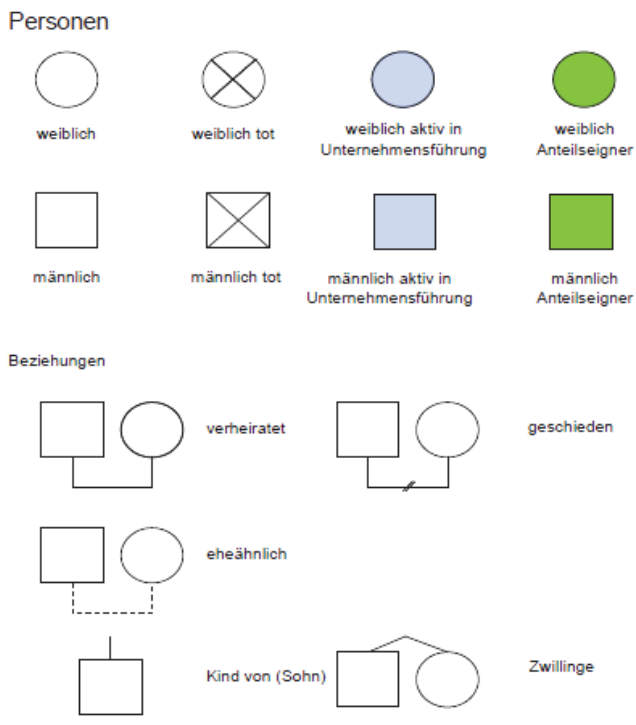

Quelle: eigene Darstellung 


\section{FAMILIE CARSTENS -CARSTENS MUSIK}

Erwin Carstens $(* 1930)$ verbringt die Kriegsjahre in Berlin und wächst in der DDR auf. Er beginnt ein Studium der Musikwissenschaften zunächst an der Humboldt Universität und wechselt dann nach West-Berlin an die Freie Universität. Er führt sein Studium jedoch nie zu Ende, stattdessen arbeitet er in diversen Musikverlagen. 1959 heiratet er eine Buchhändlerin und die beiden bekommen zwei Kinder, erst einen Sohn und fünf Jahre später eine Tochter. Die Familie ist mittler-weile nach Süddeutschland um-gesiedelt, da Erwin dort eine Stelle in einem großen Musik-verlag ange-

\begin{tabular}{|c|c|}
\hline \multicolumn{2}{|c|}{ Firmenprofil } \\
\hline Gründungsjahr: & frühe 2000er Jahre \\
\hline Tätigkeitsfeld: & Musikverlag \\
\hline Mitarbeiterzahl: & 7 \\
\hline Umsatz: & ca. 500.000 EUR \\
\hline Region: & Süddeutschland \\
\hline Eigentümerstruktur: & Bianca Carstens $100 \%$ \\
\hline Geschäftsführung: & Bianca Carstens \\
\hline Aktuelle Nachfolge: & $\begin{array}{l}\text { Neugründung } \\
\text { (nachdem das FU an Externe } \\
\text { verkauft wurde) }\end{array}$ \\
\hline
\end{tabular}
treten hat. Innerhalb kurzer Zeit wird er Verlagsleiter. 1974 geht es dem Unternehmen wirtschaftlich sehr schlecht, als ein sehr großer Konzern ihnen ein Angebot unterbreitet. Erwin kümmert sich um den Verkauf des Verlagshauses, gliedert jedoch eine kleine Sparte aus, mit der er sein eigenes Musikverlagshaus gründet. Im Jahr darauf lassen sich Erwin und seine Frau scheiden. 1976 lernt Erwin seine zwanzig Jahre jüngere Partnerin kennen, mit der er bis heute liiert ist. Sie arbeitet über Jahrzehnte hinweg in seinem Verlagshaus mit. Der Sohn von Erwin wird Pilot bei der Bundeswehr und ist darüber hinaus künstlerisch interessiert. Seine Tochter Bianca arbeitet bereits während ihrer Schulzeit im Musikverlag mit und beginnt im Anschluss an ihr Abitur eine Ausbildung beim Vater. Die folgenden Jahre reist Bianca viel und absolviert diverse Praktika und Jobs in Australien, Neuseeland und den USA. Schließlich beginnt sie Anglistik und Musik in Berlin zu studieren. Anfang der 1990er Jahre tritt Bianca eine leitende Stelle im Unternehmen des Vaters an. Kurz darauf wird sie von ihrem damaligen Freund schwanger. Einige Zeit nach der Geburt ihres Sohnes trennt sie sich jedoch von ihm. 1996 lernt Bianca ihren heutigen Ehemann, den Industriefachwirt Jan kennen, die beiden bekommen noch zwei weitere Söhne. Jan steckt für die Karriere seiner Frau zurück. Er schlägt berufliche Chancen aus, um am Wohnort der Familie verweilen zu können und übernimmt für einige Zeit die Kindererziehung. Die Familie kauft sich 2000 ein kleines Haus in der Nähe des Musikverlages. 
Abbildung A 2: Genogramm der Familie Carstens

\section{Familienkonstellation}

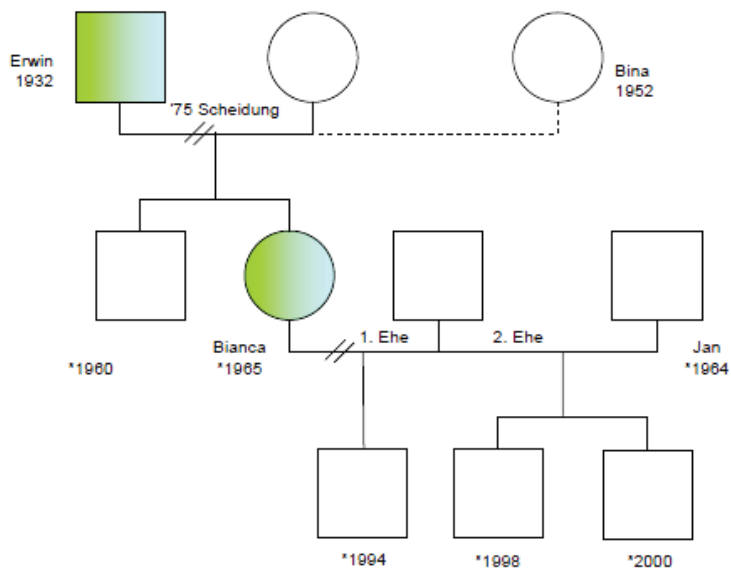

Quelle: eigene Darstellung

2003 unterbreitet ein weiterer großer Musikkonzern Erwin ein Kaufangebot für sein Unternehmen, in dem auch Arbeitsplätze sowohl für ihn als auch seine Tochter enthalten sind. Erwin willigt mit Blick auf die Finanzierung seines Ruhestandes ein. Bianca fühlt sich unwohl mit den Konzernstrukturen und gründet im Jahr 2004 - mit einer Reihe von bestehenden Kontakten zu bekannten Musikern - ihren eigenen Musikverlag. Etwas später beginnt Jan im internationalen Vertrieb mitzuarbeiten. Erwin geht 2005 in den Ruhestand. Seitdem führt er ein paar eigene Projekte fort und steht seiner Tochter beratend zur Seite.

Die Fallgeschichte stellt den Stand von 2009 dar. Interviews wurden mit Erwin, Bianca und Jan Carstens geführt. 


\section{FAMILIE CLAAS -UNTERNEHMENSGRUPPE CLAAS UMWELTTECHNIK}

Rainer Claas $(* 1923)$ dient als Soldat im zweiten Weltkrieg. Nach seiner Rückkehr heiratet er und 1949 wird sein erster Sohn Cornelius geboren. Nachdem Rainer als Maurer keine Arbeit findet beschließt er 1951, sich mit dem Recycling von Altpapier und Kleidung selbständig zu machen. Gemeinsam mit seiner Frau sammeln sie mit Pferd und Wagen die Materialien ein. Sie beziehen mit der Firma ein Ruinengrundstück. Der ältere Bru-der von Rainer, der im Krieg beide Beine verlor, hilft ebenfalls im Betrieb mit. In den folgenden Jahren werden Claudia und Carsten geboren. Die drei Kinder wachsen sehr eng mit dem Unternehmen auf. Die Eltern sprechen täglich von den betrieblichen Vorgängen und die Kinder verbringen viel Zeit dort. Die Partnerschaft der Eltern sowie das Verhältnis zu den Kindern gestalten sich konfliktreich. Während ihrer Jugendzeit (68er Generation) sind die Kinder politisch links engagiert. Besonders die beiden Älteren, Cornelius und Claudia, stellen sich gegen den patriarchischen Vater. Ihre Rebellion endet mit dem Rauswurf aus dem Elternhaus. Der älteste Sohn versucht sich von nun an immer wieder mit unternehmerischen Projekten, während die Schwester zunächst Kinderkrankenschwester lernt und nebenbei ihr Abitur nachholt. Auch Carsten absolviert eine Lehre zum Speditionskaufmann und legt dann das Wirtschaftsabitur auf dem zweiten Bildungsweg ab. Als Mitte der siebziger Jahre die Firma von Cornelius nicht gut läuft, beginnt er für seinen Vater zu arbeiten. Ihr Verhältnis ist angespannt und schwierig. Zur gleichen Zeit nimmt Claudia ein Psychologiestudium auf, während Carsten nach dem Zivildienst Sozialpädagogik studiert. Das Unternehmen des Vaters entwickelt sich stetig zu einem größeren Umwelttechnikbetrieb. Mitte der achtziger Jahre steigt Cornelius nach vielen Streitereien aus dem Familienunternehmen aus. Einige Monate danach bittet der Vater seinen jüngsten Sohn Carsten, in die Firma einzusteigen. Gemeinsam legen Vater und Sohn der Mutter nahe, ihre Tätigkeit in der Buchhaltung niederzulegen. Drei Jahre später bittet Carsten den Vater, sich endgültig aus dem operativen Geschäft zurückzuziehen und nur noch als Eigentümer auf das Unternehmen einzuwirken. Der Vater willigt ein, installiert aber einen Beirat, den er selbst bestimmen kann, aus Wirtschaftsberater, Bankdirektor und Steuerberater, die dem Sohn von nun an zur Seite stehen sollen. 
Abbildung A 3: Genogramm der Familie Claas

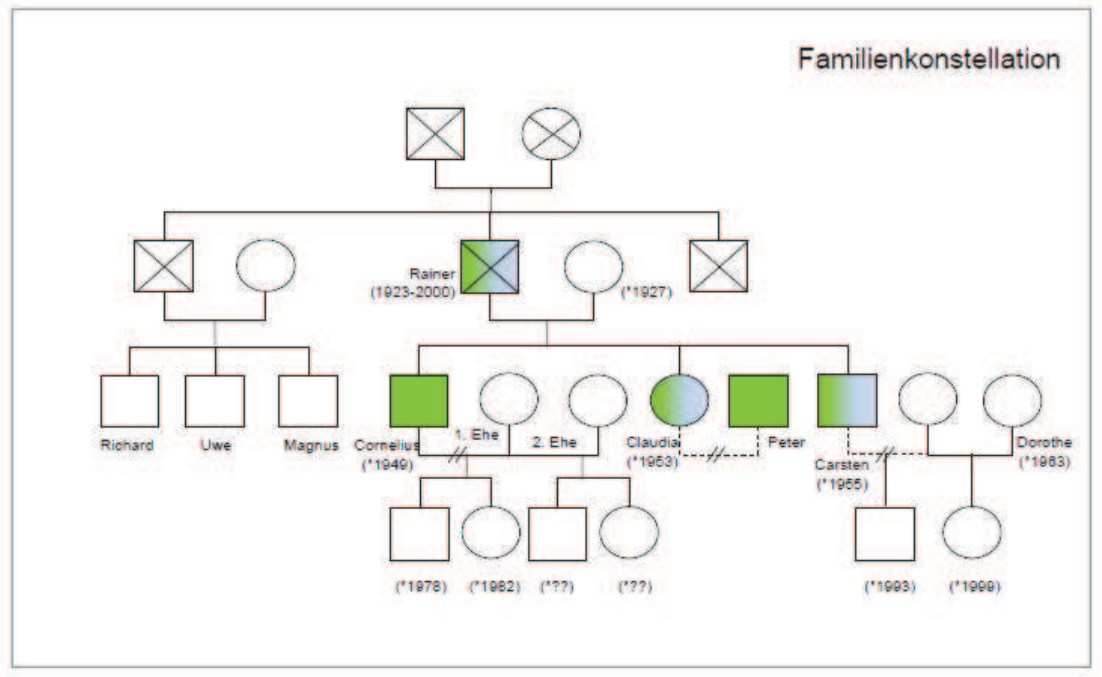

Quelle: eigene Darstellung

Auch Claudia beginnt für ein Unternehmen, an dem der Vater Anteile hält, aber nicht operativ involviert ist, zu arbeiten. Etwas später wird sie dort Geschäftsführerin, bis sie der Bruder in das Hauptgeschäft holt. Die Geschwister teilen sich die Sparten auf. Claudias Lebenspartner Peter wird Assistent ihres Vaters. Der älteste Bruder geht in der Zwischenzeit mit seiner Firma insolvent und gründet ein neues Unternehmen.

1999 findet eine Umstrukturierung des Unternehmens statt. Die Sparten werden in rechtlich selbständige Firmen aufgeteilt, die alle unter dem Dach einer Holding zusammenlaufen. Carsten fusioniert in seiner Sparte mit einem anderen Betrieb und holt einen externen Geschäftsführer zusätzlich an Bord. 2000 verstirbt Vater Rainer unerwartet an einem Herzinfarkt. Jedes der drei Kinder erbt ein Drittel der Holding Gesellschaft. Die Mutter erhält eine Betriebsrente. Cornelius will daraufhin wieder in das Unternehmen einsteigen, doch die beiden jüngeren Geschwister verhindern dies und zahlen ihn aus. Cornelius investiert das Geld in sein Unternehmen, geht jedoch ein Jahr später erneut insolvent. Er wird von nun an als Händler auf Provision tätig und arbeitet heute wieder mit dem Familienunternehmen zusammen. Peter ist, trotzdem er sich von Claudia trennte, nun Geschäftsführer der Holding.

Die Fallgeschichte stellt den Stand von 2009 dar. Interviews wurden mit Claudia und Carsten Claas geführt. 


\section{FAmilie Clemens -Clemens TeChNiK AG}

Justus Clemens stammt aus einer im Wagenbau tätigen Familie. Als jüngster Sohn erhält er eine Auszahlung (weichender Erbe), die er in einen kleinen Laden für mechanische Haushalts- und Gewerbeprodukte investiert. Mit der Übergabe an seinen ältesten Sohn Justus wird die Produkt-palette erweitert und mit der eigenen Fertigung begonnen. 1890 übernimmt Conrad Clemens die Firma seines Vaters und richtet eine zusätzliche Werkstatt ein. Das Unternehmen wächst stetig. Anfang der 1920er Jahre wird eine eigene Betriebs-krankenkasse, nach dem ersten Weltkrieg eine erste internationale Fertigungsstätte in Italien gegründet.

\begin{tabular}{|ll|}
\hline \multicolumn{2}{|c|}{ Firmenprofil } \\
Gründungsjahr: & frühe 1840er Jahre \\
Tätigkeitsfeld: & Technikproduzent \\
Mitarbeiterzahl: & ca. 39.000 \\
Umsatz: & ca. 4 Mrd \\
Region: & Mitteldeutschland \\
Eigentümerstruktur: & Es halten die Kinder von: \\
& Stiefbruder 1\% \\
& Leopold Clemens 51\% \\
& $\begin{array}{l}\text { Schwester } 48 \% \\
\text { (10 Anteilshaber) }\end{array}$ \\
Vorstandsvorsitz: & Leopold Clemens \\
Aktuelle Nachfolge: & Leopold (5. Gen) scheidet in \\
& 2 Jahren aus. Die Nachfolger \\
& stehen noch nicht fest. \\
\hline
\end{tabular}

Nach dem frühen Tod von Conrad im Krisenjahr 1929 gehen die Unternehmensanteile an seine drei Söhne über. Die beiden jüngeren treten in den Betrieb als kaufmännische und technische Leiter ein. Der Älteste hingegen wird Gynäkologe und genießt einen hohen Lebensstandard. Seine Brüder lösen ihn gegen eine Altersvorsorge aus. Oscar Clemens ist verheiratet und hat drei Kinder aus erster Ehe und einen älteren Stiefsohn aus zweiter Ehe. Die Familie lebt bürgerlich in der Kleinstadt, in der auch das Unternehmen angesiedelt ist. Die Kinder besuchen die örtlichen Schulen und sind im Verein engagiert. Oscar hält eine Reihe von hohen Ämtern auf Verbandsebene inne. Er legt großen Wert auf eine gute Erziehung und Ausbildung seiner Kinder. Beispielsweise erhalten diese zusätzlichen Sprachunterricht. Sein jüngerer Bruder ist ebenfalls verheiratet und bekommt im Alter von 54 Jahren eine Tochter. In der Firma wird der Internationalisierungskurs fortgesetzt. Die Kinder erleben den Erfolg des Unternehmens mit. Sehr früh übertragen Oscar und sein Bruder die ersten Unternehmensanteile an ihre Kinder: Leopold, sein Stiefbruder und seine Cousine werden Anteilseigner der Clemens Technik, seine beiden Geschwister an internationalen Tochterunternehmen. Nach seinem Abitur lernt Leopold zunächst in einer Bank (wie sein Vater) und arbeitet im Anschluss für zwei Jahre in England und den USA. Leopold absolviert den Wehrdienst und übernimmt anschließend die Leitung eines neu gekauften Unternehmens in Brasilien. Anfang der 1970er Jahre erfolgt die Umwandlung des Unternehmens in eine AG (nur Familienmitglieder sind Aktionäre). Im Jahr darauf kehrt Leopold nach Europa zurück und tritt als Mitglied des Vorstandes in das Familienunternehmen ein. Zur gleichen Zeit wird auch sein älterer Stiefbruder im Unternehmen tätig. 
Abbildung A 4: Genogramm der Familie Clemens

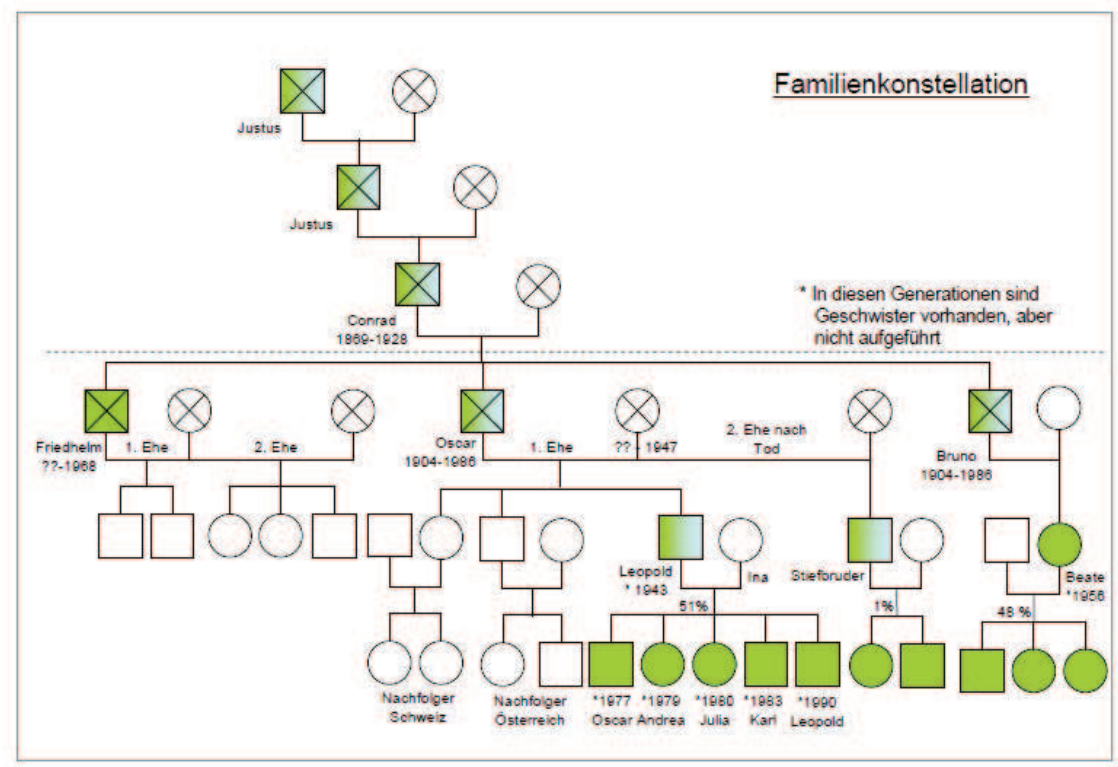

Quelle: eigene Darstellung

1975 heiratet Leopold und sie lassen sich in seiner Heimatstadt nieder. Zwei Jahre später erhält er die kaufmännische Gesamtverantwortung und wird Vorstandsvorsitzender des stark wachsenden Unternehmens. In den folgenden Jahren bekommen Ilona und Leopold fünf Kinder. Zudem engagiert sich Leopold partei- und verbandspolitisch (gleiche Partei, gleiche Verbände, später auch ähnliche Ämter wie sein Vater). Ihre Kinder besuchen die örtlichen Schulen und sind im Sportverein aktiv (Leistungssport). Sie werden regelmäßig auf Sommercamps und Schüleraustausch geschickt. Zudem verbringen alle Kinder einen Teil ihrer Ausbildung im Ausland: Die älteren Söhne absolvieren ihren Wehrdienst bei der Deutsch-Französischen Brigade. Oscar Clemens studiert nach einer Banklehre Finanzierung in England und steigt nach einem Trainee-Programm in die brasilianische Niederlassung ein. Andrea studiert Jura, absolviert ihr Referendariat als Rechtsanwältin im Ruhrgebiet und arbeitet schließlich noch in den USA. Julia studiert zunächst Modedesign in London und absolviert einen MBA bevor sie in der PR einer größeren Stiftung arbeitet. Karl studiert Maschinenbau und arbeitet später in Indien. Und Leopold junior geht nach seinem Abitur nach Neuseeland. Auch ihre Cousins sind beruflich erfolgreich. Anfang der 90er Jahre beginnt der materielle Transfer an die nächste Generation. Anteile werden in kleinen Schenkungspaketen an alle Kinder übergeben. 
Auf dem jährlichen Familientag besprechen sie den Geschäftsbericht. Leopold gibt bekannt, dass er in zwei Jahren seine Aktivitäten im Unternehmen aufgeben wird. Ein Nachfolger ist noch nicht bestimmt.

Die Fallgeschichte stellt den Stand von 2009 dar. Interviews wurden mit Leopold, Ilona, Andrea und Julia Clemens geführt. 


\section{FAMILIE HAGEMANN -FRISCH UND LECKER OHG}

Samuel Hagemann wird 1939 als jüngster Sohn einer ostdeutschen Unternehmerfamilie geboren, die eine kleine Marmeladenfabrik betreibt. In den Kriegsjahren wird die Fabrik schwer beschädigt. Bei der Einschulung von Samuel stellt man eine gravierende Sehbehinderung fest, die später auf einem Auge operativ behoben werden kann. Er lernt erst sehr spät Lesen und Schreiben und nimmt am Unterricht überwie-gend verbal teil. Nach dem Krieg baut der Vater die Fabrik erst wieder auf, entschließt sich dann jedoch nach Westberlin zu gehen. Seine Frau und seine Kinder sollten ihm ursprünglich folgen, verbleiben aber bei den Großeltern in der Ostzone. Der Vater arbeitete später in einem großen Kaufhaus in Frankfurt am Main. Die Familie ist von nun an getrennt. Samuel schließt die 8. Klasse gut ab und kann anschließend eine Ausbildung zum Verkäufer in der zentralen Ausbildungsstelle des „Konsum“ in Leipzig absolvieren. Seine sechs Jahre ältere Schwester ist nach dem Abitur ebenfalls im Handel tätig und sein älterer Bruder arbeitet als Bauzeichner. Samuel lebt während dieser Zeit in einem Internat. 1958 lernt Samuel seine spätere Frau Martha kennen, die als Sekretärin in der Berufsschule arbeitet. 1960 heiraten die beiden und bekommen ihren Sohn Timo. In dieser Handelsorganisation macht Samuel über verschiedene Stationen (auch mit kurzzeitigen Unterbrechungen) eine Karriere, die insgesamt 35 Jahre andauert. Er ist Abteilungsleiter, Bereichsleiter und schließlich sogar stellvertretender Direktor. Samuel ist Ansager für Modeschauen, organisiert Weihnachts- und Kinderveranstaltungen. Zudem qualifiziert er sich per Fernstudium zum Pressereferenten und Betriebswirt weiter. Die Familie zieht 1966 in eine größere Wohnung und besitzt einen Schrebergarten. Sie leben gut in der DDR. Ihr Sohn Timo macht eine Berufsausbildung mit Abitur in einem Geräte und Reglerwerk in Teltow. Anschließend studiert er Anlagenbau (Chemie). Die folgenden Jahre arbeitet er in verschiedenen Kombinaten und Werken, bis 1989 die Wende das Leben der Familie grundlegend verändert: 
Abbildung A 5: Genogramm der Familie Hagemann

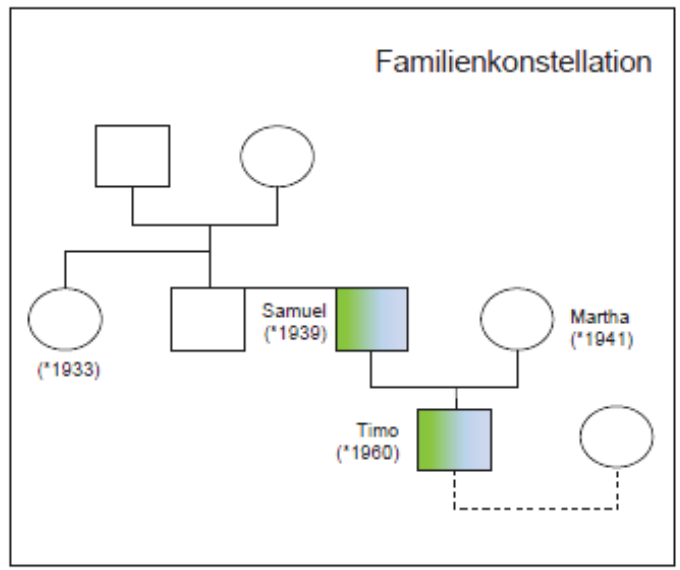

Quelle: eigene Darstellung

Das Werk, bei dem Timo angestellt war, wird aufgekauft und geschlossen. Er findet in seinem Bereich keine Arbeit mehr und wird Jugendclubleiter. Sein Vater kündigt nach angespanntem Verhältnis in den letzten Jahren 1990 seine Stelle und macht sich mit einer Beratungsfirma für Handelsorganisationen selbständig. Unter anderem arbeitet er auch als freier Mitarbeiter für die Kette „Frisch und Lecker“. Kurz darauf hat Samuel die Möglichkeit eine kleinere Ladenfläche anzumieten und eröffnet selbst eine Franchise-Niederlassung der Supermarktkette. Sein Sohn wird offiziell Marktleiter. Martha übernimmt das Personalwesen und die Buchhaltung. Nach langen Verhandlungen gelingt es Samuel schließlich Ende der 1990er Jahre einen wesentlich größeren Supermarkt anzumieten. Der Vater gibt seine beratende Tätigkeit auf und arbeitet nun voll in dem neuen Markt. Er organisiert regelmäßig größere Veranstaltungen für Spenden- und Werbezwecke. 2002 eröffnet schließlich Timo einen weiteren Supermarkt in einem anderen Stadtteil. Er führt diesen bis 2006 erfolgreich. Als sein Vater sich zur Ruhe setzen will, verkauft Timo seinen Laden und übernimmt den Supermarkt des Vaters. 2007 geht Samuel mit 68 Jahren in den Ruhestand und ist von nun an nur noch beratend für den Sohn tätig. Die Mutter arbeitet weiterhin aktiv mit. Die Fallgeschichte stellt den Stand von 2007 dar. Interviews wurden mit Samuel und Timo Hagemann geführt. 


\section{FAMILIE TREUNINGER -LOEBER \& SOHN GMBH}

Seit Jahrhunderten baut Familie Treuninger Geräte für den bäuerlichen Bedarf. Sie stehen dem württembergischen Pietis-mus nahe. Mitte des 19. Jahrhunderts fällt die Werkstatt aufgrund einer Reihe von frühen Todesfällen für kurze Zeit in „externe“ Hände: Die Witwe Anna von P.M. Treu-ninger heiratet Gustav Loeber, der Anfang der 1840er Jahre auf Basis der bestehenden Werkstatt und Vertriebskanäle seine eigene Firma gründet. Im Jahr 1870 erfolgt die erste familieninterne Übergabe: Gustav Loebers Stiefsohn Adam Treuninger tritt in das Unternehmen ein (von nun an „Loeber \& Sohn“).

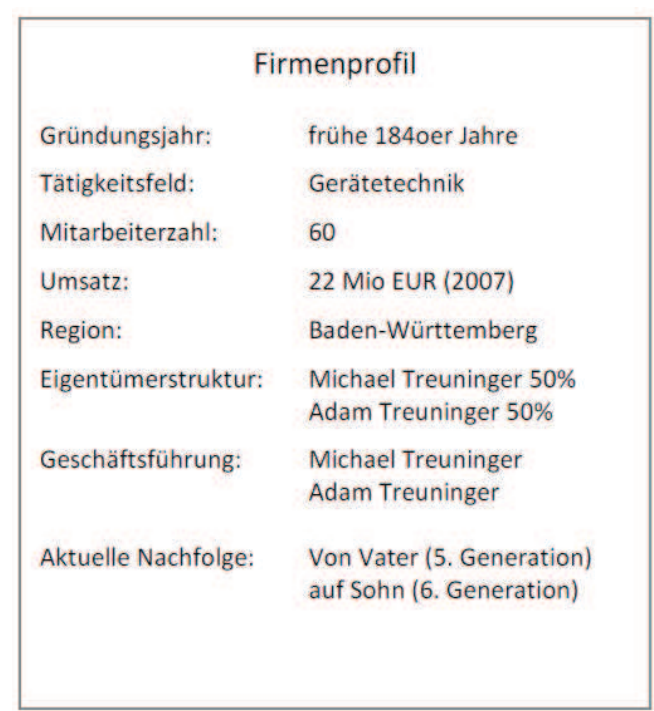

Adam forciert die Mechani-sierung und baut den Handwerksbetrieb in einen Industriebetrieb um. Er errichtet ein Fabrikgelände mit Wohnung, auf dem die Familie für die nächsten Jahrzehnte ihren Lebensmittelpunkt haben wird. 1903 setzt sich Adam zur Ruhe und sein Sohn Gustav übernimmt in dritter Generation das Unternehmen. Die Unternehmensentwicklung verläuft über die beiden Weltkriege und die Zeit der Weimarer Republik recht turbulent. Ende der 1930er Jahre treten die beiden Söhne Paul und Walter in die Firma ein; Gustav übergibt die Leitung damit an die vierte Generation. Mit dem Kriegsausbruch steigt die Firma in die Rüstungsproduktion ein. Die beiden Brüder und weitere wesentliche Mitarbeiter verbleiben im Unternehmen und ziehen nicht in den Krieg. Doch im Juli 1944 trifft eine Bombe die Firma, dabei werden Paul und Walter tödlich verletzt. Die Produktion steht in der Folge für ein gutes Jahr still und einige Maschinen werden demontiert. Der Besitz des Unternehmens verteilt sich weiterhin unter den Familienangehörigen, doch die aktive Leitung des Unternehmens übernehmen in den nächsten Jahren Fremdgeschäftsführer. Michael Treuninger wird 1937 als erster Sohn von drei Kindern des Walter Treuninger geboren. Er wächst in der großzügigen Fabrikwohnung auf, die sich seine Mutter mit seinen Großeltern und einer Tante teilen. Nach der zehnten Klasse absolviert Michael eine Ausbildung zum Metallbauer und studiert auf einer Ingenieurhochschule. 1960 tritt er als Assistent der Fremdgeschäftsführung in das marode Familienunternehmen ein und übernimmt drei Jahre später, er ist Mitte zwanzig, die persönliche Haftung. 
Abbildung A 6: Genogramm der Familie Treuninger

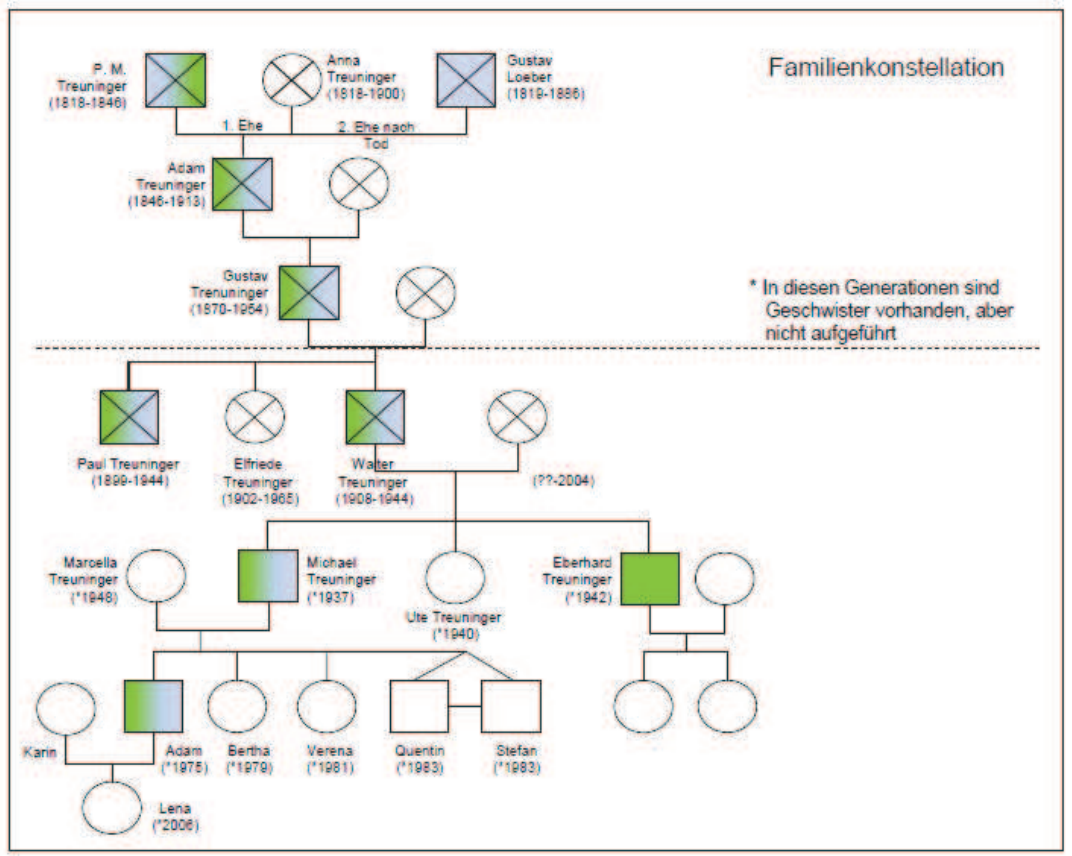

Quelle: eigene Darstellung

Über eine Kontaktanzeige lernt Michael seine spätere Ehefrau Marcella kennen. Sie ist studierte Medizinerin und übt ihren Beruf bis zur Geburt des ersten Sohnes Adam aus. Es folgen vier weitere Kinder. Marcella engagiert sich stark in der Firma, initiiert neue Projekte und durchläuft (fast) alle Abteilungen. Das Unternehmen und die Geschichte der Familie sind fester Bestandteil des familiären Alltags. Der älteste Sohn Adam schließt 1995 das Gymnasium $\mathrm{ab}$ und beginnt Betriebswirtschaft zu studieren. Nach einer Anstellung in der Nähe von Berlin plant er mit dem Vater den Einstieg in das Familienunternehmen. Adam absolviert eine Reihe von Praktika in Partnerbetrieben des Unternehmens in Europa, bis er schließlich im Jahr 2005 eine Stelle als Assistent des Vaters antritt. Im gleichen Jahr heiratet Adam eine alte Schulfreundin und sie bekommen eine Tochter. 2006 gründet Adam zusätzlich eine eigene Firma mit einem verwandten Produktspektrum. 2008 kauft er den Anteil seines Onkels am Unternehmen ab.Die ältere Tochter der Familie studiert zunächst Medizin und schwenkt später auf Psychologie um. Die jüngere 
Tochter studiert ebenfalls Betriebswirtschaft und arbeitet heute in London. Die Zwillinge studieren Maschinenbau. Während Michael noch einige Zeit im Unternehmen arbeiten möchte, hofft Marcella darauf, sich mit der älteren Tochter selbständig machen zu können.

Die Fallgeschichte stellt den Stand von 2008 dar. Interviews wurden mit Michael, Marcella und Adam Treuninger geführt. 


\section{FAMILIE LÖSCH -METALL IN FORM GMBH}

Jannik Lösch wird 1958 als zweites Kind und erster Sohn geboren. Sein Vater ist promovierter Mediziner, übt seinen Beruf jedoch nie aus. Er tritt nach dem Studium in das Unternehmen seines Vaters ein. Als Vertriebsmann im industriellen Bereich ist der Vater selten zu Hause in Hannover. Die Kinder erhalten bereits minderjährig erste Anteile am Unternehmen und bei erfolgreichem Geschäfts-jahr auch eine Dividende. In der Familie wird über die Entwicklung der Firma, Geld und Unternehmertum gesprochen. Die Kinder sind auf den Messen aktiv und haben Kundenkontakt. Im Garten finden Eigentümerversammlungen statt. Die Eltern interessieren

\begin{tabular}{|c|c|}
\hline \multicolumn{2}{|c|}{ Firmenprofil } \\
\hline Gründungsjahr: & frühe 1980er Jahre \\
\hline Tätigkeitsfeld: & Automobilzulieferer \\
\hline Mitarbeiterzahl: & $\begin{array}{l}440 \text { in Deutschland } \\
\text { und Polen }\end{array}$ \\
\hline Umsatz: & 41 Mio EUR (2007) \\
\hline Region: & Brandenburg \\
\hline Eigentümerstruktur: & $\begin{array}{l}\text { Familie Lösch 51\% } \\
\text { davon Jannik 10\% } \\
\text { Private Equity 49\% }\end{array}$ \\
\hline Geschäftsführung: & $\begin{array}{l}\text { Jannik Lösch } \\
\text { Thorsten Feger (extern) }\end{array}$ \\
\hline Aktuelle Nachfolge: & Von Vater auf Sohn \\
\hline
\end{tabular}
sich für Kultur, der Vater sammelt Kunst. Jannik entdeckt bereits während seiner Zeit auf dem Gymnasium das Theater für sich. In dieser Zeit übernimmt der Vater die Vertretung für einen größeren Kunden in Berlin und die Familie begleitet ihn oft dorthin. Nach dem Abitur beginnt Jannik Theaterwissenschaften in Berlin zu studieren. Er finanziert das Studium aus den Ausschüttungen sowie aus Gelegenheitsjobs z.B. als Busfahrer. Nach seinem Abschluss schlägt er sich mit Engagements an Theatern, der Organisation von Ausstellungen sowie Zeitungsartikeln durch. In der Zwischenzeit meldet der Kunde von Janniks Vater in Berlin Konkurs an. Gemeinsam mit einem Partner entschließt sich der Vater dazu, einen Teil der Firma selbständig weiter zu führen. Von nun an ist er für zwei Tage in der Stadt und baut das Unternehmen neu auf. Mitte der achtziger Jahre wird Jannik eine Stelle am Theater in einer norddeutschen Stadt angeboten. Nach vier Jahren ist Jannik unzufrieden mit der Theaterwelt und dem hohen Arbeitsaufkommen. Er beginnt eine Promotion, kündigt seine Stelle, spielt mit dem Gedanken nach Australien auszuwandern und wägt eine Reihe von anderen Optionen ab. Währenddessen zahlt Janniks Vater seinen Partner nach Spannungen aus. Er will sich nun ganz um die Firma in Berlin kümmern und gibt seine Stelle im Familienunternehmen auf. Sein Schwiegersohn (der Mann der jüngsten Tochter) übernimmt seinen Posten. Zudem bittet der Vater Janniks älteste Schwester, die eine Managementposition in der Modebranche innehatte, ihn im Unternehmen zu unterstützen, doch sie entscheidet sich dann anders. Ein Jahr später gründet sein Vater ein zweites Partnerunternehmen im Brandenburgischen. 


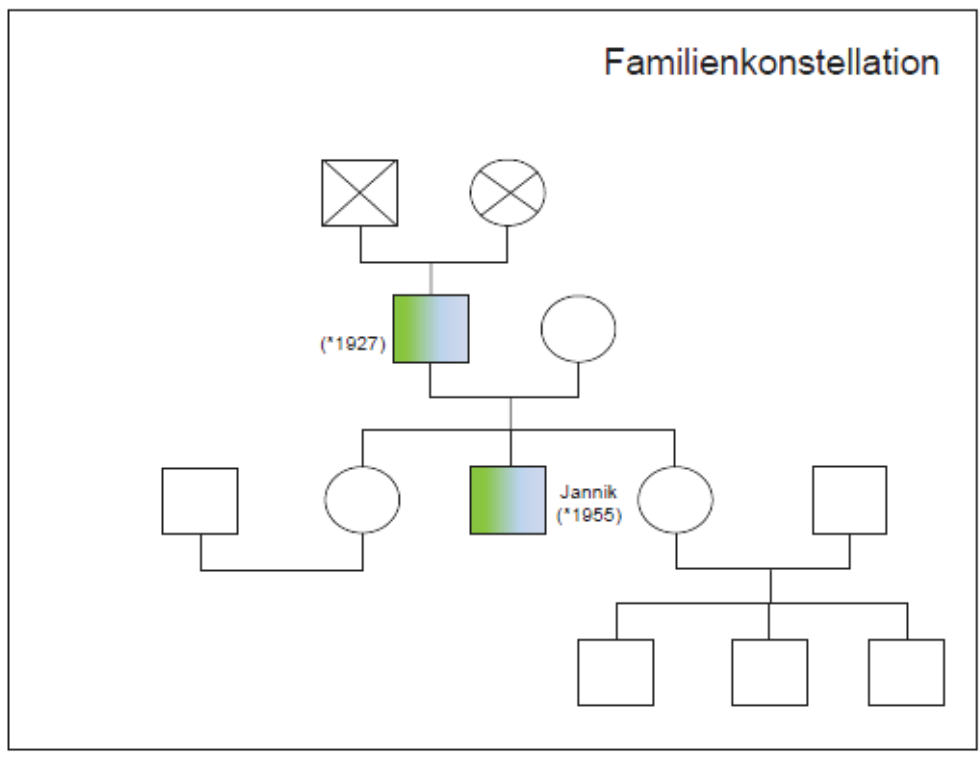

Quelle: eigene Darstellung

Er benötigt dafür einen pro forma Geschäftsführer und bittet Jannik dies für ihn zu übernehmen. Jannik engagiert sich jedoch stärker als ursprünglich gedacht. Er bittet schließlich seinen Vater, die Stelle als Geschäftsführer tatsächlich ausfüllen zu dürfen. Jannik durchläuft die verschiedenen Abteilungen und besucht die Abendschule. Mitte der neunziger Jahre wird er offiziell Geschäftsführer. Das Unternehmen übersteht die Krisenzeiten in der Automobilbranche. Anfang der 2000er Jahre eröffnen sie einen neuen Standort in Polen. Der Vater von Jannik ist ab 2003 nicht mehr operativ tätig. Für eine größere Investition holt sich Jannik schließlich Kapital über einen Private Equity Investor, der von nun an $48 \%$ des Unternehmens besitzt. Die restlichen Anteile gehören weiterhin seinem Vater, der testamentarisch geregelt hat, dass Jannik diese einmal erben wird. Zudem hat Jannik sich über die letzten Jahre 10\% der Anteile des Vaters erkauft. Jannik selbst hat keine Familie und keine Ambitionen das Unternehmen innerhalb seiner erweiterten Familie weiterzugeben. Die Fallgeschichte stellt den Stand von 2008 dar. Es wurde ein Interview mit Jannik Lösch geführt. 


\section{FAMILIE NAUMANN -WEINGUT NAUMANN}

Das Weingut Naumann befindet sich seit den frühen 1780er Jahren im Besitz der Familie. 1864 erbaute der Eigentümer in dritter Genera-tion ein Haus inmitten des Weingutes. In der vierten Generation besitzt seine Tochter das Weingut, die mit einem reichen Unternehmer verheiratet ist. Im 1. Weltkrieg fällt ihr ältester Sohn. Ferdinand, der zweite männliche Nachkomme, stirbt ebenso jung, hinterlässt aber Frau und zwei Kinder: Werner und Amalia. Werner musste nach dem Tod seines Vaters sein begonnenes Studium abbrech-en, zieht mit seiner Frau nach Berlin und wird dort als kaufmännischer Angestellter tätig. Für ihn unerwartet erben er und seine deutlich jüngere Schwester 1927 zu gleichen Teilen das Weingut von der Großmutter. Amalia steht zu diesem Zeitpunkt noch unter der Vormundschaft ihres Ehemannes, einem Anwalt, der zum Verkauf des Weingutes drängt. Werner lehnt ab und führt in der Folgezeit neben seiner Angestelltentätigkeit mit Hilfe eines Verwalters das Weingut in der Pfalz. Im Zweiten Weltkrieg dient Werner als Soldat. In der Zwischenzeit übernimmt seine Frau die kaufmännische Leitung des Gutes. Nach den turbulenten Kriegsjahren stirbt 1948 der Gutsverwalter. Daraufhin zieht Werner mit seinem Sohn Felix Walter $\left({ }^{*} 1932\right)$ auf das Gut. Sie leben sehr beengt mit sieben anderen Familien, die hier eine Notunterkunft gefunden haben. Werner Naumann übernimmt von nun an die Führung des Betriebes, ist aber auch weiterhin berufstätig als kaufmännischer Angestellter. Felix Walter schließt 1953 sein Abitur ab. Nach einer Ausbildung zum Groß- und Außenhandelskaufmann studiert er Volks- und Betriebswirtschaft in Frankfurt und Uppsala, er promoviert in Wien. Im Anschluss an eine Tätigkeit als wissenschaftlicher Assistent beginnt er in gehobener Position in einem Organ der Weinbranche zu arbeiten. Er heiratet Ottilie, die aus einer enteigneten Adelsfamilie aus den Ostgebieten stammt und zieht mit ihr zu Felix Walters Familie auf das Gut. Sie bekommen drei Söhne und eine Tochter. 1982 stirbt Werner Naumann, der seinen Anteil des Gutes vollständig an Felix Walter vererbt. Es kommt zu Streitigkeiten innerhalb der Familie über die Zukunft des Weingutes: Felix Walter schlägt ein Angebot eines Vetters aus, eine Ablöse für seine Anteile zu erhalten und einen externen Geschäftsführer einzusetzen. 
Abbildung A 8: Genogramm der Familie Naumann

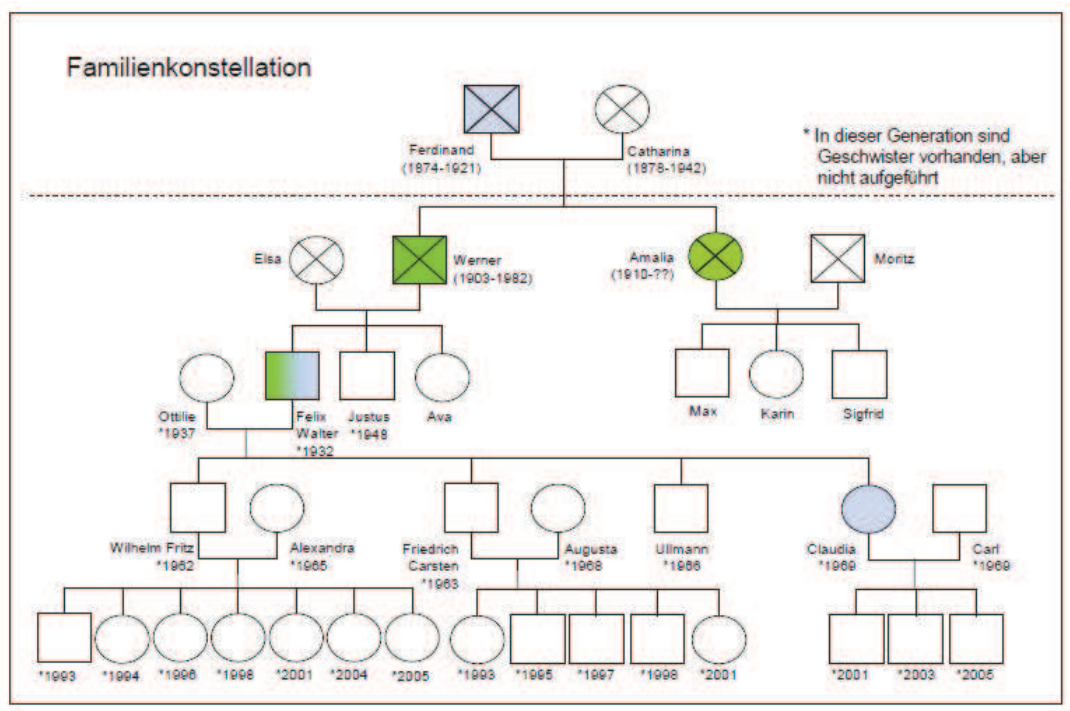

Quelle: eigene Darstellung

Stattdessen kauft er den fünfzig Prozentanteil der Tante per Kredit zurück. Seine Frau unterstützt ihn bei der kaufmännischen Leitung des Weingutes. Die Familie von Felix Walter lebt weiter auf dem Gut. Die Söhne, insbesondere die beiden älteren, sind auf dem Gut engagiert und werden als potentielle Nachfolger angesehen. Der Vater legt Wert auf ihre berufliche Selbständigkeit und alle drei Söhne studieren Jura. Die Tochter absolviert eine Ausbildung zur Physiotherapeutin - wie schon ihre Mutter. 1990 thematisiert der zweite Sohn die Möglichkeit einer Nachfolge, doch der Vater will selbst noch einige Jahre das Weingut führen. 1997 geht Felix Walter nach 35 Jahren Tätigkeit als Angestellter im öffentlichen Dienst in den Ruhestand. Von nun an engagiert er sich Vollzeit als Geschäftsführer im Weingut. Sein ältester Sohn ist als Rechtsanwalt und Notar in Berlin tätig, hat sieben Kinder und lebt in Berlin. Der zweitgeborene Sohn ist ebenfalls Rechtsanwalt und betreibt mit seiner Frau einen Hof in Brandenburg, sie haben fünf Kinder. Auch der jüngste Sohn ist als Anwalt in Berlin tätig und ledig. Claudia, die Tochter der Familie, hat nach einem Studium der Germanistik eine zeitlang im Verlagswesen gearbeitet. Inzwischen hat sie einen Unternehmer geheiratet, die beiden haben drei Kinder.2005 erwerben Claudia und ihre Familie einen größeren Hof ganz in der Nähe des Weingutes. Nach aufwendiger Renovierung verlagern sie ihren Lebensmittelpunkt in die Pfalz. Claudia hat in den letzten Jahren bereits ihren Vater bei seinen Tätigkeiten im Weingut unterstützt. Sie kümmert sich um ihre Kinder und ist in der Kirchengemeinde aktiv. Sie wird in den 
nächsten Jahren allmählich die Nachfolge ihres mittlerweile 76-jährigen Vaters antreten.

Die Fallgeschichte stellt den Stand von 2008 dar. Es wurden Interviews mit Felix Walter, Wilhelm Fritz und Friedrich Carsten Naumann sowie mit Claudia Nentzick geführt. 


\section{FAMILIE NiEST -NIEST MASCHINEN GMBH}

Konrad Niest wird im Jahr 1904 in Ostpreußen geboren. Nach einer Ausbildung zum Elektriker geht er 1923 nach Berlin. Mit einem jüdischen Elektroingenieur macht er sich selbständig: sie reparieren und vertreiben Maschinen. 1936 emigriert sein Partner nach Brasilien. Im Jahr darauf wird Konrad Beauftragter des Wirtschaftsministeriums. 1938 hei-ratet Konrad und das Paar bekommt eine Tochter. Über die Kriegsjahre wird der Betrieb durch Bomben schwer beschädigt und unter russischer Besatzung demontiert. Die Familie von Konrad lebt in dieser Zeit außerhalb von Berlin. 1945 ist sie erneut schwanger, doch Mut-

\begin{tabular}{|c|c|}
\hline \multicolumn{2}{|c|}{ Firmenprofil } \\
\hline Gründungsjahr: & späte 1920er Jahre \\
\hline Tätigkeitsfeld: & Vertrieb von Maschinen \\
\hline Mitarbeiterzahl: & 60 \\
\hline Umsatz: & 10,5 Millionen \\
\hline Region: & Berlin \\
\hline Eigentümerstruktur: & $\begin{array}{l}\text { Konrad Niest (Vater) 60\% } \\
\text { Malte Niest (Sohn) 10\% } \\
\text { Onkel 10\% } \\
\text { Sandra Niest (Tochter) 10\% } \\
\text { Stiefmutter 10\% }\end{array}$ \\
\hline Geschäftsleitung: & Konrad \& Malte Niest \\
\hline Aktuelle Nachfolge: & $\begin{array}{l}\text { Großmutter (1. Gen) und } \\
\text { Vater (2. Gen) auf Sohn/ } \\
\text { Enkel (3. Gen). }\end{array}$ \\
\hline
\end{tabular}
ter und Sohn versterben im Wochenbett. In der Nachkriegszeit baut Konrad das Unternehmen wieder auf. Seit 1940 arbeitet Gudrun $(* 1922$ in Berlin) bei ihm in der Buchhaltung. Die beiden werden ein Paar und heiraten im Jahr 1948. Sie ziehen gemeinsam mit seiner Tochter und ihren Eltern in ein großes Haus. 1950 wird Konrad (junior), ein paar Jahre später ein weiterer Sohn geboren. Die Eltern arbeiten im Unternehmen während sich die Großmutter (mütterlicherseits) um die drei Kinder kümmert. Der Betrieb entwickelt sich in den folgenden Jahren positiv: Sie ziehen auf ein größeres Grundstück, bauen Lagerhallen und Werkstätten an. Die Söhne gehen auf das Gymnasium und arbeiten in den Ferien im Unternehmen (Büro und Werkstatt) und am Wochenende im Olympiastadion. Nach dem Abitur beginnen die beiden Brüder Wirtschaftsingenieurwesen an der TU Berlin zu studieren. 1975 tritt Konrad (junior) in das Unternehmen des Vaters ein. Er heiratet im Jahr darauf seine Jugendliebe (Lehrerin). Sein jüngerer Bruder entscheidet sich das Studium zwar zu beenden, den Beruf aber nie auszuüben. Stattdessen wird er Medizin studieren und sich als Frauenarzt in einer anderen Stadt niederlassen. Konrad (junior) wird 1979 zunächst Vater von Malte und zwei Jahre später von Sandra Niest. Kurz darauf erleidet Konrad (senior) mehrere Schlaganfälle und verstirbt 1984. Das bis dahin als Personengesellschaft geführte Unternehmen wird nun in eine GmbH umgewandelt mit Gudrun und Konrad (junior) als Geschäftsführer. Konrad beginnt seiner Halbschwester ihren 10\% Anteil über eine monatliche Rente abzukaufen. Mit dem Fall der Mauer und der Wiedervereinigung ändert sich die Marktsituation grundlegend. Das Unternehmen entwickelt sich konstant. 
Abbildung A 9: Genogramm der Familie Niest

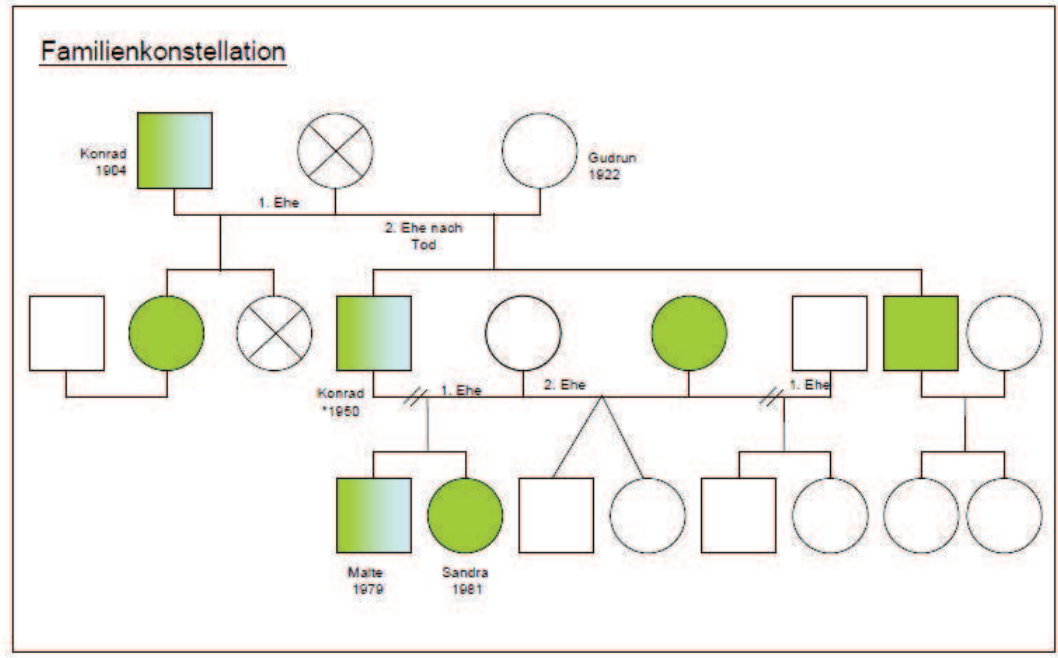

Quelle: eigene Darstellung

1994 trennt sich Konrads Ehefrau von ihm und er lebt mit den beiden Kindern alleine. Ein paar Monate später lernt er seine neue Lebensgefährtin kennen, die selbst zwei Kinder ( 3 und 4 Jahre) mit in die Beziehung bringt. Als sie heiraten und noch Zwillinge bekommen, ziehen Konrads leibliche Kinder (16 und 14 Jahre) in eine eigene Wohnung im gleichen Stadtteil. Malte arbeitet nebenbei im Unternehmen. Nachdem er das Abitur geradeso bestanden hat, leistet er den Grundwehrdienst in einer Nahkampfeinheit. Anschließend nimmt er ein Studium zum Wirtschaftsingenieur an der Technischen Universität Berlin auf. Sandra beginnt ein wenig später Politologie und Publizistik zu studieren. Malte absolviert eine Reihe von unternehmensnahen Praktika und lernt Englisch, Französisch und Spanisch. Direkt nach dem Studium tritt er in das Familienunternehmen ein. Seine Schwester zeigt kein Interesse am Unternehmen. 2006 verschenkt Konrad Unternehmensanteile an seine zweite Ehefrau, Malte und Sandra - sie erhalten jeweils 10\%. Im Jahr darauf wird Malte Niest als Geschäftsführer im Handelsregister eingetragen und Gudrun Niest gibt nach 67 Jahren ihre Tätigkeit im Unternehmen auf (doch sie behält ihr Büro). Das Unternehmen wächst stark und mehrere Niederlassungen werden gegründet. Während der Finanzkrise 2008 stellt das Unternehmen auf Kurzarbeit um. Konrad (junior) will noch ca. 6-7 Jahre arbeiten und sich dann zur Ruhe setzen. Die Fallgeschichte stellt den Stand von 2008 dar. Interviews wurden mit Gudrun, Konrad (junior) und Malte Niest geführt. 


\section{FAMILIE Vester/WAGNER - PfÄLZER MÜHLE}

Barbara Vester (geboren 1950 in Berlin) macht nach dem Abitur eine Ausbildung in der Hotellerie. Sie arbeitet in verschiedenen größeren Städten in Deutschland. 1973 lernt sie ihren späteren Mann kennen, der als stellvertretender Küchenchef bei dem gleichen Arbeitgeber beschäftigt ist. Nach der Geburt des zweiten Sohnes entscheidet sich die Familie dazu, sich in der Gastronomie selbständig zu machen. Sie führen zunächst einen TennisClub und später ein HotelRestaurant. Der unternehmerische Erfolg bleibt aus und der Ehemann von Barbara nimmt zusätzlich eine Stelle als Koch an. Auf dem Heimweg verunglückt er tödlich bei einem Verkehrsunfall. Barbara entscheidet sich dazu, den Betrieb abzugeben und ernährt die Familie mit diversen Jobs und Tätigkeiten im Gastronomiebereich. Zur besseren Vereinbarung von Familie und Beruf nimmt sie nach ein paar Jahren schließlich eine Stelle als Telefonverkäuferin an und lernt auf diese Weise ihren zweiten Ehemann Florian Wagner kennen. Dieser wird als jüngstes Kind einer Winzerfamilie geboren und besucht nach einer Ausbildung zum Koch die Hotelfachschule. Für einige Jahre arbeitet er als Geschäftsführer von diversen Stadthallen, bis er eine davon als selbständiger Pächter übernimmt. Zudem ist Florian stark auf Verbandsebene engagiert. Die beiden kaufen sich nach wenigen Monaten ein Haus und Barbara beginnt in seinem Restaurant zu arbeiten. Sie sind beruflich stark eingebunden. Oscar und Andreas besuchen ab 1990 ein Internat, beide helfen an den Wochenenden im Unternehmen aus. Oscar beginnt nach der Hauptschulreife eine Ausbildung zum Restaurantfachmann und arbeitet in diversen Hotels und auf einem Kreuzfahrtschiff. Andreas studiert Betriebswirtschaft und arbeitet ebenfalls in der Hotelbranche. Die beiden Brüder üben Ehrenämter in Branchenverbänden aus. Ende der 1990er Jahre übernehmen Barbara und Florian ein zusätzliches Restaurant, die „Alte Eiche" in einem nahe gelegenen Ort. Sowohl Oscar als auch Andreas arbeiten immer wieder für einige Monate in einem der beiden Betriebe mit. In dieser Zeit interessieren sich die Eltern bereits für das Gelände der „Pfälzer Mühle“ und unterbreiten der Stadt ein Kaufangebot, erhalten jedoch den Zuschlag nicht. 2004 beginnt Oscar fest als Restaurantleiter im alten Betrieb der Eltern zu arbeiten. 
Abbildung A 10: Genogramm der Familie Vester/Wagner

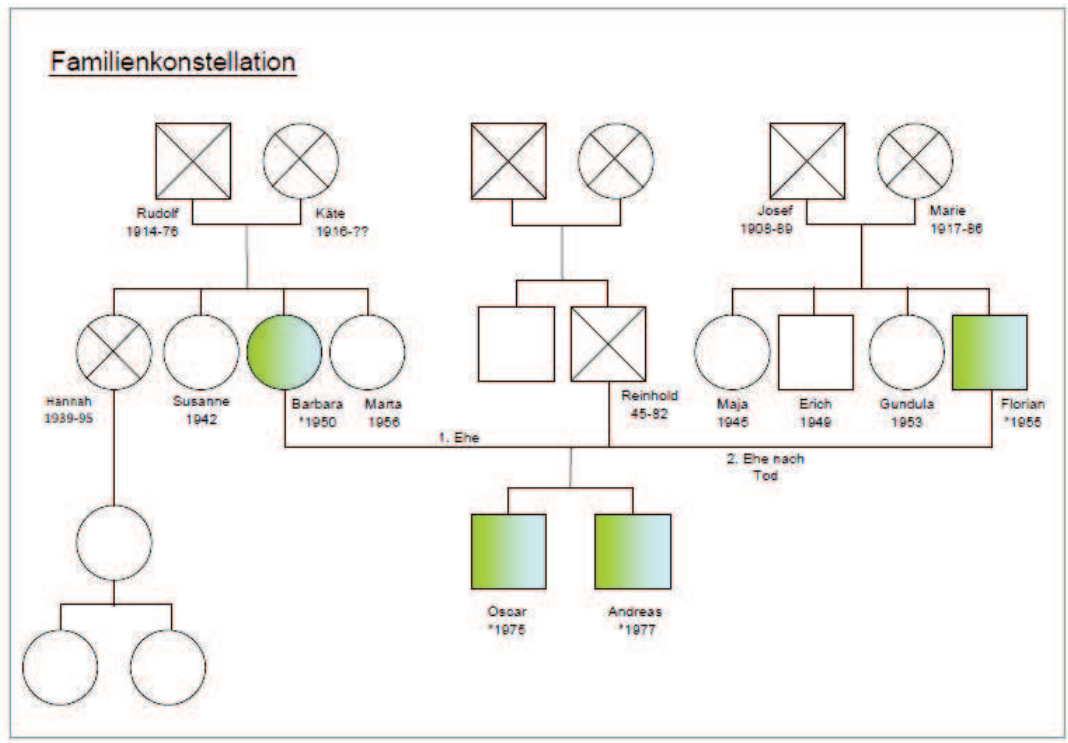

Quelle: eigene Darstellung

Ein paar Jahre später gelingt es Barbara und Florian, die „Pfälzer Mühle“, ein Gelände mit mehreren Restaurants, zu erwerben. Die Bank erteilt den dafür notwendigen Kredit lediglich, wenn eine entsprechende Nachfolgeregelung getroffen wird. Sowohl Oscar als auch Andreas entscheiden sich dafür gemeinsam mit den Eltern den Gastronomiebetrieb zu gründen und zu leiten. Alle vier werden gleichberechtigte Teilhaber und Geschäftsführer. Die Familie übernimmt sehr langfristige Kreditverträge. Nach einem größeren Umbau wird die Mühle eröffnet und das alte Restaurant aufgegeben. Jedes der vier Familienmitglieder ist für ein spezifisches Aufgabengebiet zuständig. Alle wohnen in unmittelbarer Nähe zum Gelände. Im Zuge der Erbregelung heiraten Barbara und Florian nach 22 Jahren. Florian adoptiert die beiden Söhne. Im Jahr darauf kündigt der Geschäftsführer der „Alten Eiche“ und Oscar übernimmt seine Position. Florian ist müde und gesundheitlich angeschlagen und will sich in ein paar Jahren aus dem Betrieb zurückziehen, während Barbara hofft, noch viele Jahre aktiv sein zu können.Die Fallgeschichte stellt den Stand von 2008 dar. Interviews wurden mit Barbara, Oscar und Andreas Vester sowie Florian Wagner geführt. 


\section{FAMILIE SINGERMAYER -SINGERMAYER GMBH}

Ende der 1830er Jahre eröffnet der yer einen Laden und Werkstatt in einer süddeutschen Stadt. Er heiratet Luise, die den Laden führt. Insgesamt haben sie elf Kinder, von denen der erst-geborene Carl sowie vier Schwestern überleben. Nach der Handelsschule geht Carl in die Lehre bei seinem Vater und anschließend auf Wanderjahre quer durch Europa. Er heiratet Therese und übernimmt 1867 das stark wachsende Geschäft des Vaters. Er errichtet am Stadtrand ein großes Fabrikgebäude und gründet neue Verkaufsfilialen. 1883 verunglückt Carl bei einem Verkehrs-unfall tödSchneidermeister Justus Singerma-

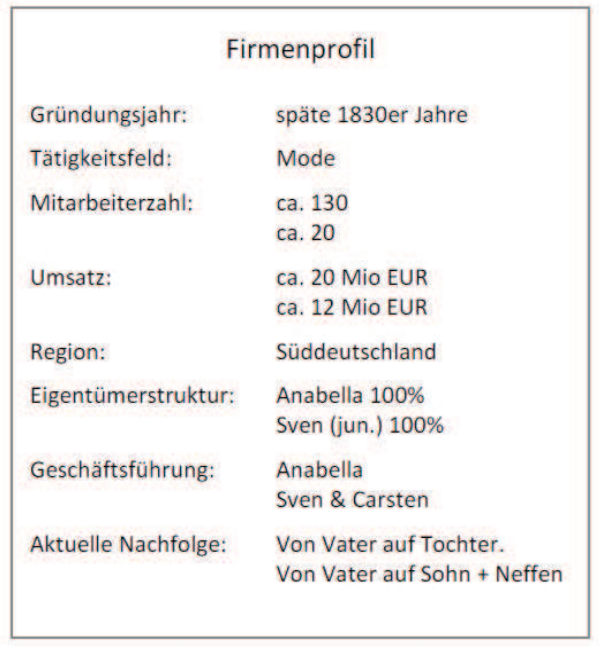

lich. Seine Frau übernimmt mit zwei leitenden Mitarbeitern kommissarisch die Führung des Unternehmens. Sein Sohn Hugo ist zu diesem Zeitpunkt 16 Jahre alt. Er absolviert eine Ausbildung und geht für vier Jahre ins Ausland und wird schließlich Geschäftsleiter des Unternehmens. Hugo heiratet Lieselotte und die beiden haben drei Kinder. Sein jüngster Sohn Hugo Franz studiert BWL und steigt als Teilhaber ins Familienunternehmen ein. In den 1920er Jahren gerät das Unternehmen in finanzielle Nöte. Lieselotte investiert einen Teil ihres Privatvermögens. Während den Weltkriegen produziert das Unternehmen Gasmasken und beschäftigt im Dritten Reich Zwangsarbeiter und KZ-Häftlinge. Hugo Franz hat vier Kinder, die während den Kriegsjahren auf einem Einödbauernhof aufwachsen. Nach dem Krieg befindet sich das Unternehmen für zwei Jahre unter der Führung eines Treuhänders (Entnazifizierung). Hugo Franz baut das völlig desolate und zerstörte Unternehmen neu auf. Seine Tochter studiert BWL, sein ältester Sohn ist im Brauereigewerbe, sein mittlerer Sohn Sven will Medizin studieren und der jüngste Sohn macht eine Ausbildung im Familienunternehmen und soll Nachfolger werden. Doch 1960 verstirbt der jüngste Sohn mit 18 Jahren beim Bergsteigen. Der Vater fragt alle Kinder nach ihren Plänen und Sven willigt ein, den Platz seines Bruders im Betrieb einzunehmen. Sven rouliert durch verschiedene Abteilungen und belegt Kurse an der Universität, dort trifft er auch seine spätere Frau Marie. Anschließend absolviert er verschiedene Praktika in England, Frankreich und Italien. 1966 übernimmt er schließlich in 5. Generation die Geschäftsführung. Sven und Marie bekommen vier Kinder und leben etwas außerhalb der Stadt. 
Abbildung A 11: Genogramm der Familie Singermayer

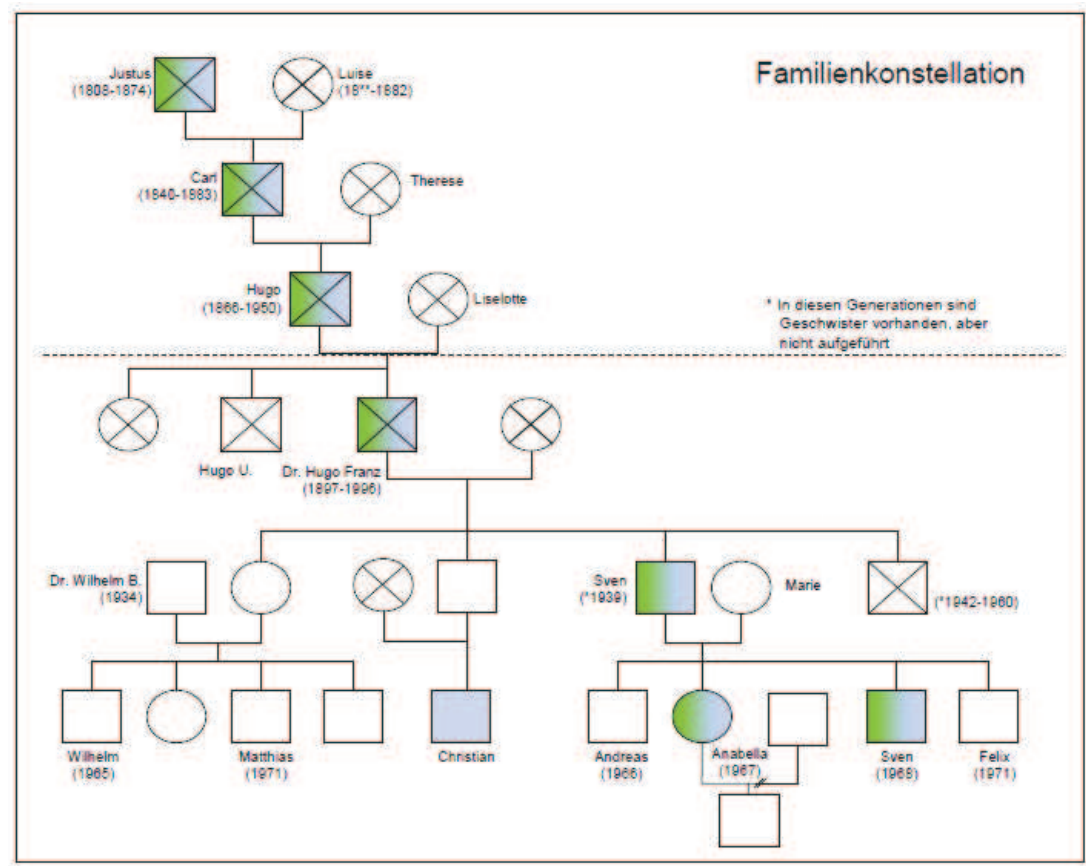

Quelle: eigene Darstellung

1972 äußert Svens älterer Bruder den Wunsch auch im Unternehmen tätig zu werden. Daraufhin gliedert er einen Teil als separate Firma aus. 1980 beginnt Marie im Bereich Unternehmenskommunikation zu arbeiten. Ihr ältester Sohn studiert Tiermedizin und betreibt später eine eigene Klink. Ihre Tochter wird nach dem Abitur schwanger. Sie absolviert allein erziehend eine Ausbildung und später ihren Handelsfachwirt im Unternehmen. Ihr jüngerer Bruder studiert BWL und arbeitet bei einer Unternehmensberatung. Der Jüngste der Familie studiert Humanmedizin. Anfang der 90er Jahre gibt Svens Bruder sein Unternehmen nach 20 Jahren auf. Er hat einen Sohn aus erster Ehe (im Kindbett verstorben) und zwei Stiefkinder aus zweiter Ehe (Die zweite Ehe ist nicht im Genogramm aufgeführt). Seine älteste Schwester ist nach Mitteldeutschland gezogen und hat dort einen Unternehmer geheiratet. Mitte der 90er Jahre bespricht Sven die Nachfolge mit allen Kindern. Außer Anabella möchte niemand das Unternehmen weiterführen. Erste Anteile und kompensatorisch Immobilien werden übertragen. 1999 entschließt sich Sven (junior) nun doch 
zum Einstieg in das Familienunternehmen. Daraufhin teilt Sven (senior) das Unternehmen in zwei Sparten auf. 2003 zieht er sich zu einem festen Stichtag aus dem Unternehmen zurück, ein Jahr später folgt ihm auch seine Frau in den Ruhestand. 2005 kommt es zu Schwierigkeiten zwischen den Geschwistern, woraufhin die wirtschaftliche und rechtliche Trennung der beiden Sparten veranlasst wird. 2006 steigt Christian, der Neffe von Sven (senior) in das Unternehmen des Sohnes als zweiter Geschäftsführer ein.

Die Fallgeschichte stellt den Stand von 2007 dar. Interviews wurden mit Sven (senior) und Anabella Singermayer geführt. 


\section{FAMILIE ÜBERMANN -MARKUS ÜBERMANN WEIN}

Mitte der 1970er Jahre heiraten Beate und Richard Übermann. Beide stammen aus landwirtschaftlich tätigen $\mathrm{Fa}$ milien. Beate hat eine Ausbildung zur Fachver-käuferin und arbeitet bei Konsum. Richard ist Weinküfer in der LPG. Mit der Hochzeit zieht das Paar auf den Hof ihrer Eltern in einem sehr kleinen Dorf in Ostdeutschland, hier leben und arbeiten sie mit vier Generationen. 1978 wird Sohn Markus geboren, 1983 kommt Tochter Nadine zur Welt. Während der DDR-Jahre arbeiten beide Vollzeit und bewirtschaften am Abend und am Wochenende den Hof mit

\section{Firmenprofil}

Gründungsjahr: frühe 1990er Jahre

Tätigkeitsfeld: Wein

Mitarbeiterzahl: $\quad 4$

Umsatz:

nicht bekannt

Region: Ostdeutschland

Eigentümerstruktur: Markus Übermann 100\%

Geschäftsführung: Markus Übermann

Aktuelle Nachfolge: Von Mutter (1. Generation) auf Sohn (2. Generation) Tieren und Gemüse. Die Kinder helfen aktiv in der Landwirtschaft mit. Mit der Wende verändert sich die Situation der Familie grundlegend: Die LPG wird in eine Genossenschaft umgewandelt. Konsum wird von Edeka übernommen und Beate Übermann verliert ihre Anstellung. Es besteht die Möglichkeit, jene Ländereien zurückzuerhalten, die vormals in Familienbesitz waren. Die Übermanns entschließen sich dazu, einige Hektar Weinberg selbständig zu bewirtschaften. Offiziell wird Beate Übermann Geschäftsführerin. Sie arbeitet sich allmählich in diese für sie neue Materie ein: übernimmt nicht nur das Kaufmännische und Organisatorische, sondern ist auch in der Weinernte und -produktion tätig. Letzteres ist das Spezialgebiet ihres Mannes, der jedoch weiterhin Vollzeit erwerbstätig bleibt. 1993 pachtet die Familie eine weitere Fläche von 3,5 Hektar und setzt ca. 11.000 Rebstöcke neu. 1995 erhalten sie die erste Auszeichnung für einen ihrer Weine. Sie bauen den Betrieb stetig um und aus. Markus absolviert nach der Realschule zunächst eine Ausbildung zum Elektriker. Anschließend zieht er ins Rheingau, um dort Winzer zu lernen. Schließlich besucht er eine Fachschule für Weinbautechnik. Während seiner Ausbildung arbeitet er viel im Unternehmen der Eltern mit. Nadine Übermann lernt im Hotelfach und besucht eine Weiterbildung zur Fach-Betriebswirtin. Auch sie hilft im elterlichen Weinbetrieb aus. 
Abbildung A 12: Genogramm der Familie Übermann

\section{Familienkonstellation}

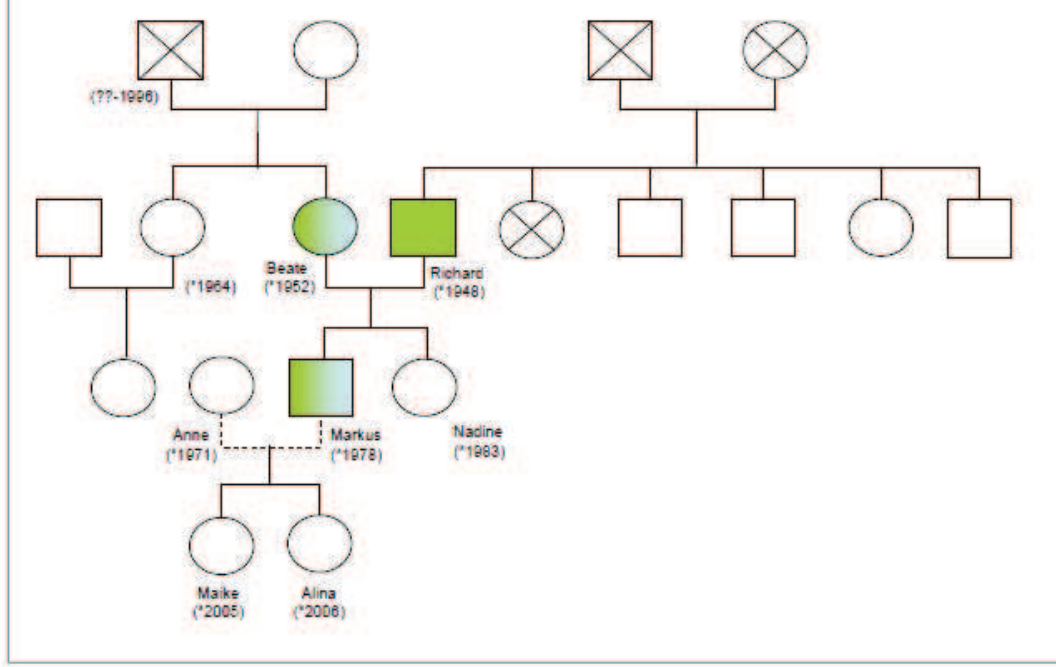

Quelle: eigene Darstellung

Im Jahr 2003 erkrankt und überlebt Beate Übermann den Brustkrebs. Sie bekommen zunächst einen Betriebshelfer gestellt, doch Markus steigt in den Betrieb ein. Er stößt eine Reihe von Neuerungen an: z.B. neue Etiketten, neue Vermarktungsstrukturen, Solaranlage, Ferienwohnungen etc. Zwei Jahre später erfolgt die offizielle Übernahme des Betriebes durch den Sohn. Markus zieht mit seiner Partnerin auf den Hof der Familie. Die beiden bekommen zwei Mädchen. Seine Freundin beginnt im Betrieb zu helfen (Computer). Nadine wollte in ihrem Weinberg (Erbausgleich) eine Gaststätte bauen und betreiben, doch erhält keine Baugenehmigung. Sie bewirtschaftet mit ihrem Freund (Gartenbauer) den Weinberg und liefert die Trauben beim Bruder ab. Heute arbeitet sie in einem größeren Unternehmen der Branche als Gästebetreuerin. Beate und Richard sind nach wie vor im Unternehmen aktiv - auch wenn sie nicht in erster Reihe stehen. Insbesondere das Expertenwissen des Vaters ist dem Sohn wertvoll, der einen lebenslangen Platz auf dem Hof für seine Eltern sieht. Die Fallgeschichte stellt den Stand von 2008 dar. Interviews wurden mit Beate, Richard und Markus Übermann geführt. 


\section{FAMILIE Von MOLINE -WeINGUT SCHLOSS QUELLENBURG}

Die Familie von Moline ist adeliger Abstammung, die Linien können über 900 Jahre zurückverfolgt werden. Ein Familienzweig siedelte im 18. Jahr-hundert in den Osten um. Dort unterhielten sie eine Reihe von Unternehmen, Land und Gebäuden. Mit der Hochzeit von Claus und Florentina von Moline gelangt das Schloss Quellenburg mit dazu gehörenden Ländereien in den Besitz der Familie. Hier haben sie ihren Lebensmittelpunkt. Claus ist Kunst interessiert und legt Wert auf gute Bildung. Seine beiden Söhne Clemens und Trauthelm wachsen mit einem Hauslehrer auf. Trauthelm von Moline erwirbt eine Vielzahl von Abschlüssen und gilt als bedeutender Unternehmer in den Ostgebieten. Wie in der Familie üblich heiratet auch er adelig. Gemeinsam mit Paulina bekommen sie zwischen 1937 und 1945 sechs Kinder. 1943 wird das Schloss Quellenburg von den Nationalsozialisten besetzt. Nach dem Ende des zweiten Weltkrieges beschließt die Familie auf dem Schloss zu verbleiben. Kurz darauf werden sie enteignet, von der russischen Besatzung verhaftet und später in ein Zuchthaus gesteckt. Der Vater verliert alle Papiere. In den Kriegswirren stirbt das zweitjüngste Kind der Familie. Schließlich wird die Familie nach Norddeutschland zur Großmutter mütterlicherseits ausgewiesen. Sie lassen sich in einer ländlichen Gegend nieder und der Vater nimmt eine Reihe von Gelegenheitsjobs an, bis er eine Anstellung als Geschäftsführer in einem Betonunternehmen erhält. Während der DDRÄra werden die Ländereien von Schloss Quellenburg von der damaligen Landwirtschaftlichen Produktions-Genossenschaft (LPG) bewirtschaftet. 1957 wird Gabriel als Nachzügler der Familie geboren. Nach dem Abitur lässt sich Gabriel zunächst als Reserveoffizier der Bundeswehr ausbilden und studiert anschließend Agrar- und Betriebswirtschaft. Dann absolvierte er ein Aufbaustudium zum Wirtschaftsingenieur und promoviert 1991. Schon während dem Studium handelt Gabriel mit hochwertigen Modeprodukten. Nach dem Studium findet er eine Anstellung als externer Geschäftsführer in einem Familienunternehmen. 
Abbildung A 13: Genogramm der Familie von Moline

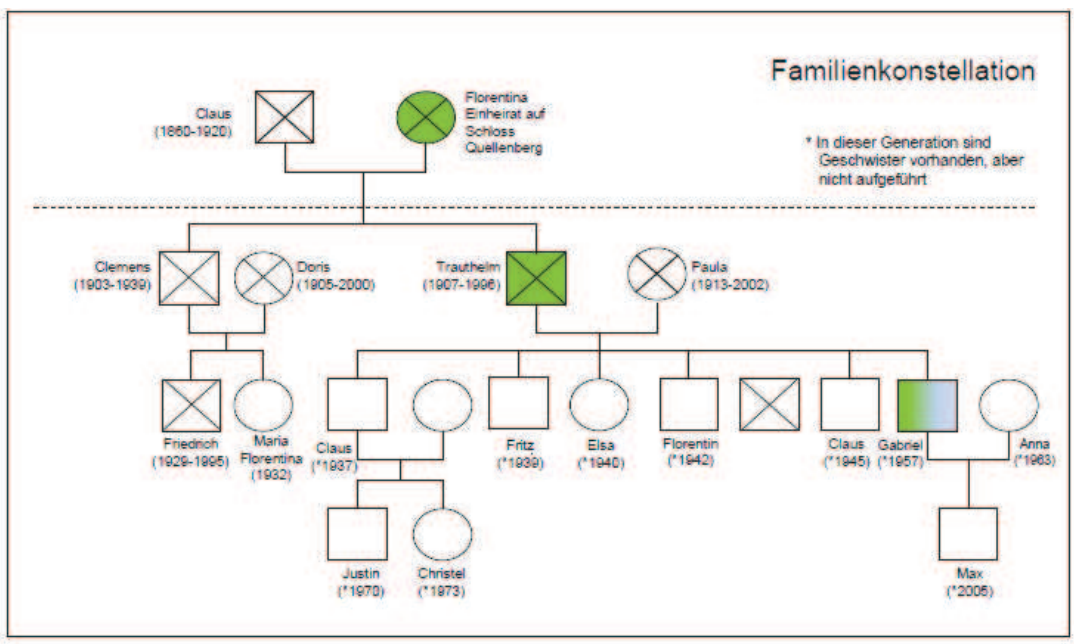

Quelle: eigene Darstellung

Auf Wunsch des Vaters besichtigt Gabriel 1990 einige der alten Besitztümer und Firmen der Familie. Er entscheidet sich dafür, einige Ländereien zu erwerben und wieder selbst zu bewirtschaften. Für diesen lan kündigt er seine Anstellung. Zudem bemüht sich Gabriel um den Rückkauf von Schloss Quellenburg. Die Verhandlungen laufen nur sehr schleppend und so kauft er 1995 einen nahe gelegenen Hof, um einen Mittelpunkt für die Landwirtschaft zu schaffen. Zwei Jahre später (und erst nach dem Tod des Vaters) gelingt es ihm schließlich doch noch, das Schloss zu erwerben. Er nutzt dies als Veranstaltungsstätte. Gabriel heiratet eine Architektin und die beiden bekommen im Jahr 2005 einen Sohn.

Die Fallgeschichte stellt den Stand von 2008 dar. Es wurde ein Interview mit Gabriel von Moline geführt. 


\section{Unsere Fachzeitschriften auf www.budrich-journals.de}

- Einzelbeiträge im Download

- $\quad$ Print + Online

Abonnements

- Online-Freischaltung über IP

- mit open access-Bereich

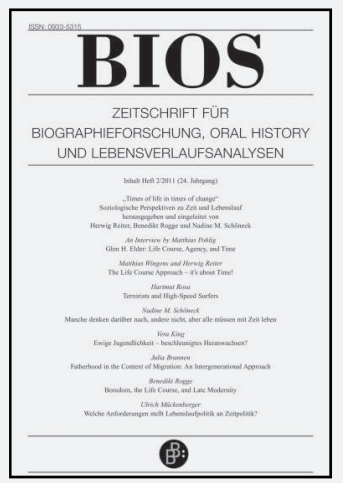

Sozialwissenschaftliche Fachzeitschriften Online!

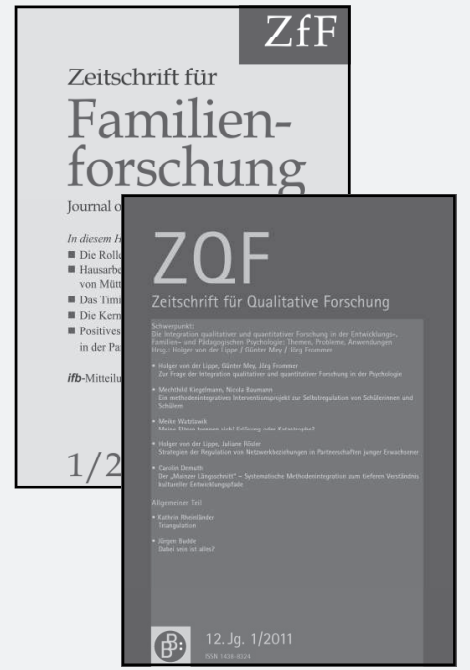

Als Abonnentln z.B. mit KombiAbo Print + Online bekommen Sie weiterhin $\mathrm{lhr}$ Heft wie gewohnt bequem nach Hause geliefert und Sie haben Zugriff auf das gesamte Online-Archiv.

Fragen Sie uns!

\section{Verlag Barbara Budrich •}

\section{Barbara Budrich Publishers}

Stauffenbergstr. 7. D-51379 Leverkusen Opladen

Tel +49 (0)2171.344.594 • Fax +49 (0)2171.344.693 •

info@budrich-journals.de • www.budrich-journals.de 